\title{
Pathogenicity of a minimal organism: Role of protein phosphorylation in
}

\section{Mycoplasma pneumoniae}

\author{
Dissertation \\ zur Erlangung des mathematisch-naturwissenschaftlichen Doktorgrades \\ „Doctor rerum naturalium“ \\ der Georg-August-Universität Göttingen
}

vorgelegt von

Sebastian Schmidl

aus Bad Hersfeld

Göttingen 2010 
Mitglieder des Betreuungsausschusses:

Referent: Prof. Dr. Jörg Stülke

Koreferent: PD Dr. Michael Hoppert

Tag der mündlichen Prüfung: 02.11.2010 
"Everything should be made as simple as possible, but not simpler."

(Albert Einstein) 


\section{Danksagung}

Zunächst möchte ich mich bei Prof. Dr. Jörg Stülke für die Ermöglichung dieser Doktorarbeit bedanken. Nicht zuletzt durch seine freundliche und engagierte Betreuung hat mir die Zeit viel Freude bereitet. Des Weiteren hat er mir alle Freiheiten zur Verwirklichung meiner eigenen Ideen gelassen, was ich sehr zu schätzen weiß.

Für die Übernahme des Korreferates danke ich PD Dr. Michael Hoppert sowie Prof. Dr. Heinz Neumann, PD Dr. Boris Görke, PD Dr. Rolf Daniel und Prof. Dr. Botho Bowien für das Mitwirken im Thesis-Komitee. Der Studienstiftung des deutschen Volkes gilt ein besonderer Dank für die finanzielle Unterstützung dieser Arbeit, durch die es mir unter anderem auch möglich war, an Tagungen in fernen Ländern teilzunehmen.

Prof. Dr. Michael Hecker und der Gruppe von Dr. Dörte Becher (Universität Greifswald) danke ich für die freundliche Zusammenarbeit bei der Durchführung von zahlreichen Proteomics-Experimenten. Ein ganz besonderer Dank geht dabei an Katrin Gronau, die mich in die Feinheiten der 2D-Gelelektrophorese eingeführt hat. Außerdem möchte ich mich bei Andreas Otto für die zahlreichen Proteinidentifikationen in den letzten Monaten bedanken. Nicht zu vergessen ist auch meine zweite Außenstelle an der Universität in Barcelona. Dr. Maria Lluch-Senar und Dr. Jaume Piñol gilt ein besonderer Dank bei der morphologischen Untersuchungen von Mutanten sowie zahlreichen lustigen Abenden auf Tagungen.

Für die gute Arbeitsatmosphäre und tatkräftige Unterstützung im Labor möchte ich mich bei Julia Busse bedanken. Speziell natürlich für die unzähligen Slot Blots. Ein besonderer Dank geht auch an Hinnerk Eilers für die vielen anregenden Diskussionen rund um die kleinen Biester. Viel Erfolg in Umeå! Meinem Nachfolger Arne Schmeisky wünsche ich alles Gute und hoffe, dass er das Mycoplasmen-Mekka weiterhin gut vertreten wird. Dr. Petra Neumann-Staubitz danke ich für die vielen unterhaltsamen Gespräche im S2-Labor. Mal sehen, ob ihr Schilderwahn einmal ein Ende nimmt! Weiterhin bedanke ich mich bei meinen alteingesessenen sowie neuen Mitstreitern Katrin Gunka, Christine Diethmaier, Christoph Wrede, Nico Pietack, Lope A. Flórez, Martin Lehnik, Frederik Meyer und Fabian Rothe, die in den letzten 3 Jahren viel zu einer entspannten und kreativen Arbeitsatmosphäre beigetragen haben. Ein großer Dank für die Erleichterung des Laboraltages geht auch an unsere „Pufferella“ Bärbel Herbst. Möge sie wieder zu ihrer alten Stärke zurückfinden! Bei der Praktikantin Sandra Appelt, 
den Bachelor-Studenten Stephanie Großhennig, Miriam Bothe und Daniel Reuß sowie den Diplomanden Meike Ridderbusch und Pavel Dutow bedanke ich mich für die tatkräftige Unterstützung bei vielen Projekten. Hoffentlich haben sie meinen kleinen Exkurs in die „Advanced and Applied Mycoplasmology“ gut überstanden!? Außerdem danke ich noch all jenen, die ich nicht namentlich erwähnen konnte, die mich aber dennoch bei der Erstellung dieser Arbeit unterstützt haben.

Mein ganz spezieller Dank gilt meiner Familie. Ohne die moralische Unterstützung meiner Eltern und meiner Schwester hätte ich weder mein Studium noch meine Doktorarbeit schaffen können. Dafür ein riesengroßes Dankeschön! Zum Schluss gilt mein Dank meiner Freundin Anke, die mich immer wieder neu motiviert und so sehr an mich glaubt. 


\section{Contents}

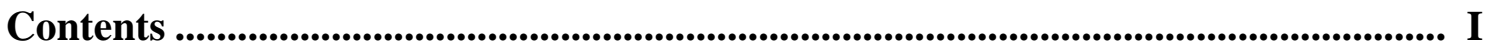

List of abbreviations ................................................................................... III

List of publications........................................................................................................... VII

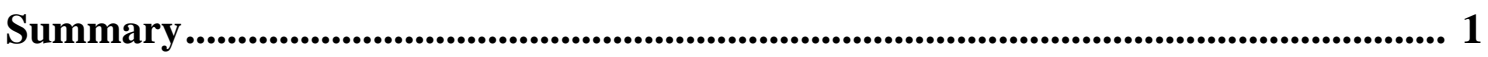

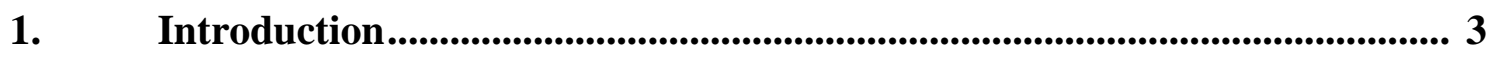

(A) Mycoplasma and Spiroplasma................................................................ 3

(B) Aims of this work .................................................................................. 30

2. The stability of cytadherence proteins in Mycoplasma pneumoniae requires activity of the protein kinase PrkC .......................................... 31

3. The phosphoproteome of the minimal bacterium

Mycoplasma pneumoniae: Analysis of the complete known

Ser/Thr kinome suggests the existence of novel kinases.............................. 52

4. In vitro phosphorylation of key metabolic enzymes from

Bacillus subtilis: PrkC phosphorylates enzymes from different

branches of basic metabolism 109

5. Upregulation of thymidine kinase activity compensates for loss of thymidylate synthase activity in Mycoplasma pneumoniae 128

6. Interactions between glycolytic enzymes of Mycoplasma pneumoniae.... 146

7. The hidden pathway: Impact of the glycerophosphodiesterase GlpQ

on virulence of Mycoplasma pneumoniae

8. Discussion 205 


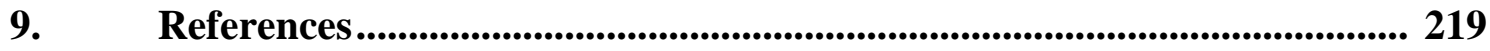

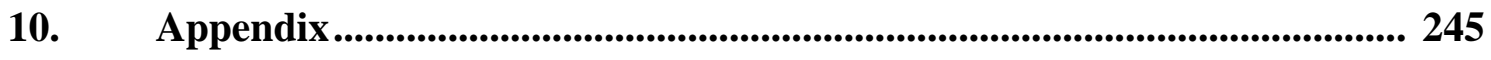

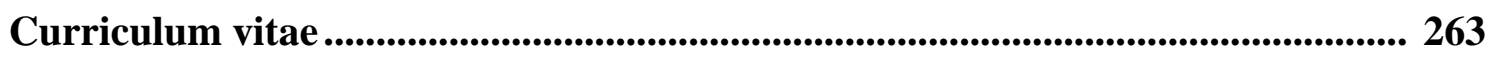




\section{List of abbreviations}

5FdUMP 5-fluorodeoxyuridine monophosphat

A

$\AA$

adenine

$\mathrm{ABC}$

ADP

Amp

Asn

ATP

B2H

bp

BSA

C

$\mathrm{Ca}^{2+}$

ångström

adenosine 5'-triphosphate binding cassette

adenosine 5'-diphosphate

ampicillin

asparagine

adenosine 5'-triphosphate

bacterial two-hybrid

base pair

bovine serum albumin

cytosine

calcium cation

Cam

chloramphenicol

CAMP

cyclic adenosine 5'-monophosphate

CDP-Star

$\mathrm{CH}_{3} \mathrm{CN}$

CHAPS

disodium 2-chloro-5-(4-methoxyspiro \{1,2-dioxetane-3,2'-(5'-chloro)

tricyclo[3.3.1.1 $1^{3,7}$ decan $\left.\}-4-y l\right)$ phenyl phosphate

acetonitrile

ChIP-chip

Cho

3-[(3-cholamidopropyl)dimethylammonio]-1-propanesulfonate

$\mathrm{Ci}$

chromatin immunoprecipitation with microarray technology

choline

$\mathrm{cm}$

curie

centimeter

$\mathrm{CM}$

$\mathrm{cm}^{2}$

CMP

$\mathrm{CO}$

cell membrane

square centimeter

$\mathrm{CO}_{2}$

$\mathrm{CoA}$

cytidine 5'-monophosphate

control

carbon dioxide

coenzyme A

COG

cluster of orthologous groups of proteins

Cys

cysteine

Da

dalton

DHAP

dihydroxyacetone phosphate

DIG

DNA

dT

digoxigenin

deoxyribonucleic acid

thymidine

dTDP

thymidine 5'-diphosphate

dTMP

DTT

thymidine 5'-monophosphate

dTTP

dithiothreitol

$\mathrm{dU}$

thymidine 5'-triphosphate

deoxyuridine

dUMP

deoxyuridine 5'-monophosphate

EDTA

Erm

ethylenediaminetetraacetic acid

erythromycine

ESI

et al.

electrospray ionization

and others

FAD flavin adenine dinucleotide 
Fig.

G

g

G3P

Glc

Glu

Gly

$\mathrm{Gm}$

GPC

GTP

$\mathrm{h}$

$\mathrm{H}$

$\mathrm{H}_{2} \mathrm{O}$

$\mathrm{HCl}$

HEPES

His

HIV

HMW

HPLC

HPrK

i.d.

i.e.

IPG

IPTG

IS

Kan

$\mathrm{kb}$

$\mathrm{KCl}$

$\mathrm{KClO}_{4}$

$\mathrm{kDa}$

$\mathrm{KOH}$

$\mathrm{kPa}$

l

LB

LC

Leu

$\mathrm{M}$

$\mathrm{M}$

$\mathrm{m} / \mathrm{z}$

$\mathrm{mg}$

$\mathrm{Mg}^{2+}$

$\mathrm{MgCl}_{2}$

$\mu \mathrm{g}$

$\mu \mathrm{l}$

$\mu \mathrm{m}$

$\mu \mathrm{M}$

min

$\mathrm{ml}$

$\mathrm{mm}$

$\mathrm{mM}$ figure

guanine

gram

glycerol 3-phosphate

glucose

glutamic acid

glycerol

gentamicin

glycerophosphorylcholine

guanosine 5'-triphosphate

hour

hydrogen

water

hydrochloric acid

4-(2-hydroxyethyl)-1-piperazineethanesulfonic acid

histidine

human immunodeficiency virus

high molecular weight

high performance liquid chromatography

HPr kinase

in diameter

that is

immobilized power of hydrogen gradient

isopropyl- $\beta$-D-1-thiogalactopyranoside

insertion sequence

kanamycin

kilobase

potassium chloride

potassium perchlorate

kilodalton

potassium hydroxide

kilopascal

liter

lysogeny broth

liquid chromatography

leucine

marker

molar

mass-to-charge ratio

milligram

magnesium cation

magnesium chloride

microgram

microliter

micrometer

micromolar

minute

milliliter

millimeter

millimolar 


\begin{tabular}{|c|c|}
\hline mmol & millimole \\
\hline MMR & multiple mutation reaction \\
\hline $\mathrm{Mn}^{2+}$ & manganese cation \\
\hline $\mathrm{MnCl}_{2}$ & manganese chloride \\
\hline MPN & Mycoplasma pneumoniae \\
\hline mRNA & messenger ribonucleic acid \\
\hline MS & mass spectrometry \\
\hline MW & molecular weight \\
\hline na & not available \\
\hline $\mathrm{NaCl}$ & sodium chloride \\
\hline $\mathrm{NAD}^{+} / \mathrm{NADH}$ & nicotinamide adenine dinucleotide (oxidized/reduced form) \\
\hline NADPH & nicotinamide adenine dinucleotide phosphate (reduced form) \\
\hline $\mathrm{NaF}$ & sodium fluoride \\
\hline $\mathrm{NaH}_{2} \mathrm{PO}_{4}$ & monosodium phosphate \\
\hline $\mathrm{NaOH}$ & sodium hydroxide \\
\hline nd & not detectable \\
\hline ng & nanogram \\
\hline $\mathrm{NH}_{4} \mathrm{HCO}_{3}$ & ammonium bicarbonate \\
\hline $\mathrm{Ni}^{2+}$ & nickel cation \\
\hline $\mathrm{nl}$ & nanoliter \\
\hline $\mathrm{nm}$ & nanometer \\
\hline nmol & nanomole \\
\hline ns & no significant difference \\
\hline N-terminal & amino-terminal \\
\hline $\mathrm{OH}$ & hydroxyl group \\
\hline ORF & open reading frame \\
\hline $\mathrm{P}$ & phosphate \\
\hline p.s.i. & pound per square inch \\
\hline PAGE & polyacrylamide gel electrophoresis \\
\hline PBS & phosphate buffered saline \\
\hline PCA & perchloric acid \\
\hline PCR & polymerase chain reaction \\
\hline PEI & polyethylenimine \\
\hline PEP & phosphoenolpyruvate \\
\hline Pfu & Pyrococcus furiosus \\
\hline $\mathrm{pH}$ & power of hydrogen \\
\hline Phe & phenylalanine \\
\hline pI & isoelectric point \\
\hline ppm & parts per million \\
\hline PrkC & protein kinase $\mathrm{C}$ \\
\hline PrpC & protein phosphatase of the PP2C family \\
\hline PRPP & phosphoribosyl pyrophosphate \\
\hline $\mathrm{pS}$ & phosphoserine \\
\hline $\mathrm{pT}$ & phosphothreonine \\
\hline PTS & phosphotransferase system \\
\hline RNA & ribonucleic acid \\
\hline RNAse & ribonuclease \\
\hline rRNA & ribosomal ribonucleic acid \\
\hline s & second \\
\hline sa & similar amount \\
\hline
\end{tabular}


SDS

Ser

Spec

SSC

Str

Strep

$\mathrm{T}$

Tab.

TAP

TCA

Tet

Thr

TLC

Tn

Tris

tRNA

Trp

TY

Tyr

U

UDP

UMP

Urd

UTP

$\mathrm{V}$

$\mathrm{vol} / \mathrm{vol}$

$v s$.

w/o

wt

wt/vol

$\mathrm{X}-\mathrm{Gal}$

zip

$\mathrm{Zn}^{2+}$

$\mathrm{ZnCl}_{2}$ sodium dodecyl sulfate

serine

spectinomycin

saline sodium citrate

streptomycin

Streptavidin (Streptomyces avidinii)

thymine

table

tandem affinity purification

tricarboxylic acid

tetracycline

threonine

thin layer chromatography

transposon

tris(hydroxymethyl)aminomethane

transfer ribonucleic acid

tryptophan

tryptophan yeast

tyrosine

uracil

uridine 5'-diphosphate

uridine 5'-monophosphate

uridine

uridine 5'-triphosphate

version

volume per volume (volume percent)

versus

without

wild type

weight per volume (percentage solution)

5-bromo-4-chloro-3-indolyl- $\beta$-D-galactopyranoside

zipper

zinc cation

zinc chloride 


\section{List of publications}

Stülke, J., H. Eilers, and S. R. Schmidl. 2009. Mycoplasma and Spiroplasma. Encyclopedia of Microbiology (M. Schaechter, ed.), Elsevier, Oxford. p. 208-219.

Schmidl, S. R., K. Gronau, C. Hames, J. Busse, D. Becher, M. Hecker, and J. Stülke. 2010. The stability of cytadherence proteins in Mycoplasma pneumoniae requires activity of the protein kinase PrkC. Infect. Immun. 78: 184-192.

Schmidl, S. R., K. Gronau, N. Pietack, M. Hecker, D. Becher, and J. Stülke. 2010. The phosphoproteome of the minimal bacterium Mycoplasma pneumoniae: Analysis of the complete known Ser/Thr kinome suggests the existence of novel kinases. Mol. Cell. Proteomics 9: 1228-1242.

Pietack, N., D. Becher, S. R. Schmidl, M. H. Saier, M. Hecker, F. M. Commichau, and J. Stülke. 2010. In vitro phosphorylation of key metabolic enzymes from Bacillus subtilis: PrkC phosphorylates enzymes from different branches of basic metabolism. J. Mol. Microbiol. Biotechnol. 18: 129-140.

Wang, L., C. Hames, S. R. Schmidl, and J. Stülke. 2010. Upregulation of thymidine kinase activity compensates for loss of thymidylate synthase activity in Mycoplasma pneumoniae. Mol. Microbiol. 77: 1502-1511.

Dutow, P., S. R. Schmidl, M. Ridderbusch, and J. Stülke. 2010. Interactions between glycolytic enzymes of Mycoplasma pneumoniae. J. Mol. Microbiol. Biotechnol. accepted.

Schmidl, S. R., A. Otto, M. Lluch-Senar, J. Piñol, J. Busse, D. Becher, and J. Stülke. The hidden pathway: Impact of the glycerophosphodiesterase GlpQ on virulence of Mycoplasma pneumoniae. PLoS Pathogens submitted. 


\section{Summary}

Mycoplasma pneumoniae is a human pathogen that belongs to the Mollicutes, a group of bacteria with the smallest genomes that are capable of independent life. The reductive evolution of the Mollicutes is reflected by their limited regulatory features for gene expression. Thus, posttranslational regulation might be important for M. pneumoniae to adapt to environmental changes. Among the very few regulatory proteins retained is the HPr kinase (HPrK), which phosphorylates the phosphocarrier protein $\mathrm{HPr}$ at the Ser-46 residue. This phosphorylation event is a major signal to trigger carbon catabolite repression in less degenerated bacteria. However, the function of $\mathrm{HPr}($ Ser-P) in M. pneumoniae is unknown. For the protein phosphatase PrpC, an implication in the dephosphorylation of $\operatorname{HPr}(\mathrm{Ser}-\mathrm{P})$ could be shown. In addition to HPrK, the M. pneumoniae prkC gene encodes another serine/threonine protein kinase C.

The determination of the complete phosphoproteome of $M$. pneumoniae by two-dimensional gel electrophoresis and mass spectrometry allowed the detection of 63 phosphorylated proteins, including many enzymes of central carbon metabolism and proteins related to host cell adhesion. It was also possible to detect 16 phosphorylation sites, among them 8 serine and 8 threonine residues. However, a comparison with the phosphoproteomes of other bacteria revealed that there is only a weak conservation of phosphorylation sites, even if the same proteins are phosphorylated in related organisms. There is only one exception: The phosphorylation of phosphosugar mutases on a conserved serine residue, which could be detected in all studied organisms from archaea and bacteria to man. In the case of the phosphosugar mutase ManB in M. pneumoniae, it could be shown that this protein undergoes autophosphorylation. In conclusion, the results indicate that protein phosphorylation seems to be highly specific for each individual organism.

For a more detailed analysis of the phosphorylation network in M. pneumoniae, the phosphoproteomes of the wild type strain and of three isogenic mutants that are affected in the two protein kinases HPrK and PrkC and in the protein phosphatase PrpC were compared. Examination of the phosphorylation profile of the hprK mutant revealed that only HPr is phosphorylated by HPrK, whereas six proteins, including the major adhesin P1 and two cytadherence proteins HMW1 and HMW3, were affected by the loss of PrkC. In contrast, inactivation of PrpC that antagonizes PrkC-dependent phosphorylation resulted in more intensive phosphorylation of the same target proteins. 
The phenotypic characterization of prkC mutant cells revealed a nonadherent growth type along with a loss of cytotoxicity toward HeLa cells. Thus, posttranslational modification of cytadherence proteins by PrkC is essential for cell adhesion and virulence in M. pneumoniae.

The phosphoproteomic analysis demonstrated that several glycolytic enzymes are subject to phosphorylation. M. pneumoniae uses glycolysis as the major pathway for the generation of energy by substrate-level phosphorylation. Using a bacterial two-hybrid approach, the enolase was identified as the central glycolytic enzyme of M. pneumoniae due to its ability to directly interact with all other glycolytic enzymes. Moreover, most of the glycolytic enzymes performed self-interactions. The results support the idea that glycolysis proceeds in a well structured manner even in a minimal organism.

In its natural habitat, $M$. pneumoniae thrives on pulmonary surfaces that are mainly composed of phosphatidylcholine. This phospholipid can be integrated directly into the cell membrane or serve as precursor for cellular processes. $M$. pneumoniae possesses two potential glycerophosphodiesterases, MPN420 (GlpQ) and MPN566, that are able to cleave deacylated phospholipids to glycerol 3-phosphate and choline. Further glycerol 3-phosphate utilization by enzymes of the glycerol metabolism is crucial for the cytotoxicity of $M$. pneumoniae due to hydrogen peroxide release. Biochemical studies showed that GlpQ is active as a glycerophosphodiesterase, whereas MPN566 has no enzymatic activity in vitro. Mutants affected in either glycerophosphodiesterase revealed that inactivation of mpn566 did not result in any phenotype. In contrast, the glpQ mutant exhibited a growth defect in glucose-supplemented medium. Moreover, the lack of GlpQ resulted in an absence of hydrogen peroxide formation in the presence of deacylated phospholipids and a loss of cytotoxicity toward HeLa cells. These observations imply that GlpQ is important for the pathogenicity of $M$. pneumoniae, but also for other functions in the cell. Indeed, proteomic and transcriptomic analyses of the wild type and the $g l p Q$ mutant strain suggested a GlpQ-dependent transcription regulation, which led to higher or lower protein amounts of the glycerol facilitator, a subunit of a metal ion ABC transporter, and three lipoproteins. Interestingly, all genes subject to GlpQ-dependent control have a conserved potential cis-acting element upstream of the coding region. Nevertheless, it is open for speculation whether GlpQ or a transcription factor that is controlled by GlpQ is responsible for this regulation. 


\section{Chapter 1}

\section{Introduction}

\section{(A) Mycoplasma and Spiroplasma}

This chapter is part of the following publication:

Stülke, J., H. Eilers, and S. R. Schmidl. 2009. Mycoplasma and Spiroplasma. Encyclopedia of Microbiology (M. Schaechter, ed.), Elsevier, Oxford. p. 208-219.

Author contributions:

This review was written by JS, HE, and SRS. SRS performed the systematics of the Mollicutes, on which the first two chapters are based as well as partial researches on biochemistry, genetics, and molecular biology of the Mollicutes. 


\section{Defining statement}

Mycoplasma and Spiroplasma species are bacteria that lack a cell wall (the Mollicutes). These organisms evolved in close association with their eukaryotic hosts, resulting in an extreme genome reduction. In this article, the biology of the Mollicutes is discussed with special emphasis on their pathogenicity, cell biology, and molecular biology.

\section{Introduction}

Mycoplasmas and spiroplasmas are two important genera of the bacterial group called Mollicutes. The name Mollicutes - soft skin - reflects the major collective characteristic of these bacteria - the lack of a cell wall - which at the same time distinguishes them from all other bacteria with the exception of the chlamydiae. The lack of a cell wall is caused by the absence of genes encoding enzymes for peptidoglycan biosynthesis. The lack of a cell wall is closely linked to another characteristic feature of the Mollicutes - their cells are usually pleomorphic. Again, there is no rule without exception: The cells of the genus Spiroplasma have a helical shape (see "Cytology of the Mollicutes").

Another important feature of the Mollicutes is their close association with eukaryotic host organisms. In nature, Mollicutes are never found as free-living organisms. Hosts are either animals including humans (Mycoplasma, Ureaplasma) or plants and insects (Spiroplasma, Phytoplasma) (Table 1). Mycoplasma species usually cause mild diseases such as atypical pneumonia (Mycoplasma pneumoniae) or nongonococcal urethritis (Mycoplasma genitalium). However, there is an interesting exception: Mycoplasma alligatoris, a pathogen of alligators, causes lethal infections. Although the infections caused by Mollicutes are rarely lethal, Mollicutes pathogenic for plants and animals cause a significant economic loss in agriculture. This is true for cattle in Africa that are infected by Mycoplasma mycoides as well as for rice crops in some regions of Southeast Asia that are infected by phytoplasmas. These losses not only have an economic dimension but also a significant effect on human nutrition in the affected regions. Mycoplasma species such as Mycoplasma hyorhinis or Acholeplasma laidlawii are major sources of cell culture contamination and have gained increasing 
interest. These infections are often discovered only late in the course of an experiment and can invalidate the scientific research.

The close association of Mollicutes with eukaryotic hosts and their adaptation to habitats with a good nutrient supply and relatively constant growth conditions led to a remarkable process of reductive genome evolution. The organism with the smallest known genome capable of independent life (if provided with rich artificial medium) is M. genitalium, a human pathogen. This organism has a genome size of only $580 \mathrm{~kb}$ and encodes about 480 proteins, as compared to about 4 million bp and 4000 genes for bacteria such as Escherichia coli or Bacillus subtilis. These small genomes made the Mollicutes important tools for the new discipline of synthetic biology (see "Genomic comparisons of Mollicutes”).

\section{The systematics of the Mollicutes}

Evolution of the Mollicutes. The analysis and comparison of 16S rRNA sequences revealed that the Mollicutes belong to the Gram-positive bacteria with genomes of low GC content. Ironically, most members of this phylum are characterized by their thick Gram-positive cell wall, and the group is therefore referred to as the Firmicutes. This bacterial phylum includes the lactic acid bacteria (such as Streptococcus and Lactobacillus), spore-forming bacteria (Bacillus and Clostridium) and their close relatives (Listeria and Staphylococcus). As can be seen in the phylogenetic tree of the Firmicutes (Fig. 1), the Mollicutes form a sister group to the large Bacillus/lactic acid bacteria group. It is believed that the first Mollicutes emerged some 600 million years ago and that significant loss of ancestral genomic sequences was a major force in the evolution of the Mollicutes.

The Mollicutes are subdivided in several ways. Three traditional classifications rely on genetic or physiological properties of the bacteria, whereas more recent classification schemes are based on the similarity of the 16S rRNA or conserved protein families. 
Tab. 1. The systematic groups of the Mollicutes.

\begin{tabular}{|c|c|c|c|c|c|}
\hline Order & Genus & Genome size & Sterol requirement & Characteristics & Habitat \\
\hline \multirow[t]{4}{*}{ Mycoplasmatales } & Mycoplasma & $580-1350 \mathrm{~kb}$ & Yes & Growth optimum: $37^{\circ} \mathrm{C}$ & Humans, animals \\
\hline & & & & UGA as Trp codon & \\
\hline & Ureaplasma & $760-1170 \mathrm{~kb}$ & Yes & Urea hydrolysis & Humans, animals \\
\hline & & & & UGA as Trp codon & \\
\hline \multirow[t]{6}{*}{ Entomoplasmatales } & Entomoplasma & $790-1140 \mathrm{~kb}$ & Yes & Growth optimum: $30^{\circ} \mathrm{C}$ & Insects, plants \\
\hline & Mesoplasma & $870-1100 \mathrm{~kb}$ & No & Growth optimum: $30^{\circ} \mathrm{C}$ & Insects, plants \\
\hline & & & & UGA as Trp codon & \\
\hline & Spiroplasma & $780-2200 \mathrm{~kb}$ & Yes & Growth optimum: $30-37^{\circ} \mathrm{C}$ & Insects, plants \\
\hline & & & & UGA as Trp codon & \\
\hline & & & & Helical motile filaments & \\
\hline \multirow[t]{2}{*}{ Anaeroplasmatales } & Anaeroplasma & $1500-1600 \mathrm{~kb}$ & Yes & Obligate anaerobes & Bovine/ovine rumen \\
\hline & Asteroleplasma & $1500 \mathrm{~kb}$ & No & Obligate anaerobes & Bovine/ovine rumen \\
\hline \multirow[t]{4}{*}{ Acholeplasmatales } & Acholeplasma & $1500-1650 \mathrm{~kb}$ & No & Growth optimum: $30-37^{\circ} \mathrm{C}$ & Animals, plants, insects \\
\hline & & & & UGA as stopp codon & \\
\hline & Phytoplasma & $640-1185 \mathrm{~kb}$ & Not known & Uncultured in vitro & Insects, plants \\
\hline & & & & UGA as stopp codon & \\
\hline
\end{tabular}


Two large groups of Mollicutes can be distinguished based on their host organisms. Although most Mollicutes infect exclusively animal hosts, there are other representatives (Spiroplasma and Phytoplasma) that are capable of infecting both plant and insect hosts. Another conventional way of classifying the Mollicutes is based on their requirement for sterols. Most genera need sterols for growth, whereas this is not the case for the members of the genus Acholeplasma (see Table 1). However, this requirement can only be determined for those Mollicutes that can be cultivated, and many (perhaps most) representatives have not yet been cultured, including all species of the genus Phytoplasma. Another peculiarity of most Mollicutes is their codon usage: They use the UGA codon to specify tryptophan rather than as a stop codon as in the universal genetic code. Only the genera Acholeplasma and Phytoplasma among the Mollicutes use UGA as a stop codon. Because this is the ancestral property, it can be assumed that Acholeplasma and Phytoplasma represent the more ancestral Mollicutes. This conclusion is supported by a phylogenetic tree based on a concatenated alignment of 30 protein families present in all Mollicutes that places the genus Phytoplasma at the bottom of the tree (Fig. 1). The genus Acholeplasma is not included in this analysis because of the lack of genome sequence information. It is interesting to note that the genus Mycoplasma is paraphyletic, and that genera such as Spiroplasma, Mesoplasma, and Ureaplasma have specific relatives among the different Mycoplasma clades (Fig. 1).

For practical reasons, the Mollicutes are grouped in four orders that do not represent the phylogenetic relationships. An overview of these taxa is provided in Table 1.

Mycoplasma. As mentioned earlier, the genus Mycoplasma is a paraphyletic collection of Mollicutes that are widespread in nature as parasites of humans, mammals, birds, reptiles, and fish. The first representative of the genus Mycoplasma was identified in 1898 as the causative agent of contagious bovine pleuropneumonia (M. mycoides). The human pathogens Mycoplasma hominis and $M$. pneumoniae were discovered in 1937 and 1944, respectively. Even now, new species are being identified: In 1981, M. genitalium was isolated from a patient suffering from nongonococcal urethritis, and more recently, Mycoplasma penetrans and Mycoplasma fermentans were found to be associated with HIV infections. 


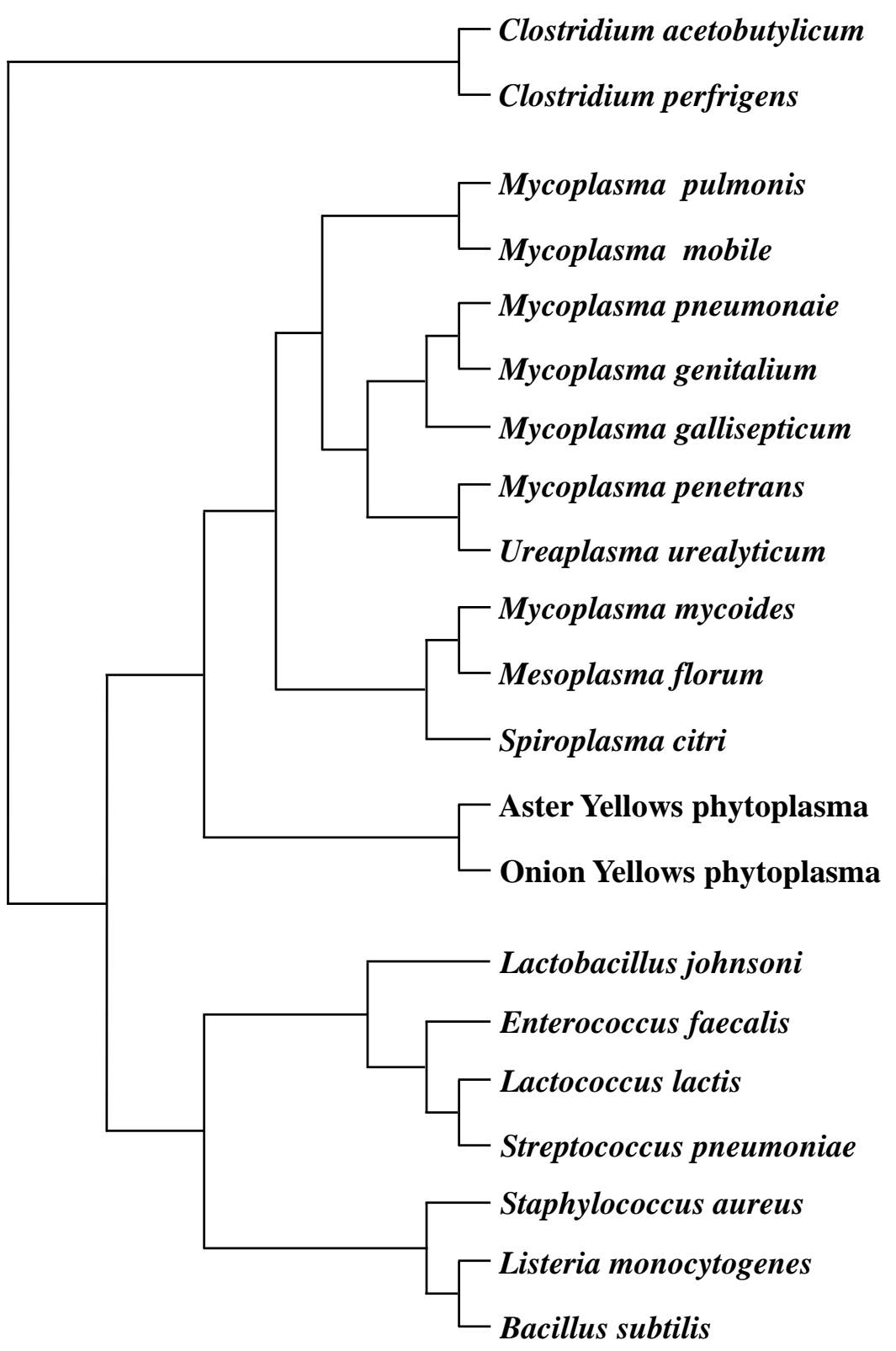

Fig. 1. Unrooted phylogenetic tree of the Firmicutes with special emphasis to the Mollicutes. The tree is based on a concatenated alignment of 31 universal protein families. Reproduced from Ciccarelli et al. (2006).

The primary habitats of human and animal mycoplasmas are the mucous surfaces of the respiratory and urogenital tracts, the eyes, the alimentary canal, and mammary glands. In addition, cell cultures are an artificial habitat for many Mycoplasma species. The mycoplasmas exhibit a rather strict host and tissue specificity, probably reflecting their highly specific metabolic demands and their parasitic lifestyle. For example, $M$. pneumoniae and $M$. genitalium are preferentially detected in the respiratory and urogenital tracts, respectively. 
If cultivated in the laboratory, mycoplasmas as well as other Mollicutes require complex media containing sugars, amino acids, nucleotides, and vitamins. It has so far been impossible to cultivate them on chemically defined media.

The complete genome sequences of ten species of the genus Mycoplasma have been determined so far. This large interest in the variability of the Mycoplasma genetic complement is stimulated by the interest in creating artificial organisms based on the Mycoplasma species (i.e., synthetic biology; see "Genomic comparisons of Mollicutes"). The genome sequences revealed the reason for the complex nutritional requirements of the mycoplasmas: They lack the genes for many biosynthetic pathways and are thus dependent on their host or on the artificial medium to provide these required nutrients. Another interesting feature revealed by genome sequences is that only very few known regulatory proteins are present. Again, this is reflective of their close adaptation to one single natural habitat and a result of the reductive evolution: While a metabolically versatile bacterium such as Pseudomonas aeruginosa that is capable of thriving in a wide variety of environments reserves as much as $10 \%$ of its genome for regulatory genes, only a handful of these genes is found in the mycoplasmas (see “Gene expression in the Mollicutes”).

Pathogenicity has been most intensively studied with $M$. pneumoniae. In contrast to most other pathogenic bacteria, M. pneumoniae and other Mollicutes do not seem to produce any exo- or endotoxins. However, a recent study suggests the formation of a protein similar to ADP-ribosylating and vacuolating cytotoxin. However, this observation has not been confirmed by other groups. A major factor contributing to cytotoxicity and thus to pathogenicity of $M$. pneumoniae is the formation of hydrogen peroxide. The synthesis of hydrogen peroxide by mycoplasmas is most strongly increased if the bacteria are supplied with glycerol. This can be attributed to the oxidase activity of the enzyme that oxidizes glycerol 3-phosphate. This enzyme, glycerol-3-phosphate oxidase, uses water rather than $\mathrm{NAD}^{+}$(as in typical glycerol-3-phosphate dehydrogenases) as the electron acceptor. The hydrogen peroxide formed by $M$. pneumoniae acts in concert with endogenous toxic oxygen molecules generated by the host cells and induces oxidative stress in the respiratory epithelium. The effects of the peroxide on the host cells include loss of reduced glutathione, denaturation of hemoglobin, peroxidation of erythrocyte lipids, and eventually the lysis of the cells. Another result of infection by $M$. pneumoniae is the release of 
proinflammatory cytokines by the host cells. It has been suggested that cytokine production leads to chronic pulmonary diseases such as bronchial asthma.

The significance of glycerol metabolism in hydrogen peroxide production and virulence has been convincingly demonstrated by a series of studies that started with an analysis of the differences between European and African strains of M. mycoides, the causative agent of contagious bovine pleuropneumonia. Glycerol transport is highly efficient in the African isolates, whereas it is barely detectable in the European isolates. Because glycerol catabolism gives rise to the formation of hydrogen peroxide, it is not surprising that hydrogen peroxide production is high in the African strains but low in the European isolates of $M$. mycoides. In consequence, the African strains are highly virulent to cattle, whereas their European relatives are harmless. It has been hypothesized that intracellular formation of large quantities of hydrogen peroxide would be toxic for the producing cells themselves. Accordingly, the cellular localization of the responsible enzyme, GlpO, was studied in M. mycoides and it was found to be located in the cell membrane. The inactivation of GlpO by antibodies results in the loss of cytotoxicity of $M$. mycoides toward bovine epithelial cells. Given that hydrogen peroxide in concentrations similar to those produced by $M$. mycoides is not cytotoxic, it was concluded that GlpO is not only inserted in the bacterial cell membrane, but also in the membrane of the host cell to inject the cytotoxic hydrogen peroxide directly into the epithelial cells. This may cause oxidative stress and subsequent cell death.

Plant pathogenic Mollicutes: Spiroplasma and Phytoplasma. The genera Spiroplasma and Phytoplasma contain plant pathogenic Mollicutes that shuttle between plant and insect hosts. Spiroplasma citri was identified in 1971 as a causative agent of citrus stubborn disease. Phytoplasmas were first described in 1967 as the probable cause of plant yellow diseases. Originally, it was speculated that these diseases are of viral origin, and only in 1967 it became clear that these pathogens are Mycoplasma-like organisms. While spiroplasmas can be cultivated in the laboratory, no cultivation of any representative of the phytoplasmas has been reported. Therefore, no valid species description for members of the genus Phytoplasma is available. Moreover, Spiroplasma cells have a spiral morphology, whereas phytoplasmas are pleomorphic. 
Spiroplasma species live in the phloem sieve tubes of their host plants. They are transmitted by insect vectors that feed on the phloem sap. Multiplication of the bacteria occurs both in the plant and in the insect hosts. The most intensively studied representative of the genus, S. citri, infects periwinkle (Catharanthus roseus) and its vector, the leafhopper Circulifer haematoceps. Unfortunately, no genome sequences of any Spiroplasma species are so far publicly available, although the Spiroplasma kunkelii genome has recently been sequenced.

The spiroplasmas are unique among the Mollicutes for their helical cell morphology, and also by their unique mechanism of locomotion. The genetic determinants for this distinct morphology and movement are so far unknown. Although the spiroplasmas have a shape that is similar to that of the members of the genus Spirillum, they are different because they do not possess flagella. Propulsion is generated by a propagation of kink pairs down the length of the cell, caused by a processive change of cell helicity. In addition, these waves of kinks seem to be initiated always by the same end of the cell suggesting cell polarity. Cell polarity can also be concluded from the results of diverse microscopic studies that showed heterogeneity of both ends: One end is tapered with a tip-like structure called terminal organelle and the other one is blunt or round.

An interesting aspect of the $S$. citri lifecycle is the differential utilization of carbohydrates as source of carbon and energy in the two hosts. S. citri possesses the genetic equipment for the utilization of sorbitol, trehalose, glucose, and fructose as carbon sources, which are mainly catabolized to acetate. The two habitats of S. citri differ significantly in their carbon source availability. While glucose and fructose are predominant in phloem sieve tubes of plants, trehalose is the major sugar in the hemolymph of the vector insect, the leafhopper $C$. haematoceps. The glucose and trehalose permeases of the $S$. citri phosphotransferase system (PTS) share a common IIA domain encoded by the crr gene, which might be involved in the rapid physiological adaptation to changing carbon supplies. The glucose and fructose found in the plant sieve tubes are both derived from the cleavage of sucrose by the plant enzyme invertase. A transposon mutagenesis study with $S$. citri revealed that mutants devoid of a functional fruR gene encoding the transcriptional activator of the fructose utilization operon are no longer phytopathogenic. The fructose operon of S. citri contains three genes, fruR, fruA, and fruK encoding the transcription activator, the fructose-specific 
permease of the PTS, and the fructose-1-phosphate kinase, respectively. Mutations in the fruA and fruK genes also resulted in decreased phytopathogenicity. However, these mutant strains could revert, and this reversion also restored severe symptoms upon plant infection. Thus, fructose utilization and pathogenicity are intimately linked in S. citri. In contrast to mutations affecting fructose utilization, a ptsG mutation abolishing glucose transport into the cell does not result in reduced pathogenicity of S. citri. The reason for the differential implication of the two sugars in pathogenicity was studied by nuclear magnetic resonance analysis and it turned out that the bacteria use fructose preferentially, whereas the glucose accumulated in the leaf cells of the infected plants. This led to the following model. In noninfected plants, both fructose and glucose are formed by invertase. Fructose inhibits this enzyme resulting in a very low activity. In contrast, no inhibition occurs in infected plants because of fructose utilization by S. citri. The accumulating glucose that is not used by the bacteria results in inhibition of photosynthesis and thus in the different symptoms.

Transmission from an infected plant to an insect vector occurs by the uptake of bacteria along with the phloem sap. Inside the leafhopper, the bacteria have to pass the intestine midgut lining to multiply in the hemolymph, and then infect the salivary glands. Infection of the salivary glands is important because transmission from the insect to a host plant occurs by inoculation of the saliva into the damaged plant during feeding. It was shown that certain adhesins are necessary for transmissibility of S. citri from an infected plant to a vector, and that the genes coding for these adhesins are located on plasmids not existing in all S. citri strains.

In contrast to the spiroplasmas whose members are pathogenic to a broad range of plants and insects, the phytoplasmas form their own group among the Mollicutes that is strictly pathogenic to plants. Like the plantpathogenic spiroplasmas, they inhabit the phloem sieve tubes of their host plants after infection by an insect vector (usually belonging to the family of Cicadelli), but they depend completely on their host and so far it has been impossible to cultivate them in vitro. However, the genome sequences of three members of this group, Candidatus Phytoplasma asteris onion yellows strain (OY-M), aster yellows Phytoplasma strain witches broom (AY-WB), and Candidatus Phytoplasma australiense have been determined. 
Compared to other members of the Mollicutes, the phytoplasmas have some unique features. They exhibit shapes that range from rounded pleomorphic cells, with an average diameter of 200-800 $\mu \mathrm{m}$, to filaments. Their genomes lack all known genes coding for cytoskeleton or flagellum elements, suggesting that translocation of cells in planta is a passive event caused by the flow of phloem sap. As other Mollicutes, the phytoplasmas lack genes for the de novo synthesis of amino acids, fatty acids, or nucleotides, but they also lack some genes considered to be essential in all bacteria, such as $f t s Z$ encoding a tubulin-like protein. As FtsZ is involved in cell division, the mechanism of division in the phytoplasmas lacking it must be completely different from that of other bacteria. Although living in an environment that is rich in carbon sources, neither of the sequenced Phytoplasma possesses genes coding for sugar-specific components of the PTS. In contrast, S. citri and S. kunkelii, which thrive in the same environment as the phytoplasmas, contain three PTS for the import of glucose, fructose, and the insect-specific sugar trehalose (see earlier). However, Phytoplasma possesses the maltose-binding protein MalE. This protein may bind other sugars as well, but genes for enzymes making these sugars available for glycolysis are absent. Sucrose, the main sugar in the phloem sap of plants, could be used as a source of carbon and energy, but in sequenced phytoplasmas the gene for sucrose phosphorylase, which is important for sucrose degradation, is absent or fragmented. In general, phytoplasmas possess fewer genes related to carbon metabolism than the other Mollicutes. Energy generation in phytoplasmas seems to be restricted to glycolysis because ATP synthases are absent. OY-M Phytoplasma contains a $\mathrm{P}_{2 \mathrm{C}}$-ATPase, which is common in eukaryotic cells but unique among prokaryotes. Another remarkable feature that makes the phytoplasmas unique among the Mollicutes is their ability to synthesize phospholipids, supporting a closer phylogenetic relationship to Acholeplasma, which do not require sterols.

\section{Biochemistry of the Mollicutes}

Cytology of the Mollicutes. The Mollicutes differ from other bacteria not only because they lack a cell wall but also by dint of their small cell sizes. A typical cell of M. pneumoniae is 1-2 $\mu \mathrm{m}$ long and 0.1-0.2 $\mu \mathrm{m}$ wide (Fig. 2). In contrast, a typical rod-shaped bacterial cell (such as E. coli or B. subtilis) is 1-4 $\mu \mathrm{m}$ in length and $0.5-1 \mu \mathrm{m}$ in diameter. 
The absence of a cell wall has serious consequences for the osmotic stability of the Mollicute cells. They are much more sensitive to changes of the osmotic conditions than bacteria possessing a cell wall. The parasitic lifestyle of the Mollicutes may be directly related to their osmotic sensitivity: The hosts provide them with osmotically constant conditions that would not be found in the external environment. For example, M. genitalium is a parasite of the human urogenital tract, and its transmission by sexual contact ensures minimal exposure of the bacteria to an external, osmotically variable, environment. With the exception of the phytoplasmas and acholeplasmas, the Mollicutes are unable to produce fatty acids for membrane biosynthesis and are therefore dependent on exogenously provided fatty acids, which are then used for phospholipid synthesis. The lack of fatty acid synthesis is accompanied by the absence of a fatty acid desaturase, which is required to adapt the membrane fluidity to lower temperatures. To overcome this difficulty, most Mollicutes incorporate large amounts of sterols, which serve as a very effective buffer of membrane fluidity (see Table 1).

The lack of a cell wall has also consequences for the cellular morphology of the Mollicutes. The cells are pleomorphic; however, they are not small amoebas! The Mollicutes exhibit a variety of morphologies, such as pear-shaped cells, flask-shaped cells with terminal tip structures (see below), filaments of various lengths, and in the case of Spiroplasma species the cells are helical.

The mycoplasmas have a flask- or club-like shape with a terminal organelle, the so-called tip structure (see Fig. 2). This tip structure is a complex and specialized attachment organelle that has evolved to facilitate the parasitic existence of the mycoplasmas. The tip structure is made up of a network of adhesins, interactive proteins, and adherence accessory proteins, which cooperate structurally and functionally to mobilize and concentrate adhesins at the tip of the cell. The major adhesin of $M$. pneumoniae is the $170 \mathrm{kDa} \mathrm{P} 1$ protein that is responsible for the interaction of the bacteria with the host cells. In addition, the tip structure is important for the internalization of intracellular Mollicutes such as $M$. penetrans and M. genitalium. M. penetrans is capable of actively entering different types of animal cells, even those with minimal phagocytic activity. This may protect the bacterial cells against the host immune system. The formation of the tip structure in $M$. pneumoniae depends on the activity of the P41 protein that serves as an anchor protein. In the absence of this protein, multiple terminal organelles form at lateral sites of the cell and 
the terminal organelles are not attached to the body of the cell. In Mycoplasma mobile, there is also a terminal structure that is referred to as the "jellyfish" structure made up of a "bell" with dozens of flexible tentacles. Several components of this structure have been identified. With the exception of the glycolytic enzyme phosphoglycerate kinase, these $M$. mobile proteins are all absent from the genome of $M$. pneumoniae suggesting that the two species found individual solutions for the assembly of the terminal organelle.

Mycoplasma species are able to glide on solid surfaces with the help of their terminal attachment organelle. Terminal organelles that are detached from the body of the $M$. pneumoniae cell are released by some mutants. These detached organelles are still capable of gliding demonstrating that this organelle acts as a novel engine that allows cellular movement. The fastest gliding Mycoplasma species, M. mobile, contains a dedicated $349 \mathrm{kDa}$ "leg” protein that is required for gliding. This protein is composed of an oval base with three successive flexible extensions that may support movement. Movement is thought to occur by repeated catching and releasing of sialic acid on solid surfaces and is driven by the hydrolysis of ATP. This ATP hydrolysis may be catalyzed by the glycolytic enzyme phosphoglycerate kinase that is part of the terminal organelle in M. mobile.

As other bacteria, the Mollicutes divide by binary fission. Again, the terminal organelle seems to be very important for this process: Cell division in M. pneumoniae is preceded by the formation of a second tip structure adjacent to the existing one. The two terminal organelles then separate leading eventually to cytokinesis. Among the proteins known to be important for bacterial cell division is the tubulin-like GTP-hydrolyzing FtsZ protein that forms a ring at the division site. Until recently, FtsZ proteins were found in any newly analyzed genome, and the fts $Z$ gene is essential in most bacteria, including E. coli and B. subtilis. Therefore, FtsZ was considered to be indispensable for all life. However, it recently turned out that some Mollicutes such as M. mobile, Ureaplasma urealyticum, and the two sequenced phytoplasmas lack fts $Z$ genes, suggesting that its function is dispensable at least in some Mollicutes. In many bacteria, the FtsA protein is required for the recruitment of the proteins that form the septum for cell division. Interestingly, this protein is absent from all the pleomorphic Mollicutes, whereas it has been detected in S. kunkelii. This may be related to the helical morphology of these bacteria. 


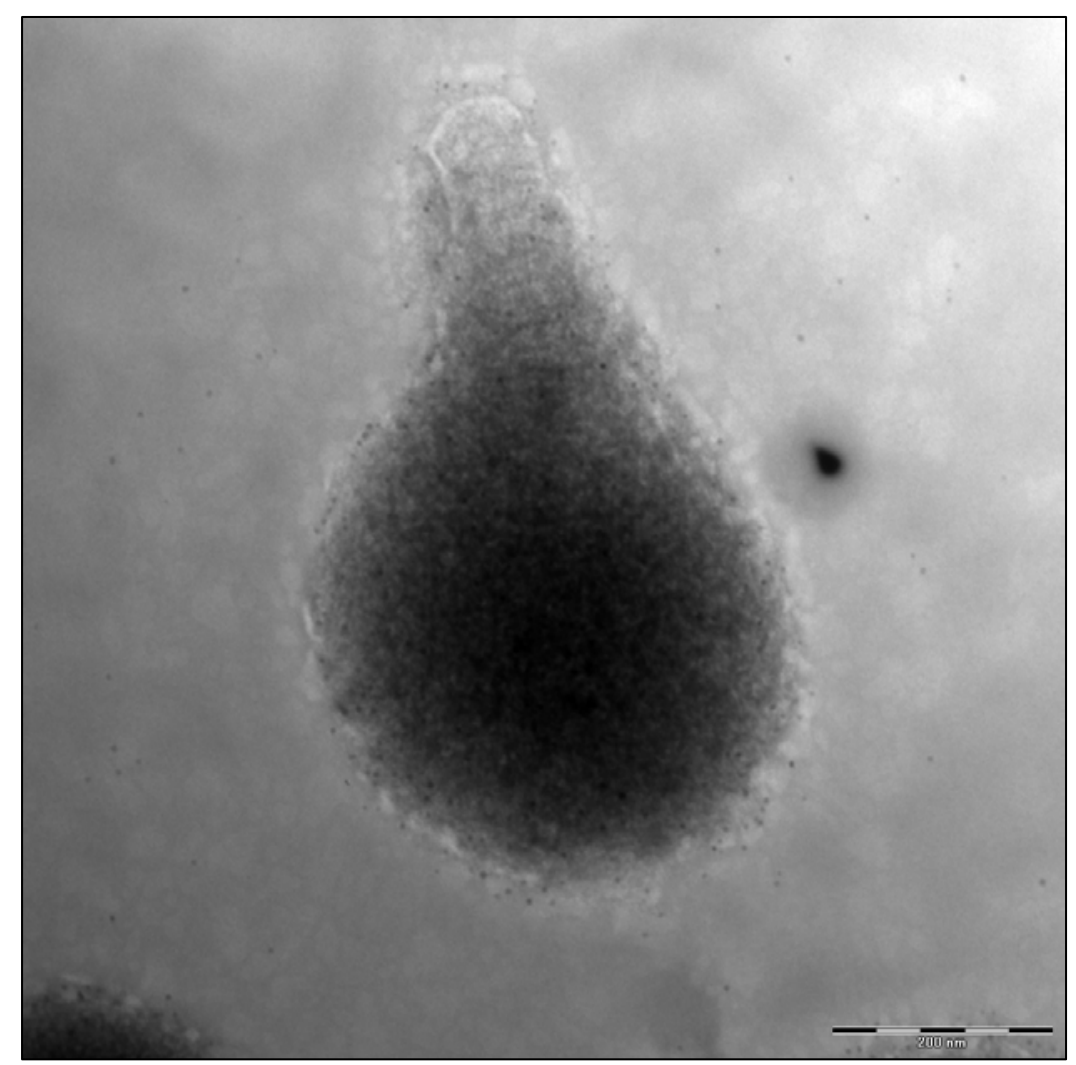

Fig. 2. Electron micrograph of a cell of $\boldsymbol{M}$. pneumoniae. The terminal organelle (also called the tip structure) is visible in the upper part of the cell. Scale bar, $200 \mathrm{~nm}$.

Metabolism of the Mollicutes. The reductive evolution of the Mollicutes is reflected in their limited metabolic properties. Of the central metabolic pathways, that is, glycolysis, the pentose phosphate shunt, and the tricarboxylic acid (TCA) cycle, only glycolysis seems to be operative in most Mollicutes. Most striking is the lack of many energy-yielding systems in the Mollicutes. No quinones or cytochromes were found in any representative. The electron transport system is flavin-terminated. Thus, ATP is produced by substrate-level phosphorylation, a less efficient mechanism as compared to oxidative phosphorylation.

As observed for M. genitalium glyceraldehyde-3-phosphate dehydrogenase, the glycolytic kinases of several Mollicute species have functions in addition to that in glycolysis. These enzymes can use not only ADP/ATP but also other nucleoside diphosphate/triphosphate couples. Thus, these enzymes (phosphofructokinase, phosphoglycerate kinase, pyruvate kinase, and acetate kinase) compensate for the lack of the normally essential $n d k$ gene encoding nucleoside diphosphate kinase that is required for nucleotide biosynthesis. 
Glycolysis is not the only source of ATP formation by substrate level phosphorylation in the Mollicutes. Pyruvate can be oxidized to acetyl-CoA by pyruvate dehydrogenase. Acetyl-CoA can be further catabolized by phosphotransacetylase and acetate kinase in an additional substrate-level phosphorylation resulting in the formation of acetate. An alternative pathway of pyruvate consumption is its reduction to lactate, leading to the regeneration of $\mathrm{NAD}^{+}$.

A recent study with $M$. pneumoniae demonstrated that glucose is the carbon source allowing the fastest growth of these bacteria. In addition, M. pneumoniae can utilize glycerol and fructose. Interestingly, mannitol is not used even though the genetic equipment to utilize this carbohydrate seems to be complete. Obviously, one or more of the required genes are not expressed or inactive.

Glucose and fructose are transported into the cells by the PTS. This system is made up of general soluble components and sugar-specific membrane-bound permeases. The general components, enzyme I and HPr, transfer a phosphate group from phosphoenolpyruvate to the sugar permease, which phosphorylates the sugar concomitant to its transport.

The arginine dihydrolase pathway can be found also in some Spiroplasma and Mycoplasma species. Arginine hydrolysis by this pathway results in the production of ornithine, ATP, $\mathrm{CO}_{2}$, and ammonia. The pathway uses three enzymes: Arginine deiminase, ornithine carbamoyl transferase, and carbamate kinase. The degradation of arginine is coupled to equimolar generation of ATP by substrate-level phosphorylation. The role of this pathway as a sole energy-generating source in mycoplasmas is questionable. However, the existence of an arginine-ornithine antiport system in Spiroplasma melliferum requiring no ATP for arginine import into the cells supports an energetic advantage in arginine utilization.

Mollicutes possess very limited metabolic and biosynthetic activities for amino acids, carbohydrates, and lipids as compared to “conventional” bacteria. M. pneumoniae scavenges nucleic acid precursors and does not synthesize purines or pyrimidines de novo. These may be provided by RNA and DNA that have been degraded by potent mycoplasmal nucleases. Furthermore, both $M$. genitalium and $M$. pneumoniae lack all the genes involved in amino acid synthesis, making them totally dependent on the exogenous supply of amino acids from the host or from the artificial culture medium. The mycoplasmas have also lost most of the genes involved in cofactor biosynthesis; 
therefore, to cultivate them in vitro, the medium has to be supplemented with essentially all the vitamins.

Being dependent on the exogenous supply of many nutrients would predict that mycoplasmas need many transport systems. Surprisingly, M. genitalium and M. pneumoniae possess a only small number of transport proteins (34 and 44 proteins, respectively) compared to the 281 transport and binding proteins annotated in E. coli and almost 400 in B. subtilis. The apparent low substrate specificity of some of the Mollicute transport systems, such as those for amino acids, may also contribute to the significant gene reduction observed.

Although Mollicutes produce hydrogen peroxide, $M$. pneumoniae and M. genitalium lack the genes dealing with oxidative stress, such as those encoding catalase, peroxidase, and superoxide dismutase. A thioredoxin reductase system, identified in the mycoplasmas, may protect them from reactive oxygen compounds.

A major problem for the research with Mollicutes is the difficulty of cultivating them in vitro. Only a minority of the Mollicutes existing in nature have been cultivated so far. For example, none of the phytoplasmas infecting insects or plants has been cultivated in vitro. To overcome the metabolic deficiencies of the mycoplasmas, complex media are used for their cultivation. The media are usually based on beef heart infusion, peptone, yeast extract, and serum with various supplements. Serum has been shown to provide, among other nutrients, fatty acids and sterols that are required for membrane synthesis. The requirement for sterols has served as an important taxonomic criterion distinguishing the sterol-nonrequiring mycoplasmas, particularly the Acholeplasma species, from the sterol-requiring ones. For most mycoplasmas, the $\mathrm{pH}$ is adjusted to a slightly alkaline value, conditions that imitate those in the eukaryotic host. A common approach to improve in vitro cultivation of fastidious mycoplasmas is based on coculture with eukaryotic cell lines (cell-assisted growth). In this way, some spiroplasmas, such as the Colorado potato beetle Spiroplasma, were first successfully cocultivated with insect cell lines.

\section{Genetics and molecular biology of the Mollicutes}

Gene expression in the Mollicutes. The basic mechanisms of gene expression have been studied poorly in the Mollicutes. They possess a conventional bacterial RNA polymerase, but unlike most other bacteria, they encode only one sigma factor of the 
RNA polymerase. Thus, diversity of promoters and RNA polymerase holoenzymes are not used for regulatory purposes in the Mollicutes. The transcription start sites have been identified for several $M$. pneumoniae genes, and it turned out that the -10 region of these promoters is similar to that recognized by the housekeeping sigma factors of other bacteria such as $E$. coli or $B$. subtilis. In contrast, there is no conserved -35 region. These observations were confirmed by a recent analysis of the sequence determinants that are required for promoter activity in front of the $M$. pneumoniae ldh gene encoding lactate dehydrogenase. The -10 region is essential for transcription initiation, whereas the -35 region could be mutated without any consequences. Thus, the single M. pneumoniae RNA polymerase holoenzyme recognizes only the -10 region for promoter recognition.

Another peculiarity of the M. pneumoniae transcription machinery is the lack of the termination factor Rho, and correspondingly, the absence of Rho-dependent transcription terminators. Surprisingly, a bioinformatic analysis of bacterial genomes and the free energy values of RNAs around the end of open reading frames suggest that the Mollicutes do also not contain functional Rho-independent transcription terminators. This raises the important question of how transcription is terminated in the Mollicutes or whether it is terminated at all. The answer came from Northern blot experiments aimed at the identification of in vivo transcripts, and this answer is ambiguous. Indeed, defined transcripts were observed in a few cases, such as the M. genitalium and M. pneumoniae ftsZ gene clusters or the $M$. pneumoniae ptsH gene. The existence of these defined transcripts implies that there are also defined transcription terminators present. However, these terminators may be very rare. This might explain the observation that unrelated genes are expressed as parts of one transcription unit in the Mollicutes. Moreover, most attempts to determine transcript sizes by Northern blot analysis in the Mollicutes have failed. This is probably the result of mRNA length polymorphisms, which prevent the detection of clearly defined RNA species.

Most genes in the Mollicutes have the same orientation on the chromosome, and the intergenic regions are usually quite short if present at all. The transcription of most of these large gene clusters is colinear with replication. This genome organization also favors polycistronic transcription of large gene clusters. 
The lack of defined mRNA species results not only from the absence of transcription terminators but also from the weak conservation of sequences that mediate transcription initiation: A -10 region made up of only Ts and As is statistically overrepresented in the AT-rich Mollicute genome. Indeed, the -10 regions predicted from the analysis of many start points occur about 2900 times in the $816 \mathrm{~kb}$ genome of M. pneumoniae. This large number of possible transcription initiation sites is also reflected by the observation of substantial antisense transcription in both $M$. genitalium and M. pneumoniae.

In bacteria, regulation is usually exerted at the level of transcription. In the Mollicutes, only one example of transcription regulation is clearly documented: This is the regulation of the $S$. citri fructose operon by the transcription activator FruR (see “Plant pathogenic Mollicutes: Spiroplasma and Phytoplasma”). Moreover, the induction of chaperone-encoding genes at elevated temperatures was demonstrated in several Mycoplasma species. By analogy to the mechanism of heat shock regulation by the repressor protein HrcA and the DNA operator element CIRCE, it was proposed that heat shock genes are under the control of HrcA in the Mollicutes. In addition to HrcA, the genomes of $M$. genitalium and $M$. pneumoniae encode only two other potential transcription factors that belong to the GntR and the Fur family, respectively. Unfortunately, the function of these regulators has so far not been studied.

It is interesting to note that $M$. pneumoniae contains only three potential regulators (less than $0.5 \%$ of all open reading frames), whereas environmental bacteria such as Streptomyces coelicolor and $P$. aeruginosa reserve about $10 \%$ of their genetic capacity to encode transcription factors. The low number of transcription factors in the Mollicutes and the weak stringency of transcription signals in the Mollicutes might therefore reflect their close adaptation to specific habitats that provide a good supply of nutrients and protect the bacteria from harmful environmental conditions. Moreover, the good supply of nutrients from external sources, that is, the host, may abolish the need for transcription regulation, that is, to switch off the expression of genes if their products are not required.

An additional mechanism of regulation is provided by riboswitches and regulatory RNAs. A guanine-specific riboswitch was detected in the untranslated region of the Mesoplasma florum guaAB operon suggesting that this RNA element governs the regulation of this operon via guanine. 
Translation is one of the most prominent activities of the Mollicute cell: As much as $15 \%$ of the genome of the Mollicutes is devoted to translation-related functions. The principal mechanisms of translation in the Mollicutes are identical to those found in other bacteria. Because of the low genomic GC content, the codon usage is strongly biased toward AT-rich codons. With the exception of Phytoplasma and Acholeplasma, the Mollicutes decode the UGA codon as tryptophan instead of using it as a stop codon as in the universal genetic code. This poses severe problems for the expression of Mollicute proteins in heterologous hosts (see "Molecular biology and genetic tools for the Mollicutes”).

The mechanisms of translation initiation seem to differ among the Mollicutes. In some organisms such as Mycoplasma capricolum and S. citri, the open reading frames are preceded by canonical Shine-Dalgarno sequences that form base pairs with the 3' end of the 16S rRNA. In contrast, many genes of M. pneumoniae and M. genitalium lack such a sequence, and moreover, leaderless mRNAs are common in these bacteria. The molecular mechanisms of translation initiation in $M$. pneumoniae and its close relatives still await elucidation.

Posttranslational protein modification. In many bacteria including the mycoplasmas, the HPr protein of the PTS cannot only be phosphorylated by enzyme I, but is also the target of a regulatory phosphorylation on Ser-46 by a metabolite-activated protein kinase, HPrK. The phosphorylation of HPr on Ser-46 in “less degenerated” Firmicutes leads to carbon catabolite repression. So far, the functions of HPrK and ATP-dependent phosphorylation of HPr have not been studied in the Mollicutes. In contrast, much work has been devoted to the biochemical characterization of HPrK from $M$. pneumoniae. Unlike its equivalent from other bacteria, this protein is active at very low ATP concentrations. As in related proteins, it contains an essential Walker A motif for ATP binding. Mutations in this region severely affect both the kinase and the phosphatase activities of the protein. Fluorescence studies revealed that the $M$. pneumoniae HPrK has a significantly higher affinity for ATP than any other HPrK studied so far. This may explain why it is active even at low ATP concentrations. The M. pneumoniae HPrK was crystallized and its structure determined. As observed for homologous proteins, it forms a hexamer with the C-terminal domains in the active center. 
In addition to HPrK, there is one other protein kinase in M. pneumoniae and many other Mollicutes, PrkC. The corresponding gene is clustered with the gene encoding a protein phosphatase of the PP2C family, PrpC. It was shown that PrpC is implicated in the dephosphorylation of $\operatorname{HPr}(\operatorname{Ser}-\mathrm{P})$. PrkC is known to phosphorylate a wide variety of proteins in other Firmicutes; however, its targets and the role of PrkC-dependent phosphorylation in the Mollicutes remain to be studied.

Protein phosphorylation seems to be important for the biology of the Mollicutes. An analysis of the $M$. genitalium proteome revealed that each identified protein is present at an average of 1.22 spots on a two-dimensional gel, suggesting posttranslational modification of about $25 \%$ of all proteins. Given the importance of protein phosphorylation in all other living organisms, it seems safe to assume that a large portion of these modified proteins is actually phosphorylated. A phosphoproteome analysis of M. genitalium and M. pneumoniae identified 5 and 3\% of the total protein complement of these bacteria, respectively, as phosphoproteins. Among these proteins are not only enzymes of central carbon metabolism such as enolase and pyruvate dehydrogenase subunits but also several cytoskeleton and cytadherence proteins. It is tempting to speculate that PrkC may catalyze these phosphorylation events.

As in other bacteria, there is protein secretion in the Mollicutes. While some exported proteins carry typical signal peptides at their N-termini, there is no signal peptidase I present in the genome of the Mollicutes. This raises the possibility that so far uncharacterized proteins are active in protein secretion in the Mollicutes.

Genomic comparisons of Mollicutes. One of the questions that have been of interest to humans since its early days is the problem of what constitutes life. Only today, in the era of genome research, are we able to attempt an answer to this question. A major milestone in defining life was the identification of key features that characterize all living things and differentiate them from nonliving matter such as viruses and prions. Among these features are metabolism, autonomous replication, communication, and evolution. With the availability of genome sequences, it has become possible to determine the genetic equipment required for independent life. The Mollicutes are of special interest in this respect because they have the smallest genomes that allow independent life, at least under laboratory conditions. 
Genome research with the Mollicutes is driven by two major challenges: (1) The identification of the minimal set of genes that is required for independent life and (2) the creation of artificial organisms that are based on this minimal gene set. The simplicity of the Mollicutes and the broad body of knowledge on their biology makes them ideal starting points for these research areas.

Several different strategies have been applied to identify the minimal gene set required for life. The most simple approach is based on the comparison of sequenced genomes of different organisms. It seems safe to assume that those genes that are conserved in different organisms are more important than those that appear only in certain species. The smallest genome of any independent living organism known so far is that of $M$. genitalium. This bacterium has a genome of $580 \mathrm{~kb}$ with 482 protein-coding genes and 39 genes coding for RNAs. M. pneumoniae has a genome of $816 \mathrm{~kb}$ with 779 genes coding for proteins and 40 RNA-coding genes. A comparison of the two genomes reveals an overlap of 477 genes common to both species. This suggests that $M$. pneumoniae is an "extended version” of $M$. genitalium. It is tempting to speculate that $M$. genitalium is further advanced on the pathway of reductive genome evolution. Indeed, some genes present in $M$. pneumoniae but not in M. genitalium such as the mannitol utilization genes are known to be nonfunctional in the former organism. Thus, $M$. genitalium seems to be very close to a true minimal organism.

A comparison of all sequenced Mollicute genomes reveals that only a small subset of their genes is part of a common gene pool. Only 156 genes are common to all Mollicute genomes that have so far been sequenced. This represents about one-third of the 482 open reading frames of $M$. genitalium. Interestingly, of the 156 genes of the Mollicute core genome, the large majority, that is, 124 genes, are shared by all Firmicutes. Thus, there is only a small set of 32 genes that is conserved in all Mollicutes but not in all Firmicutes. However, even these genes are shared by many members of the Firmicutes thus precluding the idea of a gene set unique to the Mollicutes. Moreover, a large fraction of the common Mollicute gene set forms the core genome of all bacteria (about 100 genes). Thus, the genome reduction of the Mollicutes obviously went down to a minimum that is absolutely required for cellular life. This is becoming clear if one takes into account that even unrelated bacteria such as E. coli ( $\gamma$-proteobacterium) and B. subtilis (Firmicute) share about 1000 genes. 
The core gene set of the Mollicutes is made up mainly of genes encoding proteins involved in essential cellular functions such as DNA topology, replication and repair, transcription, RNA modification and degradation, translation, protein folding, secretion, modification or degradation (Table 2). In addition, seven genes encoding potential GTP-binding proteins are conserved in all Mollicute genomes. A few conserved metabolic genes encode proteins involved in glycolysis, metabolite and ion transport, nucleotide, lipid, phosphate, and amino acid metabolism. Interestingly, not a single protein of completely unknown function is conserved among all Mollicutes. Moreover, the genes common to all Mollicutes act in the central processes of life. This implies that there are no genes common to all Mollicutes that are required for Mollicute-specific activities such as the formation of the terminal organelle. This is in good agreement with earlier studies that demonstrated a large variability in the protein composition of this organelle.

A second approach to determine the minimal gene set required for life uses an experimental setup. Global transposon mutagenesis studies with $M$. genitalium and M. pneumoniae revealed dispensable genes. For M. genitalium, about 100 genes could be disrupted. This implies that the remaining 382 genes are essential. In addition, five genes that are part of groups of redundant genes seem to be essential. It is believed that these 387 genes (plus the RNA-coding genes) constitute the essential gene set of M. genitalium. The difference between the 156 genes in the core gene set of the Mollicutes and the 387 genes that are essential for M. genitalium suggests that many of the additional genes are important under the specific ecological conditions of M. genitalium. This idea is supported by the presence of 110 genes of unknown function among the essential genes. This finding clearly demonstrates how much remains to be learned about the biology of $M$. genitalium, and surely about the other Mollicutes as well.

With information on the minimal gene set in hand, the logical next step will be to construct artificial organisms with this set of genes. In 2007 and 2008, two important technological steps have been made on the way to the construction of such minimal artificial life: First, the replacement of one genome by another, a process called genome transplantation, was demonstrated. Genomic DNA of M. mycoides large colony was used to replace the genome of $M$. capricolum by polyethylene glycol-mediated transformation. The second major achievement was the chemical synthesis and 
assembly of the M. genitalium chromosome. Thus, an artificial chromosome can be synthesized and this DNA can be introduced into a living cell to provide the environment for the expression of this genome. The generation of an artificial minimal Mycoplasma-derived organism ("Mycoplasma laboratorium”) would be the logical next step and the ultimate proof of both these technologies and of our understanding for the minimal equipment of a living cell.

Tab. 2. The core gene set of the Mollicutes.

\begin{tabular}{ll}
\hline Function & Number of genes \\
\hline Information pathways - Protein & 38 \\
\hline Ribosomal proteins & 11 \\
Translation factors & 19 \\
Amino acyl tRNA synthetases & 2 \\
Chaperones & 3 \\
Proteolysis & 1 \\
Protein modification & 5 \\
Protein secretion & 7 \\
\hline Information pathways - RNA & 8 \\
\hline Transcription & 5 \\
RNA modification & 7 \\
RNA degradation and maturation & 8 \\
\hline Information pathways - DNA & 3 \\
\hline Replication & 7 \\
Repair & 8 \\
DNA topology & 1 \\
\hline Metabolism & 6 \\
\hline Basic carbon and energy metabolism & 1 \\
Amino acid metabolism & \\
Nucleotide biosynthesis & \\
Pyrophosphatase & \\
Lipid metabolism & \\
\hline Miscellaneous functions & \\
\hline Transport & \\
GTP-binding proteins & \\
Unknown proteins & \\
\hline & \\
\hline
\end{tabular}


Molecular biology and genetic tools for the Mollicutes. The detailed genetic analysis of the Mollicutes has been hampered for a long time by the lack of genetic tools that allow the efficient expression of UGA-containing Mollicute genes in heterologous hosts for purification and subsequent biochemical analysis, the stable introduction of foreign genetic material into a Mollicute cell, and either the targeted construction or the targeted isolation of desired mutant strains. During the past few years considerable progress has been made in the field of Mollicute genetics, making these organisms accessible for genetic studies.

The occurrence of UGA codons in the genes of Mollicutes has often prevented their expression in heterologous hosts for detailed biochemical analysis, because they serve as stop codons in E. coli and other expression hosts. To circumvent this problem, a variety of different but rather dissatisfying strategies had been employed, including the expression of UGA-containing genes in opal suppressor strains of E. coli, or in S. citri that also reads UGA as a tryptophan codon. As long as only few UGA codons are present in a gene, their sequential replacement by standard site-directed mutagenesis strategies might also be taken into consideration. However, the latter approach is time-consuming and cost-intensive with an increasing number of UGA codons. Recently, a strategy referred to as multiple mutation reaction (MMR) allowing the simultaneous replacement of multiple UGA codons in a single-step reaction was developed. This strategy is based on the use of 5'-phosphorylated oligonucleotides containing the desired mutations in a polymerase chain reaction (PCR). During the elongation steps, the external amplification primers are extended. As the mutation primers are designed to hybridize more strongly to their targets, the elongated amplification primers can then be ligated to the 5' ends of the mutation primer by a thermostable DNA ligase, yielding a DNA strand that contains the desired mutation. With this strategy, the simultaneous introduction of up to nine mutations in one single step is possible.

The majority of genetic tools that are well established in model organisms are unavailable for Mollicutes. Therefore, transposons are in common use for a variety of purposes. In combination with smart screening systems, they were used for the disruption of genes but also as carriers for the introduction of genetic material into the chromosome. The transposons Tn916 and Tn4001 and their improved derivatives can be used in Mollicutes. These transposons were originally isolated from Enterococcus 
faecalis and Staphylococcus aureus, respectively, and have a broad host range. Tn916 is a conjugative $18 \mathrm{~kb}$ transposable element that contains the xis-Tn/int-Tn genes for excision/integration, followed by the tet $M$ tetracycline resistance determinant and a set of genes (tra) required for intercellular transfer. Tn916 does not generate target duplications at its integration site, because it transposes by an excision/integration mechanism that is based on staggered nicks in the donor DNA. Tn4001 is a $4.5 \mathrm{~kb}$ composite transposon consisting of two identical IS256 elements flanking the gentamicin/kanamycin/tobramycin resistance conferring aac-aphD gene. Tn4001 has been used for transforming several Mycoplasma species. To increase the stability of transposon insertion mutants, mini-transposons on the basis of Tn4001 were constructed that have the transposase gene outside the transposable elements to prevent reexcision of the transposon after the first transposition event.

Until very recently, the targeted construction of gene knockout mutants via homologous recombination has only been reported in a few Mollicutes such as M. genitalium, Mycoplasma gallisepticum, Mycoplasma pulmonis, and A. laidlawii. In the absence of homologous recombination, the only remaining way to obtain gene knockouts is transposon mutagenesis. Because of the randomness of integration, the screening of large transposon mutant libraries for the loss or gain of a specific phenotype is required to isolate a gene knockout of interest. If no screenable phenotype can be expected to be associated with a gene of interest, the only known feature of the desired gene knockout is the specific DNA junction between the gene of interest and the transposon. Based on this idea, a strategy referred to as "haystack mutagenesis" has been designed that allows the targeted isolation of any viable transposon insertion strain out of an ordered library of transposon mutants. The concept of haystack mutagenesis is based on a saturating transposon mutagenesis to ensure that each dispensable gene is disrupted at a desired confidence level. Once the required number of transposon mutants has been isolated, they are arranged in pools of a reasonable size. These pools can then be screened by PCR using a gene-specific oligonucleotide and another one specific to the transposon for identifying the pool that contains the desired insertion. Subsequently, a similar screen at the level of the individual clones of the positive pool will identify the mutant of interest. This strategy has already been used for the isolation of several M. pneumoniae mutants. Alternatively, transposon mutant libraries can be screened for mutants that exhibit an interesting phenotype, such as loss of gliding motility. 
The use of transposons is accompanied by the problem of changes of the genetic context at the site of integration that may cause undesired side effects. To avoid this problem, autonomously replicating plasmids have always been the vehicle of choice. Some early studies reported the isolation of naturally occurring plasmids from M. mycoides. These are small cryptic plasmids with a size in the range of 1.7-1.9 kb coding for replication functions only. Based on one of these plasmids, M. mycoides-E. coli shuttle vectors were developed. Further developments of artificial plasmid vectors were stimulated, when the first genome sequences became available that allowed the determination of the origins of replication of Mycoplasma chromosomes. Plasmid replicons have been constructed that contain the oriC sequences from M. mycoides, M. capricolum, and Mycoplasma agalactiae. Remarkably, a certain host specificity was observed for oriC plasmids, hampering the prediction the oriC compatibility between different Mycoplasma species and the derived plasmids. Nevertheless, with the genome sequence of many mycoplasmas at hand, the construction of stably replicating oriC plasmids for any desired Mycoplasma can be expected in the near future.

In the past there have been a couple of studies aimed at the definition of mycoplasmal promoters. The lack of clarity concerning the nature of gene expression/regulation signals in Mollicutes (see "Gene expression in the Mollicutes”) can only be answered in experiments that make use of promoter reporter systems. Such reporter systems based on the promoterless lacZ gene or on fluorescent proteins have been developed and used. They are used in two ways: The reporter genes can be randomly introduced into the chromosome to isolate random fusions with promoters; alternatively, the fusions can be prepared on plasmid vectors before their introduction into the genome. This second possibility allows the analysis of mutant promoter variants.

At present all required tools for the application of standard genetics to mycoplasmas are available. The biochemical in vitro analysis of individual proteins is no longer hampered by the genetic code of these organisms. Thus, interesting proteins can be easily studied. Similarly, antigenic surface proteins, which are often very large and thus contain many UGA codons can now easily be produced in heterologous hosts in sufficient amounts to be tested as vaccine candidates. Using the existing reporter systems, it will be possible to refine the mycoplasmal promoter concept, to discover 
regulatory DNA sequences and, ultimately, unravel the signal transduction mechanisms that mediate the adaptive responses seen in a wide variety of DNA microarray analyses, but which are not yet understood at the molecular level. To confirm in vitro findings with purified proteins, targeted disruption of desired genes can presently be carried out in various representatives of the genus Mycoplasma, either by homologous recombination or by facilitated screening methods such as haystack mutagenesis.

\section{Accompanying feature}

Additional resources on the Mollicutes (key references, genome information, labs working on the Mollicutes, and information on important methods) can be found on an accompanying web page (http://tinyurl.com/3vw8ca).

\section{Further reading}

Barré et al. (2004) Nucleic Acids Res. 32: D307-D310.

Bové et al. (2003) Annu. Rev. Phytopathol. 41: 483-500.

Christensen et al. (2005) Trends Plant Sci. 10: 526-535.

Ciccarelli et al. (2006) Science 311: 1283-1287.

Gibson et al. (2008) Science 319: 1215-1220.

Glass et al. (2006) Proc. Natl. Acad. Sci. USA 103: 425-430.

Halbedel and Stülke (2007) Int. J. Med. Microbiol. 297: 37-44.

Krause and Balish (2004) Mol. Microbiol. 51: 917-924.

Lee et al. (2000) Annu. Rev. Microbiol. 54: 221-255.

Pollack (2001) Trends Microbiol. 9: 169-175.

Razin and Herrmann (2002) Kluwer Academic/Plenum Publishers, New York.

Sirand-Pugnet et al. (2007) Res. Microbiol. 158: 754-766.

Waites and Talkington (2004) Clin. Microbiol. Rev. 17: 697-728. 


\section{(B) Aims of this work}

The close adaptation of M. pneumoniae to human mucosal surfaces did not only result in a reductive evolution of metabolic capabilities but also affected the regulation of protein biosynthesis and activity. The most prominent regulatory modification of a protein both in bacteria and in eukaryotes is posttranslational modification via protein phosphorylation (Boekhorst et al., 2008; Jers et al., 2008).

In M. pneumoniae, the phosphorylation of HPr by HPrK was the first regulatory event and the first covalent protein modification described for any Mycoplasma strain (Halbedel et al., 2004; Merzbacher et al., 2003; Steinhauer et al., 2002). Later on, the implication of PrpC in $\operatorname{HPr}(\mathrm{Ser}-\mathrm{P})$ dephosphorylation was discovered (Halbedel et al., 2006) and phylogenetic studies suggested that PrkC may be another protein kinase in M. pneumoniae. A recent publication demonstrated that many proteins of M. pneumoniae are subject to protein phosphorylation (Su et al., 2007). Interestingly, the phosphorylation of HPr escaped the authors of this study. Moreover, these authors do not have the mutants affected in kinases and the phosphatase.

To understand the role of HPrK and other enzymes implicated in protein phosphorylation/dephosphorylation, it was therefore intended to analyze the phosphoproteome of the M. pneumoniae wild type strain and of three isogenic mutants that are affected in the two protein kinases HPrK and PrkC and in the protein phosphatase PrpC using two-dimensional gel electrophoresis. Similarly, the regulatory output of protein phosphorylation was studied more directly by unraveling the phosphorylation effect on cell morphology and virulence.

In a second part of this work two proteins encoding paralogous glycerophosphodiesterases (GlpQ/MPN420 and MPN566) were to be characterized. These enzymes cleave glycerol 3-phosphate diesters present in phospholipids to produce glycerol 3-phosphate that can be utilized by enzymes of the glycerol metabolism (Hames et al., 2009). A first experiment was the analysis of the biochemical activity of the two glycerophosphodiesterases in vitro. To analyze the impact of glycerophosphodiesterases on the pathogenicity of M. pneumonaie, glpQ and mpn566 mutant strains had to be isolated. These mutants allowed the verification of hydrogen peroxide production and their cytotoxicity toward HeLa cells. Furthermore, a detailed proteome analysis of the mutants should provide the basis for a better understanding of regulatory mechanisms of gene expression in M. pneumoniae. 


\section{Chapter 2}

\section{The stability of cytadherence proteins in Mycoplasma pneumoniae requires activity of the protein kinase PrkC}

The work described in this chapter was published in:

Schmidl, S. R., K. Gronau, C. Hames, J. Busse, D. Becher, M. Hecker, and J. Stülke. 2010. The stability of cytadherence proteins in Mycoplasma pneumoniae requires activity of the protein kinase PrkC. Infect. Immun. 78: 184-192.

Author contributions:

This study was designed and interpreted by SRS, $\mathrm{CH}$, and JS. $\mathrm{CH}$ isolated the prkC transposon mutant. SRS performed all experiments, but JB contributed substantially to the expression analysis of cytadherence genes. The proteomic analysis was done in collaboration with KG, DB, and MH, University of Greifswald. SRS and JS wrote the paper. 


\section{Abstract}

Mycoplasma pneumoniae belongs to the Mollicutes, a group of bacteria that have strongly reduced genomes, but that are nevertheless capable of independent life. With only three transcription factors, the regulatory features of these bacteria are very limited. Thus, posttranslational regulation might be important for M. pneumoniae. In addition to the highly specific HPr kinase, the $M$. pneumoniae prkC gene encodes the serine/threonine protein kinase C. In order to study the function(s) of this kinase, we isolated an M. pneumoniae mutant affected in PrkC. This mutation resulted in nonadherent growth and loss of cytotoxicity. Examination of the phosphorylation profile of the prkC mutant suggested that phosphorylation of cytadherence proteins was affected by the loss of this kinase. In contrast, inactivation of the prpC gene affecting the protein phosphatase that antagonizes PrkC-dependent phosphorylation resulted in more intensive phosphorylation of the cytadherence proteins HMW1 and HMW3 of the major adhesin P1 and of the surface protein MPN474. Moreover, loss of PrkC affects not only the phosphorylation state of the cytadherence proteins but also their intracellular accumulation. However, the expression of the corresponding genes was not affected by PrkC, suggesting that PrkC-dependent phosphorylation results in stabilization of the cytadherence proteins. The HMW proteins and P1 are part of the so-called terminal organelle of $M$. pneumoniae that is involved in gliding motility, cell division, and adhesion to host epithelial tissues. Our observations suggest that the posttranslational modification of cytadherence proteins by PrkC is essential for the development and function of the M. pneumoniae terminal organelle.

\section{Introduction}

Mycoplasma pneumoniae belongs to the Mollicutes, i.e., cell wall-less bacteria. These organisms are among the smallest self-replicating living beings capable of a host-independent existence. M. pneumoniae is a pathogenic bacterium that causes atypical pneumonia and extrapulmonary infections, such as autoimmune disorders, asthma, and arthritis (Atkinson et al., 2008; Stülke et al., 2009; Waites and Talkington, 2004). 
M. pneumoniae and its close relative Mycoplasma genitalium have recently attracted much attention, not only with respect to the elucidation of pathogenicity mechanisms, but also because the small genomes of these bacteria define the lower limit of naturally existing independent life. The analysis of the minimal gene complement of M. pneumoniae and M. genitalium is one of the sources of synthetic biology, a new discipline of biology (Gibson et al., 2008; Glass et al., 2006).

However, life is not static and not determined only by a defined set of genes. A very important feature is the control of biological activities in response to changing environmental conditions. Only this control enables organisms to adapt to different habitats and to survive suboptimal conditions. In $M$. pneumoniae, the regulatory potential seems to be rather limited. In bacteria, regulation of gene expression is achieved mainly at the level of transcription, by alternative sigma factors of the RNA polymerase, by transcription repressors and activators, or by RNA switches. The annotation of the $M$. pneumoniae genome revealed only one sigma factor and three putative transcriptional repressors. No two-component regulatory system is present (Dandekar et al., 2000). This set of transcription factors corresponds to only $0.5 \%$ of the protein-coding genes of $M$. pneumoniae. In contrast, versatile environmental bacteria, such as Streptomyces coelicolor and Pseudomonas aeruginosa, reserve as much as $10 \%$ of their coding capacity for transcription regulation (Greenberg, 2000).

A second way to control biochemical activities is the posttranslational modification of proteins, and protein phosphorylation is the most common protein modification. For a long time, it has been thought that bacteria prefer to phosphorylate proteins on histidine and aspartate residues, whereas eukaryotes phosphorylate serine, threonine, and tyrosine residues. In the past few years, protein phosphorylation on these amino acids was found to be common in bacteria as well (Jers et al., 2008).

In $M$. pneumoniae, protein phosphorylation has been studied with the phosphotransferase system (PTS). This system uses the phosphate group of phosphoenolpyruvate to phosphorylate consecutively its components, enzyme I, HPr, the glucose permease, and the incoming glucose (Halbedel et al., 2004). The HPr protein of the PTS is not only subject to enzyme I-dependent phosphorylation on His-15, but it is also phosphorylated on Ser-46 by the HPr kinase at the expense of ATP (Steinhauer et al., 2002). The molecular role of HPr phosphorylation on Ser-46 has not yet been identified with $M$. pneumoniae; in other bacteria this phosphorylation is crucial 
for carbon catabolite repression (Görke and Stülke, 2008). Dephosphorylation of $\mathrm{HPr}($ Ser-P) in M. pneumoniae requires the protein phosphatase PrpC, the product of the MPN247 gene (Halbedel et al., 2006). This gene is clustered with another gene that potentially encodes a protein kinase (PrkC). This gene cluster is conserved in all Firmicutes, i.e., in gram-positive bacteria with a low GC content of their genomic DNA (this group includes the Mollicutes). In other Firmicutes, PrkC has been shown to be a protein kinase involved in many functions, such as phosphorylation of glycolytic enzymes, virulence, and germination (Faucher et al., 2008; Kristich et al., 2007; Lomas-Lopez et al., 2007; Shah et al., 2008). However, the potential protein kinase PrkC of M. pneumoniae has so far not been the subject of any studies.

To date, phosphorylation of HPr by the HPr kinase is the best-studied protein phosphorylation event of M. pneumoniae (Allen et al., 2003; Halbedel and Stülke, 2005; Halbedel et al., 2006; Merzbacher et al., 2004; Steinhauer et al., 2002). However, in addition to HPr, several other proteins are phosphorylated in these bacteria. Among these proteins are HMW1 and HMW2, large cytadherence proteins that were shown to be phosphorylated on serine and threonine residues (Dirksen et al., 1994; Krebes et al., 1995). Recently, a proteomic approach was used to identify phosphorylated proteins in M. genitalium and M. pneumoniae (Su et al., 2007). This study identified 18 phosphoproteins in $M$. pneumoniae, among them the surface protein MPN474, the cytadherence protein HMW3, and several metabolic enzymes. Unfortunately, none of the previously identified phosphoproteins was found by the phosphoproteome approach. Until now, it has not been known, which kinases are responsible for these phosphorylation events. Moreover, the function of these phosphorylations has not been addressed.

In this work, we have studied the function of PrkC in M. pneumoniae by genetic and proteomic methods. The analysis of a prkC mutant revealed that this kinase is required for adherent growth of the cells on solid surfaces and for cytotoxicity toward eukaryotic cells. Moreover, we provide evidence that PrkC-dependent protein phosphorylation is crucial for the stability of a group of high molecular weight cytadherence proteins. 


\section{Materials and Methods}

Bacterial strains, oligonucleotides, and growth conditions. The M. pneumoniae strains used in this study were M. pneumoniae M129 (ATCC 29342) in the 32nd broth passage and its isogenic mutant derivatives GPM11 (prkC::mini-Tn, $\mathrm{Gm}^{\mathrm{R}}$ ), GPM51 (hprK::mini-Tn, Gm²) (Halbedel et al., 2006), GPM68 (prpC::mini-Tn, $\mathrm{Gm}^{\mathrm{R}}$ ) (Halbedel et al., 2006), and GPM70 (mpn474::mini-Tn, $\mathrm{Gm}^{\mathrm{R}}$ ) (Hegermann et al., 2008). The oligonucleotides used in this study are listed in Table S1 in the supplemental material. M. pneumoniae was grown at $37^{\circ} \mathrm{C}$ in $150-\mathrm{cm}^{2}$ tissue culture flasks containing $100 \mathrm{ml}$ of modified Hayflick medium with glucose $[1 \%(\mathrm{wt} / \mathrm{vol})]$ as the carbon source as described previously (Halbedel et al., 2004). Strains harboring transposon insertions were cultivated in the presence of $80 \mathrm{~g} / \mathrm{ml}$ gentamicin.

Preparation of whole cell extracts. A 100-ml culture was washed twice with cold phosphate-buffered saline (PBS) $(137 \mathrm{mM} \mathrm{NaCl}, 2.7 \mathrm{mM} \mathrm{KCl,} 10 \mathrm{mM}$ sodium hydrogen phosphate, $2 \mathrm{mM}$ potassium dihydrogenphosphate, $\mathrm{pH}$ 7.4). The cells were harvested with $1.5 \mathrm{ml}$ PBS by scraping them off the surface of the flask. The cells were then centrifuged for $4 \mathrm{~min}$ at $15,000 \times \mathrm{g}$ at $4^{\circ} \mathrm{C}$. The pellet was resuspended in $150 \mu \mathrm{l}$ of PBS. The protein concentration was determined and adjusted to $1 \mu \mathrm{g} / \mathrm{l}$ in PBS containing sodium dodecyl sulfate-polyacrylamide gel electrophoresis (SDS-PAGE) loading dye. Cells of the M. pneumoniae prkC mutant GPM11 were harvested by centrifugation of the culture. The pellets were washed twice with $1.5 \mathrm{ml} \mathrm{PBS}$, and the cells were resuspended as described above.

Visualization of protein phosphorylation. In order to detect phosphorylated proteins, cell extracts were separated on 6\% SDS polyacrylamide gels. The gels were subsequently stained with Pro-Q Diamond (Invitrogen) and Flamingo fluorescent dye (Bio-Rad) to visualize the phosphoproteins and all proteins, respectively (Eymann et al., 2007). Pro-Q staining was detected using a Molecular Imager FX (Bio-Rad). Flamingo signals were detected using a Typhoon 9400 image scanner. Relative quantification of the phosphosignal intensities was performed using Image $\mathrm{J}$ software v1.42 (Abramoff et al., 2004).

Protein identification by MS. Gel pieces were washed twice with $200 \mu \mathrm{l}$ $20 \mathrm{mM} \mathrm{NH} \mathrm{HCO}_{3} / 30 \%$ (vol/vol) acetonitrile for $30 \mathrm{~min}$ at $37^{\circ} \mathrm{C}$ and dried in a vacuum centrifuge (Concentrator 5301, Eppendorf). Trypsin solution (10 ng/ $\mu \mathrm{l}$ trypsin in $20 \mathrm{mM}$ ammonium bicarbonate) was added until gel pieces stopped swelling, and 
digestion was allowed to proceed for 16 to $18 \mathrm{~h}$ at $37^{\circ} \mathrm{C}$. Peptides were extracted from gel pieces by incubation in an ultrasonic bath for $15 \mathrm{~min}$ in $20 \mu \mathrm{l}$ high-performance liquid chromatography-grade water and transferred into micro vials for mass spectrometric analysis. Peptides were separated by liquid chromatography and measured online by electrospray ionization mass spectrometry (MS) using a nanoAcquity ultra-performance liquid chromatography system (Waters, Milford, MA) coupled to an LTQ Orbitrap mass spectrometer (Thermo Fisher Scientific, Waltham, MA). Peptides were desalted onto a trap column (Symmetry $\mathrm{C}_{18}$, Waters). Elution was performed in an analytical column (BEH130 $\mathrm{C}_{18}$, Waters) with a binary gradient of buffer $\mathrm{A}$ [0.1\% (vol/vol) acetic acid] and B [100\% (vol/vol) acetonitrile, 0.1\% (vol/vol) acetic acid] over a period of $80 \mathrm{~min}$ with a flow rate of $400 \mathrm{nl} / \mathrm{min}$. The LTQ Orbitrap was operated in data-dependent tandem MS (MS-MS) mode using multistage activation for phospho-relevant masses. Proteins were identified by searching all MS-MS spectra in .dta format against all M. pneumoniae proteins (extracted from the NCBI database [http://www.ncbi.nlm.nih.gov/sites/entrez?Db=genome\&Cmd=Retrieve\&dopt=Protein+ Table\&list_uids=113]) using SEQUEST (Bioworks 3.3.1/Sequest version 2.7, revision 11; Thermo Electron). Initial mass tolerances for peptide identification on MS and MS-MS peaks were $10 \mathrm{ppm}$ and $1 \mathrm{Da}$, respectively. Up to two missed tryptic cleavages were allowed. Methionine oxidation (+15.99492 Da) was set as a variable modification. Proteins were identified by at least two peptides by applying a stringent SEQUEST filter (Xcorr versus charge state of 1.90 for singly charged ions, 2.2 for doubly charged ions, 3.3 for triply charged ions, and 3.75 for higher-charged ions). Phosphorylated peptides that passed this filter were examined manually and accepted only when b- or y-ions confirmed the phosphorylation site.

Western blot analysis. For Western blot analysis, M. pneumoniae cell extracts were separated on SDS polyacrylamide gels (6\% to 12\%). After electrophoresis, the proteins were transferred onto a polyvinylidene difluoride membrane (Bio-Rad) by electroblotting. The proteins of interest were detected with polyclonal antibodies raised against these proteins. The antibodies used in this study are listed in Table S2 in the supplemental material. Antibodies were visualized by using anti-rabbit immunoglobulin G-alkaline phosphatase secondary antibodies (Promega) and the CDP-Star detection system (Roche Diagnostics). The quantification was performed using Image J software v1.42 (Abramoff et al., 2004). 
Southern blot analysis. For the preparation of $M$. pneumoniae chromosomal DNA, cells of a 100-ml culture were harvested as described previously (Halbedel et al., 2004). The cell pellet was resuspended in $750 \mu \mathrm{l} 50 \mathrm{mM}$ Tris- $\mathrm{HCl}, \mathrm{pH}$ 8.0, and $25 \mathrm{mM}$ EDTA, and RNase A was added to a final concentration of $25 \mu \mathrm{g} / \mathrm{ml}$. After an incubation step at $37^{\circ} \mathrm{C}$ for $15 \mathrm{~min}, 50 \mu \mathrm{l}$ proteinase $\mathrm{K}(25 \mathrm{mg} / \mathrm{ml})$ and $75 \mu \mathrm{l} 10 \% \mathrm{SDS}$ were added. The mixture was incubated at $50^{\circ} \mathrm{C}$ until the lysate was clarified and subsequently cooled down on ice. To precipitate debris, $300 \mu \mathrm{l} 5 \mathrm{M} \mathrm{NaCl}$ was added, and the mixture was incubated for $20 \mathrm{~min}$ on ice. The precipitate was pelleted by centrifugation ( $25 \mathrm{~min}, 15,000 \times \mathrm{g}, 4^{\circ} \mathrm{C}$ ), and the resulting supernatant was mixed with $500 \mu \mathrm{l}$ isopropanol to precipitate the chromosomal DNA. The DNA pellet was washed with $70 \%$ ethanol and finally resolved in $300 \mu$ l Tris-EDTA buffer. Digests of chromosomal DNA were separated using 1\% agarose gels, transferred onto a positively charged nylon membrane (Roche Diagnostics) (Sambrook et al., 1989), and probed with digoxigenin (DIG)-labeled riboprobes obtained by in vitro transcription with T7 RNA polymerase (Roche Diagnostics) using PCR-generated fragments as templates. Primer pairs for the amplification of prkC and aac-ahpD gene fragments were CH74/SH89 and SH62/SH63, respectively (see Table S1 in the supplemental material). The reverse primers contained a T7 RNA polymerase recognition sequence. In vitro RNA labeling, hybridization, and signal detection were carried out according to the manufacturer's instructions (DIG RNA labeling kit and detection chemicals, Roche Diagnostics).

Analysis of mRNA amounts. Preparation of total $M$. pneumoniae RNA was done as described previously (Halbedel et al., 2004). For slot blot analysis, serial twofold dilutions of the RNA extract in $10 \times$ SSC $(1 \times$ SSC is $0.15 \mathrm{M} \mathrm{NaCl}$ plus $0.015 \mathrm{M}$ sodium citrate) ( $2 \mu \mathrm{g}$ to $0.25 \mu \mathrm{g}$ ) were blotted onto a positively charged nylon membrane using a PR 648 slot blot manifold (Amersham Biosciences). Equal amounts of yeast tRNA (Roche) and M. pneumoniae chromosomal DNA served as controls. DIG-labeled riboprobes were obtained by in vitro transcription from PCR products that cover open reading frame internal sequences using T7 RNA polymerase (Roche). The reverse primers used to generate the PCR products contained a T7 promoter sequence (see Table S1 in the supplemental material). The quantification was performed using Image J software v1.42 (Abramoff et al., 2004). 
HeLa cell cytotoxicity assay. HeLa cells were grown in 24-well plates with $2.5 \times 10^{4}$ cells per well in $700 \mu \mathrm{l}$ Dulbecco modified Eagle medium for $24 \mathrm{~h}$ at $37^{\circ} \mathrm{C}$ and $5 \% \mathrm{CO}_{2}$. The $M$. pneumoniae cultures were grown for $96 \mathrm{~h}$ at $37^{\circ} \mathrm{C}$. The M. pneumoniae cells were then washed three times with $67.6 \mathrm{mM}$ HEPES, $\mathrm{pH} 7.3$; $140 \mathrm{mM} \mathrm{NaCl}$; and $7 \mathrm{mM} \mathrm{MgCl}_{2}$. The M. pneumoniae cells were resuspended with a 0.4- by 20-mm needle. Depending on the size of the pellet, they were resuspended in 5 to $8 \mathrm{ml}$ buffer. The cell suspensions were adjusted to an $A_{550}$ of 0.1 and centrifuged for $5 \mathrm{~min}$ at $15,000 \times \mathrm{g}$ at $4^{\circ} \mathrm{C}$. The pellet was resuspended in $125 \mu \mathrm{l}$ of modified Hayflick medium with a 0.4 - by 20 -mm needle. The cells were then pipetted onto the lawn of HeLa cells and incubated for $2 \mathrm{~h}$ at $37^{\circ} \mathrm{C}$ and $5 \% \mathrm{CO}_{2}$. Then the supernatant was removed and replaced by $700 \mu \mathrm{l}$ Dulbecco modified Eagle medium, and the cells were incubated. The cytotoxicity assays were performed in triplicate.

\section{Results}

Isolation of an $M$. pneumoniae prkC mutant. To get more insights into the function of the protein kinase PrkC (MPN248), we attempted to isolate prkC mutants. This was done using "haystack mutagenesis" (Halbedel et al., 2006). This strategy is based on an ordered collection of pooled random transposon insertion mutants that can be screened for junctions between the transposon and the gene of interest due to transposon insertion. Sixty-four pools containing 2,976 individual mutants (Halbedel et al., 2006) were used in a PCR to detect junctions between the prkC gene and the mini-transposon using the oligonucleotides CH35 and SH29 for prkC and the mini-transposon, respectively (Fig. 3A). For one pool that gave a positive signal, colony PCR with the 50 individual mutants resulted in the identification of one prkC mutant. The presence of the transposon insertion in $p r k C$ was verified by Southern blot analysis using a prkC-specific probe (Fig. 3B). To test whether this strain contained only a unique transposon insertion, we did another Southern blotting using a probe specific for the aac-aphD resistance gene present on the mini-transposon. As shown in Fig. 3B, only a single band hybridizing with this probe was detected; moreover, this fragment had the same size as that of the XhoI fragment hybridizing to the prkC probe (Fig. 3B). The isolated prkC mutant strain was designated GPM11. The position of the transposon insertion in the prkC gene of $M$. pneumoniae GPM11 was determined by DNA sequencing. The prkC gene was disrupted between nucleotides 313 and 314, resulting in 
a truncated protein of 106 amino acids, with one additional amino acid and the following stop codon encoded by the inserted mini-transposon. The disruption destroyed the kinase domain of PrkC (amino acids 80 to 256), suggesting that no active PrkC is present in the mutant strain.

A

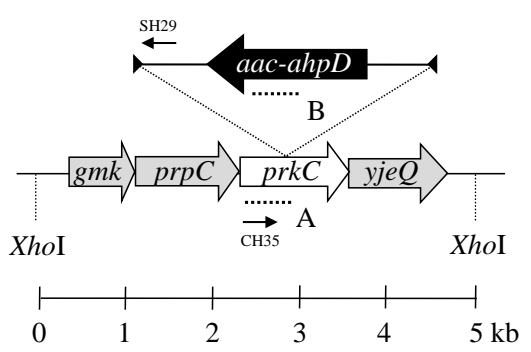

B
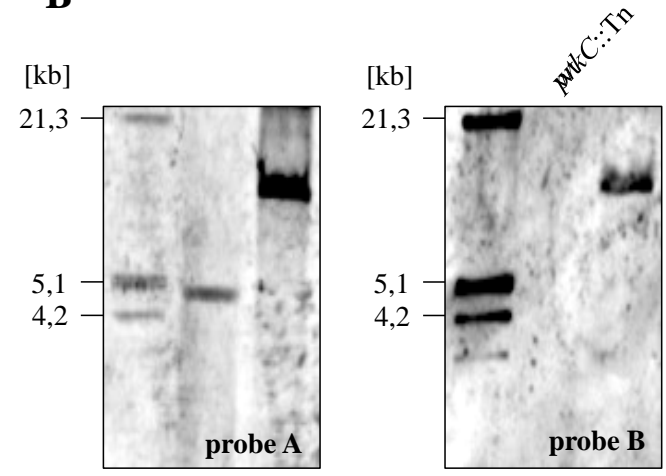

Fig. 3. Isolation of a $M$. pneumoniae prkC transposon insertion mutant. (A) Schematic representation of the genomic region surrounding the $p r k C$ gene in $M$. pneumoniae and site of the transposon insertion in the prkC knockout strain GPM11. The annealing sites of oligonucleotides used for the determination of the transposon insertion site are indicated by arrows. Probes hybridizing to internal fragments of the prkC and the aac-ahpD genes are depicted as dotted lines. (B) Southern blot analysis to confirm the single insertion of the mini-transposon into the prkC gene of strain GPM11. Chromosomal DNA of the wild type and of strain GPM11 was digested using XhoI. Blots were hybridized with the prkC-specific probe (left) and a probe hybridizing to the $a a_{c}-a h p D$ gene of the mini-transposon (right). DIG-labeled DNA molecular size marker III (Roche Applied Science) served as a standard.

Implication of PrkC in adherent growth and cytotoxicity. For an initial characterization of the prkC mutant strain GPM11, we observed growth of this strain in modified Hayflick medium. A comparison of the growths of the wild type and the prkC mutant strain revealed that both strains grow at the same rate in the presence of glucose as the carbon source (data not shown). However, in contrast to its isogenic wild type, GPM11 was unable to attach to the surface of the culture flask, and the cells grew as a suspension in liquid medium. 
This loss of adherent growth suggested that the prkC mutant might also be impaired in cytotoxicity. To assess the cytotoxicity of the prkC mutant strain, we infected confluently grown HeLa cell cultures with $M$. pneumoniae cells (multiplicity of infection of two). The cytotoxicity of the prkC mutant was compared to that of wild type strain M129 and M. pneumoniae GPM68, which is affected in $p r p C$, the protein phosphatase that is thought to be the antagonist of PrkC. As shown in Fig. 4, the HeLa cells underwent lysis after 6 days upon infection with wild type $M$. pneumoniae. In contrast, a large portion of viable cells was observed after infection of the cell culture with prkC mutant GPM11. In contrast, cytotoxicity induced by $\operatorname{prpC}$ mutant strain GPM68 was equivalent to that of the wild type strain (Fig. 4B). These data clearly demonstrate that PrkC is required for host cell damage, whereas PrpC is not. This suggests that PrkC-dependent protein phosphorylation is an important factor for cytotoxicity.

A

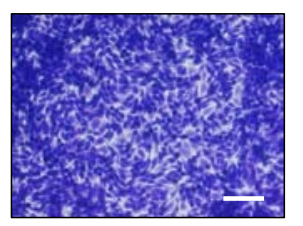

control

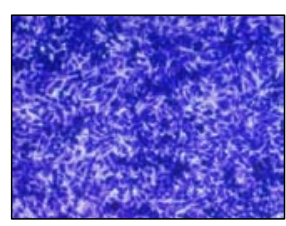

prkC::Tn

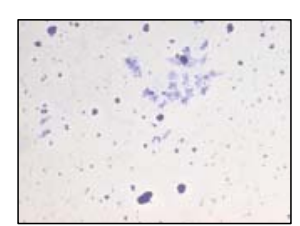

wt

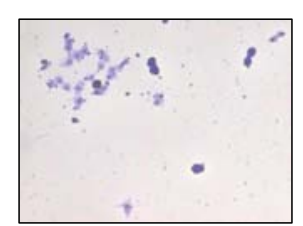

$\operatorname{prp} C:: \operatorname{Tn}$
B

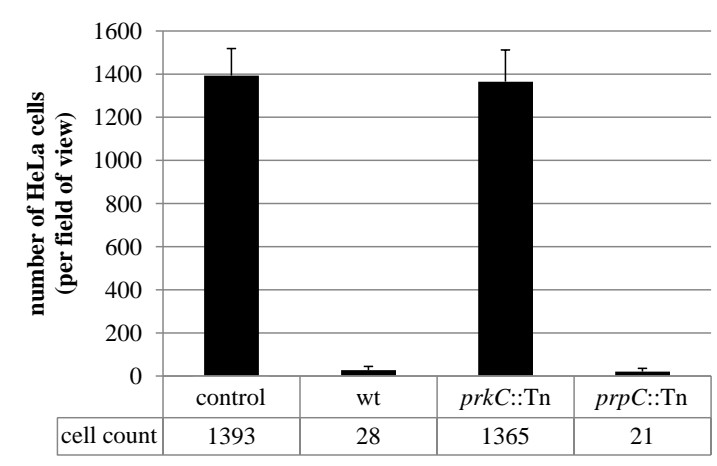

Fig. 4. Cytotoxicity of $M$. pneumoniae toward HeLa cell cultures. (A) Infection assay with the M. pneumoniae prkC::Tn strain GPM11 and the prpC::Tn strain GPM68. HeLa cell culture without M. pneumoniae (left top; control); HeLa cell culture incubated with wild type M. pneumoniae (right top; wt); HeLa cell culture incubated with the $\operatorname{prkC}$ or $\operatorname{prpC}$ mutant (bottom; prkC::Tn or prpC::Tn, respectively). After 6 days, HeLa cell cultures were stained with crystal violet and photographed. Scale bar, $0.1 \mathrm{~mm}$. (B) Quantification of HeLa cells after infection with wild type M. pneumoniae or the prkC or prpC mutant. The cell count of surviving cells is indicated as the number of viable cells per field of view, quantified by crystal violet staining after 6 days of incubation. An uninfected HeLa cell culture served as a control. Error bars indicate the standard deviation (based on three independent experiments). 
Identification of proteins that are subject to PrkC-dependent phosphorylation. The loss of adherent growth and cytotoxicity in the prkC mutant suggested that proteins that are phosphorylated by PrkC might be involved in these functions. To identify such proteins, we separated whole cell extracts of $M$. pneumoniae by SDS-PAGE. The proteins were consecutively stained with Pro-Q Diamond, a dye for the detection of Ser/Thr/Tyr-phosphorylated proteins, and with Flamingo fluorescence stain for the detection of the total proteins. For this analysis, we compared the wild type strain M129 and the prkC mutant GPM11. As a control, we used the prpC mutant GPM68. Since PrpC is thought to be the antagonist of PrkC, we expected to observe a more intense phosphorylation of PrkC target proteins with this mutant. In addition, we used a mutant affected in the other identified protein kinase of $M$. pneumoniae, HPrK (GPM51), and a mutant deficient in the surface protein MPN474, which is not thought to be implicated in protein phosphorylation (GPM70) (Krebes et al., 1995). Several of the proteins that are involved in cytadherence in $M$. pneumoniae are very large proteins, such as HMW1, HMW2, and HMW3. It has been shown that these proteins are phosphorylated in M. pneumoniae (Krebes et al., 1995; Su et al., 2007). Therefore, we decided to analyze specifically the phosphorylation pattern of large proteins by using $6 \%$ SDS-polyacrylamide gels. A comparison of the total protein patterns in the wild type and the prkC mutant strain revealed that the quantity of four proteins was severely reduced (Fig. 5A). These protein bands were identified by MS as the three high molecular weight proteins HMW1, HMW2, and HMW3 and the major adhesin P1. The amounts of these four proteins in the $\operatorname{prp} C$ mutant were indistinguishable from those of the wild type strain, suggesting that PrkC is necessary for their accumulation in the cell. The quantitative analysis of the Pro-Q Diamond signal intensities for the detection of phosphorylated proteins revealed that the bands of these proteins were absent or much weaker in the prkC mutant (Fig. 5B and C). In contrast, the phosphorylation signals of HMW1, HMW3, and P1 were more intense in the $\operatorname{prpC}$ phosphatase mutant than in the wild type (Fig. 5C). This observation indicated the accumulation of phosphorylated protein that cannot be dephosphorylated by the cognate protein phosphatase. In turn, this suggests that these proteins are targets of PrkC-dependent phosphorylation and that phosphorylation plays a role in their accumulation. In addition, one protein with an apparent molecular mass of $160 \mathrm{kDa}$ accumulates in the $\operatorname{prpC}$ mutant and shows also a 
new very strong phosphorylation signal in this mutant (Fig. 5B). This protein is the acidic surface protein MPN474.

A

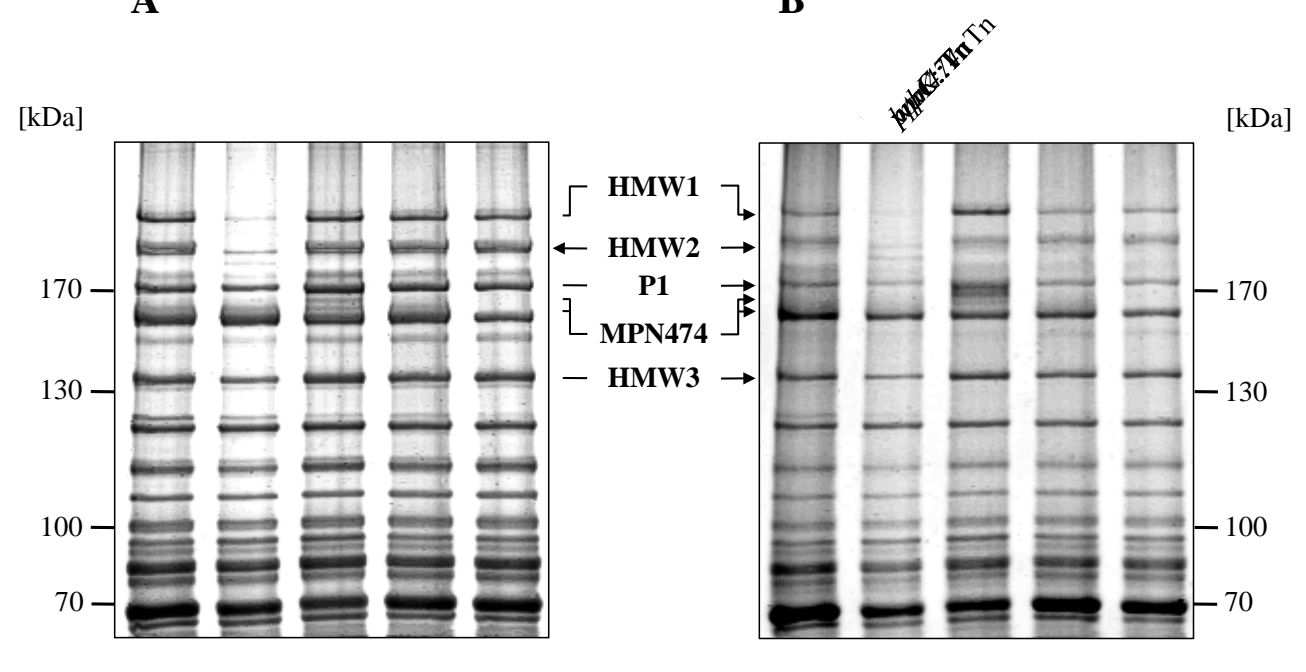

C
B

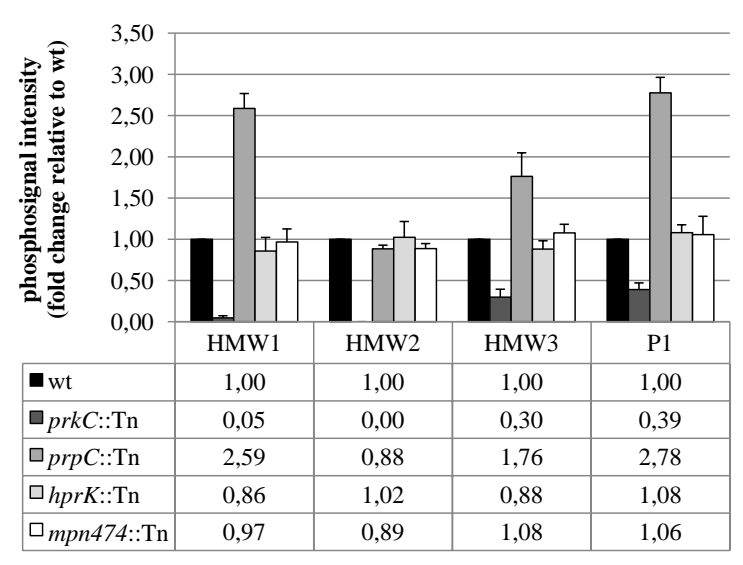

Fig. 5. Comparison of protein profile and in vivo phosphorylation patterns in M. pneumoniae wild type and mutant cells. Whole cell extracts of the M. pneumoniae wild type and different mutant strains were analyzed by SDS-PAGE and stained with Pro-Q Diamond (Invitrogen) and Flamingo fluorescent dye (Bio-Rad) for visualization of phosphoproteins and all proteins, respectively. Ten $\mu g$ of extract was applied to each lane. Interesting protein bands were cut out and identified by MS. Protein bands with changes in the phosphorylation signal or the protein amount are indicated by arrows. Total protein (A) (Flamingo fluorescent stain); phosphoproteins (B) (Pro-Q Diamond stain). (C) Relative quantification of phosphosignal intensity. The graph shows the changes relative to the phosphorylation signal of the corresponding protein in the M. pneumoniae wild type. Error bars indicate the standard deviation (based on three independent experiments). Note that the protein amount of HMW1-3 and P1 is also decreased in the prkC mutant. 
Impact of PrkC on the accumulation of cytadherence proteins. It has been shown that HMW2 mutants of $M$. pneumoniae are impaired in the accumulation of other cytadherence proteins, such as HMW1, HMW3, and MPN309 (P65) (Balish et al., 2003). Since the amounts of the HMW proteins were reduced in the prkC mutant, we determined the cellular amounts of other cytadherence proteins by use of specific polyclonal antibodies raised against these proteins (Table 3 provides a summary of the results). As controls, we used the elongation factor G (FusA) and the MPN567 (P200) protein, which is required for gliding motility (Jordan et al., 2007). The amounts of both proteins were virtually identical in all strains tested in this study (Fig. 6C).

Tab. 3. Summary of Western blot analyses with a prkC::Tn mutant (GPM11).

\begin{tabular}{|c|c|c|c|c|}
\hline $\begin{array}{l}\text { M. pneumoniae } \\
\text { locus tags }\end{array}$ & $\begin{array}{l}\text { SwissProt } \\
\text { accession no. }\end{array}$ & Protein & $\begin{array}{l}\text { Signal in Western } \\
\text { blot }(\%)^{a}\end{array}$ & Protein function \\
\hline MPN141 & P11311 & P1 & 35 & Adhesin \\
\hline MPN142 & Q50341 & P40 & 40 & Involved in cytadherence \\
\hline MPN227 & P75544 & FusA & 100 & Elongation factor $\mathrm{G}$ \\
\hline MPN309 & P53663 & P65 & 9 & Adhesin-related protein \\
\hline MPN310 & P75471 & HMW2 & 0 & $\begin{array}{l}\text { Cytadherence high } \\
\text { molecular weight protein } 2\end{array}$ \\
\hline MPN310 & P75471 & $\begin{array}{l}\text { HMW2-S } \\
(\mathrm{P} 28)\end{array}$ & 0 & $\begin{array}{l}\text { Adhesin-related protein, } \\
\text { HMW2 fragment }\end{array}$ \\
\hline MPN311 & P75470 & P41 & 8 & Adhesin-related protein \\
\hline MPN312 & P75469 & P24 & 41 & Adhesin-related protein \\
\hline MPN447 & Q50365 & HMW1 & 20 & $\begin{array}{l}\text { Cytadherence high } \\
\text { molecular weight protein } 1\end{array}$ \\
\hline MPN452 & Q50360 & HMW3 & 22 & $\begin{array}{l}\text { Cytadherence high } \\
\text { molecular weight protein } 3\end{array}$ \\
\hline MPN453 & P75330 & P30 & 34 & Adhesin \\
\hline MPN474 & P75310 & MPN474 & 104 & $\begin{array}{l}\text { Coiled coil protein, } \\
\text { putative structural protein } \\
\text { involved in cytosceleton }\end{array}$ \\
\hline MPN567 & P75211 & P200 & 96 & Involved in cytadherence \\
\hline
\end{tabular}

\footnotetext{
${ }^{\mathrm{a}}$ The protein amount in the prkC mutant relative to that in the wild type strain (100\%) is indicated.
} 
A

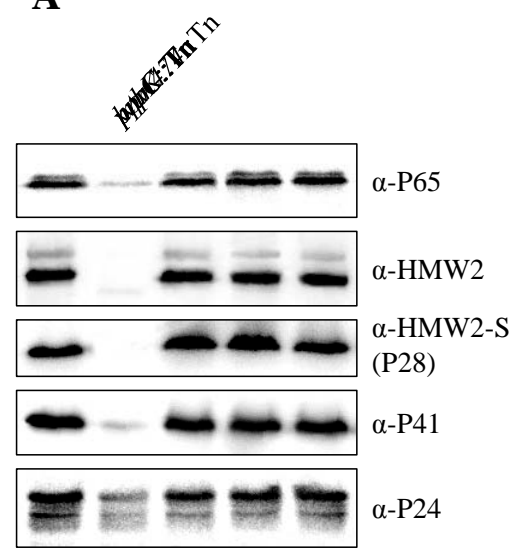

B
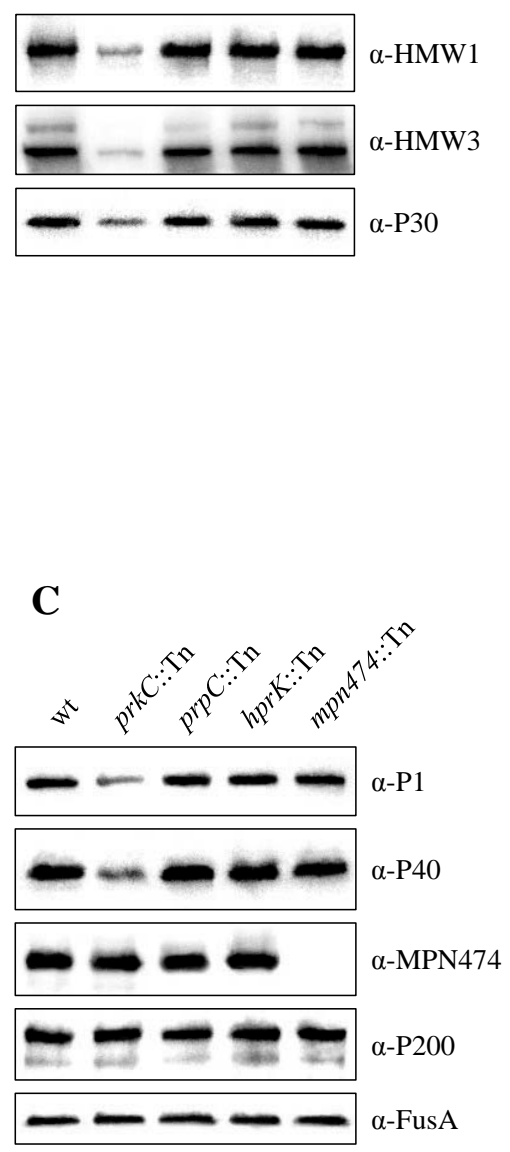
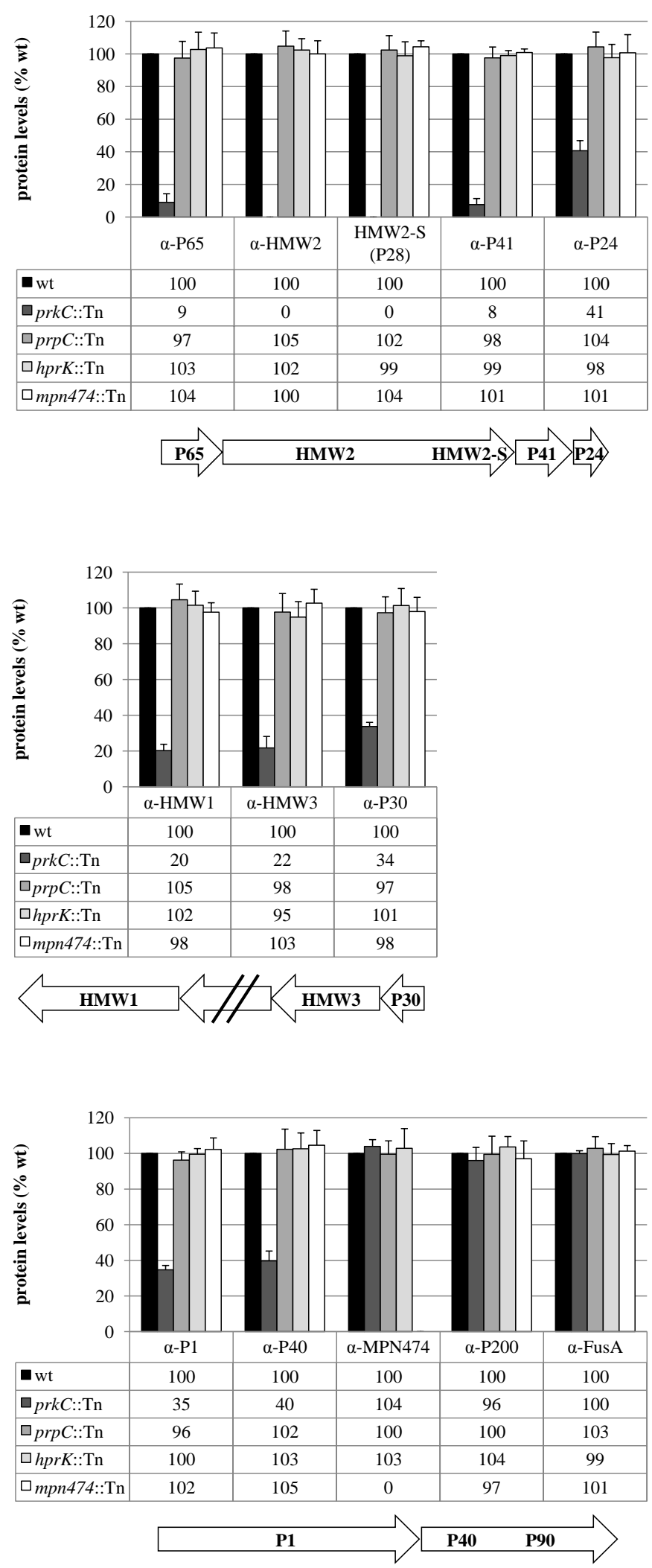
Fig. 6. Western blot analyses of expression profiles of $M$. pneumoniae cytadherence proteins. Whole cell extracts of the $M$. pneumoniae wild type and different mutant strains were separated by SDS-PAGE and blotted onto a polyvinylidene difluoride membrane. Cytadherence proteins were detected by using polyclonal rabbit antibodies raised against proteins of the P65 operon (A), the HMW gene cluster (B), and major adhesins (C). A polyclonal rabbit antibody raised against M. pneumoniae elongation factor G (FusA) was used as the control. Ten $\mu \mathrm{g}$ of extract was applied to each lane. The names of antibodies are given next to each blot. $\alpha$, anti. HMW1, HMW2, HMW3, MPN474, P1, and P200 were analyzed by $6 \%$ SDS-PAGE, and the other proteins by 12\% SDS-PAGE. The graphs show the quantification of Western blot signals relative to that for the $M$. pneumoniae wild type. Error bars indicate the standard deviation (based on three independent experiments). The organization of the different operons is shown by arrows. Note that there are four genes between $h m w 3$ and $h m w 1$, which is indicated (panel B). Moreover, mpn474, p200, and fusA are not part of operons (panel C).

First, we studied the proteins encoded by one gene cluster containing the gene encoding HMW2 (the so-called P65 operon). As shown in Fig. 6A, the cellular amounts of all these proteins are significantly reduced in the prkC mutant. In fact, the HMW2 protein and HMW2-S (also called P28), a second translation product of the same mRNA (Boonmee et al., 2009), are nearly completely absent in the prkC mutant strain. The accumulation of all the proteins encoded by the gene cluster is not affected by a disruption of the prpC gene (Fig. 6A). Thus, the phosphorylation of these proteins seems to be essential for their accumulation. The so-called HMW gene cluster encodes the HMW1, HMW3, and MPN453 (P30) proteins. As observed for HMW2, the two high molecular weight proteins encoded by this gene cluster are much less abundant in the prkC mutant, whereas none of the other tested mutations had any effect. The accumulation of MPN453 was also somewhat reduced but to a lesser extent (Fig. 6B). Finally, we assayed the amounts of the major adhesins MPN141 (P1) and MPN142 (P40) and of the surface protein MPN474, which was shown to be phosphorylated by PrkC (Fig. 5). As shown for the large cytadherence proteins, many fewer adhesins were detected in the prkC mutant than in the wild type or all other strains. In contrast, the cellular amounts of MPN474 were not affected by the prkC mutation. As expected, the antibodies do not cross-react with any protein in the MPN247 mutant strain GPM70.

Transcription of the genes encoding cytadherence proteins in mutants affecting protein phosphorylation. The virtual absence of cytadherence proteins in the prkC mutant can be explained by two alternative scenarios: (1) The expression of the corresponding genes and the accumulation of their mRNAs might require some kind of 
PrkC-dependent protein phosphorylation and (2) the proteins might be translated to a lesser extent or be subject to rapid degradation. To distinguish between these possibilities, we compared the amounts of the mRNAs of the cytadherence genes in the wild type strain M129 and in our set of mutants that are affected in protein (de)phosphorylation. For this purpose, we isolated RNA from cultures grown in modified Hayflick medium supplemented with glucose and performed slot blot analyses.

A

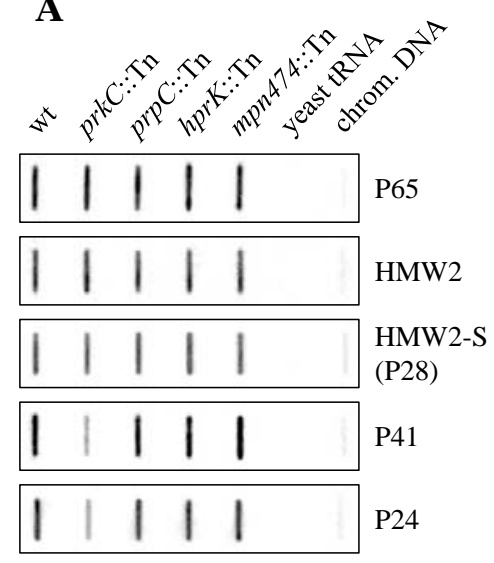

B

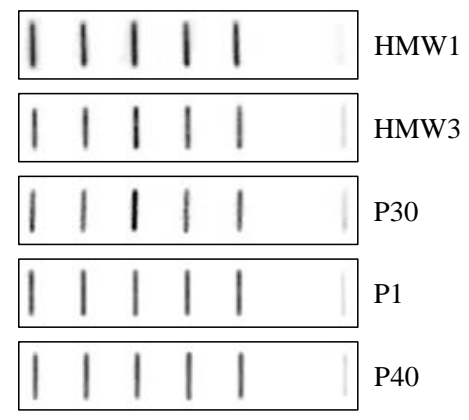

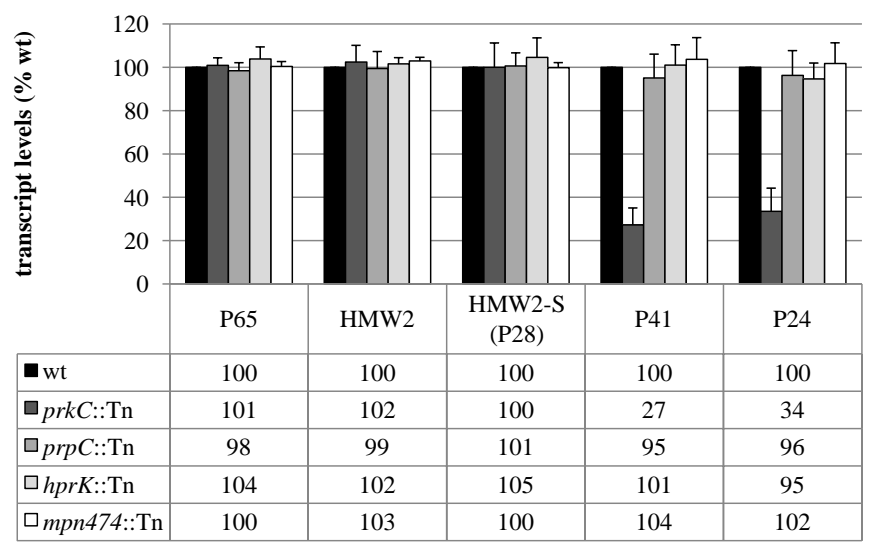

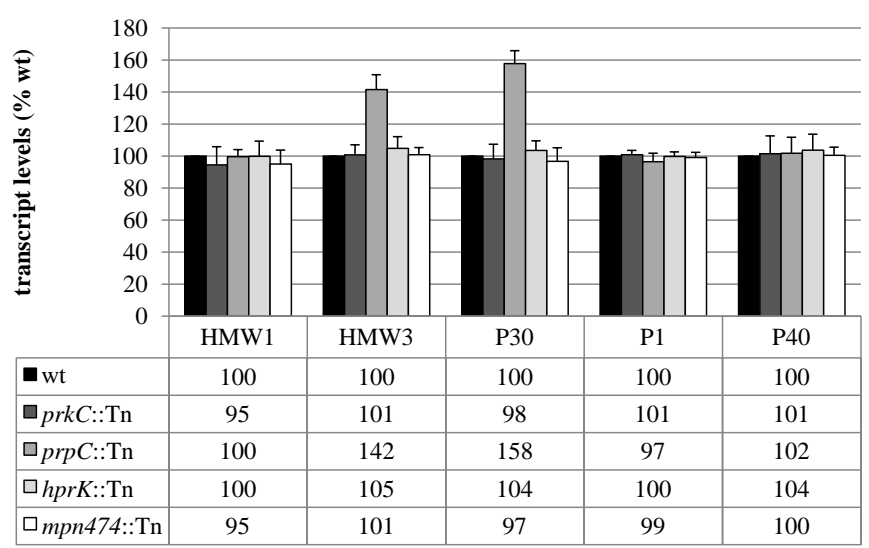

Fig. 7. Expression of cytadherence genes in M. pneumoniae wild type and mutant cells. Slot blot analyses with whole RNA extracts of the M. pneumoniae wild type and different mutant strains. A dilution series of RNA extracts was blotted onto a positively charged nylon membrane and probed with a DIG-labeled riboprobe specific for an internal part of a particular open reading frame. Names of riboprobes are given next to each blot. Signals obtained with $1 \mu \mathrm{g}$ of RNA are shown. Yeast tRNA and M. pneumoniae chromosomal DNA served as controls. Expression profiles of the genes of the P65 operon are shown (A), and the analyses of cytadherence genes of the HMW gene cluster and the major adhesins are also shown (B). The graphs show the quantification of transcript levels relative to that for the M. pneumoniae wild type. Error bars indicate the standard deviation (based on three independent experiments). 
For the P65 operon, we observed similar amounts of mRNA for the two proximal genes of the operon, MPN309 (encoding P65) and hmw2 (encoding HMW2) in the wild type and in the kinase and phosphatase mutants (Fig. 7A). This observation is in sharp contrast to the strongly reduced amounts of the corresponding proteins in the prkC mutant (Fig. 7A). For the two distal genes of the operon, MPN311 (P41) and MPN312 (P24), we observed a slight reduction of mRNA amounts in the prkC mutant (Fig. 7A). These reduced mRNA levels might explain the weak reduction of P24 protein accumulation, but they are not sufficient to account for the strongly reduced P41 amounts in the prkC mutant strain GPM11.

The genes of the HMW and the adhesin (P1 and P40) operons were all expressed at similar levels in the wild type as well as in the $\operatorname{prk} C, h p r K$, and $\operatorname{prpC}$ mutant strains (Fig. 7B). Again, the mRNA amounts were not affected by the $p r k C$ mutation, whereas the protein amounts were strongly reduced (Fig. 6C). This is in good agreement with the observations for the P65 operon and supports the conclusion that PrkC-dependent protein phosphorylation is implicated in the stability of the cytadherence and adhesion proteins in $M$. pneumoniae.

\section{Discussion}

Protein phosphorylation is a major mechanism to control the activities of proteins. This posttranslational modification has been observed with all organisms, for which it has been studied, including the minimal bacteria $M$. genitalium and M. pneumoniae (Su et al., 2007; this work). However, the physiological consequences of protein phosphorylation events in bacteria, as well as the responsible kinases, have remained largely unknown.

So far, only a few classes of serine/threonine protein kinases have been identified and characterized for bacteria. M. pneumoniae contains two of these protein kinases, HPrK and PrkC (Steinhauer et al., 2002; this work). While HPrK is generally thought to phosphorylate only one specific substrate, the HPr protein of the phosphotransferase system, a variety of substrates have been proposed for PrkC and its homologues in different gram-positive bacteria. In Bacillus subtilis, PrkC was shown to phosphorylate the elongation factors Tu and G, the small-ribosome-associated GTPase CpgA, and the potential stressosome component YezA (Absalon et al., 2009; Shah et al., 2008). PrkC is required for germination of $B$. subtilis spores in response to 
muropeptides (Shah et al., 2008). The extracellular PASTA domain of PrkC senses these muropeptides (Shah et al., 2008). Since M. pneumoniae neither sporulates nor possesses a peptidoglycan cell wall, it is not surprising that the PrkC protein of this bacterium does not contain a PASTA domain. Thus, it is safe to assume that M. pneumoniae PrkC has a function(s) that differs completely from that described for B. subtilis. Indeed, this work provides evidence that PrkC is required for adhesive growth of $M$. pneumoniae, and a prkC mutant has lost cytotoxicity. This is reminiscent of observations that have been made with several other pathogenic Firmicutes. In Streptococcus pyogenes, the homolog of PrkC is required for adherence to host cells and for invasion (Jin and Pancholi, 2006). Similarly, the kinase of Enterococcus faecalis is implicated in persistence in the intestine of mice (Kristich et al., 2007). In contrast, PrkC of Staphylococcus aureus was reported to phosphorylate glycolytic enzymes (Lomas-Lopez et al., 2007).

While the importance of PrkC-dependent protein phosphorylation is well established for many bacteria, the molecular consequences of the primary phosphorylation events have not been studied so far. Most often, protein phosphorylation results in a shift of a protein's activity (Johnson and Barford, 1993).

In this work, we observed that PrkC-dependent phosphorylation of large cytadherence proteins is required for the stability of these complexes. It is unknown, which of these proteins is responsible for the destabilization of cytadherence proteins in the prkC mutant, but our studies revealed that HMW1, HMW3, the major adhesin P1, and the surface protein MPN474 are subject to PrkC-dependent phosphorylation. This conclusion is supported not only by the absence of these proteins in cell extracts of the prkC mutant but also by the increased intensity of phosphorylation signals for these proteins in the $p r p C$ mutant strain. PrpC is the protein phosphatase that reverses PrkC-dependent protein phosphorylation. Thus, our work confirms and extends earlier reports on the phosphorylation of cytadherence proteins (Krebes et al., 1995; Su et al., 2007). We cannot exclude, however, the possibility that an additional kinase(s) might phosphorylate HMW3 and P1 (Fig. 5C). It is well established that the cytadherence proteins show reciprocal dependency in their stabilities, i.e., mutations in one of the corresponding genes result in destabilization and delocalization of the other proteins of the attachment structure (Balish and Krause, 2006; Popham et al., 1997; Willby et al., 2004). Specifically, HMW1 was shown to be required for HMW2 and P1 localization 
and stability. In turn, HMW2 is necessary for the stabilization of HMW3 and P65. As shown in this work, it has been suggested previously that the interdependence of the proteins of the attachment organelle does not involve reduced transcription (Popham et al., 1997). Taken together, these findings establish that phosphorylation of the HMW proteins by PrkC is a prerequisite for their stability and perhaps for their proper localization in the attachment structure. This is the first time that protein phosphorylation was shown to increase the stability of a protein.

M. pneumoniae has only limited regulatory potential. These bacteria may use, in addition to three potential transcription regulators, protein phosphorylation to adapt to changes in environmental conditions. An analysis of the $M$. pneumoniae genome revealed the presence of two genes for serine/threonine kinases, i.e., hprK and prkC, encoding the HPr kinase and protein kinase C (PrkC), respectively. Based on our present knowledge, it appears that these two proteins represent the total serine/threonine kinome of $M$. pneumoniae. It will be interesting to study how PrkC activity is triggered and whether other proteins are targets of this pleiotropic protein kinase.

\section{Acknowledgments}

We are grateful to Richard Herrmann for the gift of antibodies. Hinnerk Eilers is acknowledged for helpful discussions. This work was supported by Deutsche Forschungsgemeinschaft and the Fonds der Chemischen Industrie. S.R.S. was supported by a personal grant from the Studienstiftung des Deutschen Volkes. 


\section{Supplemental material}

Tab. S1. Oligonucleotides used in this study.

\begin{tabular}{|c|c|}
\hline Oligonucleotide & Sequence $\left(5^{\prime} \rightarrow 3^{\prime}\right)^{a}$ \\
\hline CH35 & GCTCAACTTGATTAATTTAAAACAATGG \\
\hline $\mathrm{CH} 74$ & CTAATACGACTCACTATAGGGAGAGGTGTTCAAAACTGTGGAGG \\
\hline SH29 & ATGAGTGAGCTAACTCACAG \\
\hline SH62 & TAGAATTTTATGGTGGTAGAG \\
\hline SH63 & CTAATACGACTCACTATAGGGAGAACACTATCATAACCACTACC \\
\hline SH89 & TAGAGCTCGATGGCACTAAATTTAAAGATTGG \\
\hline SS60 & GCTACTGCATACGATCCCAATC \\
\hline SS61 & CTAATACGACTCACTATAGGGAGACATAGTAAGCGTTGGGATCGG \\
\hline SS62 & GACACTGGGTTTGATGATGGG \\
\hline SS63 & CTAATACGACTCACTATAGGGAGACCTGGTTAGCTGCAATCTGTTC \\
\hline SS64 & GAAGCGCAACCAACTAACGC \\
\hline SS65 & CTAATACGACTCACTATAGGGAGAGTTGTTGGGACAGATCAGCAC \\
\hline SS66 & GATGATGAAGCTGACATCATCATAG \\
\hline SS67 & CTAATACGACTCACTATAGGGAGAGTGTATTCCGTGCCACCAATAAC \\
\hline SS68 & CTAACACTAAAACGGGTACGAATTG \\
\hline SS69 & CTAATACGACTCACTATAGGGAGAGCCACAAGTGTTTCTGATGTCAC \\
\hline SS70 & CTGAGGAAAATCCCGAACAGATC \\
\hline SS71 & CTAATACGACTCACTATAGGGAGAGCTGGTACATCTGCTTCCAAAC \\
\hline SS72 & GCCAACAGCGACATTAACAGC \\
\hline SS73 & CTAATACGACTCACTATAGGGAGACAGTATCACTGCAGAGCTTATAAAC \\
\hline SS74 & GTATGGTAAGCTAGCACAAAAGATC \\
\hline SS75 & CTAATACGACTCACTATAGGGAGAGTTGATCAGCTTGCTGTTCGG \\
\hline SS76 & CTTGGATTCTCATCCTCACCG \\
\hline SS77 & CTAATACGACTCACTATAGGGAGAGGTGCAGCCCCACTCAAAC \\
\hline SS78 & GCGAGCGGGTGGTTCG \\
\hline SS79 & CTAATACGACTCACTATAGGGAGAGCTTGTAAGCACTCGCTTCC \\
\hline SS80 & CCTGAATGCTAGTGCTGTTCAG \\
\hline SS81 & CTAATACGACTCACTATAGGGAGAGTTGAGGTGGGAAACCAGTAC \\
\hline SS86 & GCACGCATCTTAAAGCTGTTGG \\
\hline SS87 & CTAATACGACTCACTATAGGGAGACACTGTCAGCGTGCTCTGC \\
\hline SS92 & GCTAGCTTCTTCACTTACCGG \\
\hline SS93 & CTAATACGACTCACTATAGGGAGAGTTAACGTTCCTTCGTTACCATTG \\
\hline
\end{tabular}

${ }^{\mathrm{a}}$ The sequence of the T7-promotor is underlined. 
Tab. S2. Antibodies used in this study.

\begin{tabular}{llll}
\hline M. pneumoniae locus tags & Protein & Antibody number & Dilution \\
\hline MPN141 & P1 & 449 & $1: 1000$ \\
MPN146 & P40 & 66450 & $1: 250$ \\
MPN227 & FusA & 66708 & $1: 3000$ \\
MPN309 & P65 & 66578 & $1: 3000$ \\
MPN310 & HMW2 & 28050 & $1: 2000$ \\
MPN310 & HMW2-S (P28) & 86236 & $1: 1000$ \\
MPN311 & P41 & 86630 & $1: 1000$ \\
MPN312 & P24 & 85910 & $1: 250$ \\
\hline MPN447 & HMW1 & 84267 & $1: 10000$ \\
MPN452 & HMW3 & 65376 & $1: 5000$ \\
MPN453 & P30 & 123 & $1: 50000$ \\
MPN474 & MPN474 & 41804 & $1: 20000$ \\
MPN567 & P200 & 65020 & $1: 10000$ \\
\hline
\end{tabular}




\section{Chapter 3}

\section{The phosphoproteome of the minimal bacterium}

Mycoplasma pneumoniae:

\section{Analysis of the complete known Ser/Thr kinome suggests the existence of novel kinases}

The work described in this chapter was published in:

Schmidl, S. R., K. Gronau, N. Pietack, M. Hecker, D. Becher, and J. Stülke. 2010. The phosphoproteome of the minimal bacterium Mycoplasma pneumoniae: Analysis of the complete known Ser/Thr kinome suggests the existence of novel kinases. Mol. Cell. Proteomics 9: 1228-1242.

Author contributions:

This study was designed and interpreted by SRS and JS. SRS performed the phosphoproteome experiments in $M$. pneumonaie. NP contributed to the investigation of the autophosphorylation event of phosphosugar mutases, especially in $B$. subtilis. The phosphoproteome analysis was done in collaboration with $\mathrm{KG}, \mathrm{MH}$, and DB, University of Greifswald. KG and DB helped substantially with the evaluation of the phosphorylation sites. SRS and JS wrote the paper. 


\section{Abstract}

Mycoplasma pneumoniae belongs to the Mollicutes, the group of organisms with the smallest genomes that are capable of host-independent life. These bacteria show little regulation in gene expression, suggesting an important role for the control of protein activities. We have studied protein phosphorylation in $M$. pneumoniae to identify phosphorylated proteins. Two-dimensional gel electrophoresis and mass spectrometry allowed the detection of 63 phosphorylated proteins, many of them enzymes of central carbon metabolism and proteins related to host cell adhesion. We identified 16 phosphorylation sites, among them 8 serine and 8 threonine residues, respectively. A phosphoproteome analysis with mutants affected in the two annotated protein kinase genes or in the single known protein phosphatase gene suggested that only one protein (HPr) is phosphorylated by the HPr kinase, HPrK, whereas four adhesion-related or surface proteins were targets of the protein kinase C, PrkC. A comparison with the phosphoproteomes of other bacteria revealed that protein phosphorylation is evolutionarily only poorly conserved. Only one single protein with an identified phosphorylation site, a phosphosugar mutase (ManB in M. pneumoniae), is phosphorylated on a conserved serine residue in all studied organisms from archaea and bacteria to man. We demonstrate that this protein undergoes autophosphorylation. This explains the strong conservation of this phosphorylation event. For most other proteins, even if they are phosphorylated in different species, the actual phosphorylation sites are different. This suggests that protein phosphorylation is a form of adaptation of the bacteria to the specific needs of their particular ecological niche.

\section{Introduction}

Bacteria of the group called Mollicutes are unique among all living organisms because of their small genome that nevertheless allows them to grow independently from any host cell. The two most intensively studied representatives of the Mollicutes are Mycoplasma genitalium and Mycoplasma pneumoniae with genome sizes of 580 and $816 \mathrm{~kb}$, respectively. The approximately 475 protein-coding and 43 RNA-coding genes of $M$. genitalium define the lower limit of the genetic equipment that permits independent life. The small genomes of the Mollicutes reflect their adaptation to rarely changing ecosystems, such as lung epithelia for M. pneumoniae (Stülke et al., 2009). 
Furthermore, M. pneumoniae has only limited metabolic capabilities: This bacterium can utilize only a few carbon sources (glucose, fructose, and glycerol) (Halbedel et al., 2004), and its only way to produce ATP is by substrate level phosphorylation in glycolysis. The citric acid cycle, respiration, and most anabolic reactions are not carried out by M. pneumoniae (Halbedel et al., 2007).

M. pneumoniae is a human pathogen that causes usually mild infections such as atypical pneumonia; however, the infections may be severe in children and elderly people. In addition, $M$. pneumoniae is involved in extrapulmonary complications, such as erythema multiforme and pediatric encephalitis (Atkinson et al., 2008; Jacobs, 1997; Waites and Talkington, 2004).

The close adaptation of $M$. pneumoniae to human mucosal surfaces did not only result in a reductive evolution of metabolic capabilities but also affected the regulation of protein biosynthesis and activity: In contrast to versatile environmental bacteria such as Pseudomonas aeruginosa that reserve about $10 \%$ of their coding capacity for regulators of gene expression, $M$. pneumoniae encodes only three potential transcription regulators (less than $0.5 \%$ of all genes) (Stülke et al., 2009). This absence of regulation at the level of gene expression suggests that the control of protein activities might play an important role in M. pneumoniae and other Mollicutes.

An important way to control the activity of a protein is posttranslational modification with protein phosphorylation being the most prominent regulatory modification both in bacteria and in eukaryotes (Boekhorst et al., 2008; Jers et al., 2008). Studies on the phosphoproteome have been performed for several bacteria, including the model organisms Escherichia coli and Bacillus subtilis. In both bacteria, about $5 \%$ of all proteins can be phosphorylated on Ser, Thr, or Tyr residues (Eymann et al., 2007; Lévine et al., 2006; Macek et al., 2008; Macek et al., 2007). Interestingly, there is a high level of species specificity with respect to the phosphoproteome: A comparison of the phosphoproteomes of B. subtilis, E. coli, and Lactococcus lactis revealed only eight proteins that are phosphorylated in all three species. Six of these proteins are involved in sugar metabolism, and two are translation factors. Of these eight proteins, only one, phosphoglucosamine mutase, is phosphorylated at a conserved residue (Soufi et al., 2008).

In M. pneumoniae, only the phosphorylation of the HPr protein of the phosphoenolpyruvate:sugar phosphotransferase system by the HPr kinase has been 
studied to some detail (Allen et al., 2003; Halbedel et al., 2004; Steinhauer et al., 2002). Once phosphorylated, HPr can be dephosphorylated by a protein phosphatase, PrpC (Halbedel et al., 2006). The corresponding prpC gene is in many Gram-positive bacteria clustered with the gene for a protein kinase, $p r k C$, suggesting that PrkC and PrpC form a functional couple with opposing activities. Evidence for this idea was provided by the observation that the two proteins from $B$. subtilis have identical targets in vitro (Absalon et al., 2009). The prpC/prkC gene cluster also exists in M. pneumoniae; however, the targets of the corresponding proteins are so far unknown. Previous studies with $M$. pneumoniae demonstrated that several proteins implicated in adhesion to the host cell are subject to protein phosphorylation in vivo (Dirksen et al., 1994; Krebes et al., 1995; Schmidl et al., 2010). These proteins, HMW1 and HMW2, are important for the virulence of $M$. pneumoniae because adhesion is the first step in the interaction with the host. A recent proteomics study resulted in the identification of 18 phosphorylated proteins in $M$. pneumoniae, among them adhesion and surface proteins and metabolic enzymes (Su et al., 2007). Unfortunately, none of the previously known phosphoproteins were detected in this study. Moreover, Ser-46 in HPr is still the only known phosphorylation site in $M$. pneumoniae (with the exception of phosphorylated His and Cys residues in phosphotransferase system proteins), and the (de)phosphorylation of HPr is the only event, for which the kinase and the phosphatase are known. Finally, nothing is known about protein tyrosine phosphorylation in M. pneumoniae and other Mollicutes.

We are interested in the elucidation of the molecular networks that allow life of M. pneumoniae as a minimal organism. The analysis of these bacteria is hampered by their slow growth and by the lack of efficient genetic systems that allow the elucidation of gene functions. We have established a technique, haystack mutagenesis, that can be used to isolate mutants of M. pneumoniae (Halbedel et al., 2006). In this work, we have studied the phosphoproteome of the $M$. pneumoniae wild type strain and of three isogenic mutants that are affected in the two protein kinases, HPr kinase (HPrK) and protein kinase C (PrkC), and in the protein phosphatase PrpC. We identified 63 phosphorylated proteins. However, most of these proteins are phosphorylated by so far unknown kinases. An in-depth analysis of the only universally conserved phosphoprotein, a phosphosugar mutase, revealed that this enzyme autophosphorylates. 


\section{Experimental procedures}

Bacterial strains and growth conditions. The $M$. pneumoniae strains used in this study were M. pneumoniae M129 (ATCC 29342) in the 32nd broth passage and its isogenic mutant derivatives GPM11 (prkC::mini-Tn, Gm ${ }^{\mathrm{R}}$ ) (Schmidl et al., 2010), GPM51 (hprK::mini-Tn, Gm ${ }^{\mathrm{R}}$ ) (Halbedel et al., 2006), and GPM68 (prpC::mini-Tn, $\mathrm{Gm}^{\mathrm{R}}$ ) (Halbedel et al., 2006). M. pneumoniae was grown at $37^{\circ} \mathrm{C}$ in $150-\mathrm{cm}^{2}$ tissue culture flasks containing $100 \mathrm{ml}$ of modified Hayflick medium with glucose $(1 \%, \mathrm{wt} / \mathrm{vol})$ as the carbon source as described previously (Halbedel et al., 2004). Strains harboring transposon insertions were cultivated in the presence of $80 \mu \mathrm{g} / \mathrm{ml}$ gentamicin. B. subtilis 168 (trpC2; laboratory collection) was grown in LB and in minimal medium containing succinate and glutamate/ammonium as basic sources of carbon and nitrogen, respectively (Faires et al., 1999). The media were supplemented with tryptophan (at $50 \mathrm{mg} / \mathrm{liter}$ ) and glucose (0.5\%, wt/vol). B. subtilis was transformed with plasmid DNA or PCR products according to the two-step protocol (Kunst and Rapoport, 1995). Transformants were selected on sporulation medium plates (Faires et al., 1999) containing erythromycin plus lincomycin (2 and $25 \mu \mathrm{g} / \mathrm{ml}$, respectively) and/or spectinomycin $(100 \mu \mathrm{g} / \mathrm{ml})$.

Preparation of cell extracts. After 4 days of incubation at $37^{\circ} \mathrm{C}$, the culture medium was removed from the $M$. pneumoniae cell layer, and the cells were washed twice with $20 \mathrm{ml}$ of cold PBS. After washing, cells were collected by scraping into $1.5 \mathrm{ml}$ of PBS and subsequent centrifugation $\left(5 \mathrm{~min}, 15,000 \times \mathrm{g}, 4^{\circ} \mathrm{C}\right.$ ). The cell pellet was resuspended in $500 \mu \mathrm{l}$ of $10 \mathrm{mM}$ Tris- $\mathrm{HCl}, \mathrm{pH}$ 7.5, and the cells were disrupted by sonication $\left(3 \times 10 \mathrm{~s}, 50\right.$ watts, $\left.4^{\circ} \mathrm{C}\right)$. Cell debris were sedimented by centrifugation (10 min, 15,000 $\times \mathrm{g}, 4^{\circ} \mathrm{C}$ ), and the resulting supernatant was centrifuged again (30 min, $24,000 \times \mathrm{g}, 4^{\circ} \mathrm{C}$ ) to remove disturbing particles. The protein concentration of the supernatant was determined using the Bio-Rad dye binding assay with bovine serum albumin as the standard.

Two-dimensional SDS-polyacrylamide gel electrophoresis. Two-dimensional separation of proteins was performed as described previously (Halbedel et al., 2007). For separation in the first dimension, IPG strips with a linear $\mathrm{pH}$ range of 4-7, 4.5-5.5, or 6-11 were used (Immobiline ${ }^{\mathrm{TM}}$ DryStrip, Amersham Biosciences). The gels were subsequently stained with Pro-Q Diamond (Invitrogen) and Flamingo ${ }^{\mathrm{TM}}$ fluorescent dye (Bio-Rad) to visualize the phosphoproteins and all proteins, respectively. Image 
analysis, spot quantification, and determination of putative phosphorylated protein spots were performed as described previously (Eymann et al., 2007). The determination of phosphorylated proteins was based on the Pro-Q/Flamingo log ratio. Phosphoprotein spots were cut from the gel and identified by mass spectroscopy.

Protein identification by mass spectrometry. Gel pieces were washed twice with $200 \mu \mathrm{l}$ of $20 \mathrm{mM} \mathrm{NH}_{4} \mathrm{HCO}_{3}, 30 \%(\mathrm{v} / \mathrm{v}) \mathrm{CH}_{3} \mathrm{CN}$ for $30 \mathrm{~min}$ at $37^{\circ} \mathrm{C}$ and dried in a vacuum centrifuge (Concentrator 5301, Eppendorf). Trypsin solution (10 ng/ $\mu$ l trypsin in $20 \mathrm{mM}$ ammonium bicarbonate) was added until gel pieces stopped swelling, and digestion was allowed to proceed for $16-18 \mathrm{~h}$ at $37^{\circ} \mathrm{C}$. Peptides were extracted from gel pieces by incubation in an ultrasonic bath for 15 min in $20 \mu \mathrm{l}$ of HPLC grade water and transferred into microvials for mass spectrometric analysis. Peptides were separated by liquid chromatography and measured on line by ESI-mass spectrometry using a nanoACQUITY UPLC ${ }^{\mathrm{TM}}$ system (Waters) coupled to an LTQ Orbitrap ${ }^{\mathrm{TM}}$ mass spectrometer (Thermo Fisher Scientific, Waltham, MA). Peptides were desalted onto a trap column (Symmetry ${ }^{\circledR} \mathrm{C}_{18}$, Waters). Elution was performed onto an analytical column (BEH130 $\mathrm{C}_{18}$, Waters) by a binary gradient of buffer A [0.1\% (v/v) acetic acid] and B [100\% (v/v) acetonitrile, $0.1 \%(\mathrm{v} / \mathrm{v})$ acetic acid] over a period of $50 \mathrm{~min}$ with a flow rate of $400 \mathrm{nl} / \mathrm{min}$. The LTQ Orbitrap was operated in data-dependent MS/MS mode using multistage activation for phosphorelevant masses. Proteins were identified by searching all MS/MS spectra in .dta format against all 689 M. pneumoniae proteins (extracted from the NCBI database) using Sorcerer $^{\mathrm{TM}}{ }_{-}$SEQUEST $^{\circledR}$ (SEQUEST version 2.7 revision 11, Thermo Electron) including Scaffold_2_05_02 (Proteome Software Inc., Portland, OR). SEQUEST was searched with a fragment ion mass tolerance of $1.00 \mathrm{Da}$ and a parent ion tolerance of $10 \mathrm{ppm}$. Up to two missed tryptic cleavages were allowed. Methionine oxidation (+15.99492 Da), cysteine carbamidomethylation (+57.021465 Da), and phosphorylation (+79.966331 Da) were set as variable modifications. For protein identification, a stringent SEQUEST filter for peptides was used (Xcorr versus charge state: 1.90 for singly, 2.2 for doubly, and 3.3 for triply charged ions and $\Delta \mathrm{Cn}$ value greater than 0.10 ), and at least two peptides per proteins were required. With this method, all identified proteins had a protein probability score greater than $99.9 \%$. Protein probabilities were assigned by the Protein Prophet algorithm (Nesvizhskii et al., 2003). Phosphorylated peptides that had a peptide probability score greater than $95.0 \%$ as specified by the Peptide Prophet algorithm 
(Keller et al., 2002) were examined manually and accepted only when b- or y-ions confirmed the identification. All spectra with Scaffold-annotated b- and y-ions series are provided as screen shots in the supplemental material (see supplemental Fig. S3).

Analysis of protein conservation and structure prediction. To address the potential conservation of phosphorylation sites, multiple sequence alignments were performed using the EXPRESSO alignment server that takes available structures into account (Armougom et al., 2006). Structural models of the phosphoproteins were created using SWISS-MODEL in the automated mode and Swiss-PdbViewer v4.0.1 (Guex and Peitsch, 1997; Kiefer et al., 2009). Ordered and disordered regions of proteins were predicted using the DisEMBL v1.5 web server (Linding et al., 2003).

Cloning procedures. E. coli DH5 $\alpha$ (Sambrook et al., 1989) was used for cloning experiments and protein expression. E. coli was grown in LB medium. LB plates were prepared by addition of $17 \mathrm{~g}$ of Bacto agar/liter (Difco) to LB (Sambrook et al., 1989). Transformation of E. coli and plasmid DNA extraction were performed using standard procedures (Sambrook et al., 1989). Restriction enzymes, T4 DNA ligase, and DNA polymerases were used as recommended by the manufacturers. DNA fragments were purified from agarose gels using the QIAquick Gel Extraction kit (Qiagen). Pfu DNA polymerase was used for the polymerase chain reaction as recommended by the manufacturer. DNA sequences were determined using the dideoxy chain termination method (Sambrook et al., 1989). Standard procedures were used to transform E. coli (Sambrook et al., 1989), and transformants were selected on LB plates containing ampicillin $(100 \mu \mathrm{g} / \mathrm{ml})$.

Plasmid constructions. Plasmids for the overexpression and purification of the phosphosugar mutases GlmM and ManB from B. subtilis and M. pneumoniae, respectively, were constructed as follows. The coding sequence of the genes was amplified by PCR with gene-specific primers (listed in supplemental Table S3) using chromosomal DNA of $B$. subtilis 168 and $M$. pneumoniae M129 as the template. The PCR products were digested as shown in supplemental Table S4 and cloned into the appropriately linearized expression vector pWH844 (Schirmer et al., 1997). These plasmids allowed the expression of the phosphosugar mutases carrying an $\mathrm{N}$-terminal His $_{6}$-tag. The resulting plasmids pGP1401 and pGP656 are listed in supplemental Table S4. In M. pneumoniae, the UGA specifies a tryptophan; however, in E. coli, it is a stop codon. The manB gene contains three UGA codons that were replaced by the 
multiple mutation reaction (Hames et al., 2005) using the phosphorylated mutagenic oligonucleotides SS109, SS110, and SS111 (see supplemental Table S3) and plasmid pGP656 as the template. The resulting plasmid was pGP657 (see supplemental Table S4). The phosphorylation sites of GlmM and ManB were replaced by alanine residues. For this purpose, the combined chain reaction (Bi and Stambrook, 1998) was applied with the mutagenic primers NP116 and SS112 (see supplemental Table S3) and plasmids pGP1401 and pGP657. The resulting plasmids are pGP1405 ( $\mathrm{glmM})$ and pGP658 (manB) (see supplemental Table S4). All plasmid inserts were verified by DNA sequencing.

The plasmids pGP400 and pGP1403 were used to express the wild type and S100A mutant forms of GlmM, respectively, in B. subtilis. For the construction of pGP400, the glmM gene was amplified using the oligonucleotides NP108/NP109. The PCR product was digested with BamHI and SalI and cloned into pBQ200 (Martin-Verstraete et al., 1994) digested with the same enzymes. For the construction of the plasmid encoding the S100A GlmM variant, mutagenesis was performed using the combined chain reaction with NP116 as the mutagenic primer and NP108/NP109. The resulting PCR product was cloned into pBQ200 as described for the wild type allele. The resulting plasmid was pGP1403.

Overexpression and purification of recombinant proteins. E. coli $\mathrm{DH} 5 \alpha$ transformed with the appropriate expression vector was used as host for the overexpression of recombinant proteins. Expression was induced by the addition of isopropyl 1-thio- $\beta$-D-galactopyranoside (final concentration, $1 \mathrm{mM}$ ) to exponentially growing cultures $\left(A_{600}\right.$ of 0.6$)$. Cells were lysed using a French press (20,000 p.s.i., 138,000 kilopascals; Spectronic Instruments). After lysis, the crude extracts were centrifuged at $15,000 \times \mathrm{g}$ for $1 \mathrm{~h}$. For purification of His $_{6}$-tagged proteins, the resulting supernatants were passed over a $\mathrm{Ni}^{2+}$ HiTrap chelating column (4-ml bed volume; GE Healthcare) followed by elution with an imidazole gradient (from 0 to $500 \mathrm{mM}$ imidazole in a buffer containing $10 \mathrm{mM}$ Tris- $\mathrm{HCl}, \mathrm{pH}$ 7.5, $600 \mathrm{mM} \mathrm{NaCl}$, $10 \mathrm{mM} \beta$-mercaptoethanol) over $30 \mathrm{ml}$ at a flow rate of $0.5 \mathrm{ml} / \mathrm{min}$. After elution, the fractions were tested for the desired protein using 12.5\% SDS-PAGE. The relevant fractions were combined and dialyzed overnight. Protein concentration was determined using the Bio-Rad dye binding assay where bovine serum albumin served as the standard. 
Assays for protein phosphorylation. Protein phosphorylation assays were carried out with purified proteins in assay buffer $[25 \mathrm{mM}$ Tris-HCl, pH 7.6, $1 \mathrm{mM}$ dithiothreitol, $0.4 \mathrm{mM}\left[\gamma_{-}{ }^{32} \mathrm{P}\right] \mathrm{ATP}(480 \mathrm{Ci} / \mathrm{mmol})$, divalent cations as indicated] using purified target proteins. The assays were carried out at $37^{\circ} \mathrm{C}$ for $30 \mathrm{~min}$ followed by thermal inactivation of the protein $\left(10 \mathrm{~min}\right.$ at $\left.95^{\circ} \mathrm{C}\right)$. The assay mixtures were analyzed on $16 \%$ SDS-polyacrylamide gels. Proteins were visualized by Coomassie staining. Radioactive protein spots were detected using a phosphorimaging system (Storm 860, GE Healthcare).

Allelic replacement of $\boldsymbol{B}$. subtilis gImM gene. To delete the chromosomal copy of the $\operatorname{glmM}$ gene, the long flanking homology PCR technique was used (Wach, 1996). Briefly, a cassette carrying the spc resistance gene was amplified from plasmid pDG1726 (Guérout-Fleury et al., 1995). DNA fragments of about 1,000 bp flanking the gImM gene at its 5' and 3' ends were amplified. The 3' end of the upstream fragment as well as the 5' end of the downstream fragment extended into $\operatorname{glm} M$ in a way that all expression signals of genes up- and downstream of the $g \operatorname{lm} M$ remained intact. The joining of the two fragments to the resistance cassette was performed in a second PCR as described previously (Jordan et al., 2006). In this reaction, we used the primer pair spec-fwd/spec-rev (Jordan et al., 2006) for the amplification and joining of the spc cassette. The PCR product was directly used to transform B. subtilis carrying plasmids that express different alleles of $g \operatorname{lmM}$. The integrity of the regions flanking the integrated resistance cassette was verified by sequencing PCR products of about 1,000 bp amplified from chromosomal DNA of the resulting mutants.

\section{Results}

Phosphoproteome of growing $M$. pneumoniae cells. In a previous study on protein phosphorylation in M. pneumoniae, no phosphorylation sites were determined, and no mutants affected in the players of protein phosphorylation were studied (Su et al., 2007). To get a more comprehensive insight into protein phosphorylation in M. pneumoniae, we decided to analyze the phosphoproteome of the wild type strain M129 and of three isogenic mutants defective in the two annotated protein kinases and the single annotated protein phosphatase of M. pneumoniae. 
A

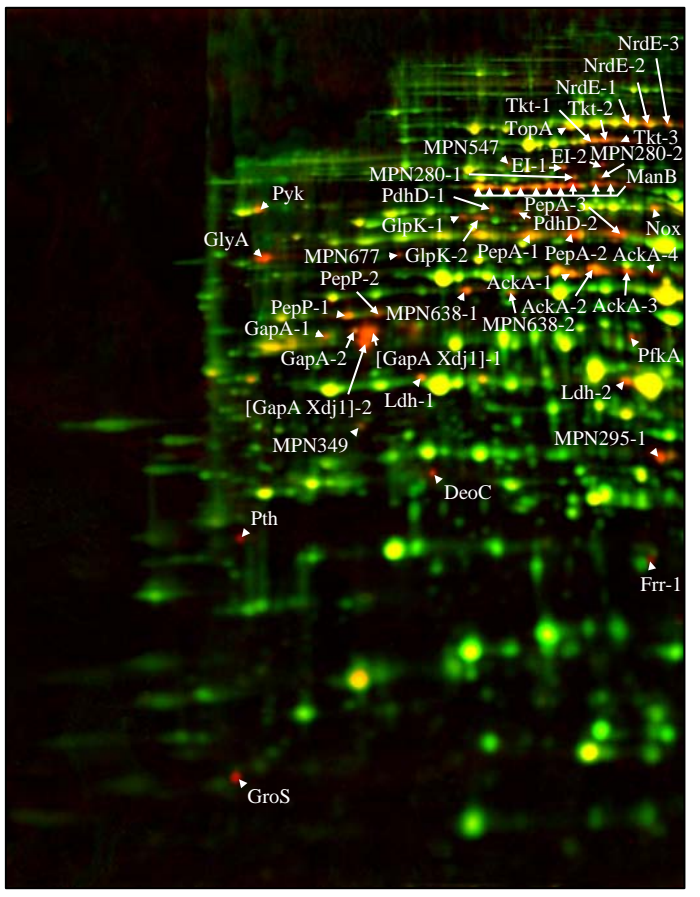

pI 11.0
B

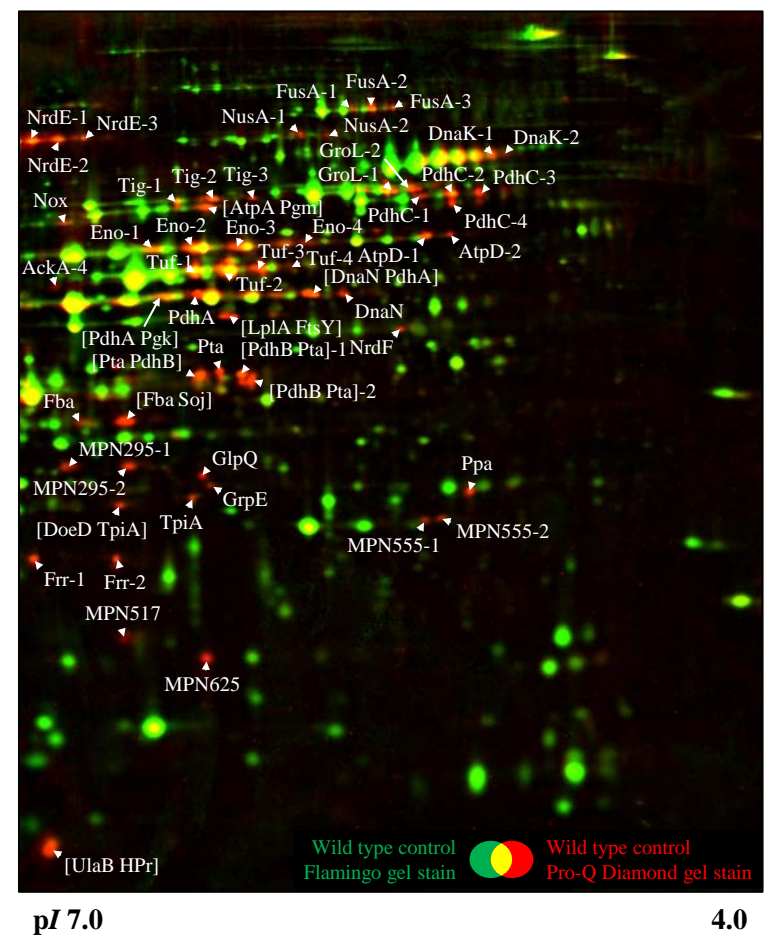

Fig. 8. Dual channel image of total protein amount (Flamingo fluorescent dye-stained; green) and phosphorylated proteins (Pro-Q Diamond-stained; red) of stationary phase $M$. pneumoniae cells. About $125 \mu \mathrm{g}$ of a total protein extract of $M$. pneumoniae grown in modified Hayflick medium supplemented with glucose were subjected to two-dimensional gel electrophoresis using either an 18-cm IPG strip with a linear pH gradient of pI 6-11 (A) or pI 4-7 (B) in the first dimension. Dual channel images were obtained using the software DECODON Delta2D v3.6. Spots with high Pro-Q Diamond/Flamingo log ratios representing phosphorylated proteins (Eymann et al., 2007) are indicated on the gel. Multiple protein spots corresponding to the same original protein are distinguished by appended digits (e.g. Tuf-1 and Tuf-2). Protein spots were cut from the gel and identified by MS/MS (see Table 4 and supplemental Table S5).

The wild type strain M129 was grown in modified Hayflick medium with glucose as the principal carbon source, and cells were harvested in the late logarithmic phase. Protein extracts were prepared as described under "Experimental procedures" and the proteins were separated by two-dimensional gel electrophoresis (see Fig. 8). Traditional two-dimensional gels cover only the neutral and acidic proteins ( $\mathrm{pH}$ range, 4-7). However, the theoretical proteome of $M$. pneumoniae shows a high number of more basic proteins that might escape detection (see supplemental Fig. S1). To include these basic proteins, we performed the isoelectric focusing in two $\mathrm{pH}$ ranges, 4-7 and 6-11. The gels were stained with Pro-Q Diamond and Flamingo fluorescent dye 
to detect the phosphorylated proteins and the all proteins, respectively. The presence of more than 700 protein spots on the gels revealed that the proteome of $M$. pneumoniae was completely covered by our system (Jaffe et al., 2004; Regula et al., 2000). The identification of phosphorylated proteins revealed 58 phosphoproteins (see Table 4). We observed 36 phosphorylated proteins in the $\mathrm{pH}$ range from 4 to 7 and 27 phosphoproteins in the $\mathrm{pH}$ range from 6 to 11 with only five proteins (i.e. AckA, Frr, MPN295, Nox, and NrdE) detected in both gel systems. Of the proteins previously known to be phosphorylated, we detected HPr and, with the exception of the ribosomal protein S2 and the adhesin-related protein P65, all 17 phosphoproteins reported by Su et al. (2007).

In a next step, we tried to identify the phosphorylation sites in all phosphorylated peptides that had a peptide probability score greater than $95.0 \%$ as specified by the Peptide Prophet algorithm (see “Experimental procedures”). This analysis identified 12 phosphorylation sites in 10 phosphoproteins (see Table 4). These phosphorylations occurred on serine (eight sites) and threonine (four sites). Two proteins possessing two phosphorylation sites, NrdE and PepA, were phosphorylated on both a serine and a threonine residue. It is interesting to note that the individual $\mathrm{NrdE}$ molecules were phosphorylated only on one site: The two phosphopeptides for this protein were identified in different protein spots. In contrast, both PepA phosphopeptides were present in the protein from one spot, suggesting that this molecule was indeed doubly phosphorylated.

Phosphoproteome in prpC mutant. The $M$. pneumoniae genome encodes one protein phosphatase, PrpC. Previously, we have shown that PrpC dephosphorylates HPr(Ser-P) (Halbedel et al., 2006). We considered that the analysis of the phosphoproteome in a $\operatorname{prpC}$ mutant might allow the identification of additional phosphorylated proteins that may have escaped our attention in the experiments with the wild type strain. Therefore, we cultivated the $\operatorname{prpC}$ mutant strain GPM68 as described above for the wild type and analyzed the protein phosphorylation pattern by two-dimensional gel electrophoresis. A comparison of the phosphoproteomes of the two strains revealed that all phosphoproteins detected in the wild type were also present in the prpC mutant. In addition, a few additional phosphoprotein spots were detected in the prpC mutant. These proteins had isoelectric points between 4 and 5 (data not shown). Therefore, we increased the resolution for these proteins by performing the isoelectric 
focusing in the $\mathrm{pH}$ range from 4.5 to 5.5. In the $\operatorname{prpC}$ mutant, we observed five phosphoprotein spots that were not present in the wild type strain. The corresponding proteins were RpoE, the $\delta$ subunit of RNA polymerase, the adhesin-related protein P41, the high molecular weight cytadherence protein HMW3, the uncharacterized protein MPN256, and the cell surface protein MPN474 (see Table 4 and Fig. 9). With the exception of RpoE, phosphorylation of these proteins seemed likely in the wild type strain, but was at the detection limit.

The analysis of the phosphorylated peptides that occur exclusively in the prpC mutant led to the identification of four additional phosphorylation sites. For P41, MPN256, and MPN474, phosphorylation occurred on a threonine residue. In addition, two different phosphorylation sites (Ser-87 and Thr-93) could be validated independently on the same phosphopeptide in the case of RpoE. However, the exact site could not be determined unambiguously, so it could only be assumed that phosphorylation occurred most likely on the threonine residue.

\section{Effect of inactivation of Ser/Thr kinases on phosphoproteome.}

M. pneumoniae encodes two known serine/threonine kinases, i.e. the HPr kinase HPrK and the protein kinase $\mathrm{C}$ PrkC. To analyze the impact of these kinases on protein phosphorylation on a global level, we analyzed the phosphoproteome in the corresponding mutant strains. As described for the wild type strain M129, the isogenic mutants GPM51 ( $p r k$ ) and GPM11 (prkC) were grown in modified Hayflick medium, and cell extracts separated by two-dimensional gel electrophoresis.

In the hprK mutant, only one phosphoprotein spot appeared to be less intense as compared with the wild type (see Fig. 10). In the wild type strain, this spot contains two phosphorylated proteins, $\mathrm{HPr}$ and UlaB, a putative EIIB component of the phosphotransferase system. In the hprK mutant GPM51, this spot contained only the phosphorylated UlaB protein, whereas HPr was absent. This observation is in good agreement with the previous report that HPrK is the only kinase for HPr (Halbedel et al., 2006). On the other hand, HPr is the only substrate that is phosphorylated by HPrK. 
A

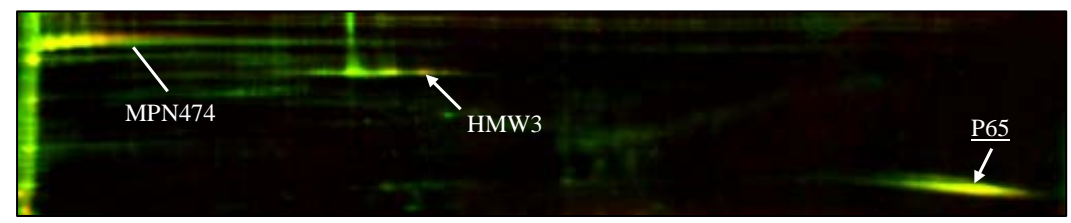

wt

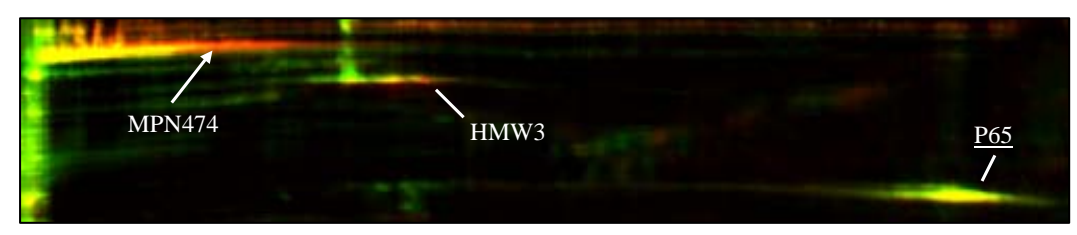

prpC::Tn

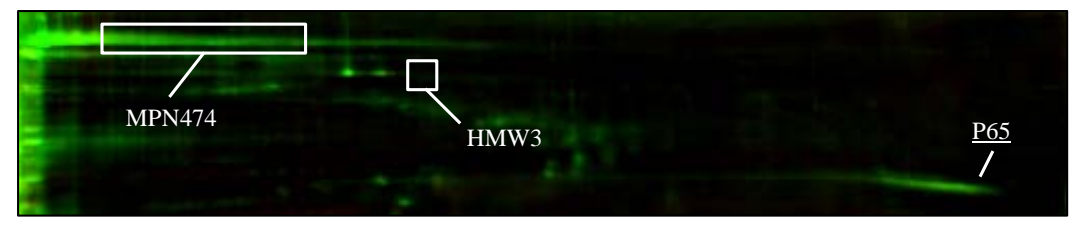

prkC::Tn

B

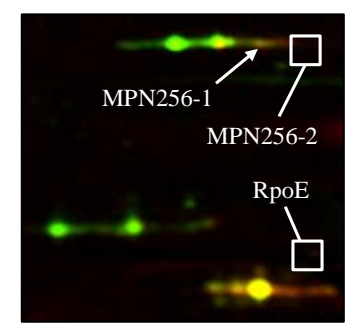

wt

C

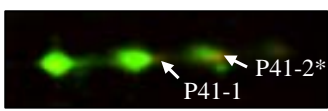

wt

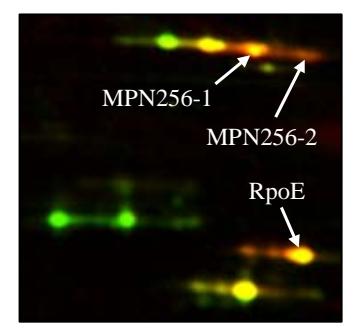

$\operatorname{prpC}:$ Tn

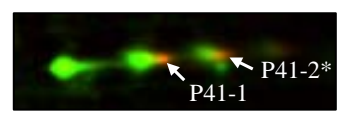

prpC::Tn

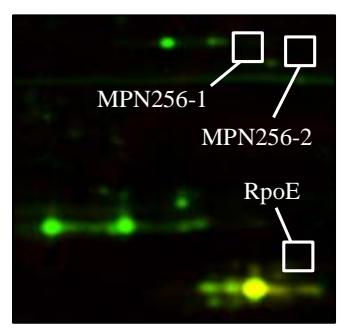

prkC::Tn

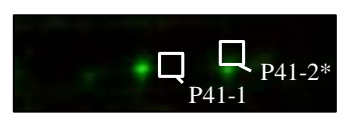

prkC::Tn

Flamingo ( Pro-Q Diamond

Fig. 9. PrpC/PrkC-dependent modification of cytadherence proteins in M. pneumoniae. Shown are sections of dual channel images of Flamingo fluorescent dye- (green) and Pro-Q Diamond-stained (red) two-dimensional gels representing selected proteins in the M. pneumoniae wild type, $\operatorname{prp} C:: \operatorname{Tn}$ mutant, and $p r k C:: T n$ mutant. Proteins were separated on an 18-cm IPG strip with a linear $\mathrm{pH}$ gradient of pI 4.5-5.5 in the first dimension. The proteins HMW3, MPN474, P65 (A), MPN256, RpoE (B), and P41 (C) are shown. Putative phosphorylated proteins in the M. pneumoniae wild type as well as proteins with increased or new phosphorylation spots in the prpC::Tn mutant are indicated. Missing phosphorylation spots in the M. pneumoniae wild type and the prkC::Tn mutant are highlighted by a box. Protein amounts of HMW3, MPN256, and P41 (all represented by a triple protein spot) seem to be reduced as well as for the P65 protein (underlined) in the $p r k C::$ Tn mutant. Note that the P41 spot " $2 *$ " is only putative because of a low Pro-Q Diamond/Flamingo log ratio in the analyzed strains. Protein spots were cut from the gel and identified by MS/MS (see Table 4 and supplemental Table S5). 
For the prkC mutant, we observed that the majority of phosphoproteins were not affected by the inactivation of this kinase. However, the phosphorylation or the accumulation of a few proteins was different in the prkC mutant as compared with the isogenic wild type. The large surface protein MPN474 was weakly phosphorylated in the wild type, and this protein was not at all phosphorylated in the prkC mutant, suggesting that MPN474 is a substrate for PrkC. This hypothesis is reinforced by the finding that MPN474 phosphorylation is strongly enhanced in the $\operatorname{prpC}$ phosphatase mutant (see Fig. 9A). Similarly, the so far uncharacterized protein MPN256 and the cytadherence proteins HMW3 and P41 were phosphorylated in the wild type but not in the prkC mutant strain GPM11. As observed with MPN474, the intensity of the phosphoprotein spot of these proteins was strongly increased in the $\operatorname{prpC}$ mutant (see Fig. 9A and B). Thus, MPN256, HMW3, and P41 are novel targets of PrkC-dependent phosphorylation in M. pneumoniae.

The prkC mutation did not only affect protein phosphorylation but also the accumulation of some proteins. MPN256, a substrate of PrkC, was much less abundant in the prkC mutant as compared with the wild type. Similarly, the adhesion proteins P41 and HMW3 were present in reduced amounts in the prkC mutant. In addition, the adhesin-related protein P65 was less abundant in the prkC mutant. However, the phosphorylation signals for this protein in the wild type and the $\operatorname{prp} C$ mutant strains were below the threshold to conclude phosphorylation.

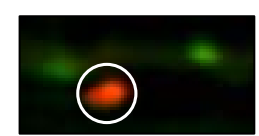

$\mathbf{w t}$

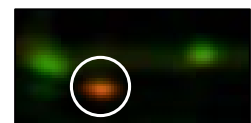

hprK::Tn

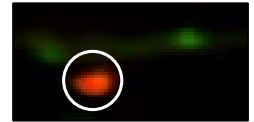

prpC::Tn

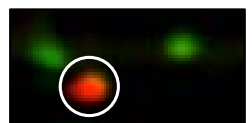

prkC::Tn

Flamingo $\bigcirc$ Pro-Q Diamond

Fig. 10. Comparison of HPr phosphorylation pattern between different M. pneumoniae strains. Section of dual channel images of Flamingo fluorescent dye- (green) and Pro-Q Diamond-stained (red) two-dimensional gels displaying the phosphorylated spot of HPr in the M. pneumoniae wild type and different mutant strains. Proteins were separated on an 18-cm IPG strip with a linear pH gradient of pI 4-7 in the first dimension. Note that the HPr spot represents a multiprotein spot containing HPr and UlaB, whereas only UlaB could be detected in the hprK::Tn mutant (much lesser intensity of the phosphorylation spot). 
Tab. 4. Phosphoproteins of M. pneumoniae.

For detailed MS/MS data, see supplemental Tables S5 and S6 and Fig. S3. pS, phosphoserine; pT, phosphothreonine.

\begin{tabular}{|c|c|c|c|c|c|c|}
\hline $\begin{array}{l}\text { Locus } \\
\text { name }\end{array}$ & $\begin{array}{l}\text { Protein } \\
\text { name }\end{array}$ & Protein function & $\mathbf{C O G}^{\mathrm{a}}$ & $\begin{array}{l}\text { Phospho spots in } \\
\text { two-dimensional gel }\end{array}$ & Phosphosite & Phosphopeptide sequence \\
\hline MPN001 & DnaN & DNA polymerase III subunit $\beta$ & $\mathrm{L}$ & 2 & & \\
\hline MPN002 & Xdj1 & DnaJ-like protein & $\mathrm{O}$ & 2 & & \\
\hline MPN024 & RpoE & RNA polymerase subunit $\delta$ & K & 1 & $\begin{array}{l}\text { [Ser-87 or } \\
\text { Thr-93] }\end{array}$ & Ip[SQAMFVT]KEIFEEGYEDLSNK \\
\hline MPN025 & Fba & Fructose-bisphosphate aldolase & $\mathrm{G}$ & 2 & & \\
\hline MPN050 & GlpK & Glycerol kinase & $\mathrm{C}$ & 2 & & \\
\hline MPN053 & $\mathrm{HPr}$ & Phosphocarrier protein & G & 1 & & \\
\hline MPN062 & DeoD & Purine-nucleoside phosphorylase & $\mathrm{F}$ & 1 & & \\
\hline MPN063 & DeoC & Deoxyribose-phosphate aldolase & $\mathrm{F}$ & 1 & & \\
\hline MPN066 & ManB & Phosphomannomutase/phosphoglucomutase & G & 10 & Ser-149 & (K)YHFDGGVNVTApSHNPK \\
\hline MPN082 & Tkt & Transketolase & G & 3 & & \\
\hline MPN120 & GrpE & Heat shock protein & $\mathrm{O}$ & 1 & & \\
\hline MPN154 & NusA & Transcription elongation factor & $\mathrm{K}$ & 2 & Ser-503 & PVVKPKpSVFSITVEADDSK \\
\hline MPN221 & Pth & Peptidyl-tRNA hydrolase & $\mathrm{J}$ & 1 & & \\
\hline MPN227 & FusA & Elongation factor $\mathrm{G}$ & $\mathrm{J}$ & 3 & & \\
\hline
\end{tabular}


Tab. 4. Continued.

\begin{tabular}{|c|c|c|c|c|c|c|}
\hline $\begin{array}{l}\text { Locus } \\
\text { name }\end{array}$ & $\begin{array}{l}\text { Protein } \\
\text { name }\end{array}$ & Protein function & $\mathrm{COG}^{\mathrm{a}}$ & $\begin{array}{l}\text { Phospho spots in } \\
\text { two-dimensional gel }\end{array}$ & Phosphosite & Phosphopeptide sequence \\
\hline MPN256 ${ }^{\mathrm{b}}$ & MPN256 & Uncharacterized protein & $\mathrm{S}$ & 2 & Thr-200 & FIDELDQIpTK \\
\hline MPN261 & TopA & DNA topoisomerase I & $\mathrm{L}$ & 1 & Ser-426 & TVApSLMADCKK \\
\hline MPN280 & MPN280 & Ribonuclease J & A & 2 & & \\
\hline MPN295 & MPN295 & Uncharacterized protein & S & 2 & Ser-63 & YGPECEKSFLSLQpSK \\
\hline MPN302 & PfkA & Phosphofructokinase & $\mathrm{G}$ & 1 & & \\
\hline MPN303 & Pyk & Pyruvate kinase & $\mathrm{G}$ & 1 & & \\
\hline MPN311 & P41 & Adhesin-related protein & M & 1 & Thr-223 & TNNSIQQLEAEIQIPpTTHIK \\
\hline MPN322 & NrdF & Ribonucleoside-diphosphate reductase subunit $\beta$ & $\mathrm{F}$ & 1 & & \\
\hline MPN324 & NrdE & Ribonucleoside-diphosphate reductase subunit $\alpha$ & $\mathrm{F}$ & 3 & $\begin{array}{l}\text { Thr-159, } \\
\text { Ser-412 }\end{array}$ & $\begin{array}{l}\text { FQPApTPTFLNAGR } \\
\text { VGNDIpSCNLGSLNIAK }\end{array}$ \\
\hline MPN331 & Tig & Trigger factor & $\mathrm{O}$ & 3 & & \\
\hline MPN349 & MPN349 & Uncharacterized protein & S & 1 & & \\
\hline MPN389 & LplA & Lipoate-protein ligase A & $\mathrm{H}$ & 1 & & \\
\hline MPN390 & PdhD & Pyruvate dehydrogenase E3 component & $\mathrm{C}$ & 2 & & \\
\hline MPN391 & PdhC & Pyruvate dehydrogenase E2 component & $\mathrm{C}$ & 4 & & \\
\hline MPN392 & PdhB & Pyruvate dehydrogenase E1 component subunit $\beta$ & $\mathrm{C}$ & 3 & & \\
\hline
\end{tabular}


Tab. 4. Continued.

\begin{tabular}{|c|c|c|c|c|c|c|}
\hline $\begin{array}{l}\text { Locus } \\
\text { name }\end{array}$ & $\begin{array}{l}\text { Protein } \\
\text { name }\end{array}$ & Protein function & $\mathrm{COG}^{\mathrm{a}}$ & $\begin{array}{l}\text { Phospho spots in } \\
\text { two-dimensional gel }\end{array}$ & Phosphosite & Phosphopeptide sequence \\
\hline MPN393 & PdhA & Pyruvate dehydrogenase E1 component subunit $\alpha$ & $\mathrm{C}$ & 3 & Ser-205 & TKLEpSAVSDLSTK \\
\hline MPN394 & Nox & NADH oxidase & $\mathrm{H}$ & 1 & & \\
\hline MPN420 & GlpQ & Glycerophosphoryldiester phosphodiesterase & $\mathrm{C}$ & 1 & & \\
\hline MPN425 & FtsY & Cell division protein homolog & $\mathrm{U}$ & 1 & & \\
\hline MPN428 & Pta & Phosphotransacetylase & $\mathrm{C}$ & 4 & & \\
\hline MPN429 & Pgk & Phosphoglycerate kinase & G & 1 & & \\
\hline MPN430 & GapA & Glyceraldehyde-3-phosphate dehydrogenase & G & 4 & Ser-245 & VPVLTGPSIVELCVALEK \\
\hline MPN434 & DnaK & Chaperone protein & $\mathrm{O}$ & 2 & & \\
\hline MPN452 & HMW3 & Cytadherence high molecular weight protein 3 & M & 1 & & \\
\hline MPN470 & PepP & Xaa-Pro aminopeptidase & $\mathrm{E}$ & 2 & Thr-317 & LLCENAVIpTIEPGIYIPSVGGIR \\
\hline MPN474 & MPN474 & Coiled coil surface protein & M & 1 & Thr-773 & QNNEELpTDKCSNIQNELHDLNR \\
\hline MPN495 & UlaB & $\begin{array}{l}\text { Ascorbate-specific phosphotransferase enzyme IIB } \\
\text { component }\end{array}$ & G & 1 & & \\
\hline MPN517 & MPN517 & Reductase homolog & $\mathrm{C}$ & 1 & & \\
\hline MPN528 & Ppa & Inorganic pyrophosphatase & $\mathrm{P}$ & 1 & & \\
\hline MPN533 & AckA & Acetate kinase & $\mathrm{C}$ & 4 & & \\
\hline
\end{tabular}


Tab. 4. Continued.

\begin{tabular}{|c|c|c|c|c|c|c|}
\hline $\begin{array}{l}\text { Locus } \\
\text { name }\end{array}$ & $\begin{array}{l}\text { Protein } \\
\text { name }\end{array}$ & Protein function & $\mathrm{COG}^{\mathrm{a}}$ & $\begin{array}{l}\text { Phospho spots in } \\
\text { two-dimensional gel }\end{array}$ & Phosphosite & Phosphopeptide sequence \\
\hline MPN547 & MPN547 & Dihydroxyacetone kinase & $\mathrm{C}$ & 1 & & \\
\hline MPN555 & MPN555 & Uncharacterized protein & S & 2 & & \\
\hline MPN572 & PepA & Cytosol aminopeptidase & $\mathrm{E}$ & 3 & $\begin{array}{l}\text { Ser-249, } \\
\text { Thr-420 }\end{array}$ & $\begin{array}{l}\text { YDMSGAAIVCpSTVLALAK } \\
\text { EGVPLIHCDIASpTASIQDLGQGVLVR }\end{array}$ \\
\hline MPN573 & GroL & 60-kDa chaperonin & $\mathrm{O}$ & 2 & & \\
\hline MPN574 & GroS & 10-kDa chaperonin & $\mathrm{O}$ & 1 & & \\
\hline MPN576 & GlyA & Serine hydroxymethyltransferase & $\mathrm{E}$ & 1 & & \\
\hline MPN598 & AtpD & ATP synthase subunit $\beta$ & $\mathrm{C}$ & 2 & & \\
\hline MPN600 & AtpA & ATP synthase subunit $\alpha$ & $\mathrm{C}$ & 1 & & \\
\hline MPN606 & Eno & Enolase & G & 4 & & \\
\hline MPN625 & MPN625 & Osmotically inducible protein C-like protein & $\mathrm{O}$ & 1 & & \\
\hline MPN627 & EI & Phosphotransferase system enzyme I & G & 2 & & \\
\hline MPN628 & Pgm & Phosphoglycerate mutase & G & 1 & & \\
\hline MPN629 & TpiA & Triose-phosphate isomerase & G & 2 & & \\
\hline MPN636 & Frr & Ribosome-recycling factor & $\mathrm{J}$ & 2 & & \\
\hline
\end{tabular}


Tab. 4. Continued.

\begin{tabular}{|c|c|c|c|c|c|c|}
\hline $\begin{array}{l}\text { Locus } \\
\text { name }\end{array}$ & $\begin{array}{l}\text { Protein } \\
\text { name }\end{array}$ & Protein function & $\mathrm{COG}^{\mathrm{a}}$ & $\begin{array}{l}\text { Phospho spots in } \\
\text { two-dimensional gel }\end{array}$ & Phosphosite & Phosphopeptide sequence \\
\hline MPN638 & MPN638 & $\begin{array}{l}\text { Putative type I restriction enzyme specificity } \\
\text { protein }\end{array}$ & $\mathrm{V}$ & 2 & & \\
\hline MPN665 & Tuf & Elongation factor TU & $\mathrm{J}$ & 4 & Thr-383 & EGGRpTVGAGSVTEVLE \\
\hline MPN674 & Ldh & L-Lactate dehydrogenase & $\mathrm{C}$ & 2 & & \\
\hline MPN677 & MPN677 & Uncharacterized protein & S & 1 & & \\
\hline MPN688 & Soj & ParA family protein & $\mathrm{D}$ & 1 & & \\
\hline
\end{tabular}

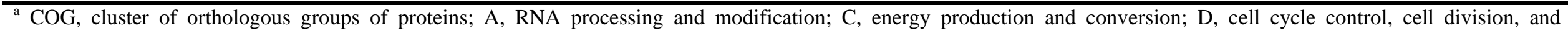

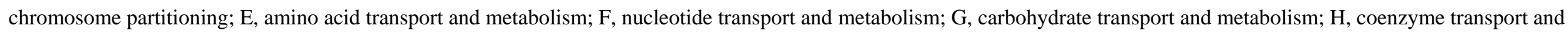

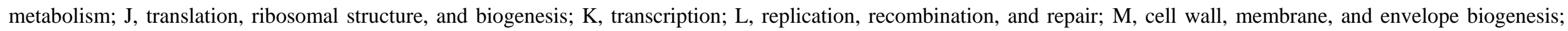

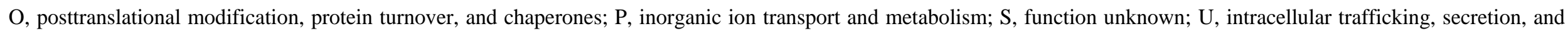
vesicular transport; $\mathrm{V}$, defense mechanisms.

${ }^{\mathrm{b}}$ Phosphoproteins only detectable in a prpC::Tn mutant (GPM68).

${ }^{\mathrm{c}} \operatorname{HPr}$ (MPN053) not detectable in a hprK::Tn mutant (GPM51). 
Classes of phosphorylated proteins. To get an impression of, which cellular processes are affected by protein phosphorylation, we grouped the proteins according to their functional categories (cluster of orthologous groups of proteins) (see Table 4). This analysis revealed that most phosphoproteins (36 proteins) are metabolic enzymes or transporters. 13 phosphorylated proteins are involved in cellular processes such as protein folding or envelope biogenesis. Nine of the phosphorylated proteins are required for the storage and processing of the genetic information, and the functions of five proteins are unknown.

Among the phosphorylated enzymes and transporters, a large number are involved in the central carbon metabolism (see Fig. 11). Of the glycolytic enzymes, all but the phosphoglucose isomerase are phosphorylated in M. pneumoniae. Moreover, all subunits of the pyruvate dehydrogenase and all three enzymes of overflow metabolism (Ldh, AckA, and Pta) are phosphoproteins. Of the enzymes of the pentose phosphate pathway, only the transketolase (Tkt) is phosphorylated in $M$. pneumoniae. In addition to glucose, $M$. pneumoniae can utilize glycerol and fructose. Although the enzymes that introduce fructose in the central metabolism are not phosphorylated, two glycerol 3-phosphate-forming enzymes are phosphoproteins.

Glycolytic enzymes are phosphorylated in many bacteria (see Table 5). A comparison of the phosphorylation pattern of these enzymes in different bacteria, however, revealed specific sets of phosphorylated glycolytic enzymes in each species. None of the glycolytic enzymes are phosphorylated in all species, and the phosphofructokinase is only phosphorylated in $M$. pneumoniae but not in any other bacterium studied so far. For some of the glycolytic phosphoproteins, the phosphorylation sites have been determined in multiple species. It is interesting to note that even if the same enzyme is phosphorylated in different species, the phosphorylation sites are not conserved. An exception is the pyruvate kinase that is phosphorylated on Ser-36 in a Gram-positive and in a Gram-negative bacterium (B. subtilis and E. coli, respectively) (Macek et al., 2008; Macek et al., 2007). However, phosphorylation of this enzyme was not detected in L. lactis (Soufi et al., 2008), a close relative of both B. subtilis and M. pneumoniae (see "Discussion”). 


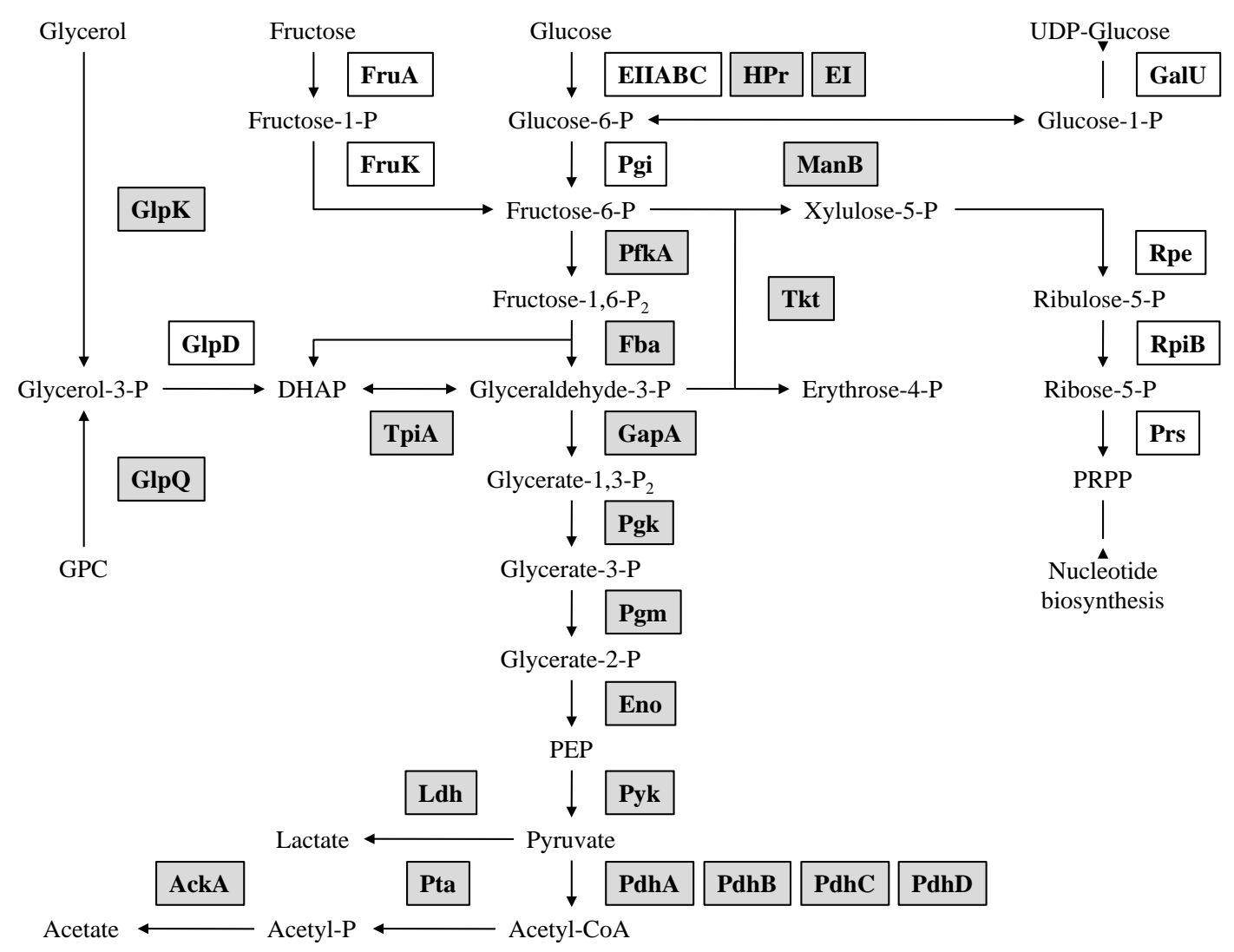

Fig. 11. Schematic illustration of phosphorylation events in central metabolic pathways of M. pneumoniae. Of the central metabolic pathways, only glycolysis is operative in M. pneumoniae, and a few carbon sources such as glucose, fructose, and glycerol can be utilized. Pyruvate can be oxidized to acetate or reduced to lactate. An incomplete pentose phosphate pathway serves to generate phosphoribosyl pyrophosphate for nucleotide biosynthesis. Phosphorylated enzymes are highlighted in gray. DHAP, dihydroxyacetone phosphate; GPC, glycerophosphorylcholine; PEP, phosphoenolpyruvate; PRPP, phosphoribosyl pyrophosphate; $\mathrm{P}$, phosphate.

Another interesting group of phosphoproteins is involved in cytadherence. These three proteins (P41, HMW3, and MPN474) were all only weakly phosphorylated in the wild type strain, but the intensity of the phosphorylation signals was strongly increased in the $p r p C$ phosphatase mutant, whereas these phosphorylation events were completely abolished in the prkC mutant. As outlined above, these observations suggest that PrkC phosphorylates these proteins. In addition to HPr, the well known target of the HPrK, these adhesion proteins and the unknown protein MPN256 are the only phosphoproteins, for which the kinase could be identified. 
Tab. 5. Phosphorylation events in glycolytic pathway of bacteria.

Bracketed letters indicate putative phosphorylation sites due to identification of the phosphopeptide.

\begin{tabular}{|c|c|c|c|c|c|}
\hline \multirow{2}{*}{ Protein } & \multicolumn{5}{|c|}{ Organism } \\
\hline & M. pneumoniae & B. subtilis & L. lactis & E. coli & C. jejuni \\
\hline Glucose-6-phosphate isomerase & No & $\begin{array}{c}\text { Yes } \\
\text { (Thr-39) }\end{array}$ & $\begin{array}{c}\text { Yes } \\
\text { (Ser-143) }\end{array}$ & $\begin{array}{c}\text { Yes } \\
\text { ([Ser-105], Thr-107) }\end{array}$ & No \\
\hline Phosphofructokinase & Yes & No & No & No & $\mathrm{No}^{\mathrm{b}}$ \\
\hline Fructose-bisphosphate aldolase & Yes & $\begin{array}{c}\text { Yes } \\
(\text { Thr-212, Thr-234) }\end{array}$ & $\begin{array}{c}\text { Yes } \\
\text { ([Thr-49, Ser-50], } \\
\text { Ser-216) }\end{array}$ & No & Yes \\
\hline Triose-phosphate isomerase & Yes & $\begin{array}{c}\text { Yes } \\
\text { (Ser-213) }\end{array}$ & No & No & Yes \\
\hline Glyceraldehyde-3-phosphate dehydrogenase & $\begin{array}{c}\text { Yes } \\
\text { (Ser-245) }\end{array}$ & $\begin{array}{c}\text { Yes } \\
\text { ([Ser-148, Ser-151, } \\
\text { Thr-153, Thr-154]) }\end{array}$ & $\begin{array}{c}\text { Yes } \\
\text { (Ser-126, Ser-211, } \\
\text { [Thr-212], Thr-321) }\end{array}$ & No & No \\
\hline
\end{tabular}


Tab. 5. Continued.

\begin{tabular}{|c|c|c|c|c|c|}
\hline \multirow{2}{*}{ Protein } & \multicolumn{5}{|c|}{ Organism } \\
\hline & M. pneumoniae & B. subtilis & L. lactis & E. coli & C. jejuni \\
\hline Phosphoglycerate kinase & Yes & $\begin{array}{c}\text { Yes } \\
\text { (Ser-183, Thr-299) }\end{array}$ & $\begin{array}{c}\text { Yes } \\
\text { (Ser-217) }\end{array}$ & $\begin{array}{c}\text { Yes } \\
\text { (Ser-192, Thr-196, } \\
\text { Thr-199) }\end{array}$ & No \\
\hline Phosphoglycerate mutase & Yes & $\begin{array}{c}\text { Yes } \\
\text { (Ser-62) }\end{array}$ & $\begin{array}{c}\text { Yes } \\
\text { (T94, S144) }\end{array}$ & $\begin{array}{c}\text { Yes } \\
\text { (S146) }\end{array}$ & No \\
\hline Enolase & Yes & $\begin{array}{c}\text { Yes } \\
\text { (Thr-141, Ser-259, } \\
\text { Tyr-281, Ser-325) }\end{array}$ & No & $\begin{array}{c}\text { Yes } \\
\text { ([Ser-372, Thr-375, } \\
\text { Thr-379]) }\end{array}$ & $\begin{array}{c}\text { Yes } \\
\text { ([Ser-180, Tyr-185]) }\end{array}$ \\
\hline Pyruvate kinase & Yes & $\begin{array}{c}\text { Yes } \\
\text { (Ser-36) }\end{array}$ & No & $\begin{array}{c}\text { Yes } \\
(\text { Ser-36) }\end{array}$ & Yes \\
\hline
\end{tabular}

${ }^{\mathrm{a}}$ C. jejuni does not encode for the glycolytic enzyme phosphofructokinase. 


\section{Autophosphorylation of single universally conserved phosphoprotein. As} mentioned above, there is little conservation of the phosphorylation sites even if the same proteins are phosphorylated in fairly closely related organisms. There is only one exception to this notion, the phosphosugar mutase ManB (according to the nomenclature for $M$. pneumoniae). Phosphosugar mutases are phosphorylated in all domains of life from the archaeon Halobacterium salinarum and bacteria such as M. pneumoniae and E. coli to eukaryotes such as yeast, Drosophila melanogaster, and humans (Aivaliotis et al., 2009; Li et al., 2007; Macek et al., 2008; Yu et al., 2007; Zhai et al., 2008).

The phosphoproteome analysis showed the presence of 10 phosphospots that do all correspond to ManB. The analysis of the phosphorylation site revealed that Ser-149 was phosphorylated in ManB of M. pneumoniae (see Fig. 12). This site was detected in all 10 spots, suggesting that ManB is subject to different posttranslational modifications that affect the isoelectric point of the protein. An alignment of the regions surrounding the phosphorylation site of these proteins revealed a very strong conservation of the region around the phosphorylated serine residue (see Fig. 13A). The motif (T/S)ASHN(P/R) is present in all proteins. This strong similarity suggests phylogenetic conservation of the phosphorylation site and also implies conservation of the phosphorylation mechanism (the kinase) and of the biological function of the phosphorylation event.

Because there are no conserved protein kinases in the three domains of life, we considered the possibility that ManB might autophosphorylate as it had been shown for the phosphoglucosamine mutase from E. coli (Jolly et al., 2000). To test this idea, we purified ManB carrying an N-terminal $\mathrm{His}_{6}$-tag and performed phosphorylation assays. As shown in Fig. 13B, the protein did indeed autophosphorylate in the presence of manganese. Only faint phosphorylation signals were observed in the presence of other divalent cations. A protein in which the phosphorylated serine residue was replaced by a non-phosphorylatable alanine did not autophosphorylate (Fig. 13C). This result demonstrates that ManB is phosphorylated on the same site in vivo and in vitro and supports the hypothesis of autophosphorylation. To demonstrate that autophosphorylation is not limited to the enzyme of $M$. pneumoniae, we also purified the phosphosugar mutase GlmM from $B$. subtilis and observed autophosphorylation for this protein as well. Again, no phosphorylation was detectable if the phosphorylated 
serine residue [Ser-100 (Eymann et al., 2007; Macek et al., 2007)] was replaced by an alanine. Thus, autophosphorylation is the common mechanism of phosphorylation of these enzymes. This explains the unique conservation of a phosphorylation site.

The phosphosugar mutases play a key role in metabolism as shown by the fact that they are often essential. This is true for GlmM from E. coli and B. subtilis (Kobayashi et al., 2003; Mengin-Lecreulx et al., 1996). To test whether ManB of $M$. pneumoniae is also encoded by an essential gene, we attempted to isolate a manB mutant from an ordered collection of transposon insertions. In this collection, each viable mutant is included with a probability of 99.999\% (Halbedel et al., 2006; Halbedel and Stülke, 2007). No transposon insertion in manB was identified, suggesting that the gene is indeed essential. This finding is in good agreement with previous reports that no transposon insertion in $\operatorname{manB}$ could be isolated in $M$. pneumoniae and M. genitalium (Glass et al., 2006; Hutchison III et al., 1999).

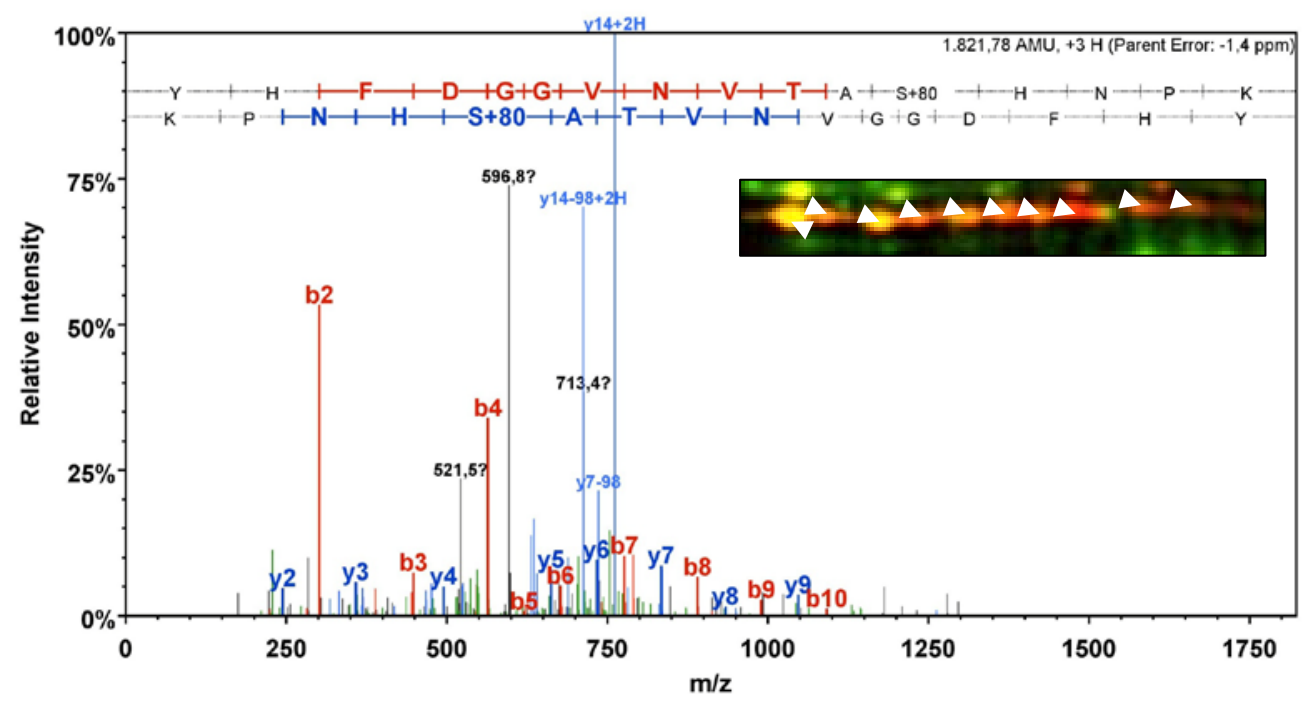

Fig. 12. MS/MS spectrum of serine-phosphorylated peptide from phosphomannomutase/ phosphoglucomutase ManB (MPN066). The peptide was measured on line by ESI-mass spectrometry using a nanoACQUITY UPLC system coupled to an LTQ Orbitrap mass spectrometer (see "Experimental procedures”). The phosphorylation site (Ser-149) is located in the conserved phosphoserine signature of phosphosugar mutases. Detection of the same phosphorylated peptide in all 10 spots of ManB (enhanced image section) suggests further posttranslational modifications that affect the isoelectric point of the ManB protein. 
A

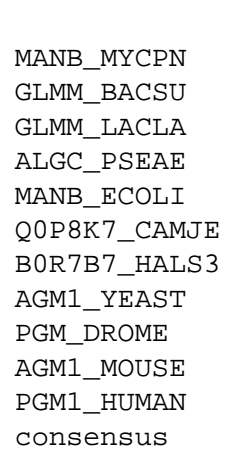

B

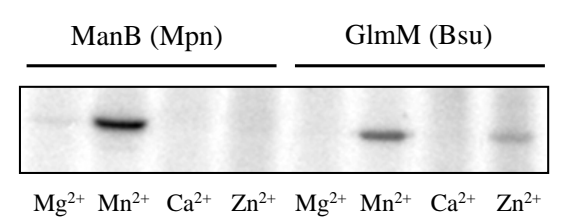

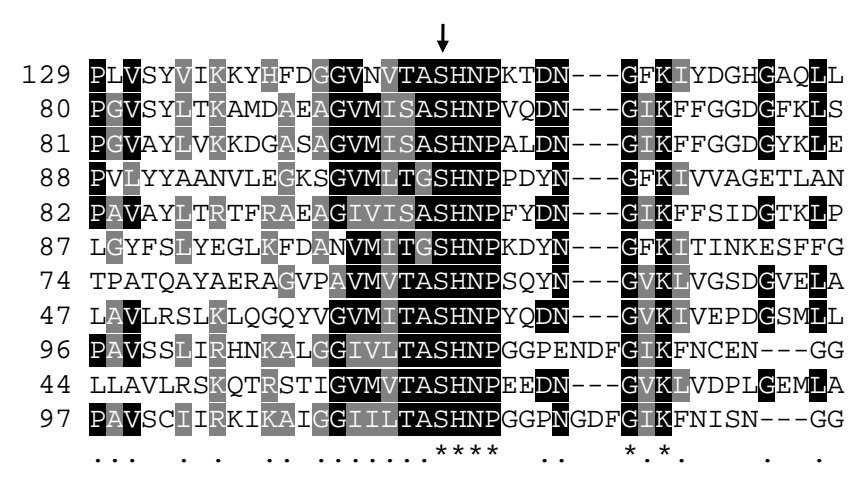

C

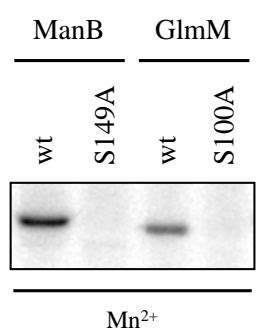

Fig. 13. Autophosphorylation on universally conserved serine residue in active site of phosphosugar mutases. (A) Multiple alignment of the conserved phosphoserine signature of phosphosugar mutases from all domains of life. Amino acids with similarities in at least two of the sequences are highlighted in gray, whereas amino acids that are identical in at least two of the sequences are depicted in a black background. The UniProtKB entry names of the aligned sequences are MANB_MYCPN (ManB, M. pneumoniae), GLMM_BACSU (GlmM; B. subtilis), GLMM_LACLA (GlmM; L. lactis), ALGC_PSEAE (AlgC; P. aeruginosa), MANB_ECOLI (ManB; E. coli), Q0P8K7_CAMJE (ManB; C. jejuni), B0R7B7_HALS3 (PMM1; H. salinarum), AGM1_YEAST (AGM1; Saccharomyces cerevisiae), PGM_DROME (PGM; D. melanogaster), AGM1_MOUSE (AGM1; Mus musculus), and PGM1_HUMAN (PGM1; Homo sapiens). The conserved active site phosphoserine is indicated by an arrow. (B) M. pneumoniae ManB and B. subtilis GlmM autophosphorylation assay in the presence of various divalent cations. About $5 \mu \mathrm{g}$ of purified $\mathrm{His}_{6}$-tagged ManB (lanes 1-4) or GlmM (lanes 4-8) were incubated in the presence of $\left[\gamma^{32} \mathrm{P}\right] \mathrm{ATP}$ in an autophosphorylation assay (see "Experimental procedures"). Each reaction mixture was analyzed by SDS-PAGE and phosphorimaging analysis. Divalent cations ( $5 \mathrm{mM}$ ) used in the assays are indicated below the lanes. Bsu, B. subtilis; Mpn, M. pneumoniae. (C) Autophosphorylation assay of M. pneumoniae ManB (wild type (wt) and S149A) and B. subtilis GlmM (wild type and S100A) recombinant proteins. The four recombinant proteins were checked for autophosphorylation as described in (B). Autophosphorylation reactions were conducted in the presence of $\mathrm{Mn}^{2+}(5 \mathrm{mM})$ as divalent cation as it showed the strongest signal in (B). 
The universal conservation of the phosphorylation site in phosphosugar mutases led us to conclude that this phosphorylation event might be essential. This hypothesis could not be addressed in $M$. pneumoniae because of the lack of genetic tools (Halbedel and Stülke, 2007). Therefore, we studied the role of this phosphorylation site for GlmM B. subtilis. The glmM gene and its variant encoding the S100A protein were established on multicopy plasmids in $B$. subtilis. In a strain expressing the wild type glmM gene from a plasmid, the chromosomal copy could be deleted by homologous recombination using a PCR fragment obtained by long flanking homology PCR. In contrast, a resistance cassette could not replace the chromosomal copy of $g \operatorname{lm} M$ when the $g \operatorname{lmM}$-S100A allele was expressed from the plasmid. Thus, the phosphorylation of GlmM on Ser-100 seems to be essential for its function and thus for the survival of the bacteria.

\section{Discussion}

The analysis of the phosphoproteome of $M$. pneumoniae revealed substantial protein serine/threonine/tyrosine phosphorylation even in this minimal organism. We identified 63 phosphoproteins that account for nearly 10\% of all proteins encoded by M. pneumoniae. This exceeds the percentage of phosphorylated proteins in other bacteria by far. For example, only $2.5 \%$ of all proteins of $B$. subtilis are known to be phosphorylated on a serine, threonine or tyrosine residue (Eymann et al., 2007; Lévine et al., 2006; Macek et al., 2007). This raises the question whether protein phosphorylation is more common in $M$. pneumoniae than in other bacteria. It is tempting to speculate that the high rate of protein phosphorylation compensates for the obvious lack of transcriptional regulation in $M$. pneumoniae. However, this lack of regulation of gene expression allows the detection of the complete phosphoproteome in a single (or a few) experiment(s). Indeed, we detected about 500 CHAPS-soluble proteins that correspond to $80 \%$ of all 635 proteins that are in the $\mathrm{pI}$ range covered in this study (see supplemental Fig. S1). In other species with complex transcriptional regulation, only about 1,000 proteins are detectable under any specific condition, whereas the rest are required under specific growth conditions (Eymann et al., 2004). For B. subtilis or E. coli, this corresponds to only $25 \%$ of all proteins. Thus, the higher coverage in our study is most probably due to the lack of transcription regulation in M. pneumoniae, but does not seem to reflect a higher impact of protein phosphorylation. 
A striking feature of the phosphoproteomes of different bacteria is the phosphorylation of enzymes of the central metabolic pathways. In $M$. pneumoniae, metabolism is strongly reduced, and the major pathways are depicted in Fig. 11. The citric acid cycle and pathways of lipid, amino acid, and nucleotide metabolisms are absent in these bacteria. As in the other Firmicutes, most glycolytic enzymes are phosphorylated in $M$. pneumoniae. Unfortunately, the functions of these phosphorylations as well as the responsible kinases are so far unknown. It is interesting that all four subunits of the pyruvate dehydrogenase are phosphorylated in $M$. pneumoniae. In $B$. subtilis, there are different results concerning the pyruvate dehydrogenase. One study reports phosphorylation of PdhB (Macek et al., 2007), whereas PdhC and PdhD were found to be phosphoproteins in another work (Eymann et al., 2007). In contrast, none of the subunits is phosphorylated in E. coli, Campylobacter jejuni, and L. lactis (Macek et al., 2008; Soufi et al., 2008; Voisin et al., 2007).

This work is the first global analysis of the effects of mutations in the complete set of annotated protein kinases on the phosphoproteome. We have shown that the HPr kinase has only one substrate, HPr. The phosphorylation of HPr on Ser-46 is a major signal to trigger carbon catabolite repression of catabolic genes and operons in B. subtilis and other Firmicutes (Görke and Stülke, 2008). If $\operatorname{HPr}($ Ser-P) had the same function in $M$. pneumoniae, one would have expected major changes in the proteome of the hprK mutant as compared with the wild type strain. However, with the exception of HPr phosphorylation (see Fig. 10), no differences were detectable. This suggests that HPr phosphorylation on Ser-46 has a function different from that in the other bacteria. This conclusion is supported by three observations. First, $\operatorname{HPr}(\operatorname{Ser}-\mathrm{P})$ acts as the cofactor for the pleiotropic transcription factor CcpA to cause catabolite repression. No protein similar to CcpA is encoded in the genome of any Mycoplasma species. Second, the phosphorylation of HPr on Ser-46 interferes with its participation in sugar transport due to the inhibition of enzyme I-dependent phosphorylation of His-15. This inhibition is relevant even in Gram-negative bacteria that also do not possess an equivalent of CcpA (Krausse et al., 2009). However, in M. pneumoniae, these two phosphorylation events are not mutually exclusive (Halbedel and Stülke, 2005). Finally, although the activity of the HPr kinase of $B$. subtilis and most other bacteria is strongly controlled by the ATP and fructose 1,6-bisphosphate concentrations, the enzyme of $M$. pneumoniae is 
constitutively active due to its high affinity to ATP (Merzbacher et al., 2004). Thus, the phosphorylation of HPr on Ser-46 seems to serve other purposes in M. pneumoniae.

In addition to the HPr kinase, $M$. pneumoniae encodes only one protein kinase, PrkC. This kinase is implicated in a multitude of cellular functions in different bacteria, among them spore germination, virulence, and control of glycolysis (Kristich et al., 2007; Lomas-Lopez et al., 2007; Shah et al., 2008). We found that PrkC has at least four targets in M. pneumoniae, the cell adhesion proteins HMW3 and P41, the cell surface protein MPN474, and the uncharacterized protein MPN256. These proteins are not phosphorylated in a prkC mutant, but their phosphorylation is enhanced if the protein phosphatase PrpC, the antagonist of PrkC, is not expressed (see Fig. 9). Moreover, HMW1 and P1, two very large proteins that were out of the detection window applied in this study, are likely to be phosphorylated by PrkC (Schmidl et al., 2010). This suggests that PrkC might be required for cell adhesion in M. pneumoniae. Indeed, a prkC mutant strain is not capable of adherent growth and has lost toxicity to human host cells, most likely due to the defect in cell adhesion (Schmidl et al., 2010). Thus, PrkC has a specific function in $M$. pneumoniae and phosphorylates a specific set of proteins.

As stated above, HPrK and PrkC are the only two annotated protein kinases in M. pneumoniae. However, they account for only five of 63 identified protein phosphorylation events. This suggests the existence of other, yet to be discovered protein kinases in M. pneumoniae. Alternatively, autophosphorylation might be more relevant than previously anticipated. Indeed, the only phosphorylation site that is universally conserved from archaea via bacteria such as $M$. pneumoniae to eukaryotes is the result of an autophosphorylation event in the catalytic site of phosphosugar mutases such as GlmM and ManB (Jolly et al., 2000; Mengin-Lecreulx et al., 1996; this work). This autophosphorylation is essential for the enzymatic activity of these enzymes, thus providing an explanation for the universal but highly exceptional conservation of the phosphorylation event at a conserved site. Interestingly, another ubiquitous protein, the heat shock protein DnaK (also called Hsp70) is also present as a phosphoprotein in all organisms that were studied so far. In E. coli and mycobacteria, DnaK is capable of autophosphorylating at a strongly conserved threonine residue (McCarty and Walker, 1991; Peake et al., 1998; Preneta et al., 2004; Zylicz et al., 1983). This site is also present in the DnaK protein of M. pneumoniae (Thr-182), but it is not conserved in the 
archaeal DnaK of $H$. salinarum (Aivaliotis et al., 2009). It is therefore possible that DnaK autophosphorylates at a conserved site in bacteria and eukaryotes, whereas another phosphorylation mechanism occurs in archaea.

In this study, we unambiguously identified 16 phosphorylation sites. Phosphorylation on serine or threonine residues is equally distributed (eight phosphorylation sites each). Serine and threonine are also the predominant phosphorylation sites in other bacteria, and they are used with similar frequency as well (Macek et al., 2008; Macek et al., 2007; Soufi et al., 2008). For eukaryotic phosphoproteins, it was shown that phosphorylations occur often in disordered regions and that the actual phosphorylation sites are not completely fixed in these regions (Iakoucheva et al., 2004; Landry et al., 2009). Indeed, seven of the 16 phosphorylation sites identified in this study are predicted to be localized in disordered regions (data not shown). An analysis of the regions surrounding the phosphorylation sites revealed the absence of defined amino acid sequence motifs. Rapid evolution and a corresponding lack of conservation of phosphorylation sites have recently also been observed for protein phosphorylation in yeast and other eukaryotes (Beltrao et al., 2009; Holt et al., 2009; Tan et al., 2009). Taken together, these findings suggest that either the responsible protein kinases are quite nonspecific or that a large number of so far unidentified protein kinases exist in $M$. pneumoniae. An alternative explanation is offered by an analysis of the positions of the phosphorylation sites: Structural information is available for seven of the phosphoproteins with identified phosphorylation sites (see supplemental Fig. S2). All nine phosphorylation sites in these proteins are buried in the protein and are thus poorly accessible for protein kinases. This would suggest that these phosphorylation events are autophosphorylations as observed for ManB. Based on the structure of the $P$. aeruginosa phosphomannomutase/phosphoglucomutase (Regni et al., 2002) (see Fig. 13), the site of autophosphorylation of ManB and GlmM is also buried in the inside of the protein.

The lack of conservation of phosphorylation sites was also observed in similar phosphoproteins from different bacteria. Only two strongly conserved phosphorylation sites were detected, Ser-46 in $\mathrm{HPr}$ and Ser-149 in ManB. Although HPr is phosphorylated by the ATP-dependent HPr kinase that is conserved in many bacteria (Steinhauer et al., 2002; see Fig. 10), ManB is subject to autophosphorylation. Even among the glycolytic enzymes, only one identified phosphorylation site was conserved 
between two species (Ser-36 in the pyruvate kinases from B. subtilis and E. coli). Because this latter site is conserved in $M$. pneumoniae, it is tempting to speculate that it is also phosphorylated in the Mollicutes. The observations reported in this work have important implications for the evolution of protein phosphorylation in bacteria: Protein phosphorylation events seem to be highly specific for each individual bacterial species; even among members of one bacterial phylum such as the Firmicutes, there is little conservation of protein phosphorylation. Moreover, one protein kinase may phosphorylate distinct sets of proteins in the different species. Finally, there may be a large number of protein kinases that have so far escaped discovery, and these kinases are likely to be specific for small groups of bacteria. In conclusion, protein phosphorylation may be one of the major features in the adaptation of bacteria to their individual ecological niche.

\section{Acknowledgments}

We are grateful to Julia Busse for excellent technical assistance. Robert Hertel is acknowledged for help with some experiments. This work was supported by grants of the Deutsche Forschungsgemeinschaft and the Fonds der Chemischen Industrie (to D. B., M. H., and J. S.). S. R. S. was supported by the Studienstiftung des Deutschen Volkes. 


\section{Supplemental material}

Tab. S3. Primers used in this study.

\begin{tabular}{|c|c|c|c|}
\hline Primer & Sequence $\left(5^{\prime} \rightarrow 3^{\prime}\right)^{\mathrm{ab}}$ & Mutation & Resulting plasmid \\
\hline NP108 & $\begin{array}{l}\text { AAAGGATCCCTCACTTATTTAAAGGAGGAAA } \\
\text { CAATCATGGGCAAGTATTTTGGAACAGACG }\end{array}$ & None & pGP400, pGP1403 \\
\hline NP109 & $\begin{array}{l}\text { TTTGTCGACCTATCACTCTAATCCCATTTCTG } \\
\text { ACCGG }\end{array}$ & None & $\begin{array}{l}\text { pGP400, pGP1401, } \\
\text { pGP1403, pGP1405 }\end{array}$ \\
\hline NP110 & GGCTATTCTCCGGAGCAGCCGATTGTCA & None & - \\
\hline NP111 & CAGGAACGGACGACCAAAAGTTTTCCCG & None & - \\
\hline NP112 & $\begin{array}{l}\text { CCTATCACCTCAAATGGTTCGCTGTAAAGGC } \\
\text { CAGCTCAGGTGTAAGCTC }\end{array}$ & None & - \\
\hline NP113 & $\begin{array}{l}\text { CCGAGCGCCTACGAGGAATTTGTATCGAGCG } \\
\text { AAGACGAAAGAGCTGTGCGA }\end{array}$ & None & - \\
\hline NP114 & CATCGCCATCGCATCAGATGCTACGACGT & None & - \\
\hline NP115 & TATAAGACGCACGTGTAATCACGTCACCATC & None & - \\
\hline NP116 & $\begin{array}{l}\text { P_GCAGAGGCGGGCGTCATGATTTCCGCTGC } \\
\text { CCATAACCCAGTGCAGGATAACGGCATCAA }\end{array}$ & T298G & pGP1403, pGP1405 \\
\hline NP117 & $\begin{array}{l}\text { AAAGGATCCATGGGCAAGTATTTTGGAACAG } \\
\text { ACG }\end{array}$ & None & pGP1401, pGP1405 \\
\hline $\begin{array}{l}\text { spec-fwd } \\
\text { (kan) }\end{array}$ & $\begin{array}{l}\text { CAGCGAACCATTTGAGGTGATAGGGACTGGC } \\
\text { TCGCTAATAACGTAACGTGACTGGCAAGAG }\end{array}$ & None & - \\
\hline $\begin{array}{l}\text { spec-rev } \\
\text { (kan) }\end{array}$ & $\begin{array}{l}\text { CGATACAAATTCCTCGTAGGCGCTCGGCGTA } \\
\text { GCGAGGGCAAGGGTTTATTGTTTTCTAAAAT } \\
\text { CTG }\end{array}$ & None & - \\
\hline SH29 & ATGAGTGAGCTAACTCACAG & None & - \\
\hline SH30 & CAATACGCAAACCGCCTC & None & - \\
\hline SS107 & $\begin{array}{l}\text { AAAGGATCCATGAACAGTAACGCATACTTGG } \\
\text { AAGC }\end{array}$ & None & pGP656-pGP658 \\
\hline SS108 & $\begin{array}{l}\text { TATACTGCAGCTAAGCTTTATCGAGGTTGAG } \\
\text { CAATC }\end{array}$ & None & pGP656-pGP658 \\
\hline SS109 & P_GAAAGTAAGGATACCTGGGAGTTAGCGCG & A846G & pGP657 \\
\hline SS110 & $\begin{array}{l}\text { P_CTTTGCGATTGCCGAATGGAATCCGCAAA } \\
\text { C }\end{array}$ & A936G & pGP657 \\
\hline SS111 & $\begin{array}{l}\text { P_GATTACTATAACTGGACGGTACCACACAC } \\
\text { CATTC }\end{array}$ & A1356G & pGP657 \\
\hline SS112 & $\begin{array}{l}\text { P_CGGTGAATGTCACTGCTGCTCATAATCCTA } \\
\text { AAACC }\end{array}$ & $\begin{array}{l}\text { A445G, } \\
\text { G446C }\end{array}$ & pGP658 \\
\hline
\end{tabular}


Tab. S3. Continued.

\begin{tabular}{llll}
\hline Primer & Sequence $\left(\mathbf{5}^{\prime} \rightarrow \mathbf{3}^{\prime}\right)^{\mathbf{a b}}$ & Mutation & Resulting plasmid \\
\hline SS113 & CAAGGCCACAATTGATTATGTCTTTG & None & - \\
SS114 & GGTAACTTAGTGGAAGTCCACTTAAC & None & - \\
\hline${ }^{\text {a }}$ Restriction sites are underlined. & \\
b The “P” at the 5' end of primer sequences indicates phosphorylation.
\end{tabular}

Tab. S4. Plasmids used for analysis of phosphosugar mutases.

\begin{tabular}{|c|c|c|c|}
\hline Plasmid & Relevant characteristics & $\begin{array}{l}\text { Used restriction } \\
\text { sites }\end{array}$ & Reference \\
\hline pBQ200 & Allows expression of proteins in $B$. subtilis & - & $\begin{array}{l}\text { Martin-Verstraete et al. } \\
\text { (1994) }\end{array}$ \\
\hline pGP400 & pBQ200-glmM & BamHI + SalI & This work \\
\hline pGP656 & pWH844-manB & BamHI + PstI & This work \\
\hline pGP657 & pWH844-manB ${ }^{\mathrm{a}}$ & BamHI + PstI & This work \\
\hline pGP658 & pWH844-manB(S149A) & BamHI + PstI & This work \\
\hline pGP1401 & pWH844-glmM & BamHI + SalI & This work \\
\hline pGP1403 & pBQ200-glmM(S100A) & BamHI + SalI & This work \\
\hline pGP1405 & pWH844-glmM(S100A) & BamHI + SalI & This work \\
\hline pWH844 & $\begin{array}{l}\text { Allows overexpression of N-terminal } \\
\mathrm{His}_{6} \text {-tag fusion proteins in E. coli DH5 } \alpha\end{array}$ & - & Schirmer et al. (1997) \\
\hline
\end{tabular}

${ }^{\mathrm{a}}$ The three TGA opal codons in the wild type manB gene are mutated to TGG. 
Tab. S5. Complete list of phosphospots identified from M. pneumoniae two-dimensional gels.

For general overview, see Table 4. pS, phosphoserine; pT, phosphothreonine.

\begin{tabular}{|c|c|c|c|c|c|c|c|c|c|c|c|c|c|}
\hline \# & Spot & $\begin{array}{l}\text { Protein } \\
\text { name }\end{array}$ & $\begin{array}{l}\text { Locus } \\
\text { name }\end{array}$ & $\begin{array}{l}\text { UniProtKB } \\
\text { accession } \\
\text { number }\end{array}$ & Protein function & $\begin{array}{l}\text { Molecular } \\
\text { weight (Da) }\end{array}$ & $\begin{array}{l}\text { Isoelectric } \\
\text { point (pI) }\end{array}$ & $\begin{array}{l}\text { Protein } \\
\text { identification } \\
\text { probability (\%) }\end{array}$ & $\begin{array}{l}\text { Number } \\
\text { of unique } \\
\text { peptides }\end{array}$ & $\begin{array}{l}\text { Coverage } \\
\text { (\%) }\end{array}$ & $\begin{array}{l}\text { Phosphorylation } \\
\text { site }\end{array}$ & Phosphopeptide sequence & Experiment $^{\mathrm{a}}$ \\
\hline 1 & AckA-1 & AckA & MPN533 & P75245 & Acetate kinase & 43672.68 & 8.05 & 100.00 & 23 & 58.97 & & & $1,2,3,4$ \\
\hline 2 & AckA-2 & AckA & MPN533 & P75245 & Acetate kinase & 43672.68 & 8.05 & 100.00 & 22 & 55.38 & & & $1,2,3,4$ \\
\hline 3 & AckA-3 & AckA & MPN533 & P75245 & Acetate kinase & 43672.68 & 8.05 & 100.00 & 18 & 50.77 & & & $1,2,3,4$ \\
\hline 4 & AckA-4 & AckA & MPN533 & P75245 & Acetate kinase & 43672.68 & 8.05 & 100.00 & 16 & 43.85 & & & $1,2,3,4$ \\
\hline \multirow[t]{2}{*}{5} & [AtpA Pgm] & AtpA & MPN600 & Q50329 & ATP synthase subunit $\alpha$. & 57339.01 & 6.14 & 100.00 & 19 & 41.12 & & & $1,2,3,4$ \\
\hline & & Pgm & MPN628 & P75167 & Phosphoglycerate mutase & 56339.53 & 6.08 & 100.00 & 14 & 36.22 & & & $1,2,3,4$ \\
\hline 6 & AtpD-1 & AtpD & MPN598 & Q50331 & ATP synthase subunit $\beta$ & 52204.62 & 5.36 & 100.00 & 28 & 86.32 & & & $1,2,3,4$ \\
\hline 7 & AtpD-2 & AtpD & MPN598 & Q50331 & ATP synthase subunit $\beta$ & 52204.62 & 5.36 & 100.00 & 22 & 71.79 & & & $1,2,3,4$ \\
\hline 8 & DeoC & DeoC & MPN063 & P09924 & Deoxyribose-phosphate aldolase & 24861.89 & 8.34 & 100.00 & 9 & 53.13 & & & $1,2,3,4$ \\
\hline \multirow[t]{2}{*}{9} & [DoeD TpiA] & DeoD & MPN062 & P75053 & Purine-nucleoside phosphorylase & 26266.66 & 6.78 & 100.00 & 15 & 77.73 & & & $1,2,3,4$ \\
\hline & & TpiA & MPN629 & P78010 & Triosephosphate isomerase & 26941.33 & 7.34 & 100.00 & 11 & 55.33 & & & $1,2,3,4$ \\
\hline 10 & DnaK-1 & DnaK & MPN434 & P75344 & Chaperone protein & 65058.30 & 5.28 & 100.00 & 34 & 51.09 & & & $1,2,3,4$ \\
\hline 11 & DnaK-2 & DnaK & MPN434 & P75344 & Chaperone protein & 65058.30 & 5.28 & 100.00 & 18 & 35.97 & & & $1,2,3,4$ \\
\hline 12 & DnaN & DnaN & MPN001 & Q50313 & DNA polymerase III subunit $\beta$ & 43829.01 & 5.74 & 100.00 & 23 & 59.74 & & & $1,2,3,4$ \\
\hline \multirow[t]{2}{*}{13} & [DnaN PdhA] & DnaN & MPN001 & Q50313 & DNA polymerase III subunit $\beta$ & 43829.01 & 5.74 & 100.00 & 28 & 76.05 & & & $1,2,3,4$ \\
\hline & & PdhA & MPN393 & P75390 & $\begin{array}{l}\text { Pyruvate dehydrogenase E1 component } \\
\text { subunit } \alpha\end{array}$ & 40568.44 & 6.22 & 100.00 & 10 & 30.45 & & & $1,2,3,4$ \\
\hline 14 & EI-1 & EI & MPN627 & P75168 & Phosphotransferase system enzyme I & 63909.70 & 7.92 & 100.00 & 31 & 47.03 & & & $1,2,3,4$ \\
\hline 15 & EI-2 & EI & MPN627 & P75168 & Phosphotransferase system enzyme I & 63909.70 & 7.92 & 100.00 & 12 & 27.62 & & & $1,2,3,4$ \\
\hline 16 & Eno-1 & Eno & MPN606 & P75189 & Enolase & 49197.47 & 6.11 & 100.00 & 24 & 64.69 & & & $1,2,3,4$ \\
\hline 17 & Eno-2 & Eno & MPN606 & P75189 & Enolase & 49197.47 & 6.11 & 100.00 & 25 & 70.18 & & & $1,2,3,4$ \\
\hline 18 & Eno-3 & Eno & MPN606 & P75189 & Enolase & 49197.47 & 6.11 & 100.00 & 20 & 60.96 & & & $1,2,3,4$ \\
\hline 19 & Eno-4 & Eno & MPN606 & P75189 & Enolase & 49197.47 & 6.11 & 100.00 & 17 & 55.26 & & & $1,2,3,4$ \\
\hline 20 & $\mathrm{Fba}$ & Fba & MPN025 & P75089 & Fructose-bisphosphate aldolase & 31049.17 & 6.44 & 100.00 & 6 & 23.26 & & & $1,2,3,4$ \\
\hline \multirow[t]{2}{*}{21} & [Fba Soj] & Fba & MPN025 & P75089 & Fructose-bisphosphate aldolase & 31049.17 & 6.44 & 100.00 & 12 & 53.82 & & & $1,2,3,4$ \\
\hline & & Soj & MPN688 & Q50314 & ParA family protein & 30061.83 & 7.26 & 100.00 & 8 & 27.78 & & & $1,2,3,4$ \\
\hline 22 & Frr-1 & Frr & MPN636 & P75161 & Ribosome-recycling factor & 21653.51 & 8.87 & 100.00 & 12 & 63.04 & & & $1,2,3,4$ \\
\hline
\end{tabular}


Tab. S5. Continued.

\begin{tabular}{|c|c|c|c|c|c|c|c|c|c|c|c|c|c|}
\hline \# & Spot & $\begin{array}{l}\text { Protein } \\
\text { name }\end{array}$ & $\begin{array}{l}\text { Locus } \\
\text { name }\end{array}$ & $\begin{array}{l}\text { UniProtKB } \\
\text { accession } \\
\text { number }\end{array}$ & Protein function & $\begin{array}{l}\text { Molecular } \\
\text { weight (Da) }\end{array}$ & $\begin{array}{l}\text { Isoelectric } \\
\text { point (pI) }\end{array}$ & $\begin{array}{l}\text { Protein } \\
\text { identification } \\
\text { probability (\%) }\end{array}$ & $\begin{array}{l}\text { Number } \\
\text { of unique } \\
\text { peptides }\end{array}$ & $\begin{array}{l}\text { Coverage } \\
\text { (\%) }\end{array}$ & $\begin{array}{l}\text { Phosphorylation } \\
\text { site }\end{array}$ & Phosphopeptide sequence & Experiment $^{\mathrm{a}}$ \\
\hline 23 & 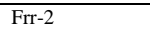 & Frr & $\begin{array}{l}\text { MPN636 } \\
\end{array}$ & P75161 & Ribosome-recycling factor & 21653.51 & 8.87 & 100.00 & 10 & 62.50 & & & $1,2,3,4$ \\
\hline 24 & FusA-1 & FusA & MPN227 & P75544 & Elongation factor G & 76451.41 & 5.43 & 100.00 & 24 & 53.49 & & & $1,2,3,4$ \\
\hline 25 & FusA-2 & FusA & MPN227 & P75544 & Elongation factor G & 76451.41 & 5.43 & 100.00 & 37 & 71.22 & & & $1,2,3,4$ \\
\hline 26 & FusA-3 & FusA & MPN227 & P75544 & Elongation factor G & 76451.41 & 5.43 & 100.00 & 45 & 76.02 & & & $1,2,3,4$ \\
\hline 27 & GapA-1 & GapA & MPN430 & P75358 & $\begin{array}{l}\text { Glyceraldehyde-3-phosphate } \\
\text { dehydrogenase }\end{array}$ & 36782.40 & 8.85 & 100.00 & 20 & 68.84 & & & $1,2,3,4$ \\
\hline 28 & GapA-2 & GapA & MPN430 & P75358 & $\begin{array}{l}\text { Glyceraldehyde-3-phosphate } \\
\text { dehydrogenase }\end{array}$ & 36782.40 & 8.85 & 100.00 & 17 & 49.85 & & & $1,2,3,4$ \\
\hline \multirow[t]{2}{*}{29} & [GapA Xdj1]-1 & GapA & MPN430 & P75358 & $\begin{array}{l}\text { Glyceraldehyde-3-phosphate } \\
\text { dehydrogenase }\end{array}$ & 36782.40 & 8.85 & 100.00 & 35 & 88.72 & S245 & VPVLTGPSIVELCVALEK & $1,2,3,4$ \\
\hline & & Xdj1 & MPN002 & Q50312 & DnaJ-like protein & 37109.23 & 9.44 & 100.00 & 13 & 36.25 & & & $1,2,3,4$ \\
\hline \multirow[t]{2}{*}{30} & [GapA Xdj1]-2 & GapA & MPN430 & P75358 & $\begin{array}{l}\text { Glyceraldehyde-3-phosphate } \\
\text { dehydrogenase }\end{array}$ & 36782.40 & 8.85 & 100.00 & 27 & 86.05 & S245 & VPVLTGPSIVELCVALEK & $1,2,3,4$ \\
\hline & & Xdj1 & MPN002 & Q50312 & DnaJ-like protein & 37109.23 & 9.44 & 100.00 & 11 & 40.78 & & & $1,2,3,4$ \\
\hline 31 & GlpK-1 & GlpK & MPN050 & P75064 & Glycerol kinase & 56555.27 & 8.72 & 100.00 & 16 & 47.83 & & & $1,2,3,4$ \\
\hline 32 & GlpK-2 & GlpK & MPN050 & P75064 & Glycerol kinase & 56555.27 & 8.72 & 100.00 & 22 & 66.34 & & & $1,2,3,4$ \\
\hline 33 & GlpQ & GlpQ & MPN420 & P75367 & $\begin{array}{l}\text { Glycerophosphoryldiester } \\
\text { phosphodiesterase }\end{array}$ & 28354.79 & 6.36 & 100.00 & 8 & 31.54 & & & $1,2,3,4$ \\
\hline 34 & GlyA & GlyA & MPN576 & P78011 & Serine hydroxymethyltransferase & 45237.55 & 9.26 & 100.00 & 15 & 36.70 & & & $1,2,3,4$ \\
\hline 35 & GroL-1 & GroL & MPN573 & P78012 & 60-kDa chaperonin & 58051.10 & 5.48 & 100.00 & 52 & 83.98 & & & $1,2,3,4$ \\
\hline 36 & GroL-2 & GroL & MPN573 & P78012 & 60-kDa chaperonin & 58051.10 & 5.48 & 100.00 & 35 & 74.03 & & & $1,2,3,4$ \\
\hline 37 & GroS & GroS & MPN574 & P75205 & 10-kDa chaperonin & 12610.67 & 9.46 & 100.00 & 4 & 35.34 & & & $1,2,3,4$ \\
\hline 38 & GrpE & GrpE & MPN120 & P78017 & Heat shock protein & 24690.28 & 8.81 & 100.00 & 12 & 46.54 & & & $1,2,3,4$ \\
\hline 39 & HMW3 & HMW3 & MPN452 & Q50360 & $\begin{array}{l}\text { Cytadherence high molecular weight } \\
\text { protein } 3\end{array}$ & 73676.4 & 4.53 & 100.00 & 20 & 37.95 & & & 2 \\
\hline 40 & Ldh-1 & Ldh & MPN674 & P78007 & L-Lactate dehydrogenase & 33866.91 & 8.76 & 100.00 & 24 & 79.49 & & & $1,2,3,4$ \\
\hline 41 & Ldh-2 & Ldh & MPN674 & P78007 & L-Lactate dehydrogenase & 33866.91 & 8.76 & 100.00 & 21 & 75.64 & & & $1,2,3,4$ \\
\hline 42 & [LplA FtsY] & LplA & MPN389 & P75394 & Lipoate-protein ligase A & 39169.02 & 6.14 & 100.00 & 25 & 67.26 & & & $1,2,3,4$ \\
\hline & & FtsY & MPN425 & P75362 & Cell division protein homolog & 38751.53 & 7.16 & 100.00 & 11 & 47.70 & & & $1,2,3,4$ \\
\hline
\end{tabular}


Tab. S5. Continued.

\begin{tabular}{|c|c|c|c|c|c|c|c|c|c|c|c|c|c|}
\hline \# & Spot & $\begin{array}{l}\text { Protein } \\
\text { name }\end{array}$ & $\begin{array}{l}\text { Locus } \\
\text { name }\end{array}$ & $\begin{array}{l}\text { UniProtKB } \\
\text { accession } \\
\text { number }\end{array}$ & Protein function & $\begin{array}{l}\text { Molecular } \\
\text { weight (Da) }\end{array}$ & $\begin{array}{l}\text { Isoelectric } \\
\text { point (pI) }\end{array}$ & $\begin{array}{l}\text { Protein } \\
\text { identification } \\
\text { probability (\%) }\end{array}$ & $\begin{array}{l}\text { Number } \\
\text { of unique } \\
\text { peptides }\end{array}$ & $\begin{array}{l}\text { Coverage } \\
(\%)\end{array}$ & $\begin{array}{l}\text { Phosphorylation } \\
\text { site }\end{array}$ & Phosphopeptide sequence & Experiment ${ }^{\mathrm{a}}$ \\
\hline 43 & ManB-1 & ManB & $\begin{array}{l}\text { MPN066 } \\
\end{array}$ & P75050 & $\begin{array}{l}\text { Phosphomannomutase/ } \\
\text { phosphoglucomutase }\end{array}$ & 63175.24 & 8.39 & 100.00 & 10 & 18.23 & S149 & YHFDGGVNVTApSHNPK & $1,2,3,4$ \\
\hline 44 & ManB-2 & ManB & MPN066 & P75050 & $\begin{array}{l}\text { Phosphomannomutase/ } \\
\text { phosphoglucomutase }\end{array}$ & 63175.24 & 8.39 & 100.00 & 20 & 52.17 & S149 & YHFDGGVNVTApSHNPK & $1,2,3,4$ \\
\hline 45 & ManB-3 & ManB & MPN066 & P75050 & $\begin{array}{l}\text { Phosphomannomutase/ } \\
\text { phosphoglucomutase }\end{array}$ & 63175.24 & 8.39 & 100.00 & 23 & 54.69 & S149 & KYHFDGGVNVTAPSHNPK & $1,2,3,4$ \\
\hline 46 & ManB-4 & ManB & MPN066 & P75050 & $\begin{array}{l}\text { Phosphomannomutase/ } \\
\text { phosphoglucomutase }\end{array}$ & 63175.24 & 8.39 & 100.00 & 32 & 59.57 & S149 & YHFDGGVNVTAPSHNPK & $1,2,3,4$ \\
\hline 47 & ManB-5 & ManB & MPN066 & P75050 & $\begin{array}{l}\text { Phosphomannomutase/ } \\
\text { phosphoglucomutase }\end{array}$ & 63175.24 & 8.39 & 100.00 & 25 & 60.47 & S149 & YHFDGGVNVTApSHNPK & $1,2,3,4$ \\
\hline 48 & ManB-6 & ManB & MPN066 & P75050 & $\begin{array}{l}\text { Phosphomannomutase/ } \\
\text { phosphoglucomutase }\end{array}$ & 63175.24 & 8.39 & 100.00 & 22 & 66.61 & S149 & YHFDGGVNVTAPSHNPK & $1,2,3,4$ \\
\hline 49 & ManB-7 & ManB & MPN066 & P75050 & $\begin{array}{l}\text { Phosphomannomutase/ } \\
\text { phosphoglucomutase }\end{array}$ & 63175.24 & 8.39 & 100.00 & 24 & 66.25 & S149 & YHFDGGVNVTApSHNPK & $1,2,3,4$ \\
\hline 50 & ManB-8 & ManB & MPN066 & P75050 & $\begin{array}{l}\text { Phosphomannomutase/ } \\
\text { phosphoglucomutase }\end{array}$ & 63175.24 & 8.39 & 100.00 & 21 & 58.12 & S149 & YHFDGGVNVTAPSHNPK & $1,2,3,4$ \\
\hline 51 & ManB-9 & ManB & MPN066 & P75050 & $\begin{array}{l}\text { Phosphomannomutase/ } \\
\text { phosphoglucomutase }\end{array}$ & 63175.24 & 8.39 & 100.00 & 26 & 66.25 & S149 & YHFDGGVNVTAPSHNPK & $1,2,3,4$ \\
\hline 52 & ManB-10 & ManB & MPN066 & P75050 & $\begin{array}{l}\text { Phosphomannomutase/ } \\
\text { phosphoglucomutase }\end{array}$ & 63175.24 & 8.39 & 100.00 & 36 & 58.12 & S149 & YHFDGGVNVTAPSHNPK & $1,2,3,4$ \\
\hline 53 & MPN256-1 & MPN256 & MPN256 & P75518 & Uncharacterized protein & 25722.9 & 4.18 & 100.00 & 14 & 67.71 & T200 & FIDELDQIpTK & 2 \\
\hline 54 & MPN256-2 & MPN256 & MPN256 & P75518 & Uncharacterized protein & 25722.9 & 4.18 & 100.00 & 7 & 48.88 & & & 2 \\
\hline 55 & MPN280-1 & MPN280 & MPN280 & P75497 & Ribonuclease J & 64045.33 & 7.57 & 100.00 & 18 & 44.29 & & & $1,2,3,4$ \\
\hline 56 & MPN280-2 & MPN280 & MPN280 & P75497 & Ribonuclease J & 64045.33 & 7.57 & 100.00 & 16 & 39.72 & & & $1,2,3,4$ \\
\hline 57 & MPN295-1 & MPN295 & MPN295 & P75482 & Uncharacterized protein & 25684.16 & 8.08 & 100.00 & 16 & 60.91 & & & $1,2,3,4$ \\
\hline 58 & MPN295-2 & MPN295 & MPN295 & P75482 & Uncharacterized protein & 25684.16 & 8.08 & 100.00 & 22 & 73.18 & $\mathrm{~s} 63$ & YGPECEKSFLSLQpSK & $1,2,3,4$ \\
\hline 59 & MPN349 & MPN349 & MPN349 & P75429 & Uncharacterized protein & 31411.30 & 8.83 & 100.00 & 12 & 52.67 & & & $1,2,3,4$ \\
\hline 60 & MPN474 & MPN474 & MPN474 & P75310 & Coiled coil surface protein & 118006.4 & 4.81 & 100.00 & 44 & 61.08 & $\mathrm{~T} 773$ & QNNEELPTDKCSNIQNELHDLNR & 2 \\
\hline 61 & MPN517 & MPN517 & MPN517 & P75270 & Reductase homolog & 18856.85 & 7.26 & 100.00 & 7 & 42.17 & & & $1,2,3,4$ \\
\hline
\end{tabular}


Tab. S5. Continued.

\begin{tabular}{|c|c|c|c|c|c|c|c|c|c|c|c|c|c|}
\hline \# & Spot & $\begin{array}{l}\text { Protein } \\
\text { name }\end{array}$ & $\begin{array}{l}\text { Locus } \\
\text { name }\end{array}$ & $\begin{array}{l}\text { UniProtKB } \\
\text { accession } \\
\text { number }\end{array}$ & Protein function & $\begin{array}{l}\text { Molecular } \\
\text { weight (Da) }\end{array}$ & $\begin{array}{l}\text { Issoelectric } \\
\text { point (pI) }\end{array}$ & $\begin{array}{l}\text { Protein } \\
\text { identification } \\
\text { probability (\%) }\end{array}$ & $\begin{array}{l}\text { Number } \\
\text { of unique } \\
\text { peptides }\end{array}$ & $\begin{array}{l}\text { Coverage } \\
(\%)\end{array}$ & $\begin{array}{l}\text { Phosphorylation } \\
\text { site }\end{array}$ & Phosphopeptide sequence & Experiment ${ }^{\mathrm{a}}$ \\
\hline 62 & MPN547 & MPN547 & MPN547 & P75231 & Dihydroxyacetone kinase & 62398.49 & 8.20 & 100.00 & 39 & 75.63 & & & $1,2,3,4$ \\
\hline 63 & MPN555-1 & MPN555 & MPN555 & P75223 & Uncharacterized protein & 22419.68 & 5.51 & 100.00 & 7 & 41.97 & & & $1,2,3,4$ \\
\hline 64 & MPN555-2 & MPN555 & MPN555 & P75223 & Uncharacterized protein & 22419.68 & 5.51 & 100.00 & 11 & 55.44 & & & $1,2,3,4$ \\
\hline 65 & MPN625 & MPN625 & MPN625 & P75170 & $\begin{array}{l}\text { Osmotical inducible protein C-like } \\
\text { protein }\end{array}$ & 15458.91 & 6.60 & 100.00 & 4 & 31.21 & & & $1,2,3,4$ \\
\hline 66 & MPN638-1 & MPN638 & MPN638 & P75159 & $\begin{array}{l}\text { Putative type I restriction enzyme } \\
\text { specificity protein }\end{array}$ & 42623.25 & 8.51 & 100.00 & 24 & 66.67 & & & $1,2,3,4$ \\
\hline 67 & MPN638-2 & MPN638 & MPN638 & P75159 & $\begin{array}{l}\text { Putative type I restriction enzyme } \\
\text { specificity protein }\end{array}$ & 42623.25 & 8.51 & 100.00 & 15 & 43.47 & & & $1,2,3,4$ \\
\hline 68 & MPN677 & MPN677 & MPN677 & P75115 & Uncharacterized protein & 50554.18 & 8.89 & 100.00 & 12 & 36.94 & & & $1,2,3,4$ \\
\hline 69 & NrdE-1 & NrdE & MPN324 & P78027 & $\begin{array}{l}\text { Ribonucleoside-diphosphate reductase } \\
\text { subunit } \alpha\end{array}$ & 82322.96 & 6.66 & 100.00 & 66 & 69.90 & T159 & FQPAPTPTFLNAGR & $1,2,3,4$ \\
\hline 70 & NrdE-2 & NrdE & MPN324 & P78027 & $\begin{array}{l}\text { Ribonucleoside-diphosphate reductase } \\
\text { subunit } \alpha\end{array}$ & 82322.96 & 6.66 & 100.00 & 54 & 61.86 & S412 & VGNDIPSCNLGSLNIAK & $1,2,3,4$ \\
\hline 71 & NrdE-3 & NrdE & MPN324 & P78027 & $\begin{array}{l}\text { Ribonucleoside-diphosphate reductase } \\
\text { subunit } \alpha\end{array}$ & 82322.96 & 6.66 & 100.00 & 17 & 23.02 & & & $1,2,3,4$ \\
\hline 72 & $\mathrm{NrdF}$ & $\mathrm{NrdF}$ & MPN322 & P75461 & $\begin{array}{l}\text { Ribonucleoside-diphosphate reductase } \\
\text { subunit } \beta\end{array}$ & 39388.04 & 5.36 & 100.00 & 19 & 71.98 & & & $1,2,3,4$ \\
\hline 73 & Nox & Nox & MPN394 & P75389 & NADH oxidase & 52841.36 & 6.70 & 100.00 & 11 & 29.85 & & & $1,2,3,4$ \\
\hline 74 & NusA-1 & NusA & MPN154 & P75591 & Transcription elongation factor & 60235.03 & 5.77 & 100.00 & 43 & 80.00 & S503 & PVVKPKpSVFSITVEADDSK & $1,2,3,4$ \\
\hline 75 & NusA-2 & NusA & MPN154 & P75591 & Transcription elongation factor & 60235.03 & 5.77 & 100.00 & 19 & 48.15 & & & $1,2,3,4$ \\
\hline 76 & P41 & P41 & MPN311 & P75470 & Adhesin-related protein & 40555.6 & 4.64 & 100.00 & 9 & 42.58 & T223 & TNNSIQQLEAEIQIPрTTHIK & 2 \\
\hline 77 & PdhA & PdhA & MPN393 & P75390 & $\begin{array}{l}\text { Pyruvate dehydrogenase E1 component } \\
\text { subunit } \alpha\end{array}$ & 40568.44 & 6.22 & 100.00 & 19 & 53.63 & & & $1,2,3,4$ \\
\hline \multirow[t]{2}{*}{78} & [PdhA Pgk] & PdhA & MPN393 & P75390 & $\begin{array}{l}\text { Pyruvate dehydrogenase E1 component } \\
\text { subunit } \alpha\end{array}$ & 40568.44 & 6.22 & 100.00 & 14 & 38.27 & S205 & TKLEPSAVSDLSTK & $1,2,3,4$ \\
\hline & & Pgk & MPN429 & P78018 & Phosphoglycerate kinase & 44184.27 & 7.26 & 100.00 & 9 & 26.65 & & & $1,2,3,4$ \\
\hline
\end{tabular}


Tab. S5. Continued.

\begin{tabular}{|c|c|c|c|c|c|c|c|c|c|c|c|c|c|}
\hline \# & Spot & $\begin{array}{l}\text { Protein } \\
\text { name }\end{array}$ & $\begin{array}{l}\text { Locus } \\
\text { name }\end{array}$ & $\begin{array}{l}\text { UniProtKB } \\
\text { accession } \\
\text { number }\end{array}$ & Protein function & $\begin{array}{l}\text { Molecular } \\
\text { weight (Da) }\end{array}$ & $\begin{array}{l}\text { Isoelectric } \\
\text { point (pI) }\end{array}$ & $\begin{array}{l}\text { Protein } \\
\text { identification } \\
\text { probability (\%) }\end{array}$ & $\begin{array}{l}\text { Number } \\
\text { of unique } \\
\text { peptides }\end{array}$ & $\begin{array}{l}\text { Coverage } \\
(\%)\end{array}$ & $\begin{array}{l}\text { Phosphorylation } \\
\text { site }\end{array}$ & Phosphopeptide sequence & Experiment $^{\mathrm{a}}$ \\
\hline \multirow[t]{2}{*}{79} & [PdhB Pta] -1 & PdhB & MPN392 & P75391 & $\begin{array}{l}\text { Pyruvate dehydrogenase E1 component } \\
\text { subunit } \beta\end{array}$ & 35891.67 & 6.54 & 100.00 & 15 & 57.19 & & & $1,2,3,4$ \\
\hline & & Pta & MPN428 & P75359 & Phosphotransacetylase & 35196.23 & 7.16 & 100.00 & 9 & 38.75 & & & $1,2,3,4$ \\
\hline \multirow[t]{2}{*}{80} & [PdhB Pta]-2 & PdhB & MPN392 & P75391 & $\begin{array}{l}\text { Pyruvate dehydrogenase E1 component } \\
\text { subunit } \beta\end{array}$ & 35891.67 & 6.54 & 100.00 & 14 & 50.76 & & & $1,2,3,4$ \\
\hline & & Pta & MPN428 & P75359 & Phosphotransacetylase & 35196.23 & 7.16 & 100.00 & 7 & 27.50 & & & $1,2,3,4$ \\
\hline 81 & PdhC-1 & PdhC & MPN391 & P75392 & Pyruvate dehydrogenase E2 component & 42370.45 & 5.43 & 100.00 & 13 & 37.06 & & & $1,2,3,4$ \\
\hline 82 & PdhC-2 & PdhC & MPN391 & P75392 & Pyruvate dehydrogenase E2 component & 42370.45 & 5.43 & 100.00 & 15 & 37.06 & & & $1,2,3,4$ \\
\hline 83 & PdhC-3 & PdhC & MPN391 & P75392 & Pyruvate dehydrogenase E2 component & 42370.45 & 5.43 & 100.00 & 11 & 33.58 & & & $1,2,3,4$ \\
\hline 84 & PdhC-4 & PdhC & MPN391 & P75392 & Pyruvate dehydrogenase E2 component & 42370.45 & 5.43 & 100.00 & 8 & 18.41 & & & $1,2,3,4$ \\
\hline 85 & PdhD-1 & PdhD & MPN390 & P75393 & Pyruvate dehydrogenase E3 component & 49406.02 & 8.14 & 100.00 & 15 & 47.05 & & & $1,2,3,4$ \\
\hline 86 & PdhD-2 & PdhD & MPN390 & P75393 & Pyruvate dehydrogenase E3 component & 49406.02 & 8.14 & 100.00 & 11 & 38.29 & & & $1,2,3,4$ \\
\hline 87 & PepA-1 & PepA & MPN572 & P75206 & Cytosol aminopeptidase & 48758.12 & 8.23 & 100.00 & 38 & 75.73 & $\begin{array}{l}\text { S249 } \\
\mathrm{T} 420\end{array}$ & $\begin{array}{l}\text { YDMSGAAIVCPSTVLALAK } \\
\text { EGVPLIHCDIASPTASIQDLGQGVLVR }\end{array}$ & $1,2,3,4$ \\
\hline 88 & РерA-2 & РерА & MPN572 & P75206 & Cytosol aminopeptidase & 48758.12 & 8.23 & 100.00 & 22 & 56.18 & & & $1,2,3,4$ \\
\hline 89 & РерA-3 & РерА & MPN572 & P75206 & Cytosol aminopeptidase & 48758.12 & 8.23 & 100.00 & 18 & 49.44 & & & $1,2,3,4$ \\
\hline 90 & PepP-1 & РерP & MPN470 & P75313 & Xаa-Pro aminopeptidase & 39598.88 & 8.81 & 100.00 & 34 & 88.70 & T317 & LLCENAVIPTIEPGIYIPSVGGIR & $1,2,3,4$ \\
\hline 91 & PepP-2 & РерP & MPN470 & P75313 & Xaa-Pro aminopeptidase & 39598.88 & 8.81 & 100.00 & 23 & 64.12 & & & $1,2,3,4$ \\
\hline 92 & PfkA & PfkA & MPN302 & P75476 & Phosphofructokinase & 35966.53 & 7.30 & 100.00 & 6 & 24.39 & & & $1,2,3,4$ \\
\hline 93 & Рра & Рра & MPN528 & P75250 & Inorganic pyrophosphatase & 21355.08 & 5.38 & 100.00 & 8 & 35.87 & & & $1,2,3,4$ \\
\hline 94 & Pta & Pta & MPN428 & P75359 & Phosphotransacetylase & 35196.23 & 7.16 & 100.00 & 16 & 57.19 & & & $1,2,3,4$ \\
\hline \multirow[t]{2}{*}{95} & [Pta PdhB] & Pta & MPN428 & P75359 & Phosphotransacetylase & 35196.23 & 7.16 & 100.00 & 16 & 60.63 & & & $1,2,3,4$ \\
\hline & & PdhB & MPN392 & P75391 & $\begin{array}{l}\text { Pyruvate dehydrogenase E1 component } \\
\text { subunit } \beta\end{array}$ & 35891.67 & 6.54 & 100.00 & 11 & 47.71 & & & $1,2,3,4$ \\
\hline 96 & Pth & Pth & MPN221 & P78034 & Peptidyl-tRNA hydrolase & 21403.30 & 9.91 & 100.00 & 6 & 38.83 & & & $1,2,3,4$ \\
\hline 97 & Pyk & Pyk & MPN303 & P78031 & Pyruvate kinase & 57232.04 & 9.45 & 100.00 & 28 & 63.19 & & & $1,2,3,4$ \\
\hline 98 & RpoE & RpoE & MPN024 & P75090 & RNA polymerase subunit $\delta$ & 17395.3 & 4.17 & 100.00 & 11 & 70.55 & [S87 or T93] & Ip[SQAMFVT]KEIFEEGYEDLSNK & 2 \\
\hline 81 & PdhC-1 & PdhC & MPN391 & P75392 & Pyruvate dehydrogenase E2 component & 42370.45 & 5.43 & 100.00 & 13 & 37.06 & & & $1,2,3,4$ \\
\hline 99 & Tig-1 & Tig & MPN331 & P75454 & Trigger factor & 51321.25 & 6.08 & 100.00 & 27 & 66.67 & & & $1,2,3,4$ \\
\hline
\end{tabular}


Tab. S5. Continued.

\begin{tabular}{|c|c|c|c|c|c|c|c|c|c|c|c|c|c|}
\hline \# & Spot & $\begin{array}{l}\text { Protein } \\
\text { name }\end{array}$ & $\begin{array}{l}\text { Locus } \\
\text { name }\end{array}$ & $\begin{array}{l}\text { UniProtKB } \\
\text { accession } \\
\text { number }\end{array}$ & Protein function & $\begin{array}{l}\text { Molecular } \\
\text { weight (Da) }\end{array}$ & $\begin{array}{l}\text { Isoelectric } \\
\text { point (pI) }\end{array}$ & $\begin{array}{l}\text { Protein } \\
\text { identification } \\
\text { probability (\%) }\end{array}$ & $\begin{array}{l}\text { Number } \\
\text { of unique } \\
\text { peptides }\end{array}$ & $\begin{array}{l}\text { Coverage } \\
(\%)\end{array}$ & $\begin{array}{l}\text { Phosphorylation } \\
\text { site }\end{array}$ & Phosphopeptide sequence & Experiment $^{\mathrm{a}}$ \\
\hline 100 & Tig-2 & Tig & MPN331 & P75454 & Trigger factor & 51321.25 & 6.08 & 100.00 & 25 & 52.03 & & & $1,2,3,4$ \\
\hline 101 & Tig-3 & Tig & MPN331 & P75454 & Trigger factor & 51321.25 & 6.08 & 100.00 & 16 & 30.18 & & & $1,2,3,4$ \\
\hline 102 & TopA & TopA & MPN261 & P78032 & DNA topoisomerase I & 81914.21 & 9.52 & 100.00 & 48 & 64.84 & S426 & TVAPSLMADCKK & $1,2,3,4$ \\
\hline 103 & TpiA & TpiA & MPN629 & P78010 & Triose-phosphate isomerase & 26941.33 & 7.34 & 100.00 & 12 & 56.97 & & & $1,2,3,4$ \\
\hline 104 & Tkt-1 & Tkt & MPN082 & P75611 & Transketolase & 72332.86 & 7.20 & 100.00 & 25 & 51.23 & & & $1,2,3,4$ \\
\hline 105 & Tkt-2 & Tkt & MPN082 & P75611 & Transketolase & 72332.86 & 7.20 & 100.00 & 20 & 37.35 & & & $1,2,3,4$ \\
\hline 106 & Tkt-3 & Tkt & MPN082 & P75611 & Transketolase & 72332.86 & 7.20 & 100.00 & 15 & 38.12 & & & $1,2,3,4$ \\
\hline 107 & Tuf-1 & Tuf & MPN665 & P23568 & Elongation factor TU & 43122.32 & 6.04 & 100.00 & 30 & 65.23 & T383 & EGGRPTVGAGSVTEVLE & $1,2,3,4$ \\
\hline 108 & Tuf-2 & Tuf & MPN665 & P23568 & Elongation factor TU & 43122.32 & 6.04 & 100.00 & 24 & 64.97 & T383 & EGGRPTVGAGSVTEVLE & $1,2,3,4$ \\
\hline 109 & Tuf-3 & Tuf & MPN665 & P23568 & Elongation factor TU & 43122.32 & 6.04 & 100.00 & 19 & 47.21 & T383 & EGGRPTVGAGSVTEVLE & $1,2,3,4$ \\
\hline 110 & Tuf-4 & Tuf & MPN665 & P23568 & Elongation factor TU & 43122.32 & 6.04 & 100.00 & 7 & 22.08 & T383 & EGGRPTVGAGSVTEVLE & $1,2,3,4$ \\
\hline \multirow[t]{2}{*}{111} & [UlaB HPr] & UlaB & MPN495 & Q9EXD8 & $\begin{array}{l}\text { Ascorbate-specific phosphotransferase } \\
\text { enzyme IIB component }\end{array}$ & 10320.41 & 8.61 & 100.00 & 4 & 55.79 & & & $1,2,3,4$ \\
\hline & & HPr & MPN053 & P75061 & Phosphocarrier protein & 9489.23 & 9.22 & 100.00 & 3 & 30.68 & & & $1,2,3$ \\
\hline
\end{tabular}

${ }^{a}$ Phosphospot detected in different M. pneumoniae strains. 1, wild type; 2, prpC::Tn mutant; 3, prkC::Tn mutant; 4, hprK::Tn mutant. 
Tab. S6. List of phosphopeptides.

For general overview, see Table 4. oM, oxidised methionine; pS, phosphoserine; pT, phosphothreonine.

\begin{tabular}{|c|c|c|c|c|c|c|c|c|c|c|c|c|c|c|c|}
\hline \# & Spot & $\begin{array}{l}\text { Protein } \\
\text { name }\end{array}$ & $\begin{array}{l}\text { Locus } \\
\text { name }\end{array}$ & $\begin{array}{l}\text { UniProtKB } \\
\text { accession } \\
\text { number }\end{array}$ & Phosphopeptide sequence & Start aa & Stop aa & $\begin{array}{l}\text { SEQUEST } \\
\text { Xcorr }\end{array}$ & $\begin{array}{l}\text { SEQUEST } \\
\text { deltaCn }\end{array}$ & $\begin{array}{l}\text { Observed } \\
\mathbf{m} / \mathbf{z}\end{array}$ & 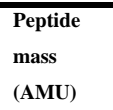 & Charge & $\begin{array}{l}\text { Peptide } \\
\text { identification } \\
\text { probability (\%) }\end{array}$ & Position & Mod. aa \\
\hline 1 & [GapA Xdj1]-1 & GapA & MPN430 & P75358 & VPVLTGPSIVELCVALEK & 239 & 255 & 2.1403 & 0.7004 & 925.4895 & 1848.9634 & 2 & 95.00 & 245 & $\mathrm{~s}$ \\
\hline 2 & [GapA Xdj1]-2 & GapA & MPN430 & P75358 & VPVLTGPSIVELCVALEK & 239 & 255 & 2.8309 & 0.7830 & 925.4899 & 1848.9641 & 2 & 95.00 & 245 & $\mathrm{~s}$ \\
\hline 3 & ManB-1 & ManB & MPN066 & P75050 & YHFDGGVNVTApSHNPK & 138 & 153 & 3.4808 & 0.7661 & 608.2682 & 1821.7811 & 3 & 95.00 & 149 & $\mathrm{~s}$ \\
\hline 4 & ManB-2 & ManB & MPN066 & P75050 & YHFDGGVNVTApSHNPK & 138 & 153 & 3.4107 & 0.8276 & 608.2686 & 1821.7824 & 3 & 95.00 & 149 & $\mathrm{~s}$ \\
\hline 5 & ManB-3 & ManB & MPN066 & P75050 & KYHFDGGVNVTApSHNPK & 137 & 153 & 4.1654 & 0.4348 & 488.4794 & 1949.8865 & 4 & 95.00 & 149 & $\mathrm{~s}$ \\
\hline 6 & ManB-4 & ManB & MPN066 & P75050 & YHFDGGVNVTAPSHNPK & 138 & 153 & 3.0082 & 0.7313 & 608.2675 & 1821.7791 & 3 & 95.00 & 149 & $\mathrm{~s}$ \\
\hline 7 & ManB-5 & ManB & MPN066 & P75050 & YHFDGGVNVTAPSHNPK & 138 & 153 & 3.3379 & 0.7312 & 608.2715 & 1821.7910 & 3 & 95.00 & 149 & $\mathrm{~s}$ \\
\hline 8 & ManB-6 & ManB & MPN066 & P75050 & YHFDGGVNVTAPSHNPK & 138 & 153 & 3.5166 & 0.7365 & 608.2682 & 1821.7811 & 3 & 95.00 & 149 & $\mathrm{~s}$ \\
\hline 9 & ManB-7 & ManB & MPN066 & P75050 & YHFDGGVNVTAPSHNPK & 138 & 153 & 3.4299 & 0.7130 & 608.2709 & 1821.7891 & 3 & 95.00 & 149 & $\mathrm{~s}$ \\
\hline 10 & ManB-8 & ManB & MPN066 & P75050 & YHFDGGVNVTAPSHNPK & 138 & 153 & 3.5219 & 0.6994 & 608.2709 & 1821.7893 & 3 & 95.00 & 149 & $\mathrm{~s}$ \\
\hline 11 & ManB-9 & ManB & MPN066 & P75050 & YHFDGGVNVTAPSHNPK & 138 & 153 & 3.1959 & 0.7766 & 608.2673 & 1821.7783 & 3 & 95.00 & 149 & $\mathrm{~s}$ \\
\hline 12 & ManB-10 & ManB & MPN066 & P75050 & YHFDGGVNVTAPSHNPK & 138 & 153 & 3.2742 & 0.7367 & 608.2702 & 1821.7871 & 3 & 95.00 & 149 & $\mathrm{~s}$ \\
\hline 13 & MPN256-1 & MPN256 & MPN256 & P75518 & FIDELDQIpTK & 192 & 201 & 1.8676 & 0.4646 & 651.3056 & 1300.5955 & 2 & 95.00 & 200 & $\mathrm{~T}$ \\
\hline 14 & MPN295-2 & MPN295 & MPN295 & P75482 & YGPECEKSFLSLQpSK & 50 & 64 & 1.8583 & 0.6774 & 898.4016 & 1794.7875 & 2 & 95.00 & 63 & $\mathrm{~s}$ \\
\hline 15 & MPN474 & MPN474 & MPN474 & P75310 & QNNEELpTDKCSNIQNELHDLNR & 767 & 788 & 3.8806 & 0.6714 & 903.0639 & 2706.1682 & 3 & 95.00 & 773 & $\mathrm{~T}$ \\
\hline 16 & NrdE-1 & NrdE & MPN324 & P78027 & FQPAPTPTFLNAGR & 155 & 167 & 1.9105 & 0.6332 & 750.3589 & 1498.7022 & 2 & 95.00 & 159 & $\mathrm{~T}$ \\
\hline 17 & NrdE-2 & NrdE & MPN324 & P78027 & VGNDIPSCNLGSLNIAK & 407 & 422 & 2.1525 & 0.3036 & 849.3960 & 1696.7763 & 2 & 95.00 & 412 & $\mathrm{~s}$ \\
\hline 18 & NusA-1 & NusA & MPN154 & P75591 & PVVKPKpSVFSITVEADDSK & 497 & 515 & 3.2040 & 0.6335 & 709.3649 & 2125.0713 & 3 & 95.00 & 503 & $\mathrm{~s}$ \\
\hline 19 & P41 & P41 & MPN311 & P75470 & TNNSIQQLEAEIQIPpTTHIK & 208 & 227 & 3.8887 & 0.7207 & 786.7265 & 2357.1560 & 3 & 95.00 & 223 & $\mathrm{~T}$ \\
\hline 20 & [PdhA Pgk] & PdhA & MPN393 & P75390 & TKLEPSAVSDLSTK & 201 & 213 & 3.4406 & 0.2305 & 729.8603 & 1457.7050 & 2 & 95.00 & 205 & $\mathrm{~s}$ \\
\hline 21 & PepA-1 & РерА & MPN572 & P75206 & YDoMSGAAIVCPSTVLALAK & 239 & 256 & 3.2690 & 0.6324 & 954.9456 & 1907.8756 & 2 & 95.00 & 249 & $\mathrm{~s}$ \\
\hline 22 & РерА-1 & РерА & MPN572 & P75206 & EGVPLIHCDIASPTASIQDLGQGVLVR & 408 & 433 & 2.1490 & 0.3303 & 924.4661 & 2770.3749 & 3 & 95.00 & 420 & $\mathrm{~T}$ \\
\hline 23 & PepP-1 & РерР & MPN470 & P75313 & LLCENAVIPTIEPGIYIPSVGGIR & 309 & 331 & 2.7570 & 0.7431 & 627.5862 & 2506.3136 & 4 & 95.00 & 317 & $\mathrm{~T}$ \\
\hline 24 & RpoE & RpoE & MPN024 & P75090 & IpSQAoMFVTKEIFEEGYEDLSNK & 86 & 107 & 4.5815 & 0.6970 & 892.0717 & 2673.1917 & 3 & 95.00 & 87 & $\mathrm{~s}$ \\
\hline 25 & RpoE & RpoE & MPN024 & P75090 & ISQAMFVPTKEIFEEGYEDLSNK & 86 & 107 & 4.9395 & 0.7017 & 886.7402 & 2657.1970 & 3 & 95.00 & 93 & $\mathrm{~T}$ \\
\hline 26 & TорА & TорА & MPN261 & P78032 & TVAPSLMADCKK & 423 & 433 & 1.9215 & 0.5958 & 623.7813 & 1245.5470 & 2 & 95.00 & 426 & $\mathrm{~s}$ \\
\hline 27 & Tuf-1 & Tuf & MPN665 & P23568 & EGGRpTVGAGSVTEVLE & 379 & 394 & 3.4741 & 0.7097 & 820.8821 & 1639.7486 & 2 & 95.00 & 383 & $\mathrm{~T}$ \\
\hline
\end{tabular}


Tab. S6. Continued.

\begin{tabular}{|c|c|c|c|c|c|c|c|c|c|c|c|c|c|c|c|}
\hline \# & Spot & $\begin{array}{l}\text { Protein } \\
\text { name }\end{array}$ & $\begin{array}{l}\text { Locus } \\
\text { name }\end{array}$ & $\begin{array}{l}\text { UniProtKB } \\
\text { accession } \\
\text { number }\end{array}$ & Phosphopeptide sequence & Start aa & Stop aa & $\begin{array}{l}\text { SEQUEST } \\
\text { Xcorr }\end{array}$ & $\begin{array}{l}\text { SEQUEST } \\
\text { deltaCn }\end{array}$ & $\begin{array}{l}\text { Observed } \\
\mathbf{m} / \mathbf{z}\end{array}$ & $\begin{array}{l}\text { Peptide } \\
\text { mass } \\
\text { (AMU) }\end{array}$ & Charge & $\begin{array}{l}\text { Peptide } \\
\text { identification } \\
\text { probability (\%) }\end{array}$ & Position & Mod. aa \\
\hline 28 & 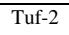 & Tuf & MPN665 & P23568 & EGGRPTVGAGSVTEVLE & 379 & 394 & 3.2175 & 0.7339 & 820.8817 & 1639.7478 & 2 & 95.00 & 383 & $\mathrm{~T}$ \\
\hline 29 & Tuf-3 & Tuf & MPN665 & P23568 & EGGRpTVGAGSVTEVLE & 379 & 394 & 2.9993 & 0.6668 & 820.8795 & 1639.7433 & 2 & 95.00 & 383 & $\mathrm{~T}$ \\
\hline 30 & Tuf-4 & Tuf & MPN665 & P23568 & EGGRPTVGAGSVTEVLE & 379 & 394 & 3.2929 & 0.7727 & 820.8883 & 1639.7609 & 2 & 95.00 & 383 & $\mathrm{~T}$ \\
\hline
\end{tabular}




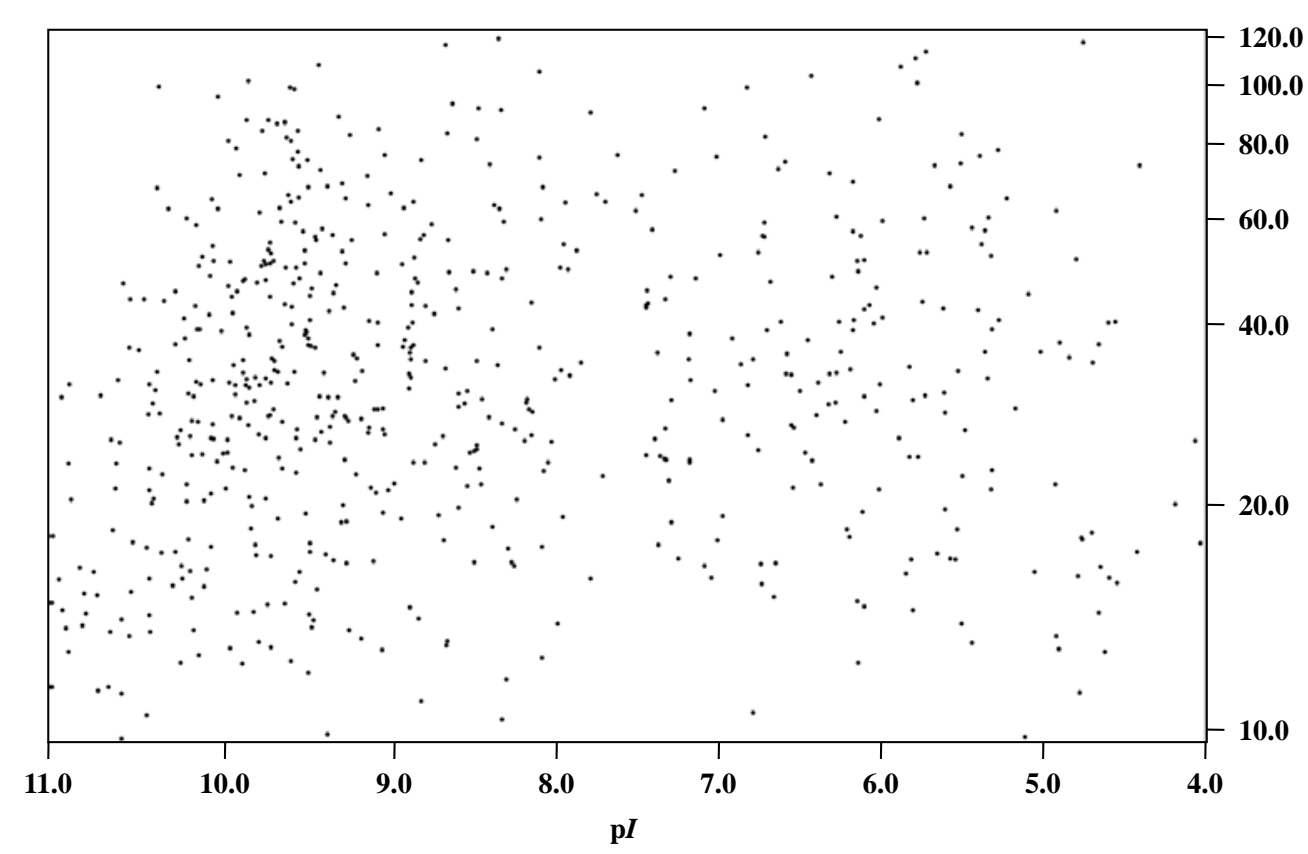

kDa

Fig. S1. Virtual two-dimensional protein map of M. pneumoniae. Based on the calculated molecular weight (MW) and theoretical isoelectric point (pI), each protein is localized in this virtual two-dimensional protein map of $M$. pneumoniae using the software JVirGel v2.2.3b (http://www.jvirgel.de/). NCBI reference sequence used for predicting MWs and pIs of the 689 proteins is NC_000912 (M. pneumoniae strain M129). The pI ranged from 4-11 and the molecular weight is adjusted from 8-125 $\mathrm{kDa}$. About 635 proteins could be visualized with this set-up. 
A

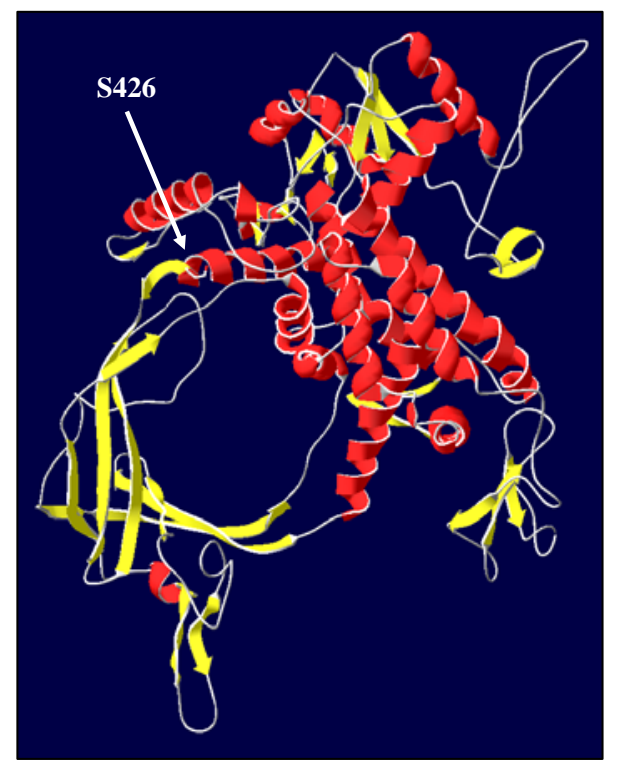

B

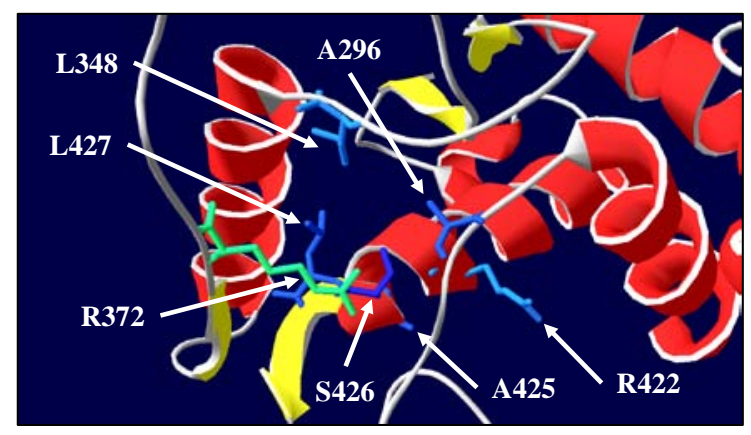

MPN324 - NrdE - Ribonucleoside-diphosphate reductase subunit alpha (T159 \& S412)

A

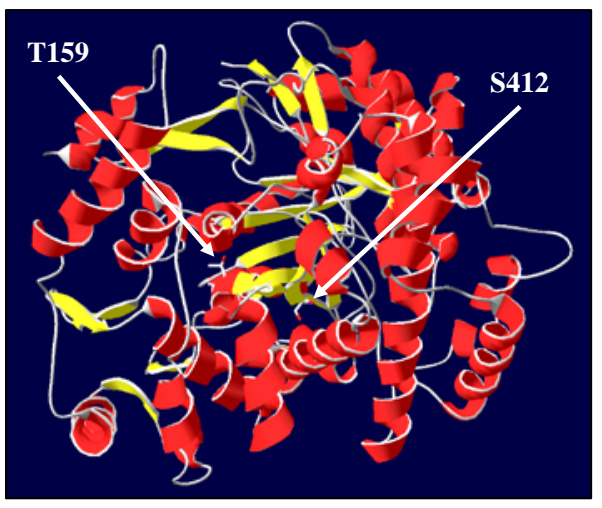

B

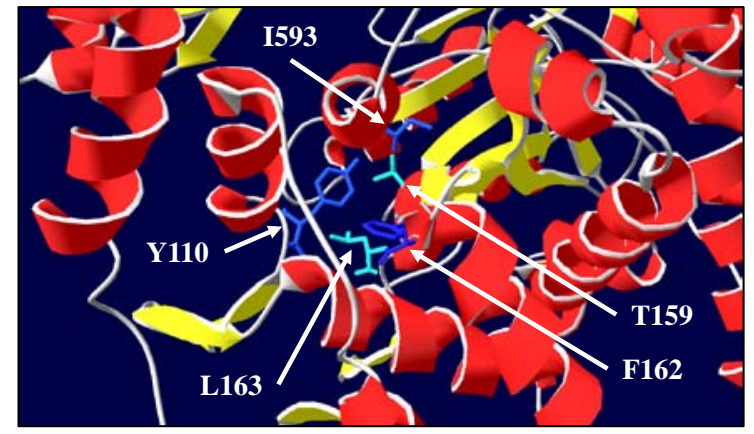

Fig. S2. Models of phosphoproteins based on homology with available structures. The models are based on the following PDB entries: 2GAI (MPN261, TopA), 1PEO (MPN324, NrdE), 1W85 (MPN393, PdhA), 1HDG (MPN430, GapA), 1PV9 (MPN470, PepP), 2J9A (MPN572, PepA), and 1OB2 (MPN665, Tuf). (A) Ribbon diagrams of phosphoproteins colored by secondary structure. As structural elements $\alpha$-helices are shown in red and $\beta$-strands in yellow. Remaining elements are grey. The corresponding phosphorylation sites are highlighted on the model. (B) Close-up view of the phosphorylation sites with surrounding residues. Residues (distance of $1 \AA$ ) that possibly affect the phosphorylation are indicated. Additionally, each amino acid is colored by its relative accessibility. Dark blue color is attributed to completely buried amino acids, whereas red color is attributed to amino acids with at least $75 \%$ of their relative surface accessibility accessible. 
MPN324 - NrdE - Ribonucleoside-diphosphate reductase subunit alpha (T159 \& S412)

A

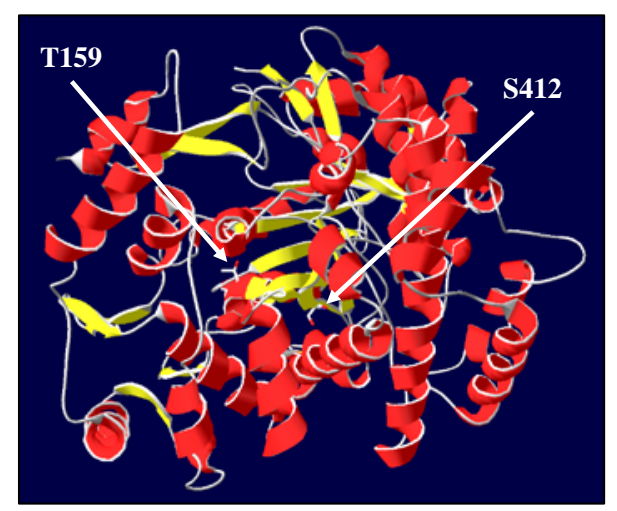

B

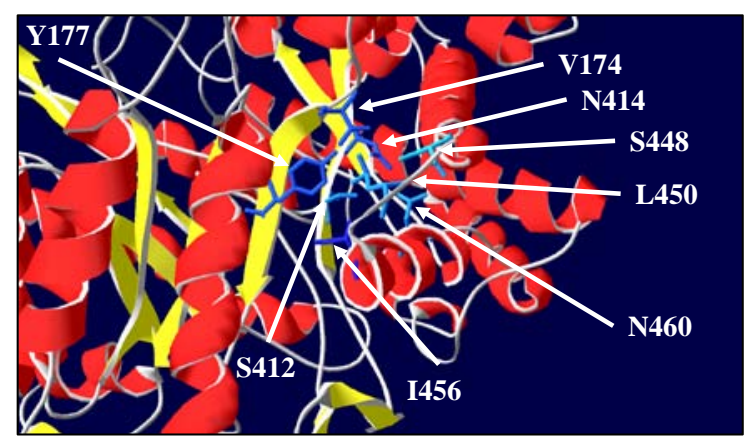

MPN393 - PdhA - Pyruvate dehydrogenase E1 component subunit alpha (S205)

A

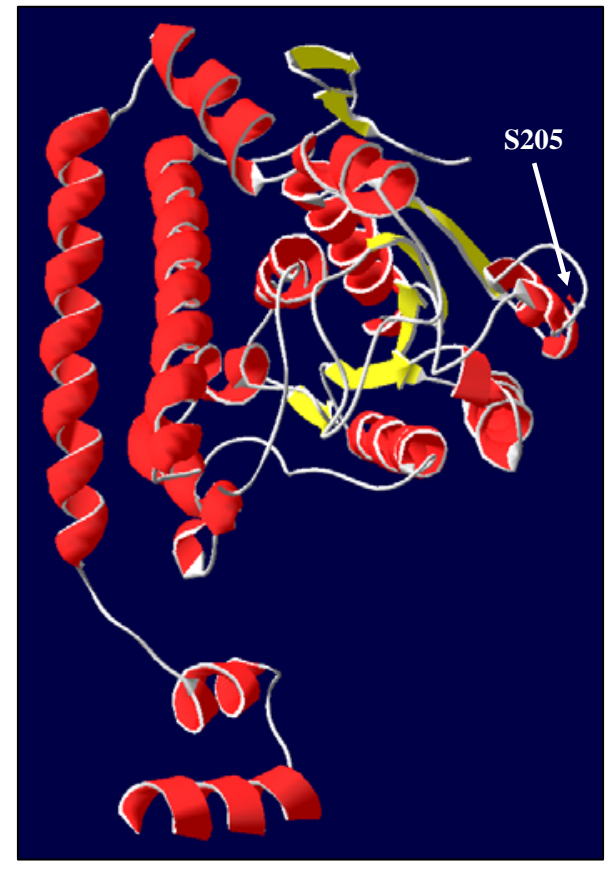

B

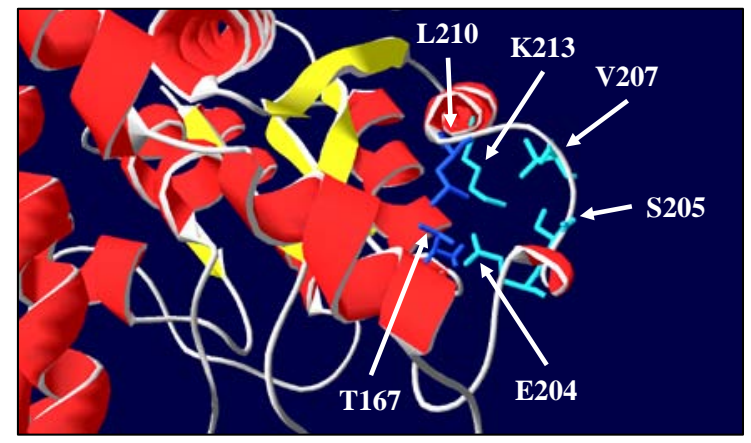

Fig. S2. Continued. 
MPN430 - GapA - Glyceraldehyde-3-phosphate dehydrogenase (S245)

A

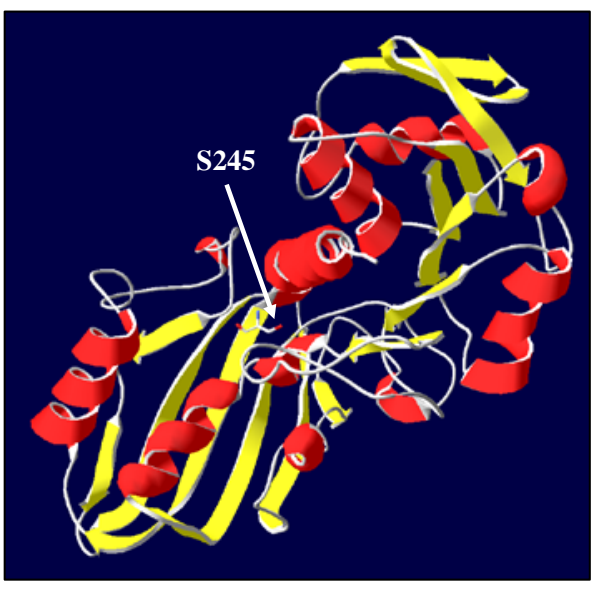

B

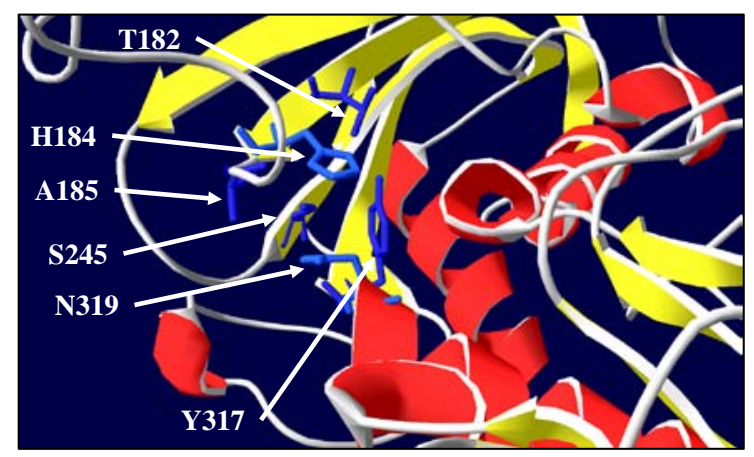

MPN470 - PepP - Xaa-Pro aminopeptidase (T317)

A

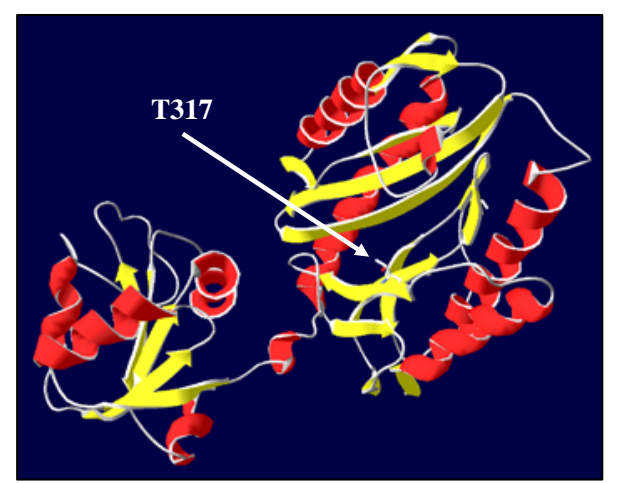

B

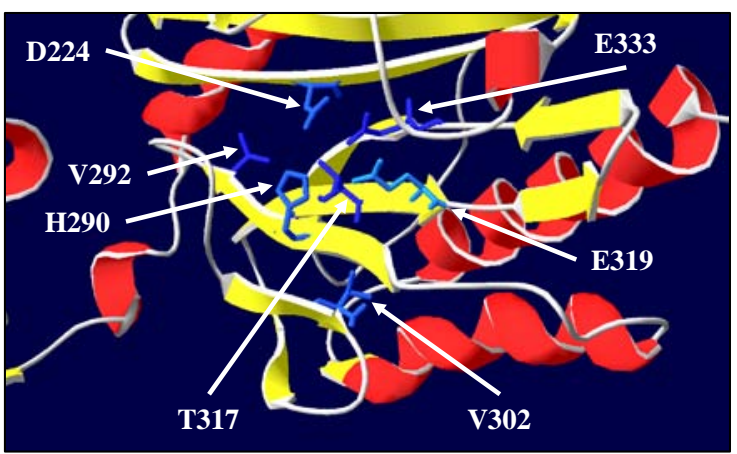

MPN572 - PepA - Cytosol aminopeptidase (S249 \& T420)

A

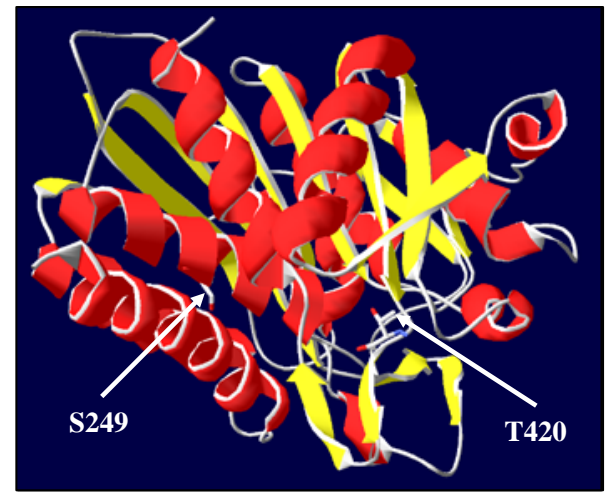

B

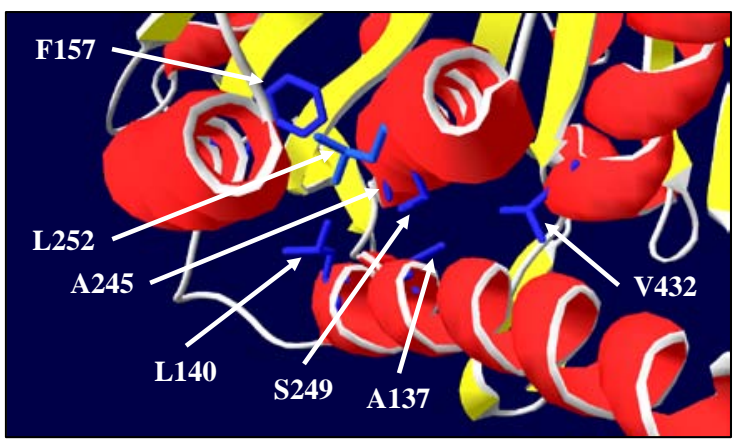

Fig. S2. Continued. 
MPN572 - PepA - Cytosol aminopeptidase (S249 \& T420)

A

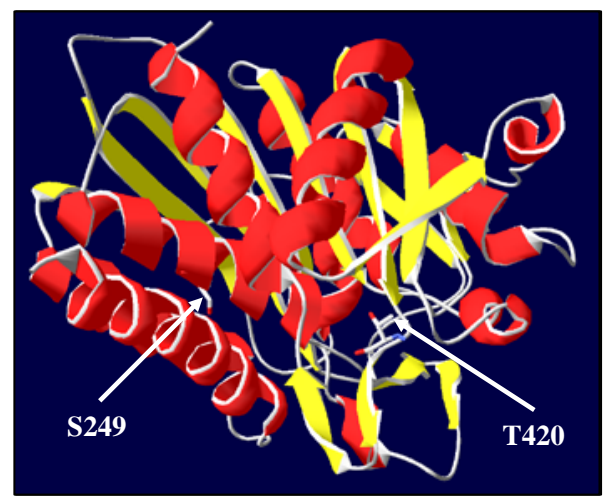

B

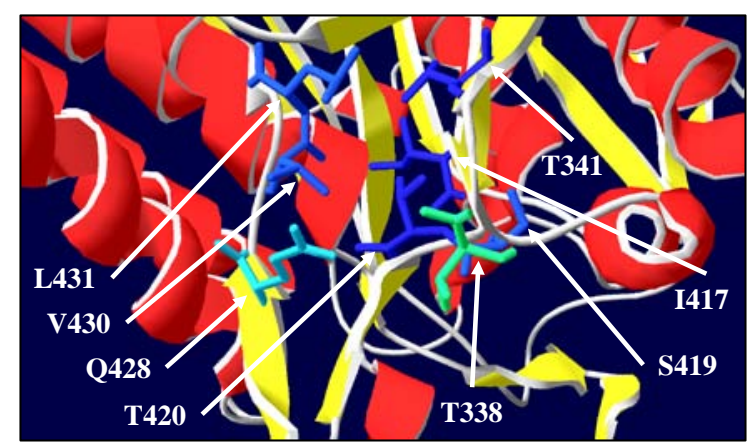

MPN665 - Tuf - Elongation factor TU (T383)

A

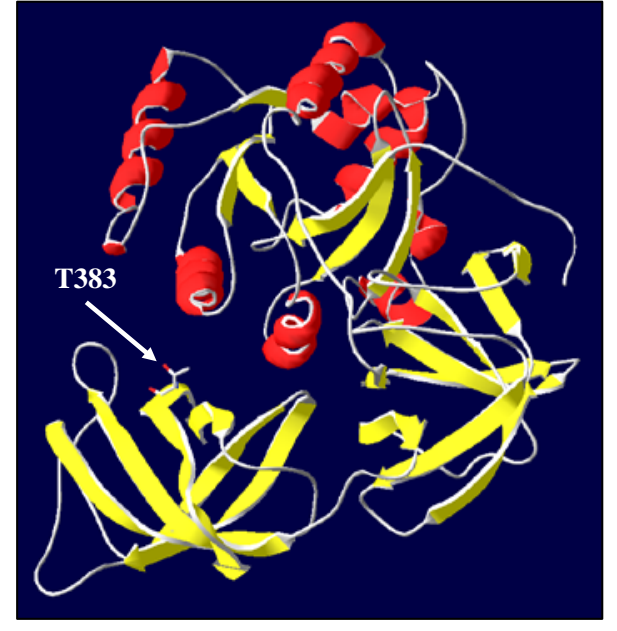

B

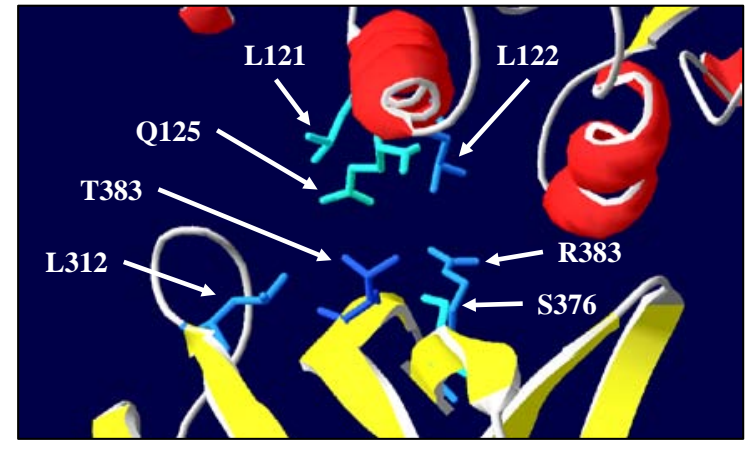

Fig. S2. Continued. 
MPN024 - RpoE - RNA polymerase subunit delta

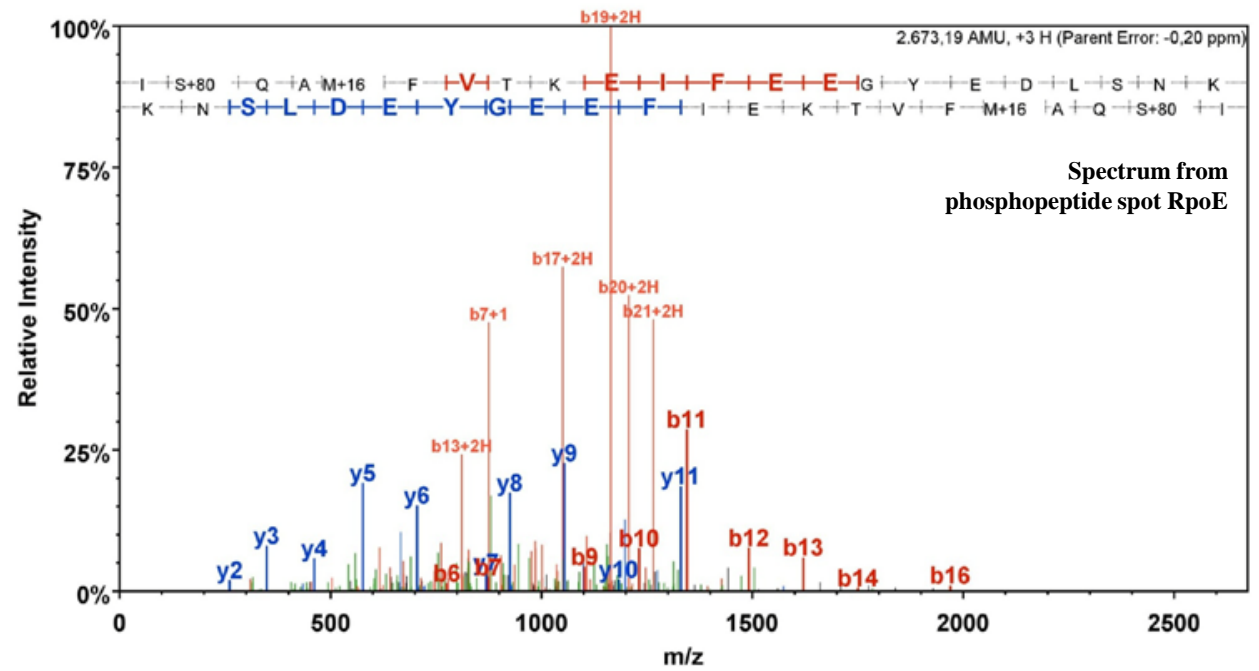

MPN024 - RpoE - RNA polymerase subunit delta

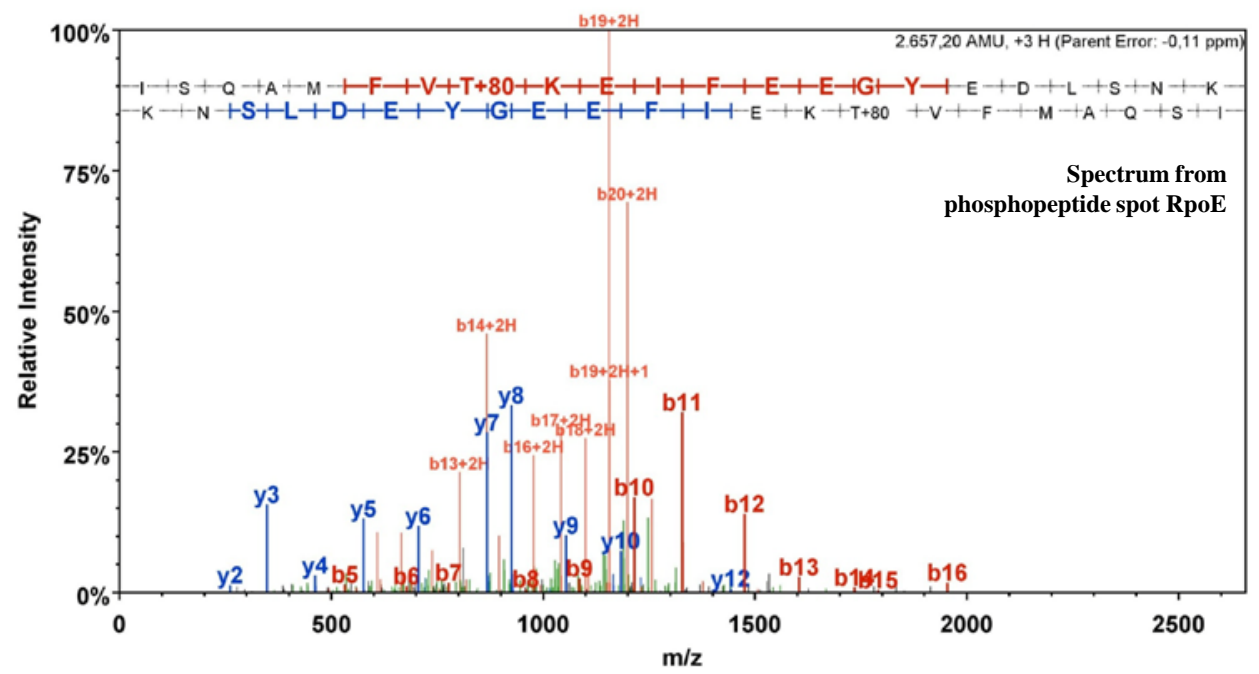

Fig. S3. Detailed MS/MS spectra of phosphorylated peptides. Peptides were measured online by ESI-mass spectrometry using a nanoACQUITY UPLC system coupled to an LTQ Orbitrap mass spectrometer (see "Experimental procedures"). Spectra are provided as screen shot with Scaffold annotated b- and y-ions series. 
MPN066 - ManB - Phosphomannomutase/phosphoglucomutase

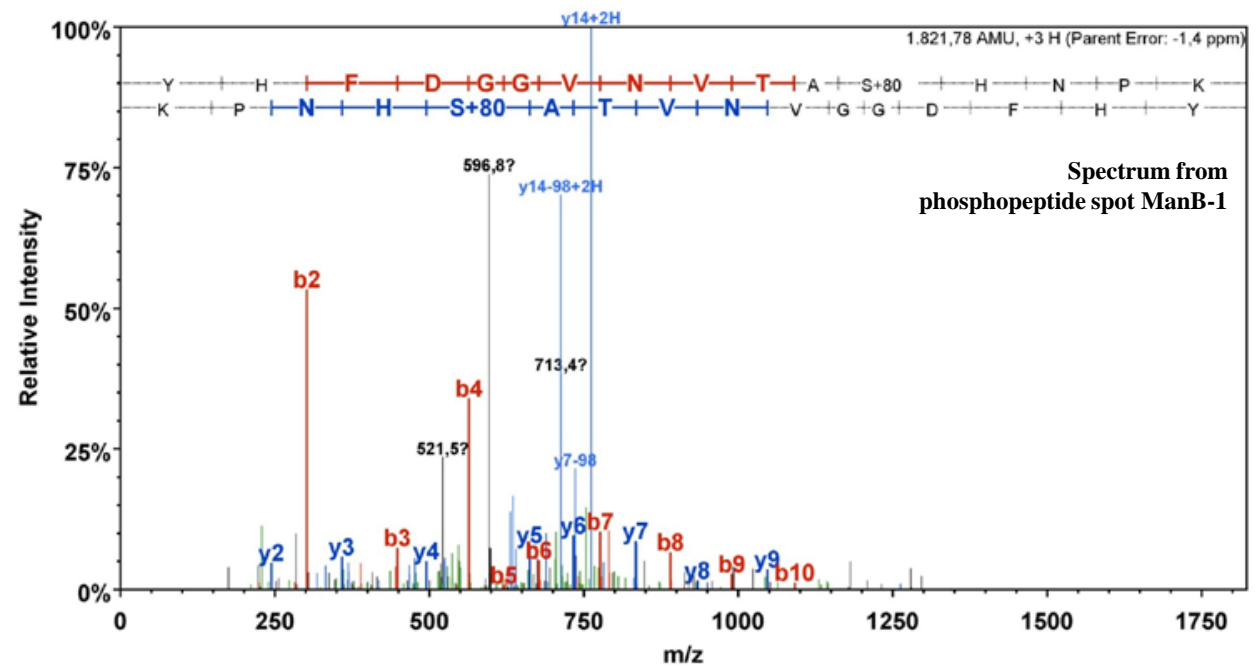

MPN066 - ManB - Phosphomannomutase/phosphoglucomutase

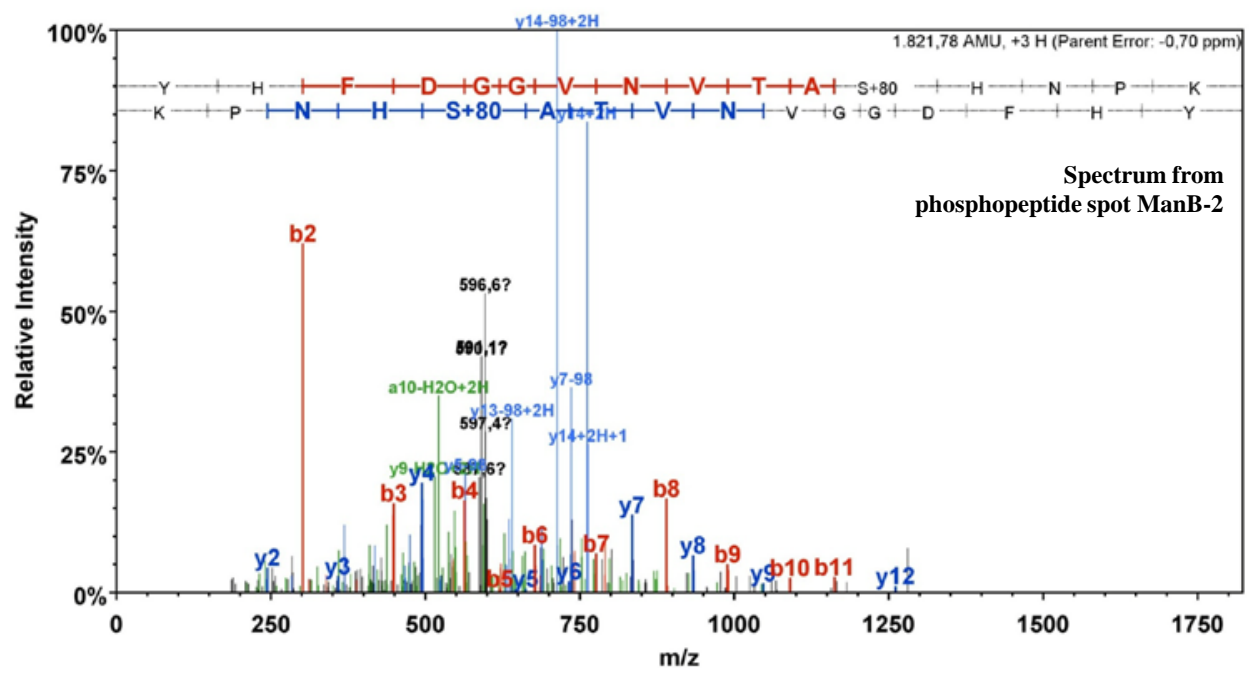

MPN066 - ManB - Phosphomannomutase/phosphoglucomutase

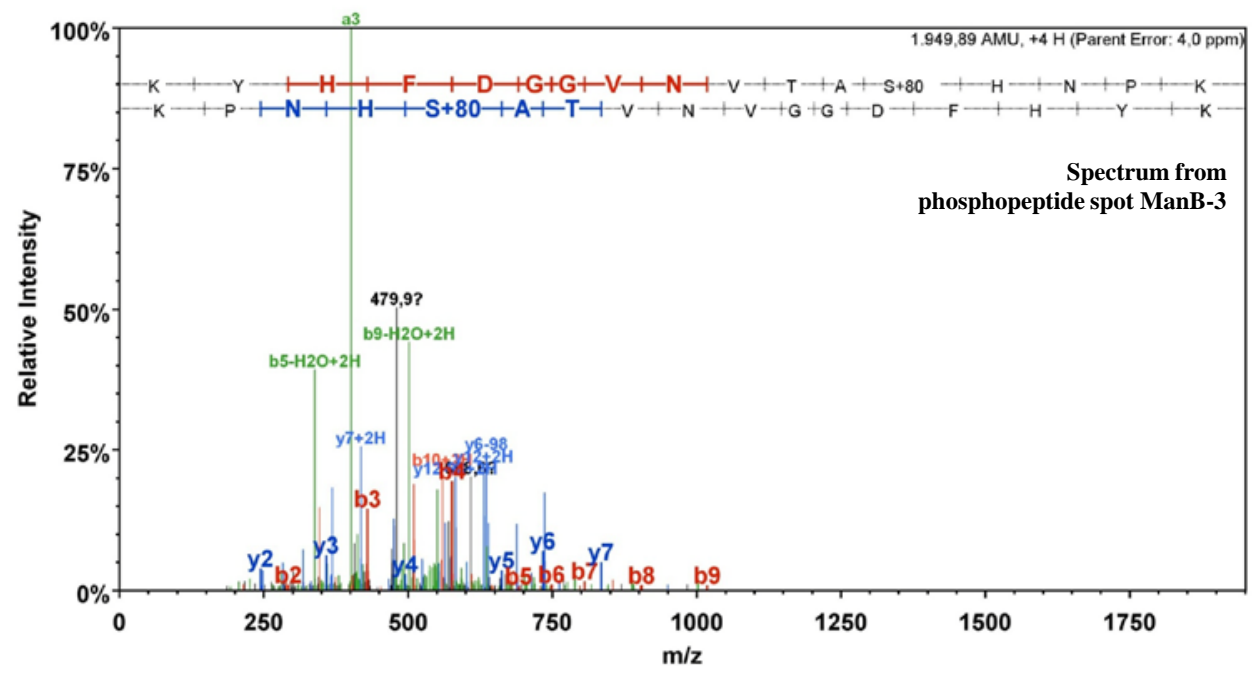

Fig. S3. Continued. 
MPN066 - ManB - Phosphomannomutase/phosphoglucomutase

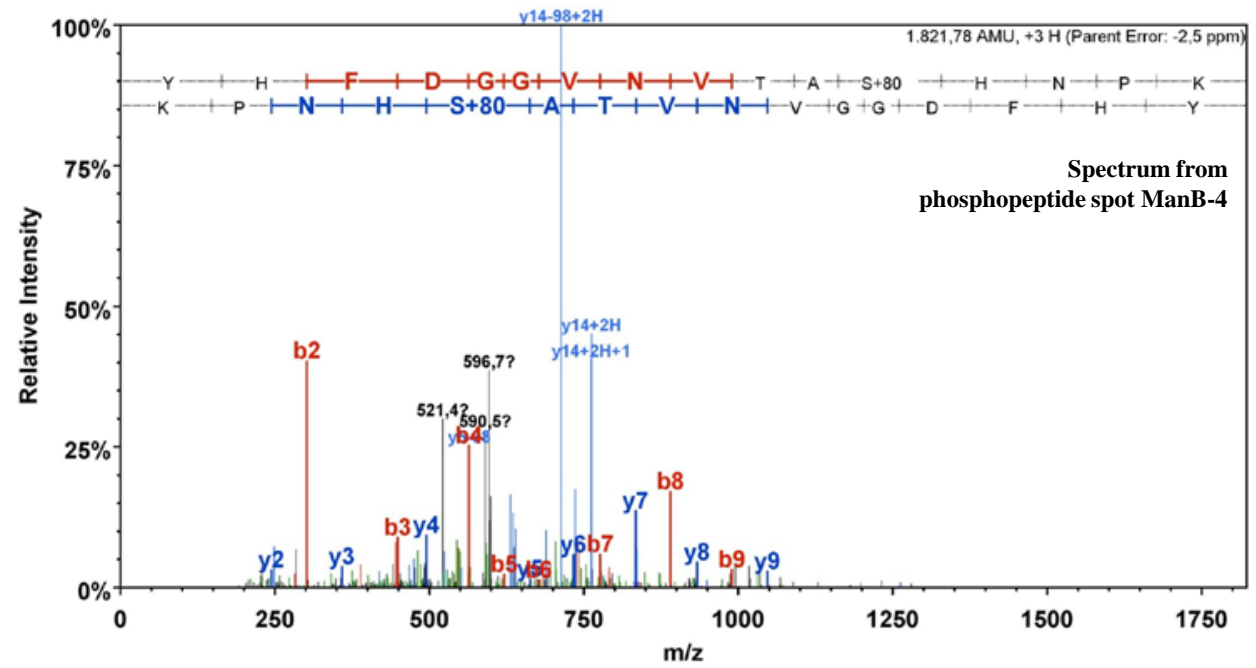

MPN066 - ManB - Phosphomannomutase/phosphoglucomutase

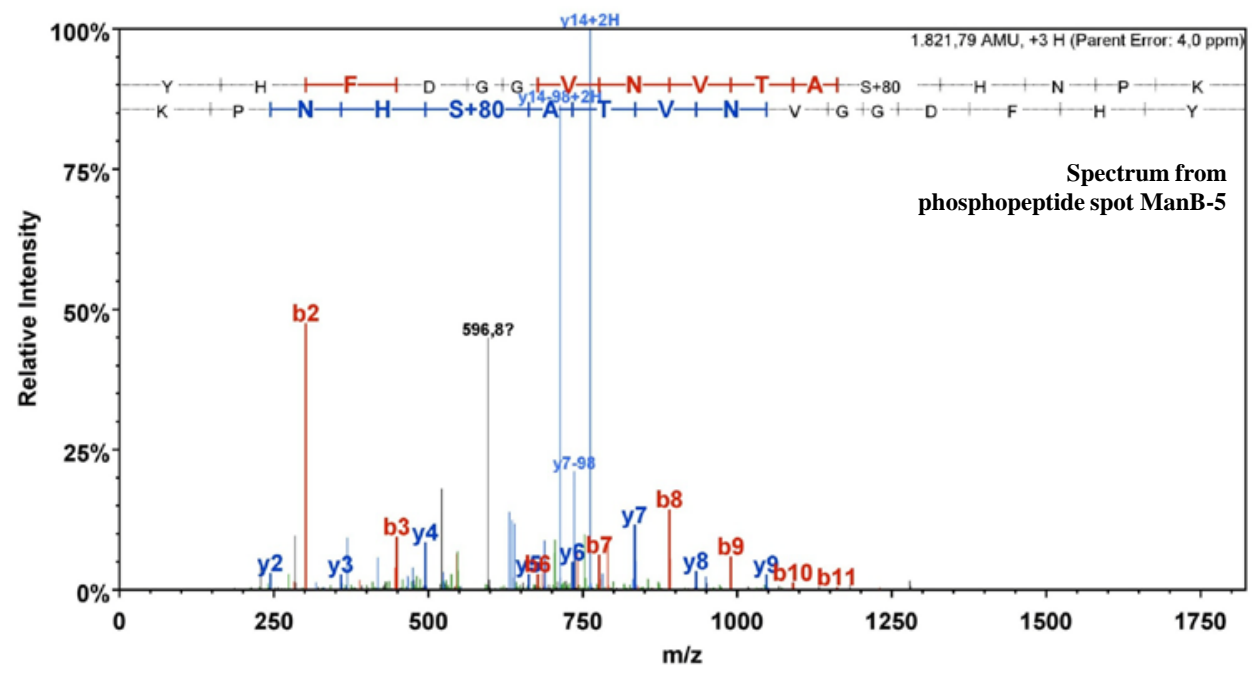

MPN066 - ManB - Phosphomannomutase/phosphoglucomutase

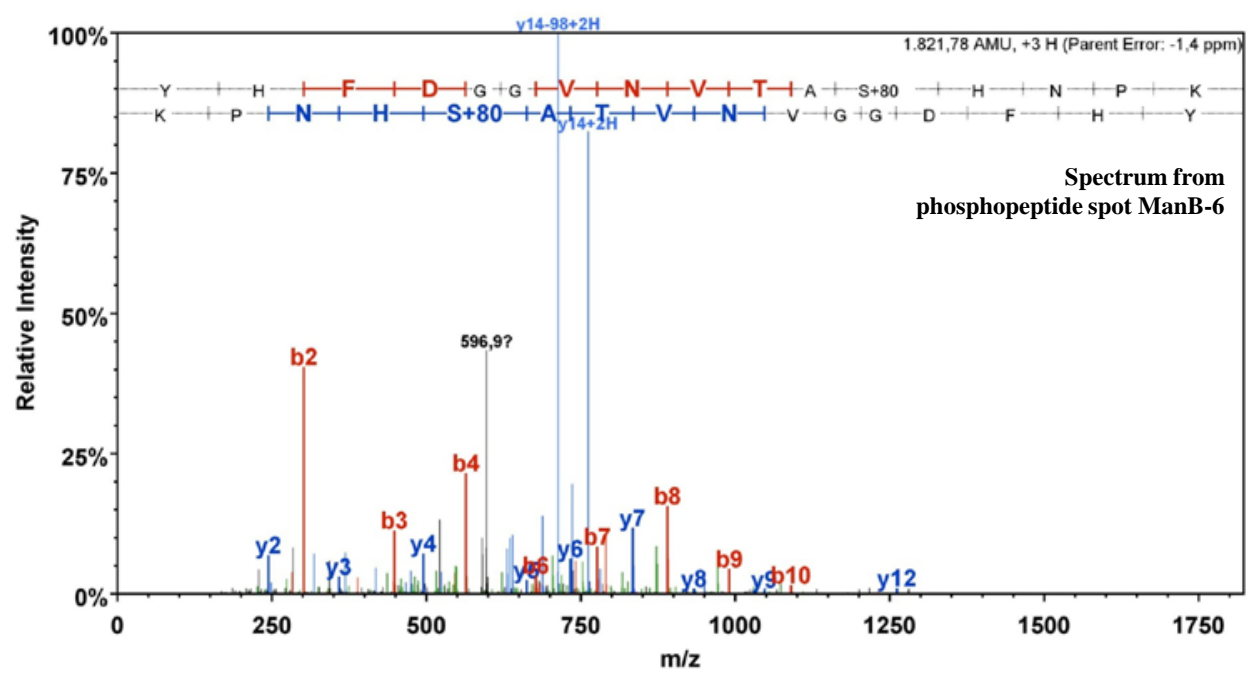

Fig. S3. Continued. 
MPN066 - ManB - Phosphomannomutase/phosphoglucomutase

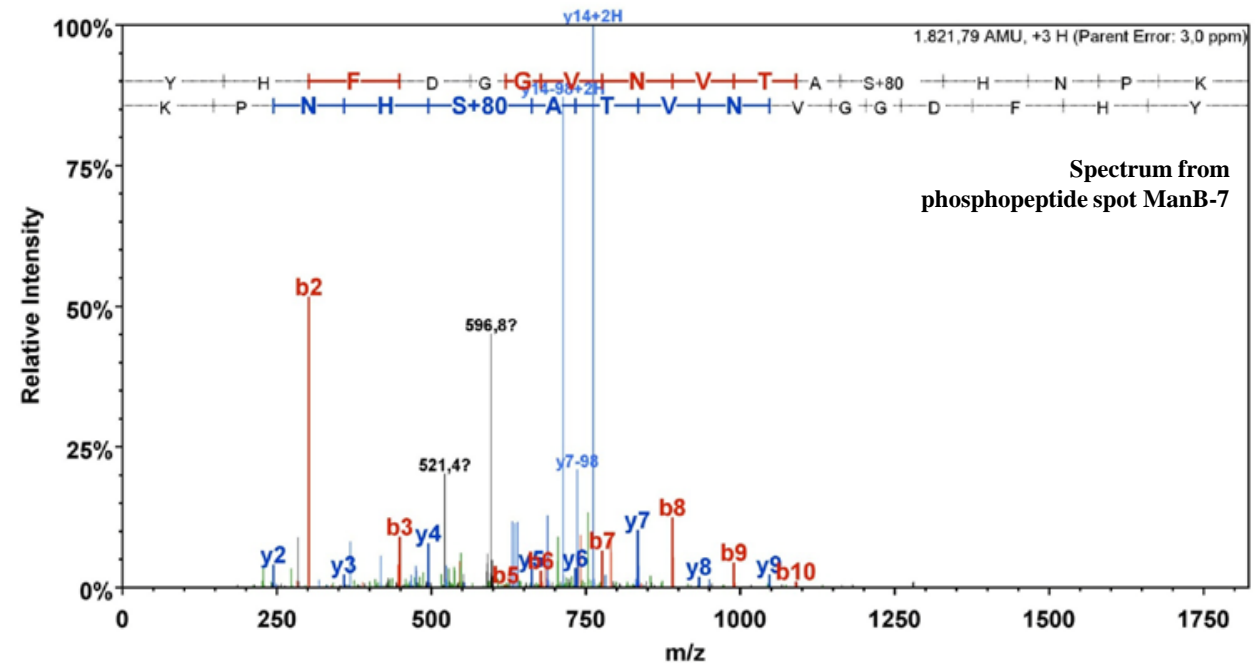

MPN066 - ManB - Phosphomannomutase/phosphoglucomutase

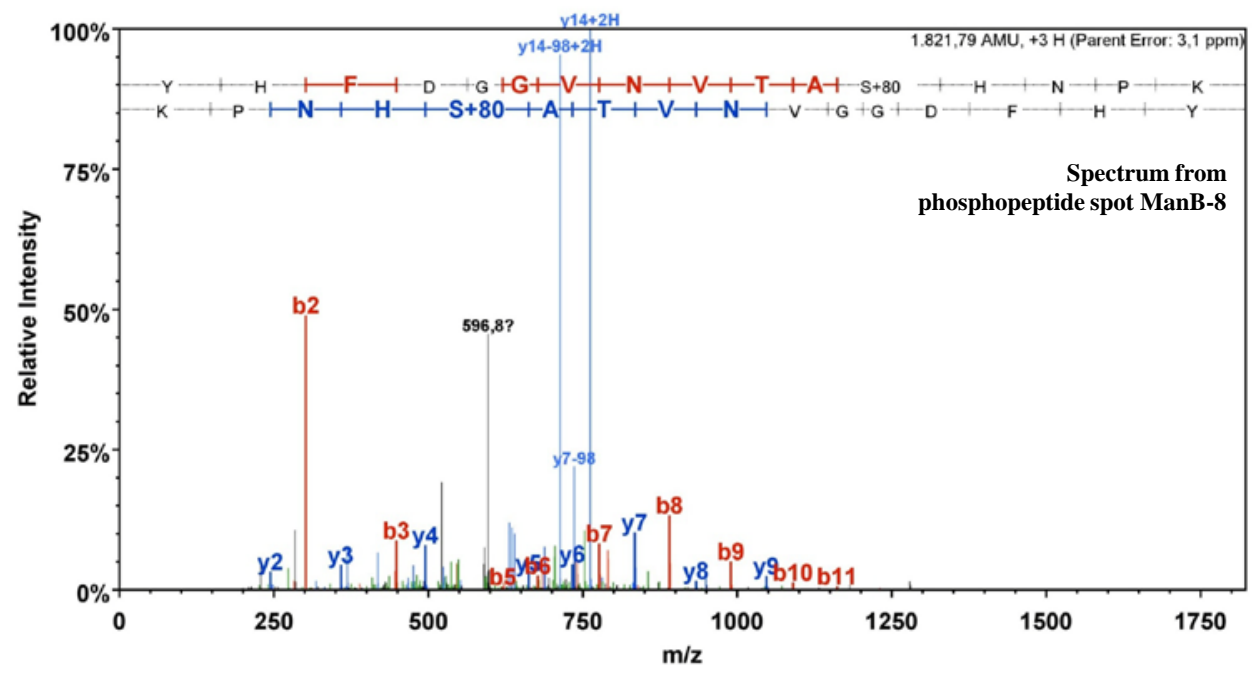

MPN066 - ManB - Phosphomannomutase/phosphoglucomutase

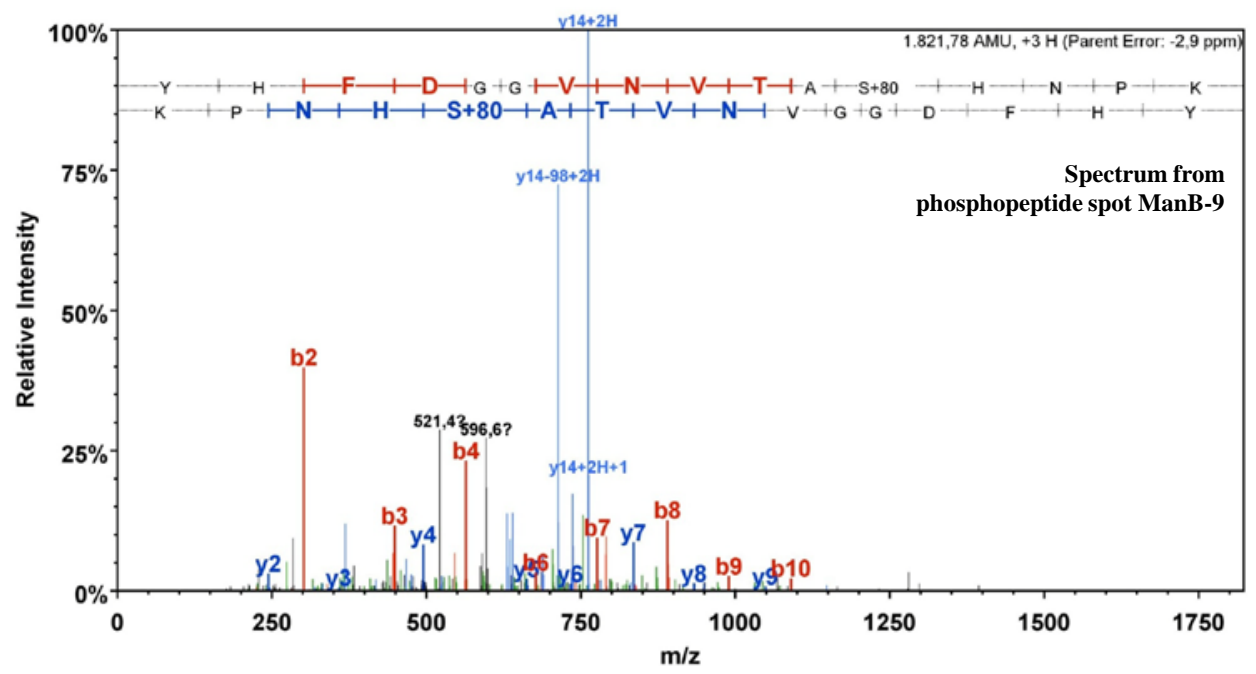

Fig. S3. Continued. 
MPN066 - ManB - Phosphomannomutase/phosphoglucomutase

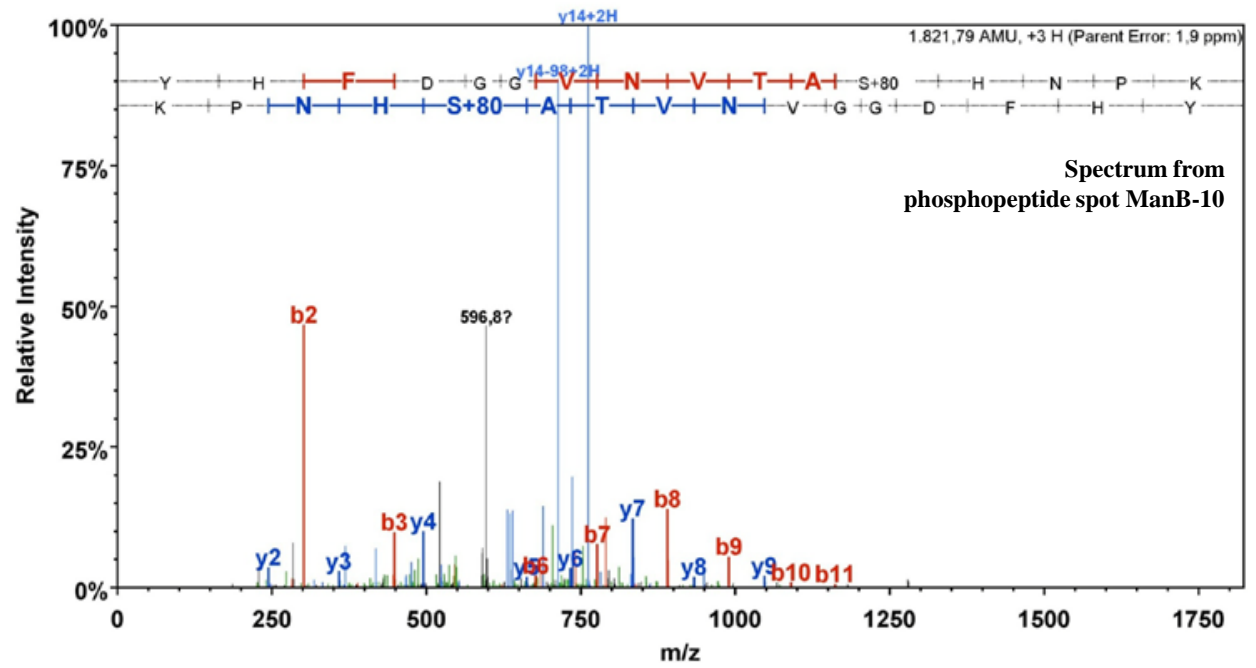

MPN154 - NusA - Transcription elongation factor

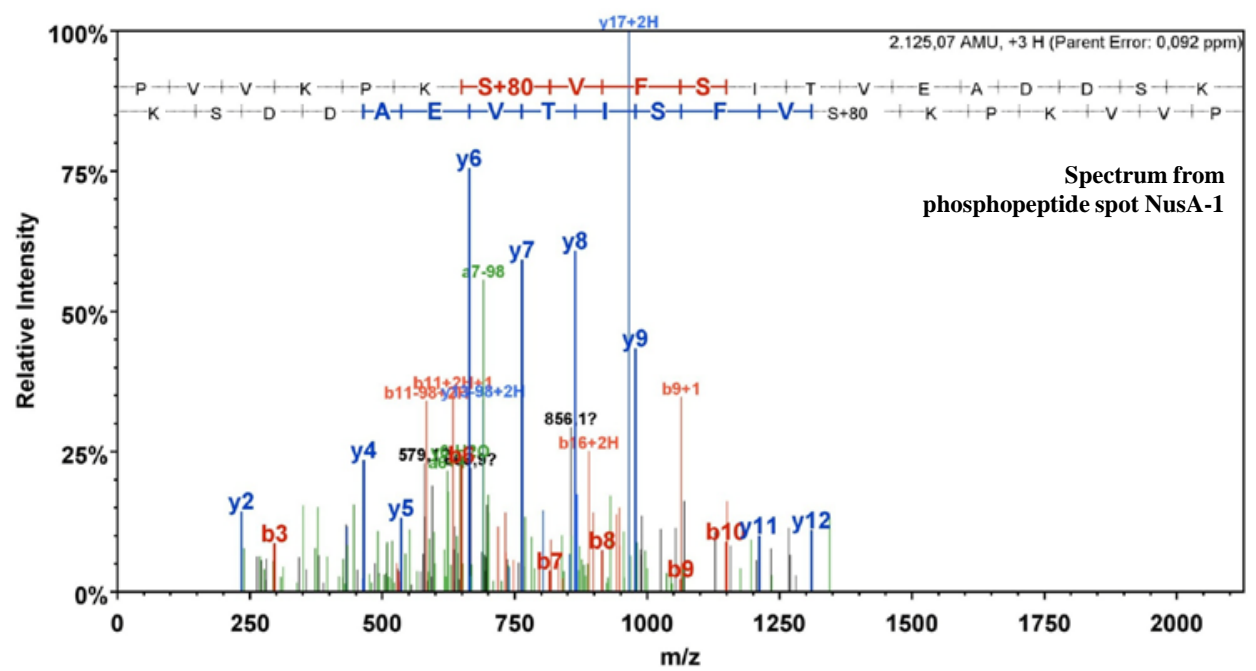

MPN256 - Uncharacterized protein

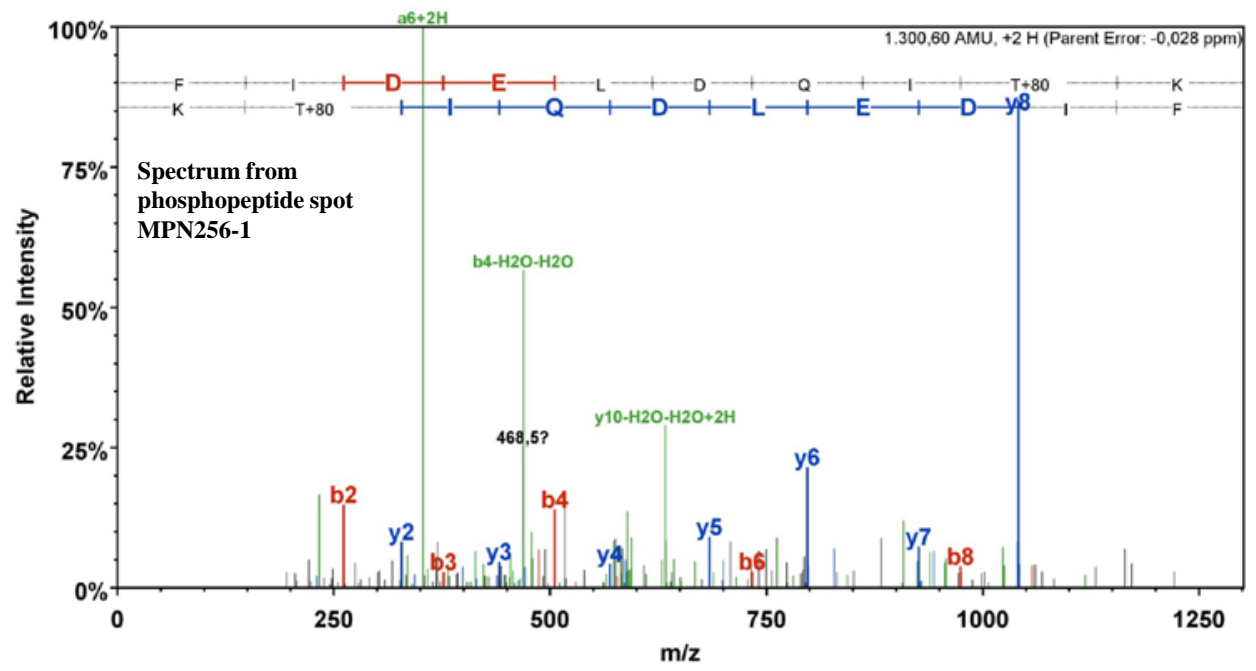

Fig. S3. Continued. 
MPN261 - TopA - DNA topoisomerase I

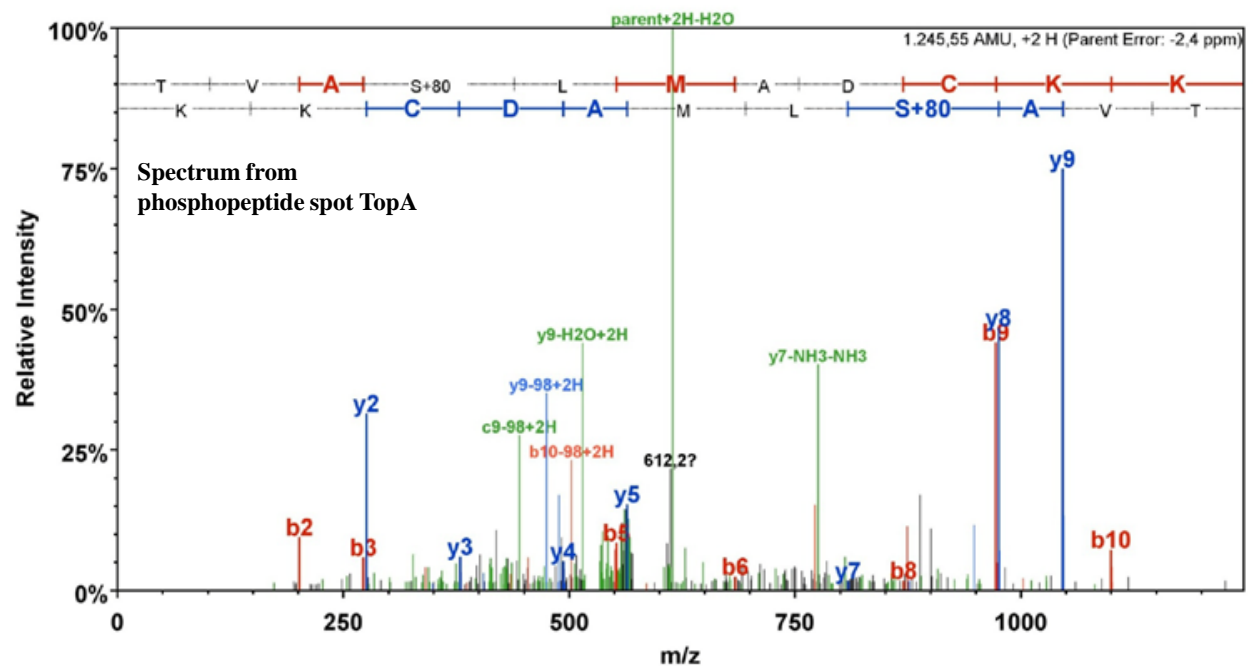

MPN295 - Uncharacterized protein

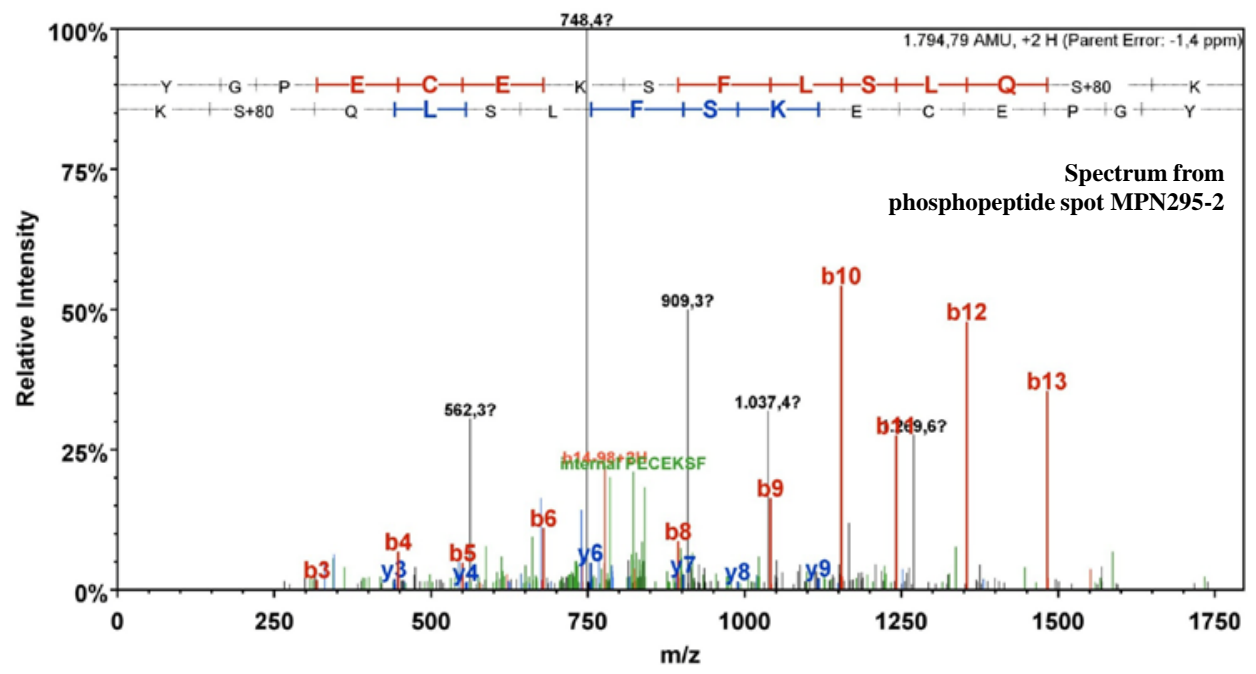

MPN311 - P41 - Adhesin-related protein

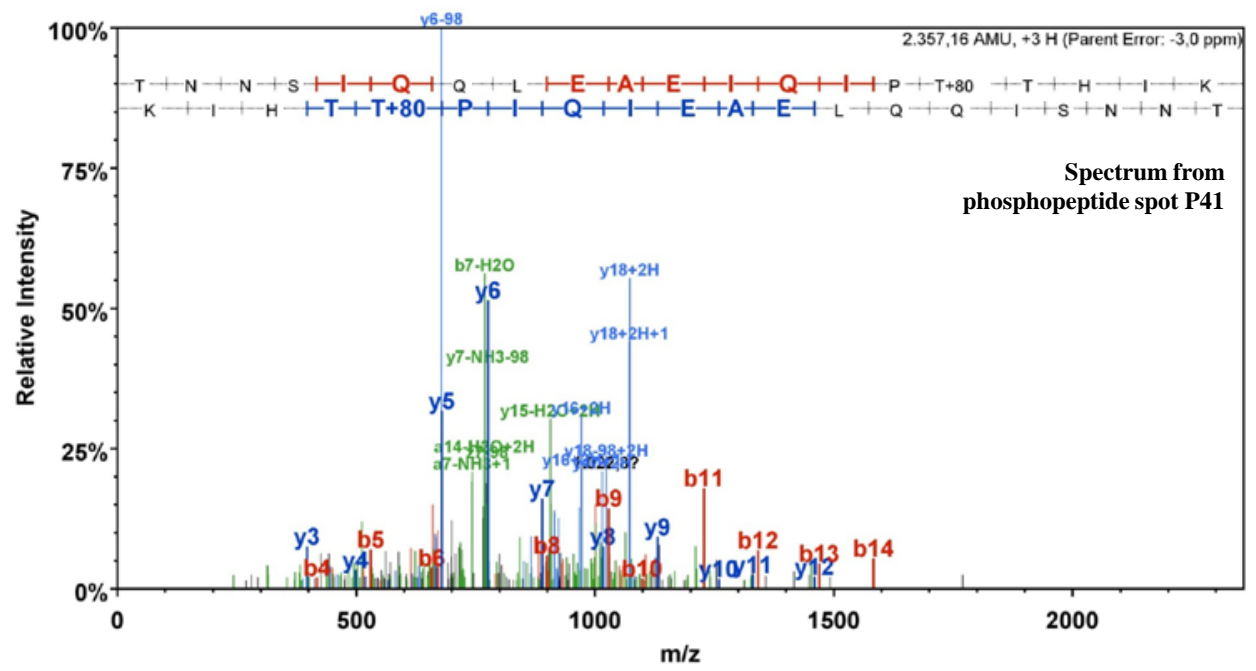

Fig. S3. Continued. 
MPN324 - NrdE - Ribonucleoside-diphosphate reductase subunit alpha

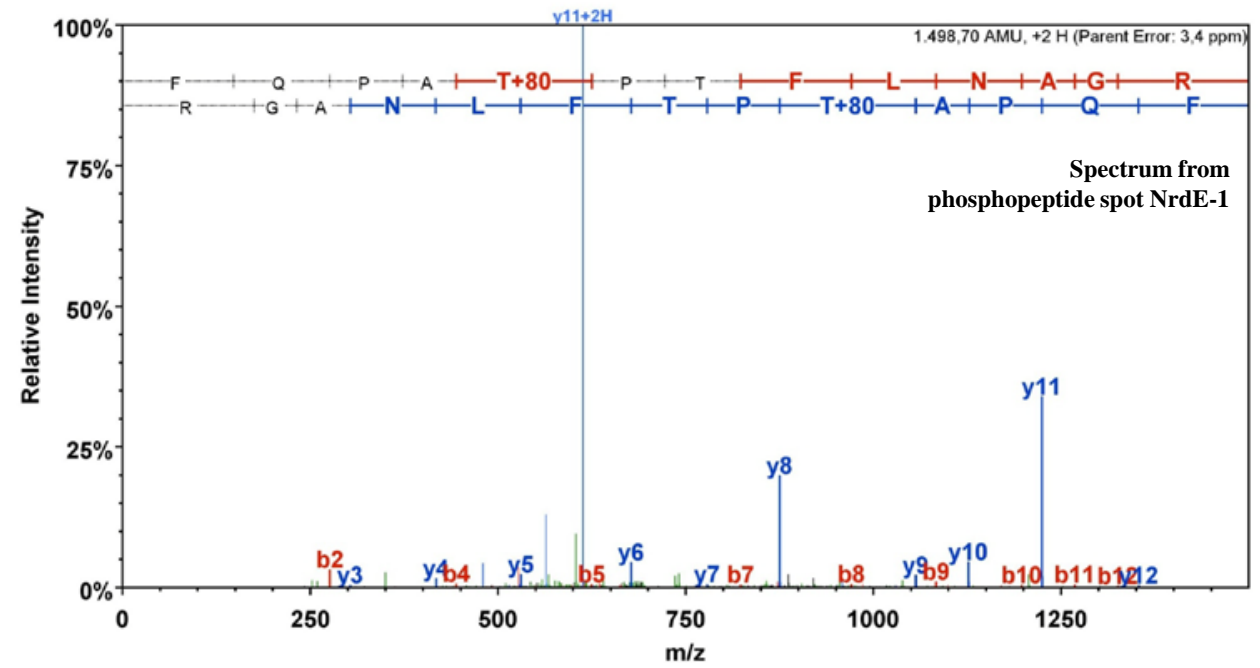

MPN324 - NrdE - Ribonucleoside-diphosphate reductase subunit alpha

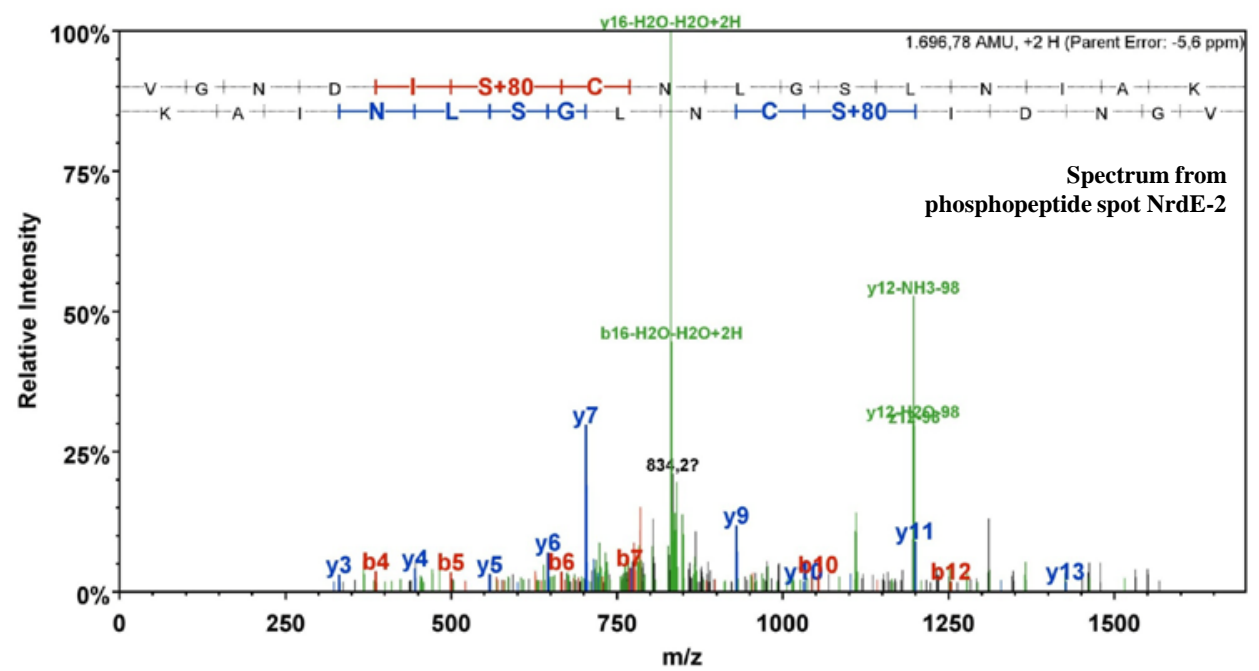

MPN393 - PdhA - Pyruvate dehydrogenase E1 component subunit alpha

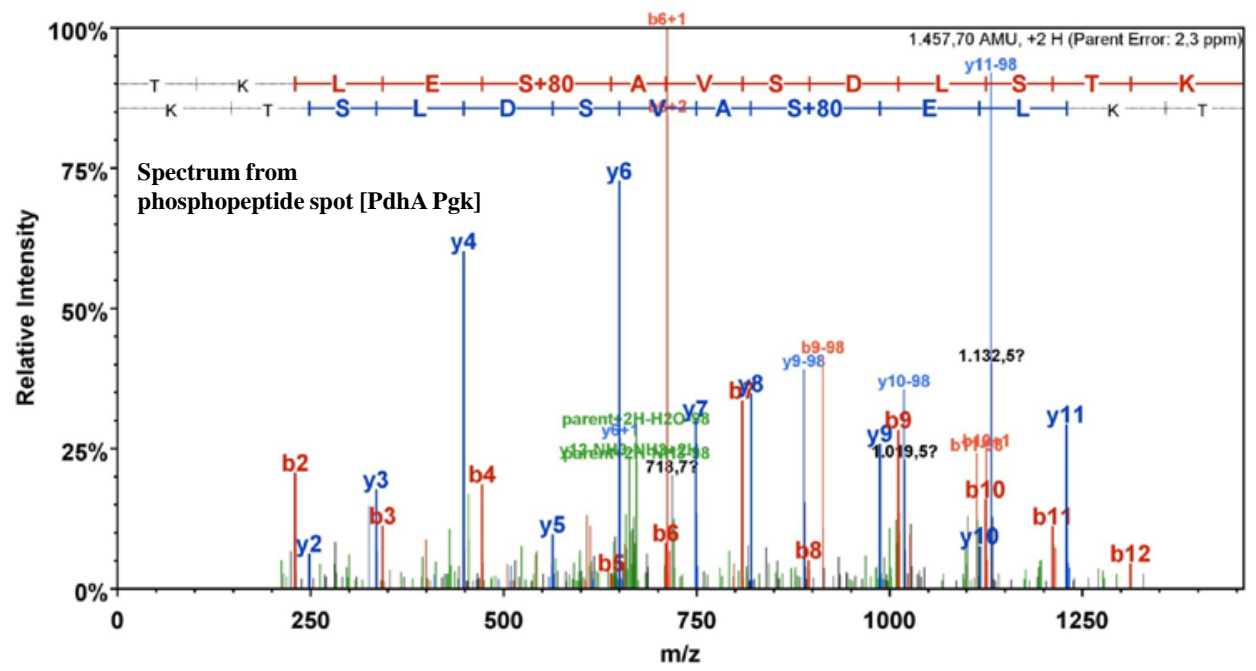

Fig. S3. Continued. 
MPN430 - GapA - Glyceraldehyde-3-phosphate dehydrogenase

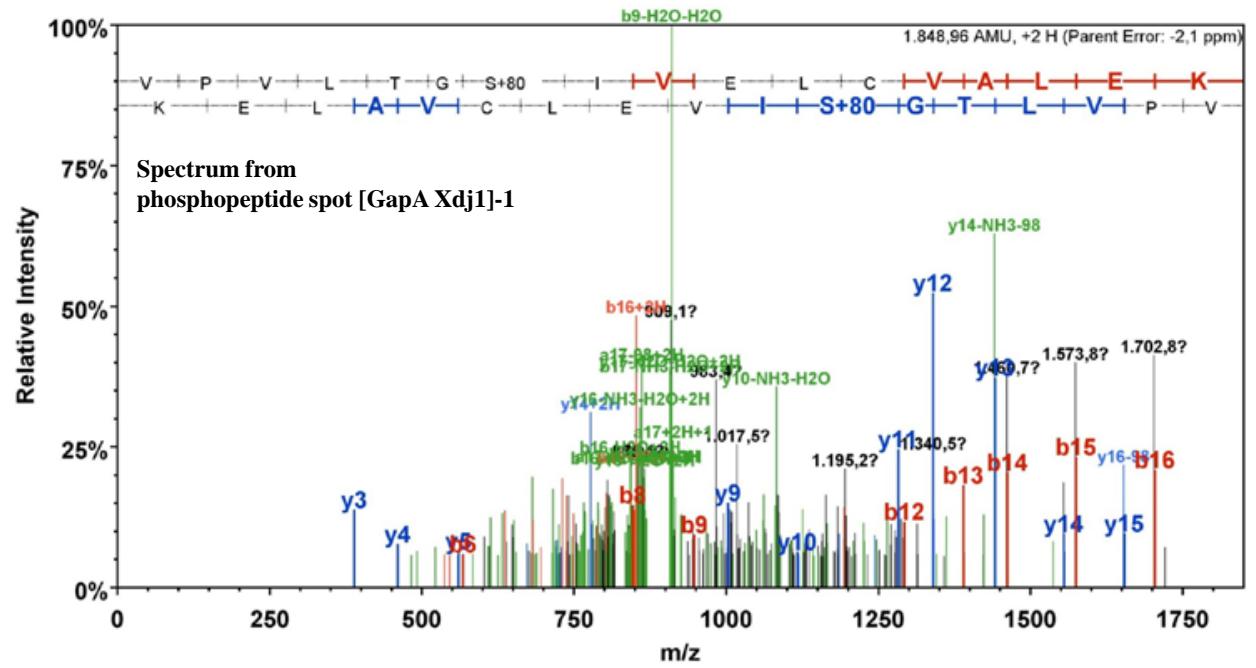

MPN430 - GapA - Glyceraldehyde-3-phosphate dehydrogenase

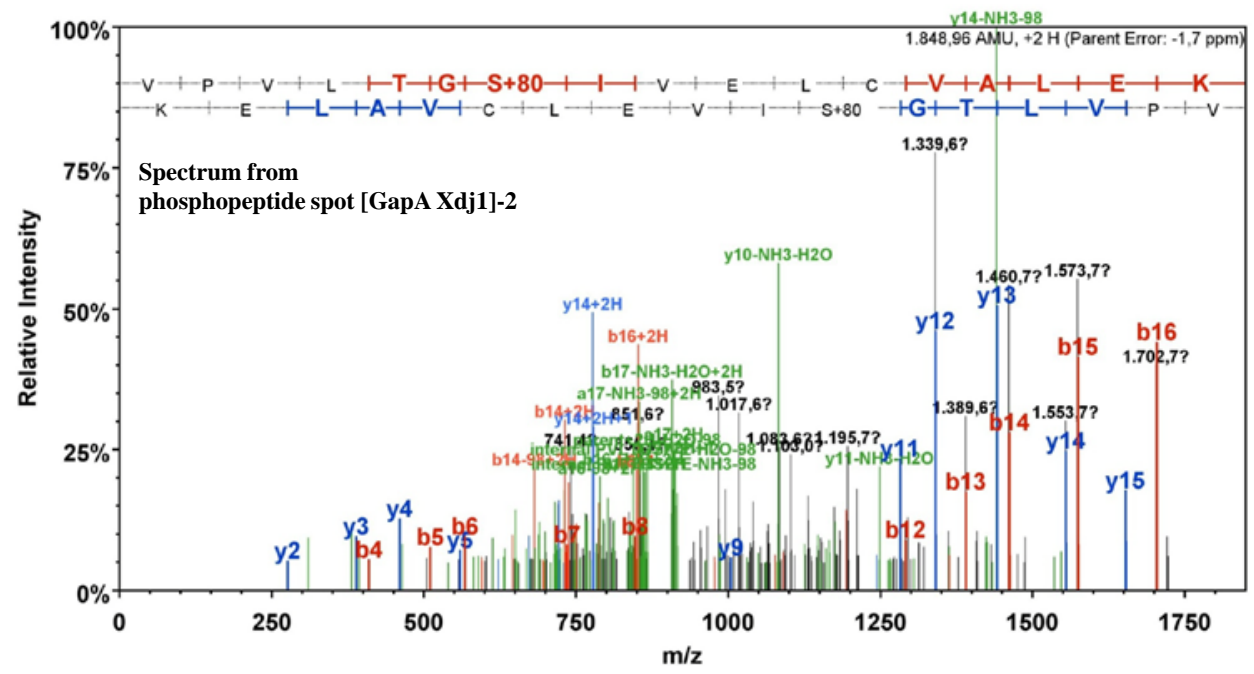

MPN470 - PepP - Xaa-Pro aminopeptidase

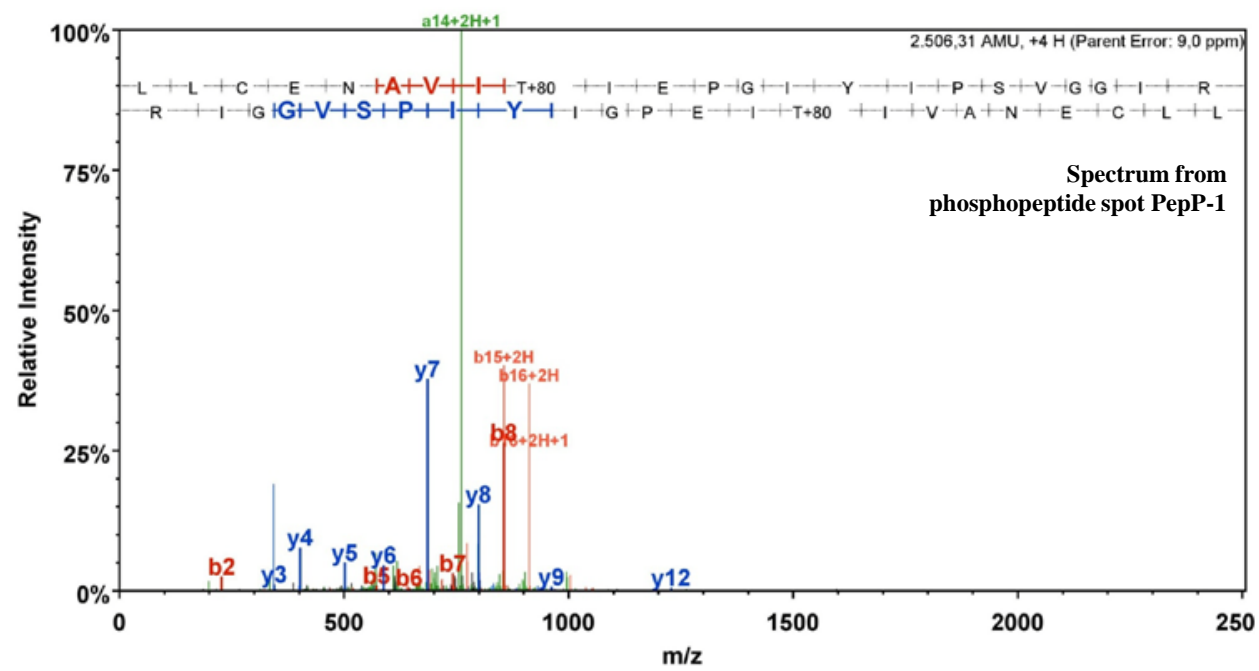

Fig. S3. Continued. 
MPN474 - Coiled coil surface protein

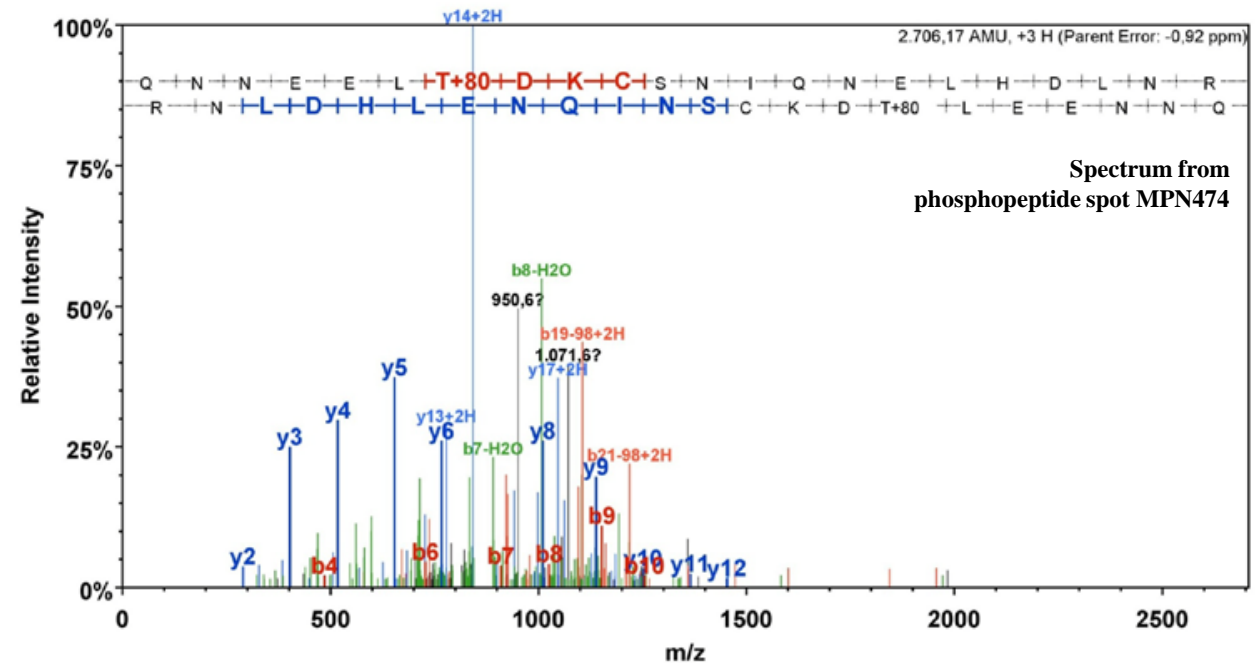

MPN572 - PepA - Cytosol aminopeptidase

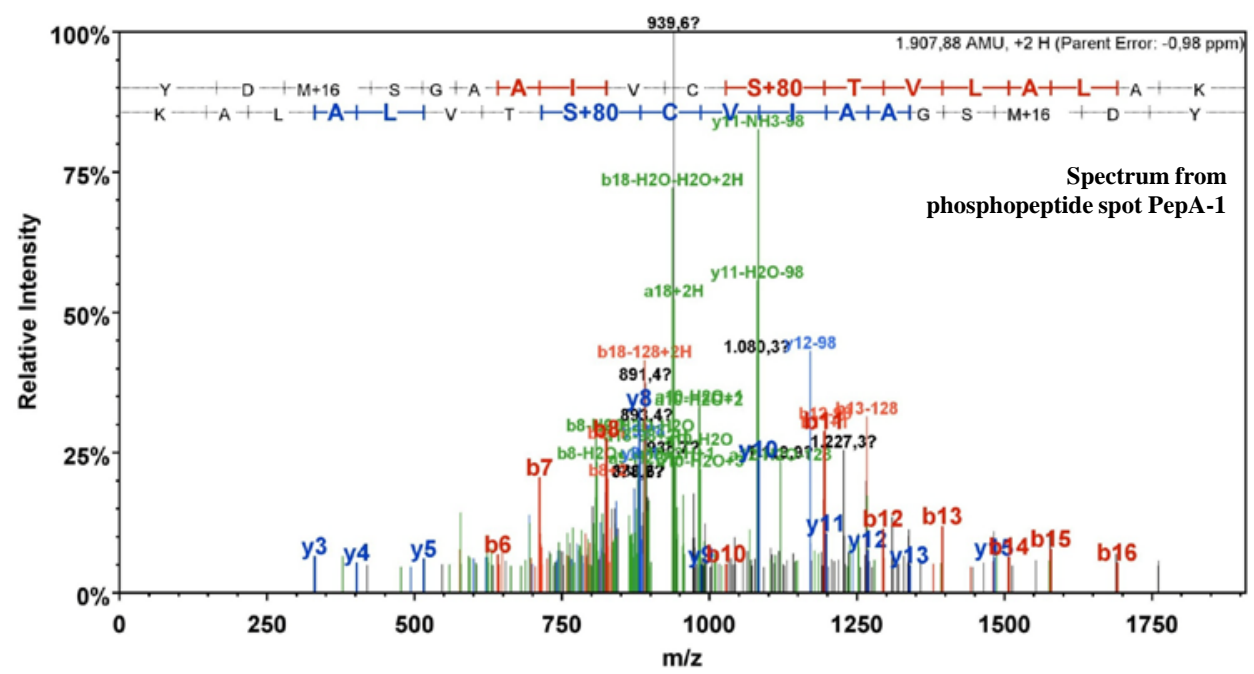

MPN572 - PepA - Cytosol aminopeptidase

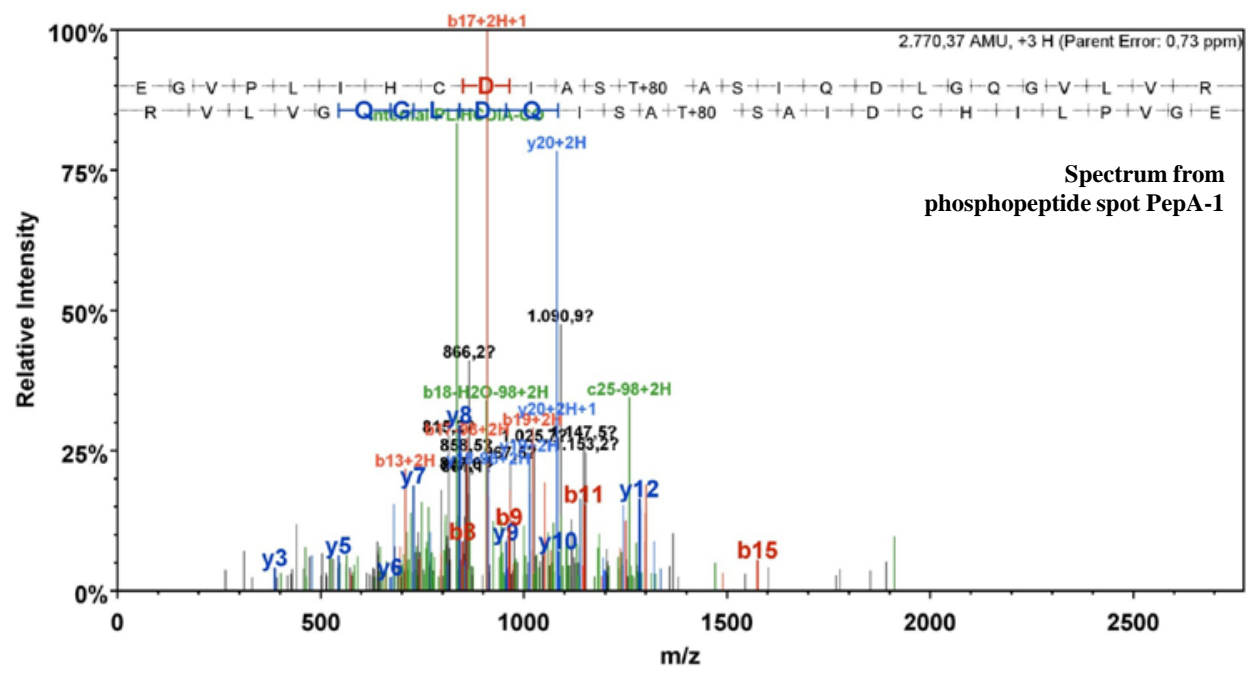

Fig. S3. Continued. 
MPN665 - Tuf - Elongation factor TU

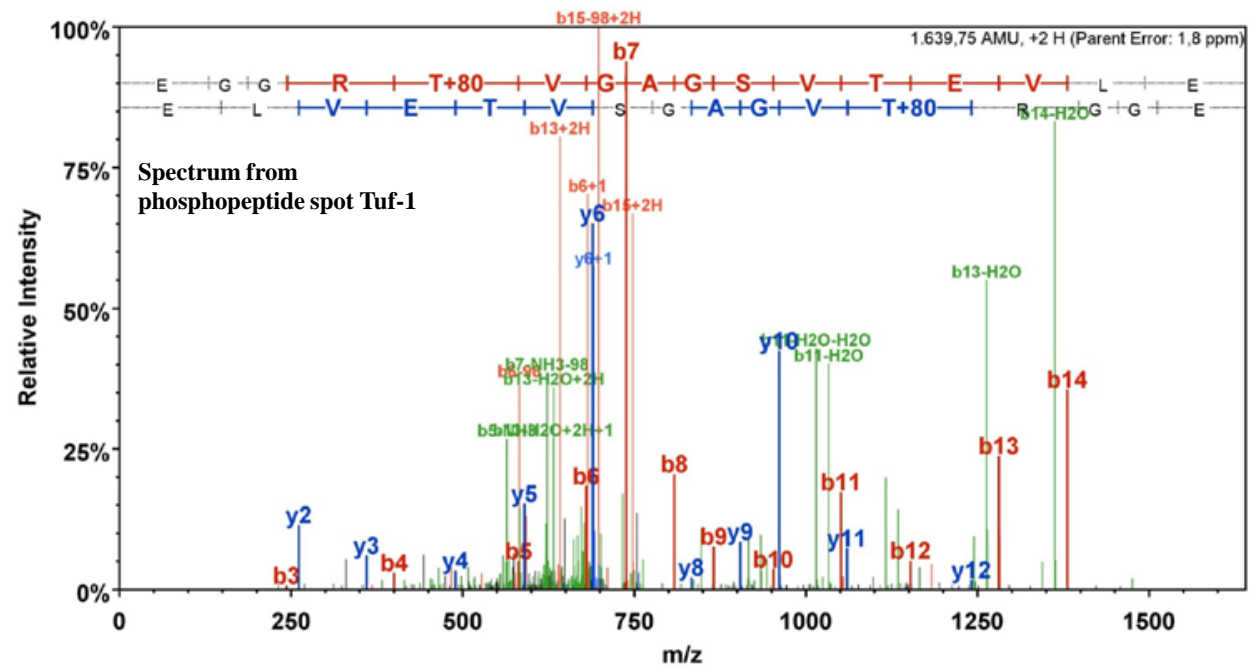

MPN665 - Tuf - Elongation factor TU

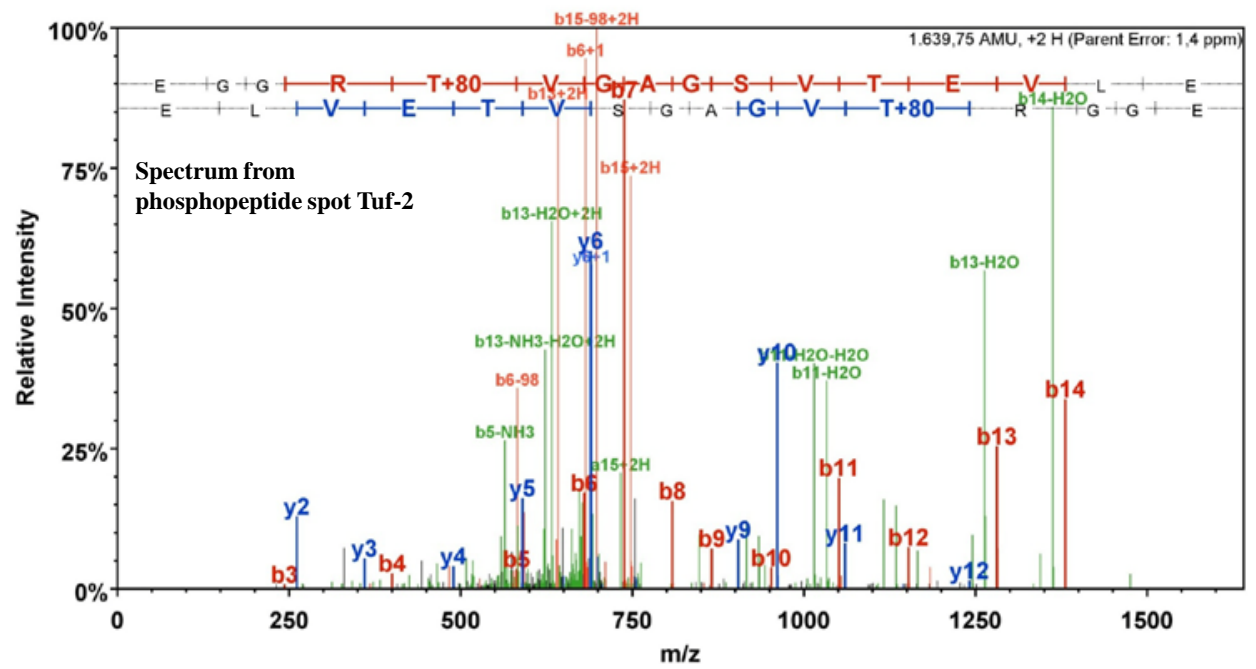

MPN665 - Tuf - Elongation factor TU

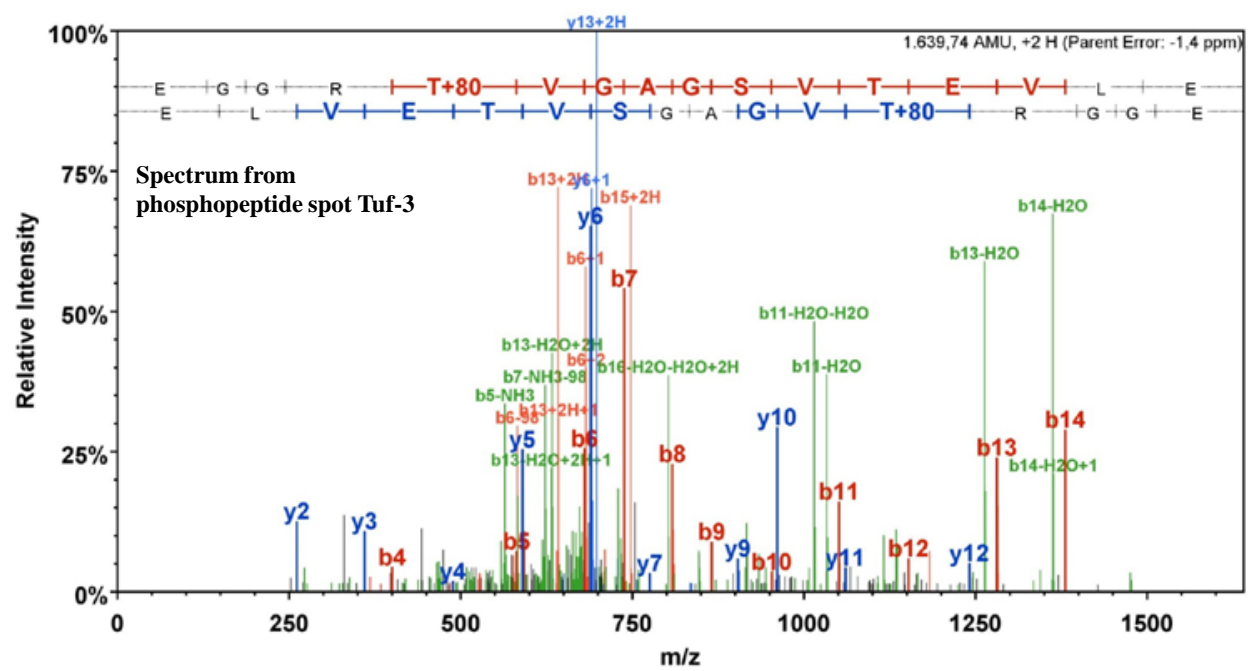

Fig. S3. Continued. 
MPN665 - Tuf - Elongation factor TU

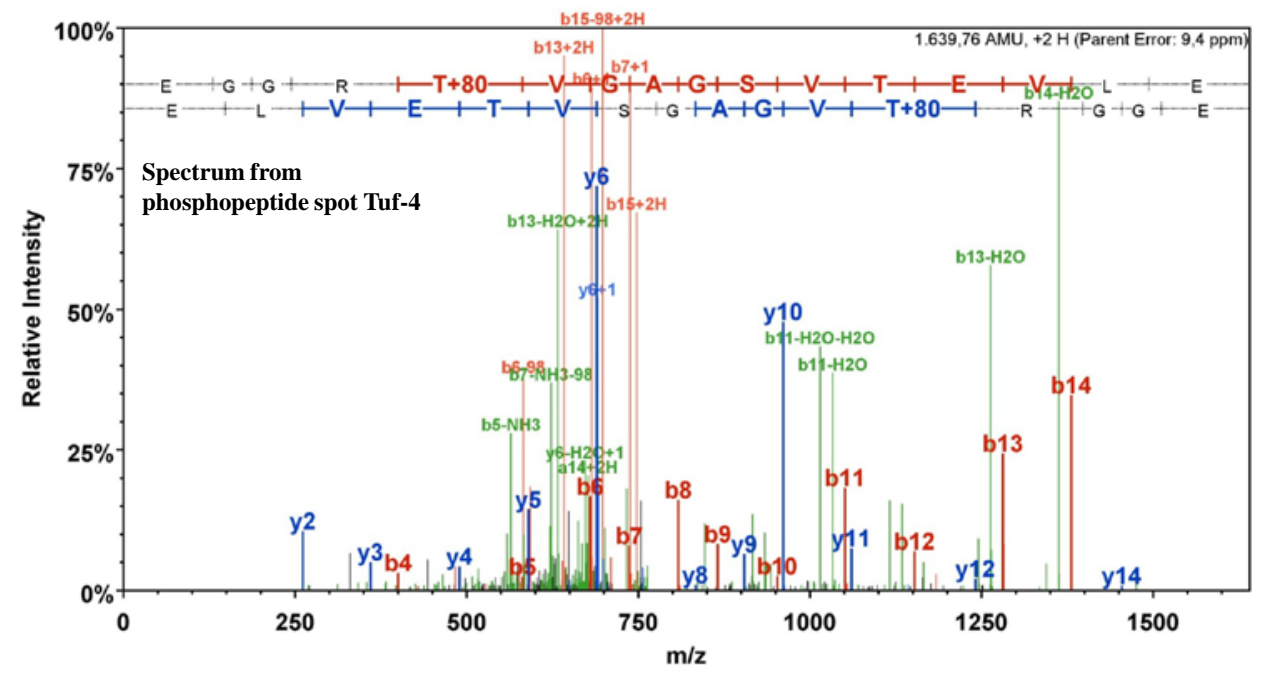

Fig. S3. Continued. 


\section{Chapter 4}

\section{In vitro phosphorylation of key metabolic enzymes from Bacillus subtilis: \\ PrkC phosphorylates enzymes from different branches of basic metabolism}

The work described in this chapter was published in:

Pietack, N., D. Becher, S. R. Schmidl, M. H. Saier, M. Hecker, F. M. Commichau, and J. Stülke. 2010. In vitro phosphorylation of key metabolic enzymes from Bacillus subtilis: PrkC phosphorylates enzymes from different branches of basic metabolism. J. Mol. Microbiol. Biotechnol. 18: 129-140.

Author contributions:

This study was designed and interpreted by NP, FMC, and JS. NP performed all in vitro phosphorylation experiments. SRS performed the phylogenetic analysis of PrkC homologues during an internship at the lab of MHS, University of California, San Diego. Determination of phosphorylation sites was done in collaboration with DB and MH, University of Greifswald. NP and JS wrote the paper. 


\section{Abstract}

Phosphorylation is an important mechanism of protein modification. In the Gram-positive soil bacterium Bacillus subtilis, about 5\% of all proteins are subject to phosphorylation, and a significant portion of these proteins is phosphorylated on serine or threonine residues. We were interested in the regulation of the basic metabolism in $B$. subtilis. Many enzymes of the central metabolic pathways are phosphorylated in this organism. In an attempt to identify the responsible protein kinase(s), we identified four candidate kinases, among them the previously studied kinase PrkC. We observed that PrkC is indeed able to phosphorylate several metabolic enzymes in vitro. Determination of the phosphorylation sites revealed a remarkable preference of PrkC for threonine residues. Moreover, PrkC often used several phosphorylation sites in one protein. This feature of PrkC-dependent protein phosphorylation resembles the multiple phosphorylations often observed in eukaryotic proteins. The HPr protein of the phosphotransferase system is one of the proteins phosphorylated by PrkC, and PrkC phosphorylates a site (Ser-12) that has recently been found to be phosphorylated in vivo. The agreement between in vivo and in vitro phosphorylation of HPr on Ser-12 suggests that our in vitro observations reflect the events that take place in the cell.

\section{Introduction}

Adaptation to changing environmental conditions is essential for life. The Gram-positive soil bacterium Bacillus subtilis is permanently exposed to changing conditions, such as nutrient limitations or various physical stresses. In principle, there are three different mechanisms by which bacteria can adapt their metabolism to the environment: (1) alterations of gene expression, (2) changing the stabilities of proteins, and (3) modulation of enzymatic activities. Regulation of gene expression is by far the most intensively studied regulatory mechanism. There is a huge collection of studies concerning the response of $B$. subtilis to environmental changes at the proteome and/or transcriptome levels (Blencke et al., 2003; Eymann et al., 2002; Moreno et al., 2001). In the past few years, when transcriptome and proteome studies became very popular, most regulatory effects were attributed to changes in transcription. However, changes in transcription are not always accompanied by corresponding changes in metabolic fluxes 
and vice versa (Schilling et al., 2007). Thus, posttranslational regulatory mechanisms require our attention to fully understand regulatory phenomena.

In the past few years, it turned out that extensive protein phosphorylation not only on histidine, but also on serine, threonine, and tyrosine residues occurs in bacteria (Deutscher and Saier, 2005; Kennelly and Potts, 1996). In B. subtilis, phosphorylation on histidine residues occurs in the phosphotransferase system (PTS) as well as in PTS-controlled transcription regulators and glycerol kinase. The sensor kinases of the two-component regulatory systems constitute a second class of His-phosphorylated proteins (Fabret et al., 1999; Reizer et al., 1999). In the PTS, some phosphotransfer domains are phosphorylated on cysteine residues from which the phosphate is transferred to the incoming sugar. In contrast, the response regulators of the two-component systems are phosphorylated on aspartate residues by their cognate sensor kinases (Fabret et al., 1999; Reizer et al., 1999). Phosphorylation on serine residues has long been studied for the HPr protein of the PTS that is phosphorylated on Ser-46 by the metabolite-controlled HPr kinase/phosphorylase. HPr(Ser-P) then binds to the transcription factor CcpA and acts as a corepressor in carbon regulation (Görke and Stülke, 2008). Regulatory phosphorylation of proteins on serine (and threonine) residues is also well established for the modulators of sigma factor activity in sporulation and stress response (Min et al., 1993; Yang et al., 1996). Protein tyrosine phosphorylation is implicated in the control of the transcription repressor CtsR, of UDP-glucose dehydrogenases and of the single-stranded DNA-binding proteins (Grangeasse et al., 2007). In total, about 200 proteins, representing 5\% of all B. subtilis proteins, are now known to be phosphorylated (Eymann et al., 2007; Lévine et al., 2006; Macek et al., 2007).

With the large number of proteins phosphorylated on serine, threonine or tyrosine, the identification of the responsible kinase(s) is getting into the spotlight. So far, the HPr kinase and the three kinases involved in controlling sigma factor activities have been studied. All these kinases seem to phosphorylate clearly defined sites and are therefore not candidates for the phosphorylation of novel targets. In addition, the protein kinase PrkC can phosphorylate several proteins, including elongation factor G and PrkC itself (Gaidenko et al., 2002; Madec et al., 2002). PrkC is a located in the cell membrane of vegetative cells and in the inner spore membrane of spores (Madec et al., 2002; Shah et al., 2008). The prkC gene is part of the prpC-prkC-cpgA operon that is 
constitutively expressed throughout growth (Iwanicki et al., 2005). Recently, stimulation of PrkC activity by muropeptides was reported. In spores, this activation of PrkC triggers germination, and prkC mutants are unable to germinate in response to muropeptides (Shah et al., 2008). Homologues of PrkC are widespread in both Gram-positive and Gram-negative bacteria (Fig. 14). This kinase is implicated in the phosphorylation of glycolytic enzymes and histone-like proteins as well as in pathogenicity in other Gram-positive bacteria (Jin and Pancholi, 2006; Kristich et al., 2007; Lomas-Lopez et al., 2007). It is interesting to note that $p r k C$ is encoded in an operon with a corresponding protein phosphatase, PrpC (Madec et al., 2002). This phosphatase is able to dephosphorylate $\operatorname{HPr}(\mathrm{Ser}-\mathrm{P})$ in B. subtilis and Mycoplasma pneumoniae (Halbedel et al., 2006; Singh et al., 2007).

We were interested in the regulation of carbon and nitrogen metabolism in $B$. subtilis. In an analysis of the transcriptome and metabolic flux responses to organic acids, we noticed that there are some cases where the changes in transcription do not match those in the metabolic fluxes. This is exemplified by the expression of the alsSD operon encoding acetolactate synthase and acetolactate decarboxylase and the corresponding acetoin production. Therefore, we considered posttranscriptional events to be important for metabolic adaptation (Schilling et al., 2007). At the same time, phosphoproteome analyses demonstrated that indeed many enzymes of basic metabolism are subject to phosphorylation in B. subtilis (Eymann et al., 2007; Lévine et al., 2006; Macek et al., 2007). Thus, protein phosphorylation might indeed play an important role for the adaptation of $B$. subtilis metabolism to changing conditions. However, the kinases responsible for these phosphorylation events have so far not been identified.

In this study, we intended to identify the kinase(s) for some of the enzymes of central metabolism by in vitro analysis of protein phosphorylation. Our work demonstrates that PrkC is able to phosphorylate several enzymes of different metabolic pathways. Moreover, PrkC was also responsible for a novel modification of the HPr protein of the PTS on Ser-12, an event previously observed in vivo (Macek et al., 2007). Thus, PrkC may play an important role in phosphorylating proteins with different functions in B. subtilis. 


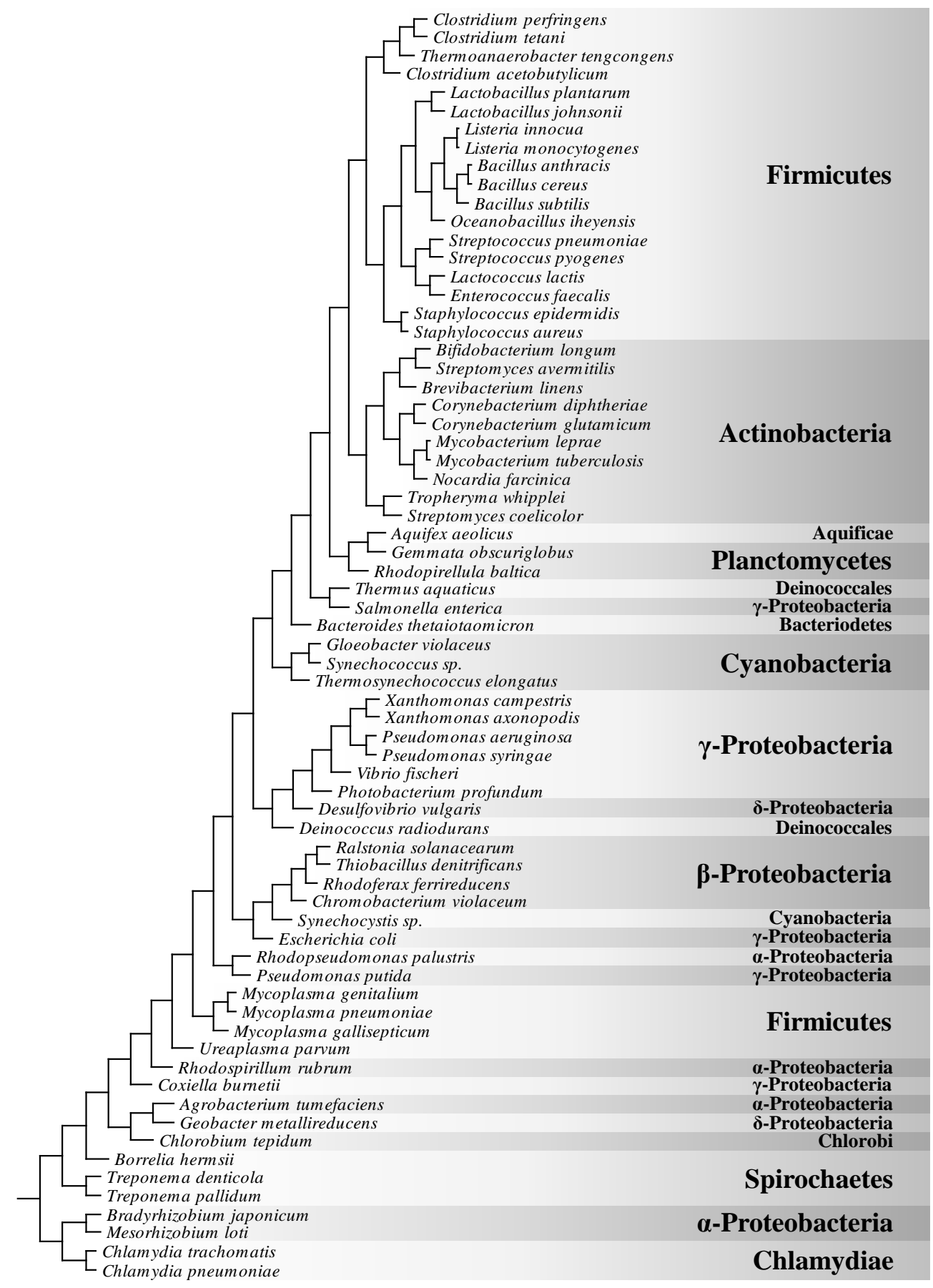

- 0.1 (substitutions / site)

Fig. 14. Phylogenetic tree of PrkC homologues. The tree is based on a multiple alignment generated with the CLUSTAL X v2.0.12. program (Larkin et al., 2007). The guide tree has been calculated using the UPGMA algorithm. The final phylogenetic tree was obtained using the Tree View X v0.5.0 program (http://darwin.zoology.gla.ac.uk/ rpage/treeviewx/). 
Tab. 6. Plasmids used in this work.

\begin{tabular}{|c|c|c|c|c|c|c|}
\hline Plasmid & Gene & Protein & Affinity tag & Oligonucleotides & Vector & References \\
\hline pAG2 & ptsH & $\mathrm{HPr}$ & $\mathrm{His}_{6}$-tag & - & - & Galinier et al. (1997) \\
\hline pGP174 & $g \ln A$ & Glutamine synthetase & Strep-tag & - & - & Heinrich et al. (2006) \\
\hline pGP205 & hprK & HPr kinase & $\mathrm{His}_{6}$-tag & - & - & Hanson et al. (2002) \\
\hline pGP371 & $p t s H^{\mathrm{a}}$ & HPr-S46A & $\mathrm{His}_{6}$-tag & SH82/SH83 & pWH844 & This work \\
\hline pGP563 & eno & Enolase & $\mathrm{His}_{6}$-tag & eno forw1/eno rev & pWH844 & This work \\
\hline pGP819 & $y w j H$ & Transaldolase & Strep-tag & NP11/NP12 & pGP172 & This work \\
\hline pGP820 & tkt & Transketolase & Strep-tag & NP05/NP06 & pGP172 & This work \\
\hline pGP821 & prkD & Protein kinase & Strep-tag & NP09/NP10 & pGP172 & This work \\
\hline pGP822 & alsD & $\alpha$-acetolactate decarboxylase & Strep-tag & NP01/NP02 & pGP172 & This work \\
\hline pGP823 & yabT & Protein kinase & Strep-tag & NP07/NP08 & pGP172 & This work \\
\hline pGP824 & $y x a L$ & Protein kinase & Strep-tag & NP13/NP14 & pGP172 & This work \\
\hline pGP825 & prkC & Protein kinase & Strep-tag & NP03/NP04 & pGP172 & This work \\
\hline pGP931 & icd & Isocitrate dehydrogenase & $\mathrm{His}_{6}$-tag & FC17/FC18 & pWH844 & This work \\
\hline pGP1100 & pyk & Pyruvate kinase & $\mathrm{His}_{6}$-tag & pyk forw1/pyk rev & pWH844 & This work \\
\hline
\end{tabular}

${ }^{\mathrm{a}}$ Indicates mutant alleles. 


\section{Results}

Identification of potential serine/threonine protein kinases in B. subtilis. The genome of $B$. subtilis encodes several proteins that are known or suspected to have serine/threonine kinase activity, among them the intensively studied HPr kinase/phosphorylase and the kinases involved in the control of sigma factor activities. In addition, the PrkC serine/threonine kinase has been studied before. Analysis of the annotated genome sequence revealed the presence of three additional potential kinases: PrkD that is similar to the kinase domain of PrkC (Madec et al., 2002), YxaL and YabT.

\section{Purification of selected potential protein kinases and phosphorylation} targets. The four genes encoding the selected proteins were cloned into the expression vector pGP172, fusing them to an N-terminal Strep-tag. The proteins were expressed and purified using their affinity for Streptactin Sepharose. PrkC and PrkD were purified to apparent homogeneity. In contrast, we were unable to purify detectable amounts of YxaL. This may be due to the fact that YxaL is a secreted protein carrying a signal peptide (Tjalsma et al., 2004). Similarly, only small amounts of YabT were obtained. Therefore, we concentrated our subsequent analyses on PrkC and PrkD.

Among the phosphoproteins that are involved in central metabolism, we selected glutamine synthetase (GlnA), enolase (Eno), transaldolase (YwjH), transketolase (Tkt), $\alpha$-acetolactate decarboxylase (AlsD), isocitrate dehydrogenase (Icd), and pyruvate kinase (Pyk). These proteins were found to be phosphorylated in vivo on serine, threonine or tyrosine (Eymann et al., 2007; Lévine et al., 2006; Macek et al., 2007). The corresponding genes were cloned into the expression vectors pWH844 and pGP172 to fuse the coding sequences to an N-terminal His $6^{-}$or Strep-tag, respectively (Table 6). All these proteins were expressed in Escherichia coli and were purified to apparent homogeneity by affinity chromatography.

Autophosphorylation of PrkC and PrkD. First, we analyzed the autophosphorylation of the selected serine/threonine kinases, PrkC and PrkD. For this purpose, we incubated the two proteins in the presence of $\left[\gamma_{-}{ }^{32} \mathrm{P}\right] \mathrm{ATP}$ and analyzed them by SDS-PAGE. As shown in Fig. 15, both proteins exhibited autophosphorylation activity. This observation is in excellent agreement with a previous report on the autophosphorylation of PrkC (Madec et al., 2003). To characterize these autophosphorylation events in more detail, we determined the phosphorylation sites by mass spectrometry using an LTQ Orbitrap mass spectrometer. For PrkC, we detected 
six phosphorylation sites: Ser-214, Thr-290, Thr-313, Thr-320 in the cytoplasmic kinase domain, and Thr-417 and Thr-498 in the extracytoplasmic domain. The phosphorylation sites in the kinase domain were also detected in a previous study that was exclusively devoted to the analysis of this domain (Madec et al., 2003). Moreover, phosphorylation of Thr-290 was found to take place in vivo (Macek et al., 2007). For PrkD, we found one phosphorylation site, Thr-174. It is interesting to note that the region surrounding this site is not conserved in PrkC. Similarly, the regions encompassing the phosphorylation sites in PrkC are not conserved in PrkD. Thus, both PrkD and PrkC are mainly autophosphorylated on threonine residues, but the actual phosphorylation sites are not conserved even though 26 and 41\% of identical and similar residues are conserved, respectively, for the N-terminal 200 amino acids of the two kinases.

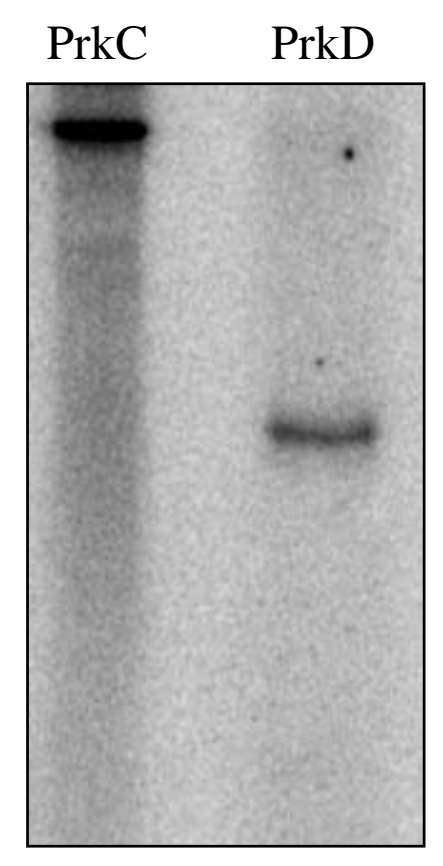

Fig. 15. Autophosphorylation of the serine/threonine kinases PrkC and PrkD. Two micrograms of each purified protein were incubated with $0.4 \mathrm{mM}\left[\gamma_{-}{ }^{32} \mathrm{P}\right] \mathrm{ATP}(480 \mathrm{Ci} / \mathrm{mmol})$ at $37^{\circ} \mathrm{C}$ for $30 \mathrm{~min}$ in assay buffer. The samples were then heated for $10 \mathrm{~min}$ at $95^{\circ} \mathrm{C}$ and loaded on a $16 \%$ SDS-PAGE gel. The gel was dried and analyzed by using a Storm 860 Molecular Imager, Molecular Dynamics.

Phosphorylation of the potential target proteins. The principal aim of this study was the investigation of phosphorylation of central metabolic enzymes of $B$. subtilis. To address this question, we incubated the putative kinase substrates with $\left[\gamma-{ }^{32} \mathrm{P}\right] \mathrm{ATP}$ and either of the two kinases. Of the seven selected proteins, four were 
phosphorylated by PrkC (Fig. 16). These were the transaldolase YwjH, the glutamine synthetase GlnA, the isocitrate dehydrogenase Icd, and the $\alpha$-acetolactate decarboxylase AlsD. In contrast, enolase, transketolase, and the pyruvate kinase PykA were not substrates for PrkC-dependent phosphorylation (data not shown). However, the phosphorylation efficiency differed for the PrkC substrates: The phosphorylation efficiency of $\mathrm{YwjH}$, GlnA, and Icd by PrkC was similar to that observed for HPrK-dependent phosphorylation of HPr (Fig. 16). In contrast, only weak signals were observed with AlsD (Fig. 16). This might be caused by differences in the number of phosphorylation sites or in different affinities of PrkC for its respective substrates. The kinase assays with PrkD revealed that only Icd and GlnA were phosphorylated by PrkD (data not shown). However, these phosphorylation signals were extremely faint, which questions their biological relevance. Thus, our analysis revealed selective phosphorylation of four enzymes of central metabolism by PrkC.

Determination of phosphorylation sites in the target proteins. In order to get new insights into PrkC substrates, we determined the phosphorylation sites of the proteins phosphorylated by PrkC. As observed for the autophosphorylation of PrkC, multiple phosphorylations with threonine as the major phosphoacceptor were observed. For the transaldolase $\mathrm{YwjH}$, we observed six sites that were phosphorylated by PrkC. The phosphorylated amino acids were scattered throughout the protein. There was one phosphorylated serine residue, Ser-55, and five phosphothreonines, i.e. Thr-26, Thr-82, Thr-125, Thr-159, and Thr-184. Similar results were obtained with glutamine synthetase. This enzyme was phosphorylated on one serine residue, Ser-207, and three threonine residues, Thr-26, Thr-147, and Thr-286. It is interesting to note that Thr-26 is located in the $\mathrm{N}$-terminal $\beta$-grasp domain of the enzyme that is thought to be involved in binding of small molecules (Burroughs et al., 2007), whereas the other phosphorylation events take place in the catalytic domain of glutamine synthetase. Isocitrate dehydrogenase is phosphorylated by PrkC on three threonine residues, Thr-138, Thr-147, and Thr-396. In contrast to the other PrkC substrates (including PrkC autophosphorylation), the $\alpha$-acetolactate decarboxylase AlsD was phosphorylated by PrkC on a single serine residue, Ser-88. 

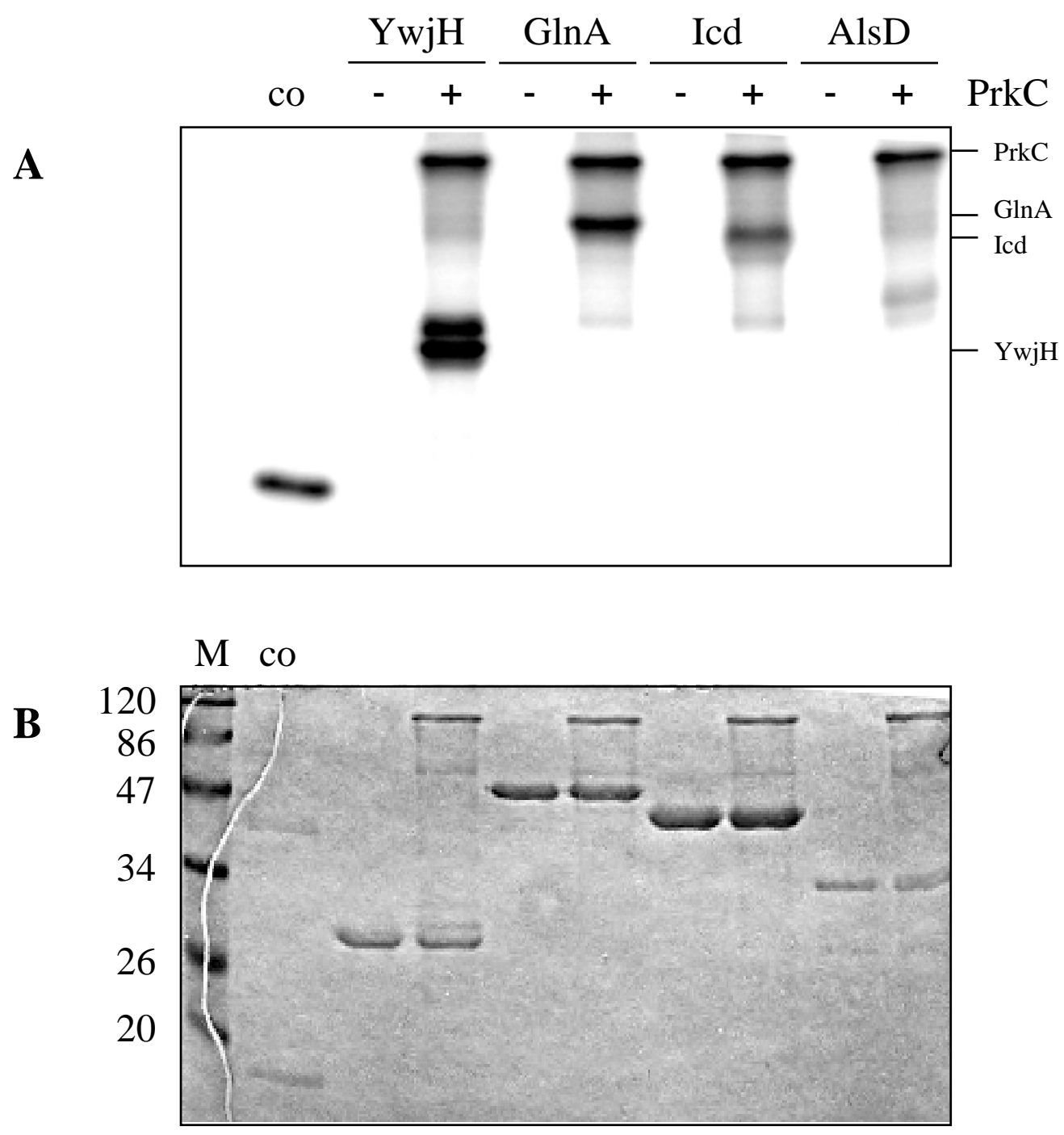

Fig. 16. Phosphorylation of potential target proteins by PrkC. Ten micrograms of the purified enzymes YwjH, GlnA, Icd, and AlsD were incubated with $0.4 \mathrm{mM}\left[\gamma^{3}{ }^{32} \mathrm{P}\right] \mathrm{ATP}(480 \mathrm{Ci} / \mathrm{mmol})$ in the absence and in the presence of $2 \mu \mathrm{g}$ PrkC at $37^{\circ} \mathrm{C}$ for $30 \mathrm{~min}$ in assay buffer. The samples were heated for $10 \mathrm{~min}$ at $95^{\circ} \mathrm{C}$ and loaded on a $16 \%$ SDS-PAGE gel. To obtain the autoradiogram, the gel was dried and analyzed using a Storm 860 Molecular Imager, Molecular Dynamics. (A) In addition, the proteins were visualized by Coomassie staining. (B) co = Control (HPr phosphorylated by HPrK). 
Phosphorylation of HPr by PrkC. A recent analysis of the B. subtilis phosphoproteome revealed a novel phosphorylation of the HPr protein of the PTS on Ser-12 (Macek et al., 2007). However, the source of this phosphorylation is not known so far. We considered the possibility that this phosphorylation might be caused by PrkC. Therefore, we assayed the phosphorylation of HPr in the presence of PrkC and $\left[\gamma-{ }^{32} \mathrm{P}\right] \mathrm{ATP}$. The HPr kinase that phosphorylates HPr on Ser-46 served as a control. As shown in Fig. 17, HPr was efficiently phosphorylated by the HPr kinase. Similarly, PrkC was capable of phosphorylating HPr. Both HPrK (with its phosphorylase activity) and PrpC, a protein phosphatase encoded in an operon with PrkC, are able to dephosphorylate HPr(Ser-P) (Mijakovic et al., 2002; Singh et al., 2007). Therefore, we considered the possibility that Ser-46 of HPr was the phosphorylation site used by HPrK as well as by PrkC. To address this problem, we used a mutant variant of the HPr protein devoid of its HPrK-dependent phosphorylation site (HPr-S46A) and tested the phosphorylation of this protein by the two kinases. As expected, the mutant protein was no longer phosphorylated by HPr kinase, thus confirming the conclusion that Ser-46 is the only phosphorylation site in HPr used by HPr kinase. In contrast, the mutant protein was still phosphorylated by PrkC, and the intensity of the phosphorylation signal was similar to that observed with the wild type protein. These observations suggest that a site (or sites) distinct from Ser-46 are the primary target(s) of PrkC-dependent phosphorylation in HPr.

The use of a different phosphorylation site by PrkC may suggest that Ser-12, a site phosphorylated in vivo (Macek et al., 2007), is the target of PrkC. This was verified by mass-spectrometric determination of the PrkC-dependent phosphorylation site (Fig. 18). Indeed, the only phosphorylated site detected in this study was Ser-12. Thus, the in vitro activity of PrkC on HPr reflects the in vivo situation. This observation suggests that PrkC is the kinase that phosphorylates HPr in vivo on Ser-12. 

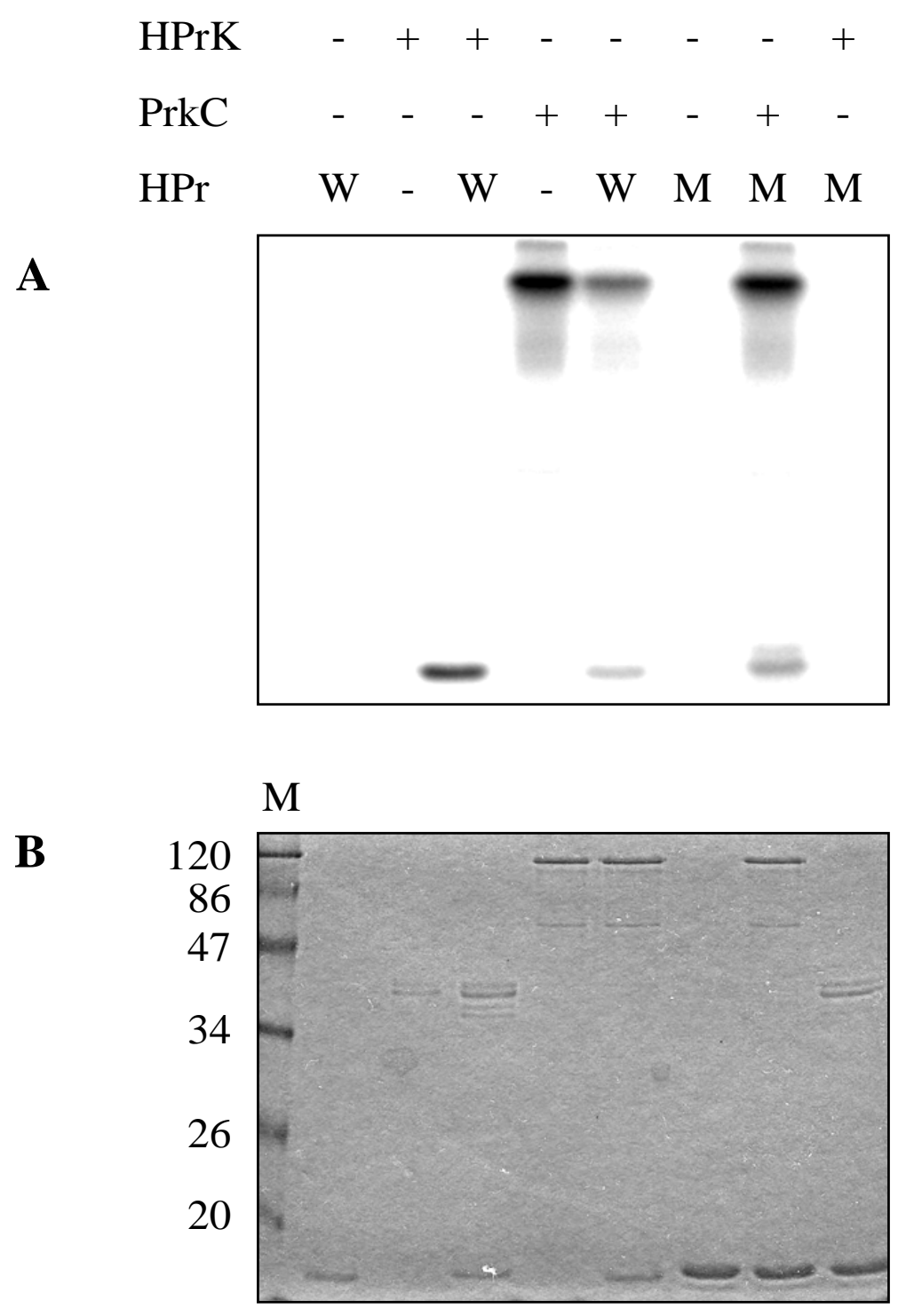

Fig. 17. Phosphorylation of wild type HPr and HPr-S46A by PrkC and HPrK. Ten micrograms of the purified wild type $\operatorname{HPr}(\mathrm{W})$ and $\operatorname{HPr}-\mathrm{S} 46 \mathrm{~A}(\mathrm{M})$ were incubated with $0.4 \mathrm{mM}\left[\gamma^{3}{ }^{32} \mathrm{P}\right] \mathrm{ATP}$ ( $480 \mathrm{Ci} / \mathrm{mmol}$ ) in the absence and in the presence of either $2 \mu \mathrm{g}$ PrkC or $\mathrm{HPrK}$ at $37^{\circ} \mathrm{C}$ for $30 \mathrm{~min}$ in assay buffer. The samples were heated for $10 \mathrm{~min}$ at $95^{\circ} \mathrm{C}$ and loaded on a $16 \%$ SDS-PAGE gel. The autoradiogram (A) and the Coomassie stained gel (B) were prepared as described in Fig. 15. 


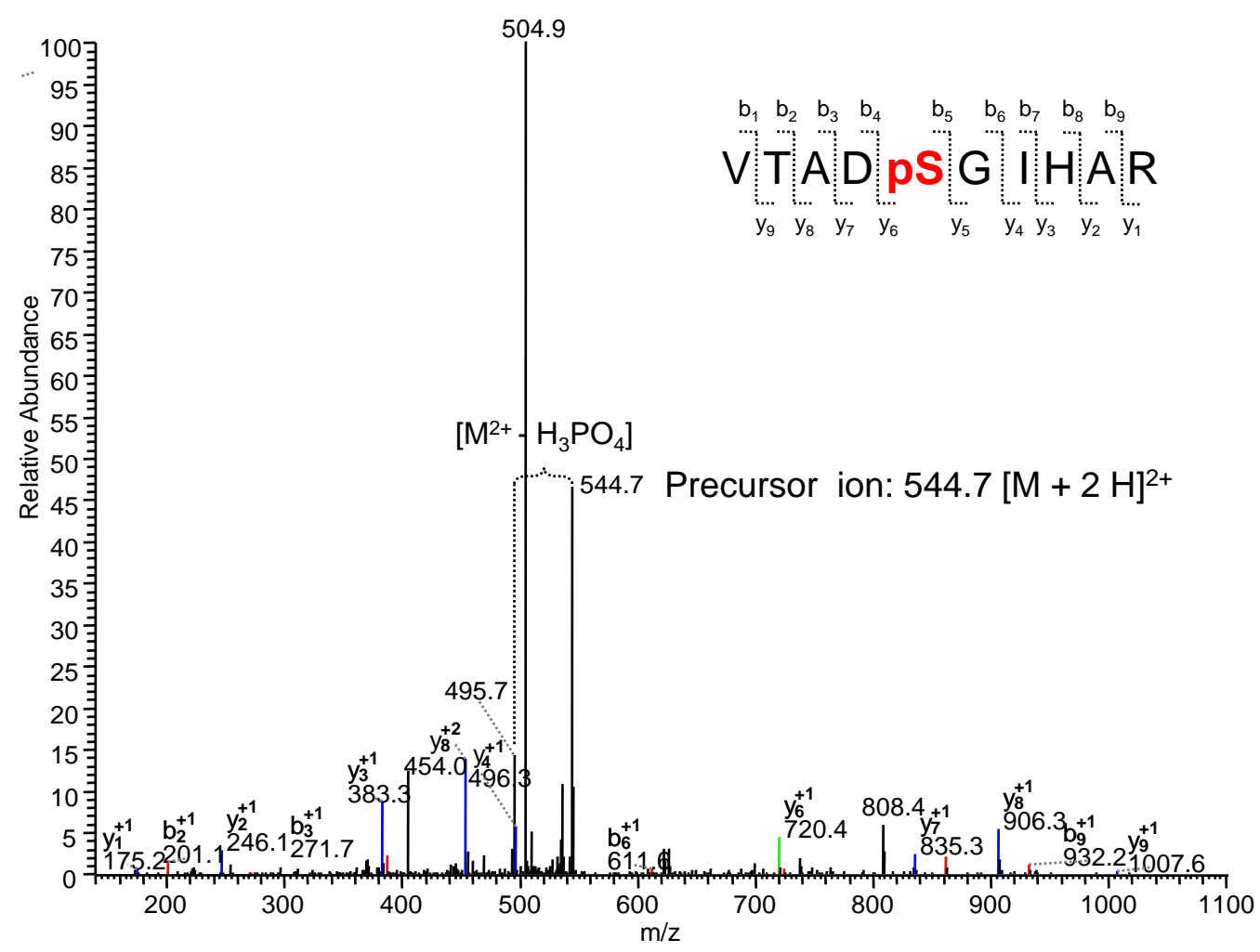

Fig. 18. Identification of the site of PrkC-dependent phosphorylation in HPr. The fragment ion spectrum of the HPr T2 peptide (VTADSGIHAR) with a precursor ion mass $544.7[\mathrm{M}+2 \mathrm{H}]^{2+}$ indicates a phosphate residue. The corresponding b- and y-ions and the formation of the ion corresponding to the neutral loss of phosphoric acid from the doubly-charged precursor ion are highlighted and demonstrate the Ser-12 phosphorylation of HPr.

\section{Discussion}

Protein phosphorylation is a very common event in all organisms (Eymann et al., 2007; Ficarro et al., 2002; Levine et al., 2006; Macek et al., 2007; Macek et al., 2008). As much as about $5 \%$ of all proteins can be phosphorylated in organisms so different as the sporulating bacterium B. subtilis and the genome minimalist Mycoplasma genitalium (Su et al., 2007). Two major questions remain to be answered: (1) Which kinases are responsible for these phosphorylation events, and (2) what is the effect of the phosphorylation on the activity of the target proteins? 
In this work, we addressed the first of these questions. In B. subtilis, only five serine/threonine kinases have been studied so far. These are HPr kinase, RsbT kinase, RsbW kinase, and SpoIIAB kinase, involved in the control of sigma factor activities and PrkC (Reizer et al., 1998; Madec et al., 2002; Min et al., 1993). In this work, we focused on PrkC and its relative, PrkD. Indeed, our in vitro experiments revealed that PrkC is able to phosphorylate several of the proteins that were found to exist as phosphoproteins in vivo.

PrkC is implicated in sporulation, biofilm formation, and germination (Gaidenko et al., 2002; Madec et al., 2002; Shah et al., 2008), but only two substrates (EF-G and PrkC itself) have been identified. Our work confirms that PrkC might be of global importance as it phosphorylates enzymes from different pathways, such as transaldolase, glutamine synthetase, isocitrate dehydrogenase, $\alpha$-acetolactate decarboxylase, and HPr. However, among the eight phosphoproteins tested for phosphorylation by PrkC, three were not targets of this kinase. A global role for PrkC is supported by studies with other bacteria. In Staphylococcus aureus, PrkC phosphorylates glycolytic enzymes (Lomas-Lopez et al., 2007), whereas the $\alpha$-subunit of RNA polymerase and the phosphoglucosamine mutase are substrates of the Streptococcus pneumoniae PrkC homolog, StkP (Novákováa et al., 2005). Moreover, PrkC homologs are important for virulence development and global gene expression in S. pneumoniae, S. aureus, M. pneumoniae, and Bacillus anthracis (Débarbouillé et al., 2009; Sasková et al., 2007; Schmidl et al., 2010; Shakir et al., 2010). Thus, PrkC can phosphorylate a wide range of proteins involved in a variety of cellular functions.

Another remarkable feature of PrkC is its obvious preference for threonine. This study presents a significant increase of the known phosphorylation sites used by PrkC. Of the sites identified here, 16 are threonines and only 5 are serine residues. In a previous study on PrkC autophosphorylation, 7 phosphorylated threonine residues and one serine residue were reported (Madec et al., 2003). Our results confirm the autophosphorylation of Ser-214 as well as of Thr-290, Thr-313, and Thr-320. We failed to detect the phosphorylation of a stretch of multiple threonine residues in the N-terminal part of PrkC, but instead we detected two phosphorylation sites in the extracellular domain of PrkC that was not included in the previous study (Madec et al., 2003). In vivo phosphorylation of PrkC was observed on Thr-290 (Macek et al., 2007), thus providing a confirmation for our in vitro study. Studies on protein phosphorylation 
by PrkC proteins from Streptococcus pyogenes also revealed the use of threonine as the preferred phosphorylation target by PrkC (Jin and Pancholi, 2006). The kinase domains of PrkC and PrkD are very similar to each other (about 30\% identical residues). However, the sites of autophosphorylation were not conserved between the two proteins although the only phosphorylation site identified for PrkD was also a threonine. A comparison of the regions around the PrkC-dependent phosphorylation sites revealed little conservation of the primary sequences, suggesting that it is not the primary sequence context that serves for recognition by PrkC.

An interesting aspect of this work is the identification of HPr as a novel target for PrkC. HPr is a paradigm of protein phosphorylation with its His-15 and Ser-46 phosphorylated by enzyme I of the PTS and by the ATP-dependent HPr kinase, respectively (Reizer et al., 1998). Analysis of the B. subtilis phosphoproteome revealed the phosphorylation of yet another site, Ser-12. We could attribute this phosphorylation event to the activity of PrkC. This finding suggests that our in vitro observations regarding PrkC-dependent protein phosphorylation reflect the in vivo activity of PrkC and the physiological interactions with the different target proteins. However, it is not known whether this phosphorylation affects the biological activity of HPr. The weak phosphorylation of Ser-12 by PrkC suggests that this phosphorylation does not affect the properties of the cellular HPr. Similar results have been obtained for the transcription antiterminator GlcT, which can be phosphorylated and inactivated to a minor degree by HPr under conditions in which its full activity is required. Again, this phosphorylation affects only a small fraction of the cellular GlcT pool and, therefore, does not influence GlcT-mediated regulation (Schmalisch et al., 2003).

Our work suggests that PrkC is a protein kinase, which may have global importance in B. subtilis. However, important questions remain to be answered: Which other proteins does PrkC phosphorylate? Phosphoproteome studies with prkC mutants might be helpful to address this question. Does PrkC-dependent phosphorylation serve for fine-tuning of the metabolism, does it tag the proteins for degradation, or does it serve completely different purposes? Finding the answers to these questions is challenging, but these answers will have a significant impact on our understanding of microbial physiology. 


\section{Materials and Methods}

Bacterial strains, oligonucleotides, and growth conditions. B. subtilis 168 (wild type, laboratory collection) and QB5223 (ptsH1) (Martin-Verstraete et al., 1995) were used to isolate chromosomal DNA for the amplification of the desired genes. E. coli DH5 and BL21(DE3)/pLysS (Sambrook et al., 1989) were used for cloning experiments and protein expression, respectively. B. subtilis was grown in TY medium (1\% wt/vol tryptone, $0.5 \% \mathrm{wt} / \mathrm{vol}$ yeast extract, and $1 \% \mathrm{wt} / \mathrm{vol} \mathrm{NaCl}$ ). E. coli was grown in lysogeny broth medium (LB medium), and transformants were selected on plates containing ampicillin (100 g/ml). LB plates were prepared by the addition of 17 g Bacto agar (Difco) per liter of LB medium. The oligonucleotides used in this study are listed in Table 7.

DNA manipulation. Transformation of E. coli and plasmid DNA extraction were performed using standard procedures (Sambrook et al., 1989). Restriction enzymes, T4 DNA ligase, and DNA polymerases were used as recommended by the manufacturers. DNA fragments were purified from agarose gels using the QIAquick PCR Purification kit (Qiagen, Germany). Pfu DNA polymerase was used for the polymerase chain reaction as recommended by the manufacturer. DNA sequences were determined using the dideoxy chain termination method (Sambrook et al., 1989). All plasmid inserts derived from PCR products were verified by DNA sequencing. Chromosomal DNA of B. subtilis was isolated as described (Martin-Verstraete et al., 1995).

Plasmid constructions. The plasmids used for the expression of tagged $B$. subtilis proteins in E. coli were constructed using the expression vectors pWH844 (Schirmer et al., 1997) or pGP172 (Merzbacher et al., 2004) that allow the fusion of the cloned proteins to an N-terminal $\mathrm{His}_{6}$ - or Strep-tag, respectively. These plasmids are listed in Table 6.

For the expression of the S46A mutant variant of the HPr proteins, plasmid pGP371 was constructed by cloning of the ptsH1 DNA fragment from B. subtilis QB5223 (Table 6). B. subtilis HPr kinase and wild type HPr were expressed using pGP205 and pAG2, respectively (Galinier et al., 1997; Hanson et al., 2002). 
Tab. 7. Oligonucleotides used in this study.

\begin{tabular}{|c|c|}
\hline Oligonucleotide & Sequence $\left(5^{\prime} \rightarrow 3^{\prime}\right)^{a}$ \\
\hline CD13 & AAACATATGGCTAGCTGGAGCCACCCGCAGTTC \\
\hline eno forw1 & AAGGATCCCCATACATTGTTGATGTTTATGCAC \\
\hline eno rev & TTTCTGCAGTTACTTGTTTAAGTTGTAGAAAGAGTTG \\
\hline FC17 & AAAGGATCCGTGGCACAAGGTGAAAAAATTAC \\
\hline FC18 & TTTCTGCAGTTATTAGTCCATGTTTTTGAT \\
\hline JS39 & TCTATCAACAGGAGTCCAAGC \\
\hline NP01 & AAAGAGCTCCGATGAAACGAGAAAGCAACATTC \\
\hline NP02 & TTTGGATCCCTATTATTCAGGGCTTCCTTCAGT \\
\hline NP03 & AAAGAGCTCCGGTGCTAATCGGCAAGC \\
\hline NP04 & TTTAGATCTTCATTATTCATCTTTCGGATACTCAAT \\
\hline NP05 & AAAGAGCTCGATGGATACAATTGAAAAGAAATCAG \\
\hline NP06 & TTTGGATCCTCATTACTTATTGATTAATGCCTTAAC \\
\hline NP07 & AAAGAGCTCGATGATGAACGACGCTTTGAC \\
\hline NP08 & TTTAGATCTTCATTAGATTAAGAAAAAGATAATA \\
\hline NP09 & AAAGAGCTCGATGGCATTAAAACTTCTAAAAAA \\
\hline NP10 & TTTGGATCCTCATTATGTGACCGATTGAATGGC \\
\hline NP11 & AAAGAGCTCGATGTTATTCTTTGTTGATACAG \\
\hline NP12 & TTTGGATCCTCATTATTTGTTCCAGTCTGCC \\
\hline NP13 & AAAGAGCTCGATGGTCAAGTCATTTCG \\
\hline NP14 & TTTGGATCCTCATTATTTCCCAAAAGCCATCAG \\
\hline NP15 & CCGGTGTTCACGGTGCGCCGCTTGG \\
\hline NP16 & CTCACСTGATTCTTTAACAGCTACAGCG \\
\hline NP17 & CTTGCTAAAGCTGCTGGCGAAAAGAAGCTG \\
\hline NP18 & ATCGTGAGCAAAACCCACGGCCAC \\
\hline NP19 & ATGTTCACGCGATCAAACCGACGTC \\
\hline NP20 & GCAGCAGCCAACTCAGCTTCCTTTCGGGC \\
\hline SH82 & ATGGATCCATGGCACAAAAAACATTTAAAG \\
\hline SH83 & ATAAAGCTTCTCGCCGAGTCCTTCG \\
\hline pWH844 fw & TATGAGAGGATCGCATCACCAT \\
\hline pyk forw1 & AAGGATCCAGAAAAACTAAAATTGTTTGTACCATCGG \\
\hline pyk rev & TTTCTGCAGTTAAAGAACGCTCGCACGG \\
\hline
\end{tabular}

${ }^{\mathrm{a}}$ The restrictions sites are underlined. 
Overexpression and purification of recombinant proteins. E. coli BL21(DE3)/pLysS was used as host for the overexpression of recombinant proteins. Expression was induced by the addition of IPTG (final concentration $1 \mathrm{mM}$ ) to exponentially growing cultures $\left(\mathrm{OD}_{600}\right.$ of 0.8$)$. Cells were lysed using a French press (20,000 p.s.i., 138,000 kPa; Spectronic Instruments, UK). After lysis, the crude extracts were centrifuged at $15,000 \mathrm{~g}$ for $1 \mathrm{~h}$. For purification of $\mathrm{His}_{6}$-tagged proteins, the resulting supernatants were passed over an $\mathrm{Ni}^{2+}$ HiTrap chelating column (5 ml bed volume, Pharmacia) followed by elution with an imidazole gradient (from 0 to $500 \mathrm{mM}$ imidazole in a buffer containing $10 \mathrm{mM}$ Tris-HCl pH 7.5, $600 \mathrm{mM} \mathrm{NaCl}$, $10 \mathrm{mM} \beta$-mercaptoethanol) over $30 \mathrm{ml}$ at a flow rate of $0.5 \mathrm{ml} / \mathrm{min}$. For proteins carrying an N-terminal Strep-tag, the crude extract was passed over a Streptactin column (IBA, Göttingen, Germany). The recombinant protein was eluted with desthiobiotin (Sigma, final concentration $2.5 \mathrm{mM}$ ).

After elution, the fractions were tested for the desired protein using 12.5\% SDS-PAGE gels. The relevant fractions were combined and dialyzed overnight. Protein concentration was determined using the Bio-Rad dye-binding assay with bovine serum albumin serving as the standard.

Assays for protein phosphorylation. Activity assays of potential protein kinases were carried out with purified proteins in assay buffer $\left[10 \mathrm{mM} \mathrm{MgCl}_{2}\right.$, $25 \mathrm{mM}$ Tris-HCl pH 7.6, $1 \mathrm{mM}$ dithiothreitol, $0.4 \mathrm{mM}\left[\gamma_{-}{ }^{32} \mathrm{P}\right] \mathrm{ATP}$ (480 Ci/mmol)] using purified target proteins. For assays of $\mathrm{HPr}$ kinase activity, fructose 1,6-bisphosphate was added to a final concentration of $25 \mathrm{mM}$. The assays were carried out at $37^{\circ} \mathrm{C}$ for 30 min followed by thermal inactivation of the enzyme $\left(10 \mathrm{~min}\right.$ at $\left.95^{\circ} \mathrm{C}\right)$. The assay mixtures were analyzed on $16 \%$ SDS-PAGE gels. Proteins were visualized by Coomassie staining.

Protein identification by mass spectrometry. Peptide preparation was carried out as recently described (Eymann et al., 2007). Excised polyacrylamide gel pieces of stained protein bands were washed with $100 \mu \mathrm{l}$ of $200 \mathrm{mM} \mathrm{NH}_{4} \mathrm{HCO}_{3}$ in $50 \% \mathrm{CH}_{3} \mathrm{CN}$ for $20 \mathrm{~min}$ at $37^{\circ} \mathrm{C}$ and dried in a vacuum centrifuge for $20 \mathrm{~min}$. In-gel digestion with trypsin (Promega, Madison, Wisc., USA) was performed overnight at $37^{\circ} \mathrm{C}$. After addition of $15 \mu$ f formic acid (5\%, v/v), samples were incubated in an ultrasonic bath for $20 \mathrm{~min}$. The supernatant was transferred into microsample vials and stored for LC-MS/MS analysis at $4^{\circ} \mathrm{C}$. 
LC-MS/MS analysis was performed using either an LTQ Orbitrap mass spectrometer (Thermo Electron, San Jose, Calif., USA) in conjunction with a nanoACQUITY UPLC ${ }^{\mathrm{TM}}$ system (Waters, Milford, Mass., USA) or a Q-Star Pulsar mass spectrometer (Applied Biosystems MDS Sciex) in conjunction with the Ettan MDLC system (GE Healthcare, Munich, Germany).

In the nanoACQUITY UPLC system, peptides were loaded onto a trapping column (nanoAcquity UPLC Column, Symmetry ${ }^{\circledR} \mathrm{C}_{18} 5 \mu \mathrm{m}, 180 \mu \mathrm{m} \times 20 \mathrm{~mm}$, Waters) and washed for $3 \mathrm{~min}$ with $99 \%$ buffer A $(0.1 \% \mathrm{v} / \mathrm{v}$ formic acid) at a flow rate of $10 \mu \mathrm{l} / \mathrm{min}$ and separated on an analytical column (BEH130 $\mathrm{C}_{18} 1.7 \mu \mathrm{m}$, $100 \mu \mathrm{m} \times 100 \mathrm{~mm}$, Waters) in a linear gradient from 99\% buffer A to 60\% buffer B ( $90 \% \mathrm{v} / \mathrm{v}$ acetonitrile, $0.1 \% \mathrm{v} / \mathrm{v}$ acetic acid) in $80 \mathrm{~min}$. The flow rate during elution was set to $1 \mu \mathrm{l} / \mathrm{min}$. In the Ettan MDLC system, the peptides were loaded onto a trapping column ( $\mu$-Precolumn, PepMap ${ }^{\mathrm{TM}}, \mathrm{C}_{18}, 300-\mu \mathrm{m}$ i.d. $\times 5$ mm, LC Packings), washed for 15 min with buffer $\mathrm{A}$ and separated onto the analytical column (PepMap, $\mathrm{C}_{18}$, $75-\mu m$ i.d. $\times 15 \mathrm{~cm}$, LC Packings) by formation of a 70-min gradient of $99 \%$ buffer $\mathrm{A}$ to $60 \%$ buffer $\mathrm{B}$ at a flow rate of $200 \mathrm{nl} / \mathrm{min}$.

The LTQ Orbitrap was employed to acquire a full Orbitrap survey scan in the range $\mathrm{m} / \mathrm{z}$ 300-2,000. Subsequently, MS/MS experiments of the three most abundant precursor ions were carried out in the LTQ. Meanwhile, the masses of the precursor ions were determined with high accuracy via single-ion mode scans in the Orbitrap cell of the mass spectrometer. The Q-Star system was used to carry out a survey scan in the mass range of $\mathrm{m} / \mathrm{z} 230-2,000$ in the first step. In a second step, up to four precursor ions were selected for a fragmentation in MS/MS experiments. Product ions were detected in the range of m/z 70-2,000. The data were analyzed as described (Eymann et al., 2007).

\section{Acknowledgments}

Oliver Schilling, Sven Halbedel, and Christina Herzberg are acknowledged for their help with some experiments. Finally, we wish to express our gratitude to Josef Deutscher and Boris Görke for their interest in this work and their valuable suggestions. This work was supported by grants of the Deutsche Forschungsgemeinschaft, the Fonds der Chemischen Industrie, and the BMBF (SYSMO, PtJ-BIUO/0313978D) to J. S. 


\section{Chapter 5}

\section{Upregulation of thymidine kinase activity compensates for loss of thymidylate synthase activity in Mycoplasma pneumoniae}

The work described in this chapter was published in:

Wang, L., C. Hames, S. R. Schmidl, and J. Stülke. 2010. Upregulation of thymidine kinase activity compensates for loss of thymidylate synthase activity in Mycoplasma pneumoniae. Mol. Microbiol. 77: 1502-1511.

Author contributions:

This study was designed and interpreted by LW. CH isolated the thy A transposon mutant. LW performed all experiments and SRS contributed to the slot blot analysis of the thymidine kinase gene. LW wrote the paper. 


\section{Summary}

Thymidylate, an essential building block of DNA, is synthesized either from deoxyuridylate by thymidylate synthase (TS) or thymidine (dT) by thymidine kinase (TK). Thymidylate kinase (TMPK) phosphorylates dTMP to dTTP. Thymidine phosphorylase (TP) catalyses reversible phosphorolysis of dT. Using transposon mutagenesis, Mycoplasma pneumoniae TS gene (thyA/mpn320) was interrupted and requirement of this enzyme was studied. We found that TK activity and transcript levels and TP activity, but not TMPK or TS activity, are growth-phase-regulated, with induction at the exponential growth phase and a decline after the stationary phase. Inactivation of thyA results in upregulation of TK transcript and a 10-fold increase in TK activity, reduced TMPK level and it had no effect on TP activity. The level of $\left[{ }^{3} \mathrm{H}\right]$-dT uptake and incorporation into DNA in the thyA mutant correlates with increases in TK activity, suggesting that $\mathrm{dT}$ uptake and metabolism is TK-dependent and that upregulation of TK activity in the thyA mutant compensates for the lack of ThyA activity. $\left[{ }^{3} \mathrm{H}\right]-\mathrm{dU}$ uptake was low compared with $\mathrm{dT}$, and incorporation of radioactivity into DNA in the thyA mutant indicates the presence of an alternative TS. Our results suggest that TK and TMPK are potential targets for the development of Mycoplasma-specific antibiotics.

\section{Introduction}

Mycoplasmas, of the class Mollicutes, phylogenetically belong to the family of Gram-positive bacteria and lack a cell wall. Several species are pathogenic to humans, including M. pneumoniae, Mycoplasma genitalium and Ureaplasma urealyticum. M. pneumoniae is spread through respiratory droplet transmission. Once attached to the mucosa, M. pneumoniae can cause pharyngitis, bronchitis, and pneumonia. M. pneumoniae has also been associated with a wide variety of extrapulmonary diseases, including central nerve system manifestation, such as encephalitis, aseptic meningitis, acute transverse myelitis, stroke, and polyradiculopathy (Narita, 2009; Narita, 2010; Tsiodras et al., 2005; Waites and Talkington, 2004). Because of the lack of a cell wall, mycoplasmas are unaffected by antibiotics targeting cell wall synthesis, such as penicillin. 
Thymidylate is an essential building block for DNA synthesis. In Mollicutes there is a general lack of enzymes in the de novo synthesis of purines and pyrimidines and therefore nucleotide biosynthesis begins with bases, nucleosides or deoxynucleosides, e.g. through the salvage pathway. As depicted in Fig. 19 thymidylate (dTMP) is synthesized either from deoxyuridylate (dUMP) by thymidylate synthase (TS), which catalyses the transfer of a methyl group from methylenetetrahydrofolate to dUMP, or from thymidine ( $\mathrm{dT}$ ) by thymidine kinase (TK), which catalyses the transfer of a gamma phosphate group from ATP to the 5 '-OH group of dT to form dTMP. Deoxyuridine (dU) can also be phosphorylated by TK to generate dUMP. Thymidylate kinase (TMPK) phosphorylates dTMP to dTDP, which can be further phosphorylated to dTTP by pyruvate kinase (Pyk) (Pollack et al., 2002) or TMPK (Wang, 2007). Both dT and $\mathrm{dU}$ can be degraded to their respective base by thymidine phosphorylase (TP) (Fig. 19). Pathway analysis of the M. pneumoniae genome by Pachkov et al. (2007) suggested that dTTP production can limit $M$. pneumoniae growth rate, even under optimal conditions, and pyrimidine metabolism appears to be more appropriate as a drug target due to its low plasticity.

A recent study using defined minimum medium showed that cytidine alone could provide all pyrimidines, and further addition of thymine promoted M. pneumoniae growth, but adding uracil had no such effect. Their study indicated that enzymes required for pyrimidine nucleotide biosynthesis, such as ribonucleotide reductase and thymidylate synthase, are active in vivo (Yus et al., 2009). However, there are no experimental data regarding the activities of $M$. pneumoniae enzymes in dTTP synthesis pathway.

Thymidine kinase (Tdk, MPN044), TMPK (MPN006), TP (MPN064) and TS (ThyA, MPN320) have been annotated in the M. pneumoniae genome. The amino acid sequences of $M$. pneumoniae TK and TMPK show high identity ( 40\%) to Ureaplasma TK and TMPK, which have been characterized in detail (Carnrot et al., 2003; Kosinska et al., 2005; Wang, 2007; Welin et al., 2004). M. pneumoniae TS (ThyA, MPN320) shows high sequence homology to mammalian and bacterial ThyA proteins. Previous studies demonstrated that TK and TMPK are essential for the survival of M. genitalium and M. pneumoniae, but that TP and TS are dispensable (Glass et al., 2006; Hutchison et al., 1999). 


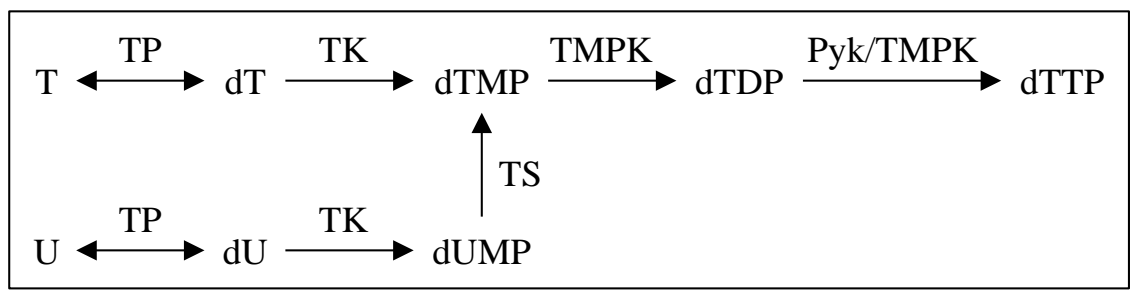

Fig. 19. Simplified dTTP synthesis pathway in mycoplasmas. In Mollicutes genomes nucleoside diphosphate kinase (Ndk), an enzyme responsible for the phosphorylation of nucleoside diphosphates to triphosphates has not been identified; however, it has been shown experimentally that glycolytic enzymes, such as Pyk (Pollack et al., 2002), or TMPK (Wang, 2007) are able to phosphorylate dTDP to dTTP, and they were suggested to replace Ndk function in Mollicutes. dT, thymidine; T, thymine; dTMP, thymidine 5'-monophosphate; dTDP, thymidine 5'-diphosphate; dTTP, thymidine 5'-triphosphate; dU, deoxyuridine; U, uracil; dUMP, deoxyuridine 5'-monophosphate; TK, thymidine kinase; TMPK, thymidylate kinase; TS, thymidylate synthase; TP, thymidine phosphorylase; Pyk, pyruvate kinase.

The transposon mutagenesis technique was used to interrupt the $M$. pneumoniae TS gene (thyA/mpn320) to study the interactions of enzymes in the dTTP synthesis pathway in relation to $M$. pneumoniae growth. We found that TK and TP activities are closely regulated to $M$. pneumoniae growth but not TS and TMPK. Inactivation of the thy $A$ gene resulted in upregulation of $t d k(\mathrm{TK})$ gene transcription and increased TK activity and reduced TS and TMPK activity. Metabolic labelling with $\left[{ }^{3} \mathrm{H}\right]-\mathrm{dT}$ shows a high level of $\left[{ }^{3} \mathrm{H}\right]-\mathrm{dT}$ uptake and incorporation in the thyA mutant. However, $\left[{ }^{3} \mathrm{H}\right]-\mathrm{dU}$ was primarily metabolized to ribonucleotides and incorporation into DNA was low. These data suggest that dT salvage through TK and TMPK is rate-limiting in dTTP synthesis and upregulation of TK activity compensated for the loss of ThyA activity in the thyA mutant.

\section{Results}

Characterization of the thy A mutant. The thyA (mpn320) mutant M. pneumoniae strain was generated by the global transposon mutagenesis procedure, as previously described (Halbedel et al., 2006). Transposon insertion site into the thy $A$ locus was after nucleotide 653. Wild type and the mutant strain were cultured according to standard protocol using modified Hayflick medium. Total proteins were extracted and TS activity was determined using $\left[{ }^{32} \mathrm{P}\right]$-dUMP as the substrate. TS activity was detected in both strains (Fig. 20A). 
The presence and absence of ThyA protein was determined by labelling with radioactive 5-fluorodeoxyuridine monophosphate (5FdUMP), which forms a covalent complex with ThyA proteins together with methylenetetrahydrofolate. Total protein extracts were incubated in a reaction mixture containing $\left[{ }^{32} \mathrm{P}\right]$-5FdUMP and methylenetetrahydrofolate. The reactions were stopped by addition of Laemmli sample buffer, and the samples were analysed by SDS-PAGE and autoradiography. A radiolabeled protein band corresponding to the size of ThyA protein (MPN320, with calculated molecular weight of $33.46 \mathrm{kDa}$ ) was seen in the wild type but not in thy $A$ mutant strain (Fig. 20B), as expected. The presence of TS activity but not ThyA protein in the thyA mutant strain indicates the presence of an alternative TS.

A

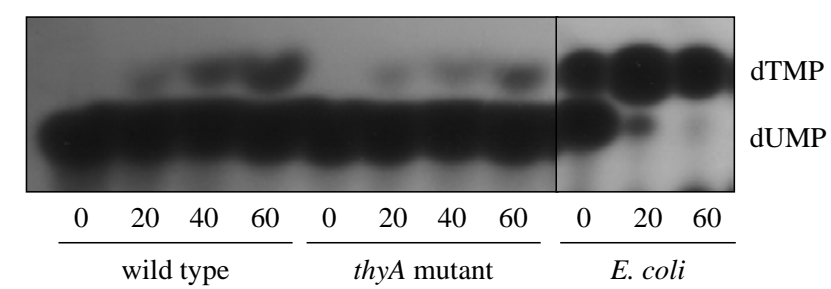

B

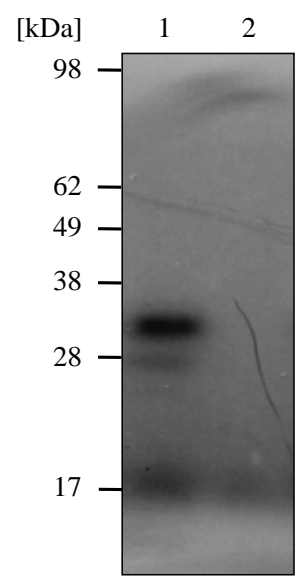

Fig. 20. Characterization of thyA mutant. (A) TS activity determined with $\left.{ }^{32} \mathrm{P}\right]$-dUMP as the labeled substrate. E. coli extracts was used as positive control. The reaction products were resolved by TLC and visualized by autoradiography. (B) Ternary complex formation of ThyA protein with $\left[{ }^{32} \mathrm{P}\right]-5 \mathrm{FdUMP}$ and methylenetetrahydrofolate. Lane 1, wild type; Lane 2, thyA mutant. 
The levels of TK, TP, TS, and TMPK activity. The activities of enzymes in dTTP synthesis were determined in protein extracts from both $M$. pneumoniae strains. TK activity was elevated ( $>10$-fold) in the thyA mutant, compared with the wild type (Table 8). TMPK activity was lower in the mutant strain as compared with wild type cells. The difference in TP activity in the thyA mutant and wild type strain was not statistically significant. There was also detectable TS activity in the thyA knockout strain (Table 8). We have also observed great variation in TK and TP activity in cultures that were harvested at different time points and/or had a different number of cells in the starting culture (inoculum size) (data not shown).

Tab. 8. Specific activities of enzymes in dTTP synthesis (nmol/(min $\mathrm{mg})$ ).

\begin{tabular}{llll}
\hline & Wild type & thy $\boldsymbol{A}$ mutant & $\boldsymbol{P}_{\text {-value }}$ \\
\hline TK & $1.10 \pm 0.07$ & $13.20 \pm 0.30$ & $<0.001$ \\
TMPK & $0.14 \pm 0.01$ & $0.083 \pm 0.003$ & 0.002 \\
TP & $2.65 \pm 0.20$ & $3.27 \pm 0.09$ & 0.170 \\
TS & $1.14 \pm 0.10$ & $0.0012 \pm 0.0004$ & $<0.001$ \\
\hline
\end{tabular}

${ }^{a}$ Statistical analysis was performed by Student's $t$-test and $P$-value $<0.05$ indicates significant difference. Results are mean \pm standard error from four to six measurements.

\section{Activities of dTTP synthesis enzymes in relation to $M$. pneumoniae growth.}

The above results warranted a further study of the levels of these enzymes at different phases of M. pneumoniae cell growth. Wild type and thyA mutant cells were cultured over an extended period (8 days) and harvested at $24 \mathrm{~h}$ intervals. M. pneumoniae growth was monitored daily by color change of the culture medium measured by $\mathrm{OD}_{550}$ value, $\mathrm{pH}$ value of the medium, and cell pellet wet weight at harvest. There was no observable difference in growth between the two strains. Total proteins were extracted and enzyme activities and proteins levels were determined.

Thymidine kinase activity and transcript levels. There was a sharp increase in TK activity from day 2 to day 3 in wild type cells and activity peaked at day 4, decreasing to basal levels thereafter (Fig. 21A). In thyA mutant cells, TK activity increased at day 2 and reached the maximum level at day 3, remaining at this level until decreasing at day 6 (Fig. 21A). Interestingly, TK activity at each time point was 4-5 times higher in thyA mutant cells compared with wild type. 
Thymidine kinase transcript levels, determined by slot blot analysis of total RNA, showed a similar pattern as TK activity profiles in both wild type and thyA mutant (Table 9 and Fig. 21B). In wild type cells there was a sharp increase in TK transcript from day 2, peaked at day 3 , and then decreased. In thy A mutant cells TK transcript level increased from day 2, reached maximum at day 3 , remained at relative high level at day 4 and 5, and then decreased to basal level at day 6 (Table 9 and Fig. 21B). At day 1 and day 2 TK transcripts in thyA mutant were at similar levels as wild type and upregulation of TK transcript levels in thyA mutant was observed from day 3 to day 6 with the exception that at day 4 the levels were similar in both strains (Table 9).

By comparing TK transcript and activity profiles, we observed that in wild type cells TK transcript levels peaked at day 3, but activity reached maximum at day 4 while in thy $A$ mutant cells both TK transcript and activity reached maximum levels at day 3 and remained at high levels from day 3 through day 5, at day 6 TK transcript decreased to basal level, but TK activity still remained at highest level. These observations indicated that there is a clear difference in transcriptional and translational regulation as well as TK protein degradation in wild type and thyA mutant.

Thymidylate kinase activity. The TMPK activity increased approximately 25\% from day 1 to 3 in wild type cells and stayed at a similar level throughout the culture period. In contrast, TMPK activity began decreasing in thy $A$ mutant strains from day 3 resulting in about a 25\% decrease from starting levels by the end of the culture period (Fig. 21C), and thus the mutant strain had lower TMPK activity at the end of the culture period compared with wild type cells (Fig. 21C). In general, TMPK activity was much lower compared with TK activity in both strains, suggesting that the second and third phosphorylation by TMPK is rate-limiting in dTTP synthesis.

The TMPK reaction products were analysed by the TLC technique and revealed that the majority ( $75 \%$ ) of the products were dTTP (data not shown). These data suggest that TMPK is capable of functioning as a nucleoside diphosphate kinase, as was demonstrated earlier with the Ureaplasma TMPK (Wang, 2007).

Thymidine phosphorylase activity. In both strains, TP activity increased during the exponential growth phase, reached a peak value at day 3 , and then decreased to the end of the culture period. In wild type and thyA mutant cells the levels of TP activity were similar (Fig. 21D). 
Tab. 9. TK transcript levels in different growth phases.

\begin{tabular}{llll}
\hline & Wild type $^{\mathbf{a}}$ & thyA mutant $^{\mathbf{a}}$ & Wild type/thyA mutant $^{\mathbf{b}}$ \\
\hline Day 1 & 1.00 & 1.00 & $1.06 \pm 0.06$ \\
Day 2 & $1.48 \pm 0.07$ & $1.53 \pm 0.05$ & $0.98 \pm 0.09$ \\
Day 3 & $3.36 \pm 0.10$ & $5.04 \pm 0.17$ & $1.63 \pm 0.12$ \\
Day 4 & $2.54 \pm 0.08$ & $2.55 \pm 0.06$ & $1.03 \pm 0.05$ \\
Day 5 & $1.03 \pm 0.08$ & $2.48 \pm 0.12$ & $2.30 \pm 0.10$ \\
Day 6 & $0.21 \pm 0.14$ & $0.47 \pm 0.11$ & $4.28 \pm 0.24$ \\
\hline
\end{tabular}

${ }^{\mathrm{a}}$ TK transcript levels relative to day 1 (set to 1.00).

b TK transcript levels in thyA mutant relative to wild type. Results are mean \pm standard error from three measurements.

A
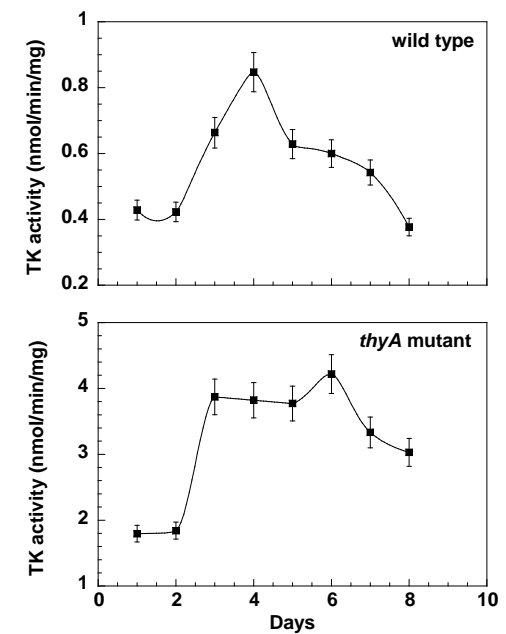

B

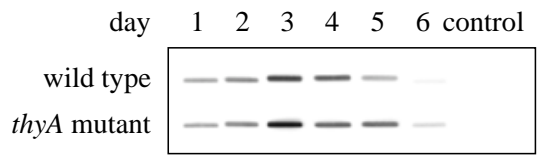

C

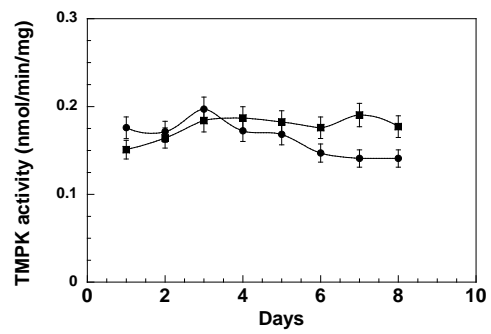

D

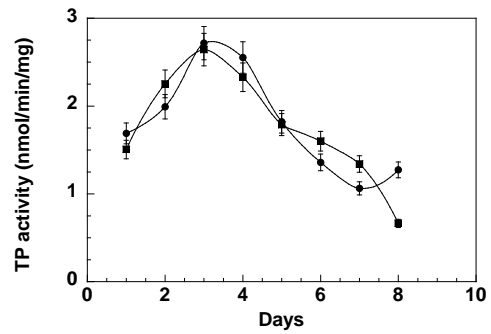

Fig. 21. TK activity (A) and transcript (B) levels in different growth phases. TK activity was determined using a radiochemical assay with $\left[{ }^{3} \mathrm{H}\right]-\mathrm{dT}$ as substrate and total proteins extracts. TK transcript levels were determined by slot blot analyses of total RNA. A dilution series of RNA was blotted onto a positively charged nylon membrane and probed with a DIG-labeled riboprobe specific for an internal part of TK. Signals obtained with $1 \mu \mathrm{g}$ of RNA are shown. Yeast tRNA (upper) and M. pneumoniae chromosomal DNA (lower) served as controls. TMPK (C) and TP (D) activity in different growth phases. Wild type (square) and thyA mutant (dot). 
Thymidylate synthase activity and protein levels. The TS activity was measured by using the tritium-release assay with $\left[5-{ }^{3} \mathrm{H}\right]$-dUMP as the labeled substrate. The activity levels in wild type cells decreased over the next 8 days, resulting in approximately $25 \%$ lower activity. In the thyA mutant strain there was detectable TS activity, approximately $0.1 \%$ of wild type TS activity in the starting cultures (Fig. 22A).

ThyA protein levels were determined by covalent labelling with $\left[{ }^{32} \mathrm{P}\right]-5 \mathrm{FdUMP}$. SDS-PAGE and autoradiography analysis revealed only one detectable radiolabeled band, which corresponded to the size of the ThyA protein in the wild type (Fig. 22B). The intensity of the ThyA protein band at each time point was similar, indicating that the ThyA protein is constitutively expressed. Therefore, the observed decrease in TS activity in wild type cells was not due to decreased protein expression. These data indicate that there may be other factors that are modulated during culture and regulate ThyA activity. As expected, no radiolabeled protein was detected in the thyA mutant strain (Fig. 22B).

$\left[{ }^{3} \mathbf{H}\right]-\mathbf{d T}$ metabolism. The uptake and metabolism of dT was measured in both M. pneumoniae strains using tritium-labeled dT (Table 10). The level of uptake was high in the thyA mutant strain, approximately fourfold compared with wild type cells. In both strains, $<1 \%$ of total uptake radioactivity was detected in the soluble nucleosides and nucleotides, and $>99 \%$ was found in the insoluble fraction. High-performance liquid chromatography (HPLC) analysis of the soluble fractions revealed that the radiolabeled substances were thymine, dT, dTMP, dTDP, and dTTP (Fig. 23A). There was also an unidentified radiolabeled peak that eluted at an earlier time in both strains, possibly a degradation product of thymine.

The absolute levels of soluble nucleoside and nucleotides were approximately four times higher in thyA mutant cells compared with wild type cells. The absolute amount of radioactivity incorporated into DNA was three times higher in the thyA mutant compared with wild type strain (Table 10).

Analysis of the medium revealed that the majority of remaining unincorporated radiolabeled compounds was $\left[{ }^{3} \mathrm{H}\right]$-thymine, indicating degradation of $\left[{ }^{3} \mathrm{H}\right]-\mathrm{dT}$ by TP (Table 10). 
A
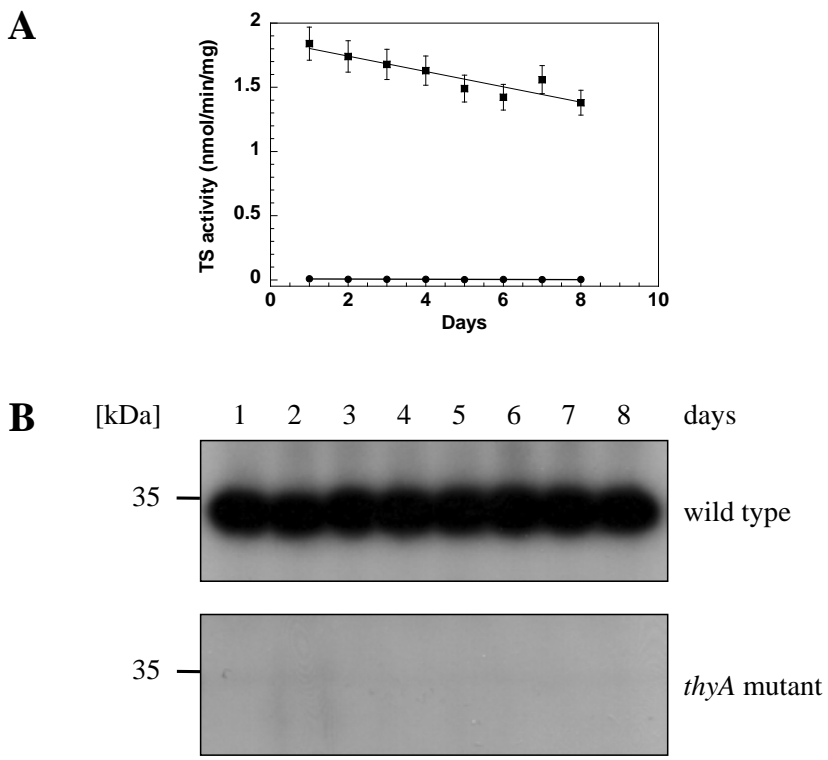

Fig. 22. TS activities (A) and protein levels (B) at different growth phases. TS activity determined using the tritium release assay. Wild type (square) and thyA mutant (dot). ThyA protein levels were determined by $\left[{ }^{32} \mathrm{P}\right]-5 \mathrm{FdUMP}$ and methylenetetrahydrofolate labelling.

A
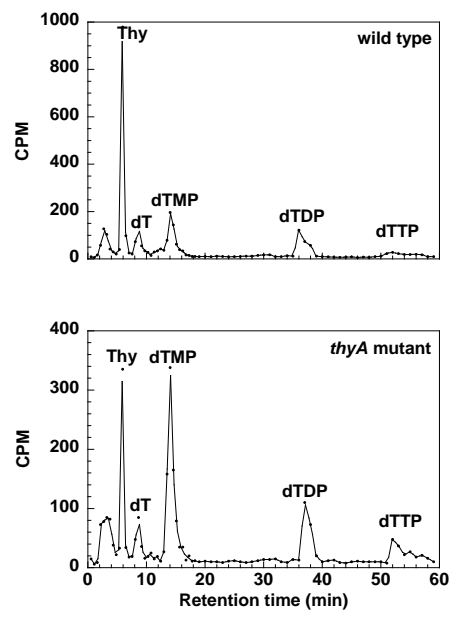

B
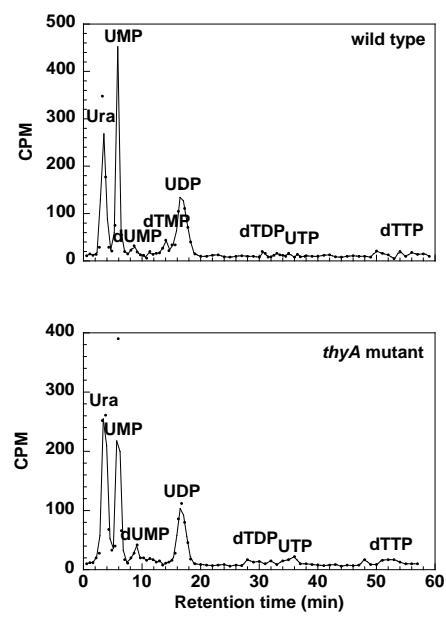

C
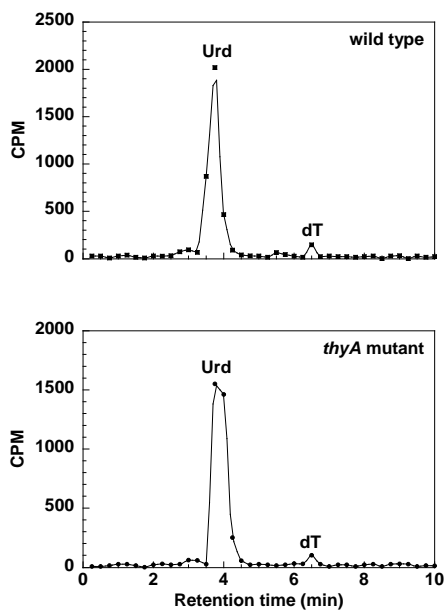

Fig. 23. HPLC analysis of acid soluble and insoluble extracts from wild type and thy $A$ mutant strains. (A) $\left[{ }^{3} \mathrm{H}\right]-\mathrm{dT}$ metabolism acid soluble extracts. (B) $\left[6-{ }^{3} \mathrm{H}\right]-\mathrm{dU}$ metabolism acid soluble extracts. HPLC fractions $(0.5 \mathrm{ml})$ were collected and the radioactivity was counted. (C) $\left[6-{ }^{3} \mathrm{H}\right]-\mathrm{dU}$ metabolism acid insoluble fractions after treatment with $\mathrm{NaOH}$ and alkaline phosphatase. HPLC fractions $(0.2 \mathrm{ml})$ were collected and the radioactivity was counted. Retention times for standards were: 3.23 min (uracil), $3.75 \mathrm{~min}$ (uridine), $4.58 \mathrm{~min}$ (dU), $5.80 \mathrm{~min}$ (UMP), $8.22 \mathrm{~min}$ (dUMP), $16.41 \mathrm{~min}$ (UDP), $36.7 \mathrm{~min}$ (UTP), 5.87 min (thymine), 8.75 min (dT), 14.35 min (dTMP), 31.9 min (dTDP), and $56.2 \mathrm{~min}$ (dTTP). 
Tab. 10. $\left[{ }^{3} \mathrm{H}\right]-\mathrm{dT}$ metabolism in wild type and thyA mutant $M$. pneumoniae strains ${ }^{\mathrm{a}}$.

\begin{tabular}{lll}
\hline & Wild type & thyA mutant \\
\hline Total uptake (\%) & $19 \pm 5$ & $82 \pm 2$ \\
PCA-soluble extracts (total counts) & $16,830 \pm 1,600$ & $60,264 \pm 6,500$ \\
\% of total uptake & $0.6 \pm 0.3$ & $0.7 \pm 0.2$ \\
NaOH-soluble fraction (total counts) & $1,455,915 \pm 15,000$ & $4,768,860 \pm 45,000$ \\
\% of total uptake & $99.4 \pm 0.3$ & $99.3 \pm 0.2$ \\
\hline Medium analysis after removal of cells & & $15 \pm 5$ \\
\hline$\left[{ }^{3} \mathrm{H}\right]-\mathrm{dT}$ (\%) & $31 \pm 13$ & $85 \pm 5$ \\
\hline$\left.{ }^{3} \mathrm{H}\right]-$ Thymine (\%) & $69 \pm 12$ & \\
\hline${ }^{a}$ Data were from four independent incubations and represented as the mean \pm standard error. Total uptake \\
was calculated as radioactivity recovered in total extracts (soluble and insoluble) as compared with total \\
radioactivity in the medium before incubation start.
\end{tabular}

[6- $\left.{ }^{3} \mathbf{H}\right]-\mathbf{d U}$ metabolism. A similar approach was performed using $\left[6-{ }^{3} \mathrm{H}\right]-\mathrm{dU}$ to determine the role of TS in ATTP synthesis in wild type and thyA mutant strains (Table 11). The level of uptake was low in the thyA mutant strain at approximately $0.57 \%$, while the level of uptake in wild type cells was at approximately $2.6 \%$. In wild type strain, approximately $5 \%$ of total uptake was found in the soluble nucleosides and nucleotides, and approximately $95 \%$ was found in the insoluble fraction. In the thy $A$ mutant strain, $10 \%$ of total uptake was incorporated into soluble nucleoside and nucleotides and $90 \%$ of the total radioactivity was recovered in the insoluble fraction. HPLC analysis of soluble nucleotides revealed that the radiolabeled compounds were predominantly uracil, UMP, and UDP, indicating that dU was degraded to uracil and further metabolized to ribonucleotides. Tritium-labeled dUMP and dTMP were detected at low levels in the wild type strain, but there was no detectable tritium-labeled dTMP in the thyA mutant strain (Fig. 23B). There was no $\left[{ }^{3} \mathrm{H}\right]$-dU detected in the soluble fractions. Trace amounts of radiolabeled dTDP and dTTP were also detected (Fig. 23B).

The absolute levels of soluble nucleotides were low in thyA mutant compared with wild type cells (Table 10). The absolute amount of radioactivity in the insoluble fraction was six times lower in the thyA mutant than in wild type cells (Table 11).

The PCA-insoluble fractions were solubilized with $\mathrm{NaOH}$, neutralized and treated with alkaline phosphatase before HPLC analysis. Two radioactive peaks were detected in both strains, one with a retention time of $3.95 \mathrm{~min}$ (Urd) and the other with a retention time of $6.35 \mathrm{~min}(\mathrm{dT})$, indicating that there was thymidylate incorporated into 
DNA even in the thyA knockout strain (Fig. 23C). The amount of $\left[{ }^{3} \mathrm{H}\right]-\mathrm{dT}$ recovered from HPLC analysis of PCA-insoluble fractions in both wild type and the thyA mutant were only $\sim 1 \%$ of the total radioactivity in these fractions, which indicated that $99 \%$ of the radioactivity originated from $\left[6-{ }^{3} \mathrm{H}\right]-\mathrm{dU}$ was incorporated into RNA as uridine ribonucleotide.

Analysis of the medium showed that the remaining radiolabeled compounds were $\left[{ }^{3} \mathrm{H}\right]$-uracil and $\left[{ }^{3} \mathrm{H}\right]-\mathrm{dU}$, indicating that $\left[{ }^{3} \mathrm{H}\right]$-dU had been degraded (Table 11).

Tab. 11. $\left[{ }^{3} \mathrm{H}\right]-\mathrm{dU}$ metabolism in wild type and thy $A$ mutant $M$. pneumoniae strains ${ }^{\mathrm{a}}$.

\begin{tabular}{lll}
\hline & Wild type & thyA mutant \\
\hline Total uptake (\%) & $2.6 \pm 0.1$ & $0.57 \pm 0.10$ \\
PCA-soluble extracts (total counts) & $15,510 \pm 987$ & $6,435 \pm 650$ \\
\% of total uptake & $4.3 \pm 0.5$ & $10.3 \pm 0.2$ \\
NaOH-soluble fraction (total counts) & $276,750 \pm 2540$ & $46,230 \pm 2,347$ \\
$\%$ of total uptake & $95.7 \pm 0.7$ & $90.3 \pm 0.4$ \\
\hline Medium analysis after removal of cells & & $48 \pm 5$ \\
\hline$\left[{ }^{3} \mathrm{H}\right]$-dU (\%) & $34 \pm 6$ & $52 \pm 5$ \\
{$\left[{ }^{3} \mathrm{H}\right]-$ Uracil (\%) } & $66 \pm 7$ & \\
\hline${ }^{a}$ Data were from three independent experiments and given as mean \pm standard error. Total uptake was \\
calculated as radioactivity recovered in total extracts (soluble and insoluble) as compared with total \\
radioactivity in the medium before incubation start.
\end{tabular}

\section{Discussion}

In mycoplasmas there are only a few enzymes involved in the biosynthesis of dTTP (Fig. 19), an essential precursor for DNA synthesis, as compared with their hosts. The corresponding mammalian enzymes, including TK, TMPK, TP, and TS, have been well studied, and their activity levels have been shown to be tightly regulated throughout the cell cycle, with the highest levels in S-phase (Gasparri et al., 2009; Ke and Chang, 2004). However, little is known regarding cell cycle regulation of bacterial enzymes. Here we show that $M$. pneumoniae TK, at both activity and transcript levels, is regulated during the growth phase, with a clear induction in the exponential growth phase and a drastic decline during the stationary phase. The M. pneumoniae TK activity profile in wild type cells correlates with the rate of DNA synthesis (Yus et al., 2009). Compared with wild type both TK activity and transcript levels were upregulated in thy $A$ mutant, and the differences seen in TK expression profiles in wild type and thy $A$ 
mutant indicate that there is a cross-talk in transcriptional and translational regulation of $t d k(T K)$ and thyA genes. Inactivation of thyA resulted in the upregulation of $t d k$ gene transcription, which leads to induction of TK protein and increased TK activity, as a mechanism to compensate the loss of thyA. The upregulation and prolonged period of high TK activity in the thy $A$ mutant strain indicates the importance of TK activity in dTTP synthesis and DNA replication in compensating for the lack of ThyA activity. In contrast to mammalian enzymes, $M$. pneumoniae TMPK activity varies only slightly during the culture period in both wild type and the thyA mutant strain. In wild type ThyA protein remained at similar at levels during culture; however, TS activity decreased over time. In the thyA mutant a very low level of TS activity was detected, indicating the presence of an alternative TS enzyme. Similar to TK, TP activity was induced at the exponential growth phase and decreased at the stationary phase. The level of TP activity was similar in wild type and thyA mutant cells. Collectively, these results suggest that ThyA and TMPK are constitutively expressed and inactivation of the thy $A$ gene results in the induction of TK.

\section{Increased salvage of $\mathrm{dT}$ in thy A mutant compensates for the lack of ThyA} activity. Metabolic labelling studies with dT and dU showed that both nucleosides or bases can be taken up, metabolized, and incorporated into DNA, confirming the activity of additional enzymes in the dTTP synthesis pathway. Furthermore, there was higher level of $\mathrm{dT}$ than $\mathrm{dU}$ uptake in wild type cells, indicating $M$. pneumoniae salvages $\mathrm{dT}$ more efficiently than $\mathrm{dU}$. Radiolabeled $\mathrm{dT}$ was converted to the corresponding nucleotides and efficiently incorporated into DNA. However, $\left[{ }^{3} \mathrm{H}\right]$-dU was recovered mainly as ribonucleotides and the amount of radioactivity in the form of dUMP and dTMP was $<5 \%$ of the total soluble fraction and total incorporation into DNA was only about $1 \%$. These data indicate that the efficiency of ribonucleotide reduction and conversion of dUMP to dTMP was low.

The level of dT uptake and incorporation into DNA in the thyA mutant correlates with increases in TK activity, suggesting that dT uptake and metabolism is TK-dependent and upregulation of TK activity in thyA mutant cells is apparently compensating for the lack of ThyA activity. Our results also indicate that the efficiency of dTTP synthesis via UDP reduction was low compared with the direct salvage of dT, explaining why thyA is not essential. 
Metabolism of dU and thymidylate synthases. The uptake of $\left[{ }^{3} \mathrm{H}\right]-\mathrm{dU}$ was low compared with dT uptake in wild type cells, and uptake and incorporation of $\left[{ }^{3} \mathrm{H}\right]-\mathrm{dU}$ into macromolecules were greatly reduced in the thyA knockout strain. HPLC analysis revealed that the radiolabeled compounds were mainly ribonucleotides and no detectable $\left[{ }^{3} \mathrm{H}\right]-\mathrm{dU}$ was present in the soluble fractions, suggesting that $\left[{ }^{3} \mathrm{H}\right]-\mathrm{dU}$ has been degraded to uracil and subsequently taken up and converted to ribonucleotides via uracil phosphoribosyltransferase (MPN033). The low level of uracil uptake in the thyA mutant indicates that inactivation of the thyA gene may affect uracil uptake and/or uracil phosphoribosyltransferase activity. This is the first experimental proof that connects the pentose phosphate pathway to nucleotide metabolism in $M$. pneumoniae. These results also explain why dU uptake is not TK-dependent.

The TS activity in the thy A mutant was only approximately $0.1 \%$ of activity in wild type cells. Incorporation of radioactive thymidylate into DNA originated from $\left[{ }^{3} \mathrm{H}\right]-\mathrm{dU}$ in thyA mutant cells, indicating the presence of an alternative $\mathrm{TS}$ in M. pneumoniae. Nonetheless, our study indicates that dTTP synthesis via ribonucleotide reduction and methylation of dUMP by ThyA is not efficient in M. pneumoniae.

Thymidine/thymine, but not uracil, promotes $M$. pneumoniae growth. Yus et al. (2009) showed that purine and pyrimidine composition in the minimum defined medium can be replaced by ribonucleosides, but not deoxyribonucleosides, and that thymidine can be metabolized to some extent, but not thymine. Furthermore, addition of thymine increases $M$. pneumoniae growth when added with cytidine. In our metabolic study, we did not observe any difference in growth rate since we used enriched medium (modified Hayflick medium). When the medium was analysed at the end of the culture period most of the radiolabeled dT and dU were degraded; therefore, both thymine and uracil were present. We showed that dT, thymine, and uracil, but not $\mathrm{dU}$, were taken up and metabolized. These data suggest that there may be specific transporters for bases and ribonucleosides and $M$. pneumoniae may lack such transporters for deoxynucleosides. Our study also indicates that the expression of TP may be influenced by nutrition status, since $M$. pneumoniae apparently was not able to degrade $\mathrm{dT}$ or $\mathrm{dU}$ and/or salvage thymine in the minimum defined medium (Yus et al., 2009). The uptake of uracil was less efficient as compared with dT/thymine and the level of dTTP synthesized through UDP reduction was very low. However, thymine/dT can be efficiently salvaged to thymidine nucleotides (dTTP) through the combined 
action of TP, TK, and TMPK, and increased dTTP synthesis promotes $M$. pneumoniae growth. Our results suggest that the slow division rate of $M$. pneumoniae is most likely due to inefficient dTTP synthesis.

Inhibition of $M$. pneumoniae dTTP synthesis as a therapeutic target. Mycoplasmas cause a wide variety of diseases and have also been associated with cancer (Narita, 2009; Narita, 2010; Waites and Talkington, 2004). Persistent Mycoplasma infection induced malignant transformation of human cells, as shown in a recent study (Namiki et al., 2009). Mycoplasma infections can reduce the effect of anticancer nucleoside analogues due to degradation by Mycoplasma enzymes (Liekens et al., 2009). Therefore, it may be beneficial to treat Mycoplasma infections in cancer patients who receive nucleoside analogues as chemotherapeutic agents. Current antibiotics used in treating Mycoplasma infections are effective but clinical antibiotic resistant Mycoplasma strains are emerging (Morozumi et al., 2010). In addition, mycoplasmas are slow growing and therefore long-term treatment is required, which may lead to the emergence of antibiotic resistance in other bacteria species. Therefore, new targets for developing specific antibiotics against Mycoplasma are needed.

DNA synthesis requires dTTP, which is an essential building block, thus, blocking dTTP synthesis would lead to thymine deficiency-induced cell death. Inhibition of pyrimidine uptake and/or conversion to thymidine nucleotides would impair Mycoplasma dTTP synthesis and lead to cell death. Therefore, TK and TMPK are potential targets for future development of antibiotics against Mycoplasma infections.

\section{Experimental procedures}

Materials. Radiolabeled compounds [methyl- ${ }^{3} \mathrm{H}$ ]-thymidine (dT, $\left.20 \mathrm{Ci} / \mathrm{mmol}\right)$ and $\left[\gamma-{ }^{32} \mathrm{P}\right]$-ATP $(3,000 \mathrm{Ci} / \mathrm{mmol})$ were purchased from PerkinElmer. [5- $\left.{ }^{3} \mathrm{H}\right]-\mathrm{dUMP}$ (deoxyuridine 5'-monophosphate, $47 \mathrm{Ci} / \mathrm{mmol}$ ) and $\left[6-{ }^{3} \mathrm{H}\right]$-deoxyuridine (dU, $45 \mathrm{Ci} / \mathrm{mmol}$ ) were bought from Moravek Biochemicals. $\left[{ }^{32} \mathrm{P}\right]-5 F d U M P,\left[{ }^{32} \mathrm{P}\right]$-dUMP, and $\left[{ }^{3} \mathrm{H}\right]$-dTMP were synthesized enzymatically using Ureaplasma thymidine kinase with $\left[{ }^{32} \mathrm{P}\right]$-ATP or $\left[{ }^{3} \mathrm{H}\right]-\mathrm{dT}$ as the labeled substrate and purified (Carnrot et al., 2003). Rabbit anti-MPN358 antibodies were produced by using synthetic peptides chosen from MPN358 sequence as antigens (GenScript Corp.) and antisera were used directly in Western blot analysis without further purification. 
Mycoplasma culture and protein extraction. $M$. pneumoniae wild type and the thyA mutant strains were cultured in modified Hayflick medium, as described previously (Halbedel et al., 2004).

Time-course study. Culture was started (day 0 ) by the addition of $1 \mathrm{ml}$ stock $\left(\mathrm{OD}_{550}, \sim 0.7\right)$ to a $25-\mathrm{cm}^{2}$ flask containing $25 \mathrm{ml}$ fresh medium and incubated at $37^{\circ} \mathrm{C}$ with $5 \% \mathrm{CO}_{2}$. Culture optical density was monitored at $550 \mathrm{~nm}$ daily and cells harvested at $24 \mathrm{~h}$ intervals by centrifugation at 7,000 g for $30 \mathrm{~min}$. Pellets were washed three times with phosphate-buffered saline (PBS) and then resuspended in protein extraction buffer. Total proteins were extracted as previously described (Wang et al., 2001). Protein concentration was determined by Bio-Rad protein assay using BSA as a standard, according to the manufacturer's protocol.

Metabolic labelling with [methyl- $\left.{ }^{3} \mathbf{H}\right]-\mathrm{dT}$ and $\left[6-{ }^{3} \mathrm{H}\right]-\mathrm{dU}$. A stationary phase culture was resuspended in $6 \mathrm{ml}$ fresh medium in a $25-\mathrm{cm}^{2}$ flask and used as stock. One milliliter stock culture was added to a $25-\mathrm{cm}^{2}$ flask containing $25 \mathrm{ml}$ fresh medium with $1 \mu \mathrm{Ci} / \mathrm{ml}$ [methyl- $\left.{ }^{3} \mathrm{H}\right]-\mathrm{dT}$ or $\left[6-{ }^{3} \mathrm{H}\right]-\mathrm{dU}\left(1 \mu \mathrm{M}\right.$ dT or dU) and incubated at $37^{\circ} \mathrm{C}$. After 5 days the cultures were harvested by centrifugation, washed three times with PBS, and divided into three aliquots and saved at $-20^{\circ} \mathrm{C}$ for further analysis. Soluble nucleotides were extracted with $100 \mu$ of $10 \%$ PCA (perchloric acid), followed by centrifuge at $16,000 \mathrm{~g}$ for $15 \mathrm{~min}$. The supernatant was transferred to a new tube and neutralized with $\mathrm{KOH}$ and the insoluble salt $\left(\mathrm{KClO}_{4}\right)$ was removed by centrifugation. The supernatant containing nucleotides and nucleosides was analysed directly by TLC and/or HPLC to determine the amount of radiolabeled nucleoside tri-, di-, and monophosphates. To extract nucleotides from the insoluble fraction, pellets were washed twice with $10 \%$ PCA, followed by addition of $100 \mu \mathrm{l} 1 \mathrm{M} \mathrm{NaOH}$ and incubation at $60^{\circ} \mathrm{C}$ for $2 \mathrm{~h}$ with shaking, and incorporation of radioactivity was then counted using a liquid scintillation counter (Beckman-Coulter). The $\mathrm{NaOH}$-soluble fraction was treated with alkaline phosphatase after neutralization and analysed by HPLC and TLC. Non-radioactive nucleosides and/or nucleotides were used as internal standards in HPLC analysis. Culture medium before and after incubation was counted for radioactivity and the radioactive components were analysed by TLC and/or HPLC. Total uptake was calculated as radioactivity recovered in total extracts (soluble and insoluble) as compared with total radioactivity in the medium before incubation start. 
The HPLC analysis was performed using reverse phase column (Source 5RPC, GE Healthcare) by isocratic elution in buffer containing $50 \mathrm{mM}$ ammonium phosphate, $\mathrm{pH}$ 6.5, $5 \mathrm{mM}$ tetrabutylammonium hydrogen sulphate and $12 \%$ methanol at a flow rate of $1 \mathrm{ml} / \mathrm{min}$. Soluble nucleotide extracts were mixed with HPLC buffer (1:4) before injection. Fractions $(0.5 \mathrm{ml}$ or $0.2 \mathrm{ml})$ were collected and mixed with $2 \mathrm{ml}$ scintillation fluid and counted. The radioactive peaks were compared with non-radioactive standards. Retention times for standards were $3.23 \mathrm{~min}$ (uracil), $3.75 \mathrm{~min}$ (uridine), 4.58 min (dU), 5.80 min (UMP), 8.22 min (dUMP), 16.41 min (UDP), 36.7 min (UTP), 5.87 min (thymine), $8.75 \mathrm{~min}$ (dT), $14.35 \mathrm{~min}$ (dTMP), $31.9 \mathrm{~min}$ (dTDP), and $56.2 \mathrm{~min}$ (dTTP). TLC analysis was performed with PEI-cellulose and developed in $0.1 \mathrm{M} \quad \mathrm{NaH}_{2} \mathrm{PO}_{4}$ for separation of thymidine nucleotides and isobutyric acid/ $\mathrm{H}_{2} \mathrm{O} /$ ammonia (66:33:1) for separation of $\mathrm{Urd}$, $\mathrm{dU}$ from other nucleosides and nucleotides as previously described (Wang, 2007).

Labelling of ThyA protein with $\left[{ }^{32} \mathbf{P}\right]-5 F d U M P$. M. pneumoniae protein extracts (30 mg) were added to a reaction mixture containing $25 \mathrm{mM} \mathrm{MgCl}_{2}$, $0.2 \mathrm{mM}$ methylenetetrahydrofolate, $0.2 \mathrm{mM}$ CMP, $20 \mathrm{mM}$ DTT, and $5 \mu \mathrm{M}\left[{ }^{32} \mathrm{P}\right]$-5FdUMP in a total volume of $25 \mathrm{ml}$. The mixtures were incubated at $37^{\circ} \mathrm{C}$ for $2 \mathrm{~h}$. The reaction was stopped by addition of Laemmli sample buffer (5 $\mu \mathrm{l})$ and $15 \mu \mathrm{l}$ ( 15 $\mu \mathrm{g}$ protein) was resolved by 12\% SDS-PAGE. After electrophoresis, gels were fixed in methanol/acetic acid and the radiolabeled protein bands were visualized by autoradiography.

Enzyme assays. The TK activity was assayed by a radiochemical method using $\left[{ }^{3} \mathrm{H}\right]-\mathrm{dT}$ as a substrate, essentially as described (Wang et al., 2001). TMPK activity was determined by using $\left[{ }^{3} \mathrm{H}\right]$-dTMP as a substrate in a reaction mixture containing 50 mM Tris-HCl pH 8.0, 0.5 mg/ml BSA, 10 mM DTT, 5 mM $\mathrm{MgCl}_{2}, 2$ mM ATP, and $10 \mu \mathrm{M}\left[{ }^{3} \mathrm{H}\right]$-dTMP. The reaction was started by addition of $M$. pneumoniae protein extracts and incubated at $37^{\circ} \mathrm{C}$. At $0,10,20$, and $30 \mathrm{~min}$ intervals a $10 \mu \mathrm{l}$ aliquot was removed and spotted onto DE-81 filter paper and allowed to dry. The unutilized substrate was removed by washing the filter papers three times in $50 \mathrm{mM}$ ammonium formate solution. Finally, the products were eluted with $0.5 \mathrm{ml}$ of $0.1 \mathrm{M} \mathrm{HCl}$ and $0.2 \mathrm{M} \mathrm{KCl}$, and mixed with $2 \mathrm{ml}$ scintillation fluid and the radioactivity counted. The specific activity was calculated as $\mathrm{nmol} /(\mathrm{min} \mathrm{mg})$ protein. 
The TS activity was determined by the tritium release assay with $\left[5-{ }^{3} \mathrm{H}\right]$-dUMP as the labeled substrate, essentially as described (Armstrong and Diasio, 1982). TLC assay was performed with $\left[{ }^{32} \mathrm{P}\right]$-dUMP as the labeled substrate in a reaction mixture containing $50 \mathrm{mM}$ Tris- $\mathrm{HCl} \mathrm{pH}$ 7.5, $5 \mathrm{mM}$ methylenetetrahydrofolate, $10 \mathrm{mM} \mathrm{MgCl}_{2}$, $50 \mathrm{mM}$ NADPH, $10 \mathrm{mM}$ DTT, $50 \mathrm{mM}$ FAD, $15 \mathrm{mM} \mathrm{NaF}$, and $5 \mu \mathrm{M}\left[{ }^{32} \mathrm{P}\right]$-dUMP. The reaction was started by addition of $M$. pneumoniae extracts, incubated at $37^{\circ} \mathrm{C}$, and at each time point a $1.5 \mu \mathrm{l}$ aliquot was removed and spotted onto a PEI-cellulose sheet. The TLC sheet was developed in isobutyric acid/ $\mathrm{H}_{2} \mathrm{O} / \mathrm{ammonia}(66: 33: 1, \mathrm{v} / \mathrm{v})$. The products were visualized by autoradiography.

The TP activity was determined using $\left[{ }^{3} \mathrm{H}\right]-\mathrm{dT}$ as substrate, as previously described (Mizutani et al., 2003). The reaction mixture contained $100 \mathrm{mM}$ potassium phosphate $\mathrm{pH}$ 7.4, $5 \mathrm{mM}$ DTT, and $100 \mu \mathrm{M}\left[{ }^{3} \mathrm{H}\right]-\mathrm{dT}$.

Analysis of TK transcript levels. Total $M$. pneumoniae RNA was prepared as described previously (Halbedel et al., 2004). For slot blot analysis serial twofold dilutions of the RNA in 10× SSC (2 mg to $0.25 \mathrm{mg}$ ) were blotted onto a positively charged nylon membrane using a PR 648 Slot Blot Manifold (Amersham Biosciences). Equal amounts of yeast tRNA (Roche) and M. pneumoniae chromosomal DNA served as controls. A DIG-labeled riboprobe were obtained by in vitro transcription of the PCR product with the primers SS32 (5'-GATTTGTGGGCCGATGTTTTCC) and SS33 (5'CTAATACGACTCACTATAGGGAGACGGTTTCGTTGCTCTTGGAC) that cover a TK (mpn044) internal sequence using T7 RNA polymerase (Roche). The reverse primer SS33 used to generate the PCR product contained a T7 promoter sequence (underlined). The quantification was performed using the Image J software v1.44b (Abramoff et al., 2004).

\section{Acknowledgments}

This work was supported by the Swedish Research Council for Environment, Agricultural Sciences, and Spatial Planning (to L. W.). 


\section{Chapter 6}

\section{Interactions between glycolytic enzymes of Mycoplasma pneumoniae}

The work described in this chapter was published in:

Dutow, P., S. R. Schmidl, M. Ridderbusch, and J. Stülke. 2010. Interactions between glycolytic enzymes of Mycoplasma pneumoniae. J. Mol. Microbiol. Biotechnol. accepted.

Author contributions:

This study was designed by SRS and JS. MR constructed the mutated glycolytic enzyme alleles and PD performed the bacterial two-hybrid analysis. SRS designed the oligonucleotides and supervised the experimental work that was accomplished by MR and PD during their diploma theses. All authors were involved in the interpretation of the collected data. SRS and JS wrote the paper. 


\begin{abstract}
With only 688 protein-coding genes, Mycoplasma pneumoniae is one of the smallest self-replicating organisms. These bacteria use glycolysis as the major pathway for ATP production by substrate-level phosphorylation suggesting that this pathway must be optimized to high efficiency. In this study, we have investigated the interactions between glycolytic enzymes using the bacterial adenylate cyclase-based two-hybrid system. We demonstrate that most of the glycolytic enzymes perform self-interactions suggesting that they form dimers or other oligomeric forms. In addition, enolase was identified as the central glycolytic enzyme of $M$. pneumoniae due to its ability to directly interact with each other glycolytic enzyme. Our results support the idea of the formation of a glycolytic complex in $M$. pneumoniae and we suggest that the formation of this complex might ensure higher fluxes through the glycolytic pathway than would be possible with isolated non-interacting enzymes.
\end{abstract}

\title{
Introduction
}

Mycoplasma pneumoniae, a cell wall-less pathogenic bacterium, is the best-studied representative of the class Mollicutes. These bacteria are derived from Gram-positive ancestors that are related to Bacillus subtilis and lactic acid bacteria. The evolution of the Mollicutes is characterized by substantial genome degeneration that reflects adaptation of the bacteria to a life in close association with eukaryotic hosts (Razin et al., 1998; Stülke et al., 2009).

M. pneumoniae causes usually mild diseases such as pneumonias: However, implication of this bacterium in arthritic, cutaneous or auto-immune diseases as well as in encephalitis was reported (Atkinson et al., 2008; Domenech et al., 2009; Jacobs, 1997; Schalock and Dinulos, 2009; Waites and Talkington, 2004). Although M. pneumoniae is highly adapted to a life on mucosal surfaces, the bacteria are able to live independently of host cells. With less than 700 genes, M. pneumoniae belongs to the smallest organisms that are capable of autonomous life (Himmelreich et al., 1996).

The small genome of $M$. pneumoniae is also reflected by its limited metabolic capabilities. The bacteria can utilize glucose, fructose or glycerol as carbon sources (Halbedel et al., 2004; Yus et al., 2009). These carbohydrates are metabolized via the glycolytic pathway, leading to the concomitant generation of ATP by substrate-level 
phosphorylation. However, M. pneumoniae does not possess an active citric acid cycle; similarly the pentose phosphate shunt is incomplete. While $M$. pneumoniae can produce ATP, nearly all anabolic pathways have been lost during degenerative evolution. Thus, M. pneumoniae depends heavily on the host cells or on organic nutrient supply from the artificial medium (Halbedel et al., 2007; Miles, 1992).

We are interested in the glycolytic pathway. M. pneumoniae encodes all glycolytic enzymes. Interestingly, some of these enzymes have multiple functions: The glycolytic kinases are also active in nucleotide phosphorylation thus replacing the nucleoside diphosphate kinase (Pollack et al., 2002). Moreover, several glycolytic enzymes are subject to phosphorylation. However, it is not known how these phosphorylation events are catalyzed and how they affect the enzymatic activities (Schmidl et al., 2010). For the regeneration of $\mathrm{NAD}^{+}, M$. pneumoniae possesses a lactate dehydrogenase; alternatively the $\mathrm{NAD}^{+}$oxidase may directly oxidize $\mathrm{NADH}$, and the latter reaction is coupled to the production of hydrogen peroxide, the major virulence factor of M. pneumoniae (Cole et al., 1968; Halbedel et al., 2007; Low and Zimkus, 1973).

For a long time it was thought that metabolic pathways take place in an unorganized chaotic way in bacterial cells. However, at the same time there have been data indicating that, e.g. tryptophan metabolism in Escherichia coli involves protein complexes (Yanofsky and Rachmeler, 1958). Similarly, glycolytic protein complexes were reported for E. coli as well as for eukaryotic cells (Mowbray and Moses, 1976; Campanella et al., 2005). The accumulating recent evidence suggests that enzymes of one pathway may be clustered to allow efficient metabolism. This was shown for purine biosynthesis and branched-chain amino acid catabolism in human cells as well as for glycolysis in B. subtilis (An et al., 2008; Commichau et al., 2009; Islam et al., 2007). Moreover, a recent in silico study supports the idea that glycolytic flux is much more efficient if the enzymes form a complex as compared to free floating enzymes (Amar et al., 2008). The analysis of protein complexes in M. pneumoniae suggests that glycolytic enzymes might also interact in this organism (Kühner et al., 2009).

The analysis of primary protein-protein interactions in M. pneumoniae and other Mollicutes is hampered by an alteration of the genetic code in these organisms: They use the UGA codon for the incorporation of tryptophan, whereas it specifies a stop in most other organisms. Due to this problem, the interactions between Mycoplasma 
proteins have so far never been studied using a two-hybrid system. We have developed a method for the quick replacement of stop codons (Hames et al., 2005). Based on this method, we have studied the interactions between all glycolytic enzymes of $M$. pneumoniae using a bacterial two-hybrid (B2H) system. Our results support the idea that these enzymes interact, and that metabolism is well structured even in a seemingly primitive organism.

\section{Results}

Organization of glycolytic genes in M. pneumoniae. In B. subtilis and other Firmicutes, genes encoding glycolytic enzymes from phosphotransferase system (PTS) proteins for glucose transport and phosphorylation to the pyruvate kinase are clustered in five operons (Ludwig et al., 2001). In contrast, the corresponding M. pneumoniae genes are scattered on the chromosome (Fig. 24). This is even the case for the PTS genes in M. pneumoniae that are clustered in most bacteria with a functional PTS sugar transport system (Barabote and Saier, 2005). In M. pneumoniae, three gene clusters with glycolytic genes are present: The $p f k A$-pyk cluster encodes the two glycolytic kinases. These genes also form an operon in $B$. subtilis. The second cluster is made up of the pgk and gapA genes encoding phosphoglycerate kinase and glyceraldehyde-3-phosphate dehydrogenase, respectively. These genes are clustered in most bacteria, and it has been suggested that the clustering of these genes might reflect physical interaction of the encoded enzymes, which catalyze consecutive reactions (Dandekar et al., 1998). The third glycolytic cluster is formed by the ptsI, pgm, and tpiA genes encoding enzyme I of the PTS, phosphoglycerate mutase, and triose-phosphate isomerase, respectively. The pgm and tpiA genes are also part of one operon in B. subtilis (Ludwig et al., 2001).

Interactions between glycolytic enzymes. In order to identify possible primary interactions between glycolytic enzymes, we made use of the $\mathrm{B} 2 \mathrm{H}$ system. In this system, the T25 and the T18 fragments of the catalytic domain of the Bordetella pertussis adenylate cyclase were fused to full-length copies of all glycolytic enzymes of M. pneumoniae. The leucine zipper of the yeast GCN4 transcription factor served as a control (Karimova et al., 1998). The results of the B2H analysis are shown in Fig. 25. As expected, the leucine zipper of GCN4 showed strong self-interaction. 


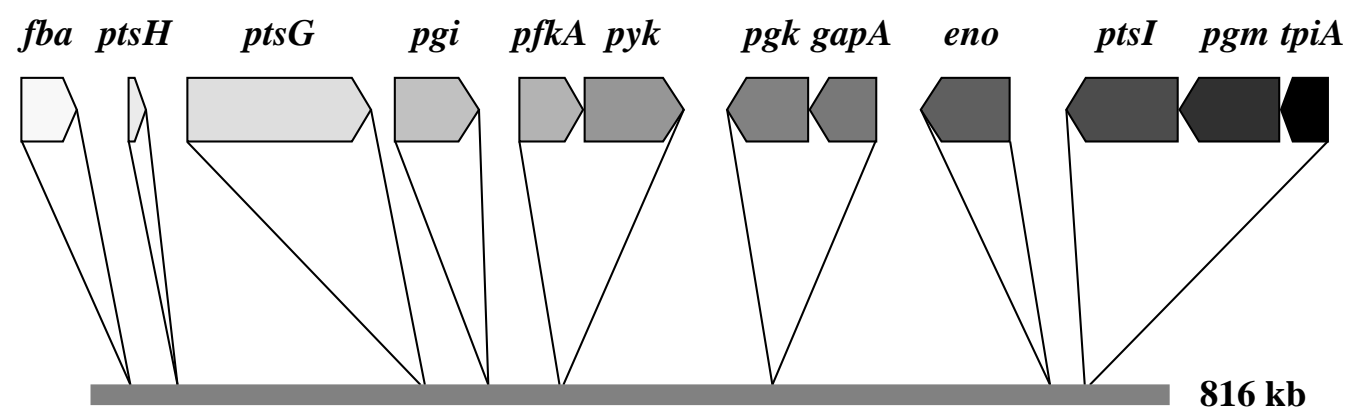

Fig. 24. Genomic localization of glycolytic genes in $\boldsymbol{M}$. pneumoniae. The $816 \mathrm{~kb}$ genome of M. pneumoniae is shown true to scale in grey. Genes coding for glycolytic enzymes are illustrated relative to their position on the genome. eno, enolase; $f b a$, fructose-bisphosphate aldolase; gapA, glyceraldehyde-3-phosphate dehydrogenase; $p f k A$, phosphofructokinase; pgi, glucose-6-phosphate isomerase; pgk, phosphoglycerate kinase; pgm, phosphoglycerate mutase; ptsG, PTS system glucose-specific EIICBA component; ptsH, phosphocarrier protein HPr; ptsI, enzyme I; pyk, pyruvate kinase; tpiA, triose-phosphate isomerase.

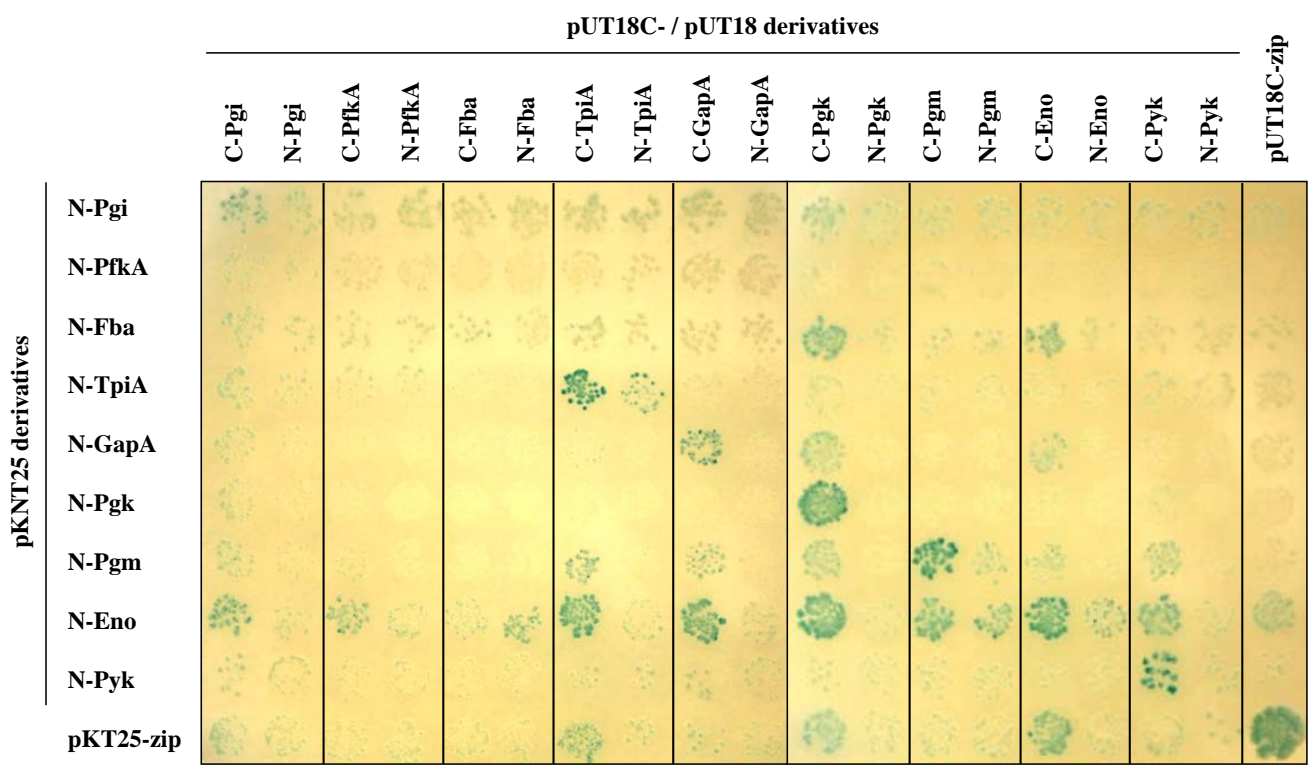

Fig. 25. Bacterial two-hybrid (B2H) analysis to identify interactions among glycolytic enzymes. All genes were cloned in the plasmids pUT18, pUT18C, and pKNT25. Plasmids pUT18 and pUT18C allow the expression of the selected enzymes fused either to the $\mathrm{N}$ - or the C-terminus of the T18 domain of the B. pertussis adenylate cyclase, respectively. Plasmid pKNT25 allows the expression of the selected enzymes fused either to the N-terminus of the T25 domain of the adenylate cyclase, respectively. The plasmids pKT25-zip and pUT18C-zip served as positive controls for complementation. The E. coli transformants were incubated for $48 \mathrm{~h}$ at $30^{\circ} \mathrm{C}$. The level of protein-protein interaction was analyzed by observation of the blue coloration of the colonies. 
As shown in Fig. 25, we observed self-interactions for all glycolytic enzymes with the exception of phosphofructokinase and fructose-bisphosphate aldolase. In addition to self-interactions, we detected several interactions between different glycolytic enzymes (see Fig. 25, Fig. 26). Of all glycolytic enzymes, enolase is involved in the largest number of interactions with other enzymes. We observed interactions of enolase with all other glycolytic enzymes. The strongest interactions were detected between enolase and triose-phosphate isomerase, glyceraldehyde-3-phosphate dehydrogenase, phosphoglycerate kinase, phosphoglycerate mutase, and pyruvate kinase. A strong interaction was also detected between phosphoglycerate kinase and fructose-bisphosphate aldolase. Weaker interactions were observed between enolase and glucose-6-phosphate isomerase, phosphofructokinase, and fructose-bisphosphate aldolase. Moreover, we observed weak interactions between glyceraldehyde-3-phosphate dehydrogenase and phosphoglycerate kinase as well as between triose-phosphate isomerase and phosphoglycerate mutase. These latter interactions reflect the genomic organization with pgk-gapA and pgm-tpiA gene clusters (see Fig. 24). Finally, faint interactions were observed between phosphoglycerate mutase on the one hand and glyceraldehyde-3-phosphate dehydrogenase, phosphoglycerate kinase, and pyruvate kinase on the other. A very weak interaction was also detectable between phosphoglycerate kinase and glucose-6-phosphate isomerase.

Thus, all glycolytic enzymes are involved in interactions with other enzymes of the pathway suggesting that glycolysis proceeds in a well organized and structured manner in M. pneumoniae.

\section{Discussion}

Glycolysis is the major pathway for the generation of energy by substrate-level phosphorylation in M. pneumoniae and most other Mollicutes. In good agreement with both the importance for cellular metabolism and the ancient origin of the glycolytic pathway is the observation that the genes encoding glycolytic enzymes are essential in many organisms and that these enzymes have additional functions that are completely unrelated to the biochemical pathway (Canback et al., 2002; Commichau et al., 2009; Kim and Kang, 2005). 


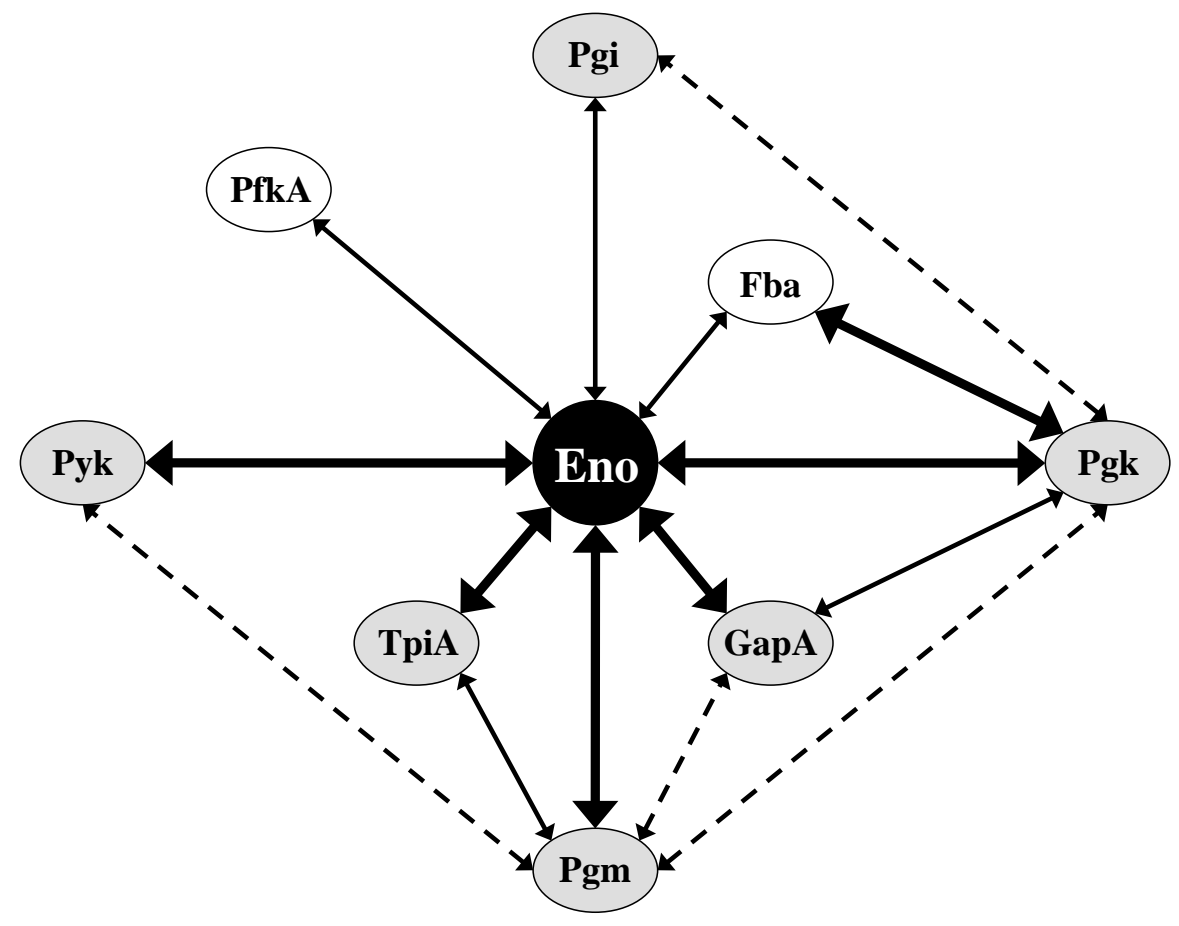

Fig. 26. Schematic summary of the outcome of the B2H analysis. The interaction network of glycolytic enzymes is depicted with the Enolase (Eno) as the core protein. Proteins that are able to form homomultimeric complexes are highlighted. Dashed arrows indicate weak interactions, whereas regular or bold black arrows point out moderate and strong interactions, respectively.

In this study, we have addressed the interactions between the glycolytic enzymes themselves. A recent proteome-level analysis of protein complexes in M. pneumoniae (Kühner et al., 2009) as well as experiments with the Gram-positive model bacterium B. subtilis (Commichau et al., 2009) provided indications for such interactions. In order to identify the primary interactions between the glycolytic enzymes, we applied the technique of two-hybrid analysis for the first time to proteins from a Mycoplasma species. Our studies revealed self-interactions that may correspond to the formation of dimers or other homo-oligomers for all glycolytic enzymes except phosphofructokinase and fructose-bisphosphate aldolase. This observation is in good agreement with the known structures of glycolytic enzymes that indicate the formation of dimers or tetramers (Erlandsen et al., 2000).

Our study indicates that enolase is of central importance in the organisation of glycolysis in $M$. pneumoniae since this is the only enzyme that is capable of interacting directly with any other glycolytic enzyme (see Fig. 26). Moreover, most of the strongest interactions involve enolase. Another interesting conclusion from this work is that the 
enzymes of the lower part of glycolysis are much more strongly embedded in the interaction network than those of the hexose phosphate part (Fig. 26). This reflects that the triose-phosphate interconversion is the key module of the whole pathway in the living world, whereas the first intermediates of this part, glyceraldehyde 3-phosphate and dihydroxyacetone phosphate may be obtained from very different sources including the upper part of glycolysis, the pentose phosphate pathway, the Entner-Doudoroff pathway, or from glycerol phosphate dehdrogenase/oxidase.

In their proteome-level analysis, Kühner et al. (2009) proposed two glycolytic complexes in $M$. pneumoniae. One of these complexes consists of glyceraldehyde-3-phosphate dehydrogenase, phosphoglycerate kinase, and enolase. Our results are in support of this complex and provide compelling evidence that these three enzymes are capable of interacting directly with each other. The second complex was suggested to be composed of glyceraldehyde-3-phosphate dehydrogenase, pyruvate kinase and fructose-bisphosphate aldolase. We did not observe primary interactions between any couple of these three proteins. However, glyceraldehyde-3-phosphate dehydrogenase, as a member of the first complex, does directly bind to enolase, and we observe direct interaction between enolase and the two other enzymes. In any case, it seems difficult to name distinct complexes from in vivo pull-down studies that have the same members. Finally, Kühner et al. (2009) suggested a complex made up of enolase, triose-phosphate isomerase, a metalloprotease (MPN569), and an unknown protein (MPN316). The interactions between the two glycolytic enzymes is supported by our analysis. It is interesting to note that enolase may be involved in interactions that are not related to glycolysis. This has also been observed in E. coli and B. subtilis. In both organisms, enolase is part of the RNA-degrading multiprotein complex called RNA degradosome (Carpousis, 2007; Commichau et al., 2009).

In conclusion, our results support the idea that glycolytic enzymes interact in M. pneumoniae. However, in contrast to previous findings, we suggest the presence of one complex of glycolytic enzymes that is organized around enolase. It is tempting to speculate that such a complex may be important for the efficiency of the glycolytic pathway, and thus for the efficiency of energy production in $M$. pneumoniae. 


\section{Experimental procedures}

Bacterial strains and growth conditions. E. coli XL1-Blue (Stratagene) and BTH101 (Karimova et al., 1998) were used for cloning experiments and B2H analyses, respectively. E. coli was grown in LB medium. LB plates were prepared by the addition of $18 \mathrm{~g}$ Bacto agar/liter (Difco) to LB medium.

DNA manipulation and transformation. Transformation of E. coli and plasmid DNA extraction were performed using standard procedures (Sambrook et al., 1989). Restriction enzymes, T4 DNA ligase, and DNA polymerases were used as recommended by the manufacturers. DNA fragments were purified from agarose gels using the Nucleospin Extract Kit (Macherey and Nagel, Germany). Phusion DNA polymerase was used for the polymerase chain reaction as recommended by the manufacturer. All primer sequences are listed in the supplemental Table S7. DNA sequences were determined using the dideoxy chain termination method (Sambrook et al., 1989). All plasmid inserts derived from PCR products were verified by DNA sequencing. E. coli transformants were selected on LB plates containing ampicillin $(100 \mu \mathrm{g} / \mathrm{ml})$ or kanamycin $(50 \mu \mathrm{g} / \mathrm{ml})$.

Plasmid constructions. The genes encoding the glycolytic enzymes from M. pneumoniae were first subcloned as follows. The coding sequence of each gene was amplified by PCR with gene specific primers using chromosomal DNA of M. pneumonia M129 as the template. The PCR products were digested as appropriate with BamHI and HindIII or with BamHI and PstI and cloned into the appropriately linearized expression vector pWH844 (Schirmer et al., 1997). These plasmids allowed the expression of the glycolytic enzymes carrying a N-terminal His 6 -tag. The resulting plasmids are listed in the supplemental Table S8. In M. pneumoniae, the UGA specifies a tryptophan; however, in E. coli it is a stop codon. With exception of $p f k A$ and $p g m$, the glycolytic genes contain one to three UGA codons that were replaced by the multiple mutation reaction (Hames et al., 2005) using phosphorylated mutagenic oligonucleotides and the former plasmids as template. The resulting final plasmids are listed in the supplemental material (see Table S8).

To obtain the plasmids for the $\mathrm{B} 2 \mathrm{H}$ analyses, the coding sequences of the glycolytic genes were amplified by PCR using the latter plasmids. The PCR products were digested as shown in the supplemental material, and the resulting fragments were cloned into each of the three plasmids pKNT25, pUT18, and pUT18C (Claessen et al., 
2008; Karimova et al., 1998), digested with the same enzymes. The resulting plasmids used for the B2H analyses are shown in the supplemental Table S8. All plasmid inserts were verified by DNA sequencing.

Bacterial two-hybrid analysis. Primary protein-protein interactions were identified by B2H analysis (Karimova et al., 1998). The B2H system is based on the interaction-mediated reconstruction of adenylate cyclase (CyaA) activity from B. pertussis in E. coli. The CyaA enzyme consists of two complementary fragments T18 and T25 that are not active when physically separated. Fusion of these fragments to interacting proteins results in functional complementation between the T18 and T25 fragments and the synthesis of cAMP. cAMP production can be monitored by measuring the $\beta$-galactosidase activity of the cAMP-CAP-dependent promoter of the E. coli lac operon. Thus, a high $\beta$-galactosidase activity reflects the interaction between the hybrid proteins. Plasmids pUT18 and pKNT25 allow the expression of proteins fused to the N-terminus of the T18 and T25 fragments of the CyaA protein, respectively, and the plasmid pUT18C allows the expression of proteins fused to the C-terminus of the T18 fragment of the CyaA protein, respectively (Claessen et al., 2008; Karimova et al., 1998). The plasmids pKT25-zip and pUT18C-zip served as positive controls for complementation. These plasmids express T18-zip and T25-zip fusion proteins that can associate due to the leucine zipper motifs resulting in an active CyaA enzyme and a high $\beta$-galactosidase activity. The plasmids constructed for the B2H assay (see Table S8) were used for cotransformations of E. coli BTH101 and the protein-protein interactions were then analyzed by plating the cells on LB plates containing ampicillin $(100 \mu \mathrm{g} / \mathrm{ml})$, kanamycin $(50 \mu \mathrm{g} / \mathrm{ml})$, X-Gal $(40 \mu \mathrm{g} / \mathrm{ml})$ and IPTG $(0.5 \mathrm{mM})$. The plates were incubated for a maximum of $60 \mathrm{~h}$ at $30^{\circ} \mathrm{C}$. The $\mathrm{B} 2 \mathrm{H}$ assays were performed in triplicate.

\section{Acknowledgments}

We are grateful to Julia Busse, Henrike Pförtner, Martin Arnold, and Frederik Meyer for helpful discussions. This work was supported by Deutsche Forschungsgemeinschaft and the Fonds der Chemischen Industrie. S. R. S. was supported by a personal grant from the Studienstiftung des Deutschen Volkes. 


\section{Supplemental material}

Tab. S7. Primers used in this study.

\begin{tabular}{|c|c|c|c|}
\hline Primer & Sequence $\left(5^{\prime} \rightarrow 3^{\prime}\right)^{\mathrm{ab}}$ & Mutation & Resulting plasmid \\
\hline \multicolumn{4}{|c|}{ Subcloning for multiple mutation reaction } \\
\hline JS39 & TCTATCAACAGGAGTCCAAGC & None & \\
\hline \multirow[t]{2}{*}{ MR04 } & AAAGGATCCATGGTTGACTTTAAAACAG & None & pGP1006, pGP1010 \\
\hline & TCCAAGC & & \\
\hline \multirow[t]{2}{*}{ MR05 } & TATAAAGCTTTTACTTTTTTTGAATATCG & None & pGP1006, pGP1010 \\
\hline & СТААТТСССАС & & \\
\hline MR06 & P_GTTAGCACAGTTCTGGGCATCATTGGG & A441G & pGP1010 \\
\hline MR07 & P_CCCAATGGCCAGCAGAACTTCAGG & A894G & pGP1010 \\
\hline \multirow[t]{2}{*}{ MR08 } & AAAGGATCCATGCATAAGAAGGTCTTAC & None & pGP1007 \\
\hline & TAGCC & & \\
\hline \multirow[t]{2}{*}{ MR09 } & TATACTGCAGTTACTTTTTACTTATTAAA & None & pGP1007 \\
\hline & AGGGAGTTTGCG & & \\
\hline \multirow[t]{2}{*}{ MR10 } & AAAGGATCCATGAGTGCACAAACTGGA & None & pGP1008 \\
\hline & ACCGATCTTTTCAAG & & \\
\hline \multirow[t]{2}{*}{ MR11 } & TATACTGCAGTTAAGCTTTTTGCGGTTTA & A1335G & pGP1008 \\
\hline & ATGTTTTTAAAGGTGTTCCAACC & & \\
\hline \multirow[t]{2}{*}{ MR19 } & AAAGGATCCATGGAAAGTAAATGGTTAA & None & pGP756, pGP762 \\
\hline & CAGTTGAC & & \\
\hline \multirow[t]{2}{*}{ MR20 } & TATAAAGCTTCTATTCACGACCAAGTTT & None & pGP756, pGP762 \\
\hline & AGCAAAC & & \\
\hline \multirow[t]{2}{*}{ MR21 } & P_GGGTAAATCATGGGCTTTGGTAGTAA & A393G & pGP762 \\
\hline & CTTC & & \\
\hline MR22 & P_CTGCTTTGCGTCACTGGCTTTACAC & A756G & pGP762 \\
\hline \multirow[t]{2}{*}{ MR23 } & AAAGGATCCATGAGTCCAAAAACAACC & None & pGP757 \\
\hline & AAAAAAATTGCC & & \\
\hline \multirow[t]{2}{*}{ MR24 } & TATAAAGCTTTTAAATAATATTTTTATTA & None & pGP757 \\
\hline & ATGACTGCAATTAAAGCGC & & \\
\hline \multirow[t]{2}{*}{ MR25 } & AAAGGATCCATGCTAGTAAACATCAAAC & None & pGP758, pGP763 \\
\hline & AAATGTTGCAAC & & \\
\hline \multirow[t]{2}{*}{ MR26 } & TATAAAGCTTCTAAGCCTTATTGGTTGA & None & pGP758, pGP763 \\
\hline & ACCACAG & & \\
\hline MR27 & P_GGCTTTACCCCGACAACTGGAAGGG & A552G & pGP763 \\
\hline \multirow[t]{2}{*}{ MR28 } & AAAGGATCCATGCGTACGAAATACCTAA & A30G & pGP759, pGP764 \\
\hline & TTGGTAACTGGAAG & & \\
\hline
\end{tabular}


Tab. S7. Continued.

\begin{tabular}{|c|c|c|c|}
\hline Primer & Sequence $\left(5^{\prime} \rightarrow 3^{\prime}\right)^{\mathrm{ab}}$ & Mutation & Resulting plasmid \\
\hline MR29 & $\begin{array}{l}\text { TATACTGCAGTTATGCATATACTTGTGCC } \\
\text { ATTACTAAAAAGTCG }\end{array}$ & None & pGP759, pGP764 \\
\hline MR30 & $\begin{array}{l}\text { P_GTGATTGCTTACGAACCAATTTGGGCA } \\
\text { ATTGGTACGG }\end{array}$ & A489G & pGP764 \\
\hline MR31 & $\begin{array}{l}\text { AAAGGATCCATGCTAGCAAAGAGTAAG } \\
\text { АСTATCC }\end{array}$ & None & pGP760, pGP765 \\
\hline MR32 & $\begin{array}{l}\text { TATACTGCAGTTAAAGCTTGGCACAATA } \\
\text { GTTAACTAC }\end{array}$ & None & pGP760, pGP765 \\
\hline MR33 & P_GCTAACCTGCCATGGGCAGAACAC & A270G & pGP765 \\
\hline MR34 & $\begin{array}{l}\text { AAAGGATCCATGATTCACCACCTAAAAC } \\
\text { GCAC }\end{array}$ & None & pGP761, pGP766 \\
\hline MR35 & $\begin{array}{l}\text { TATACTGCAGTTATAAGCTAATGATCTT } \\
\text { ATTGTGAAATACTC }\end{array}$ & None & pGP761, pGP766 \\
\hline MR36 & $\begin{array}{l}\text { P_GCTATGGACGCTAGCAATGTTAGATG } \\
\text { AC }\end{array}$ & A69G & pGP766 \\
\hline MR37 & P_CAAGGTGCCTTACTGGCAACGGTAC & A864G & pGP766 \\
\hline MR38 & P_CAGTGAGTTTTGGAAGCAGGTGGTG & A1167G & pGP766 \\
\hline pWH844 fw & TATGAGAGGATCGCATCACCAT & None & \\
\hline \multicolumn{4}{|c|}{ Bacterial two-hybrid analysis } \\
\hline FC146 & CGATGCGTTCGCGATCCAGGC & None & - \\
\hline FC147 & CCAGCCTGATGCGATTGCTGCAT & None & - \\
\hline FC148 & GTCACCCGGATTGCGGCGG & None & - \\
\hline FC149 & GCTGGCTTAACTATGCGGCATCAGA & None & - \\
\hline M13 puc rev & GGAAACAGCTATGACCATG & None & - \\
\hline PD05 & $\begin{array}{l}\text { AAATCTAGAGATGGAAAGTAAATGGTTA } \\
\text { ACAGTTGACAC }\end{array}$ & None & $\begin{array}{l}\text { pGP1553, pGP1563, } \\
\text { pGP1573 }\end{array}$ \\
\hline PD06 & $\begin{array}{l}\text { TTTGGTACCCGTTCACGACCAAGTTTAG } \\
\text { CAAACATTAAC }\end{array}$ & None & $\begin{array}{l}\text { pGP1553, pGP1563, } \\
\text { pGP1573 }\end{array}$ \\
\hline PD07 & $\begin{array}{l}\text { AAATCTAGAGATGAGTCCAAAAACAACC } \\
\text { AAAAAAATTGCC }\end{array}$ & None & $\begin{array}{l}\text { pGP1554, pGP1564, } \\
\text { pGP1574 }\end{array}$ \\
\hline PD08 & $\begin{array}{l}\text { TTTGGTACCCGAATAATATTTTTATTAAT } \\
\text { GACTGCAATTAAAGCGC }\end{array}$ & None & $\begin{array}{l}\text { pGP1554, pGP1564, } \\
\text { pGP1574 }\end{array}$ \\
\hline PD09 & $\begin{array}{l}\text { AAATCTAGAGATGCTAGTAAACATCAAA } \\
\text { CAAATGTTGCAAC }\end{array}$ & None & $\begin{array}{l}\text { pGP1555, pGP1565, } \\
\text { pGP1575 }\end{array}$ \\
\hline PD10 & $\begin{array}{l}\text { TTTGAGCTCCGAGCCTTATTGGTTGAACC } \\
\text { ACAGAG }\end{array}$ & None & $\begin{array}{l}\text { pGP1555, pGP1565, } \\
\text { pGP1575 }\end{array}$ \\
\hline
\end{tabular}


Tab. S7. Continued.

\begin{tabular}{|c|c|c|c|}
\hline Primer & Sequence $\left(5^{\prime} \rightarrow 3^{\prime}\right)^{\mathrm{ab}}$ & Mutation & Resulting plasmid \\
\hline PD11 & $\begin{array}{l}\text { AAATCTAGAGATGCGTACGAAATACCTA } \\
\text { ATTGGTAAC }\end{array}$ & None & $\begin{array}{l}\text { pGP1556, pGP1566, } \\
\text { pGP1576 }\end{array}$ \\
\hline PD12 & $\begin{array}{l}\text { TTTGGTACCCGTGCATATACTTGTGCCAT } \\
\text { TACTAAAAAGTC }\end{array}$ & None & $\begin{array}{l}\text { pGP1556, pGP1566, } \\
\text { pGP1576 }\end{array}$ \\
\hline PD13 & $\begin{array}{l}\text { AAATCTAGAGATGCTAGCAAAGAGTAA } \\
\text { GACTATCCG }\end{array}$ & None & $\begin{array}{l}\text { pGP1557, pGP1567, } \\
\text { pGP1577 }\end{array}$ \\
\hline PD14 & $\begin{array}{l}\text { TTTGAATTCCGAAGCTTGGCACAATAGT } \\
\text { TAACTACCC }\end{array}$ & None & $\begin{array}{l}\text { pGP1557, pGP1567, } \\
\text { pGP1577 }\end{array}$ \\
\hline PD15 & $\begin{array}{l}\text { AAATCTAGAGATGGTTGACTTTAAAACA } \\
\text { GTCCAAGC }\end{array}$ & None & $\begin{array}{l}\text { pGP1558, pGP1568, } \\
\text { pGP1578 }\end{array}$ \\
\hline PD16 & $\begin{array}{l}\text { TTTGAATTCCGCTTTTTTTGAATATCGCT } \\
\text { AATTCCCACTAG }\end{array}$ & None & $\begin{array}{l}\text { pGP1558, pGP1568, } \\
\text { pGP1578 }\end{array}$ \\
\hline PD17 & $\begin{array}{l}\text { AAATCTAGAGATGCATAAGAAGGTCTTA } \\
\text { CTAGCC }\end{array}$ & None & $\begin{array}{l}\text { pGP1559, pGP1569, } \\
\text { pGP1579 }\end{array}$ \\
\hline PD18 & $\begin{array}{l}\text { TTTGAATTCCGCTTTTTACTTATTAAAAG } \\
\text { GGAGTTTGCG }\end{array}$ & None & $\begin{array}{l}\text { pGP1559, pGP1569, } \\
\text { pGP1579 }\end{array}$ \\
\hline PD19 & $\begin{array}{l}\text { AAATCTAGAGATGAGTGCACAAACTGGA } \\
\text { ACCG }\end{array}$ & None & $\begin{array}{l}\text { pGP1560, pGP1570, } \\
\text { pGP1580 }\end{array}$ \\
\hline PD20 & $\begin{array}{l}\text { TTTGAATTCCGAGCTTTTTGCGGTTTAAT } \\
\text { GTTTTTAAAGGTG }\end{array}$ & None & $\begin{array}{l}\text { pGP1560, pGP1570, } \\
\text { pGP1580 }\end{array}$ \\
\hline PD21 & $\begin{array}{l}\text { AAATCTAGAGATGATTCACCACCTAAAA } \\
\text { CGCAC }\end{array}$ & None & $\begin{array}{l}\text { pGP1561, pGP1571, } \\
\text { pGP1581 }\end{array}$ \\
\hline PD22 & $\begin{array}{l}\text { TTTGGTACCCGTAAGCTAATGATCTTATT } \\
\text { GTGAAATACTCC }\end{array}$ & None & $\begin{array}{l}\text { pGP1561, pGP1571, } \\
\text { pGP1581 }\end{array}$ \\
\hline
\end{tabular}

\footnotetext{
${ }^{\mathrm{a}}$ Restriction sites are underlined.

b The "P" at the 5' end of primer sequences indicates phosphorylation.
} 
Tab. S8. Plasmids used for analysis of glycolytic enzymes.

\begin{tabular}{|c|c|c|c|}
\hline Plasmid & Relevant characteristics $^{\mathbf{a}}$ & $\begin{array}{l}\text { Used restriction } \\
\text { sites }\end{array}$ & Reference \\
\hline \multicolumn{4}{|c|}{ Subcloning for multiple mutation reaction } \\
\hline pGP756 & pWH844-pgi & BamHI + HindIII & This work \\
\hline pGP757 & pWH844-pfkA & BamHI + HindIII & This work \\
\hline pGP758 & pWH844-fba & BamHI + HindIII & This work \\
\hline pGP759 & pWH844-tpiA(A30G) & BamHI + PstI & This work \\
\hline pGP760 & pWH844-gapA & BamHI + PstI & This work \\
\hline pGP761 & pWH844-pyk & BamHI + PstI & This work \\
\hline pGP762 & pWH844-pgi(A393G+A756G) & BamHI + HindIII & This work \\
\hline pGP763 & pWH844-fba(A552G) & BamHI + HindIII & This work \\
\hline pGP764 & pWH844-tpiA(A30G+A489G) & BamHI + PstI & This work \\
\hline pGP765 & pWH844-gapA(A270G) & BamHI + PstI & This work \\
\hline pGP766 & pWH844-pyk(A69G+A864G+A1167G) & BamHI + PstI & This work \\
\hline pGP1006 & pWH844-pgk & BamHI + HindIII & This work \\
\hline pGP1007 & pWH844-pgm & BamHI + PstI & This work \\
\hline pGP1008 & pWH844-eno(A1335G) & BamHI + PstI & This work \\
\hline pGP1010 & pWH844-pgk(A441G+A894G) & BamHI + HindIII & This work \\
\hline pWH844 & $\begin{array}{l}\text { Allows overexpression of N-terminal } \\
\text { His }_{6} \text {-tag fusion proteins in E. coli DH5 } \alpha \\
\left(\mathrm{Amp}^{\mathrm{R}}\right)\end{array}$ & - & Schirmer et al. (1997) \\
\hline \multicolumn{4}{|c|}{ Bacterial two-hybrid analysis } \\
\hline pGP1553 & pUT18C-pgi(A393G+A756G) & $X b a \mathrm{I}+K p n \mathrm{I}$ & This work \\
\hline pGP1554 & pUT18C-pfkA & $X b a I+K p n I$ & This work \\
\hline pGP1555 & pUT18C-fba(A552G) & $X b a \mathrm{I}+S a c \mathrm{I}$ & This work \\
\hline pGP1556 & pUT18C-tpiA(A30G+A489G) & $X b a \mathrm{I}+K p n I$ & This work \\
\hline pGP1557 & pUT18C-gapA(A270G) & $X b a \mathrm{I}+E c o R I$ & This work \\
\hline pGP1558 & pUT18C-pgk(A441G+A894G) & $X b a \mathrm{I}+$ EcoRI & This work \\
\hline pGP1559 & pUT18C-pgm & $X b a \mathrm{I}+$ EcoRI & This work \\
\hline pGP1560 & pUT18C-eno(A1335G) & $X b a \mathrm{I}+$ EcoRI & This work \\
\hline pGP1561 & pUT18C-pyk $(A 69 G+A 864 G+A 1167 G)$ & $X b a \mathrm{I}+K p n I$ & This work \\
\hline pGP1563 & pUT18-pgi(A393G+A756G) & $X b a \mathrm{I}+K p n I$ & This work \\
\hline pGP1564 & pUT18-pfkA & $X b a \mathrm{I}+K p n I$ & This work \\
\hline pGP1565 & pUT18-fba(A552G) & $X b a \mathrm{I}+S a c \mathrm{I}$ & This work \\
\hline pGP1566 & pUT18-tpiA(A30G+A489G) & $X b a \mathrm{I}+K p n \mathrm{I}$ & This work \\
\hline pGP1567 & pUT18-gapA(A270G) & $X b a \mathrm{I}+$ EcoRI & This work \\
\hline pGP1568 & pUT18-pgk(A441G+A894G) & $X b a \mathrm{I}+$ EcoRI & This work \\
\hline pGP1569 & pUT18-pgm & $X b a \mathrm{I}+E c o R I$ & This work \\
\hline
\end{tabular}


Tab. S8. Continued.

\begin{tabular}{|c|c|c|c|}
\hline Plasmid & Relevant characteristics $^{a}$ & $\begin{array}{l}\text { Used restriction } \\
\text { sites }\end{array}$ & Reference \\
\hline pGP1570 & pUT18-eno(A1335G) & XbaI + EcoRI & This work \\
\hline pGP1571 & pUT18-pyk(A69G+A864G+A1167G) & $X b a I+K p n I$ & This work \\
\hline pGP1573 & pKNT25-pgi(A393G+A756G) & $X b a \mathrm{I}+K p n I$ & This work \\
\hline pGP1574 & pKNT25-pfkA & $X b a \mathrm{I}+K p n I$ & This work \\
\hline pGP1575 & pKNT25-fba(A552G) & $X b a \mathrm{I}+\mathrm{SacI}$ & This work \\
\hline pGP1576 & pKNT25-tpiA(A30G+A489G) & $X b a \mathrm{I}+K p n \mathrm{I}$ & This work \\
\hline pGP1577 & pKNT25-gapA(A270G) & $X b a \mathrm{I}+$ EcoRI & This work \\
\hline pGP1578 & pKNT25-pgk(A441G+A894G) & $X b a I+E c o R I$ & This work \\
\hline pGP1579 & pKNT25-pgm & $X b a \mathrm{I}+$ EcoRI & This work \\
\hline pGP1580 & pKNT25-eno(A1335G) & $X b a \mathrm{I}+$ EcoRI & This work \\
\hline pGP1581 & pKNT25-pyk $(A 69 G+A 864 G+A 1167 G)$ & $X b a \mathrm{I}+K p n \mathrm{I}$ & This work \\
\hline pKNT25 & $P_{l a c}-\mathrm{mcs}-c y a A\left(\operatorname{Kan}^{\mathrm{R}}\right)$ & - & Claessen et al. (2008) \\
\hline pKT25-zip & $P_{l a c}-c y a A-z i p\left(\operatorname{Kan}^{\mathrm{R}}\right)$ & - & Karimova et al. (1998) \\
\hline pUT18 & $P_{l a c}-\mathrm{mcs}-c y a A\left(\mathrm{Amp}^{\mathrm{R}}\right)$ & - & Karimova et al. (1998) \\
\hline pUT18C & $P_{\text {lac }}$-cyaA-mcs $\left(\mathrm{Amp}^{\mathrm{R}}\right)$ & - & Karimova et al. (1998) \\
\hline pUT18C-zip & $P_{\text {lac }}$-cyaA-zip $\left(\mathrm{Amp}^{\mathrm{R}}\right)$ & - & Karimova et al. (1998) \\
\hline
\end{tabular}

${ }^{\mathrm{a}}$ Resistance gene abbreviations as follows: Amp, ampicillin; Kan, kanamycin. 


\section{Chapter 7}

\section{The hidden pathway: \\ Impact of the glycerophosphodiesterase GlpQ on virulence of Mycoplasma pneumoniae}

The work described in this chapter was submitted for publication in:

Schmidl, S. R., A. Otto, M. Lluch-Senar, J. Piñol, J. Busse, D. Becher, and J. Stülke. The hidden pathway: Impact of the glycerophosphodiesterase GlpQ on virulence of Mycoplasma pneumoniae. PLoS Pathogens submitted.

Author contributions:

This study was designed and interpreted by SRS. The cell morphology experiments were accomplished by MLS and JP, University of Barcelona. SRS performed all biochemical and physiological researches, but JB contributed substantially to the slot blot analyses. The proteomic analysis was done in collaboration with $\mathrm{AO}$ and $\mathrm{DB}$, University of Greifswald. SRS and JS wrote the paper. 


\section{Abstract}

Mycoplasma pneumoniae is the causative agent of atypical pneumonia. The formation of hydrogen peroxide, a product of glycerol metabolism, is the major factor that damages the host cells. Phosphatidylcholine is the major carbon source available on lung epithelia, and their utilization requires the cleavage of deacylated phospholipids to glycerol 3-phosphate and choline. M. pneumoniae possesses two potential glycerophosphodiesterases, MPN420 (GlpQ) and MPN566. In this work, the function of these proteins was analyzed by biochemical, genetic, and physiological studies. The results indicate that only GlpQ is an active glycerophosphodiesterase. MPN566 has no enzymatic activity and the inactivation of the gene did not result in any detectable phenotype. Inactivation of the $g l p Q$ gene resulted in reduced growth in medium with glucose as the carbon source, in loss of hydrogen peroxide production when phosphatidylcholine was present, and in a complete loss of cytotoxicity toward HeLa cells. Moreover, the $g l p Q$ mutant strain exhibited a reduced gliding velocity. A comparison of the proteomes of the wild type and the $g l p Q$ mutant strain revealed that this enzyme is also implicated in the control of gene expression. Several proteins were present in higher or lower amounts in the mutant. This regulation by GlpQ is exerted at the level of transcription as determined by mRNA slot blot analyses. All genes subject to GlpQ-dependent control have a conserved potential cis-acting element upstream of the coding region. This element overlaps the promoter in the case of the genes that are repressed in a GlpQ-dependent manner and it is located upstream of the promoter for GlpQ-activated genes. We may suggest that GlpQ acts as a trigger enzyme that measures the availability of its product glycerol 3-phosphate and uses this information to differentially control gene expression.

\section{Author summary}

Mycoplasma pneumoniae serves as a model organism for bacteria with very small genomes that are nonetheless independently viable. These bacteria infect the human lung and cause a specific pneumonia. The major virulence determinant of $M$. pneumoniae is hydrogen peroxide that is generated during the utilization of glycerol 3-phosphate, which might be derived from free glycerol or from the degradation of phospholipids. Indeed, lecithin is the by far most abundant carbon source 
on lung epithelia. In this study, we made use of the recent availability of methods to isolate mutants of $M$. pneumoniae and characterized the enzyme that generates glycerol 3-phosphate from deacylated lecithin (glycerophosphorylcholine). This enzyme, called GlpQ, is essential for the formation of hydrogen peroxide when the bacteria are incubated with glycerophosphorylcholine. Moreover, M. pneumoniae is unable to cause any damage to the host cells in the absence of GlpQ. This underlines the important role of phospholipid metabolism for the virulence of $M$. pneumoniae. We observed that GlpQ in addition to its enzymatic activity does also control the expression of several genes, among them the glycerol transporter. Thus, GlpQ is central to the normal physiology and to pathogenicity of the minimal pathogen M. pneumoniae.

\section{Introduction}

Pathogenic bacteria have developed a large battery of enzymes and mechanisms for extracting nutrients from their hosts, and the requirement for nutrient acquisition can be regarded as one of the driving forces for virulence (Eisenreich et al., 2010; Görke and Stülke, 2008; Sonenshein, 2007). In consequence, the metabolic capabilities of a pathogen reflect its adaptation to a particular niche in a particular host.

Mycoplasma pneumoniae is the causative agent of atypical pneumonia and several additional infections including encephalitis, aseptic meningitis, acute transverses myelitis, stroke, and polyradiculopathy (Narita, 2009; Narita, 2010; Tsiodras et al., 2005; Waites and Talkington, 2004). These bacteria are members of the phylogenetic group of Mollicutes that are characterized by an extreme reductive evolution that results in the smallest genomes that allow independent life. Moreover, the Mollicutes have lost the cell wall and most metabolic pathways, since they obtain the building blocks for their cellular macromolecules from the host tissue. However, even in these minimal pathogens, there is a close relation between metabolism and virulence [for review see Halbedel et al. (2007)]. M. pneumoniae thrives at the surfaces of lung epithelia. Thus, these bacteria must have evolved to utilize the carbon sources present in this niche. The pulmonary surfactant is composed of about $90 \%$ phospholipids and $10 \%$ proteins (Veldhuizen et al., 1998). This suggests that phospholipids play a major role in the nutrition of M. pneumoniae. 
Glycerophospholipids, the major building blocks of the cell membrane in bacteria and eukaryotes, are degraded in several steps. First, the fatty acids are cleaved off from the phospholipids resulting in the formation of glycerophosphodiesters. In these molecules, the phosphate group of glycerol 3-phosphate is linked to another compound, called the head group. In eukaryotes, choline is by far the most abundant head group, and lecithin, the choline-containing phospholipid accounts for about $80 \%$ of all phospholipids in human lung cells (Veldhuizen et al., 1998). In the second step, the choline head group is cleaved off due to the activity of a glycerophosphodiesterase resulting in the formation of glycerol 3-phosphate that can feed into glycolysis after oxidation to dihydroxyacetone phosphate (see Fig. 27). In M. pneumoniae, the latter reaction is catalyzed by the glycerol-3-phosphate oxidase GlpD (Hames et al., 2009). GlpD transfers the electrons to water resulting in the formation of hydrogen peroxide, the major virulence factor of M. pneumoniae (Somerson et al., 1965). In consequence, the virulence of $M$. pneumoniae glpD mutant cells is severely attenuated (Hames et al., 2009).

While the metabolism of glycerol has been well studied in M. pneumoniae and other Mollicutes such as Mycoplasma mycoides (Bischof et al., 2009; Hames et al., 2009; Pilo et al., 2005), only little is known about the glycerophosphodiesterases required for lipid utilization. Many bacteria encode multiple glycerophosphodiesterases. In Escherichia coli, both enzymes are enzymatically active in lipid degradation; however, they are differentially regulated, with GlpQ and UgpQ being induced in the presence of glycerol 3-phosphate and under conditions of phosphate starvation, respectively (Ohshima et al., 2008; Wong and Kwan, 1992). In Bacillus subtilis, out of three putative glycerophosphodiesterases, only GlpQ has been studied. The corresponding gene is under dual control and its expression is induced when phosphate becomes limiting and glycerol is available (Antelmann et al., 2000; Nilsson et al., 1994). Moreover, glpQ expression is repressed if more favourable carbon sources such as glucose are present (Blencke et al., 2003). In Haemophilus influenzae, another bacterium thriving in the respiratory tract, the glycerophosphodiesterase is involved in pathogenicity. The enzyme generates choline, which in turn is used for the biosynthesis of the bacterial lipopolysaccharide layer - a major virulence determinant of Gram-negative bacteria (Fan et al., 2001; Forsgren et al., 2008). Similarly, glycerophosphodiesterase activity is implicated on virulence of different Borrelia 
species. The enzyme is only present in the relapsing fever group and may help the bacteria to reach a higher cell density in the blood of the host as compared to Lyme disease spirochetes (Schwan et al., 2003).

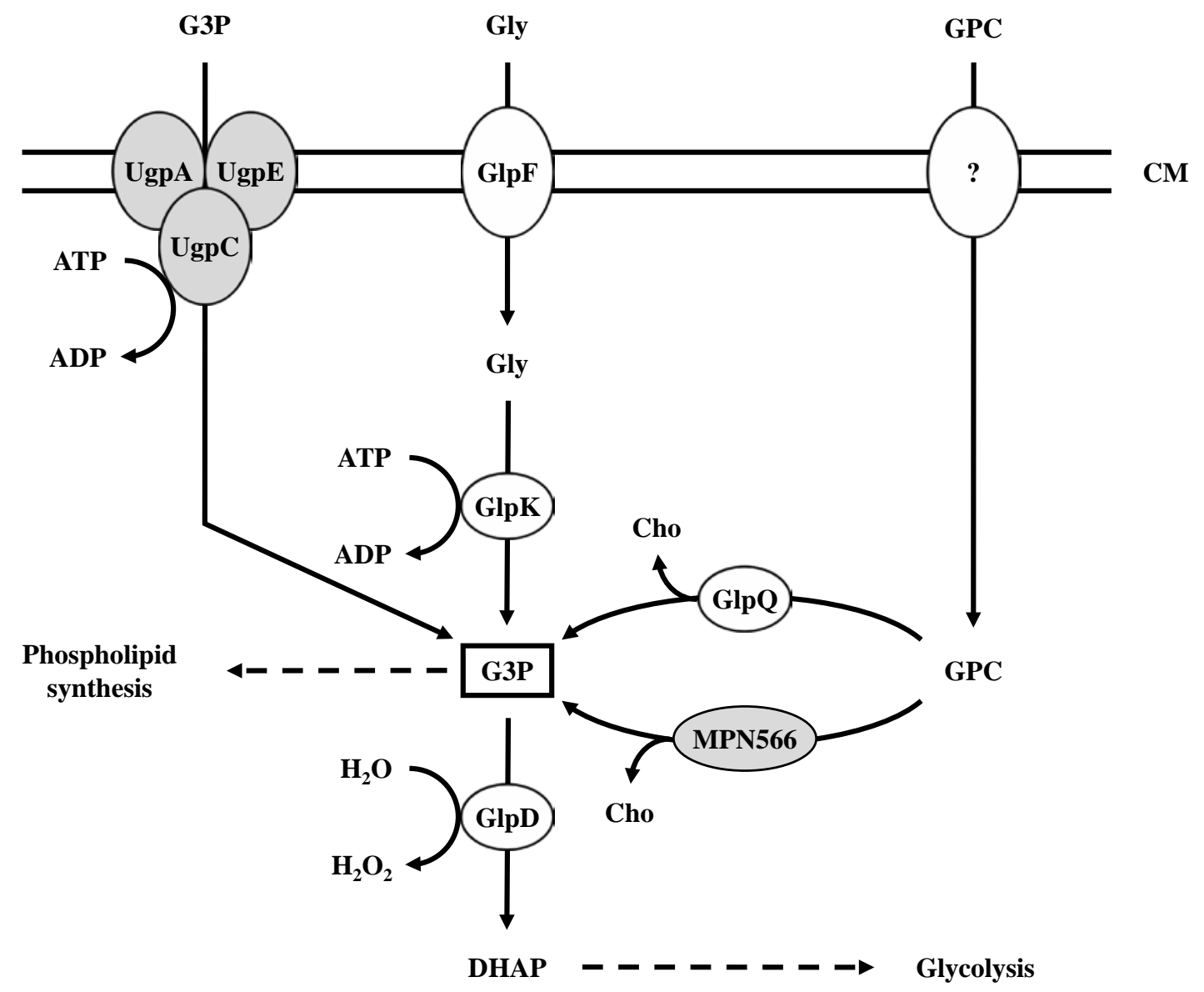

Fig. 27. Schematic illustration of the machinery for uptake and conversion of carbohydrates leading to the formation of glycerol 3-phosphate in M. pneumoniae. UgpC (MPN134), UgpA (MPN135), and UgpE (MPN136) form a ABC transport system for glycerol 3-phosphate, whereas GlpF (MPN043) is the glycerol uptake facilitator. The glycerol kinase GlpK (MPN050) and the glycerol-3-phosphate oxidase GlpD (MPN051) metabolize glycerol to the glycolytic intermediate dihydroxyacetone phosphate. Hydrogen peroxide formation by GlpD is crucial for the cytotoxic effects of M. pneumoniae. GlpQ (MPN420) and MPN566 encode two paralogous glycerophosphodiesterases that are able to metabolize glycerophosphorylcholine to glycerol 3-phosphate and choline. The uptake system for glycerophosphorylcholine is so far unknown. Proteins highlighted in grey seem not to fulfill the predicted function (this work). Cho, choline; CM, cell membrane; DHAP, dihydroxyacetone phosphate; G3P, glycerol 3-phosphate; GPC, glycerophosphorylcholine; Gly, glycerol. 
In many bacteria, central enzymes of metabolism do not only serve their catalytic function, but in addition, they are also involved in signal transduction. In this way, the information on the availability of important metabolites can be directly determined by the enzyme in charge of their conversion, and this information is then often transferred to the transcription machinery. Collectively, such enzymes were termed trigger enzymes (Commichau and Stülke, 2008). They can control gene expression by directly acting as DNA- or RNA-binding transcription factors as the E. coli proline dehydrogenase and the aconitase or by controlling the activity of transcription factors by covalent modification or a regulatory protein-protein interaction as observed for several sugar permeases of the bacterial phosphotransferase system and the B. subtilis glutamate dehydrogenase, respectively (Beinert et al., 1996; Commichau et al., 2007; Schmalisch et al., 2003; Zhu and Becker, 2003).

In this work, we have analyzed the role of the two potential glycerophosphodiesterases encoded in the genome of $M$. pneumoniae. Biochemical and physiological studies demonstrate that one of the two proteins, GlpQ, is a functional glycerophosphodiesterase. GlpQ is essential for hydrogen peroxide formation in the presence of deacylated phospholipids as the carbon source and, in consequence, for cytotoxicity. Moreover, GlpQ may act as a trigger enzyme by controlling the expression of a set of genes encoding lipoproteins, the glycerol facilitator, and a metal ion ABC transporter.

\section{Results}

\section{Identification of $M$. pneumoniae genes encoding potential} glycerophosphodiesterases. Since phospholipids are the most abundant potential carbon sources for $M$. pneumoniae living at lung epithelial surfaces, we considered the possibility that these bacteria synthesize enzymes that cleave the polar head groups from the glycerophosphodiesters to produce glycerol 3-phosphate that can be utilized by the enzymes of glycerol metabolism (Hames et al., 2009) (Fig. 27). Two genes that potentially encode such enzymes are present in the genome of $M$. pneumoniae, i.e. mpn420 (renamed to $g l p Q$ ) and mpn566. An alignment of the corresponding proteins to glycerophosphodiesterases from other bacteria is shown in the supporting information (Fig. S4). 
Enzymatic activities of the potential glycerophosphodiesterases. In order to assess the biochemical properties and physiological relevance of the putative glycerophosphodiesterases, their corresponding genes, glpQ and mpn566, were cloned into the expression vector pGP172, thus allowing a fusion of the proteins to a $\mathrm{N}$-terminal Strep-tag facilitating purification. The recombinant proteins were purified and the activities were first determined using glycerophosphorylcholine (GPC) as the substrate and a set of divalent cations. As shown in Fig. 28, purified GlpQ was active against GPC, and the activity was highest in the presence of magnesium ions (10 mM). Manganese and zinc ions did also support activity, although to a lesser extent (Fig. 28). In contrast, the enzyme was inactive in the presence of calcium and cobalt ions (data not shown). The activity assay with purified MPN566 revealed no activity with GPC, irrespective of the cation present in the assay (data not shown). We did also test the activity of both proteins with glycerophosphorylethanolamine and glycerophosphorylglycerol. However, neither protein was active with any of these substrates. Thus, our data demonstrate that GlpQ is active as a glycerophosphodiesterase, whereas MPN566 does not exhibit such an activity.

The two proteins GlpQ and MPN566 share 58\% identical residues. Thus, it seems surprising that MPN566 was inactive in the enzymatic assay. However, several residues that are known to be important for the activity of glycerophosphodiesterases are conserved in GlpQ but not in MPN566 (Fig. S4). These residues include Trp-36 and Glu-38 as well as the conserved HD motif (Asn-51 and Leu-52 in MPN566) and Phe-110. Interestingly, a similar arrangement with two GlpQ-like proteins is also observed in Mycoplasma genitalium, and as in M. pneumoniae, one protein has all the conserved residues characteristic for glycerophosphodiesterases, whereas the second protein has similar deviations from the consensus as MPN566 (Fig. S4). In order to test whether a restoration of the conserved residues would also convert MPN566 to a biologically active glycerophosphodiesterase, we replaced the five amino acids that differ from the consensus by those residues present in GlpQ. The resulting mutant allele was cloned into pGP172, and the protein purified. Unfortunately, this protein was highly unstable and purification was impossible. 


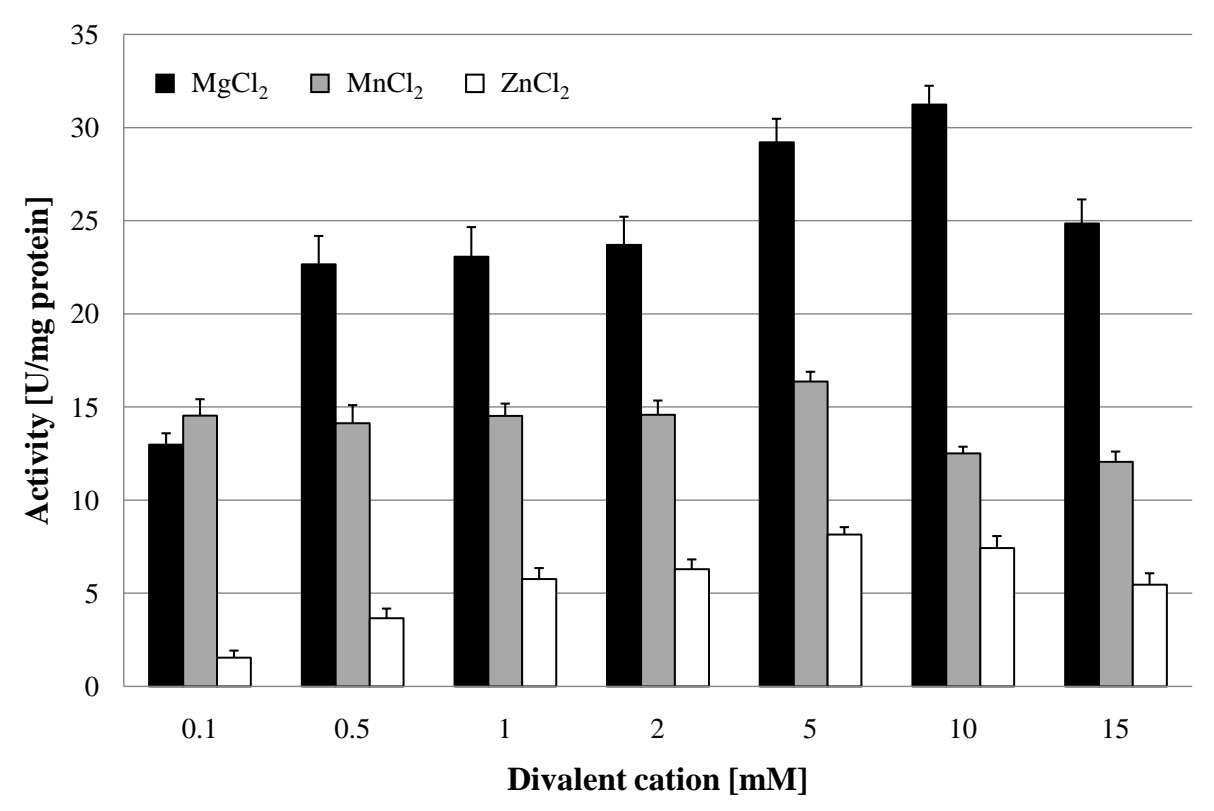

Fig. 28. Effect of divalent cations on GlpQ activity. GlpQ activity was measured in the presence of $0.5 \mathrm{mM}$ glycerophosphorylcholine and various concentrations of divalent cations. Error bars indicate standard deviation (based on three independent experiments).

Construction of $g l p Q$ and mpn566 mutants. The analysis of mutants is one of the most powerful tools for studying gene functions and bacterial physiology. The isolation of desired $M$. pneumoniae mutants became possible only recently by the introduction of the "Haystack mutagenesis" (Halbedel and Stülke, 2007). To get more insights into the physiological role of the glycerophosphodiesterases GlpQ and its paralogous MPN566, we attempted to isolate mutants affected in the corresponding genes.

The strategy of "Haystack mutagenesis" is based on an ordered collection of pooled random transposon insertion mutants that can be screened for junctions between the transposon and the gene of interest due to transposon insertion. The 64 pools were used in a PCR to detect junctions between the glpQ or mpn566 genes and the mini-transposon using the oligonucleotides SS35 and SS40 (for the respective genes) and SH30 (for the mini-transposon) (Halbedel et al., 2006) (Fig. 29). Positive signals were obtained for both genes. From pools that gave a positive signal, colony PCR with the 50 individual mutants resulted in the identification of the desired $g l p Q$ and mpn566 mutants. The presence of the transposon insertion in both genes was verified by Southern blot analysis (Fig. 29). To test whether these strains contained only unique transposon insertions, we did another Southern blot using a probe specific for the 
aac-aphD resistance gene present on the mini-transposon. As shown in Fig. 29, only one single band hybridizing with this probe was detected in each strain, moreover, this fragment had the same size as the AgeI or PstI/SacI fragment hybridizing to the $\operatorname{glpQ}$ and mpn566 probe, respectively (Fig. 29). The isolated $g l p Q$ and mpn566 mutant strains were designated as GPM81 and GPM82. The position of the transposon insertion in the two genes was determined by DNA sequencing. The glpQ gene was disrupted between nucleotides 517 and 518, resulting in a truncated protein of 172 amino acids with one additional amino acid and the following stop codon encoded by the inserted mini-transposon. The disruption of the mpn566 gene was located between nucleotides 157 and 158, resulting in a truncated protein of 52 amino acids with one additional amino acid and the following stop codon.

Contributions of GlpQ and MPN566 to growth and motility. First, we compared the ability of the wild type strain and the two mutant strains to utilize glucose and glycerol as the single carbon sources (Fig. 30). As an additional control, we used the $g l p D$ mutant strain GPM52. This strain is defective in glycerol-3-phosphate oxidase and therefore unable to utilize glycerol as the only carbon source (Hames et al., 2009). As shown in Fig. 30A, the wild type and the glpD and mpn566 mutant strains grew well with glucose. In contrast, the glpQ mutant GPM81 grew more slowly and did not reach the final biomass as compared to the other strains. As reported previously, the wild type strain exhibited very slow growth with glycerol as the only carbon source (Halbedel et al., 2004). In this respect, the glpQ and mpn566 mutants were indistinguishable from the wild type. As reported previously, the $g l p D$ mutant strain did not grow at all in glycerol-containing medium (Hames et al., 2009). In conclusion, the active glycerophosphodiesterase GlpQ is required for maximal growth in the presence of glucose, whereas its absence does not interfere with the slow growth in the presence of glycerol. It is therefore tempting to speculate that some glycerophosphodiesters in the Hayflick medium support growth (see “Discussion”).

Since the disruption of $g l p Q$ affected the growth properties of the bacteria, we wondered whether this might reflect changes in cell morphology and in the movement of the bacteria. The morphology of the wild type and mutant bacteria was analyzed by scanning electron microscopy, and no significant differences were detected (Fig. S5). An analysis of the gliding velocities of the three strains revealed that the wild type strain glided with a velocity of $0.32 \pm 0.09 \mu \mathrm{m} / \mathrm{s}$, whereas the glpQ and mpn566 mutants 
exhibited velocities of $0.2 \pm 0.08 \mu \mathrm{m} / \mathrm{s}$ and $0.3 \pm 0.1 \mu \mathrm{m} / \mathrm{s}$, respectively. Thus, the active glycerophosphodiesterase GlpQ is required for efficient gliding movement of the bacteria.

A

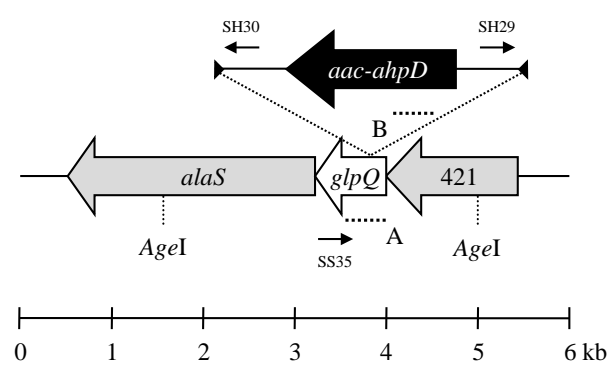

B

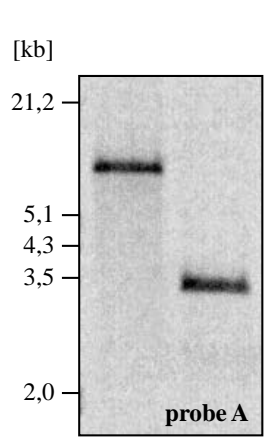

C
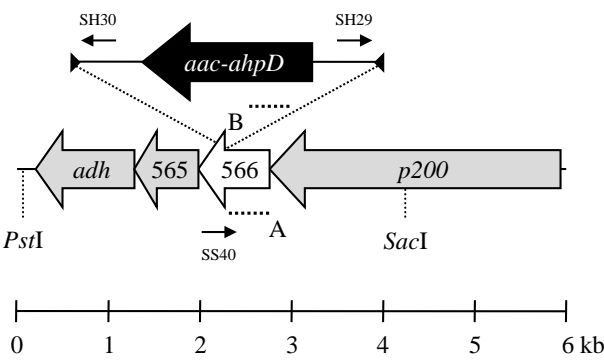

D

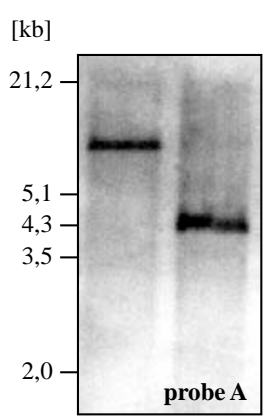

Fig. 29. Isolation of $M$. pneumoniae glycerophosphodiesterases transposon insertion mutants.

(A, C) Schematic representation of the genomic region surrounding the glpQ and mpn566 gene (both designated as glycerophosphodiesterases) in M. pneumoniae and site of the transposon insertion in the knockout strains GPM81 and GPM82, respectively. The annealing sites of oligonucleotides used for the determination of the transposon insertion site are indicated by arrows. Probes hybridizing to internal fragments of the glycerophosphodiesterases and the aac-ahpD genes are depicted as dotted lines. (B, D) Southern blot analysis to confirm the single insertion of the mini-transposon into the $g l p Q$ and mpn566 gene of the strains GPM81 and GPM82, respectively. Chromosomal DNAs of the wild type and both glycerophosphodiesterases were digested as indicated. Blots were hybridized with the respective glycerophosphodiesterase-specific probe (left) and a probe hybridizing to the aac-ahpD gene of the mini-transposon (right). 
A

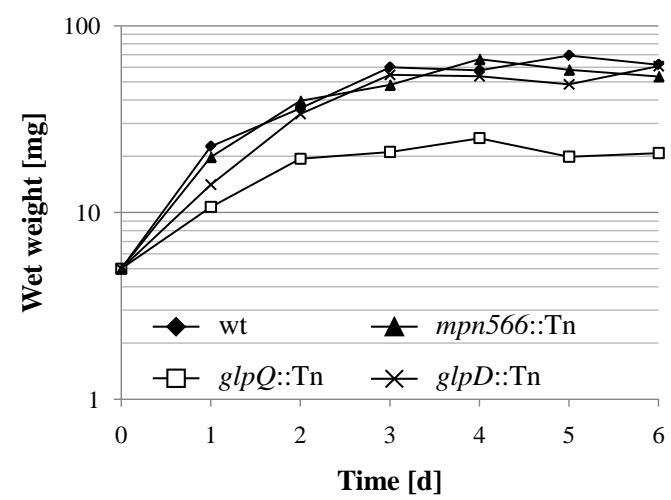

B

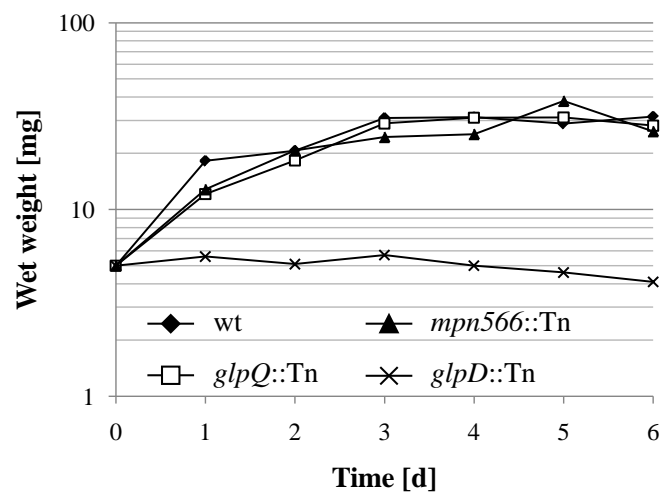

Fig. 30. Growth of $M$. pneumoniae in modified Hayflick medium containing different carbon sources. One hundred milliliters of medium were inoculated with $5 \mathrm{mg}$ of $M$. pneumoniae wild type (wt), glpQ::Tn, mpn566::Tn, and glpD::Tn [control; Hames et al. (2009)] mutant cells and incubated for up to six days at $37^{\circ} \mathrm{C}$ in $150-\mathrm{cm}^{2}$ cell culture flasks. Glucose (A) and glycerol (B) were added to a final concentration of $1 \%(\mathrm{wt} / \mathrm{vol})$. Attached cells were collected by scraping and growth was monitored by determination of the wet weight of the cell pellets. All measurements were done three times. Results are from a representative experiment.

\section{Implication of GlpQ and MPN566 in hydrogen peroxide production and} cytotoxicity. The utilization of glycerol or glycerophosphodiesters results in the generation of hydrogen peroxide, the major cytotoxic product of $M$. pneumoniae. We asked therefore whether the glpQ and mpn566 disruptions would affect hydrogen peroxide formation and if so, whether it also affects cytotoxicity. Hydrogen peroxide formation was assayed in $M$. pneumoniae cultures that contained glucose, glycerol, GPC, glycerol 3-phosphate or no carbon source. In the absence of an added carbon source, neither the wild type strain nor the mutants formed substantial amounts of hydrogen peroxide (Fig. 31). Similarly, essentially no hydrogen peroxide was produced in the presence of glucose. If glycerol was available, maximal hydrogen peroxide formation $(9.5 \mathrm{mg} / \mathrm{l})$ was observed in the wild type strain. In the $g l p D$ mutant that served as a control, no hydrogen peroxide was formed. This is in good agreement with previous reports on the increase of hydrogen peroxide generation in the presence of glycerol and its dependence on a functional glycerol-3-phosphate oxidase (Hames et al., 2009). The hydrogen peroxide production in the $g l p Q$ and mpn566 mutants was similar to that observed in the wild type strain. This result reflects that the metabolite glycerol is downstream from the glycerophosphodiesterase activity. In the presence of GPC, the 
wild type strain produced similar amounts of hydrogen peroxide $(9 \mathrm{mg} / \mathrm{l})$ as in the presence of glycerol. In contrast, no hydrogen peroxide formation was detected for the glpQ mutant GPM81, whereas the disruption of mpn566 did not have any effect on the production of hydrogen peroxide (Fig. 31). This result is in good agreement with the enzymatic activities of the two proteins: GlpQ is the only active glycerophosphodiesterase in M. pneumoniae, and no glycerol 3-phosphate, the substrate of GlpD, can be formed in its absence, whereas MPN566 is dispensable for the utilization of GPC. We also tested the ability of the $M$. pneumoniae strains to form hydrogen peroxide in the presence of glycerophosphorylethanolamine and glycerophosphorylglycerol. These compounds did not stimulate hydrogen peroxide in any of the strains tested (data not shown). This is in excellent agreement with the result of the enzyme assay that suggested that neither GlpQ nor MPN566 is able to degrade these substances. Finally, we tested whether hydrogen peroxide was formed in the presence of glycerol 3-phosphate. As shown in Fig. 31, no significant formation of hydrogen peroxide was observed in any of the strains tested. This suggests that the uptake of glycerol 3-phosphate is rather inefficient.

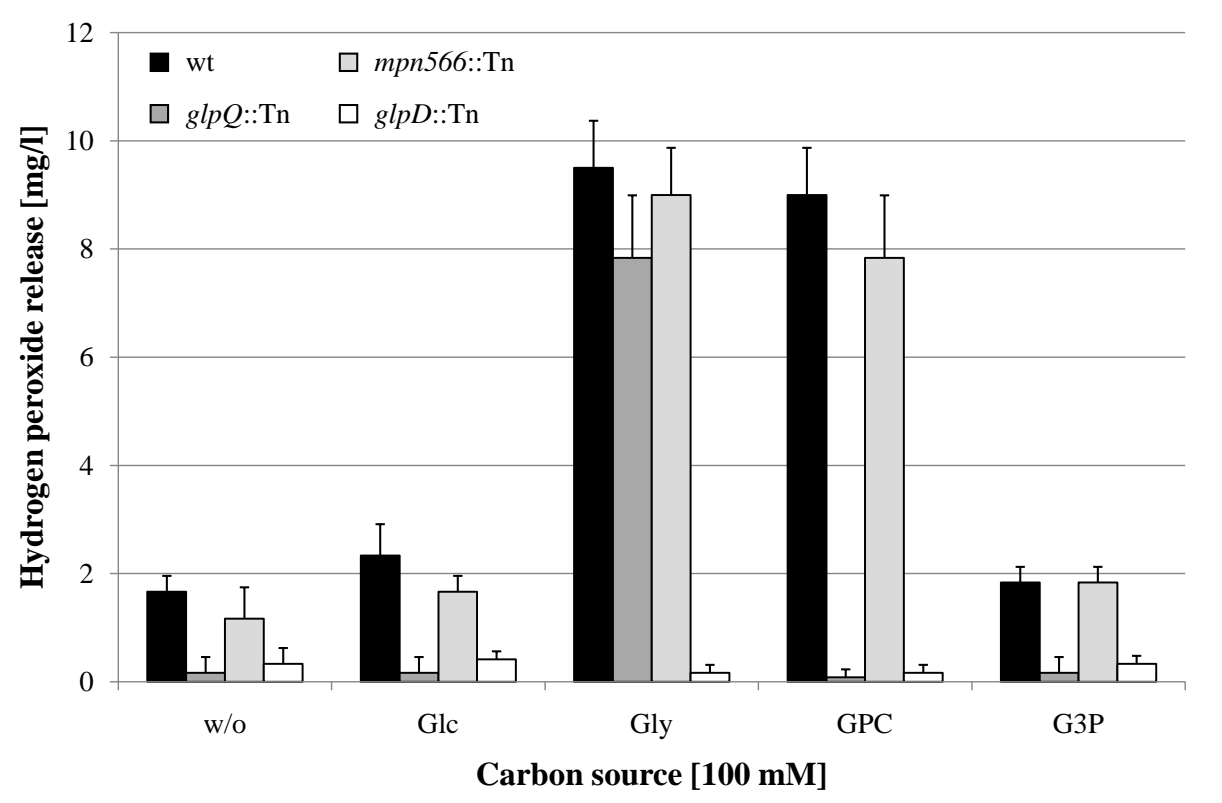

Fig. 31. Examination of M. pneumoniae hydrogen peroxide release. Hydrogen peroxide production of M. pneumoniae wild type (wt), glpQ::Tn, mpn566::Tn, and glpD::Tn [control; Hames et al. (2009)] mutant strains was measured in the presence of different carbon sources (100 $\mu \mathrm{M})$ after 2 h. Error bars indicate standard deviation (based on three independent experiments). G3P, glycerol 3-phosphate; GPC, glycerophosphorylcholine; Glc, glucose; Gly, glycerol; w/o, without addition of any carbon source. 
To assess the cytotoxicity of the different $M$. pneumoniae strains, we infected confluently grown HeLa cell cultures with $M$. pneumoniae cells (multiplicity of infection: 2). The cytotoxicity of the mutants was compared to that of the wild type strain and M. pneumoniae GPM52 that is affected in $g l p D$. As shown in Fig. 32, the HeLa cells had undergone lysis after four days upon infection with wild type M. pneumoniae. As observed previously, a large portion of viable cells was observed after infection of the cell culture with the glpD mutant GPM52 (Hames et al., 2009). For the glpQ mutant GPM81, nearly all HeLa cells had survived the infection suggesting that GlpQ is essential for cytotoxicity. In contrast, cytotoxicity induced by the mpn566 mutant strain GPM82 was equivalent to that of the wild type strain (Fig. 32). These data clearly demonstrate that the active glycerophosphodiesterase GlpQ is required for host cell damage, whereas the inactive enzyme MPN566 is not. Moreover, they support the assumption that hydrogen peroxide formation is the major factor that contributes to host cell damage.

A

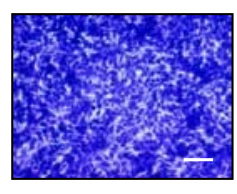

control

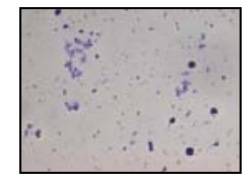

$\mathbf{w t}$

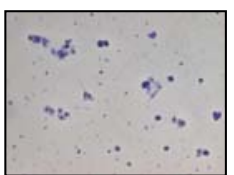

mpn566::Tn

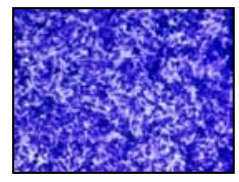

glpQ::Tn

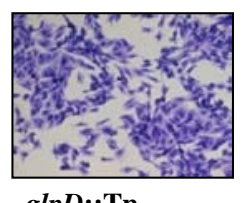

glpD::Tn
B

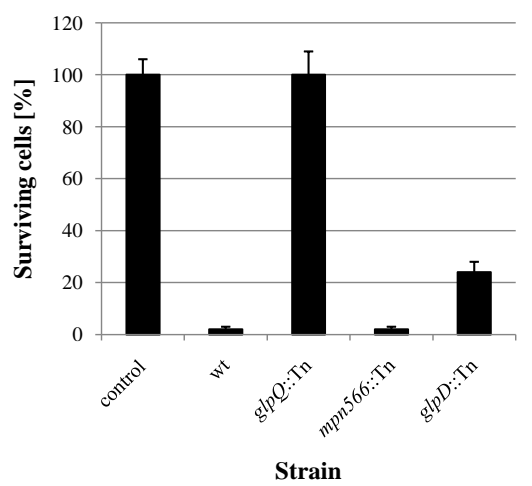

Fig. 32. Cytotoxicity of M. pneumoniae toward HeLa cell cultures. (A) Infection assay to verify cytotoxic effects of M. pneumoniae glpQ::Tn and mpn566::Tn mutant strains. HeLa cells were infected with M. pneumoniae wild type (wt), glpQ::Tn, and mpn566::Tn mutant cells. As control served two HeLa cell cultures: One without addition of M. pneumoniae cells and another after infection with the $g l p D:: T n$ mutant strain (Hames et al., 2009). After four days, HeLa cell cultures were stained with crystal violet and photographed. All pictures are shown at the same magnification. Scale bar, $0.1 \mathrm{~mm}$. (B) Quantification of HeLa cells after infection with different M. pneumoniae strains. The cell count of surviving cells is indicated in percent as the number of viable cells per field of view, quantified by crystal violet staining after four days of incubation. An uninfected HeLa cell culture served as control. Error bars indicate standard deviation (based on three independent experiments). 
The role of GlpQ in gene expression. As reported above, the glpQ mutant exhibits multiple phenotypes related to motility, metabolism, and pathogenicity. We asked therefore whether some of the effects are due to changes in the proteome of the glpQ mutant GPM81. To answer this question, we compared the total protein profiles of the wild type strain and the $g l p Q$ and mpn566 mutants, GPM81 and GPM82, respectively, after growth in glucose and glycerol (Fig. 33). While the protein patterns in the mpn566 mutant were indistinguishable from the wild type strain under both conditions, several differences were noted for the $g l p Q$ mutant.

To identify those proteins that exhibit altered accumulation in the glpQ mutant, the total proteins of the wild type and the glpQ mutant strains were identified by mass spectrometry. For the protein extracts from glucose-grown cells, 532 different proteins were identified. This corresponds to about $77 \%$ of the theoretical proteome of M. pneumoniae. In the presence of glycerol, 473 proteins corresponding to $69 \%$ of the theoretical proteome were identified. The differences in protein expression between glucose- and glycerol-grown cells as well as proteins that could not be detected at all are summarized in Tables S9 and S10. A detailed list of the differences of the protein profiles between the wild type strain and the glpQ mutant is presented in Tables S11 and S12. As expected, the GlpQ protein was detected in the protein extracts of the wild type strain but not in those of the glpQ mutant strain. In glucose-grown cells, 33 and 21 proteins were in elevated and reduced amounts, respectively, in the glpQ mutant. The strongest increase was observed for the glycerol facilitator GlpF and the uncharacterized lipoprotein MPN162. A strongly reduced accumulation was observed for the lipoprotein MPN506. In the presence of glycerol, five induced and five repressed proteins were detected. These proteins were subject to a similar regulation as in glucose-grown cells and they are the only proteins with identical regulation under both conditions (Table 12)

It has been shown before that changes at the proteome level may result from altered gene expression or from changes in protein stability (Halbedel et al., 2007; Schmidl et al., 2010). Therefore, we studied the expression of the genes corresponding to the most prominently regulated proteins and of genes encoding potential regulators, transport systems, and potential pathogenicity factors. For this purpose, we isolated RNA from cultures grown in modified Hayflick medium supplemented with glucose and performed slot blot analyses (Fig. 34 and S6). 
Table 12. Proteins with GlpQ-dependent expression pattern.

For detailed information on proteome and transcript changes in the glpQ mutant GPM81, see Tables S11 and S12.

\begin{tabular}{|c|c|c|c|c|c|c|}
\hline \multirow{2}{*}{ Locus name } & \multirow{2}{*}{ Protein name } & \multirow{2}{*}{ Protein function } & \multicolumn{2}{|c|}{ Fold-change in the presence of glucose ${ }^{a}$} & \multicolumn{2}{|c|}{ Fold-change in the presence of glycerol ${ }^{\mathrm{a}}$} \\
\hline & & & Protein level & Transcript level & Protein level & Transcript level \\
\hline MPN043 & GlpF & Glycerol uptake facilitator & $10.79 \pm 1.06$ & $5.69 \pm 0.36$ & $5.83 \pm 0.76$ & $3.32 \pm 0.34$ \\
\hline MPN162 & - & Uncharacterized lipoprotein & $9.96 \pm 0.97$ & $5.23 \pm 0.23$ & $5.41 \pm 0.83$ & $2.81 \pm 0.47$ \\
\hline MPN284 & - & Uncharacterized lipoprotein & ns & $0.25 \pm 0.05$ & $0.36 \pm 0.02$ & $0.35 \pm 0.04$ \\
\hline MPN433 & CbiO & Metal ion ABC transporter ATP-binding protein & ns & $7.56 \pm 0.39$ & $5.66 \pm 0.49$ & $2.92 \pm 0.24$ \\
\hline MPN506 & - & Uncharacterized lipoprotein & $0.10 \pm 0.03$ & $0.07 \pm 0.05$ & $0.18 \pm 0.04$ & $0.16 \pm 0.02$ \\
\hline
\end{tabular}

${ }^{\mathrm{a}}$ Fold-change cut off $\geq 2.0$ and $\leq 0.5$, respectively ( $g l p Q$ mutant strain $v s$. wild type). Abbreviation: ns, no significant difference. 

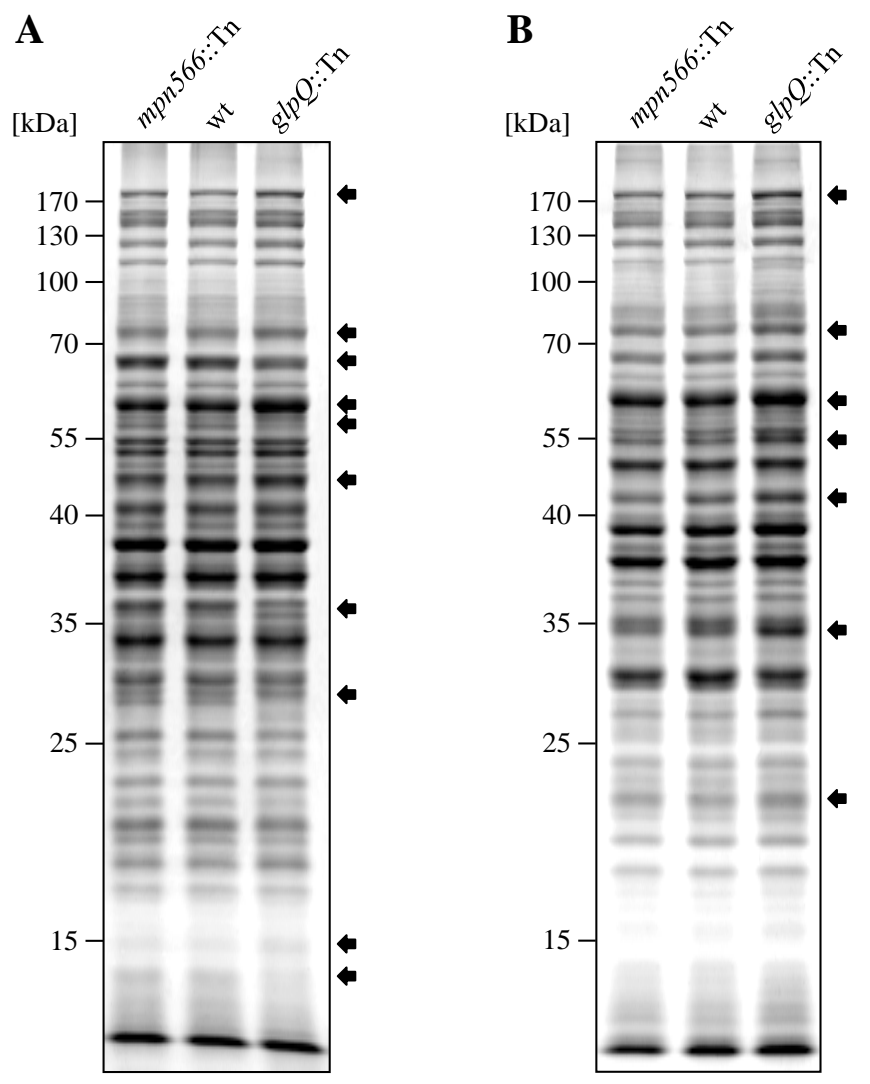

Fig. 33. Comparison of protein profile between $M$. pneumoniae wild type and both glycerophosphodiesterase mutant strains. Whole cell extracts of $M$. pneumoniae wild type (wt), glpQ::Tn, and mpn566::Tn mutant strains were analyzed by 12\% SDS-PAGE and stained with Coomassie Brillant Blue R250 dye (Serva) for visualization of proteins. $15 \mu \mathrm{g}$ of extract was applied to each lane. M. pneumoniae strains were grown in modified Hayflick medium containing either glucose (A) or glycerol (B) as sole carbon source (1\% wt/vol). Complete lanes were cut out (into 15 pieces) and identified by mass spectrometry. Protein bands with prominent changes in the amount are indicated by arrows.

These studies demonstrated that the regulation of the glycerol facilitator GlpF and the lipoproteins MPN162 and MPN506 occurs at the level of transcription (Table 12). Moreover, our results confirmed the higher expression of $g l p F$ and $m p n 162$ and the repression of mpn506 in the glpQ mutant. For the other proteins that were induced in the presence of glucose, with exception of plsC and mpn566 (nearly twofold higher transcript levels), similar accumulation of the mRNAs compared to the protein amount was observed (Table S11 and Fig. S6). In contrast, for the proteins that were present in reduced amounts in glucose-grown cells, no changes of the corresponding mRNAs were observed for all transport proteins. Interestingly, the lipoprotein MPN083 showed a similar pattern at the level of transcription as the induced proteins and the 
ribonucleoside-diphosphate reductase (encoded by $n r d F I E$ ) was the only protein with reduced mRNA amounts, however changes in transcript level were not significant (Table S11 and Fig. S6).

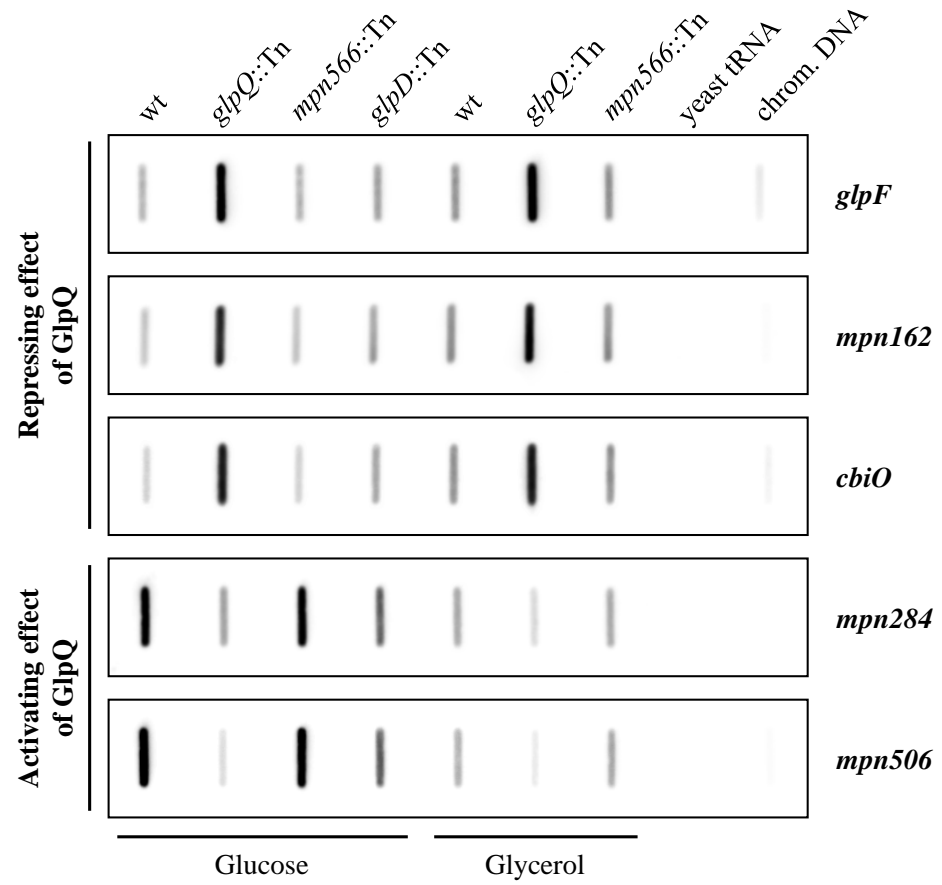

Fig. 34. Transcription analysis of GlpQ-dependent genes in M. pneumoniae. Slot blots were performed with whole RNA extracts of $M$. pneumoniae wild type (wt), glpQ::Tn, mpn566::Tn, and glpD::Tn [control; Hames et al. (2009)] mutant strains grown in modified Hayflick medium containing either glucose or glycerol as sole carbon source (1\% wt/vol). A dilution series of RNA extracts was blotted onto a positively charged nylon membrane and probed with a DIG-labeled riboprobe specific for an internal part of a particular open reading frame. Names of riboprobes are given next to each blot. Signals obtained with $1 \mu \mathrm{g}$ of RNA are shown. Yeast tRNA and M. pneumoniae chromosomal DNA served as controls. For detailed information on differences of transcript levels see Tables 12, S11, and S12.

Identification of a potential target site for GlpQ-dependent regulation. The proteome and transcription analyses identified three genes that are significantly regulated - either induced or repressed - in a GlpQ-dependent manner. An inspection of the upstream region of these genes revealed the presence of a common palindromic DNA motif (Fig. 35). To exclude the possibility that this motif is randomly distributed in the genome of $M$. pneumoniae because of the extremely AT-rich consensus sequence, we tested its presence in the genome using the GLAM2SCAN algorithm (Frith et al., 
2008). In 15 cases (matching score cut Đf 20 ), this potential motif was located upstream of open reading frames, among them the three genes mentioned above. Therefore, the expression of the remaining 12 genes was tested by slot blot analyses, and for two of these genes, cbiO and mpn284, a significant accumulation and reduction of the mRNA, respectively, was observed (data not shown; Fig. 34). Interestingly, the corresponding proteins, a subunit of a metal ion $\mathrm{ABC}$ transporter $\mathrm{CbiO}$ and the uncharacterized lipoprotein MPN284 were found to be present in higher or lower amounts in the glpQ mutant in glycerol-grown cell. Thus, there is a very good agreement between the regulatory effect of GlpQ at the proteome level, the regulation at the level of transcription, and the presence of the cis-acting element.

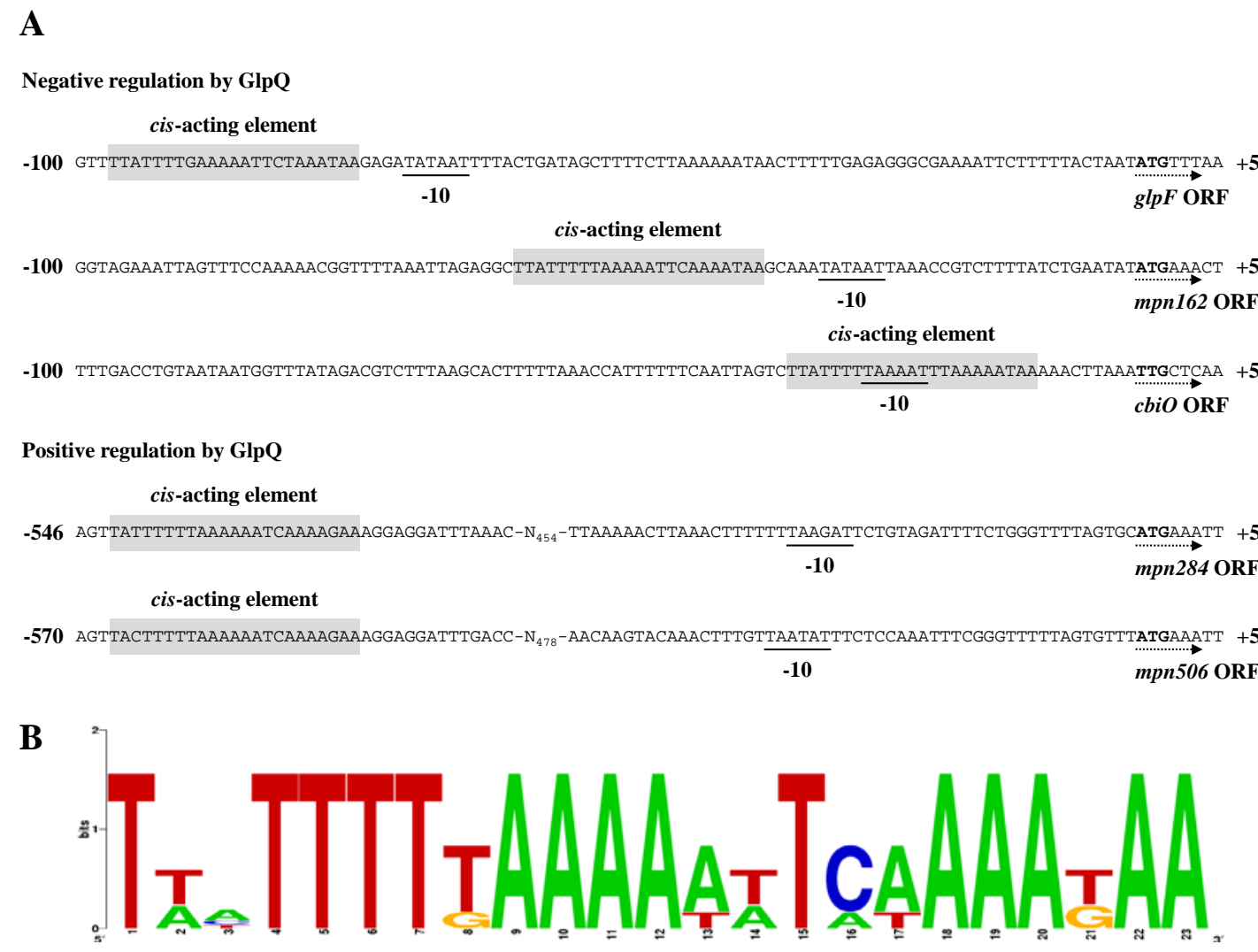

Fig. 35. Transcriptional organization of GlpQ-dependent genes in M. pneumoniae. (A) Nucleotide sequence of promoter regions of M. pneumoniae glpF (mpn043), mpn162, cbiO (mpn433), mpn284, and mpn506 genes. Promoter sequences are numbered relative to the 5' end. Directions of the open reading frames (ORF) are indicated by dotted arrows and predicted ATG/TTG start codons are highlighted by bold type. The respective -10 motifs are underlined. GlpQ-dependent cis-acting elements (palindromic DNA motifs) are indicated by grey shading. (B) Consensus sequence of the GlpQ-dependent palindromic DNA motif in M. pneumoniae. The sequence logo was created using WebLogo v2.8.2 (Crooks et al., 2004) based on all five cis-acting elements mentioned in A. 


\section{Discussion}

This work establishes that the glycerophosphodiesterase GlpQ of M. pneumoniae is essential for cytotoxicity of these bacteria. This is in excellent agreement with previous reports that carbon metabolism is intimately linked to virulence in pathogenic bacteria, including $M$. pneumoniae and other Mollicutes (Görke and Stülke, 2008; Halbedel et al., 2007; Sonenshein, 2007). The utilization of glycerol and phospholipids plays a particularly important role in virulence of Mycoplasma species: Hydrogen peroxide, the only known cytotoxic substance produced by these bacteria, is generated as a product of glycerol metabolism, and both $g l p D$ and glpQ mutants are severely affected in pathogenicity (Hames et al., 2009; this work). In M. mycoides, pathogenicity is associated with the presence of a highly efficient ABC transporter for glycerol. Non-pathogenic strains of $M$. mycoides rely on the less efficient glycerol facilitator for glycerol uptake (Vilei and Frey, 2001).

In M. pneumoniae, GlpQ is not only important for virulence but also for growth in the commonly used medium in the laboratory, i.e. modified Hayflick medium with glucose as the added carbon source (see Fig. 30A). This observation is in good agreement with a recent analysis of the $M$. pneumoniae metabolism that suggested that glycerol is essential for growth of M. pneumoniae (Yus et al., 2009). Accordingly, no difference between the wild type strain and the $g l p Q$ mutant was observed during growth in the presence of glycerol (see Fig. 30B).

In addition to GlpQ, M. pneumoniae encodes a second paralogous protein. However, as shown in this work, this protein does not exhibit enzymatic activity nor does the inactivation of the corresponding gene (mpn566) cause any detectable phenotypes. This lack of detectable activity of MPN566 is easily explained by the lack of conservation of amino acid residues that are essential for the activity as a glycerophosphodiesterase. Interestingly, a very similar arrangement with two glpQ-like genes is also present in M. genitalium and Mycoplasma alligatoris. Based on the conservation of the catalytically important residues (see Fig. S4), there is an active and an inactive enzyme in $M$. genitalium, as observed here for $M$. pneumoniae. In M. alligatoris, both potential glycerophosphodiesterases contain all the important amino acids suggesting that both proteins are enzymatically active. It is tempting to speculate that the possession of two active glycerophosphodiesterases is related to the fact that M. alligatoris is the only Mollicute that causes fatal infections (Brown et al., 2001). In 
the syphilis spirochete, Treponema pallidum, one glpQ-like gene is present, however, the encoded protein is not active as a glycerophosphodiesterase. Again, the inactivity is most likely caused by the lack of conservation of functionally important amino acids (Shevchenko et al., 1997; Stebeck et al., 1997). The presence of inactive GlpQ-like proteins in several pathogens, including a spirochete and M. genitalium, the bacterium with the smallest genome, suggests that these pseudo-enzymes have other functions that have yet to be identified. Unfortunately, the experiments reported in this study did not give any hints as to a putative function of MPN566.

Many proteins have activities in addition to their primary functions. On one hand, this allows gene duplication and specialization to non-related functions of similar proteins. On the other hand, a protein may acquire a second useful activity and act as a so-called moonlighting protein (Jeffery, 1999). The former is very common and might apply to the putative functional specialization of GlpQ and MPN566. In contrast, the latter phenomenon is true for all trigger enzymes that measure the availability of their respective metabolites and transduce this information to the regulatory machinery of the cell. In mammals, a glycerophosphodiesterase controls the development of skeletal muscles independent from its enzymatic activity (Okazaki et al., 2010). Our results suggest that GlpQ might also have such a second activity. Indeed, the expression of the glycerol facilitator GlpF, a lipoprotein, and the ATP-binding subunit of a metal ion $\mathrm{ABC}$ transporter are strongly overexpressed in the glpQ mutant, whereas two uncharacterized lipoproteins are less expressed in the mutant. Interestingly, the genes that are under negative control of GlpQ are more strongly expressed in the presence of glycerol as the carbon source (as compared to glucose). In contrast, the two lipoprotein genes mpn284 and mpn506 that require GlpQ for expression are only weakly expressed in the presence of glycerol, but they are strongly induced if glucose is used as the carbon source. These observations might be explained as follows: In the presence of glucose, only little glycerol or glycerol 3-phosphate (the product of the reaction catalyzed by GlpQ) is present in the cell. Free GlpQ might then directly bind DNA or trigger the DNA-binding activity of another, yet unknown transcription factor, resulting in repression or activation of the two sets of genes. In the presence of glycerol, glycerol 3-phosphate would be formed due to the activity of glycerol kinase, and this metabolite might then prevent GlpQ from its regulatory activity. As a result, those genes that are subject to GlpQ-dependent repression ( $g l p F, m p n 162$, and $c b i O$ ) are stronger 
expressed than in the presence of glucose, whereas the GlpQ-activated genes (mpn284 and mpn506) would be less expressed. Finally, in the glpQ mutant, the former set of GlpQ-repressed genes is highly constitutively expressed, and only a very low level of transcription can be detected for the two GlpQ-dependent lipoprotein genes. Since glycerol 3-phosphate is the product of the glycerophosphodiesterase reaction, this metabolite is an excellent candidate for detection by GlpQ. Moreover, the $g l p Q$ gene is constitutively expressed and the GlpQ protein was detected in $M$. pneumoniae cells irrespective of the carbon source used in similar amounts in this study (Güell et al., 2009; this study). Thus, GlpQ is available in the cell under all conditions to cause regulation. In a recent study on the phosphoproteome of $M$. pneumoniae, phosphorylation of GlpQ was observed (Schmidl et al., 2010), however, the functional relevance of this modification is unknown.

As observed for several other transcription regulators, GlpQ causes both transcription repression and transcription activation. The location of the putative cis-acting element correlates perfectly with the regulatory effect: Those genes that are subject to negative control by GlpQ have this element overlapping or in the very close vicinity of the -10 region of the promoters. This element is the only conserved promoter element in $M$. pneumoniae and it is sufficient for transcription initiation (Güell et al., 2009; Halbedel et al., 2007). Binding of GlpQ (or of a transcription factor that is controlled by GlpQ) would prevent a productive interaction with RNA polymerase and therefore cause transcription repression. On the other hand, the cis-acting elements that may be involved in the regulation of the GlpQ-activated genes are located upstream of the promoters. This is usually the case for binding sites of transcription activators and fits perfect with the observed regulation.

Our future work will focus on the elucidation of the mechanism(s) by which GlpQ controls gene expression. Moreover, we will address the functions of the lipoproteins that are subject by glycerol- and GlpQ-dependent regulation. 


\section{Materials and Methods}

Bacterial strains and growth conditions. The $M$. pneumoniae strains used in this study were M. pneumoniae M129 (ATCC 29342) in the 32nd broth passage, and its isogenic mutant derivatives GPM52 ( $g l p D:: \operatorname{mini}-\mathrm{Tn}, \mathrm{Gm}^{\mathrm{R}}$ ) (Hames et al., 2009), GPM81 (glpQ::mini-Tn, Gm ${ }^{\mathrm{R}}$ ), and GPM82 (mpn566::mini-Tn, Gm ${ }^{\mathrm{R}}$ ). M. pneumoniae was grown at $37^{\circ} \mathrm{C}$ in $150-\mathrm{cm}^{2}$ tissue culture flasks containing $100 \mathrm{ml}$ of modified Hayflick medium as described previously (Halbedel et al., 2004). Carbon sources were added to a final concentration of $1 \%$ (wt/vol). Growth curves were obtained by determining the wet weight of $M$. pneumoniae cultures as described previously (Halbedel et al., 2004). Strains harboring transposon insertions were cultivated in the presence of $80 \mu \mathrm{g} / \mathrm{ml}$ gentamicin. Escherichia coli DH5 $\alpha$ and BL21(DE3)/pLysS (Sambrook et al., 1989) were used as host for cloning and recombinant protein expression, respectively. The sequences of the oligonucleotides used in this study are listed in Table S13.

Construction of plasmids for the expression of potential glycerophosphodiesterases. The $M$. pneumoniae genes encoding proteins similar to glycerophosphodiesterases ( $g l p Q$ and mpn566) were amplified with chromosomal DNA as the template and the primer pairs SS34/SS35 and SS39/SS40, respectively. The PCR products were digested with SacI and BamHI and cloned into the expression vector pGP172 that allows the fusion of the target proteins to a Strep-tag at their N-terminus (Merzbacher et al., 2004). The resulting plasmids were pGP1018 and pGP1020. Since the $g l p Q$ gene contains three TGA codons that are recognized as stop codons in E. coli, these codons were replaced by TGG specifying tryptophan as in $M$. pneumoniae. For this purpose we applied the multiple mutation reaction (Hames et al., 2005) using the phosphorylated mutagenesis primers SS36, SS37, and SS38 and the external primers SS34 and SS35. The PCR product was digested and cloned into pGP172 as described above. The resulting expression vector was pGP1019. The plasmids pGP1019 and pGP1020 allowed the purification of the potential $M$. pneumoniae glycerophosphodiesterases (GlpQ and MPN566) carrying a N-terminal Strep-tag.

A mutant variant of MPN566 was obtained by the multiple mutation reaction using pGP1020 as the template and the phosphorylated mutagenesis primers SS192, SS193, and SS194 and the external primers SS39 and SS40. The PCR product was cloned into pGP172 as described above and the resulting plasmid was pGP661. 
Protein purification. The potential glycerophosphodiesterases were overexpressed in E. coli BL21(DE3)/pLysS. Expression was induced by the addition of IPTG (final concentration $1 \mathrm{mM}$ ) to exponentially growing cultures ( $\mathrm{OD}_{600}$ of 0.8 ). Cells were lysed using a french press (20.000 p.s.i., 138,000 kPa, two passes, Spectronic Instruments, UK). After lysis the crude extracts were centrifuged at 15,000 $\mathrm{g}$ for 60 min. The crude extract was passed over a Streptactin column (IBA, Göttingen, Germany). The recombinant proteins were eluted with desthiobiotin (IBA, final concentration $2.5 \mathrm{mM}$ ).

After elution the fractions were tested for the desired protein using 12\% SDS-PAGE. The relevant fractions were combined and dialyzed overnight. Protein concentration was determined according to the method of Bradford using the Bio-Rad dye-binding assay where Bovine serum albumin served as the standard.

\section{Assays of glycerophosphodiesterase enzymatic activity.} Glycerophosphodiesterase activity was measured in a coupled spectrophotometric assay as described previously (Larson et al., 1983). The enzyme assay is based on the formation of glycerol 3-phosphate and the subsequent oxidation by the glycerol-3-phosphate dehydrogenase and the formation of NADH. Briefly, $5 \mu \mathrm{g}$ of glycerophosphodiesterase were incubated with $20 \mathrm{U}$ of rabbit muscle glycerol-3-phosphate dehydrogenase (Sigma) in a $0.9 \mathrm{M}$ glycine-hydrazine buffer containing $0.5 \mathrm{mM}$ glycerophosphodiesters and $0.5 \mathrm{mM} \mathrm{NAD}^{+}$in a volume of $1 \mathrm{ml}$. Divalent cations were added as indicated. NADH formation was determined photospectrometrically at $340 \mathrm{~nm}$.

Determination of in vivo hydrogen peroxide production. The hydrogen peroxide production in $M$. pneumoniae was determined using the Merckoquant peroxide test (Merck, Darmstadt, Germany) as previously described (Hames et al., 2009). Briefly, growing cells were resuspended in assay buffer and after incubation for $1 \mathrm{~h}$ at $37^{\circ} \mathrm{C}$, glucose, glycerol, glycerol 3-phosphate or glycerophosphodiesters (final concentration $100 \mu \mathrm{M}$ ) were added to one aliquot. An aliquot without any added carbon source served as the control. The test strips were dipped into the suspensions for $1 \mathrm{~s}$ and subsequently read. 
Preparation and separation of whole cell extracts. Whole cell extracts of the different M. pneumoniae strains were prepared as described previously (Schmidl et al., 2010). In order to analyze the complete proteome, $15 \mu \mathrm{g}$ of the cell extracts were separated by one-dimensional 12\% SDS-PAGE and the gels subsequently stained with Coomassie Brillant Blue R250 dye (Serva). For protein identification, each running lane was cut out into 15 pieces followed by a separate analysis by mass spectrometry. The proteome analyses were performed in triplicate.

Protein identification by mass spectrometry. Gel pieces were washed twice with $200 \mu \mathrm{l} 20 \mathrm{mM} \mathrm{NH}_{4} \mathrm{HCO}_{3} / 30 \%$ (v/v) acetonitrile for $30 \mathrm{~min}$, at $37^{\circ} \mathrm{C}$ and dried in a vacuum centrifuge (Concentrator 5301, Eppendorf). Trypsin solution (10 ng/ $\mu$ l trypsin in $20 \mathrm{mM}$ ammonium bicarbonate) was added until gel pieces stopped swelling and digestion was allowed to proceed for 16 to $18 \mathrm{~h}$ at $37^{\circ} \mathrm{C}$. Peptides were extracted from gel pieces by incubation in an ultrasonic bath for 15 min in $20 \mu$ l HPLC grade water and transferred into micro vials for mass spectrometric analysis.

The tryptic digested proteins obtained from the one-dimensional SDS-PAGE gel pieces were subjected to a reversed phase column chromatography (Waters BEH $1.7 \mu \mathrm{m}, 100-\mu \mathrm{m}$ i.d. $\times 100$ mm, Waters Corporation, Milford, Mass., USA) operated on a nanoACQUITY UPLC ${ }^{\mathrm{TM}}$ (Waters Corporation, Milford, Mass., USA). Peptides were first concentrated and desalted on a trapping column (Waters nanoACQUITY UPLC column, Symmetry $\mathrm{C}_{18}, 5 \mu \mathrm{m}, 180 \mu \mathrm{m} \times 20 \mathrm{~mm}$, Waters Corporation, Milford, Mass., USA) for $3 \mathrm{~min}$ at a flow rate of $1 \mathrm{ml} / \mathrm{min}$ with $0.1 \%$ acetic acid. Subsequently the peptides were eluted and separated with a non-linear 80-min gradient from $5-60 \%$ acetonitrile in $0.1 \%$ acetic acid at a constant flow rate of $400 \mathrm{nl} / \mathrm{min}$. MS and MS/MS data were acquired with the LTQ Orbitrap $^{\mathrm{TM}}$ mass spectrometer (Thermo Fisher, Bremen, Germany) equipped with a nanoelectrospray ion source. After a survey scan in the Orbitrap $(r=30,000)$, MS/MS data were recorded for the five most intensive precursor ions in the linear ion trap. Singly charged ions were not taken into account for MS/MS analysis.

Tandem mass spectra were extracted using Sorcerer ${ }^{\mathrm{TM}}$ v3.5 (Sage-N Research). All MS/MS samples were analyzed using SEQUEST ${ }^{\circledR}$ (Thermo Fisher Scientific, San Jose, CA, USA; version 2.7, revision 11). Database searching was performed against a target decoy database of $M$. pneumoniae with added common laboratory contaminant proteins. Cleavage specificity for full tryptic cleavage and a maximum of 2 missed 
cleavages was assumed. SEQUEST was run with a fragment ion mass tolerance of $1.00 \mathrm{Da}$ and a parent ion tolerance of $10 \mathrm{ppm}$. Oxidation of methionine (+15.99492 Da) and phosphorylation of serine/threonine/tyrosine (+79.966331 Da) were specified in SEQUEST as variable modifications. Proteins were identified by at least two peptides applying a stringent SEQUEST filter (Xcorr vs. charge state: 1.8 for singly, 2.2 for doubly, 3.3 for triply, and 3.5 for higher charged ions). To address protein amount differences between the $M$. pneumoniae wild type and mutant strains, fold-changes were calculated by comparing number of assigned spectra for each protein (mutant $v s$. wild type strain).

Southern blot analysis. $M$. pneumoniae chromosomal DNA was prepared as described previously (Halbedel et al., 2006). Finally, digests of chromosomal DNA were separated using 1\% agarose gels and transferred onto a positively charged nylon membrane (Roche Diagnostics) (Sambrook et al., 1989) and probed with Digoxigenin (DIG)-labeled riboprobes obtained by in vitro transcription with T7 RNA polymerase (Roche Diagnostics) using PCR-generated fragments as templates. Primer pairs for the amplification of $g l p Q$, mpn566, and aac-ahpD gene fragments were SS42/SS43, SS44/SS45, and SH62/ SH63, respectively (Table S13). The reverse primers contained a T7 RNA polymerase recognition sequence. In vitro RNA labelling, hybridisation, and signal detection were carried out according to the manufacturer's instructions (DIG RNA labeling Kit and detection chemicals, Roche Diagnostics).

Analysis of mRNA amounts. Preparation of total $M$. pneumoniae RNA was done as previously described (Halbedel et al., 2004). For slot blot analysis, serial twofold dilutions of the RNA extract in $10 \times$ SSC $(2 \mu \mathrm{g}-0.25 \mu \mathrm{g})$ were blotted onto a positively charged nylon membran using a PR 648 Slot Blot Manifold (Amersham Biosciences). Equal amounts of yeast tRNA (Roche) and M. pneumoniae chromosomal DNA served as controls. DIG-labeled riboprobes were obtained by in vitro transcription from PCR products that cover ORF internal sequences using T7 RNA polymerase (Roche). The reverse primers used to generate the PCR products contained a T7 promoter sequence (Table S13). The quantification was performed using the Image $\mathrm{J}$ software v1.44c (Abramoff et al., 2004). 
HeLa cell cytotoxicity assay. Infection of HeLa cell cultures with M. pneumoniae cells were done as described previously (Hames et al., 2009; Schmidl et al., 2010). After four days upon infection, HeLa cells cultures were stained with crystal violet and photographed. The cytotoxicity assays were performed in triplicate.

Scanning electron microscopy. After growing of $M$. pneumoniae cultures in $5 \mathrm{ml}$ culture to mid-log phase, the cells were scraped off and passed ten times through a syringe. Then, $20 \mu \mathrm{l}$ of this cell suspension were inoculated to $2 \mathrm{ml}$ of modified Hayflick medium in a Lab-Tek chamber slide (Nunc). After growing cells to mid-log phase, the medium was removed and the cells were washed three times with PBS and fixed with $1 \%$ glutaraldehyde for $1 \mathrm{~h}$. The samples were washed three times with PBS and then dehydrated sequentially with $30,50,70,90$, and 100\% ethanol for $10 \mathrm{~min}$ each. Immediately, the critical point dried of samples was performed (K850 critical point drier; Emitech Ashfort, United Kingdom) and sputter coated with $20 \mathrm{~nm}$ of gold. Samples were observed using a Hitachi S-570 (Tokyo, Japan) scanning electron microscope.

Microcinematography. After passing through a syringe, cells grown in a $5 \mathrm{ml}$ culture, $20 \mu \mathrm{l}$ of disaggregated cells were inoculated to $2 \mathrm{ml}$ of modified Hayflick medium including 3\% gelatine in $14 \mathrm{~mm}$ glass bottom culture dishes plates (MatTek). Cell movement was examined at $37^{\circ} \mathrm{C}$ using a Nikon Eclipse TE 2000-E microscope, and images were captured at intervals of $2 \mathrm{~s}$ for a total of 2 min with a digital sight DS-SMC Nikon camera controlled by NIS-Elements BR software. Tracks from 50 individual motile cells corresponding to 2 min of observation and 2 separated experiments were analyzed to determine the gliding velocity and gliding motile patterns.

\section{Acknowledgments}

We are grateful to Stephanie Großhennig and Daniel Reuß for the help with some experiments. This work was supported by grants from the Deutsche Forschungsgemeinschaft and the Fonds der Chemischen Industrie. S. R. S. was supported by a personal grant from the Studienstiftung des Deutschen Volkes. 


\section{Supporting information}

Table S9. Proteins with differential expression pattern.

\begin{tabular}{|c|c|c|c|c|c|c|c|c|}
\hline $\begin{array}{l}\text { Locus } \\
\text { name }\end{array}$ & $\begin{array}{l}\text { Protein } \\
\text { name }\end{array}$ & $\begin{array}{l}\text { UniProtKB } \\
\text { accession } \\
\text { number }\end{array}$ & $\begin{array}{l}\text { Locus } \\
\text { name }\end{array}$ & $\begin{array}{l}\text { Protein } \\
\text { name }\end{array}$ & $\begin{array}{l}\text { UniProtKB } \\
\text { accession } \\
\text { number }\end{array}$ & $\begin{array}{l}\text { Locus } \\
\text { name }\end{array}$ & $\begin{array}{l}\text { Protein } \\
\text { name }\end{array}$ & $\begin{array}{l}\text { UniProtKB } \\
\text { accession } \\
\text { number }\end{array}$ \\
\hline \multicolumn{9}{|c|}{ Only identified in the presence of glucose } \\
\hline MPN012 & - & P75101 & MPN293 & LspA & P75484 & MPN502 & - & P75285 \\
\hline MPN030 & - & P75084 & MPN333 & - & P75445 & MPN504 & - & P75282 \\
\hline MPN035 & - & P75079 & MPN339 & - & P75439 & MPN505 & - & P75281 \\
\hline MPN068 & SecE & P75048 & MPN345 & HsdR & P75433 & MPN507 & - & P75279 \\
\hline MPN074 & SmpB & P75043 & MPN350 & PlsY & P75428 & MPN512 & - & P75274 \\
\hline MPN095 & - & P75597 & MPN351 & - & P75427 & MPN537 & MucB & P75241 \\
\hline MPN096 & - & P75596 & MPN385 & - & P75397 & MPN564 & Adh & P75214 \\
\hline MPN100 & - & P75592 & MPN404 & - & P75380 & MPN569 & - & P75209 \\
\hline MPN116 & RpmI & P75447 & MPN406 & - & P75378 & MPN575 & - & P75204 \\
\hline MPN130 & - & P75345 & MPN411 & - & P75373 & MPN582 & - & P75198 \\
\hline MPN136 & UgpE & P75262 & MPN414 & - & P75372 & MPN588 & - & Q50339 \\
\hline MPN137 & - & P75261 & MPN431 & - & P75357 & MPN589 & - & Q50338 \\
\hline MPN138 & - & P75260 & MPN435 & - & P75343 & MPN603 & AtpE & Q59550 \\
\hline
\end{tabular}


Table S9. Continued.

\begin{tabular}{|c|c|c|c|c|c|c|c|c|}
\hline $\begin{array}{l}\text { Locus } \\
\text { name }\end{array}$ & $\begin{array}{l}\text { Protein } \\
\text { name }\end{array}$ & $\begin{array}{l}\text { UniProtKB } \\
\text { accession } \\
\text { number }\end{array}$ & $\begin{array}{l}\text { Locus } \\
\text { name }\end{array}$ & $\begin{array}{l}\text { Protein } \\
\text { name }\end{array}$ & $\begin{array}{l}\text { UniProtKB } \\
\text { accession } \\
\text { number }\end{array}$ & $\begin{array}{l}\text { Locus } \\
\text { name }\end{array}$ & $\begin{array}{l}\text { Protein } \\
\text { name }\end{array}$ & $\begin{array}{l}\text { UniProtKB } \\
\text { accession } \\
\text { number }\end{array}$ \\
\hline MPN145 & - & P75141 & MPN440 & - & P75338 & MPN605 & - & Q50325 \\
\hline MPN146 & - & P75140 & MPN450 & - & Q50362 & MPN615 & HsdS & P75180 \\
\hline MPN151 & - & P75035 & MPN455 & CtaD & P75328 & MPN624 & RpmB & P75171 \\
\hline MPN152 & - & P75034 & MPN460 & KtrB & P75323 & MPN651 & MtlA & P75146 \\
\hline MPN163 & - & P75582 & MPN471 & RpmG & P78015 & MPN657 & - & P75134 \\
\hline MPN178 & RpsN & Q50305 & MPN482 & - & Q9EXD7 & MPN659 & TrmD & P75132 \\
\hline MPN214 & - & P75555 & MPN494 & UlaC & P75292 & MPN675 & - & P75117 \\
\hline MPN222 & Tils & P75549 & MPN495 & UlaB & Q9EXD8 & MPN682 & RpmH & P78006 \\
\hline MPN253 & PgsA & P75520 & MPN496 & UlaA & P75291 & & & \\
\hline \multicolumn{9}{|c|}{ Only identified in the presence of glycerol } \\
\hline MPN057 & PotC & P75057 & MPN365 & - & P75416 & MPN488 & - & P75297 \\
\hline MPN114 & Cpt2 & P75448 & MPN417 & P69 & P75369 & MPN640 & - & P75157 \\
\hline
\end{tabular}


Table S10. Proteins not detected in proteome analysis.

\begin{tabular}{|c|c|c|c|c|c|c|c|c|}
\hline $\begin{array}{l}\text { Locus } \\
\text { name }\end{array}$ & $\begin{array}{l}\text { Protein } \\
\text { name }\end{array}$ & $\begin{array}{l}\text { UniProtKB } \\
\text { accession } \\
\text { number }\end{array}$ & $\begin{array}{l}\text { Locus } \\
\text { name }\end{array}$ & $\begin{array}{l}\text { Protein } \\
\text { name }\end{array}$ & $\begin{array}{l}\text { UniProtKB } \\
\text { accession } \\
\text { number }\end{array}$ & $\begin{array}{l}\text { Locus } \\
\text { name }\end{array}$ & $\begin{array}{l}\text { Protein } \\
\text { name }\end{array}$ & $\begin{array}{l}\text { UniProtKB } \\
\text { accession } \\
\text { number }\end{array}$ \\
\hline MPN010 & - & P75103 & MPN206 & - & P75570 & MPN466 & - & P75317 \\
\hline MPN014 & DnaE & P75099 & MPN212 & - & P75557 & MPN467 & - & P75316 \\
\hline MPN037 & - & P75077 & MPN242 & SecG & Q9EXD0 & MPN468 & - & P75315 \\
\hline MPN038 & - & P75076 & MPN249 & EngC & P75523 & MPN485 & - & P75300 \\
\hline MPN039 & - & P75075 & MPN270 & - & Q9EXD1 & MPN486 & - & P75299 \\
\hline MPN040 & - & P75074 & MPN274 & - & P75503 & MPN497 & - & P75290 \\
\hline MPN041 & - & P75073 & MPN282 & - & P75495 & MPN500 & - & P75287 \\
\hline MPN042 & - & P75072 & MPN283 & - & P75494 & MPN503 & - & P75283 \\
\hline MPN048 & - & P75066 & MPN285 & PrrB & P75492 & MPN508 & - & P75278 \\
\hline MPN049 & - & P75065 & MPN286 & - & P75491 & MPN510 & - & P75276 \\
\hline MPN054 & - & P75060 & MPN289 & HsdS1B & P75488 & MPN511 & - & P75275 \\
\hline MPN056 & PotB & P75058 & MPN290 & - & P75487 & MPN513 & - & P75273 \\
\hline MPN069 & RpmG2 & P56850 & MPN304 & ArcA & P75475 & MPN514 & - & P75272 \\
\hline MPN085 & - & P75608 & MPN305 & ArcA & P75475 & MPN525 & - & P75253 \\
\hline MPN086 & - & P75607 & MPN306 & ArcB & P75473 & MPN527 & - & P75251 \\
\hline MPN087 & - & P75606 & MPN313 & - & P75468 & MPN534 & - & P75244 \\
\hline
\end{tabular}


Table S10. Continued.

\begin{tabular}{|c|c|c|c|c|c|c|c|c|}
\hline $\begin{array}{l}\text { Locus } \\
\text { name }\end{array}$ & $\begin{array}{l}\text { Protein } \\
\text { name }\end{array}$ & $\begin{array}{l}\text { UniProtKB } \\
\text { accession } \\
\text { number }\end{array}$ & $\begin{array}{l}\text { Locus } \\
\text { name }\end{array}$ & $\begin{array}{l}\text { Protein } \\
\text { name }\end{array}$ & $\begin{array}{l}\text { UniProtKB } \\
\text { accession } \\
\text { number }\end{array}$ & $\begin{array}{l}\text { Locus } \\
\text { name }\end{array}$ & $\begin{array}{l}\text { Protein } \\
\text { name }\end{array}$ & $\begin{array}{l}\text { UniProtKB } \\
\text { accession } \\
\text { number }\end{array}$ \\
\hline MPN088 & - & P75605 & MPN334 & BcrA & P75444 & MPN535 & RuvA & P75243 \\
\hline MPN089 & HsdS & P75604 & MPN335 & - & P75443 & MPN536 & RuvB & P75242 \\
\hline MPN091 & - & P75602 & MPN343 & - & P75435 & MPN540 & RpmF & P75238 \\
\hline MPN092 & - & P75600 & MPN346 & - & P75432 & MPN565 & - & P75213 \\
\hline MPN093 & - & P75599 & MPN347 & HsdR & P75431 & MPN570 & - & P75208 \\
\hline MPN097 & - & P75595 & MPN363 & - & P75418 & MPN571 & LcnDR3 & P75207 \\
\hline MPN098 & - & P75594 & MPN364 & - & P75417 & MPN577 & - & P75203 \\
\hline MPN099 & - & P75593 & MPN366 & - & P75415 & MPN578 & - & P75202 \\
\hline MPN101 & - & P75568 & MPN367 & - & P75414 & MPN579 & - & P75201 \\
\hline MPN102 & - & P75567 & MPN369 & - & P75412 & MPN580 & - & P75200 \\
\hline MPN103 & - & P75566 & MPN370 & - & P75411 & MPN581 & - & P75199 \\
\hline MPN107 & - & P75562 & MPN371 & - & P75410 & MPN583 & - & P75197 \\
\hline MPN108 & - & P75561 & MPN373 & - & P75408 & MPN584 & - & P75196 \\
\hline MPN110 & - & P75452 & MPN374 & - & P75407 & MPN586 & - & P75194 \\
\hline MPN111 & - & P75451 & MPN375 & - & P75406 & MPN587 & - & P75193 \\
\hline MPN112 & - & P75450 & MPN388 & - & Q9EXD4 & MPN590 & - & Q50337 \\
\hline
\end{tabular}


Table S10. Continued.

\begin{tabular}{|c|c|c|c|c|c|c|c|c|}
\hline $\begin{array}{l}\text { Locus } \\
\text { name }\end{array}$ & $\begin{array}{l}\text { Protein } \\
\text { name }\end{array}$ & $\begin{array}{l}\text { UniProtKB } \\
\text { accession } \\
\text { number }\end{array}$ & $\begin{array}{l}\text { Locus } \\
\text { name }\end{array}$ & $\begin{array}{l}\text { Protein } \\
\text { name }\end{array}$ & $\begin{array}{l}\text { UniProtKB } \\
\text { accession } \\
\text { number }\end{array}$ & $\begin{array}{l}\text { Locus } \\
\text { name }\end{array}$ & $\begin{array}{l}\text { Protein } \\
\text { name }\end{array}$ & $\begin{array}{l}\text { UniProtKB } \\
\text { accession } \\
\text { number }\end{array}$ \\
\hline MPN113 & - & P75449 & MPN403 & - & P75381 & MPN593 & - & Q50334 \\
\hline MPN127 & - & P75348 & MPN405 & - & P75379 & MPN594 & - & P75191 \\
\hline MPN128 & - & P75347 & MPN409 & - & P75375 & MPN612 & - & P75183 \\
\hline MPN129 & - & P75346 & MPN412 & - & Q9EXD5 & MPN613 & - & P75182 \\
\hline MPN131 & - & P75267 & MPN413 & - & Q9EXD6 & MPN614 & - & P75181 \\
\hline MPN132 & - & P75266 & MPN437 & - & P75341 & MPN626 & - & P75169 \\
\hline MPN143 & - & P75143 & MPN438 & - & P75340 & MPN633 & - & P75164 \\
\hline MPN144 & - & P75142 & MPN439 & - & P75339 & MPN634 & - & P75163 \\
\hline MPN147 & - & P75139 & MPN441 & - & P75337 & MPN635 & - & P75162 \\
\hline MPN149 & - & P75037 & MPN442 & - & P75336 & MPN637 & CdsA & P75160 \\
\hline MPN150 & - & P75036 & MPN448 & - & Q50364 & MPN644 & - & P75153 \\
\hline MPN160 & - & P75585 & MPN451 & Come3 & Q50361 & MPN648 & - & P75149 \\
\hline MPN188 & RpmJ & P52864 & MPN457 & - & P75326 & MPN649 & - & P75148 \\
\hline MPN201 & - & Q50287 & MPN458 & - & P75325 & MPN650 & - & P75147 \\
\hline MPN202 & - & Q50286 & MPN462 & - & P75321 & MPN654 & - & P75137 \\
\hline MPN203 & - & Q50284 & MPN463 & - & P75320 & MPN666 & - & P75125 \\
\hline
\end{tabular}


Table S10. Continued.

\begin{tabular}{|c|c|c|c|c|c|c|c|c|}
\hline $\begin{array}{l}\text { Locus } \\
\text { name }\end{array}$ & $\begin{array}{l}\text { Protein } \\
\text { name }\end{array}$ & $\begin{array}{l}\text { UniProtKB } \\
\text { accession } \\
\text { number }\end{array}$ & $\begin{array}{l}\text { Locus } \\
\text { name }\end{array}$ & $\begin{array}{l}\text { Protein } \\
\text { name }\end{array}$ & $\begin{array}{l}\text { UniProtKB } \\
\text { accession } \\
\text { number }\end{array}$ & $\begin{array}{l}\text { Locus } \\
\text { name }\end{array}$ & $\begin{array}{l}\text { Protein } \\
\text { name }\end{array}$ & $\begin{array}{l}\text { UniProtKB } \\
\text { accession } \\
\text { number }\end{array}$ \\
\hline MPN204 & - & P75572 & MPN464 & - & P75319 & MPN676 & - & P75116 \\
\hline MPN205 & - & P75571 & MPN465 & - & P75318 & MPN681 & RnpA & P75111 \\
\hline
\end{tabular}


Table S11. Summary of proteome and transcript analysis in the glpQ mutant GPM81 in the presence of glucose.

\begin{tabular}{|c|c|c|c|c|c|c|c|c|}
\hline \multirow{2}{*}{$\begin{array}{l}\text { Locus } \\
\text { name }\end{array}$} & \multirow{2}{*}{$\begin{array}{l}\text { Protein } \\
\text { name }\end{array}$} & \multirow{2}{*}{$\begin{array}{l}\text { UniProtKB } \\
\text { accession } \\
\text { number }\end{array}$} & \multirow[b]{2}{*}{ Protein function } & \multirow[b]{2}{*}{$\mathrm{COG}^{\mathrm{a}}$} & \multirow{2}{*}{$\begin{array}{l}\text { Molecular } \\
\text { weight } \\
\text { (kDa) }\end{array}$} & \multirow{2}{*}{$\begin{array}{l}\text { Isoelectric } \\
\text { point } \\
\text { (pI) }\end{array}$} & \multicolumn{2}{|c|}{ Fold-change $^{b}$} \\
\hline & & & & & & & $\begin{array}{l}\text { Protein } \\
\text { level }\end{array}$ & $\begin{array}{l}\text { Transcript } \\
\text { level }\end{array}$ \\
\hline \multicolumn{9}{|c|}{ Induced expression } \\
\hline MPN023 & MetG & P75091 & Methionyl-tRNA synthetase & $\mathrm{J}$ & 59.26 & 6.63 & $2.25 \pm 0.22$ & na \\
\hline MPN043 & GlpF & P75071 & Glycerol uptake facilitator & $\mathrm{G}$ & 28.31 & 9.33 & $10.79 \pm 1.06$ & $5.69 \pm 0.36$ \\
\hline MPN060 & MetK & P78003 & S-adenosylmethionine synthetase & $\mathrm{J}$ & 42.56 & 6.07 & $2.33 \pm 0.11$ & na \\
\hline MPN162 & - & P75583 & Uncharacterized lipoprotein & $\mathrm{S}$ & 36.10 & 6.19 & $9.96 \pm 0.97$ & $5.23 \pm 0.23$ \\
\hline MPN179 & RpsH & Q50304 & 30S ribosomal protein S8 & $\mathrm{J}$ & 15.88 & 10.32 & $2.63 \pm 0.33$ & na \\
\hline MPN191 & RpoA & Q50295 & RNA polymerase subunit $\alpha$ & $\mathrm{K}$ & 36.66 & 6.98 & $2.30 \pm 0.04$ & $2.18 \pm 0.17$ \\
\hline MPN209 & PacL & P78036 & Cation-transporting P-type ATPase & $\mathrm{P}$ & 94.97 & 6.38 & $2.49 \pm 0.26$ & na \\
\hline MPN221 & Pth & P78034 & Peptidyl-tRNA hydrolase & $\mathrm{J}$ & 21.42 & 9.52 & $2.43 \pm 0.14$ & na \\
\hline MPN223 & HPrK & P75548 & HPr kinase phosphorylase & $\mathrm{O}$ & 35.24 & 8.89 & $2.90 \pm 0.29$ & $2.83 \pm 0.09$ \\
\hline MPN239 & - & P75532 & HTH-type transcriptional regulator & K & 25.85 & 9.50 & $2.53 \pm 0.17$ & $3.16 \pm 0.14$ \\
\hline MPN244 & DisA & P75528 & Uncharacterized protein & $\mathrm{L}$ & 22.73 & 9.38 & $2.72 \pm 0.30$ & $3.39 \pm 0.19$ \\
\hline MPN247 & PrpC & P75525 & Protein phosphatase & $\mathrm{O}$ & 29.69 & 8.57 & $2.43 \pm 0.18$ & $2.91 \pm 0.12$ \\
\hline MPN248 & PrkC & P75524 & Serine threonine-protein kinase & $\mathrm{O}$ & 44.88 & 9.08 & $2.33 \pm 0.11$ & na \\
\hline MPN254 & CinA & Q9EXC9 & Uncharacterized protein & $\mathrm{S}$ & 16.96 & 8.81 & $2.60 \pm 0.27$ & $2.11 \pm 0.10$ \\
\hline MPN266 & Spx & P75509 & Transcriptional regulator & $\mathrm{K}$ & 16.81 & 9.81 & $2.71 \pm 0.13$ & $2.23 \pm 0.07$ \\
\hline
\end{tabular}


Table S11. Continued.

\begin{tabular}{|c|c|c|c|c|c|c|c|c|}
\hline \multirow{2}{*}{$\begin{array}{l}\text { Locus } \\
\text { name }\end{array}$} & \multirow{2}{*}{$\begin{array}{l}\text { Protein } \\
\text { name }\end{array}$} & \multirow{2}{*}{$\begin{array}{l}\text { UniProtKB } \\
\text { accession } \\
\text { number }\end{array}$} & \multirow[b]{2}{*}{ Protein function } & \multirow[b]{2}{*}{$\mathrm{COG}^{\mathrm{a}}$} & \multirow{2}{*}{$\begin{array}{l}\text { Molecular } \\
\text { weight } \\
\text { (kDa) }\end{array}$} & \multirow{2}{*}{$\begin{array}{l}\text { Isoelectric } \\
\text { point } \\
\text { (pI) }\end{array}$} & \multicolumn{2}{|c|}{ Fold-change $^{b}$} \\
\hline & & & & & & & $\begin{array}{l}\text { Protein } \\
\text { level }\end{array}$ & $\begin{array}{l}\text { Transcript } \\
\text { level }\end{array}$ \\
\hline MPN279 & LepA & P75498 & GTP-binding protein & $\mathrm{J}$ & 66.16 & 7.58 & $2.34 \pm 0.18$ & na \\
\hline MPN299 & PlsC & P75479 & 1-acyl-sn-glycerol-3-phosphate acyltransferase & I & 30.42 & 9.79 & $3.20 \pm 0.35$ & $6.43 \pm 0.31$ \\
\hline MPN300 & ScpA & P75478 & Segregation and condensation protein A & $\mathrm{U}$ & 59.52 & 8.15 & $2.45 \pm 0.18$ & na \\
\hline MPN338 & - & P75440 & Uncharacterized protein & $\mathrm{S}$ & 74.34 & 5.60 & $2.51 \pm 0.23$ & na \\
\hline MPN340 & PcrA & P75438 & DNA helicase II & $\mathrm{L}$ & 60.53 & 6.23 & $2.43 \pm 0.21$ & na \\
\hline MPN357 & LigA & P78021 & DNA ligase & $\mathrm{L}$ & 73.97 & 8.40 & $2.58 \pm 0.30$ & na \\
\hline MPN359 & - & P75421 & Uncharacterized protein & $\mathrm{S}$ & 30.56 & 9.67 & $2.34 \pm 0.24$ & na \\
\hline MPN372 & - & P75409 & ADP-ribosylating toxin CARDS & $\mathrm{V}$ & 68.06 & 5.63 & $2.91 \pm 0.21$ & $2.72 \pm 0.20$ \\
\hline MPN407 & - & P75377 & Lipase & I & 101.09 & 5.81 & $2.13 \pm 0.11$ & na \\
\hline MPN408 & - & P75376 & Uncharacterized lipoprotein & $\mathrm{S}$ & 83.35 & 9.16 & $3.57 \pm 0.26$ & na \\
\hline MPN425 & FtsY & P75362 & Cell division protein homolog & $\mathrm{D}$ & 38.78 & 6.85 & $2.34 \pm 0.21$ & na \\
\hline MPN433 & $\mathrm{CbiO}$ & P75355 & Metal ion ABC transporter ATP-binding protein & $\mathrm{P}$ & 30.77 & 8.41 & ns & $7.56 \pm 0.39$ \\
\hline MPN456 & - & P75327 & Uncharacterized lipoprotein & $\mathrm{S}$ & 110.52 & 6.33 & $2.35 \pm 0.18$ & na \\
\hline MPN518 & - & P75269 & Uncharacterized protein & $\mathrm{S}$ & 40.72 & 8.93 & $3.42 \pm 0.12$ & na \\
\hline MPN544 & - & P75234 & Uncharacterized protein & $\mathrm{S}$ & 76.77 & 7.50 & $5.14 \pm 0.48$ & na \\
\hline MPN552 & - & P75226 & Uncharacterized protein & $\mathrm{S}$ & 30.91 & 5.66 & $4.04 \pm 0.41$ & na \\
\hline
\end{tabular}


Table S11. Continued.

\begin{tabular}{|c|c|c|c|c|c|c|c|c|}
\hline \multirow{2}{*}{$\begin{array}{l}\text { Locus } \\
\text { name }\end{array}$} & \multirow{2}{*}{$\begin{array}{l}\text { Protein } \\
\text { name }\end{array}$} & \multirow{2}{*}{$\begin{array}{l}\text { UniProtKB } \\
\text { accession } \\
\text { number }\end{array}$} & \multirow[b]{2}{*}{ Protein function } & \multirow[b]{2}{*}{$\mathrm{COG}^{\mathrm{a}}$} & \multirow{2}{*}{$\begin{array}{l}\text { Molecular } \\
\text { weight } \\
\text { (kDa) }\end{array}$} & \multirow{2}{*}{$\begin{array}{l}\text { Isoelectric } \\
\text { point } \\
\text { (pI) }\end{array}$} & \multicolumn{2}{|c|}{ Fold-change $^{b}$} \\
\hline & & & & & & & $\begin{array}{l}\text { Protein } \\
\text { level }\end{array}$ & $\begin{array}{l}\text { Transcript } \\
\text { level }\end{array}$ \\
\hline MPN566 & - & P75212 & Similar to glycerophosphoryldiester phosphodiesterase & $\mathrm{S}$ & 27.72 & 9.24 & $2.89 \pm 0.16$ & $4.98 \pm 0.24$ \\
\hline MPN625 & - & P75170 & Osmotical inducible protein C-like protein & $\mathrm{O}$ & 15.47 & 6.50 & $2.56 \pm 0.13$ & na \\
\hline MPN664 & DegV & P75127 & Uncharacterized protein & $\mathrm{S}$ & 26.84 & 9.11 & $2.63 \pm 0.30$ & na \\
\hline \multicolumn{9}{|c|}{ Repressed expression } \\
\hline MPN015 & - & P75098 & Uncharacterized protein & $\mathrm{S}$ & 33.45 & 9.89 & $0.27 \pm 0.02$ & na \\
\hline MPN018 & Pmd1 & P75095 & ABC transporter ATP-binding protein & $\mathrm{S}$ & 68.93 & 9.07 & $0.20 \pm 0.05$ & sa \\
\hline MPN019 & MsbA & P75094 & ABC transporter ATP-binding protein & $\mathrm{S}$ & 71.15 & 9.52 & $0.17 \pm 0.04$ & na \\
\hline MPN055 & PotA & P75059 & Spermidine putrescine import ATP-binding protein & $\mathrm{E}$ & 65.13 & 9.10 & $0.26 \pm 0.02$ & na \\
\hline MPN083 & - & P75610 & Uncharacterized lipoprotein & $\mathrm{S}$ & 59.97 & 7.69 & nd & $2.81 \pm 0.16$ \\
\hline MPN084 & - & P75609 & Uncharacterized lipoprotein & $\mathrm{S}$ & 59.56 & 5.98 & $0.32 \pm 0.03$ & na \\
\hline MPN090 & - & P75603 & Adhesin P1 & M & 37.44 & 9.52 & $0.47 \pm 0.03$ & na \\
\hline MPN134 & UgpC & P75264 & sn-glycerol-3-phosphat transport system permease & G & 66.47 & 9.11 & $0.39 \pm 0.06$ & sa \\
\hline MPN258 & YjcW & P75516 & Sugar ABC transporter ATP-binding protein & $\mathrm{S}$ & 64.87 & 9.55 & $0.21 \pm 0.04$ & sa \\
\hline MPN259 & - & P75515 & Sugar ABC transporter permease & $\mathrm{S}$ & 58.59 & 9.71 & $0.22 \pm 0.06$ & na \\
\hline MPN260 & $\mathrm{RbsC}$ & P75514 & Sugar ABC transporter permease & $\mathrm{S}$ & 33.49 & 9.42 & $0.37 \pm 0.01$ & na \\
\hline MPN284 & - & P75493 & Uncharacterized lipoprotein & $\mathrm{S}$ & 87.17 & 9.16 & ns & $0.25 \pm 0.05$ \\
\hline
\end{tabular}


Table S11. Continued.

\begin{tabular}{|c|c|c|c|c|c|c|c|c|}
\hline \multirow{2}{*}{$\begin{array}{l}\text { Locus } \\
\text { name }\end{array}$} & \multirow{2}{*}{$\begin{array}{l}\text { Protein } \\
\text { name }\end{array}$} & \multirow{2}{*}{$\begin{array}{l}\text { UniProtKB } \\
\text { accession } \\
\text { number }\end{array}$} & \multirow[b]{2}{*}{ Protein function } & \multirow[b]{2}{*}{$\mathrm{COG}^{\mathrm{a}}$} & \multirow{2}{*}{$\begin{array}{l}\text { Molecular } \\
\text { weight } \\
\text { (kDa) }\end{array}$} & \multirow{2}{*}{$\begin{array}{l}\text { Isoelectric } \\
\text { point } \\
\text { (pI) }\end{array}$} & \multicolumn{2}{|c|}{ Fold-change $^{b}$} \\
\hline & & & & & & & $\begin{array}{l}\text { Protein } \\
\text { level }\end{array}$ & $\begin{array}{l}\text { Transcript } \\
\text { level }\end{array}$ \\
\hline MPN322 & NrdF & P75461 & Ribonucleoside-diphosphate reductase subunit $\beta$ & $\mathrm{F}$ & 39.41 & 5.47 & $0.38 \pm 0.05$ & ns \\
\hline MPN323 & NrdI & P75460 & Ribonucleoside-diphosphate reductase stimulatory protein & $\mathrm{F}$ & 17.15 & 7.79 & $0.29 \pm 0.06$ & na \\
\hline MPN324 & NrdE & P78027 & Ribonucleoside-diphosphate reductase subunit $\alpha$ & $\mathrm{F}$ & 82.38 & 6.63 & $0.40 \pm 0.05$ & na \\
\hline MPN420 & GlpQ & P75367 & Glycerophosphoryldiester phosphodiesterase & $\mathrm{C}$ & 28.37 & 6.32 & nd & na \\
\hline MPN449 & - & Q50363 & Uncharacterized protein & $\mathrm{S}$ & 50.55 & 9.32 & $0.10 \pm 0.04$ & na \\
\hline MPN498 & UlaF & P75289 & L-ribulose-5-phosphate 4-epimerase & $\mathrm{C}$ & 27.09 & 6.44 & $0.20 \pm 0.04$ & sa \\
\hline MPN506 & - & P75280 & Uncharacterized lipoprotein & $\mathrm{S}$ & 87.50 & 9.24 & $0.10 \pm 0.03$ & $0.07 \pm 0.05$ \\
\hline MPN684 & - & P75109 & ABC transporter permease & $\mathrm{S}$ & 209.45 & 8.23 & $0.32 \pm 0.03$ & sa \\
\hline MPN685 & CysA & Q50316 & ABC transport ATP-binding protein & $\mathrm{S}$ & 32.24 & 9.69 & $0.21 \pm 0.04$ & na \\
\hline
\end{tabular}

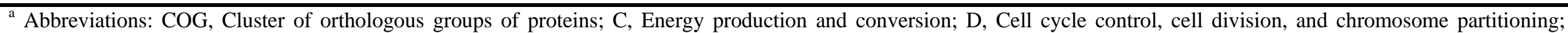

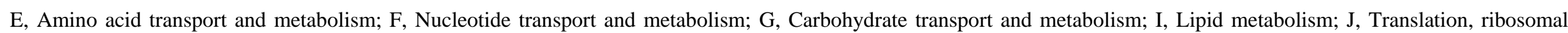

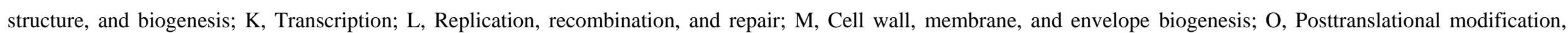

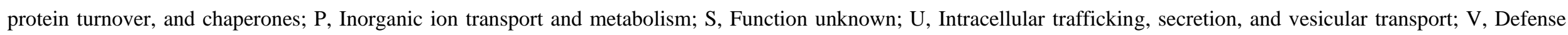
mechanisms.

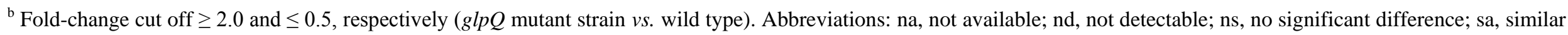
amount. 
Table S12. Summary of proteome and transcript analysis in the glpQ mutant GPM81 in the presence of glycerol.

\begin{tabular}{|c|c|c|c|c|c|c|c|c|}
\hline \multirow{2}{*}{$\begin{array}{l}\text { Locus } \\
\text { name }\end{array}$} & \multirow{2}{*}{$\begin{array}{l}\text { Protein } \\
\text { name }\end{array}$} & \multirow{2}{*}{$\begin{array}{l}\text { UniProtKB } \\
\text { accession } \\
\text { number }\end{array}$} & \multirow[b]{2}{*}{ Protein function } & \multirow[b]{2}{*}{$\mathrm{COG}^{\mathrm{a}}$} & \multirow{2}{*}{$\begin{array}{l}\text { Molecular } \\
\text { weight } \\
\text { (kDa) }\end{array}$} & \multirow{2}{*}{$\begin{array}{l}\text { Isoelectric } \\
\text { point } \\
\text { (pI) }\end{array}$} & \multicolumn{2}{|c|}{ Fold-change $^{b}$} \\
\hline & & & & & & & $\begin{array}{l}\text { Protein } \\
\text { level }\end{array}$ & $\begin{array}{l}\text { Transcript } \\
\text { level }\end{array}$ \\
\hline \multicolumn{9}{|c|}{ Induced expression } \\
\hline MPN043 & GlpF & P75071 & Glycerol uptake facilitator & G & 28.31 & 9.33 & $5.83 \pm 0.76$ & $3.32 \pm 0.34$ \\
\hline MPN162 & - & P75583 & Uncharacterized lipoprotein & S & 36.10 & 6.19 & $5.41 \pm 0.83$ & $2.81 \pm 0.47$ \\
\hline MPN433 & $\mathrm{CbiO}$ & P75355 & Metal ion ABC transporter ATP-binding protein & $\mathrm{P}$ & 30.77 & 8.41 & $5.66 \pm 0.49$ & $2.92 \pm 0.24$ \\
\hline MPN444 & - & P75334 & Uncharacterized lipoprotein & $\mathrm{S}$ & 146.28 & 7.89 & $2.74 \pm 0.09$ & na \\
\hline MPN489 & - & P75296 & Uncharacterized lipoprotein & $\mathrm{S}$ & 143.06 & 9.14 & $2.40 \pm 0.20$ & na \\
\hline \multicolumn{9}{|c|}{ Repressed expression } \\
\hline MPN284 & - & P75493 & Uncharacterized lipoprotein & $\mathrm{S}$ & 87.17 & 9.16 & $0.36 \pm 0.02$ & $0.35 \pm 0.04$ \\
\hline MPN288 & - & P75489 & Uncharacterized lipoprotein & $\mathrm{S}$ & 86.89 & 9.07 & $0.29 \pm 0.03$ & na \\
\hline MPN420 & GlpQ & P75367 & Glycerophosphoryldiester phosphodiesterase & $\mathrm{C}$ & 28.37 & 6.32 & nd & na \\
\hline MPN506 & - & P75280 & Uncharacterized lipoprotein & $\mathrm{S}$ & 87.50 & 9.24 & $0.18 \pm 0.04$ & $0.16 \pm 0.02$ \\
\hline MPN673 & - & P75118 & Uncharacterized protein & $\mathrm{S}$ & 19.47 & 9.16 & $0.21 \pm 0.03$ & na \\
\hline
\end{tabular}

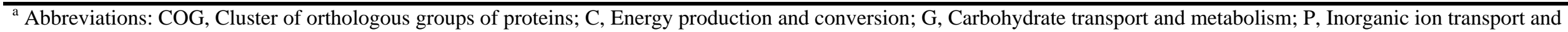
metabolism; S, Function unknown.

${ }^{\mathrm{b}}$ Fold-change cut off $\geq 2.0$ and $\leq 0.5$, respectively ( $g l p Q$ mutant strain vs. wild type). Abbreviations: na, not available; nd, not detectable. 
Table S13. Oligonucleotides used in this study.

\begin{tabular}{|c|c|c|}
\hline Oligonucleotide & Sequence $\left(5^{\prime} \rightarrow 3^{\prime}\right)^{\text {ab }}$ & Description \\
\hline CH61 & ATGCCAAATCCTGTTAGATTTGTTTAC & mpn372 probe $\mathrm{fw}$ \\
\hline CH63 & CTAATACGACTCACTATAGGGAGAGCCGGGTGGTGAGCATG & mpn372 probe rev \\
\hline SH03 & GAGTACCCGGATTAAAGCGGG & hprK probe fw \\
\hline SH04 & CTAATACGACTCACTATAGGGAGACATTAACTGGATTTCGGTGCGCTG & hprK probe rev \\
\hline SH05 & CAGGTAACGGTGCTGGTTCG & $g l p F$ probe fw \\
\hline SH06 & CTAATACGACTCACTATAGGGAGAATTGCAGTACCAGTAGCGGC & glpF probe rev \\
\hline SH29 & ATGAGTGAGCTAACTCACAG & Screening primer for transposon insertions \\
\hline SH30 & CAATACGCAAACCGCCTC & Screening primer for transposon insertions \\
\hline SH62 & TAGAATTTTATGGTGGTAGAG & aac-ahpD probe fw \\
\hline SH63 & CTAATACGACTCACTATAGGGAGAACACTATCATAACCACTACC & $a a c-a h p D$ probe rev \\
\hline SH66 & AAAGTCGACATGGACAGCACCAACCAAAAC & prpC probe fw (SalI) \\
\hline SH73 & CTAATACGACTCACTATAGGGAGAGACCATCAGAGCACAACAG & prpC probe rev \\
\hline SS34 & AAAGAGCTCGATGCTTAAACGACAACTTCTGCTAGC & glpQ gene $\mathrm{fw}(\mathrm{SacI})$ \\
\hline SS35 & TATAGGATCCTTACACTTCAAACTTCTTGTTGGCAATTTG & glpQ gene rev (BamHI) \\
\hline SS36 & P_GCCTTTTTGTTTTGGACGAAAAAGCAGTTCCAAG & glpQ A507G \\
\hline SS37 & P_CAGTATCTCCATCCCTGGACAAACATTTACG & $g l p Q$ A576G \\
\hline SS38 & P_CCTTTAGGGCTGTGGACGCTTAACAGTG & glpQ A639G \\
\hline SS39 & AAAGAGCTCGATGCGCAAACAGTTTTTAATTGCACAC & mpn566 gene fw (SacI) \\
\hline
\end{tabular}


Table S13. Continued.

\begin{tabular}{|c|c|c|}
\hline Oligonucleotide & Sequence $\left(5^{\prime} \rightarrow 3^{\prime}\right)^{\text {ab }}$ & Description \\
\hline SS40 & TATAGGATCCTTAGTAAAGTTGTGCTGCTATTTGAAATTTAAC & mpn566 gene rev (BamHI) \\
\hline SS42 & CAACTTCTGCTAGCACACCG & glpQ probe fw \\
\hline SS43 & CTAATACGACTCACTATAGGGAGAGCTATTTGGTAGTTGGGGTTAATG & glpQ probe rev \\
\hline SS44 & GCAAACAGTTTTTAATTGCACACCG & mpn566 probe fw \\
\hline SS45 & CTAATACGACTCACTATAGGGAGAGCTCTTTAACTTTTCGTTGAGGTAC & mpn566 probe rev \\
\hline SS123 & GAATCAGTTTCTCCСTTAGAATATGC & $n r d F$ probe fw \\
\hline SS124 & CTAATACGACTCACTATAGGGAGAGTCTTTCCCGGTGTAATAGGG & $n r d F$ probe rev \\
\hline SS127 & CCAACAGCGCTTTTATTCTCGG & mpn083 probe fw \\
\hline SS128 & CTAATACGACTCACTATAGGGAGAGGACATTAGGTTTGGTGTACTTAC & mpn083 probe rev \\
\hline SS129 & CCTTGTTAGTTGCGCCACAC & mpn162 probe fw \\
\hline SS130 & CTAATACGACTCACTATAGGGAGACACCTTCGTGGTGATCATGATC & mpn162 probe rev \\
\hline SS131 & GGCTTAGTCATCCACACTTGG & ulaF probe fw \\
\hline SS132 & CTAATACGACTCACTATAGGGAGACCACTGCATCCTTGCCATTC & ulaF probe rev \\
\hline SS135 & GGTTTTACCCGTTTTTGTGTTAATGC & pls $C$ probe fw \\
\hline SS136 & CTAATACGACTCACTATAGGGAGAGCCCGATTTAAACTCACCAATTTG & plsC probe rev \\
\hline SS137 & CСGTTACATTCTCСТTAАAАTTCAАAG & mpn239 probe fw \\
\hline SS138 & CTAATACGACTCACTATAGGGAGAGCTCTACCACAATGCCGTTG & mpn239 probe rev \\
\hline SS139 & ATGCTTAAGAAAAAAGTTAATAATGATGCTG & spx probe fw \\
\hline
\end{tabular}


Table S13. Continued.

\begin{tabular}{|c|c|c|}
\hline Oligonucleotide & Sequence $\left(5^{\prime} \rightarrow 3^{\prime}\right)^{\mathrm{ab}}$ & Description \\
\hline SS140 & CTAATACGACTCACTATAGGGAGATTACTTCTTTACTGTACGCACTTTAGG & spx probe rev \\
\hline SS141 & CCGCTTTTCACCTTTGCACAG & pmd1 probe fw \\
\hline SS142 & CTAATACGACTCACTATAGGGAGAGAGTTAGTGGCAATAGCAAAGGC & pmd1 probe rev \\
\hline SS143 & GCTAATGTAGATGTTAACCTCACG & $y j c W$ probe $\mathrm{fw}$ \\
\hline SS144 & CTAATACGACTCACTATAGGGAGACTCAAAACAGCGGTTGGTTCATC & $y j c W$ probe rev \\
\hline SS145 & CCTGATTTATGACAAAGAAGGTAACC & mpn684 probe fw \\
\hline SS146 & CTAATACGACTCACTATAGGGAGAGGGATTAGTCTCTAACCAAAACTG & mpn684 probe rev \\
\hline SS147 & GAATATTGTAGTCGACTTTGGTGAG & ugp $C$ probe $\mathrm{fw}$ \\
\hline SS148 & CTAATACGACTCACTATAGGGAGAGGTTAGCTTCCAGTTCACTCTG & ugpC probe rev \\
\hline SS168 & GCTAAATCATAAGCTCACCATTGC & $\operatorname{cin} A$ probe fw \\
\hline SS169 & CTAATACGACTCACTATAGGGAGAGCTGCATCTTGTTCTCTTAAACC & $\operatorname{cin} A$ probe rev \\
\hline SS170 & CTAACCСCAACTACGGCATC & rpoA probe fw \\
\hline SS171 & CTAATACGACTCACTATAGGGAGACCCCAAAGAATTAATCATTTCACGG & rpoA probe rev \\
\hline SS172 & CTTAATCTTAGCGCTTACCTTGTTG & disA probe fw \\
\hline SS173 & CTAATACGACTCACTATAGGGAGAGTTTTAATCACGCCGCGCAC & disA probe rev \\
\hline SS176 & СТСААТTTTAGCСТСАААСССААС & cbiO probe fw \\
\hline SS177 & CTAATACGACTCACTATAGGGAGACCGCTTTAGTTTGCTAGCTAGC & cbiO probe rev \\
\hline SS190 & GCTGCCTGTGGTACAAAGG & mpn506 probe fw \\
\hline
\end{tabular}


Table S13. Continued.

\begin{tabular}{|c|c|c|}
\hline Oligonucleotide & Sequence $\left(5^{\prime} \rightarrow 3^{\prime}\right)^{\text {ab }}$ & Description \\
\hline SS191 & CTAATACGACTCACTATAGGGAGAGTACCTTCTTTCTTTTTAGCGTTGTTC & mpn506 probe rev \\
\hline SS192 & P_GATGGTTTGGAGATGGATGTGCAACTCAC & mpn566 T106G/G107A/A114T \\
\hline SS193 & P_CGTTTCGCTTTCTCTTGCTTGAAATTAAGGGCG & mpn566 T328G/T329A/T330A \\
\hline SS194 & P_GTAGTCACTCATGATGACAACTATAAGGTAGGAAATAAAACC & mpn566 A151C/T154G/T155A/A156T \\
\hline SS199 & CСАATTAAAGCTCСТTCAAAGAATGG & mpn284 probe fw \\
\hline SS200 & CTAATACGACTCACTATAGGGAGACGATCGTATTGTTCGTCATTAATTTTG & mpn284 probe rev \\
\hline
\end{tabular}

${ }^{a}$ Restriction sites and T7-promotors are underlined, respectively.

b The "P" at the 5' end of primer sequences indicates phosphorylation. 


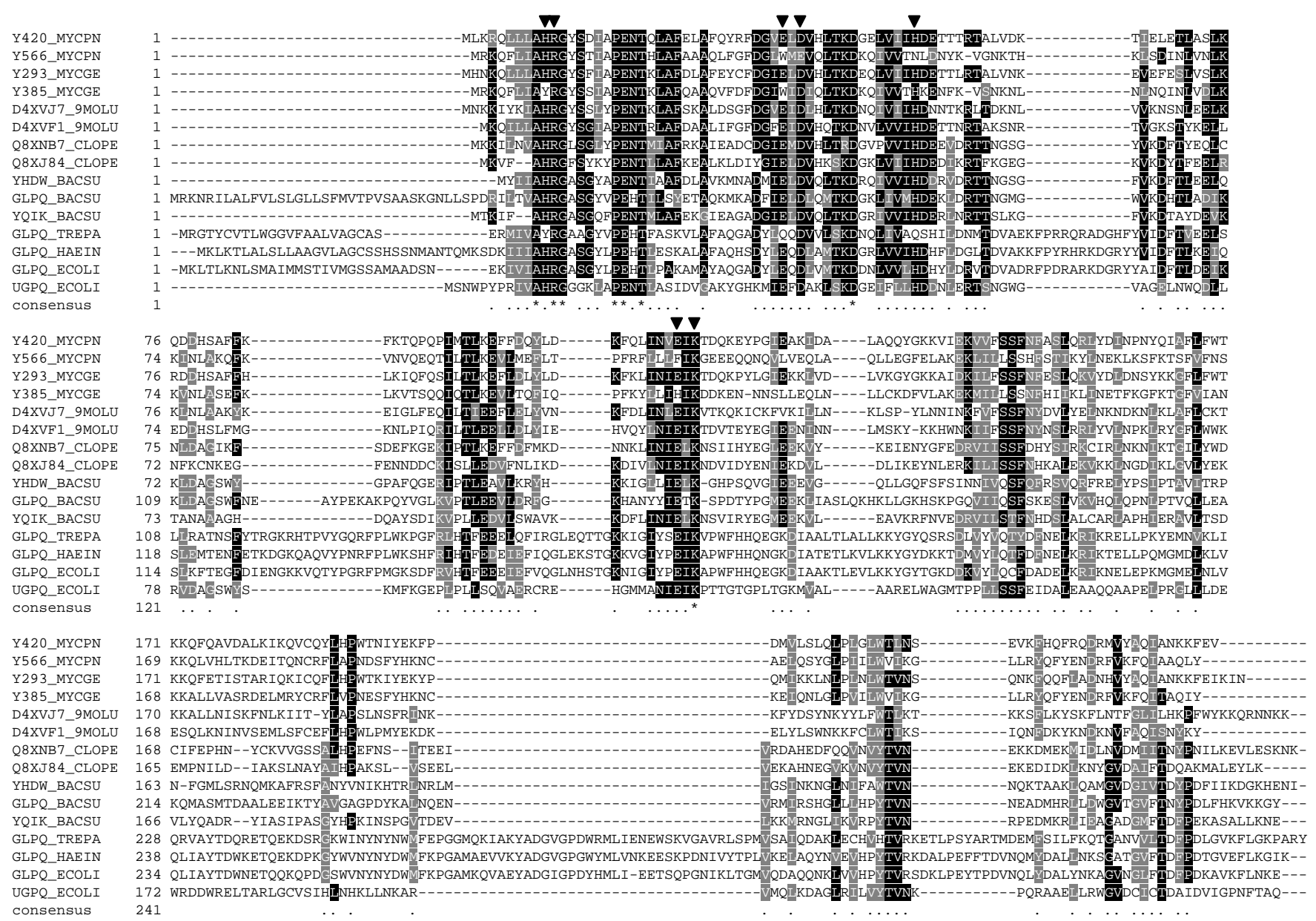


Fig. S4. Multiple sequence alignment of GIpQ and MPN566 from M. pneumoniae with orthologous glycerophosphodiesterases of other bacteria. The multiple sequence alignment was performed using ClustalW (http://www.ch.embnet.org/software/ClustalW.html) and represented with BOXSHADE v3.21 (http://www.ch.embnet.org/software/BOX_form.html). Black shading indicates $\geq 80 \%$ identity and grey shading stands for $\geq 80 \%$ similarity. Amino acids that constitute to the strictly conserved active site structure are depicted by black arrows. The UniProtKB entry names of the aligned sequences are Y420_MYCPN (GlpQ, M. pneumoniae), Y566_MYCPN (MPN566, M. pneumoniae), Y293_MYCGE (MG_293, M. genitalium), Y385_MYCGE (MG_385, M. genitalium), D4XVJ7_9MOLU (MALL_0582, M. alligatoris), D4XVF1_9MOLU (MALL_0631, M. alligatoris), Q8XNB7_CLOPE (GlpQ, Clostridium perfringens), Q8XJ84_CLOPE (GlpQ, C. perfringens), YHDW_BACSU (YhdW, B. subtilis), GLPQ_BACSU (GlpQ, B. subtilis), YQIK_BACSU (YqiK, B. subtilis), GLPQ_TREPA (GlpQ, T. pallidum), GLPQ_HAEIN (GlpQ, H. influenzae), GLPQ_ECOLI (GlpQ, E. coli), and UGPQ_ECOLI (UgpQ, E. coli).

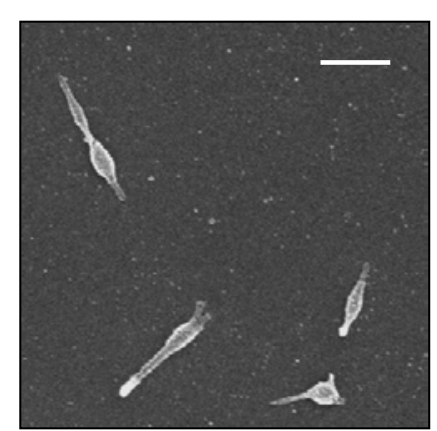

wt

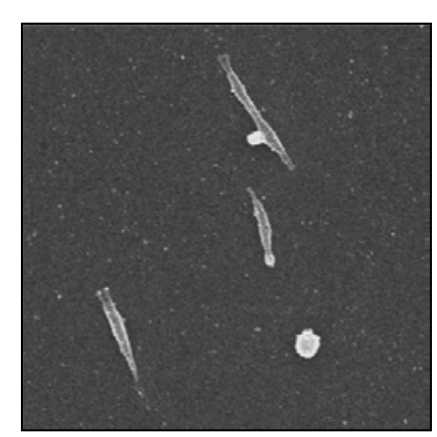

glpQ::Tn

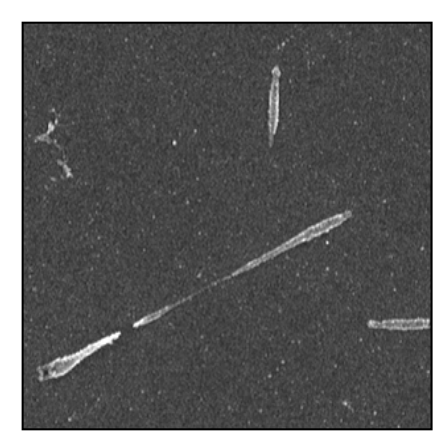

mpn566::Tn

Fig. S5. Scanning electron microscopy analyses of $M$. pneumoniae. Morphology and cell division of M. pneumoniae wild type (wt), glpQ::Tn, and mpn566::Tn mutant strains were compared with each other. All pictures are shown at the same magnification. Scale bar, $1.0 \mu \mathrm{m}$. 
A

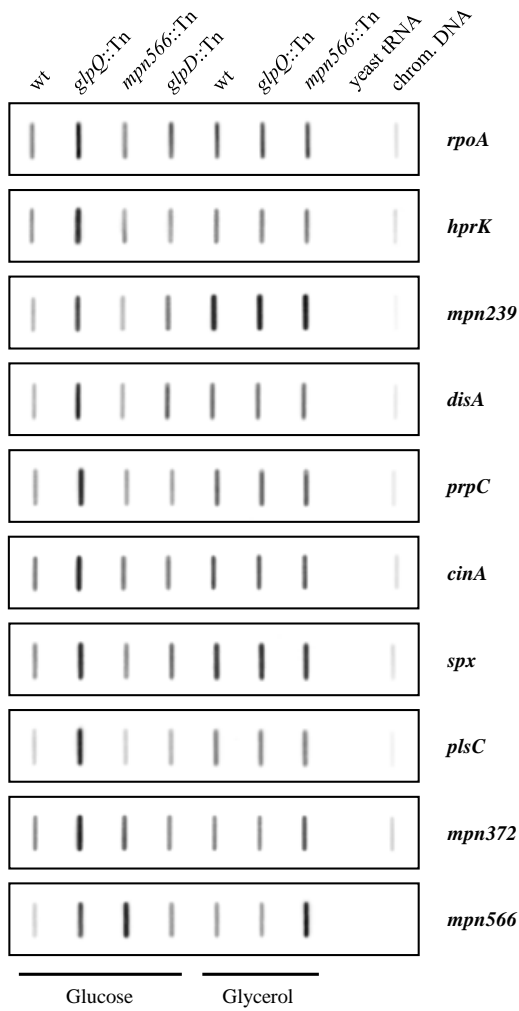

B

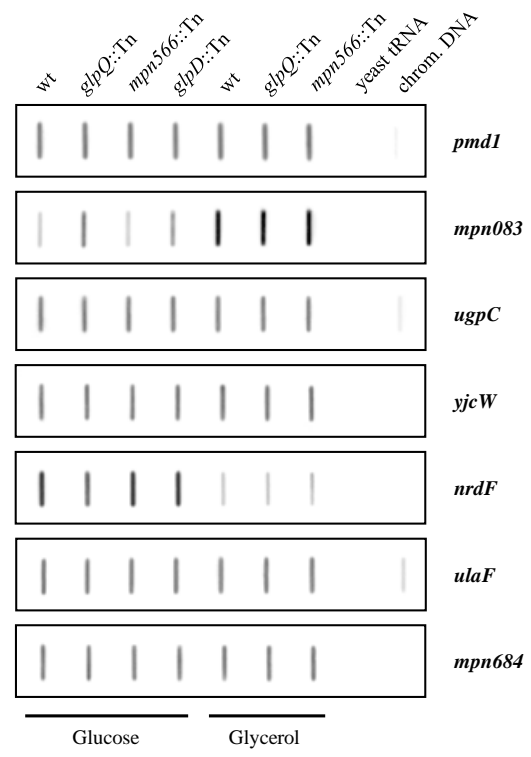

Fig. S6. Transcription analysis of interesting genes with protein amount changes in the glpQ mutant. Slot blots were performed with whole RNA extracts of M. pneumoniae wild type (wt), glpQ::Tn, mpn566::Tn, and glpD::Tn [control; Hames et al. (2009)] mutant strains grown in modified Hayflick medium containing either glucose or glycerol as sole carbon source (1\% wt/vol). A dilution series of RNA extracts was blotted onto a positively charged nylon membrane and probed with a DIG-labeled riboprobe specific for an internal part of a particular open reading frame. Names of riboprobes are given next to each blot. Signals obtained with $1 \mu \mathrm{g}$ of RNA are shown. Yeast tRNA and M. pneumoniae chromosomal DNA served as controls. Genes which had a significant higher protein amount in the $g l p Q:: T n$ mutant with glucose as carbon source are shown in (A) and genes with significant lower protein amounts in (B). For detailed information on changes of transcript levels see Table S11 and S12. 
$\underline{\text { Chapter } 8}$

Discussion 


\section{Protein phosphorylation in a minimal organism and its impact on virulence}

Although much work has been devoted to the Mollicutes in the past few years, only little is known about their regulatory mechanisms. One major question is, how Mycoplasma pneumoniae cells recognize whether they are approaching a host tissue or not, and which changes in protein activities and/or gene expressions are caused. The most prominent regulatory mechanism both in bacteria and in eukaryotes is the control of protein activity by protein phosphorylation (Boekhorst et al., 2008; Jers et al., 2008). Bacteria possess two different classes of phosphorylation events. In addition to histidine and aspartate phosphorylation as part of two-component signal transduction (Hoch, 2000; Stock et al., 2000), serine/threonine/tyrosine phosphorylation is also present and comes more and more into the spotlight. Phosphoproteome studies have been performed for several bacteria, including the model organisms Escherichia coli and Bacillus subtilis (Eymann et al., 2007; Lévine et al., 2006; Macek et al., 2008; Macek et al., 2007) or pathogens like Klebsiella pneumoniae or Streptococcus pneumoniae (Lin et al., 2009; Sun et al., 2010).

The analysis of the phosphoproteome of $M$. pneumoniae revealed substantial protein serine/threonine/tyrosine phosphorylation even in this minimal organism. In total 63 phosphorylated proteins could be identified that account for nearly $10 \%$ of all proteins encoded by $M$. pneumoniae. Compared to only $2.5 \%$ of all proteins of $B$. subtilis that are known to be phosphorylated on a serine, threonine or tyrosine residue (Eymann et al., 2007; Lévine et al., 2006; Macek et al., 2007), it can be speculated that protein phosphorylation occurs more frequently in $M$. pneumoniae than in other bacteria. This might be related to the lack of an obvious transcription regulation machinery in M. pneumoniae (Dandekar et al., 2000; Himmelreich et al., 1996). Nevertheless, the size of all studied bacterial phosphoproteomes so far is remarkably similar suggesting a conservation of phosphorylated proteins. A comparison of the phosphoproteomes revealed a high proportion of phosphoproteins, including glycolytic enzymes and elongation factors that are conserved throughout the bacterial kingdom (Soufi et al., 2008; this work). However, phosphorylation sites are hardly conserved and only one site, a serine residue of phosphosugar mutases, is actually identical in all living organisms from archaea and bacteria to man (Soufi et al., 2008; this work). This observation might be due to the fact that site specific phosphorylation coevolved with 
the adaptation of an organism to an individual ecological niche. Furthermore, the phosphorylation process might also partially result from gene transfer as many eukaryote-like kinases have been found in bacteria by metagenomic approaches (Yooseph et al., 2007). This suggests that regulation via protein phosphorylation developed relatively late in the evolution. In general, eukaryotic phosphosites are more conserved throughout the eukaryotic domain (Gnad et al., 2010). An explanation for this could be that in eukaryotes phosphorylation regulates many key processes including cell growth, proliferation, differentiation, and immune response (Hubbard and Miller, 2007; Pawson and Scott, 2005), processes which are not present in bacteria.

The knowledge about phosphorylation events and their regulation is crucial to understand the functional biology of an organism. M. pneumoniae contains two protein kinases, the HPr kinase (HPrK) and a serine/threonine protein kinase C (PrkC) (Steinhauer et al., 2002; this work). For HPrK, only an implication in the phosphorylation of the HPr protein could be observed. In B. subtilis and other Firmicutes, the phosphorylation of HPr on Ser-46 is a major signal to trigger carbon catabolite repression (Görke and Stülke, 2008). In this case, $\operatorname{HPr}(\operatorname{Ser}-\mathrm{P})$ acts as the cofactor for the pleiotropic transcription factor CcpA to regulate the expression of catabolic genes and operons. However, no such protein is encoded in the genome of M. pneumoniae suggesting a different role for $\mathrm{HPr}(\mathrm{Ser}-\mathrm{P})$. This idea is supported by the fact that also the proteome of the hprK mutant was similar to the wild type strain.

The kinase PrkC is implicated in various cellular processes in different bacteria, among them spore germination, virulence, and control of glycolysis (Kristich et al., 2007; Lomas-Lopez et al., 2007; Shah et al., 2008). In M. pneumoniae, six target proteins could be identified, including the major adhesin P1, two large cytadherence proteins HMW1 and HMW2, the adhesin-related protein P41, the coiled coil surface protein MPN474, and a protein of unknown function MPN256 (Fig. 36A). Phosphorylation of most of these proteins was reported earlier (Krebes et al., 1995; Su et al., 2007). However, the corresponding kinase had not been identified. The HMW proteins and P1 are part of the so-called terminal organelle of $M$. pneumoniae that is involved in gliding motility, cell division, and adhesion to host epithelial tissues (Balish and Krause, 2006; Miyata, 2010). PrkC-dependent phosphorylation seems to be required for the stability of this protein complex due to the observation that an inactivation of the kinase led not only to a loss of protein phosphorylation, but also to a 
decrease of the protein amount of the target proteins and also additional cytadherence proteins. It is known that the cytadherence proteins show a reciprocal dependency in their stabilities (Balish and Krause, 2006). Specifically, HMW1 was shown to be required for HMW2 and P1 localization and stability (Willby et al., 2004). In turn, HMW2 is necessary for the stabilization of HMW3 and P65 (Fisseha et al., 1999). As shown in this work, it has been suggested previously that the interdependence of the proteins of the attachment organelle is regulated posttranslationally (Popham et al., 1997). In conclusion, PrkC is essential for cell adhesion in M. pneumoniae, which is also reflected by a nonadherent growth type of the prkC mutant strain. Furthermore, this mutant has lost cytotoxicity toward HeLa cells, most likely due to defects in cell adhesion. Interestingly, it has been reported that the $\operatorname{prp} C$ mutant has a reduced gliding velocity (Hasselbring et al., 2006). Indeed, phosphorylation of the six PrkC target proteins was enhanced in the $\operatorname{prpC}$ mutant strain suggesting that PrpC is the protein phosphatase that reverses PrkC-dependent protein phosphorylation in $M$. pneumoniae (see Fig. 36A). Thus, the antagonistic PrkC/PrpC pair has a specific function in M. pneumoniae and phosphorylates/dephosphorylates a specific set of proteins. Nevertheless, PrpC is implicated in two more dephosphorylation events. First in the dephosphorylation of HPr(Ser-P) (Halbedel et al., 2006) and in addition, PrpC seems also to dephosphorylate RpoE, the RNA polymerase subunit $\delta$ (Fig. 36B). Unfortunately, the role of both dephosphorylation events is unknown.

As already mentioned, HPrK and PrkC are the only two annotated protein kinases in $M$. pneumoniae. However, these known kinases were not responsible for the majority of phosphorylation events, since only seven phosphoproteins were affected by the loss of one of these two kinases. This suggests the existence of other, yet to be discovered protein kinases in $M$. pneumoniae. Alternatively, autophosphorylation of proteins might be more relevant than previously anticipated. Indeed, it could be shown in this work that the only universally conserved phosphorylation event in all living organisms is the result of an autophosphorylation in the catalytic site of phosphosugar mutases such as B. subtilis GlmM (Ser-100) and M. pneumoniae ManB (Ser-149). For the orthologue of both phosphosugar mutases in E. coli, it was established that the protein is only active in the phosphorylated form (Jolly et al., 2000; Mengin-Lecreulx et al., 1996). Thus, autophosphorylation is essential for the enzymatic activity of these enzymes. In general, mutases are widespread over all phyla of life 
suggesting a conserved reaction mechanism. Therefore, it is not surprising to find autophosphorylation of mutases in other organisms like the archaeon Sulfolobus solfataricus or the mouse (Gururaj et al., 2004; Potters et al., 2003). Autophosphorylation was also identified for the GTP-binding protein Era in E. coli or the mammalian glyceraldehyde-3-phosphate dehydrogenase (Kawamoto and Caswell, 1986; Sood et al., 1994). Another protein group where autophosphorylation events seem to be very common, are the chaperones. The ubiquitous heat shock protein DnaK (also called Hsp70) is present as a phosphoprotein in all studied organisms so far. In E. coli and mycobacteria, DnaK is capable of autophosphorylating at a strongly conserved threonine residue (McCarty and Walker, 1991; Peake et al., 1998; Preneta et al., 2004; Zylicz et al., 1983). This site is also present in the DnaK protein of M. pneumoniae (Thr-182) suggesting a possible autophosphorylation mechanism.

A
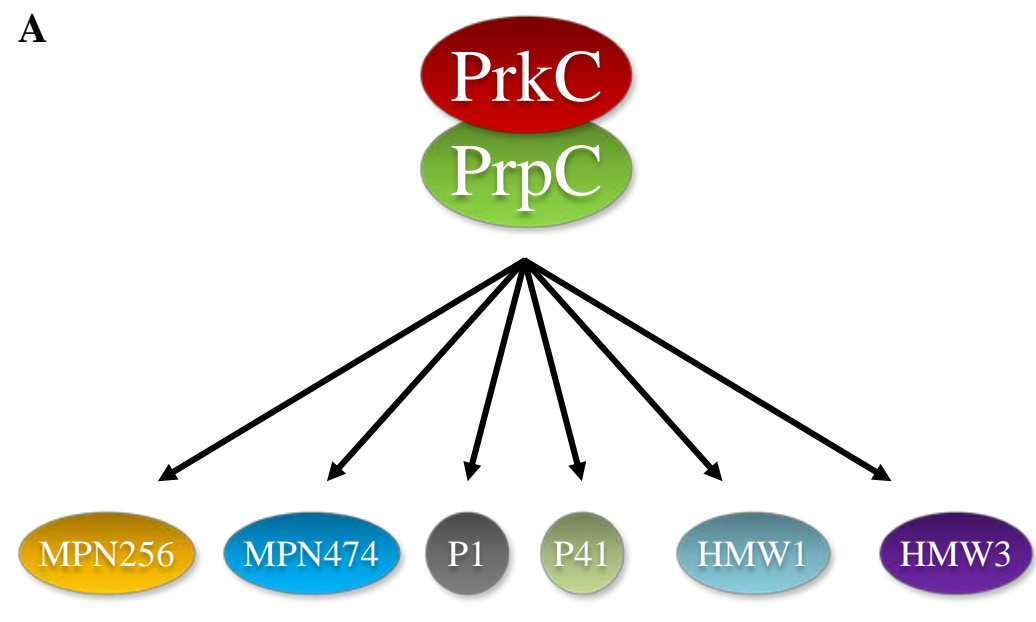

B
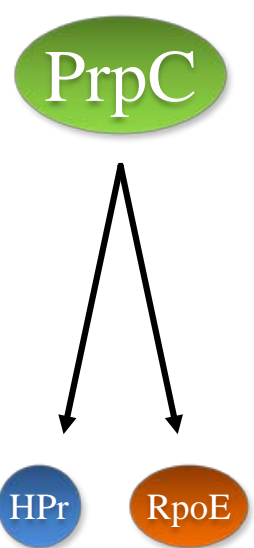

Fig 36. Overview of PrkC/PrpC-dependent phosphorylation events in M. pneumoniae. (A) Proteins affected by the antagonistic kinase/phosphatase pair. Loss of the Ser/Thr kinase PrkC results in an absence of protein phosphorylation probably leading to the observed decrease in protein amount of the proteins (protein amount of MPN474 is not influenced). In contrast, the proteins are more intensively phosphorylated in the prpC mutant. HMW1, cytadherence high molecular weight protein 1; HMW3, cytadherence high molecular weight protein 3; MPN256, uncharacterized protein; MPN474, coiled coil surface protein; P1, major adhesin; P41, adhesin-related protein. (B) Additional proteins dephosphorylated by PrpC. HPr is phosphorylated by HPrK at the conserved Ser-46 residue, whereas the phosphorylation mechanism of RpoE is unknown. HPr, phosphocarrier protein; RpoE, RNA polymerase subunit $\delta$. 


\section{Glycerophosphodiesterases: Multiple roles for universal proteins}

M. pneumoniae exhibits a hemolytic activity. While hemolysis by bacterial pathogens is usually caused by extracellular enzymes, the hemolysin of $M$. pneumoniae was identified as hydrogen peroxide (Somerson et al., 1965). The synthesis of hydrogen peroxide by $M$. pneumoniae is strongly increased if the cells are supplied with glycerol (Low, 1971). This can be attributed to the oxidase activity of the glycerol-3-phosphate oxidase GlpD that oxidizes glycerol 3-phosphate (G3P). This enzyme uses molecular oxygen rather than $\mathrm{NAD}^{+}$(as in typical glycerol-3-phosphate dehydrogenases) as the sink for electrons (Hames et al., 2009). Three potential pathways for the acquisition of G3P exist in M. pneumoniae. Glycerol may cross the cell membrane, mediated by the glycerol facilitator GlpF, and be phosphorylated by the glycerol kinase GlpK. Furthermore, $M$. pneumoniae can acquire G3P by direct uptake via the ABC transport system UgpAEC or hydrolysis of glycerophosphodiesters, an abundant degradation product of phospholipids, by glycerophosphodiesterase activity. While the first pathway is thought to be the most prominent one, it could be shown in this work that direct uptake of G3P seems not to occur in $M$. pneumoniae. Nevertheless, two potential glycerophosphodiesterases are present in the genome of M. pneumoniae, i.e. glpQ (mpn420) and mpn566 (Dandekar et al., 2000; Himmelreich et al., 1996). Interestingly, the pulmonary surfactant, the natural habitat of $M$. pneumoniae, is composed of about $90 \%$ phospholipids and $10 \%$ proteins (Veldhuizen et al., 1998) indicating that glycerophosphodiesters might play a major role in the life of this minimal organism.

A first attempt to characterize the two potential glycerophosphodiesterases of M. pneumoniae was to assess the biochemical properties of both enzymes. Enzymatic assays with the purified enzymes revealed that $M$. pneumoniae GlpQ has an enzymatic activity rather similar to the UgpQ of E. coli instead of the GlpQ of this organism (Larson et al., 1983; Ohshima et al., 2008; this work). In contrast, MPN566 was not active at all. M. pneumoniae GlpQ and E. coli UgpQ activity is highest in the presence of magnesium ions suggesting that this is the preferred cation (Ohshima et al., 2008; this work). In general, glycerophosphodiesterases exhibit a calcium-dependent enzymatic activity as it has been reported for the GlpQ of E. coli and different Borrelia species or the so-called protein D of Haemophilus influenzae (Larson et al., 1983; Munson and Sasaki, 1993; Schwan et al., 2003). Nevertheless, the E. coli GlpQ is also active in the presence of magnesium ions but to a much lesser extent (Larson et al., 
1983), whereas M. pneumoniae GlpQ and E. coli UgpQ are completely inactive with calcium ions (Ohshima et al., 2008; this work). While E. coli UgpQ accumulates under conditions of phosphate starvation (Ohshima et al., 2008), both M. pneumoniae enzymes are constitutively expressed in the wild type strain (Güell et al., 2009). Similar regulations compared to the UgpQ of $E$. coli were observed for the glycerophosphodiesterases of $B$. subtilis, Corynebacterium glutamicum, and Streptomyces coelicolor suggesting that the proteins allow the utilization of glycerophosphodiesters as a source of phosphate (Antelmann et al., 2000; Ishige et al., 2003; Santos-Beneit et al., 2009). Moreover, B. subtils glpQ expression is also induced when glycerol is available and repressed if a more favorable carbon sources such as glucose is present, respectively (Blencke et al., 2003; Nilsson et al., 1994). In Staphylococcus aureus, the GlpQ orthologue is regulated by the two-component system SaeRS, which is known to act on virulence gene expression (Rogasch et al., 2006).

M. pneumoniae GlpQ and MPN566 share 58\% identical residues. It could be shown for the glycerophosphodiesterase of Thermoanaerobacter tengcongensis that amino acid mutations in the active site affect protein activity by interfering with metal ion or substrate binding (Shi et al., 2007). In contrast to M. pneumoniae GlpQ, the active site structure is only poorly conserved in MPN566, not including all three essential metal ion binding residues. Thus, MPN566 does not seem to act as a glycerophosphodiesterase suggesting a different function in $M$. pneumoniae or the protein itself reflects the reductive evolution of this organism. The same phenomena were observed for the glycerophosphodiesterase of Treponema pallidum, whose active site is also poorly conserved (Shevchenko et al., 1997; Stebeck et al., 1997). It has been proposed that the T. pallidum GlpQ orthologue is a periplasmic lipoprotein associated with the spirochete's peptidoglycan-cytoplasma membrane complex (Shevchenko et al., 1999). Interestingly, most studied glycerophosphodiesterases are cytoplasmic or periplasmic enzymes. So far there are only two exceptions: Protein D of H. influenzae, which is membrane bound, and the extracellular GlpQ of B. subtilis (Janson et al., 1992; Voigt et al., 2009). This unusual localization could be explained by the fact that both proteins are synthesized as a precursor with a signal peptide (Wu and Tokunaga, 1986). In case of Protein $\mathrm{D}$, the signal peptide is modified by covalent binding of fatty acids to a cysteine residue, which becomes the amino terminus after cleavage of the signal sequence (Janson et al., 1992). In eukaryotes, glycerophosphodiesterases are also 
membrane proteins that form a large family with roles in motor neuron differentiation, modulation of cell growth or amino acid homeostasis (Corda et al., 2009; Kopp et al., 2010; Van der Rest et al., 2004; Yanaka, 2007).

To get more insights into the physiological role of the two paralogous glycerophosphodiesterases of $M$. pneumoniae, mutants lacking the ability to express either GlpQ or MPN566 were isolated in this work. No phenotypic differences could be detected between the $M$. pneumoniae wild type and the mpn566 mutant strain. In contrast, GlpQ is crucial for the pathogenicity of $M$. pneumoniae, since a glpQ mutant exhibited a complete loss of cytotoxicity toward HeLa cells. This might be due to the enzymatic activity itself because hydrogen peroxide release in the presence of glycerophosphorylcholine was completely gone in this mutant suggesting rather deacylated phospholipids than glycerol are used for G3P production in vivo. An implication of glycerophosphodiesterases in the pathogenicity has also been reported for different Borrelia species (Schwan et al., 2003). It is assumed that glycerophosphodiesterase activity permits only the relapsing-fever group of spirochetes to acquire G3P from phospholipids. This metabolic advantage may contribute in some extent to their ability to achieve higher cell densities in the blood than do the Lyme disease spirochetes (Schwan et al., 2003). Similar effects are observed in H. influenzae. Here, protein $\mathrm{D}$ is involved in the pathogenesis of upper respiratory tract infections due to nontypeable $H$. influenzae (Forsgren et al., 2008). A proposed mechanism is that the protein D glycerophosphodiesterase activity itself is the virulence factor, in this way that protein D hydrolyzes degraded phosphatidylcholine from host epithelia cells to obtain free choline for lipopolysaccharides on the bacteria cell surface that in turn contribute to pathogenicity (Fan et al., 2001). In M. pneumoniae, G3P rather than choline is important for the pathogenicity of this organism but also for phospholipid synthesis. Two major phospholipids were detected in the membrane of $M$. pneumoniae, phosphatidylcholine and phosphatidylglycerol (Plackett et al., 1969). However, only phosphatidylglycerol is synthesized de novo using G3P as precursor, whereas phosphatidylcholine seems to be directly integrated into the $M$. pneumoniae cell membrane from artificial media or host cells (Plackett et al., 1969). The importance of G3P in phospholipid synthesis is also reflected by the proteome changes in M. pneumoniae glpQ mutant cells growing with glucose as sole carbon source. Under this condition, 33 and 21 proteins were present in elevated and reduced amounts, 
respectively. In contrast, only five induced and five repressed proteins were detected in the presence of glycerol suggesting a complementation effect compared to glucose-grown cells. Indeed, nearly all proteins with reduced protein amounts in medium supplemented with glucose as carbon source are transport proteins indicating defects in cell membrane assembly, since transcription regulation of these proteins did not appear. Moreover, the M. pneumoniae glpQ mutant strain exhibited a growth defect in the presence of glucose and did not reach the final biomass as compared to the wild type strain, whereas growth with glycerol as sole carbon source was not affected. An implication of the glycerophosphodiesterase-like protein SHV3 in cell wall organization was already shown for the plant Arabidopsis thaliana (Hayashi et al., 2008).

The protein amount of five proteins was up- or down-regulated in the M. pneumoniae glpQ mutant strain in a carbon source independent manner, including the glycerol facilitator GlpF, a subunit of a metal ion ABC transporter CbiO, and three lipoproteins (MPN162, MPN287, and MPN506). For these proteins, it could be shown that regulation appears on transcription level indicating a GlpQ-dependent regulon in M. pneumoniae. A possible explanation for this result could be that $M$. pneumoniae GlpQ acts as a trigger enzyme (Commichau and Stülke, 2008), which measures the availability of its product G3P and uses this information to differentially control gene expression by direct binding to DNA operator elements. Alternatively, the protein may bind as a cofactor to a transcription regulator to regulate gene expression similar to the role of $\operatorname{HPr}($ Ser-P) in carbon catabolite repression in the Firmicutes (Görke and Stülke, 2008). Indeed, GlpQ was identified in the M. pneumoniae phosphoproteome analysis. However, the phosphorylation site and the function of this phosphorylation event is unknown. In addition, a recent analysis of protein-protein-interactions in M. pneumoniae provided no clear evidence for an interaction of GlpQ with other proteins. Only a self-interaction of this protein was reported (Kühner et al., 2009). Non-enzymatic functions of glycerophosphodiesterases have also been reported for the mammalian GDE5. In this case, the protein negatively regulates skeletal muscle development even without enzymatic activity (Okazaki et al., 2010). 


\section{GlpQ-dependent transcription regulation in $M$. pneumoniae: Mechanism and function?}

In bacteria, regulation mostly appears on the transcription level (Campbel et al., 2008; Hoskisson and Rigali, 2008; Segal and Ron, 1998). However, only two examples of transcription regulation are reported in the Mollicutes so far. This is the regulation of the Spiroplasma citri fructose operon by the transcription activator FruR (Gaurivaud et al., 2001) and the control of heat shock genes in M. pneumoniae by the repressor protein HrcA and the DNA operator element CIRCE (Chang et al., 2008; Weiner III et al., 2003). Moreover, the induction of chaperone-encoding genes at elevated temperatures was also observed in several other Mycoplasma species (Dascher et al., 1990; Madsen et al., 2006; Musatovova et al., 2006).

The analysis of the $M$. pneumoniae glpQ mutant strain revealed several differences in the protein amount of proteins, many triggered by changes at the transcription level. However, it was proposed that the higher proportion of regulatory events with glucose as sole carbon source are mostly due to a lack of G3P instead of the missing glycerophosphodiesterase GlpQ. Interestingly, the overlap of proteins and the corresponding genes, respectively, that exhibited a regulation independent from the carbon source, was relatively low. As mentioned before, these proteins were the glycerol facilitator GlpF, a subunit of a metal ion $\mathrm{ABC}$ transporter $\mathrm{CbiO}$, and the three lipoproteins MPN162, MPN287, and MPN506. The observation that another way to acquire G3P from glycerol was strongly up-regulated in the M. pneumoniae glpQ mutant strain confirms the crucial factor of G3P accumulation in the life of this organism. Furthermore, CbiO and MPN162 showed an increase in transcription level, whereas the two other lipoproteins were down-regulated in the M. pneumoniae glpQ mutant strain. An inspection of the upstream region of all five genes revealed the presence of a common palindromic DNA motif, which has strong similarity to the motif of the virulence gene regulator CovR in group A Streptococcus (Churchward, 2007). A possible explanation for this differential regulation pattern of the five genes could be suspected in the localization of the inverted repeat sequence in relation to the -10 region of the gene. For all up-regulated genes, the motif adjoins or overlaps with the -10 region, whereas a $\sim 500$ bases spacer is present in case of the down-regulated ones. In addition to HrcA, the genome of $M$. pneumoniae encodes only two other typical transcription factors that belong to the GntR and the Fur family, respectively 
(Dandekar et al., 2000; Himmelreich et al., 1996). However, it is open for speculation whether these two proteins are involved in this regulation process.

It is interesting to note that $M$. pneumoniae contains almost 70 potential lipoproteins (10\% of all open reading frames) (Hallamaa et al., 2006), whereas environmental bacteria such as $E$. coli and $B$. subtilis reserve less than one percent of their genetic capacity to encode these proteins (Blattner et al., 1997; Kunst et al., 1997). The high number of lipoproteins in $M$. pneumoniae might therefore reflect the close adaptation to the human host. It is reported that $M$. pneumoniae lipoproteins act as surface antigens because of their modulin activity, which makes them a preferred target of the host immune response (Chambaud et al., 1999; You et al., 2006; Zuo et al., 2009). Similar lipoprotein functions were also documented in S. aureus, where lipoproteins seem to play an important role in pathogen recognition and host innate defense mechanisms against infections (Bubeck Wardenburg et al., 2006). For M. pneumoniae MPN162 and its homologue in Mycoplasma genitalium, it could already be shown that these lipoproteins activate the nuclear factorkB, a transcription regulator involved in immune response, through toll-like receptors (Shimizu et al., 2007; Shimizu et al., 2008). Moreover, MslA, the homologue of MPN284 and MPN506, which share a similarity of $76 \%$, plays a role in Mycoplasma gallisepticum virulence, although the mechanism is unknown (Szczepanek et al., 2010). Further studies of lipoproteins in the Mollicutes suggest functions in adhesion and invasion of mammalian cells as well (Liu et al., 2006; Zeiman et al., 2008; Zimmermann et al., 2010). Nevertheless, Mycoplasma lipoproteins seem not to be constitutively expressed as different expression patterns after contact with human host cells could be observed (Cecchini et al., 2007; Hallamaa et al., 2008; Madsen et al., 2008).

In a first attempt to identify functions of GlpQ-dependent regulated proteins, M. pneumoniae mutant strains lacking the lipoproteins MPN161, MPN284 or MPN506 were isolated, while glpF and cbiO are essential (Hames et al., 2009; unpublished data). It turned out that the mpn284 mutant possessed a decreased cytotoxicity toward HeLa cells (Fig. 37A and B). Interestingly, this phenotype was very similar to that of the M. pneumoniae glpD mutant (Hames et al., 2009). In addition, only the mpn284 mutant exhibited an effect in hydrogen peroxide release, in that way that no formation occurred with glycerol as carbon source (Fig. 37C). This raises the question if decreased cytotoxicity toward HeLa cells is due to defects in hydrogen peroxide release with 
glycerol. Nevertheless, deacylated phospholipids rather than glycerol are most likely available upon infection. A recent analysis of protein-protein-interactions in M. pneumoniae revealed also two potential interaction partners of MPN284, including the thymidylate synthase ThyA and a putative phosphosugar facilitator MPN076 (Kühner et al., 2009). However, it is not known, how these three proteins are related to each other. Summing up, the first results showed that the lipoprotein MPN284 has a role in the pathogenicity of $M$. pneumoniae.

A

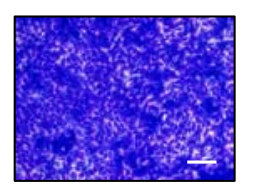

control

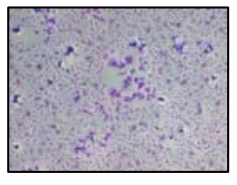

wt

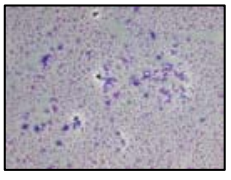

mpn162::Tn

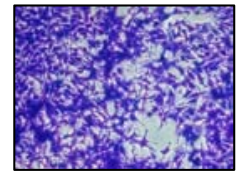

mpn284::Tn

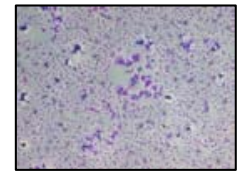

mpn506::Tn
B

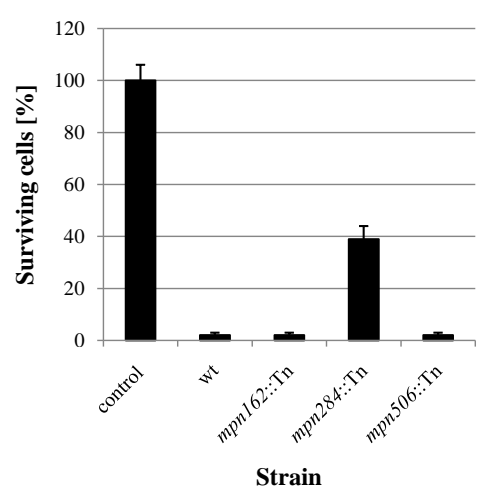

C

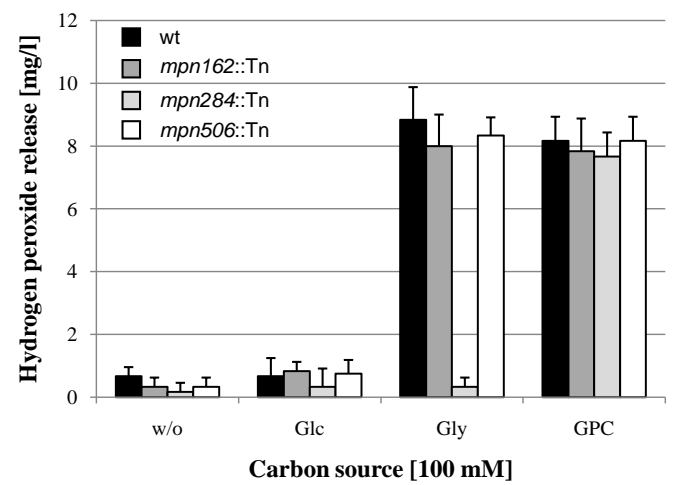

Fig. 37. Analysis of the pathogenicity of M. pneumoniae lipoprotein mutants. (A) Infection assay to verify cytotoxic effects of $M$. pneumoniae lipoprotein mutants. HeLa cells were infected with M. pneumoniae wild type (wt), mpn162::Tn, mpn284::Tn, and mpn506::Tn mutant cells. As control served a HeLa cell culture without addition of M. pneumoniae cells. After four days, HeLa cell cultures were stained with crystal violet and photographed. All pictures are shown at the same magnification. Scale bar, $0.1 \mathrm{~mm}$. (B) Quantification of HeLa cells after infection with different M. pneumoniae strains. The cell count of surviving cells is indicated in percent as the number of viable cells per field of view, quantified by crystal violet staining after four days of incubation. An uninfected HeLa cell culture served as control. Error bars indicate standard deviation (based on three independent experiments). (C) Examination of M. pneumoniae hydrogen peroxide release. Hydrogen peroxide production of M. pneumoniae wild type (wt), mpn162::Tn, mpn284::Tn, and mpn506::Tn mutant strains were measured in the presence of different carbon sources $(100 \mu \mathrm{M})$ after $2 \mathrm{~h}$. Error bars indicate standard deviation (based on three independent experiments). GPC, glycerophosphorylcholine; Glc, glucose; Gly, glycerol; w/o, without addition of any carbon source. 


\section{Outlook}

The significance of PrkC/PrpC-dependent protein phosphorylation in the pathogenicity of $M$. pneumoniae was demonstrated in this work. In addition to one HPrK target protein, six proteins are phosphorylated by the kinase PrkC. However, this is only a small group of proteins compared to over 60 identified phosphoproteins in total. Thus, the genome of $M$. pneumoniae has to encode far more than the two annotated kinases or autophosphorylation as shown for the phosphosugar mutase ManB is more common as thought so far. To identify rather cryptic kinases or potential proteins with moonlighting functions in phosphorylation, it could be helpful to analyze the phosphoproteome of the smaller ancestor $M$. genitalium. The phosphoproteome overlap between these two minimal organisms might give hints for the identification of other phosphorylation mechanisms. An evidence for a prominent role of moonlighting proteins in minimal organisms give the current smallest cellular genome of the insect symbiont Hodgkinia cicadicola, which consists of just about $144 \mathrm{~kb}$ (McCutcheon et al., 2009). Interestingly, the genome encodes for only 15 tRNAs to assemble the 20 amino acids required for protein synthesizes. It is difficult to understand, how this could work, but probably several tRNAs fulfill multiple functions in this organism.

A potential candidate of a moonlighting protein, in this special case a trigger enzyme, is the glycerophosphodiesterase GlpQ in $M$. pneumoniae. The current results indicate beside the enzymatic function a role in transcription regulation of a putative virulence regulon. Indeed, it could be shown that the protein binds DNA in vitro, but rather unspecific (unpublished data). Therefore, a useful approach should be the direct expression of a recombinant version of the protein in $M$. pneumoniae in order to elucidate the DNA-binding activity of the enzyme by techniques such as ChIP-chip. The use of a smaller protein-tag as the TAP-tag by Kühner et al. (2009) may also allow the identification of GlpQ interaction partners if the protein is not the direct transcription regulator. Moreover, the purification of GlpQ enhances the possibility to detect the phosphorylation site, which might allow a general knowledge of the regulation process itself. 
For a more detailed understanding of the interconnection between the lipoprotein MPN284 and the pathogenicity of $M$. pneumoniae, the glycerol uptake system of M. pneumoniae should be reconstituted with or without MPN284 in a B. subtilis glpF mutant. This approach allows the verification, whether MPN284 modulates directly the glycerol uptake or later regulations are responsible for the absence of hydrogen peroxide formation with glycerol as carbon source. Furthermore, it might be interesting to compare the proteome of mpn284 mutant cells grown in the presence of glycerol to the M. pneumoniae wild type strain. 
Chapter 9

\section{References}




\section{References}

Abramoff, M. D., P. J. Magelhaes, and S. J. Ram. 2004. Image processing with ImageJ. Biophotonics Internat. 11: 36-42.

Absalon, C., M. Obuchowski, E. Madec, D. Delattre, I. B. Holland, and S. J. Séror. 2009. CpgA, EF-Tu and the stressosome protein YezB are substrates of the Ser/Thr kinase/phosphatase couple, PrkC/PrpC, in Bacillus subtilis. Microbiology 155: 932-943.

Aivaliotis, M., B. Macek, F. Gnad, P. Reichelt, M. Mann, and D. Oesterhelt. 2009. Ser/Thr/Tyr protein phosphorylation in the archaeon Halobacterium salinarum A representative of the third domain of life. PLoS ONE 4: e4777.

Allen, G. S., K. Steinhauer, W. Hillen, J. Stülke, and R. G. Brennan. 2003. Crystal structure of HPr kinase/phosphatase from Mycoplasma pneumonia. J. Mol. Biol. 326: 1203-1217.

Amar, P., G. Legent, M. Thellier, C. Ripoll, G. Bernot, T. Nystrom, M. H. Saier Jr., and V. Norris. 2008. A stochastic automaton shows how enzyme assemblies may contribute to metabolic efficiency. BMC Syst. Biol. 2: 27.

An, S., R. Kumar, E. D. Sheets, and S. J. Benkovic. 2008. Reversible compartmentalization of de novo purine biosynthetic complexes in living cells. Science 320: 103-106.

Antelmann, H., C. Scharf, and M. Hecker. 2000. Phosphate starvation-inducible proteins of Bacillus subtilis: Proteomics and transcriptional analysis. J. Bacteriol. 182: 4478-4490.

Armougom, F., S. Moretti, O. Poirot, S. Audic, P. Dumas, B. Schaeli, V. Keduas, and C. Notredame. 2006. Expresso: Automatic incorporation of structural information in multiple sequence alignments using 3D-Coffee. Nucleic Acids Res. 34: W604-608.

Armstrong, R. D., and R. B. Diasio. 1982. Improved measurement of thymidylate synthetase activity by a modified tritium-release assay. J. Biochem. Biophys. Methods 6: 141-147.

Atkinson, T. P., M. F. Balish, and K. B. Waites. 2008. Epidemiology, clinical manifestations, pathogenesis and laboratory detection of Mycoplasma pneumoniae infections. FEMS Microbiol. Rev. 32: 956-973. 
Balish, M. F., and D. C. Krause. 2006. Mycoplasmas: A distinct cytoskeleton for wall-less bacteria. J. Mol. Microbiol. Biotechnol. 11: 244-255.

Balish, M. F., S. M. Ross, M. Fisseha, and D. C. Krause. 2003. Deletion analysis identifies key functional domains of the cytadherence-associated protein HMW2 of Mycoplasma pneumonia. Mol. Microbiol. 50: 1507-1516.

Barabote, R. D., and M. H. Saier Jr. 2005. Comparative genomic analyses of the bacterial phosphotransferase system. Microbiol. Mol. Biol. Rev. 69: 608-634.

Barré, A., A. de Daruvar, and A. Blanchard. 2004. MolliGen, a database dedicated to the comparative genomics of Mollicutes. Nucleic Acids Res. 32: D307-D310.

Beinert, H., M. C. Kennedy, and C. D. Stout. 1996. Aconitase as iron-sulfur protein, enzyme, and iron-regulatory protein. Chem. Rev. 96: 2335-2374.

Beltrao, P., J. C. Trinidad, D. Fiedler, A. Roguev, W. A. Lim, K. M. Shokat, A. L. Burlingame, and N. J. Krogan. 2009. Evolution of phosphoregulation: Comparison of phosphorylation patterns across yeast species. PLoS Biol. 7: e1000134.

Bi, W., and P. J. Stambrook. 1998. Site-directed mutagenesis by combined chain reaction. Anal. Biochem. 256: 137-140.

Bischof, D. F., E. M. Vilei, and J. Frey. 2009. Functional and antigenic properties of GlpO from Mycoplasma mycoides subsp. mycoides SC: Characterization of a flavine adenine dinucleotide-binding site deletion mutant. Vet. Res. 40: 35.

Blattner, F. R., G. Plunkett III, C. A. Bloch, N. T. Perna, V. Burland, M. Riley, J. Collado-Vides, J. D. Glasner, C. K. Rode, G. F. Mayhew, et al. 1997. The complete genome sequence of Escherichia coli K-12. Science 277: 1453-1462.

Blencke, H. M., G. Homuth, H. Ludwig, U. Mäder, M. Hecker, and J. Stülke. 2003. Transcriptional profiling of gene expression in response to glucose in Bacillus subtilis: Regulation of the central metabolic pathways. Metab. Engn. 5: 133-149.

Boekhorst, J., B. van Breukelen, A. J. Heck Jr., and B. Snel. 2008. Comparative phosphoproteomics reveals evolutionary and functional conservation of phosphorylation across eukaryotes. Genome Biol. 9: R144.

Boonmee, A., T. Ruppert, and R. Herrmann. 2009. The gene mpn310 (hmw2) from Mycoplasma pneumoniae encodes two proteins, HMW2 and HMW2-s, which differ in size but use the same reading frame. FEMS Microbiol. Lett. 290: 174-181. 
Bové, J. M., J. Renaudin, C. Saillard, X. Foissac, and M. Garnier. 2003. Spiroplasma citri, a plant pathogenic Mollicute: Relationship with its two hosts, the plant and the leafhopper vector. Annu. Rev. Phytopathol. 41: 483-500.

Brown, D. R., J. M Farley, L. A. Zacher, J. M. Carlton, T. L. Clippinger, J. G. Tully, and M. B. Brown. 2001. Mycoplasma alligatoris sp. nov., from American alligators. Int. J. Syst. Evol. Microbiol. 51: 419-424.

Bubeck Wardenburg, J., W. A. Williams, and D. Missiakas. 2006. Host defenses against Staphylococcus aureus infection require recognition of bacterial lipoproteins. Proc. Natl. Acad. Sci. USA 103: 13831-13836.

Burroughs, A. M., S. Balaji, L. M. Iyer, and L. Aravind. 2007. Small but versatile: The extraordinary functional and structural diversity of the beta-grasp fold. Biol. Direct. 2: 18.

Campanella, M. E., H. Chu, and P. S. Low. 2005. Assembly and regulation of a glycolytic enzyme complex on the human erythrocyte membrane. Proc. Natl. Acad. Sci. USA 102: 2402-2407.

Campbel, E. A., L. F. Westblade, and S. A. Darst. 2008. Regulation of bacterial RNA polymerase sigma factor activity: A structural perspective. Curr. Opin. Microbiol. 11: 121-127.

Canback, B., S. G. Andersson, and C. G. Kurland. 2005. The global phylogeny of glycolytic enzymes. Proc. Natl. Acad. Sci. USA 99: 6097-6102.

Carnrot, C., R. Wehelie, S. Eriksson, G. Bölske, and L. Wang. 2003. Molecular characterization of thymidine kinase from Ureaplasma urealyticum: Nucleoside analogues as potent inhibitors of Mycoplasma growth. Mol. Microbiol. 50: 771-780.

Carpousis, A. J. 2007. The RNA degradosome of Escherichia coli: An mRNA-degrading machine assembled on RNase E. Annu. Rev. Microbiol. 61: $71-87$

Cecchini, K. R., T. S. Gorton, and S. J. Geary. 2007. Transcriptional responses of Mycoplasma gallisepticum strain $\mathrm{R}$ in association with eukaryotic cells. J. Bacteriol. 189: 5803-5807.

Chambaud, I., H. Wróblewski, and A. Blanchard. 1999. Interactions between Mycoplasma lipoproteins and the host immune system. Trends Microbiol. 7: 493-499. 
Chang, L. J., W. H. Chen, F. C. Minion, and D. Shiuan. 2008. Mycoplasmas regulate the expression of heat-shock protein genes through CIRCE-HrcA interactions. Biochem. Biophys. Res. Commun. 367: 213-218.

Christensen, N. M., K. B. Axelsen, M. Nicolaisen, and A. Schulz. 2005. Phytoplasmas and their interactions with hosts. Trends Plant Sci. 10: 526-535.

Churchward, G. 2007. The two faces of Janus: Virulence gene regulation by CovR/S in group A streptococci. Mol. Microbiol. 64: 34-41.

Ciccarelli, F. D., T. Doerks, C. von Mering, C. J. Creevey, B. Snel B, and P. Bork. 2006. Toward automatic reconstruction of a highly resolved tree of life. Science 311: 1283-1287.

Claessen, D., R. Emmins, L. W. Hamoen, R. A. Daniel, J. Errington, and D. H. Edwards. 2008. Control of the cell elongation-division cycle by shuttling of PBP1 protein in Bacillus subtilis. Mol. Microbiol. 68: 1029-1046.

Cole, B. C., J. R. Ward, and C. H. Martin. 1968. Hemolysin and peroxide activity of Mycoplasma species. J. Bacteriol. 95: 2022-2030.

Commichau, F. M., and J. Stülke. 2008. Trigger enzymes: Bifunctional proteins active in metabolism and in controlling gene expression. Mol. Microbiol. 67: 692-702.

Commichau, F. M., C. Herzberg, P. Tripal, O. Valerius, and J. Stülke. 2007. A regulatory protein-protein interaction governs glutamate biosynthesis in Bacillus subtilis: The glutamate dehydrogenase RocG moonlights in controlling the transcription factor GltC. Mol. Microbiol. 65: 642-654.

Commichau, F. M., F. M. Rothe, C. Herzberg, E. Wagner, D. Hellwig, M. Lehnik-Habrink, E. Hammer, U. Völker, and J. Stülke. 2009. Novel activities of glycolytic enzymes in Bacillus subtilis: Interactions with essential proteins involved in mRNA processing. Mol. Cell. Proteomics 8: 1350-1360.

Corda, D., T. Kudo, P. Zizza, C. Iurisci, E. Kawai, N. Kato, N. Yanaka, and S. Mariggiò. 2009. The developmentally regulated osteoblast phosphodiesterase GDE3 is glycerophosphoinositol-specific and modulates cell growth. J. Biol. Chem. 284: 24848-24856.

Crooks, G. E., G. Hon, J. M. Chandonia, and S. E. Brenner. 2004. WebLogo: A sequence logo generator. Genome Res. 14: 1188-1190. 
Dandekar, T., B. Snel, M. Huynen, and P. Bork. 1998. Conservation of gene order: A fingerprint of proteins that physically interact. Trends Biochem. Sci. 23: 324-328.

Dandekar, T., M. Huynen, J. T. Regula, B. Ueberle, C. U. Zimmermann, M. A. Andrade, T. Doerks, L. Sánchez-Pulido, B. Snel, M. Suyama, et al. 2000. Re-annotating the Mycoplasma pneumoniae genome sequence: Adding value, function and reading frames. Nucl. Acids Res. 28: 3278-3288.

Dascher, C. C., S. K. Poddar, and J. Maniloff. 1990. Heat shock response in mycoplasmas, genome-limited organisms. J. Bacteriol. 172: 1823-1827.

Débarbouillé, M., S. Dramsi, O. Dussurget, M. A. Nahori, E. Vaganay, G. Jouvion, A. Cozzone, T. Msadek, and B. Duclos. 2009. Characterization of a serine/threonine kinase involved in virulence of Staphylococcus aureus. J. Bacteriol. 191: 4070-4081.

Deutscher, J., and M. H. Saier Jr. 2005. Ser/Thr/Tyr phosphorylation in bacteria - For long time neglected, now well established. J. Mol. Microbiol. Biotechnol. 9: 125-131.

Dirksen, L. B., K. A. Krebes, and D. C. Krause. 1994. Phosphorylation of cytadherence-accessory proteins in Mycoplasma pneumonia. J. Bacteriol. 176: 7499-7505.

Domenech, C., N. Leveque, B. Lina, F. Najioullah, and D. Floret. 2009. Role of Mycoplasma pneumoniae in pediatric encephalitis. Eur. J. Clin. Microbiol. Infect. Dis. 28: 91-94.

Dutow, P., S. R. Schmidl, M. Ridderbusch, and J. Stülke. 2010. Interactions between glycolytic enzymes of Mycoplasma pneumoniae. J. Mol. Microbiol. Biotechnol. accepted.

Eisenreich, W., T. Dandekar, J. Heesemann, and W. Goebel. 2010. Carbon metabolism of intracellular bacterial pathogens and possible links to virulence. Nat. Rev. Microbiol. 8: 401-412.

Erlandsen, H., E. E. Abola, and R. C. Stevens. 2000. Combining structural genomics and enzymology: Completing the picture in metabolic pathways and enzyme active sites. Curr. Opin. Struct. Biol. 10: 719-730.

Eymann, C., A. Dreisbach, D. Albrecht, J. Bernhardt, D. Becher, S. Gentner, T. Tam Le, K. Büttner, G. Buurman, C. Scharf, et al. 2004. A comprehensive proteome map of growing Bacillus subtilis cells. Proteomics 4: 2849-2876. 
Eymann, C., D. Becher, J. Bernhardt, K. Gronau, A. Klutzny, and M. Hecker. 2007. Dynamics of protein phosphorylation on Ser/Thr/Tyr in Bacillus subtilis. Proteomics 7: 3509-3526.

Eymann, C., G. Homuth, C. Scharf, and M. Hecker. 2002. Bacillus subtilis functional genomics: Global characterization of the stringent response by proteome and transcriptome analysis. J. Bacteriol. 184: 2500-2520.

Fabret, C., V. A. Feher, and J. A. Hoch. 1999. Two-component signal transduction in Bacillus subtilis: How one organism sees its world. J. Bacteriol. 181: 1975-1983.

Faires, N., S. Tobisch, S. Bachem, I. Martin-Verstraete, M. Hecker, and J. Stülke. 1999. The catabolite control protein CcpA controls ammonium assimilation in Bacillus subtilis. J. Mol. Microbiol. Biotechnol. 1: 141-148.

Fan, X., H. Goldfine, E. Lysenko, and J. N. Weiser. 2001. The transfer of choline from the host to the bacterial cell surface requires glpQ in Haemophilus influenzae. Mol. Microbiol. 41: 1029-1036.

Faucher, S. P., C. Viau, P. P. Gros, F. Daigle, and H. Le Moual. 2008. The prpZ gene cluster encoding eukaryotic-type Ser/Thr protein kinases and phosphatases is repressed by oxidative stress and involved in Salmonella enterica serovar Typhi survival in human macrophages. FEMS Microbiol. Lett. 281: 160-166.

Ficarro, S. B., M. L. McClelland, P. T. Stukenberg, D. J. Burke, M. M. Ross, J. Shabanowitz, D. F. Hunt, and F. M. White. 2002. Phosphoproteome analysis by mass spectrometry and its application to Saccharomyces cerevisiae. Nat. Biotechnol. 20: 301-305.

Fisseha, M., H. W. Göhlmann, R. Herrmann, and D. C. Krause. 1999. Identification and complementation of frameshift mutations associated with loss of cytadherence in Mycoplasma pneumoniae. J. Bacteriol. 181: 4404-4410.

Forsgren, A., K. Riesbeck, and H. Janson. 2008. Protein D of Haemophilus influenzae: A protective nontypeable $H$. influenzae antigen and a carrier for pneumococcal conjugate vaccines. Clin. Infect. Dis. 46: 726-731.

Frith, M. C., N. F. Saunders, B. Kobe, and T. L. Bailey. 2008. Discovering sequence motifs with arbitrary insertions and deletions. PLoS Comput. Biol. 4: e1000071.

Gaidenko, T. A., T. J. Kim, and C. W. Price. 2002. The PrpC serine-threonine phosphatase and PrkC kinase have opposing physiological roles in stationary-phase Bacillus subtilis cells. J. Bacteriol. 184: 6109-6114. 
Galinier, A., J. Haiech, M. C. Kilhoffer, M. Jaquinod, J. Stülke, J. Deutscher, and I. Martin-Verstraete. 1997. The Bacillus subtilis crh gene encodes a HPr-like protein involved in carbon catabolite repression. Proc. Natl. Acad. Sci. USA 94: 8439-8444.

Gasparri, F., N. Wang, S. Skog, A. Galvani, and S. Eriksson. 2009. Thymidine kinase 1 expression defines an activated G1 state of the cell cycle as revealed with site-specific antibodies and ArrayScan assays. Eur. J. Cell Biol. 88: 779-785.

Gaurivaud, P., F. Laigret, M. Garnier, and J. M. Bové. 2001. Characterization of FruR as a putative activator of the fructose operon of Spiroplasma citri. FEMS Microbiol. Lett. 198: 73-78.

Gibson, D. G., G. A. Benders, C. Andrews-Pfannkoch, E. A. Denisova, H. Baden-Tillson, J. Zaveri, T. B. Stockwell, A. Brownley, D. W. Thomas, M. A. Algire, et al. 2008. Complete chemical synthesis, assembly, and cloning of a Mycoplasma genitalium genome. Science 319: 1215-1220.

Glass, J. I., N. Assad-Garcia, N. Alperovich, S. Yooseph, M. R. Lewis, M. Maruf, C. A. Hutchison III, H. O. Smith, and J. C. Venter. 2006. Essential genes of a minimal bacterium. Proc. Natl. Acad. Sci. USA 103: 425-430.

Gnad, F., F. Forner, D. F. Zielinska, E. Birney, J. Gunawardena, and M. Mann. 2010. Evolutionary constraints of phosphorylation in eukaryotes, prokaryotes, and mitochondria. Mol. Cell. Proteomics in press (PMID: 20688971).

Görke , B., and J. Stülke. 2008. Carbon catabolite repression in bacteria: Many ways to make the most out of nutrients. Nat. Rev. Microbiol. 6: 613-624.

Grangeasse, C., A. J. Cozzone, J. Deutscher, and I. Mijakovic. 2007. Tyrosine phosphorylation: An emerging regulatory device of bacterial physiology. Trends Biochem. Sci. 32: 86-94.

Greenberg, E. P. 2000. Bacterial genomics. Pump up the versatility. Nature 406: 947-948.

Güell, M., V. van Noort, E. Yus, W. H. Chen, J. Leigh-Bell, K. Michalodimitrakis, T. Yamada, M. Arumugam, T. Doerks, S. Kühner, et al. 2009. Transcriptome complexity in a genome-reduced bacterium. Science 326: 1268-1271.

Guérout-Fleury, A. M., K. Shazand, N. Frandsen, and P. Stragier. 1995. Antibiotic resistance cassettes for Bacillus subtilis. Gene 167: 335-336. 
Guex, N., and M. C. Peitsch. 1997. SWISS-MODEL and the Swiss-PdbViewer: An environment for comparative protein modeling. Electrophoresis 18: 2714-2723.

Gururaj, A., C. J. Barnes, R. K. Vadlamudi, and R. Kumar. 2004. Regulation of phosphoglucomutase 1 phosphorylation and activity by a signaling kinase. Oncogene 23: 8118-8127.

Halbedel, S., and J. Stülke. 2005. Dual phosphorylation of Mycoplasma pneumoniae HPr by Enzyme I and HPr kinase suggests an extended phosphoryl group susceptibility of HPr. FEMS Microbiol. Lett. 247: 193-198.

Halbedel, S., and J. Stülke. 2006. Probing in vivo promoter activities in Mycoplasma pneumoniae: A system for generation of single-copy reporter constructs. Appl. Environ. Microbiol. 72: 1696-1699.

Halbedel, S., and J. Stülke. 2007. Tools for the genetic analysis of Mycoplasma. Int. J. Med. Microbiol. 297: 37-44.

Halbedel, S., C. Hames, and J. Stülke. 2004. In vivo activity of enzymatic and regulatory components of the phosphoenolpyruvate:sugar phosphotransferase system in Mycoplasma pneumoniae. J. Bacteriol. 186: 7936-7943.

Halbedel, S., C. Hames, and J. Stülke. 2007. Regulation of carbon metabolism in the Mollicutes and its relation to virulence. J. Mol. Microbiol. Biotechnol. 12: 147-154.

Halbedel, S., C. Hames, and J. Stülke. 2007. Regulation of carbon metabolism in the Mollicutes and its relation to virulence. J. Mol. Microbiol. Biotechnol. 12: 147-154.

Halbedel, S., H. Eilers, B. Jonas, J. Busse, M. Hecker, S. Engelmann, and J. Stülke. 2007. Transcription in Mycoplasma pneumoniae: Analysis of the promoters of the ackA and ldh genes. J. Mol. Biol. 371: 596-607.

Halbedel, S., J., Busse, S. R. Schmidl, and J. Stülke. 2006. Regulatory protein phosphorylation in Mycoplasma pneumoniae: A PP2C-type phosphatase serves to dephosphorylate HPr(Ser-P). J. Biol. Chem. 281: 26253-26259.

Hallamaa, K. M., G. F. Browning, and S. L. Tang. 2006. Lipoprotein multigene families in Mycoplasma pneumoniae. J. Bacteriol. 188: 5393-5399. 
Hallamaa, K. M., S. L. Tang, N. Ficorilli, and G. F. Browning. 2008. Differential expression of lipoprotein genes in Mycoplasma pneumoniae after contact with human lung epithelial cells, and under oxidative and acidic stress. BMC Microbiol. 8: 124.

Hames, C., S. Halbedel, and J. Stülke. 2005. Multiple-mutation reaction: A method for simultaneous introduction of multiple mutations into the glpK gene of Mycoplasma pneumoniae. Appl. Environ. Microbiol. 71: 4097-4100.

Hames, C., S. Halbedel, M. Hoppert, J. Frey, and J. Stülke. 2009. Glycerol metabolism is important for cytotoxicity of Mycoplasma pneumoniae. J. Bacteriol. 191: 747-753.

Hanson, K. G., K. Steinhauer, J. Reizer, W. Hillen, and J. Stülke. 2002. HPr kinase/phosphatase of Bacillus subtilis: Expression of the gene and effects of mutations on enzyme activity, growth, and carbon catabolite repression. Microbiology 148: 1805-1811.

Hasselbring, B. M., C. A. Page, E. S. Sheppard, and D. C. Krause. 2006. Transposon mutagenesis identifies genes associated with Mycoplasma pneumoniae gliding motility. J. Bacteriol. 188: 6335-6345.

Hayashi, S., T. Ishii, T. Matsunaga, R. Tominaga, T. Kuromori, T. Wada, K. Shinozaki, and T. Hirayama. 2008. The glycerophosphoryl diester phosphodiesterase-like proteins SHV3 and its homologs play important roles in cell wall organization. Plant Cell Physiol. 49: 1522-1535.

Hegermann, J., S. Halbedel, R. Dumke, J. Regula, R. R. Gaboulline, F. Mayer, J. Stülke, and R. Herrmann. 2008. The acidic, glutamine-rich MPN474 protein of Mycoplasma pneumoniae is surface exposed and covers the complete cell. Microbiology 154: 1185-1192.

Heinrich, A., K. Woyda, K. Brauburger, G. Meiss, C. Detsch, J. Stülke, and K. Forchhammer. 2006. Interaction of the membrane-bound GlnK-AmtB complex with the master regulator of nitrogen metabolism TnrA in Bacillus subtilis. J. Biol. Chem. 281: 34909-34917.

Himmelreich, R., H. Hilbert, H. Plagens, E. Pirkl, B. C. Li, and R. Herrmann. 1996. Complete sequence analysis of the genome of the bacterium Mycoplasma pneumoniae. Nucleic Acids Res. 24: 4420-4449. 
Hoch, J. A. 2000. Two-component and phosphorelay signal transduction. Curr. Opin. Microbiol. 3: 165-170.

Holt, L. J., B. B. Tuch, J. Villén, A. D. Johnson, S. P. Gygi, and D. O. Morgan. 2009. Global analysis of Cdk1 substrate phosphorylation sites provides insights into evolution. Science 325: 1682-1686.

Hoskisson, P. A., and S. Rigali. 2008. Chapter 1: Variation in form and function the helix-turn-helix regulators of the GntR superfamily. Adv. Appl. Microbiol. 69: $1-22$.

Hubbard, S. R., and W. T. Miller. 2007. Receptor tyrosine kinases: Mechanisms of activation and signaling. Curr. Opin. Cell Biol. 19: 117-123.

Hutchison III, C. A., S. C. Peterson, S. R. Gill, R. T. Cline, O. White, C. M. Fraser, H. O. Smith, and J. C. Venter. 1999. Global transposon mutagenesis and a minimal Mycoplasma genome. Science 286: 2165-2169.

Iakoucheva, L. M., P. Radivojac, C. J. Brown, T. R. O’Connor, J. G. Sikes, Z. Obradovic, and A. K. Dunker. 2004. The importance of intrinsic disorder for protein phosphorylation. Nucleic Acids Res. 32: 1037-1049.

Ishige, T., M. Krause, M. Bott, V. F. Wendisch, and H. Sahm. 2003. The phosphate starvation stimulon of Corynebacterium glutamicum determined by DNA microarray analyses. J. Bacteriol. 185: 4519-4529.

Islam, M. M., R. Wallin, R. M. Wynn, M. Conway, H. Fujii, J. A. Mobley, D. T. Chuang, and S. M. Hutson. 2007. A novel branched-chain amnio acid metabolon. Protein-protein interactions in a supramolecular complex. J. Biol. Chem. 282: 11893-11903.

Iwanicki, A., K. Hinc, S. Seror, G. Wegrzyn, and M. Obuchowski. 2005. Transcription in the prpC-yloQ region in Bacillus subtilis. Arch. Microbiol. 183: 421-430.

Jacobs, E. 1997. Mycoplasma infections of the human respiratory tract. Wien. Klin. Wochenschr. 109: 574-577.

Jaffe, J. D., H. C. Berg, and G. M. Church. 2004. Proteogenomic mapping as a complementary method to perform genome annotation. Proteomics 4: 59-77.

Janson, H., L. O. Hedén, and A. Forsgren. 1992. Protein D, the immunoglobulin D-binding protein of Haemophilus influenzae, is a lipoprotein. Infect. Immun. 60: $1336-1342$. 
Jeffery, C. J. 1999. Moonlighting proteins. Trends Biochem. Sci. 24: 8-11.

Jers, C., B. Soufi, C. Grangeasse, J. Deutscher, and I. Mijakovic. 2008. Phosphoproteomics in bacteria: Towards a systemic understanding of bacterial phosphorylation networks. Exp. Rev. Proteomics 5: 619-627.

Jin, H., and V. Pancholi. 2006. Identification and biochemical characterization of a eukaryotic-type serine/threonine kinase and its cognate phosphatase in Streptococcus pyogenes: Their biological functions and substrate identification. J. Mol. Biol. 357: 1351-1372.

Johnson, L. N., and D. Barford. 1993. The effects of phosphorylation on the structure and function of proteins. Annu. Rev. Biophys. Biomol. Struct. 22: 199-232.

Jolly, L., F. Pompeo, J. van Heijenoort, F. Fassy, and D. Mengin-Lecreulx. 2000. Autophosphorylation of phosphoglucosamine mutase from Escherichia coli. J. Bacteriol. 182: 1280-1285.

Jordan, J. L., H. Y. Chang, M. F. Balish, L. S. Holt, S. R. Bose, B. M. Hasselbring, R. H. Waldo III, T. M. Krunkosky, and D. C. Krause. 2007. Protein P200 is dispensable for Mycoplasma pneumoniae hemadsorption but not gliding motility or colonization of differentiated bronchial epithelium. Infect. Immun. 75: 518-522.

Jordan, S., A. Junker, J. D. Helmann, and T. Mascher. 2006. Regulation of LiaRS-dependent gene expression in Bacillus subtilis: Identification of inhibitor proteins, regulator binding sites, and target genes of a conserved cell envelope stress-sensing two-component system. J. Bacteriol. 188: 5153-5166.

Karimova, G., J. Pidoux, A. Ullmann, and D. Ladant. 1998. A bacterial two-hybrid system based on a reconstituted signal transduction pathway. Proc. Natl. Acad. Sci. USA 95: 5752-5756.

Kawamoto, R. M., and A. H. Caswell. 1986. Autophosphorylation of glyceraldehydephosphate dehydrogenase and phosphorylation of protein from skeletal muscle microsomes. Biochemistry 25: 657-661.

Ke, P. Y., and Z. F. Chang. 2004. Mitotic degradation of human thymidine kinase 1 is dependent on the anaphase-promoting complex/cyclosome-CDH1-mediated pathway. Mol. Cell. Biol. 24: 514-526. 
Keller, A., A. I. Nesvizhskii, E. Kolker, and R. Aebersold. 2002. Empirical statistical model to estimate the accuracy of peptide identifications made by MS/MS and database search. Anal. Chem. 74: 5383-5392.

Kennelly, P. J., and M. Potts. 1996. Fancy meeting you here! A fresh look at “prokaryotic” protein phosphorylation. J. Bacteriol. 178: 4759-4764.

Kiefer, F., K. Arnold, M. Künzli, L. Bordoli, and T. Schwede. 2009. The SWISS-MODEL Repository and associated resources. Nucleic Acids Res. 37: D387-D392.

Kim, J. W., and C. V. Dang. 2005. Multifaceted roles of glycolytic enzymes. Trends Biochem. Sci. 30: 142-150.

Kobayashi, K., S. D. Ehrlich, A. Albertini, G. Amati, K. K. Andersen, M. Arnaud, K. Asai, S. Ashikaga, S. Aymerich, P. Bessieres, et al. 2003. Essential Bacillus subtilis genes. Proc. Natl. Acad. Sci. USA 100: 4678-4683.

Kopp, F., T. Komatsu, D. K. Nomura, S. A. Trauger, J. R. Thomas, G. Siuzdak, G. M. Simon, and B. F. Cravatt. 2010. The glycerophospho metabolome and its influence on amino acid homeostasis revealed by brain metabolomics of GDE1(-/-) mice. Chem. Biol. 17: 831-840.

Kosinska, U., C. Carnrot, S. Eriksson, L. Wang, and H. Eklund. 2005. Structure of the substrate complex of thymidine kinase from Ureaplasma urealyticum and investigations of possible drug targets for the enzyme. FEBS J. 272: 6365-6372.

Krause, D. C., and M. F. Balish. 2004. Cellular engineering in a minimal microbe: Structure and assembly of the terminal organelle of Mycoplasma pneumoniae. Mol. Microbiol. 51: 917-924.

Krauße, D., K. Hunold, B. Kusian, O. Lenz, J. Stülke, B. Bowien, and J. Deutscher. 2009. Essential role of the hprK gene in Ralstonia eutropha H16. J. Mol. Microbiol. Biotechnol. 17: 146-152.

Krebes, K. A., L. B. Dirksen, and D. C. Krause. 1995. Phosphorylation of Mycoplasma pneumoniae cytadherence-accessory proteins in cell extracts. J. Bacteriol. 177: 4571-4574.

Kristich, C. J., C. L. Wells, and G. M. Dunny. 2007. A eukaryotic-type Ser/Thr kinase in Enterococcus faecalis mediates antimicrobial resistance and intestinal persistence. Proc. Natl. Acad. Sci. USA 104: 3508-3513. 
Kühner, S., V. van Noort, M. J. Betts, A. Leo-Macias, C. Batisse, M. Rode, T. Yamada, T. Maier, S. Bader, P. Beltran-Alvarez, et al. 2009. Proteome organization in a genome-reduced bacterium. Science 326: 1235-1240.

Kunst, F., and G. Rapoport. 1995. Salt stress is an environmental signal affecting degradative enzyme synthesis in Bacillus subtilis. J. Bacteriol. 177: 2403-2407.

Kunst, F., N. Ogasawara, I. Moszer, A. M. Albertini, G. Alloni, V. Azevedo, M. G. Bertero, P. Bessières, A. Bolotin, S. Borchert, et al. 1997. The complete genome sequence of the Gram-positive bacterium Bacillus subtilis. Nature 390: 249-256.

Landry, C. R., E. D. Levy, and S. W. Michnick. 2009. Weak functional constraints on phosphoproteomes. Trends Genet. 25: 193-197.

Larkin, M. A., G. Blackshields, N. P. Brown, R. Chenna, P. A. McGettigan, H. McWilliam, F. Valentin, I. M. Wallace, A. Wilm, R. Lopez, et al. 2007. Clustal W and Clustal X version 2.0. Bioinformatics 23: 2947-2948.

Larson, T. J., M. Ehrmann, and W. Boos. 1983. Periplasmic glycerophosphodiester phosphodiesterase of Escherichia coli, a new enzyme of the glp regulon. J. Biol. Chem. 258: 5428-5432.

Lee, I. M., R. E. Davis, and D. E. Gundersen-Rindal. 2000. Phytoplasma: Phytopathogenic Mollicutes. Annu. Rev. Microbiol. 54: 221-255.

Lévine, A., F. Vannier, C. Absalon, L. Kuhn, P. Jackson, E. Scrivener, V. Labas, J. Vinh, P. Courtney, J. Garin, and S. J. Séror. 2006. Analysis of the dynamic Bacillus subtilis Ser/Thr/Tyr phosphoproteome implicated in a wide variety of cellular processes. Proteomics 6: 2157-2173.

Li, X., S. A. Gerber, A. D. Rudner, S. A. Beausoleil, W. Haas, J. Villén, J. E. Elias, and S. P. Gygi. 2007. Large-scale phosphorylation analysis of alpha-factor-arrested Saccharomyces cerevisiae. J. Proteome Res. 6: 1190-1197.

Liekens, S., A. Bronckaers, and J. Balzarini. 2009. Improvement of purine and pyrimidine antimetabolite-based anticancer treatment by selective suppression of Mycoplasma-encoded catabolic enzymes. Lancet Oncol. 10: 628-635.

Lin, M. H., T. L. Hsu, S. Y. Lin, Y. J. Pan, J. T. Jan, J. T. Wang, K. H. Khoo, and S. H. Wu. 2009. Phosphoproteomics of Klebsiella pneumoniae NTUH-K2044 reveals a tight link between tyrosine phosphorylation and virulence. Mol. Cell. Proteomics 8: 2613-2623. 
Linding, R., L. J. Jensen, F. Diella, P. Bork, T. J. Gibson, and R. B. Russell. 2003. Protein disorder prediction: Implications for structural proteomics. Structure 11: 1453-1459.

Liu, W. B., J. Z. Zhang, B. H. Jiang, T. T. Ren, M. M. Gong, L. Meng, and C. C. Shou. 2006. Lipoprotein p37 from Mycoplasma hyorhinis inhibiting mammalian cell adhesion. J. Biomed. Sci. 13: 323-331.

Lomas-Lopez, R., P. Paracuellos, M. Riberty, A. J. Cozzone, and B. Duclos. 2007. Several enzymes of the central metabolism are phosphorylated in Staphylococcus aureus. FEMS Microbiol. Lett. 272: 35-42.

Low, I. E. 1971. Effect of medium on $\mathrm{H}_{2} \mathrm{O}_{2}$ levels and peroxidase-like activity by Mycoplasma pneumoniae. Infect. Immun. 3: 80-86.

Low, I. E., and S. M. Zimkus. 1973. Reduced nicotinamide adenine dinucleotide oxidase activity and $\mathrm{H}_{2} \mathrm{O}_{2}$ formation of Mycoplasma pneumoniae. J. Bacteriol. 116: $346-354$.

Ludwig, H., G. Homuth, M. Schmalisch, F. M. Dyka, M. Hecker, and J. Stülke. 2001. Transcription of glycolytic genes and operons in Bacillus subtilis: Evidence for the presence of multiple levels of control of the gapA operon. Mol. Microbiol. 41: 409-422.

Macek, B., F. Gnad, B. Soufi, C. Kumar, J. V. Olsen, I. Mijakovic, and M. Mann. 2008. Phosphoproteome analysis of E. coli reveals evolutionary conservation of bacterial Ser/Thr/Tyr phosphorylation. Mol. Cell. Proteomics 7: 299-307.

Macek, B., I. Mijakovic, J. V. Olsen, F. Gnad, C. Kumar, P. R. Jensen, and M. Mann. 2007. The serine/threonine/tyrosine phosphoproteome of the model bacterium Bacillus subtilis. Mol. Cell. Proteomics 6: 697-707.

Madec, E., A. Laszkiewicz, A. Iwanicki, M. Obuchowski, and S. Séror. 2002. Characterization of a membrane-linked Ser/Thr protein kinase in Bacillus subtilis, implicated in developmental processes. Mol. Microbiol. 46: 571-586.

Madec, E., A. Stensballe, S. Kjellström, L. Cladière, M. Obuchowski, O. N. Jensen, and S. Séror. 2003. Mass spectrometry and site-directed mutagenesis identify several autophosphorylated residues required for the activity of PrkC, a Ser/Thr kinase from Bacillus subtilis. J. Mol. Biol. 330: 459-472. 
Madsen, M. L., D. Nettleton, E. L. Thacker, R. Edwards, and F.C. Minion. 2006. Transcriptional profiling of Mycoplasma hyopneumoniae during heat shock using microarrays. Infect. Immun. 74: 160-166.

Madsen, M. L., S. Puttamreddy, E. L. Thacker, M. D. Carruthers, and F. C. Minion. 2008. Transcriptome changes in Mycoplasma hyopneumoniae during infection. Infect. Immun. 76: 658-663.

Martin-Verstraete, I., J. Stülke, A. Klier, and G. Rapoport. 1995. Two different mechanisms mediate catabolite repression of the Bacillus subtilis levanase operon. J. Bacteriol. 177: 6919-6927.

Martin-Verstraete, I., M. Débarbouillé, A. Klier, and G. Rapoport. 1994. Interaction of wild-type truncated LevR of Bacillus subtilis with the upstream activating sequence of the levanase operon. J. Mol. Biol. 241: 178-192.

McCarty, J. S., and G. C. Walker. 1991. DnaK as a thermometer: Threonine-199 is site of autophosphorylation and is critical for ATPase activity. Proc. Natl. Acad. Sci. USA 88: 9513-9517.

McCutcheon, J. P., B. R. McDonald, and N. A. Moran. 2009. Convergent evolution of metabolic roles in bacterial co-symbionts of insects. Proc. Natl. Acad. Sci. USA 106: 15394-15399.

Mengin-Lecreulx, D., and J. van Heijenoort. 1996. Characterization of the essential gene glmM encoding phosphoglucosamine mutase in Escherichia coli. J. Biol. Chem. 271: 32-39.

Merzbacher, M., C. Detsch, W. Hillen, and J. Stülke. 2004. Mycoplasma pneumoniae HPr kinase/phosphorylase: Assigning functional roles to the P-loop and the HPrK/P signature sequence motif. Eur. J. Biochem. 271: 367-374.

Mijakovic, I., S. Poncet, A. Galiner, V. Monedero, S. Fieulaine, J. Janin, S. Nessler, J. A. Marquez, K. Scheffzek, S. Hasenbein, et al. 2002. Pyrophosphate-producing protein dephosphorylation by $\mathrm{HPr}$ kinase/phosphorylase: A relic of early life? Proc. Natl. Acad. Sci. USA 99: 13442-13447.

Miles, R. J. 1992. Catabolism in Mollicutes. J. Gen. Microbiol. 138: 1773-1783.

Min, K. T., C. M. Hilditch, B. Diederich, J. Errington, and M. D. Yudkin. 1993. Sigma F, the first compartment-specific transcription factor of B. subtilis, is regulated by an anti-sigma factor that is also a protein kinase. Cell 27: 735-742. 
Miyata, M. 2010. Unique centipede mechanism of Mycoplasma gliding. Annu. Rev. Microbiol. in press (PMID: 20533876).

Mizutani, Y., H. Wada, O. Yoshida, M. Fukushima, M. Nakao, and T. Miki. 2003. The significance of thymidine phosphorylase/platelet-derived endothelial cell growth factor activity in renal cell carcinoma. Cancer 98: 730-736.

Moreno, M. S., B. L. Schneider, R. R. Maile, W. Weyler, and M. H. Saier Jr. 2001. Catabolite repression mediated by the CcpA protein in Bacillus subtilis: Novel modes of regulation revealed by whole-genome analysis. Mol. Microbiol. 39: 1366-1381.

Morozumi, M., T. Takahashi, and K. Ubukata. 2010. Macrolide-resistant Mycoplasma pneumoniae: Characteristics of isolates and clinical aspects of community-acquired pneumonia. J. Infect. Chemother. 16: 78-86.

Mowbray, J., and V. Moses. 1976. The tentative identification in Escherichia coli of a multi-enzyme complex with glycolytic activity. Eur. J. Biochem. 66: 25-36.

Munson Jr., R. S., and K. Sasaki. 1993. Protein D, a putative immunoglobulin D-binding protein produced by Haemophilus influenzae, is glycerophosphodiester phosphodiesterase. J. Bacteriol. 175: 4569-4571.

Musatovova, O., S. Dhandayuthapani, and J. B. Baseman. 2006. Transcriptional heat shock response in the smallest known self-replicating cell, Mycoplasma genitalium. J. Bacteriol. 188: 2845-2855.

Namiki, K., S. Goodison, S. Porvasnik, R. W. Allan, K. A. Iczkowski, C. Urbanek, L. Reyes, N. Sakamoto, and C. J. Rosser. 2009. Persistent exposure to Mycoplasma induces malignant transformation of human prostate cells. PLoS ONE 4: e6872.

Narita, M. 2009. Pathogenesis of neurologic manifestations of Mycoplasma pneumoniae infection. Pediatr. Neurol. 41: 159-166.

Narita, M. 2010. Pathogenesis of extrapulmonary manifestations of Mycoplasma pneumoniae infection with special reference to pneumonia. J. Infect. Chemother. 16: 162-169.

Nesvizhskii, A. I., A. Keller, E. Kolker, and R. Aebersold. 2003. A statistical model for identifying proteins by tandem mass spectrometry. Anal. Chem. 75: 4646-4658. 
Nilsson, R. P., L. Beijer, and B. Rutberg. 1994. The glpT and $g l p Q$ genes of the glycerol regulon in Bacillus subtilis. Microbiology 140: 723-730.

Nováková, L., L. Sasková, P. Pallová, J. Janecek, J. Novotná, A. Ulrych, J. Echenique, M. C. Trombe, and P. Branny. 2005. Characterization of a eukaryotic type serine/threonine protein kinase and protein phosphatase of Streptococcus pneumoniae and identification of kinase substrates. FEBS J. 272: 1243-1254.

Ohshima, N., S. Yamashita, N. Takahashi, C. Kuroishi, Y. Shiro, and K. Takio. 2008. Escherichia coli cytosolic glycerophosphodiester phosphodiesterase (UgpQ) requires $\mathrm{Mg}^{2+}, \mathrm{Co}^{2+}$ or $\mathrm{Mn}^{2+}$ for its enzyme activity. J. Bacteriol. 190: 1219-1223.

Okazaki, Y., N. Ohshima, I. Yoshizawa, Y. Kamei, S. Mariggiò, K. Okamoto, M. Maeda, Y. Nogusa, Y. Fujioka, T. Izumi, et al. 2010. A novel glycerophosphodiester phosphodiesterase, GDE5, controls skeletal muscle development via a non-enzymatic mechanism. J. Biol. Chem. 285: 27652-27663.

Pachkov, M., T. Dandekar, J. Korbel, P. Bork, and S. Schuster. 2007. Use of pathway analysis and genome context methods for functional genomics of Mycoplasma pneumoniae nucleotide metabolism. Gene 396: 215-225.

Pawson, T., and J. D. Scott. 2005. Protein phosphorylation in signaling - 50 years and counting. Trends Biochem. Sci. 30: 286-290.

Peake, P., N. Winter, and W. Britton. 1998. Phosphorylation of Mycobacterium leprae heat-shock 70 protein at threonine 175 alters its substrate binding characteristics. Biochim. Biophys. Acta 1387: 387-394.

Pietack, N., D. Becher, S. R. Schmidl, M. H. Saier, M. Hecker, F. M. Commichau, and J. Stülke. 2010. In vitro phosphorylation of key metabolic enzymes from Bacillus subtilis: PrkC phosphorylates enzymes from different branches of basic metabolism. J. Mol. Microbiol. Biotechnol. 18: 129-140.

Pilo, P., E. M. Vilei, E. Peterhans, L. Bonvin-Klotz, M. H. Stoffel, D. Dobbelaere, and J. Frey. 2005. A metabolic enzyme as a primary virulence factor of Mycoplasma mycoides subsp. mycoides small colony. J. Bacteriol. 187: 6824-6831. 
Plackett, P., B. P. Marmion, E. J. Shaw, and R. M. Lemcke. 1969. Immunochemical analysis of Mycoplasma pneumoniae. 3. Separation and chemical identification of serologically active lipids. Aust. J. Exp. Biol. Med. Sci. 47: 171-195.

Pollack, J. D. 2001. Ureaplasma urealyticum: An opportunity for combinatorial genomics. Trends Microbiol. 9: 169-175.

Pollack, J. D., M. A. Myers, T. Dandekar, and R. Herrmann. 2002. Suspected utility of enzymes with multiple activities in the small genome Mycoplasma species: The replacement of the missing "household” nucleoside diphosphate kinase gene and activity by glycolytic kinases. OMICS 6: 247-258.

Popham, P. L., T. W. Hahn, K. A. Krebes, and D. C. Krause. 1997. Loss of HMW1 and HMW3 in noncytadhering mutants of Mycoplasma pneumoniae occurs post-translationally. Proc. Natl. Acad. Sci. USA 94: 13979-13984.

Potters, M. B., B. T. Solow, K. M. Bischoff, D. E. Graham, B. H. Lower, R. Helm, and P. J. Kennelly. 2003. Phosphoprotein with phosphoglycerate mutase activity from the archaeon Sulfolobus solfataricus. J. Bacteriol. 185: 2112-2121.

Preneta, R., K .G. Papavinasasundaram, A. J. Cozzone, and B. Duclos. 2004. Autophosphorylation of the $16 \mathrm{kDa}$ and $70 \mathrm{kDa}$ antigens (Hsp 16.3 and Hsp 70) of Mycobacterium tuberculosis. Microbiology 150: 2135-2141.

Razin, S., and R. Herrmann. 2002. Molecular biology and pathogenicity of mycoplasmas (eds.), Kluwer Academic/Plenum Publishers, New York.

Razin, S., D. Yogev, and Y. Naot. 1998. Molecular biology and pathogenicity of mycoplasmas. Microbiol. Mol. Biol. Rev. 62: 1094-1156.

Regni, C., P. A. Tipton, and L. J. Beamer. 2002. Crystal structure of PMM/PGM: An enzyme in the biosynthetic pathway of $P$. aeruginosa virulence factors. Structure 10: $269-279$.

Regula, J. T., B. Ueberle, G. Boguth, A. Görg, M. Schnölzer, R. Herrmann, and R. Frank. 2000. Towards a two-dimensional proteome map of Mycoplasma pneumoniae. Electrophoresis 21: 3765-3780.

Reizer, J., C. Hoischen, F. Titgemeyer, C. Rivolta, R. Rabus, J. Stülke, D. Karamata, M. H. Saier Jr., and W. Hillen. 1998. A novel bacterial protein kinase that controls carbon catabolite repression. Mol. Microbiol. 27: 1157-1169. 
Reizer, J., S. Bachem, A. Reizer, M. Arnaud, M. H. Saier Jr., and J. Stülke. 1999. Novel phosphotransferase system genes revealed by genome analysis - The complete complement of PTS proteins encoded within the genome of Bacillus subtilis. Microbiology 145: 3419-3429.

Rogasch, K., V. Rühmling, J. Pané-Farré, D. Höper, C. Weinberg, S. Fuchs, M. Schmudde, B. M. Bröker, C. Wolz, M. Hecker, and S. Engelmann. 2006. Influence of the two-component system SaeRS on global gene expression in two different Staphylococcus aureus strains. J. Bacteriol. 188: 7742-7758.

Sambrook, J., E. F. Fritsch, and T. Maniatis. 1989. Molecular cloning: A laboratory manual, 2nd ed. Cold Spring Harbor Laboratory, Cold Spring Harbor, N.Y.

Santos-Beneit, F., A. Rodríguez-García, A. K. Apel, and J. F. Martín. 2009. Phosphate and carbon source regulation of two PhoP-dependent glycerophosphodiester phosphodiesterase genes of Streptomyces coelicolor. Microbiology 155: 1800-1811.

Sasková, L., L. Nováková, M. Basler, and P. Branny. 2007. Eukaryotic-type serine/threonine protein kinase StkP is a global regulator of gene expression in Streptococcus pneumoniae. J. Bacteriol. 189: 4168-4179.

Schalock, P. C., and J. G. Dinulos. 2009. Mycoplasma pneumoniae-induced cutaneous disease. Int. J. Dermatol. 48: 673-680.

Schilling, O., O. Frick, C. Herzberg, A. Ehrenreich, E. Heinzle, C. Wittmann, and J. Stülke. 2007. Transcriptional and metabolic responses of Bacillus subtilis to the availability of organic acids: Transcription regulation is important but not sufficient to account for metabolic adaptation. Appl. Env. Microbiol. 73: 499-507.

Schirmer, F., S. Ehrt, and W. Hillen. 1997. Expression, inducer spectrum domain structure, and function of MopR, the regulator of phenol degradation in Acinetobacter calcoaceticus NCIB8250. J. Bacteriol. 179: 1329-1336.

Schmalisch, M. H., S. Bachem, and J. Stülke. 2003. Control of the Bacillus subtilis antiterminator protein GlcT by phosphorylation: Elucidation of the phosphorylation chain leading to inactivation of GlcT. J. Biol. Chem. 278: 51108-51115. 
Schmidl, S. R., K. Gronau, C. Hames, J. Busse, D. Becher, M. Hecker, and J. Stülke. 2010. The stability of cytadherence proteins in Mycoplasma pneumoniae requires activity of the protein kinase PrkC. Infect. Immun. 78: 184-192.

Schmidl, S. R., K. Gronau, N. Pietack, M. Hecker, D. Becher, and J. Stülke. 2010. The phosphoproteome of the minimal bacterium Mycoplasma pneumoniae: Analysis of the complete known Ser/Thr kinome suggests the existence of novel kinases. Mol. Cell. Proteomics 9: 1228-1242.

Schwan, T. G., J. M Battisti, S. F. Porcella, S. J. Raffel, M. E. Schrumpf, E. R. Fischer, J. A. Carroll, P. E. Stewart, P. Rosa, and G. A. Somerville. 2003. Glycerol-3-phosphate acquisition in spirochetes: Distribution and biological activity of glycerophosphodiester phosphodiesterase (GlpQ) among Borrelia species. J. Bacteriol. 185: 1346-1356.

Segal, G., and E. Z. Ron. 1998. Regulation of heat-shock response in bacteria. Ann. NY Acad. Sci. 851: 147-151.

Shah, I. M., M. H. Laaberki, D. L. Popham, and J. Dworkin. 2008. A eukaryotic-like Ser/Thr kinase signals bacteria to exit dormancy in response to peptidoglycan fragments. Cell 135: 486-496.

Shakir, S. M., K. M. Bryant, J. L. Larabee, E. E. Hamm, J. Lovchik, C. R. Lyons, and J. D. Ballard. 2010. Regulatory interactions of a virulence-associated serine/threonine phosphatase-kinase pair in Bacillus anthracis. J. Bacteriol. 192: 400-409.

Shevchenko, D. V., D. R. Akins, E. J. Robinson, M. Li, O. V. Shevchenko, and J. D. Radolf. 1997. Identification of homologs for thioredoxin, peptidyl prolyl cis-trans isomerase, and glycerophosphodiester phosphodiesterase in outer membrane fractions from Treponema pallidum, the syphilis spirochete. Infect. Immun. 65: 4179-4189.

Shevchenko, D. V., T. J. Sellati, D. L. Cox, O. V. Shevchenko, E. J. Robinson, and J. D. Radolf. 1999. Membrane topology and cellular location of the Treponema pallidum glycerophosphodiester phosphodiesterase (GlpQ) ortholog. Infect. Immun. 67: 2266-2276. 
Shi, L., J. F. Liu, X. M. An, and D. C. Liang. 2007. Crystal structure of glycerophosphodiester phosphodiesterase (GDPD) from Thermoanaerobacter tengcongensis, a metal ion-dependent enzyme: Insight into the catalytic mechanism. Proteins 72: 280-288.

Shimizu, T., Y. Kida, and K. Kuwano. 2007. Triacylated lipoproteins derived from Mycoplasma pneumoniae activate nuclear factor-kappaB through toll-like receptors 1 and 2. Immunology 121: 473-483.

Shimizu, T., Y. Kida, and K. Kuwano. 2008. A triacylated lipoprotein from Mycoplasma genitalium activates NF-kappaB through Toll-like receptor 1 (TLR1) and TLR2. Infect Immun. 76: 3672-3678.

Singh, K. D., S. Halbedel, B. Görke, and J. Stülke. 2007. Control of the phosphorylation state of the HPr protein of the phosphotransferase system in Bacillus subtilis: Implication of the protein phosphatase PrpC. J. Mol. Microbiol. Biotechnol. 13: 165-171.

Sirand-Pugnet, P., C. Citti, A. Barré, and A. Blanchard. 2007. Evolution of Mollicutes: Down a bumpy road with twists and turns. Res. Microbiol. 158: 754-766.

Somerson, N. L., B. E. Walls, and R. M. Chanock. 1965. Hemolysin of Mycoplasma pneumoniae: Tentative identification as the peroxide. Science 150: 226-228.

Somerson, N. L., D. Taylor-Robinson, and R. M. Chanock. 1963. Hemolyin production as an aid in the identification and quantitation of Eaton agent (Mycoplasma pneumoniae). Am. J. Hyg. 77: 122-128.

Sonenshein, A. L. 2007. Control of key metabolic intersections in Bacillus subtilis. Nat. Rev. Microbiol. 5: 917-927.

Sood, P., C. G. Lerner, T. Shimamoto, Q. Lu, and M. Inouye. 1994. Characterization of the autophosphorylation of Era, an essential Escherichia coli GTPase. Mol. Microbiol. 12: 201-208.

Soufi, B., F. Gnad, P. R. Jensen, D. Petranovic, M. Mann, I. Mijakovic, and B. Macek. 2008. The Ser/Thr/Tyr phosphoproteome of Lactococcus lactis IL1403 reveals multiply phosphorylated proteins. Proteomics 8: 3486-3493. 
Stebeck, C. E., J. M. Shaffer, T. W. Arroll, S. A. Lukehart, and W. C. van Voorhis. 1997. Identification of the Treponema pallidum subsp. pallidum glycerophosphodiester phosphodiesterase homologue. FEMS Microbiol. Lett. 154: 303-310.

Steinhauer, K., T. Jepp, W. Hillen, and J. Stülke. 2002. A novel mode of control of Mycoplasma pneumoniae HPr kinase/phosphatase activity reflects its parasitic lifestyle. Microbiology 148: 3277-3284.

Stock, A. M., V. L. Robinson, and P. N. Goudreau. 2000. Two-component signal transduction. Annu. Rev. Biochem. 69: 183-215.

Stülke, J., H. Eilers, and S. R. Schmidl. 2009. Mycoplasma and Spiroplasma. Encyclopedia of Microbiology (M. Schaechter, ed.), Elsevier, Oxford. p. 208-219.

Su, H. C., C. A. Hutchison III, and M. C. Giddings. 2007. Mapping phosphoproteins in Mycoplasma genitalium and Mycoplasma pneumoniae. BMC Microbiol. 7: 63.

Sun, X., F. Ge, C. L. Xiao, X. F. Yin, R. Ge, L. H. Zhang, and Q. Y. He. 2010. Phosphoproteomic analysis reveals the multiple roles of phosphorylation in pathogenic bacterium Streptococcus pneumoniae. J. Proteome Res. 9: 275-282.

Szczepanek, S. M., S. Frasca Jr., V. L. Schumacher, X. Liao, M. Padula, S. P. Djordjevic, and S. J. Geary. 2010. Identification of lipoprotein MslA as a neoteric virulence factor of Mycoplasma gallisepticum. Infect. Immun. 78: 3475-3483.

Tan, C. S., B. Bodenmiller, A. Pasculescu, M. Jovanovic, M. O. Hengartner, C. Jørgensen, G. D. Bader, R. Aebersold, T. Pawson, and R. Linding. 2009. Comparative analysis reveals conserved protein phosphorylation networks implicated in multiple diseases. Sci. Signal. 2: ra39.

Tjalsma, H., H. Antelmann, J. D. H. Jongbloed, P. G. Braun, E. Darmon, R. Dorenbos, J. Y. Dubois, H. Westers, G. Zanen, W. J. Quax, et al. 2004. Proteomics of protein secretion by Bacillus subtilis: Separating the "secrets" of the secretome. Microbiol. Mol. Biol. Rev. 68: 207-233.

Tsiodras, S., I. Kelesidis, T. Kelesidis, E. Stamboulis, and H. Giamarellou. 2005. Central nervous system manifestations of Mycoplasma pneumoniae infection. J. Infect. 51: 343-354. 
Van der Rest, B., N. Rolland, A. M. Boisson, M. Ferro, R. Bligny, and R. Douce. 2004. Identification and characterization of plant glycerophosphodiester phosphodiesterase. Biochem. J. 379: 601-607.

Veldhuizen, R., K. Nag, S. Orgeig, and F. Possmayer. 1998. The role of lipids in pulmonary surfactant. Biochim. Biophys. Acta 1408: 90-108.

Vilei, E. M., and J. Frey. 2001. Genetic and biochemical characterization of glycerol uptake in Mycoplasma mycoides subsp. mycoides SC: Its impact on $\mathrm{H}_{2} \mathrm{O}_{2}$ production and virulence. Clin. Diagn. Lab. Immunol. 8: 85-92.

Voigt, B., H. Antelmann, D. Albrecht, A. Ehrenreich, K. H. Maurer, S. Evers, G. Gottschalk, J. M. van Dijl, T. Schweder, and M. Hecker. 2009. Cell physiology and protein secretion of Bacillus licheniformis compared to Bacillus subtilis. J. Mol. Microbiol. Biotechnol. 16: 53-68.

Voisin, S., D. C. Watson, L. Tessier, W. Ding, S. Foote, S. Bhatia, J. F. Kelly, and N. M. Young. 2007, The cytoplasmic phosphoproteome of the Gram-negative bacterium Campylobacter jejuni: Evidence for modification by unidentified protein kinases. Proteomics 7: 4338-4348.

Wach, A. 1996. PCR-synthesis of marker cassettes with long flanking homology regions for gene disruptions in S. cerevisiae. Yeast 12: 259-265.

Waites, K. B., and D. F. Talkington. 2004. Mycoplasma pneumoniae and its role as a human pathogen. Clin. Microbiol. Rev. 17: 697-728.

Wang, L. 2007. The role of Ureaplasma nucleoside monophosphate kinases in the synthesis of nucleoside triphosphates. FEBS J. 274: 1983-1990.

Wang, L., C. Hames, S. R. Schmidl, and J. Stülke. 2010. Upregulation of thymidine kinase activity compensates for loss of thymidylate synthase activity in Mycoplasma pneumoniae. Mol. Microbiol. 77: 1502-1511.

Wang, L., J. Westerberg, G. Bölske, and S. Eriksson. 2001. Novel deoxynucleoside-phosphorylating enzymes in mycoplasmas: Evidence for efficient utilization of deoxynucleosides. Mol. Microbiol. 42: 1065-1073.

Weiner III, J., C. U. Zimmerman, H. W. Göhlmann, and R. Herrmann. 2003. Transcription profiles of the bacterium Mycoplasma pneumoniae grown at different temperatures. Nucleic Acids Res. 31: 6306-6320. 
Welin, M., U. Kosinska, N. E. Mikkelsen, C. Carnrot, C. Zhu, L. Wang, S. Eriksson, B. Munch-Petersen, and H. Eklund. 2004. Structures of thymidine kinase 1 of human and mycoplasmic origin. Proc. Natl. Acad. Sci. USA 101: 17970-17975.

Willby, M. J., M. F. Balish, S. M. Ross, K. K. Lee, J. L. Jordan, and D. C. Krause. 2004. HMW1 is required for stability and localization of HMW2 to the attachment organelle of Mycoplasma pneumoniae. J. Bacteriol. 186: 8221-8228.

Wong, K. K., and H. S. Kwan. 1992. Transcription of glpT of Escherichia coli K12 is regulated by anaerobiosis and fnr. FEMS Microbiol. Lett. 73: 15-18.

Wu, H. C., and M. Tokunaga. 1986. Biogenesis of lipoproteins in bacteria. Curr. Top. Microbiol. Immunol. 125: 127-157.

Yanaka, N. 2007. Mammalian glycerophosphodiester phosphodiesterases. Biosci. Biotechnol. Biochem. 71: 1811-1818.

Yang, X., C. M. Kang, M. S. Brody, and C. W. Price. 1996. Opposing pairs of serine protein kinases and phosphatases transmit signals of environmental stress to activate a bacterial transcription factor. Genes Dev. 10: 2265-2275.

Yanofsky, C., and M. Rachmeler. 1958. The exclusion of free indole as an intermediate in the biosynthesis of tryptophan in Neurospora crassa. Biochim. Biophys. Acta 28: 640-641.

Yooseph, S., G. Sutton, D. B. Rusch, A. L. Halpern, S. J. Williamson, K. Remington, J. A. Eisen, K. B. Heidelberg, G. Manning, W. Li, et al. 2007. The "Sorcerer II" global ocean sampling expedition: Expanding the universe of protein families. PLoS Biol. 5: e16.

You, X. X., Y. H. Zeng, and Y. M. Wu. 2006. Interactions between Mycoplasma lipid-associated membrane proteins and the host cells. J. Zhejiang Univ. Sci. B 7: $342-350$.

Yu, L. R., Z. Zhu, K. C. Chan, H. J. Issaq, D. S. Dimitrov, and T. D. Veenstra. 2007. Improved titanium dioxide enrichment of phosphopeptides from HeLa cells and high confident phosphopeptide identification by cross-validation of MS/MS and MS/MS/MS spectra. J. Proteome Res. 6: 4150-4162. 
Yus, E., T. Maier, K. Michalodimitrakis, V. van Noort, T. Yamada, W. H. Chen, J. A. Wodke, M. Güell, S. Martínez, R. Bourgeois, et al. 2009. Impact of genome reduction on bacterial metabolism and its regulation. Science 326: 1263-1268.

Zeiman, E., M. Tarshis, and S. Rottem. 2008. Mycoplasma penetrans under nutritional stress: Influence on lipid and lipoprotein profiles and on the binding to and invasion of HeLa cells. FEMS Microbiol. Lett. 287: 243-249.

Zhai, B., J. Villén, S. A. Beausoleil, J. Mintseris, and S. P. Gygi. 2008. Phosphoproteome analysis of Drosophila melanogaster embryos. J. Proteome Res. 7: 1675-1682.

Zhu, W., and D. F. Becker. 2003. Flavin redox state triggers conformational changes in the PutA protein from Escherichia coli. Biochemistry 42: 5469-5477.

Zimmerman, C. U., and R. Herrmann. 2005. Synthesis of a small, cysteine-rich, 29 amino acids long peptide in Mycoplasma pneumoniae. FEMS Microbiol. Lett. 253: 315-321.

Zimmermann, L., E. Peterhans, and J. Frey. 2010. RGD motif of lipoprotein T, involved in adhesion of Mycoplasma conjunctivae to lamb synovial tissue cells. J. Bacteriol. 192: 3773-3779.

Zuo, L. L., Y. M. Wu, and X. X. You. 2009. Mycoplasma lipoproteins and Toll-like receptors. J. Zhejiang Univ. Sci. B 10: 67-76.

Zylicz, M., J. H. LeBowitz, R. McMacken, and C. Georgopoulos. 1983. The DnaK protein of Escherichia coli possesses an ATPase and autophosphorylating activity and is essential in an in vitro DNA replication system. Proc. Natl. Acad. Sci. USA 80: 6431-6435. 
Chapter 10

Appendix 


\section{Oligonucleotides}

Tab. S14. Oligonucleotides.

\begin{tabular}{|c|c|c|}
\hline Name & Sequence $\left(5^{\prime} \rightarrow 3^{\prime}\right)^{\mathrm{ab}}$ & Description $^{c}$ \\
\hline CD13 & AAACATATGGCTAGCTGGAGCCACCCGCAGTTC & $\begin{array}{l}\text { Sequencing of pGP172 } \\
\text { constructs fw (NdeI) }\end{array}$ \\
\hline CH35 & GCTCAACTTGATTAATTTAAAACAATGG & $\begin{array}{l}\text { Screening for } p r k C:: T n \\
\text { strain } \mathrm{fw}\end{array}$ \\
\hline CH61 & ATGCCAAATCCTGTTAGATTTGTTTAC & mpn372 probe fw \\
\hline CH63 & $\begin{array}{l}\text { CTAATACGACTCACTATAGGGAGAGCCGGGTGGTGA } \\
\text { GCATG }\end{array}$ & mpn372 probe rev \\
\hline CH74 & $\begin{array}{l}\text { CTAATACGACTCACTATAGGGAGAGGTGTTCAAAAC } \\
\text { TGTGGAGG }\end{array}$ & prkC probe rev \\
\hline eno forw1 & AAGGATCCCCATACATTGTTGATGTTTATGCAC & $e^{e n o} o_{\mathrm{Bsu}} \mathrm{fW}(\mathrm{BamHI})$ \\
\hline eno rev & TTTCTGCAGTTACTTGTTTAAGTTGTAGAAAGAGTTG & $e^{e n o} o_{\mathrm{Bsu}} \mathrm{rev}($ PstI) \\
\hline FC17 & AAAGGATCCGTGGCACAAGGTGAAAAAATTAC & $i c d_{\mathrm{Bsu}} \mathrm{fw}(\mathrm{BamHI})$ \\
\hline FC18 & TTTCTGCAGTTATTAGTCCATGTTTTTGAT & $i c d_{\mathrm{Bsu}}$ rev (PstI) \\
\hline FC146 & CGATGCGTTCGCGATCCAGGC & $\begin{array}{l}\text { Sequencing of pUT18 } \\
\text { constructs fw }\end{array}$ \\
\hline FC147 & CCAGCCTGATGCGATTGCTGCAT & $\begin{array}{l}\text { Sequencing of pKNT25 } \\
\text { constructs } \mathrm{fw}\end{array}$ \\
\hline FC148 & GTCACCCGGATTGCGGCGG & $\begin{array}{l}\text { Sequencing of pUT18C } \\
\text { constructs rev }\end{array}$ \\
\hline FC149 & GCTGGCTTAACTATGCGGCATCAGA & $\begin{array}{l}\text { Sequencing of pUT18C } \\
\text { constructs fw }\end{array}$ \\
\hline JS39 & TCTATCAACAGGAGTCCAAGC & $\begin{array}{l}\text { Sequencing of pWH844 } \\
\text { constructs rev }\end{array}$ \\
\hline $\begin{array}{l}\text { M13 puc } \\
\text { rev }\end{array}$ & GGAAACAGCTATGACCATG & $\begin{array}{l}\text { Sequencing pKNT25 and } \\
\text { pUT18 constructs rev }\end{array}$ \\
\hline MR04 & AAAGGATCCATGGTTGACTTTAAAACAGTCCAAGC & pgk gene fw (BamHI) \\
\hline MR05 & $\begin{array}{l}\text { TATAAAGCTTTTACTTTTTTTGAATATCGCTAATTCCC } \\
\text { AC }\end{array}$ & pgk gene rev (HindIII) \\
\hline MR06 & P_GTTAGCACAGTTCTGGGCATCATTGGG & pgk A441G \\
\hline MR07 & P_CCCAATGGCCAGCAGAACTTCAGG & pgk A894G \\
\hline MR08 & AAAGGATCCATGCATAAGAAGGTCTTACTAGCC & pgm gene fw (BamHI) \\
\hline MR09 & $\begin{array}{l}\text { TATACTGCAGTTACTTTTTACTTATTAAAAGGGAGTT } \\
\text { TGCG }\end{array}$ & pgm gene rev (PstI) \\
\hline MR10 & $\begin{array}{l}\text { AAAGGATCCATGAGTGCACAAACTGGAACCGATCTT } \\
\text { TTCAAG }\end{array}$ & eno gene fw (BamHI) \\
\hline
\end{tabular}


Tab. S14. Continued.

\begin{tabular}{|c|c|c|}
\hline Name & Sequence $\left(5^{\prime} \rightarrow 3^{\prime}\right)^{\mathrm{ab}}$ & Description $^{c}$ \\
\hline \multirow[t]{2}{*}{ MR11 } & TATACTGCAGTTAAGCTTTTTGCGGTTTAATGTTTTTA & eno gene rev $(P s t \mathrm{I})+$ \\
\hline & AAGGTGTTCCAACC & A1335G \\
\hline MR19 & AAAGGATCCATGGAAAGTAAATGGTTAACAGTTGAC & pgi gene fw (BamHI) \\
\hline MR20 & TATAAAGCTTCTATTCACGACCAAGTTTAGCAAAC & pgi gene rev (HindIII) \\
\hline MR21 & P_GGGTAAATCATGGGCTTTGGTAGTAACTTC & pgi A393G \\
\hline MR22 & P_CTGCTTTGCGTCACTGGCTTTACAC & pgi A756G \\
\hline MR23 & $\begin{array}{l}\text { AAAGGATCCATGAGTCCAAAAACAACCAAAAAAATT } \\
\text { GCC }\end{array}$ & pfkA gene fw (BamHI) \\
\hline MR24 & $\begin{array}{l}\text { TATAAAGCTTTTAAATAATATTTTTATTAATGACTGC } \\
\text { AATTAAAGCGC }\end{array}$ & pfkA gene rev (HindIII) \\
\hline MR25 & $\begin{array}{l}\text { AAAGGATCCATGCTAGTAAACATCAAACAAATGTTG } \\
\text { CAAC }\end{array}$ & fba gene fw (BamHI) \\
\hline MR26 & TATAAAGCTTCTAAGCCTTATTGGTTGAACCACAG & fba gene rev (HindIII) \\
\hline MR27 & P_GGCTTTACCCCGACAACTGGAAGGG & fba A552G \\
\hline MR28 & $\begin{array}{l}\text { AAAGGATCCATGCGTACGAAATACCTAATTGGTAAC } \\
\text { TGGAAG }\end{array}$ & $\begin{array}{l}\text { tpiA gene } \mathrm{fw}(\mathrm{BamHI})+ \\
\text { A30G }\end{array}$ \\
\hline MR29 & $\begin{array}{l}\text { TATACTGCAGTTATGCATATACTTGTGCCATTACTAA } \\
\text { AAAGTCG }\end{array}$ & tpiA gene rev (PstI) \\
\hline MR30 & $\begin{array}{l}\text { P_GTGATTGCTTACGAACCAATTTGGGCAATTGGTAC } \\
\text { GG }\end{array}$ & tpiA A489G \\
\hline MR31 & AAAGGATCCATGCTAGCAAAGAGTAAGACTATCC & gapA gene fw (BamHI) \\
\hline MR32 & TATACTGCAGTTAAAGCTTGGCACAATAGTTAACTAC & gapA gene rev (PstI) \\
\hline MR33 & P_GCTAACCTGCCATGGGCAGAACAC & gapA A270G \\
\hline MR34 & AAAGGATCCATGATTCACCACCTAAAACGCAC & pyk gene fw (BamHI) \\
\hline MR35 & $\begin{array}{l}\text { TATACTGCAGTTATAAGCTAATGATCTTATTGTGAAA } \\
\text { TACTC }\end{array}$ & pyk gene rev (PstI) \\
\hline MR36 & P_GCTATGGACGCTAGCAATGTTAGATGAC & pyk A69G \\
\hline MR37 & P_CAAGGTGCCTTACTGGCAACGGTAC & pyk A864G \\
\hline MR38 & P_CAGTGAGTTTTGGAAGCAGGTGGTG & pyk A1167G \\
\hline NP01 & AAAGAGCTCCGATGAAACGAGAAAGCAACATTC & als $D_{\text {Bsu }}$ fw (SacI) \\
\hline NP02 & TTTGGATCCCTATTATTCAGGGCTTCCTTCAGT & $a l s D_{\mathrm{Bsu}} \operatorname{rev}($ BamHI) \\
\hline NP03 & AAAGAGCTCCGGTGCTAATCGGCAAGC & $p r k C_{\mathrm{Bsu}} \mathrm{fw}(\mathrm{SacI})$ \\
\hline NP04 & TTTAGATCTTCATTATTCATCTTTCGGATACTCAAT & $p r k C_{\mathrm{Bsu}} \operatorname{rev}(\mathrm{BglII})$ \\
\hline NP05 & AAAGAGCTCGATGGATACAATTGAAAAGAAATCAG & $t k t_{\mathrm{Bsu}} \mathrm{fw}(\mathrm{SacI})$ \\
\hline NP06 & TTTGGATCCTCATTACTTATTGATTAATGCCTTAAC & $t k t_{\mathrm{Bsu}} \mathrm{rev}(\mathrm{BamHI})$ \\
\hline NP07 & AAAGAGCTCGATGATGAACGACGCTTTGAC & $y a b T_{\mathrm{Bsu}} \mathrm{fw}(\mathrm{S} a c \mathrm{I})$ \\
\hline NP08 & TTTAGATCTTCATTAGATTAAGAAAAAGATAATA & $y a b T_{\mathrm{Bsu}} \operatorname{rev}(\mathrm{Bg} l \mathrm{II})$ \\
\hline
\end{tabular}


Tab. S14. Continued.

\begin{tabular}{|c|c|c|}
\hline Name & Sequence $\left(5^{\prime} \rightarrow 3^{\prime}\right)^{\mathrm{ab}}$ & Description $^{c}$ \\
\hline NP09 & AAAGAGCTCGATGGCATTAAAACTTCTAAAAAA & $p r k D_{\mathrm{Bsu}} \mathrm{fW}(\mathrm{SacI})$ \\
\hline NP10 & TTTGGATCCTCATTATGTGACCGATTGAATGGC & $\operatorname{prk} D_{\mathrm{Bsu}} \mathrm{rev}($ BamHI) \\
\hline NP11 & AAAGAGCTCGATGTTATTCTTTGTTGATACAG & $y w j H_{\mathrm{Bsu}} \mathrm{fw}(\mathrm{SacI})$ \\
\hline NP12 & TTTGGATCCTCATTATTTGTTCCAGTCTGCC & $y w j H_{\text {Bsu }}$ rev $($ BamHI) \\
\hline NP13 & AAAGAGCTCGATGGTCAAGTCATTTCG & $y x a L_{\mathrm{Bsu}} \mathrm{fw}(\mathrm{SacI})$ \\
\hline NP14 & TTTGGATCCTCATTATTTCCCAAAAGCCATCAG & $y x a L_{\mathrm{Bsu}}$ rev $($ BamHI) \\
\hline NP15 & CCGGTGTTCACGGTGCGCCGCTTGG & $t k t_{\mathrm{Bsu}}$ sequencing fw \\
\hline NP16 & CTCACCTGATTCTTTAACAGCTACAGCG & $t k t_{\mathrm{Bsu}}$ sequencing rev \\
\hline NP17 & CTTGCTAAAGCTGCTGGCGAAAAGAAGCTG & prk $C_{\mathrm{Bsu}}$ sequencing $\mathrm{fw}$ \\
\hline NP18 & ATCGTGAGCAAAACCCACGGCCAC & $p r k C_{\mathrm{Bsu}}$ sequencing rev \\
\hline NP19 & ATGTTCACGCGATCAAACCGACGTC & $y x a L_{\mathrm{Bsu}}$ sequencing $\mathrm{fw}$ \\
\hline NP20 & GCAGCAGCCAACTCAGCTTCCTTTCGGGC & $\begin{array}{l}\text { Sequencing of pGP172 } \\
\text { constructs rev }\end{array}$ \\
\hline NP108 & $\begin{array}{l}\text { AAAGGATCCCTCACTTATTTAAAGGAGGAAACAATC } \\
\text { ATGGGCAAGTATTTTGGAACAGACG }\end{array}$ & $\begin{array}{l}g \operatorname{glm}_{\mathrm{Bsu}}+\mathrm{RBS}_{\text {gapA }} \mathrm{fw} \\
(\text { BamHI) }\end{array}$ \\
\hline NP109 & TTTGTCGACCTATCACTCTAATCCCATTTCTGACCGG & $g l m M_{\mathrm{Bsu}}$ rev (SalI) \\
\hline NP110 & GGCTATTCTCCGGAGCAGCCGATTGTCA & $\begin{array}{l}\text { glm } M_{\mathrm{Bsu}} \mathrm{LFH}-\mathrm{PCR} \mathrm{fw} \\
\text { (up-fragment) }\end{array}$ \\
\hline NP111 & CAGGAACGGACGACCAAAAGTTTTCCCG & $\begin{array}{l}\text { glmM } M_{\mathrm{Bsu}} \mathrm{LFH}-\mathrm{PCR} \mathrm{fw} \\
\text { (up-fragment, } \\
\text { sequencing) }\end{array}$ \\
\hline NP112 & $\begin{array}{l}\text { CCTATCACCTCAAATGGTTCGCTGTAAAGGCCAGCTC } \\
\text { AGGTGTAAGCTC }\end{array}$ & $\begin{array}{l}\text { glm } M_{\mathrm{Bsu}} \mathrm{LFH}-\mathrm{PCR} \text { rev } \\
\text { (up-fragment) }\end{array}$ \\
\hline NP113 & $\begin{array}{l}\text { CCGAGCGCCTACGAGGAATTTGTATCGAGCGAAGAC } \\
\text { GAAAGAGCTGTGCGA }\end{array}$ & $\begin{array}{l}\text { glmM } M_{\mathrm{Bsu}} \mathrm{LFH-PCR} \mathrm{fw} \\
\text { (down- fragment) }\end{array}$ \\
\hline NP114 & CATCGCCATCGCATCAGATGCTACGACGT & $\begin{array}{l}\text { glmM } M_{\mathrm{Bsu}} \text { LFH-PCR rev } \\
\text { (down-fragment) }\end{array}$ \\
\hline NP115 & TATAAGACGCACGTGTAATCACGTCACCATC & $\begin{array}{l}\text { glmM } M_{\mathrm{Bsu}} \mathrm{LFH-PCR} \text { rev } \\
\text { (down-fragment, } \\
\text { sequencing) }\end{array}$ \\
\hline NP116 & $\begin{array}{l}\text { P_GCAGAGGCGGGCGTCATGATTTCCGCTGCCCATAA } \\
\text { CCCAGTGCAGGATAACGGCATCAA }\end{array}$ & $g \operatorname{lm} M_{\mathrm{Bsu}} \mathrm{T} 298 \mathrm{G}$ \\
\hline NP117 & AAAGGATCCATGGGCAAGTATTTTGGAACAGACG & $\operatorname{glm} M_{\mathrm{Bsu}} \mathrm{fW}(\mathrm{BamHI})$ \\
\hline PD05 & $\begin{array}{l}\text { AAATCTAGAGATGGAAAGTAAATGGTTAACAGTTGA } \\
\text { CAC }\end{array}$ & pgi gene fw (XbaI) \\
\hline PD06 & $\begin{array}{l}\text { TTTGGTACCCGTTCACGACCAAGTTTAGCAAACATTA } \\
\text { AC }\end{array}$ & pgi gene rev (KpnI) \\
\hline
\end{tabular}


Tab. S14. Continued.

\begin{tabular}{|c|c|c|}
\hline Name & Sequence $\left(5^{\prime} \rightarrow 3^{\prime}\right)^{\mathrm{ab}}$ & Description $^{c}$ \\
\hline PD07 & $\begin{array}{l}\text { AAATCTAGAGATGAGTCCAAAAACAACCAAAAAAAT } \\
\text { TGCC }\end{array}$ & $p f k A$ gene fw $(X b a I)$ \\
\hline PD08 & $\begin{array}{l}\text { TTTGGTACCCGAATAATATTTTTATTAATGACTGCAA } \\
\text { TTAAAGCGC }\end{array}$ & $p f k A$ gene rev $(K p n I)$ \\
\hline PD09 & $\begin{array}{l}\text { AAATCTAGAGATGCTAGTAAACATCAAACAAATGTT } \\
\text { GCAAC }\end{array}$ & $f b a$ gene $\mathrm{fw}(\mathrm{XbaI})$ \\
\hline PD10 & TTTGAGCTCCGAGCCTTATTGGTTGAACCACAGAG & fba gene rev (SacI) \\
\hline PD11 & $\begin{array}{l}\text { AAATCTAGAGATGCGTACGAAATACCTAATTGGTAA } \\
\text { C }\end{array}$ & tpiA gene fw $(X b a I)$ \\
\hline PD12 & $\begin{array}{l}\text { TTTGGTACCCGTGCATATACTTGTGCCATTACTAAAA } \\
\text { AGTC }\end{array}$ & tpiA gene rev (KpnI) \\
\hline PD13 & AAATCTAGAGATGCTAGCAAAGAGTAAGACTATCCG & gapA gene fw (XbaI) \\
\hline PD14 & TTTGAATTCCGAAGCTTGGCACAATAGTTAACTACCC & gapA gene rev (EcoRI) \\
\hline PD15 & AAATCTAGAGATGGTTGACTTTAAAACAGTCCAAGC & pgk gene fw $(X b a I)$ \\
\hline PD16 & $\begin{array}{l}\text { TTTGAATTCCGCTTTTTTTGAATATCGCTAATTCCCAC } \\
\text { TAG }\end{array}$ & pgk gene rev (EcoRI) \\
\hline PD17 & AAATCTAGAGATGCATAAGAAGGTCTTACTAGCC & pgm gene fw $(X b a I)$ \\
\hline PD18 & $\begin{array}{l}\text { TTTGAATTCCGCTTTTTACTTATTAAAAGGGAGTTTG } \\
\text { CG }\end{array}$ & pgm gene rev (EcoRI) \\
\hline PD19 & AAATCTAGAGATGAGTGCACAAACTGGAACCG & eno gene fw (XbaI) \\
\hline PD20 & $\begin{array}{l}\text { TTTGAATTCCGAGCTTTTTGCGGTTTAATGTTTTTAAA } \\
\text { GGTG }\end{array}$ & eno gene rev (EcoRI) \\
\hline PD21 & AAATCTAGAGATGATTCACCACCTAAAACGCAC & pyk gene fw (XbaI) \\
\hline PD22 & $\begin{array}{l}\text { TTTGGTACCCGTAAGCTAATGATCTTATTGTGAAATA } \\
\text { CTCC }\end{array}$ & pyk gene rev (KpnI) \\
\hline SH03 & GAGTACCCGGATTAAAGCGGG & hprK probe fw \\
\hline SH04 & $\begin{array}{l}\text { CTAATACGACTCACTATAGGGAGACATTAACTGGAT } \\
\text { TTCGGTGCGCTG }\end{array}$ & hprK probe rev \\
\hline SH05 & CAGGTAACGGTGCTGGTTCG & glpF probe fw \\
\hline SH06 & $\begin{array}{l}\text { CTAATACGACTCACTATAGGGAGAATTGCAGTACCA } \\
\text { GTAGCGGC }\end{array}$ & glpF probe rev \\
\hline SH29 & ATGAGTGAGCTAACTCACAG & $\begin{array}{l}\text { Screening primer for } \\
\text { transposon insertions }\end{array}$ \\
\hline SH30 & CAATACGCAAACCGCCTC & $\begin{array}{l}\text { Screening primer for } \\
\text { transposon insertions }\end{array}$ \\
\hline SH62 & TAGAATTTTATGGTGGTAGAG & aac-ahpD probe fw \\
\hline
\end{tabular}


Tab. S14. Continued.

\begin{tabular}{|c|c|c|}
\hline Name & Sequence $\left(5^{\prime} \rightarrow 3^{\prime}\right)^{\mathrm{ab}}$ & Description $^{c}$ \\
\hline SH63 & $\begin{array}{l}\text { CTAATACGACTCACTATAGGGAGAACACTATCATAA } \\
\text { CСАCTACC }\end{array}$ & aac-ahpD probe rev \\
\hline SH66 & AAAGTCGACATGGACAGCACCAACCAAAAC & prpC probe fw (SalI) \\
\hline SH73 & $\begin{array}{l}\text { CTAATACGACTCACTATAGGGAGAGACCATCAGAGC } \\
\text { ACAACAG }\end{array}$ & prpC probe rev \\
\hline SH82 & ATGGATCCATGGCACAAAAAACATTTAAAG & $p t s H_{\text {Bsu }}$ fw (BamHI) \\
\hline SH83 & ATAAAGCTTCTCGCCGAGTCCTTCG & $p t s H_{\text {Bsu }}$ rev (HindIII) \\
\hline SH89 & TAGAGCTCGATGGCACTAAATTTAAAGATTGG & prkC probe $\mathrm{fw}$ \\
\hline $\begin{array}{l}\text { spec-fwd } \\
\text { (kan) }\end{array}$ & $\begin{array}{l}\text { CAGCGAACCATTTGAGGTGATAGGGACTGGCTCGCT } \\
\text { AATAACGTAACGTGACTGGCAAGAG }\end{array}$ & $\begin{array}{l}\text { Construction of } B \text {. subtilis } \\
\text { mutants by LFH-PCR fw }\end{array}$ \\
\hline $\begin{array}{l}\text { spec-rev } \\
\text { (kan) }\end{array}$ & $\begin{array}{l}\text { CGATACAAATTCCTCGTAGGCGCTCGGCGTAGCGAG } \\
\text { GGCAAGGGTTTATTGTTTTCTAAAATCTG }\end{array}$ & $\begin{array}{l}\text { Construction of B. subtilis } \\
\text { mutants by LFH-PCR rev }\end{array}$ \\
\hline SS29 & $\begin{array}{l}\text { AAAGAGCTCGATGATTAACATCGATCCCCATTTTATT } \\
\text { C }\end{array}$ & mpn549 gene fw (SacI) \\
\hline SS30 & TATAGGATCCTTAGCTGTTGACATGCTTTTGTTTGG & $\begin{array}{l}\text { mpn549 gene rev } \\
(\text { BamHI) }\end{array}$ \\
\hline SS31 & P_GAAGACATTAAGAAGTGGATTGGTTCCATTCG & mpn549 A807G \\
\hline SS32 & GATTTGTGGGCCGATGTTTTCC & $t d k$ probe $\mathrm{fw}$ \\
\hline SS33 & $\begin{array}{l}\text { CTAATACGACTCACTATAGGGAGACGGTTTCGTTGCT } \\
\text { CTTGGAC }\end{array}$ & $t d k$ probe rev \\
\hline SS34 & AAAGAGCTCGATGCTTAAACGACAACTTCTGCTAGC & glpQ gene fw (SacI) \\
\hline SS35 & $\begin{array}{l}\text { TATAGGATCCTTACACTTCAAACTTCTTGTTGGCAAT } \\
\text { TTG }\end{array}$ & glpQ gene rev (BamHI) \\
\hline SS36 & P_GCCTTTTTGTTTTGGACGAAAAAGCAGTTCCAAG & glpQ A507G \\
\hline SS37 & P_CAGTATCTCCATCCCTGGACAAACATTTACG & glpQ A576G \\
\hline SS38 & P_CCTTTAGGGCTGTGGACGCTTAACAGTG & glpQ A639G \\
\hline SS39 & $\begin{array}{l}\text { AAAGAGCTCGATGCGCAAACAGTTTTTAATTGCACA } \\
\text { C }\end{array}$ & mpn566 gene fw (SacI) \\
\hline SS40 & $\begin{array}{l}\text { TATAGGATCCTTAGTAAAGTTGTGCTGCTATTTGAAA } \\
\text { TTTAAC }\end{array}$ & $\begin{array}{l}\text { mpn566 gene rev } \\
\text { (BamHI) }\end{array}$ \\
\hline SS41 & CGCAACAATTTAAAGAACTGACTTAGC & $\begin{array}{l}\text { Screening for mpn549::Tn } \\
\text { strain rev }\end{array}$ \\
\hline SS42 & CAACTTCTGCTAGCACACCG & glpQ probe $\mathrm{fw}$ \\
\hline SS43 & $\begin{array}{l}\text { CTAATACGACTCACTATAGGGAGAGCTATTTGGTAG } \\
\text { TTGGGGTTAATG }\end{array}$ & glpQ probe rev \\
\hline SS44 & GCAAACAGTTTTTAATTGCACACCG & mpn566 probe fw \\
\hline
\end{tabular}


Tab. S14. Continued.

\begin{tabular}{|c|c|c|}
\hline Name & Sequence $\left(5^{\prime} \rightarrow 3^{\prime}\right)^{\mathrm{ab}}$ & Description $^{c}$ \\
\hline SS45 & $\begin{array}{l}\text { CTAATACGACTCACTATAGGGAGAGCTCTTTAACTTT } \\
\text { TCGTTGAGGTAC }\end{array}$ & mpn566 probe rev \\
\hline SS46 & $\begin{array}{l}\text { 5AAAGTCGACAGATAGATGGATGTGAGACAAACATG } \\
\text { AAGAAGATTCAAGTAGTCGTTAAAG }\end{array}$ & ptsH gene fw (SalI) \\
\hline SS47 & $\begin{array}{l}\text { 5AAACTGCAGTTAAATAACTTGGTGTTTTTCTAAAAC } \\
\text { TGC }\end{array}$ & ptsH gene rev (PstI) \\
\hline SS48 & $\begin{array}{l}\text { 5AAACTGCAGAGATAGATGGATGTGAGACAAACATG } \\
\text { AAAAAGTTATTAGTCAAGGAGTTAATC }\end{array}$ & hprK gene fw (PstI) \\
\hline SS49 & $\begin{array}{l}\text { AAAAAGCTTAGAACCGGTAGATCTAGACTGCTACTA } \\
\text { ACACTAGGATTCATC }\end{array}$ & $\begin{array}{l}\text { hprK gene rev (HindIII/ } \\
\text { BshTI/XbaI) }\end{array}$ \\
\hline SS50 & $\begin{array}{l}\text { CTAATACGACTCACTATAGGGAGAGCACTAGCGGCA } \\
\text { ACGACG }\end{array}$ & $\begin{array}{l}\text { mpn162 promoter } \\
\text { fragment probe }(+56) \text { rev }\end{array}$ \\
\hline SS51 & GGGAAGAAAAGATCCGTAAGTTTG & $\begin{array}{l}\text { Screening for mpn162::Tn } \\
\text { strain fw }\end{array}$ \\
\hline SS52 & GTTTGCCGCTTGCAGGCC & $\begin{array}{l}\text { Screening for mpn162::Tn } \\
\text { strain rev }\end{array}$ \\
\hline SS53 & $\begin{array}{l}\text { AAAGGATCCTTGTCCATTCGTAAAACTGGCATAGTAT } \\
\text { TTC }\end{array}$ & engC gene $\mathrm{fw}(\mathrm{BamHI})$ \\
\hline SS54 & $\begin{array}{l}\text { TATAAAGCTTTTAATTAATCAACTTTAGATAGCTCTC } \\
\text { ATAAAGCCAC }\end{array}$ & $\begin{array}{l}\text { engC gene rev (HindIII) + } \\
\text { A804G }\end{array}$ \\
\hline SS55 & P_CTACAGTGGAAGCGCGATTTTAAACTGTTAGTTGG & engC A108G \\
\hline SS56 & $\begin{array}{l}\text { P_CTAATTGAACCCAAGATTAACTGGCAACAGTTGTT } \\
\text { TAAATTG }\end{array}$ & engC A267G \\
\hline SS57 & ATGAATGATACTGACAAGAAGTTCCC & $\begin{array}{l}\text { Screening for } h m w 2:: T n \\
\text { strain } \mathrm{fw} 1\end{array}$ \\
\hline SS58 & GATGAATCATCACGCCGAATTGC & $\begin{array}{l}\text { Screening for } h m w 2:: T n \\
\text { strain } \mathrm{fw} 2\end{array}$ \\
\hline SS59 & TTATTTAGCTGCTTTTTGGGCAATTAG & $\begin{array}{l}\text { Screening for } h m w 2:: T n \\
\text { strain rev }\end{array}$ \\
\hline SS60 & GCTACTGCATACGATCCCAATC & p65 probe $\mathrm{fw}$ \\
\hline SS61 & $\begin{array}{l}\text { CTAATACGACTCACTATAGGGAGACATAGTAAGCGT } \\
\text { TGGGATCGG }\end{array}$ & p65 probe rev \\
\hline SS62 & GACACTGGGTTTGATGATGGG & $h m w 2$ probe $1 \mathrm{fw}$ \\
\hline SS63 & $\begin{array}{l}\text { CTAATACGACTCACTATAGGGAGACCTGGTTAGCTG } \\
\text { CAATCTGTTC }\end{array}$ & hmw2 probe 1 rev \\
\hline SS64 & GAAGCGCAACCAACTAACGC & $h m w 2$ probe $2 \mathrm{fw}$ \\
\hline
\end{tabular}


Tab. S14. Continued.

\begin{tabular}{|c|c|c|}
\hline Name & Sequence $\left(5^{\prime} \rightarrow 3^{\prime}\right)^{\mathrm{ab}}$ & Description $^{c}$ \\
\hline SS65 & $\begin{array}{l}\text { CTAATACGACTCACTATAGGGAGAGTTGTTGGGACA } \\
\text { GATCAGCAC }\end{array}$ & $h m w 2$ probe 2 rev \\
\hline SS66 & GATGATGAAGCTGACATCATCATAG & $p 41$ probe $\mathrm{fw}$ \\
\hline SS67 & $\begin{array}{l}\text { CTAATACGACTCACTATAGGGAGAGTGTATTCCGTG } \\
\text { CСАCСAATAAC }\end{array}$ & $p 41$ probe rev \\
\hline SS68 & CTAACACTAAAACGGGTACGAATTG & p24 probe $\mathrm{fw}$ \\
\hline SS69 & $\begin{array}{l}\text { CTAATACGACTCACTATAGGGAGAGCCACAAGTGTT } \\
\text { TCTGATGTCAC }\end{array}$ & p24 probe rev \\
\hline SS70 & CTGAGGAAAATCCCGAACAGATC & $h m w 1$ probe $1 \mathrm{fw}$ \\
\hline SS71 & $\begin{array}{l}\text { CTAATACGACTCACTATAGGGAGAGCTGGTACATCT } \\
\text { GCTTCCAAAC }\end{array}$ & $h m w 1$ probe 1 rev \\
\hline SS72 & GCCAACAGCGACATTAACAGC & $h m w 1$ probe $2 \mathrm{fw}$ \\
\hline SS73 & $\begin{array}{l}\text { CTAATACGACTCACTATAGGGAGACAGTATCACTGC } \\
\text { AGAGCTTATAAAC }\end{array}$ & $h m w 1$ probe 2 rev \\
\hline SS74 & GTATGGTAAGCTAGCACAAAAGATC & $h m w 3$ probe fw \\
\hline SS75 & $\begin{array}{l}\text { CTAATACGACTCACTATAGGGAGAGTTGATCAGCTT } \\
\text { GCTGTTCGG }\end{array}$ & $h m w 3$ probe rev \\
\hline SS76 & CTTGGATTCTCATCCTCACCG & p1 probe $1 \mathrm{fw}$ \\
\hline SS77 & $\begin{array}{l}\text { CTAATACGACTCACTATAGGGAGAGGTGCAGCCCCA } \\
\text { СTCAAAC }\end{array}$ & p1 probe 1 rev \\
\hline SS78 & GCGAGCGGGTGGTTCG & $p 1$ probe $2 \mathrm{fw}$ \\
\hline SS79 & $\begin{array}{l}\text { CTAATACGACTCACTATAGGGAGAGCTTGTAAGCAC } \\
\text { TCGCTTCC }\end{array}$ & p1 probe 2 rev \\
\hline SS80 & CCTGAATGCTAGTGCTGTTCAG & $p 30$ probe $\mathrm{fw}$ \\
\hline SS81 & $\begin{array}{l}\text { CTAATACGACTCACTATAGGGAGAGTTGAGGTGGGA } \\
\text { AACCAGTAC }\end{array}$ & p30 probe rev \\
\hline SS82 & CAAAGGTAGTTAATGTAGATCAGGTAG & eng $C$ probe $\mathrm{fw}$ \\
\hline SS83 & $\begin{array}{l}\text { CTAATACGACTCACTATAGGGAGAGTTGGGCTGCTA } \\
\text { ATTGTTTGAGG }\end{array}$ & eng $C$ probe rev \\
\hline SS84 & GTTGCAGTAGATCAATAAAAAGAGGG & $\begin{array}{l}\text { Screening for } c b i O:: T n \\
\text { strain } \mathrm{fw}\end{array}$ \\
\hline SS85 & GCTTTTATCAACAAAGACACTAAGATTG & $\begin{array}{l}\text { Screening for } c b i O:: T n \\
\text { strain rev }\end{array}$ \\
\hline SS86 & GCACGCATCTTAAAGCTGTTGG & $p 28$ probe $\mathrm{fw}$ \\
\hline SS87 & $\begin{array}{l}\text { CTAATACGACTCACTATAGGGAGACACTGTCAGCGT } \\
\text { GCTCTGC }\end{array}$ & p28 probe rev \\
\hline SS88 & GCTTCTCCAATTCGCGGCG & $h m w 2$ sequencing rev 1 \\
\hline
\end{tabular}


Tab. S14. Continued.

\begin{tabular}{|c|c|c|}
\hline Name & Sequence $\left(5^{\prime} \rightarrow 3^{\prime}\right)^{\mathrm{ab}}$ & Description $^{c}$ \\
\hline SS89 & CGCCGCGAATTGGAGAAGC & $h m w 2$ sequencing fw 1 \\
\hline SS90 & GTTCGATTTGCTGGAACTCCTG & $h m w 2$ sequencing rev 2 \\
\hline SS91 & CGCCAGTCTTTAGAGCATGAC & $h m w 2$ sequencing fw 2 \\
\hline SS92 & GCTAGCTTCTTCACTTACCGG & $p 40$ probe $\mathrm{fw}$ \\
\hline SS93 & $\begin{array}{l}\text { CTAATACGACTCACTATAGGGAGAGTTAACGTTCCTT } \\
\text { CGTTACCATTG }\end{array}$ & p 40 probe rev \\
\hline SS94 & GCGTCAAAGTAGTACTTGGGAG & $\begin{array}{l}\text { Screening for mpn256::Tn } \\
\text { strain } \mathrm{fw}\end{array}$ \\
\hline SS95 & GACATCAGGGTTAATTTGAGCAATC & $\begin{array}{l}\text { Screening for mpn256::Tn } \\
\text { strain rev }\end{array}$ \\
\hline SS96 & CGACTGATGGTTGGCTCTAC & $\begin{array}{l}\text { Screening for } g l p Q:: T n \\
\text { strain } \mathrm{fw}\end{array}$ \\
\hline SS97 & GCTAACCGTTTACTCGGTGG & $\begin{array}{l}\text { Screening for } g l p Q:: T n \\
\text { strain rev }\end{array}$ \\
\hline SS98 & CAGTTAATAGGCAACGAAGACTATG & $\begin{array}{l}\text { Screening for } m p n 566:: T n \\
\text { strain } \mathrm{fw}\end{array}$ \\
\hline SS99 & CACTAGTAAGAACCACAGTAGTAC & $\begin{array}{l}\text { Screening for mpn566::Tn } \\
\text { strain rev }\end{array}$ \\
\hline SS100 & GAGTAGCTCACAATGACCCTG & $\begin{array}{l}\text { Screening for } g l p K:: T n \\
\text { strain } \mathrm{fw}\end{array}$ \\
\hline SS101 & GGTGGAGCAGAAGATAAGCAG & $\begin{array}{l}\text { Screening for } g l p K:: T n \\
\text { strain rev }\end{array}$ \\
\hline SS102 & GTTAATTAATGGGAGGATAAGCAAGC & $\begin{array}{l}\text { Screening for } g l p F:: T n \\
\text { strain } \mathrm{fw}\end{array}$ \\
\hline SS103 & CCGACAAGATTTGTAAGTTAGATGC & $\begin{array}{l}\text { Screening for } g l p F:: T n \\
\text { strain rev }\end{array}$ \\
\hline SS104 & AAAGGATCCATGGCACTAAATTTAAAGATTGGTGAC & prkC gene fw (BamHI) \\
\hline SS105 & $\begin{array}{l}\text { TATAAAGCTTTTATAGGTAATTACTTACCATTAGTTC } \\
\text { ACG }\end{array}$ & $\begin{array}{l}\text { prkC catalytic domain rev } \\
\text { (HindIII) }\end{array}$ \\
\hline SS106 & TATAAAGCTTTTACGAATGGACAACTACCCCAG & prkC gene rev (HindIII) \\
\hline SS107 & AAAGGATCCATGAACAGTAACGCATACTTGGAAGC & manB gene fw (BamHI) \\
\hline SS108 & TATACTGCAGCTAAGCTTTATCGAGGTTGAGCAATC & manB gene rev (PstI) \\
\hline SS109 & P_GAAAGTAAGGATACCTGGGAGTTAGCGCG & manB A846G \\
\hline SS110 & P_CTTTGCGATTGCCGAATGGAATCCGCAAAC & manB A936G \\
\hline SS111 & P_GATTACTATAACTGGACGGTACCACACACCATTC & manB A1356G \\
\hline SS112 & P_CGGTGAATGTCACTGCTGCTCATAATCCTAAAACC & manB A445G/G446C \\
\hline
\end{tabular}


Tab. S14. Continued.

\begin{tabular}{|c|c|c|}
\hline Name & Sequence $\left(5^{\prime} \rightarrow 3^{\prime}\right)^{\mathrm{ab}}$ & Description $^{c}$ \\
\hline SS113 & CAAGGCCACAATTGATTATGTCTTTG & $\begin{array}{l}\text { Screening for manB::Tn } \\
\text { strain } \mathrm{fw}\end{array}$ \\
\hline SS114 & GGTAACTTAGTGGAAGTCCACTTAAC & $\begin{array}{l}\text { Screening for manB::Tn } \\
\text { strain rev }\end{array}$ \\
\hline SS115 & GAATTAAACAGTAAGCTAACCGGG & mpn256 probe fw \\
\hline SS116 & $\begin{array}{l}\text { CTAATACGACTCACTATAGGGAGACAATAAACTTAG } \\
\text { AAAAGTCCATGGTTG }\end{array}$ & mpn256 probe rev \\
\hline SS117 & GTATTTTGAGAGTTAGTGCCTTTCC & $\begin{array}{l}\text { Screening for } f t s Y:: T n \\
\text { strain } \mathrm{fw}\end{array}$ \\
\hline SS118 & CTCGCGTAATTGGGGATCTG & $\begin{array}{l}\text { Screening for } f t s Y:: T n \\
\text { strain rev }\end{array}$ \\
\hline SS119 & GATCAGGAGACAAATACATTCGAAC & $\begin{array}{l}\text { Screening for mpn349::Tn } \\
\text { strain fw }\end{array}$ \\
\hline SS120 & GTGCCTTGGTTAGATTACCTTTAC & $\begin{array}{l}\text { Screening for mpn349::Tn } \\
\text { strain rev }\end{array}$ \\
\hline SS121 & CACTAAGGTGTACGGTGATTTTAAG & $n r d E$ probe fw \\
\hline SS122 & $\begin{array}{l}\text { CTAATACGACTCACTATAGGGAGAGACCAATTGATT } \\
\text { CСATGTTGTCTTC }\end{array}$ & $n r d E$ probe rev \\
\hline SS123 & GAATCAGTTTCTCCCTTAGAATATGC & $n r d F$ probe fw \\
\hline SS124 & $\begin{array}{l}\text { CTAATACGACTCACTATAGGGAGAGTCTTTCCCGGTG } \\
\text { TAATAGGG }\end{array}$ & $n r d F$ probe rev \\
\hline SS125 & GATATCAAAATAGTCGACGCTAGTG & nrdI probe fw \\
\hline SS126 & $\begin{array}{l}\text { CTAATACGACTCACTATAGGGAGACTTGTTCTACATC } \\
\text { GTTTTTCGTGC }\end{array}$ & nrdI probe rev \\
\hline SS127 & CCAACAGCGCTTTTATTCTCGG & mpn083 probe fw \\
\hline SS128 & $\begin{array}{l}\text { CTAATACGACTCACTATAGGGAGAGGACATTAGGTT } \\
\text { TGGTGTACTTAC }\end{array}$ & mpn083 probe rev \\
\hline SS129 & CCTTGTTAGTTGCGCCACAC & mpn162 probe fw \\
\hline SS130 & $\begin{array}{l}\text { CTAATACGACTCACTATAGGGAGACACCTTCGTGGT } \\
\text { GATCATGATC }\end{array}$ & mpn162 probe rev \\
\hline SS131 & GGCTTAGTCATCCACACTTGG & ulaF probe fw \\
\hline SS132 & $\begin{array}{l}\text { CTAATACGACTCACTATAGGGAGACCACTGCATCCTT } \\
\text { GCCATTC }\end{array}$ & ulaF probe rev \\
\hline SS133 & CTGCTGTAGCTCATGTGGAAC & licA probe $\mathrm{fw}$ \\
\hline SS134 & $\begin{array}{l}\text { CTAATACGACTCACTATAGGGAGACTCAAAGTCAAT } \\
\text { TAAAACGACCTGC }\end{array}$ & licA probe rev \\
\hline SS135 & GGTTTTACCCGTTTTTGTGTTAATGC & pls $C$ probe fw \\
\hline
\end{tabular}


Tab. S14. Continued.

\begin{tabular}{|c|c|c|}
\hline Name & Sequence $\left(5^{\prime} \rightarrow 3^{\prime}\right)^{\mathrm{ab}}$ & Description $^{c}$ \\
\hline SS136 & $\begin{array}{l}\text { CTAATACGACTCACTATAGGGAGAGCCCGATTTAAA } \\
\text { CTCACCAATTTG }\end{array}$ & plsC probe rev \\
\hline SS137 & CCGTTACATTCTCCTTAAAATTCAAAG & mpn239 probe fw \\
\hline SS138 & $\begin{array}{l}\text { CTAATACGACTCACTATAGGGAGAGCTCTACCACAA } \\
\text { TGCCGTTG }\end{array}$ & mpn239 probe rev \\
\hline SS139 & ATGCTTAAGAAAAAAGTTAATAATGATGCTG & spx probe $\mathrm{fw}$ \\
\hline SS140 & $\begin{array}{l}\text { CTAATACGACTCACTATAGGGAGATTACTTCTTTACT } \\
\text { GTACGCACTTTAGG }\end{array}$ & spx probe rev \\
\hline SS141 & CCGCTTTTCACCTTTGCACAG & pmd1 probe fw \\
\hline SS142 & $\begin{array}{l}\text { CTAATACGACTCACTATAGGGAGAGAGTTAGTGGCA } \\
\text { ATAGCAAAGGC }\end{array}$ & pmd1 probe rev \\
\hline SS143 & GCTAATGTAGATGTTAACCTCACG & $y j c W$ probe $\mathrm{fw}$ \\
\hline SS144 & $\begin{array}{l}\text { CTAATACGACTCACTATAGGGAGACTCAAAACAGCG } \\
\text { GTTGGTTCATC }\end{array}$ & $y c j W$ probe rev \\
\hline SS145 & CCTGATTTATGACAAAGAAGGTAACC & mpn684 probe fw \\
\hline SS146 & $\begin{array}{l}\text { CTAATACGACTCACTATAGGGAGAGGGATTAGTCTC } \\
\text { TAACCAAAACTG }\end{array}$ & mpn684 probe rev \\
\hline SS147 & GAATATTGTAGTCGACTTTGGTGAG & ugp $C$ probe $\mathrm{fw}$ \\
\hline SS148 & $\begin{array}{l}\text { CTAATACGACTCACTATAGGGAGAGGTTAGCTTCCA } \\
\text { GTTCACTCTG }\end{array}$ & ugpC probe rev \\
\hline SS149 & GTTCAAGGTCCGCGGTAGC & oppF probe fw \\
\hline SS150 & $\begin{array}{l}\text { CTAATACGACTCACTATAGGGAGAGTAGGGCAAACT } \\
\text { TGTTAATCGTAG }\end{array}$ & oppF probe rev \\
\hline SS151 & GCCTAGTGGGATAATTAATAGAAATAAG & $\begin{array}{l}\text { glpF promoter fragment } \\
(-321) \mathrm{fw}\end{array}$ \\
\hline SS152 & CGGCACCAATCCAACGTGG & $\begin{array}{l}\text { glpF promoter fragment } \\
(+49) \text { rev }\end{array}$ \\
\hline SS153 & GAGTTAATGTTAGACAATGGGCAAG & $\begin{array}{l}\text { mpn239 promoter } \\
\text { fragment }(-247) \mathrm{fw}\end{array}$ \\
\hline SS154 & GGAGAATGTAACGGTAAATTATTTGAC & $\begin{array}{l}\text { mpn239 promoter } \\
\text { fragment }(+58) \text { rev }\end{array}$ \\
\hline SS155 & $\begin{array}{l}\text { AAAGAATTCGCCTAGTGGGATAATTAATAGAAATAA } \\
\text { G }\end{array}$ & $\begin{array}{l}\text { glpF promoter fragment } \\
(-321) \mathrm{fw}(E c o R I)\end{array}$ \\
\hline SS156 & TATAGGATCCCCTAAAAATTCGGCACCAATCC & $\begin{array}{l}\text { glpF promoter fragment } \\
(+59) \text { rev }(\text { BamHI) }\end{array}$ \\
\hline SS157 & AAACAATTGGAGTTAATGTTAGACAATGGGCAAG & $\begin{array}{l}\text { mpn239 promoter } \\
\text { fragment (-247) fw (MfeI) }\end{array}$ \\
\hline
\end{tabular}


Tab. S14. Continued.

\begin{tabular}{|c|c|c|}
\hline Name & Sequence $\left(5^{\prime} \rightarrow 3^{\prime}\right)^{\mathrm{ab}}$ & Description $^{c}$ \\
\hline SS158 & $\begin{array}{l}\text { TATAGGATCCCTTTGAATTTTAAGGAGAATGTAACG } \\
\text { G }\end{array}$ & $\begin{array}{l}\text { mpn239 promoter } \\
\text { fragment }(+71) \text { rev } \\
(\text { BamHI })\end{array}$ \\
\hline SS159 & $\begin{array}{l}\text { TATAGCGGCCGCTTACACTTCAAACTTCTTGTTGGCA } \\
\text { ATTTGTG }\end{array}$ & glpQ gene rev (NotI) \\
\hline SS160 & $\begin{array}{l}\text { TATAGCGGCCGCTTAGTAAAGTTGTGCTGCTATTTGA } \\
\text { AATTTAACAAAG }\end{array}$ & mpn566 gene rev (NotI) \\
\hline SS161 & AAAGGATCCGCAGGTTAGCTGATAAGTTTAGGAG & $\begin{array}{l}\text { glpQ promoter fragment } \\
(-495) \mathrm{fw}(\text { BamHI) }\end{array}$ \\
\hline SS162 & AAAGGATCCGGGGAATGGGTATGAGCTGG & $\begin{array}{l}\text { mpn566 promoter } \\
\text { fragment }(-492) \mathrm{fw} \\
(\text { BamHI) }\end{array}$ \\
\hline SS163 & AAAGGATCCGTTAATAATGATGATTGAAGC & $\begin{array}{l}\text { ackA promoter fragment } \\
(-224) \mathrm{fw}(\text { BamHI) }\end{array}$ \\
\hline SS164 & AAACTGCAGATGCTTAAACGACAACTTCTGCTAGC & glpQ gene fw (PstI) \\
\hline SS165 & AAACTGCAGATGCGCAAACAGTTTTTAATTGCACAC & mpn566 gene fw (PstI) \\
\hline SS166 & CTTCTACCGGGTTTTCCATGC & $\begin{array}{l}\text { Screening for } d e o A:: T n \\
\text { strain } \mathrm{fw}\end{array}$ \\
\hline SS167 & CGCACTACGTTCCGCACAC & $\begin{array}{l}\text { Screening for } d e o A:: T n \\
\text { strain rev }\end{array}$ \\
\hline SS168 & GCTAAATCATAAGCTCACCATTGC & $\operatorname{cin} A$ probe $\mathrm{fw}$ \\
\hline SS169 & $\begin{array}{l}\text { CTAATACGACTCACTATAGGGAGAGCTGCATCTTGTT } \\
\text { СТCTTAAACC }\end{array}$ & $\operatorname{cin} A$ probe rev \\
\hline SS170 & CTAACCCCAACTACGGCATC & rpoA probe fw \\
\hline SS171 & $\begin{array}{l}\text { CTAATACGACTCACTATAGGGAGACCCCAAAGAATT } \\
\text { AATCATTTCACGG }\end{array}$ & rpoA probe rev \\
\hline SS172 & CTTAATCTTAGCGCTTACCTTGTTG & disA probe fw \\
\hline SS173 & $\begin{array}{l}\text { CTAATACGACTCACTATAGGGAGAGTTTTAATCACG } \\
\text { CCGCGCAC }\end{array}$ & disA probe rev \\
\hline SS174 & GAAATTTAAGTATGGTGCTACCC & mpn363 probe fw \\
\hline SS175 & $\begin{array}{l}\text { CTAATACGACTCACTATAGGGAGAGTTAGGTGATAA } \\
\text { CTTGGAGAGATC }\end{array}$ & mpn363 probe rev \\
\hline SS176 & СТСААТTTTAGССТСАААСССААС & cbiO probe fw \\
\hline SS177 & $\begin{array}{l}\text { CTAATACGACTCACTATAGGGAGACCGCTTTAGTTTG } \\
\text { CTAGCTAGC }\end{array}$ & cbiO probe rev \\
\hline SS178 & GTTGGTGCCTTCTTTACССТC & mpn407 probe fw \\
\hline
\end{tabular}


Tab. S14. Continued.

\begin{tabular}{|c|c|c|}
\hline Name & Sequence $\left(5^{\prime} \rightarrow 3^{\prime}\right)^{\mathrm{ab}}$ & Description $^{c}$ \\
\hline SS179 & $\begin{array}{l}\text { CTAATACGACTCACTATAGGGAGACTTACCTTCAAA } \\
\text { CCGAGCACTAG }\end{array}$ & mpn407 probe rev \\
\hline SS180 & CTGCATGTGGTGCTAGGGG & mpn408 probe fw \\
\hline SS181 & $\begin{array}{l}\text { CTAATACGACTCACTATAGGGAGACTTTTAATCATTT } \\
\text { GAGCACCGTTTTCC }\end{array}$ & mpn408 probe rev \\
\hline SS182 & CCGCTTATCATTCCCGCAAC & p37 probe fw \\
\hline SS183 & $\begin{array}{l}\text { CTAATACGACTCACTATAGGGAGACCTCTGTAGAAA } \\
\text { TTGATTAGTTTGTTTG }\end{array}$ & p37 probe rev \\
\hline SS184 & CTAGATAGTTTTTTACCAGCAATTGTG & $m t l F$ probe $f w$ \\
\hline SS185 & $\begin{array}{l}\text { CTAATACGACTCACTATAGGGAGACATTAAAAGGAC } \\
\text { AAGATGAACTTAGCC }\end{array}$ & $m t l F$ probe rev \\
\hline SS186 & CCCTTTTTGTACTATAAGCTTGCC & lspA probe $\mathrm{fw}$ \\
\hline SS187 & $\begin{array}{l}\text { CTAATACGACTCACTATAGGGAGACTGAATTAAAAA } \\
\text { GGAGAGGAACAGAC }\end{array}$ & IspA probe rev \\
\hline SS188 & CGCCAAATCACGTCCTGTTC & mpn294 probe fw \\
\hline SS189 & $\begin{array}{l}\text { CTAATACGACTCACTATAGGGAGACACAAGAATTGG } \\
\text { CACAACTAATAATG }\end{array}$ & mpn294 probe rev \\
\hline SS190 & GCTGCCTGTGGTACAAAGG & mpn506 probe fw \\
\hline SS191 & $\begin{array}{l}\text { CTAATACGACTCACTATAGGGAGAGTACCTTCTTTCT } \\
\text { TTTTAGCGTTGTTC }\end{array}$ & mpn506 probe rev \\
\hline SS192 & P_GATGGTTTGGAGATGGATGTGCAACTCAC & $\begin{array}{l}\text { mpn566 T106G/G107A/ } \\
\text { A114T }\end{array}$ \\
\hline SS193 & P_CGTTTCGCTTTCTCTTGCTTGAAATTAAGGGCG & $\begin{array}{l}\text { mpn566 T328G/T329A/ } \\
\text { T330A }\end{array}$ \\
\hline SS194 & $\begin{array}{l}\text { P_GTAGTCACTCATGATGACAACTATAAGGTAGGAA } \\
\text { ATAAAACC }\end{array}$ & $\begin{array}{l}\text { mpn566 A151C/T154G/ } \\
\text { T155A/A156T }\end{array}$ \\
\hline SS195 & CAAAGCCAATGTCAACGGCAC & mpn131 probe fw \\
\hline SS196 & $\begin{array}{l}\text { CTAATACGACTCACTATAGGGAGACATTTTTGGTCGG } \\
\text { TGGAATTAAACTTAC }\end{array}$ & mpn131 probe rev \\
\hline SS197 & GCTGTTGGTATTTTTATTCTTTCACTAAAC & mpn149 probe fw \\
\hline SS198 & $\begin{array}{l}\text { CTAATACGACTCACTATAGGGAGAGAGGGAGCCACT } \\
\text { GTTAGTTG }\end{array}$ & mpn149 probe rev \\
\hline SS199 & CСAATTAAAGCTCCTTCAAAGAATGG & mpn284 probe fw \\
\hline SS200 & $\begin{array}{l}\text { CTAATACGACTCACTATAGGGAGACGATCGTATTGTT } \\
\text { CGTCATTAATTTTG }\end{array}$ & mpn284 probe rev \\
\hline SS201 & ССАААССССААТСССТСААААС & mpn370 probe fw \\
\hline
\end{tabular}


Tab. S14. Continued.

\begin{tabular}{|c|c|c|}
\hline Name & Sequence $\left(5^{\prime} \rightarrow 3^{\prime}\right)^{\mathrm{ab}}$ & Description $^{c}$ \\
\hline SS202 & $\begin{array}{l}\text { CTAATACGACTCACTATAGGGAGACCGTGAGGGGGA } \\
\text { GGGAC }\end{array}$ & mpn370 probe rev \\
\hline SS203 & GGAATGGATATAAGGGGGTCG & mpn468 probe fw \\
\hline SS204 & $\begin{array}{l}\text { CTAATACGACTCACTATAGGGAGACTTCTTGAACACT } \\
\text { GATCGAATTACC }\end{array}$ & mpn468 probe rev \\
\hline SS205 & GACATTGTTAAAAAGCTTGAATAAGGTTC & $\begin{array}{l}\text { mpn162 promoter } \\
\text { fragment }(-145) \mathrm{fw}\end{array}$ \\
\hline SS206 & GCACTAGCGGCAACGACG & $\begin{array}{l}\text { mpn162 promoter } \\
\text { fragment }(+56) \text { rev }\end{array}$ \\
\hline SS207 & CACACAGGATCTAATAAATTTCAAGAG & $\begin{array}{l}\text { Screening for mpn506::Tn } \\
\text { strain fw }\end{array}$ \\
\hline SS208 & GGCTTTAAAAGAGCTCTTGCAAAC & $\begin{array}{l}\text { Screening for mpn506::Tn } \\
\text { strain rev }\end{array}$ \\
\hline SS209 & GGAAAATTCAAATAGAAACTATAACACACAG & $\begin{array}{l}\text { Screening for mpn284::Tn } \\
\text { strain fw }\end{array}$ \\
\hline SS210 & GTAAGTTTTAATCTCCACGATAGTAAC & $\begin{array}{l}\text { Screening for mpn284::Tn } \\
\text { strain rev }\end{array}$ \\
\hline SS211 & $\begin{array}{l}\text { CTAATACGACTCACTATAGGGAGACGGCACCAATCC } \\
\text { AACGTGG }\end{array}$ & $\begin{array}{l}\text { glpF promoter fragment } \\
\text { probe }(+49) \text { rev }\end{array}$ \\
\hline $\begin{array}{l}\text { pWH844 } \\
\text { fw }\end{array}$ & TATGAGAGGATCGCATCACCAT & $\begin{array}{l}\text { Sequencing of pWH844 } \\
\text { constructs fw }\end{array}$ \\
\hline pyk forw1 & $\begin{array}{l}\text { AAGGATCCAGAAAAACTAAAATTGTTTGTACCATCG } \\
\text { G }\end{array}$ & $p y k_{\mathrm{Bsu}} \mathrm{fw}(\mathrm{BamHI})$ \\
\hline pyk rev & TTTCTGCAGTTAAAGAACGCTCGCACGG & $p y k_{\mathrm{Bsu}}$ rev (PstI) \\
\hline
\end{tabular}

\footnotetext{
${ }^{\mathrm{a}}$ The sequence of the T7-promotor is underlined.

b The "P” at the 5' end of primer sequences indicates phosphorylation.

${ }^{\mathrm{c}}$ Unless otherwise indicated, all genes are $M$. pneumonaie specific.
} 


\section{Plasmids}

Tab. S15. Plasmids.

\begin{tabular}{|c|c|c|c|}
\hline Name & Relevant characteristics $^{\mathrm{ab}}$ & Used restriction sites & Reference \\
\hline pAG2 & pQE30-pts $H_{\text {Bsu }}\left(\mathrm{Amp}^{\mathrm{R}}\right)$ & - & Galinier et al. (1997) \\
\hline pBQ200 & $\begin{array}{l}\text { Allows expression of proteins in } \\
\left.\text { B. subtilis (Amp }{ }^{\mathrm{R}} / \mathrm{Erm}^{\mathrm{R}}\right)\end{array}$ & - & $\begin{array}{l}\text { Martin-Verstraete et al. } \\
\text { (1994) }\end{array}$ \\
\hline pGP172 & $\begin{array}{l}\text { Allows overexpression of N-terminal } \\
\text { Strep-tag fusion proteins in } \\
\text { E. coli BL21(DE3)/pLysS }\left(\mathrm{Amp}^{\mathrm{R}}\right)\end{array}$ & - & $\begin{array}{l}\text { Merzbacher et al. } \\
\text { (2004) }\end{array}$ \\
\hline pGP174 & pGP172-gln $A_{\mathrm{Bsu}}$ & - & Heinrich et al. (2006) \\
\hline pGP205 & pWH844-hprK $K_{\mathrm{Bsu}}$ & - & Hanson et al. (2002) \\
\hline pGP353 & $\begin{array}{l}\text { pMT85 with promoterless lacZ gene } \\
\left(\mathrm{Gm}^{\mathrm{R}} / \mathrm{Kan}^{\mathrm{R}}\right)\end{array}$ & - & $\begin{array}{l}\text { Halbedel and Stülke } \\
\text { (2006) }\end{array}$ \\
\hline pGP371 & $\begin{array}{l}\text { pWH844-ptsH } H_{\mathrm{Bsu}}[\mathrm{S} 46 \mathrm{~A}] \\
(\mathrm{SH} 82+\mathrm{SH} 83)\end{array}$ & BamHI + HindIII & Pietack et al. (2010) \\
\hline pGP400 & pBQ200-glmM $M_{\text {Bsu }}(N P 108 / N P 109)$ & BamHI + SalI & Schmidl et al. (2010) \\
\hline pGP563 & pWH844-eno ${ }_{\mathrm{Bsu}}$ (eno forw1/eno rev) & BamHI + PstI & Pietack et al. (2010) \\
\hline pGP656 & pWH844-manB (SS107/SS108) & BamHI + PstI & Schmidl et al. (2010) \\
\hline pGP657 & pWH844-manB ${ }^{\mathrm{c}}$ (SS107-SS111) & BamHI + PstI & Schmidl et al. (2010) \\
\hline pGP658 & $\begin{array}{l}\text { pWH844-manB[S149A }]^{\mathrm{c}} \\
(\mathrm{SS} 107-\mathrm{SS} 112)\end{array}$ & BamHI + PstI & Schmidl et al. (2010) \\
\hline pGP659 & $\begin{array}{l}\text { pGP353-glpF(-321...+59) } \\
\text { (SS155/SS156) }\end{array}$ & EcoRI + BamHI & - \\
\hline pGP660 & $\begin{array}{l}\text { pGP353-mpn239(-247...71) } \\
\text { (SS157/SS158) }\end{array}$ & EcoRI + BamHI & - \\
\hline pGP661 & $\begin{array}{l}\text { pGP172-mpn566[W36E/E38D/ } \\
\text { N51H/L52D/F110E] } \\
\text { (SS39/SS40/SS192-SS194) }\end{array}$ & $\mathrm{SacI}+\mathrm{BamHI}$ & This work \\
\hline pGP756 & pWH844-pgi (MR19/MR20) & BamHI + HindIII & Dutow et al. (2010) \\
\hline pGP757 & pWH844-pfkA (MR23/MR24) & BamHI + HindIII & Dutow et al. (2010) \\
\hline pGP758 & pWH844-fba (MR25/MR26) & BamHI + HindIII & Dutow et al. (2010) \\
\hline pGP759 & pWH844-tpiA (MR28/MR29) & BamHI + PstI & Dutow et al. (2010) \\
\hline pGP760 & pWH844-gapA (MR31/MR32) & BamHI + PstI & Dutow et al. (2010) \\
\hline pGP761 & pWH844-pyk (MR34/MR35) & BamHI + PstI & Dutow et al. (2010) \\
\hline pGP762 & pWH844-pgic (MR19-MR22) & BamHI + HindIII & Dutow et al. (2010) \\
\hline pGP763 & pWH844-fba ${ }^{\mathrm{c}}$ (MR25-MR27) & BamHI + HindIII & Dutow et al. (2010) \\
\hline pGP764 & pWH844-tpiAc (MR28-MR30) & BamHI + PstI & Dutow et al. (2010) \\
\hline pGP765 & pWH844-gapAc (MR31-MR33) & BamHI + PstI & Dutow et al. (2010) \\
\hline
\end{tabular}


Tab. S15. Continued.

\begin{tabular}{|c|c|c|c|}
\hline Name & Relevant characteristics $^{\text {ab }}$ & Used restriction sites & Reference \\
\hline pGP766 & pWH844-pyk (MR34-MR38) & BamHI + PstI & Dutow et al. (2010) \\
\hline pGP819 & pGP172-ywjH $H_{\text {Bsu }}(\mathrm{NP} 11 / \mathrm{NP} 12)$ & SacI + BamHI & Pietack et al. (2010) \\
\hline pGP820 & pGP172-tkt $t_{\text {Bsu }}(\mathrm{NP05/NP06)}$ & SacI + BamHI & Pietack et al. (2010) \\
\hline pGP821 & pGP172-prkD $D_{\mathrm{Bsu}}(\mathrm{NP} 09 / \mathrm{NP} 10)$ & $\mathrm{SacI}+\mathrm{BamHI}$ & Pietack et al. (2010) \\
\hline pGP822 & pGP172-als $D_{\text {Bsu }}(\mathrm{NP} 01 / \mathrm{NP} 02)$ & SacI + BamHI & Pietack et al. (2010) \\
\hline pGP823 & pGP172-yabT $T_{\mathrm{Bsu}}(\mathrm{NP} 07 / \mathrm{NP} 08)$ & SacI + BamHI & Pietack et al. (2010) \\
\hline pGP824 & pGP172-yxaL $L_{\mathrm{Bsu}}(\mathrm{NP13} / \mathrm{NP14})$ & SacI + BamHI & Pietack et al. (2010) \\
\hline pGP825 & pGP172-prkC $C_{\mathrm{Bsu}}(\mathrm{NP03/NP04)}$ & SacI + BamHI & Pietack et al. (2010) \\
\hline pGP931 & pWH844-icd ${ }_{\mathrm{Bsu}}(\mathrm{FC} 17 / \mathrm{FC} 18)$ & BamHI + PstI & Pietack et al. (2010) \\
\hline pGP1006 & pWH844-pgk (MR04/MR05) & BamHI + HindIII & Dutow et al. (2010) \\
\hline pGP1007 & pWH844-pgm (MR08/MR09) & BamHI + PstI & Dutow et al. (2010) \\
\hline pGP1008 & pWH844-eno ${ }^{c}$ (MR10/MR11) & BamHI + PstI & Dutow et al. (2010) \\
\hline pGP1010 & pWH844-pgk (MR04-MR07) & BamHI + HindIII & Dutow et al. (2010) \\
\hline pGP1016 & pGP172-mpn549 (SS29/SS30) & SacI + BamHI & - \\
\hline pGP1017 & pGP172-mpn549 (SS29-SS31) & SacI + BamHI & - \\
\hline pGP1018 & pGP172-glpQ (SS34/SS35) & SacI + BamHI & This work \\
\hline pGP1019 & pGP172-glpQ ${ }^{\mathrm{C}}$ (SS34-SS38) & SacI + BamHI & This work \\
\hline pGP1020 & pGP172-mpn566 (SS39/SS40) & SacI + BamHI & This work \\
\hline pGP1100 & pWH844-pyk $k_{\mathrm{Bsu}}$ (pyk forw1/pyk rev) & BamHI + PstI & Pietack et al. (2010) \\
\hline pGP1401 & pWH844-glmM $M_{\text {Bsu }}(\mathrm{NP109/NP117)}$ & BamHI + SalI & Schmidl et al. (2010) \\
\hline pGP1403 & $\begin{array}{l}\text { pBQ200-glmM } M_{\text {Bsu }}[\mathrm{S} 100 \mathrm{~A}] \\
\text { (NP108/NP109/NP116) }\end{array}$ & BamHI + SalI & Schmidl et al. (2010) \\
\hline pGP1405 & $\begin{array}{l}\text { pWH844-glmM } M_{\text {Bsu }}[\mathrm{S} 100 \mathrm{~A}] \\
\text { (NP108/NP116/NP117) }\end{array}$ & BamHI + SalI & Schmidl et al. (2010) \\
\hline pGP1553 & pUT18C-pgic (PD05/PD06) & $X b a \mathrm{I}+K p n \mathrm{I}$ & Dutow et al. (2010) \\
\hline pGP1554 & pUT18C-pfkA (PD07/PD08) & $X b a \mathrm{I}+K p n \mathrm{I}$ & Dutow et al. (2010) \\
\hline pGP1555 & pUT18C-fba (PD09/PD10) & $X b a \mathrm{I}+\mathrm{SacI}$ & Dutow et al. (2010) \\
\hline pGP1556 & pUT18C-tpiAc (PD11/PD12) & $X b a \mathrm{I}+K p n \mathrm{I}$ & Dutow et al. (2010) \\
\hline pGP1557 & pUT18C-gap $A^{\mathrm{c}}$ (PD13/PD14) & $X b a \mathrm{I}+E c o R I$ & Dutow et al. (2010) \\
\hline pGP1558 & pUT18C-pgk (PD15/PD16) & $X b a \mathrm{I}+E c o R I$ & Dutow et al. (2010) \\
\hline pGP1559 & pUT18C-pgm (PD17/PD18) & $X b a \mathrm{I}+E c o R I$ & Dutow et al. (2010) \\
\hline pGP1560 & pUT18C-eno ${ }^{c}$ PD19/PD20) & $X b a \mathrm{I}+E c o R I$ & Dutow et al. (2010) \\
\hline pGP1561 & pUT18C-pyk ${ }^{\mathrm{c}}$ (PD21/PD22) & $X b a \mathrm{I}+K p n \mathrm{I}$ & Dutow et al. (2010) \\
\hline pGP1563 & pUT18-pgic (PD05/PD06) & $X b a \mathrm{I}+K p n \mathrm{I}$ & Dutow et al. (2010) \\
\hline pGP1564 & pUT18-pfkA (PD07/PD08) & $X b a \mathrm{I}+K p n \mathrm{I}$ & Dutow et al. (2010) \\
\hline pGP1565 & pUT18-fba $(\mathrm{PD} 09 / \mathrm{PD} 10)$ & $X b a \mathrm{I}+\mathrm{SacI}$ & Dutow et al. (2010) \\
\hline pGP1566 & pUT18-tpiA (PD11/PD12) & $X b a \mathrm{I}+K p n \mathrm{I}$ & Dutow et al. (2010) \\
\hline
\end{tabular}


Tab. S15. Continued.

\begin{tabular}{|c|c|c|c|}
\hline Name & Relevant characteristics $^{\text {ab }}$ & Used restriction sites & Reference \\
\hline pGP1567 & pUT18-gapA ${ }^{\mathrm{c}}$ (PD13/PD14) & XbaI + EcoRI & Dutow et al. (2010) \\
\hline pGP1568 & pUT18-pgk ${ }^{\mathrm{c}}$ (PD15/PD16) & $X b a \mathrm{I}+E c o R I$ & Dutow et al. (2010) \\
\hline pGP1569 & pUT18-pgm (PD17/PD18) & $X b a \mathrm{I}+E c o R I$ & Dutow et al. (2010) \\
\hline pGP1570 & pUT18-eno ${ }^{c}$ (PD19/PD20) & $X b a \mathrm{I}+E c o R I$ & Dutow et al. (2010) \\
\hline pGP1571 & pUT18-pyk (PD21/PD22) & $X b a \mathrm{I}+K p n \mathrm{I}$ & Dutow et al. (2010) \\
\hline pGP1573 & pKNT25-pgic (PD05/PD06) & $X b a \mathrm{I}+K p n \mathrm{I}$ & Dutow et al. (2010) \\
\hline pGP1574 & pKNT25-pfkA (PD07/PD08) & $X b a \mathrm{I}+K p n \mathrm{I}$ & Dutow et al. (2010) \\
\hline pGP1575 & pKNT25- $f b a^{c}$ (PD09/PD10) & $X b a \mathrm{I}+\mathrm{SacI}$ & Dutow et al. (2010) \\
\hline pGP1576 & pKNT25-tpiA (PD11/PD12) & $X b a \mathrm{I}+K p n \mathrm{I}$ & Dutow et al. (2010) \\
\hline pGP1577 & pKNT25-gapA $A^{\mathrm{c}}(\mathrm{PD} 13 / \mathrm{PD} 14)$ & $X b a \mathrm{I}+E c o R I$ & Dutow et al. (2010) \\
\hline pGP1578 & pKNT25-pgk ${ }^{\mathrm{c}}$ (PD15/PD16) & $X b a \mathrm{I}+E c o R I$ & Dutow et al. (2010) \\
\hline pGP1579 & pKNT25-pgm (PD17/PD18) & $X b a \mathrm{I}+$ EcoRI & Dutow et al. (2010) \\
\hline pGP1580 & pKNT25-eno $^{\mathrm{c}}$ (PD19/PD20) & $X b a I+E c o R I$ & Dutow et al. (2010) \\
\hline pGP1581 & pKNT25-pyk $k^{\mathrm{c}}(\mathrm{PD} 21 / \mathrm{PD} 22)$ & $X b a \mathrm{I}+K p n \mathrm{I}$ & Dutow et al. (2010) \\
\hline pKNT25 & $P_{l a c}-\mathrm{mcs}-c y a A\left(\mathrm{Kan}^{\mathrm{R}}\right)$ & - & Claessen et al. (2008) \\
\hline $\begin{array}{l}\text { pKT25- } \\
\text { zip }\end{array}$ & $P_{\text {lac }}$ cyaA-zip $\left(\mathrm{Kan}^{\mathrm{R}}\right)$ & - & Karimova et al. (1998) \\
\hline pMT85 & $\begin{array}{l}\text { Mini-transposon delivery vector for } \\
\text { M. pneumoniae }\left(\mathrm{Gm}^{\mathrm{R}} / \operatorname{Kan}^{\mathrm{R}}\right)\end{array}$ & - & $\begin{array}{l}\text { Zimmerman and } \\
\text { Herrmann (2005) }\end{array}$ \\
\hline pUT18 & $P_{l a c}-\mathrm{mcs}-c y a A\left(\mathrm{Amp}^{\mathrm{R}}\right)$ & - & Karimova et al. (1998) \\
\hline pUT18C & $P_{l a c}-c y a A-m c s\left(\mathrm{Amp}^{\mathrm{R}}\right)$ & - & Karimova et al. (1998) \\
\hline $\begin{array}{l}\text { pUT18C- } \\
\text { zip }\end{array}$ & $P_{l a c}-c y a A-z i p\left(\mathrm{Amp}^{\mathrm{R}}\right)$ & - & Karimova et al. (1998) \\
\hline pWH844 & $\begin{array}{l}\text { Allows overexpression of } \mathrm{N} \text {-terminal } \\
\text { His }_{6} \text {-tag fusion proteins in } \\
\text { E. coli DH5 } \alpha\left(\mathrm{Amp}^{\mathrm{R}}\right)\end{array}$ & - & Schirmer et al. (1997) \\
\hline
\end{tabular}

\footnotetext{
a Resistance abbreviations as follows: Amp, ampicillin; Erm, erythromycin; Gm, gentamicin; Kan, kanamycin.

${ }^{\mathrm{b}}$ Unless otherwise indicated, all genes are M. pneumonaie specific.

${ }^{c}$ All TGA opal codons in the wild type gene are mutated to TGG.

${ }^{\mathrm{d}}$ Only one TGA opal codon in the forward primer region is mutated to TGG.
} 


\section{Strains}

Tab. S16. Strains.

\begin{tabular}{|c|c|c|}
\hline Name & Genotype $^{\mathrm{a}}$ & Reference/Construction ${ }^{b}$ \\
\hline \multicolumn{3}{|l|}{ Bacillus subtilis } \\
\hline 168 & $\operatorname{trpC2}$ & Laboratory collection \\
\hline GP588 & $\begin{array}{l}\text { trpC2 } \Delta g l m M:: s p e c ; \text { complemented with } \\
\text { pGP400 }\end{array}$ & Schmidl et al. (2010) \\
\hline \multicolumn{3}{|l|}{ Escherichia coli } \\
\hline BL21(DE3)/pLysS & $\begin{array}{l}\left.\text { F- ompT hsdSB (rB- } \mathrm{mB}^{-}\right) \text {gal dcm (DE3) } \\
\text { pLysS }\left(\mathrm{Cam}^{\mathrm{R}}\right)\end{array}$ & Sambrook et al. (1989) \\
\hline BTH101 & $\begin{array}{l}\text { F- cya-99 araD139 galE15 galK16 rpsL1 } \\
\left(\mathrm{Str}^{\mathrm{R}}\right) \text { hsdR2 mcrA1 mcrB1 }\end{array}$ & Karimova et al. (2005) \\
\hline DH5 $\alpha$ & $\begin{array}{l}\text { F- } \text { Ф80lacZ } \triangle \text { M15 } \triangle(\text { lacZYA-argF }) \mathrm{U} 169 \\
\text { recA1 endA1 hsdR17 }\left(\mathrm{rK}^{-} \mathrm{mK}^{+}\right) \text {phoA supE44 } \\
\lambda \text { - thi-1 gyrA96 relA1 }\end{array}$ & Sambrook et al. (1989) \\
\hline XL1-Blue & $\begin{array}{l}\text { recA1 endA1 gyrA96 thi-1 hsdR17 supE44 } \\
\left.\text { relA1 lac [F' proAB lacI }{ }^{\mathrm{q}} \mathrm{Z} \Delta M 15 \text { Tn10 }\left(\text { Tet }^{\mathrm{R}}\right)\right]\end{array}$ & Stratagene \\
\hline \multicolumn{3}{|l|}{ Mycoplasma pneumoniae } \\
\hline GPM09 & M129 thyA::Tn4001m & Wang et al. (2010) \\
\hline GPM11 & M129 prkC::Tn4001m & Schmidl et al. (2010) \\
\hline GPM51 & M129 hprK::Tn4001m & Halbedel et al. (2006) \\
\hline GPM52 & M129 glpD::Tn4001m & Hames et al. (2009) \\
\hline GPM68 & M129 prpC::Tn4001m & Halbedel et al. (2006) \\
\hline GPM70 & M129 mpn474::Tn4001m & Hegermann et al. (2008) \\
\hline GPM80 & M129 mpn256::Tn4001m & pMT85 $\rightarrow$ M129 \\
\hline GPM81 & M129 mpn420::Tn4001m & This work \\
\hline GPM82 & M129 mpn566::Tn4001m & This work \\
\hline GPM84/1...GPM84/10 & $\begin{array}{l}\text { 1-5 Glc } g l p F(-321 \ldots+59)-\operatorname{lacZ} \\
\text { 6-10 Gly } \operatorname{glpF}(-321 \ldots+59)-\text { lacZ }\end{array}$ & pGP659 $\rightarrow$ M129 \\
\hline GPM85/1...GPM85/9 & $\begin{array}{l}\text { 1-5 Glc mpn239(-247...71)-lacZ } \\
\text { 6-9 Gly mpn239(-247...+71)-lacZ }\end{array}$ & pGP660 $\rightarrow$ M129 \\
\hline GPM86 & M129 mpn162::Tn4001m & pMT85 $\rightarrow$ M129 \\
\hline GPM87 & M129 mpn284::Tn4001m & pMT85 $\rightarrow$ M129 \\
\hline GPM88 & M129 mpn506::Tn4001m & pMT85 $\rightarrow$ M129 \\
\hline M129 (ATCC 29342) & Wild type & Somerson et al. (1963) \\
\hline
\end{tabular}

\footnotetext{
${ }^{a}$ Resistance abbreviations as follows: Cam, chloramphenicol; Spec, spectinomycin; Str, streptomycin; Tet, tetracycline.

${ }^{\mathrm{b}}$ The arrow indicates transformation.
} 


\section{Curriculum vitae}

Personal information

Name:

Sebastian Schmidl

Date of birth:

27th July, 1981

Place of Birth:

Bad Hersfeld, Germany

Nationality:

German

Parents:

Roland Schmidl and Petra Schmidl, née Haas

Sibling:

Saskia Schmidl

\section{School education}

1988 - 1992

Grundschule Friedlos, Ludwigsau

1992 - 1998

Gesamtschule Geistal, Bad Hersfeld

1998 - 2001

Modellschule Obersberg, Bad Hersfeld

$06 / 2001$

University-entrance diploma (Abitur)

\section{Community service}

2001-2002

Deutsches Rotes Kreuz, Bad Hersfeld

\section{Scientific education}

10/2002 - 09/2007

Study of biology, University of Göttingen

Diploma thesis in the group of Prof. Dr. Jörg Stülke, University of Göttingen

Thesis title:

"Control of the HPr kinase activity of

Mycoplasma pneumoniae”

$11 / 2007-11 / 2010$

PhD project:

"Pathogenicity of a minimal organism: Role of protein phosphorylation in Mycoplasma pneumoniae” Dept. of General Microbiology (Prof. Dr. Jörg Stülke), University of Göttingen

03/2008 - 11/2010 Awarded a grant from the Studienstiftung des deutschen Volkes 